\title{
Schutzziele im Umgang mit Naturrisiken in der Schweiz
}

\section{Report}

Author(s):

Hess, Josef Th.

Publication date:

2011

Permanent link:

https://doi.org/10.3929/ethz-a-005970532

Rights / license:

In Copyright - Non-Commercial Use Permitted 
Josef Th. Hess

\section{Schutzziele im Umgang mit Naturrisiken in der Schweiz}


Josef Th. Hess

Schutzziele im Umgang mit Naturrisiken in der Schweiz 
Abhandlung zur Erlangung des Titels Doktor der Wissenschaften der ETH Zürich

Diss. ETH No. 17956

angenommen auf Antrag von

Prof. Dr. Hans Rudolf Heinimann, Referent

Prof. Dr. Hansjörg Seiler, Korreferent

Dr. Kurt Hollenstein, Korreferent

\section{This work is licensed under a creative commons license}

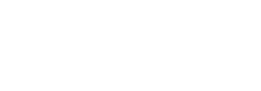

Bibliografische Informationen der Deutschen Nationalbibliothek Die Deutsche Nationalbibliothek verzeichnet diese Publikation in der Deutschen Nationalbibliografie; detaillierte bibliografische Daten sind im Internet abrufbar über http://dnb.d-nb.de.

ISBN 978-3-7281-3282-6 (Print-Ausgabe)

Download open access:

ISBN 978-3-7281-3390-8/DOI 10.3218/3390-8

www.vdf.ethz.ch

(C) 2011

vdf Hochschulverlag AG an der ETH Zürich 
Für meine Frau Luzia, unsere Kinder Karin und José

und

meinen Vater Josef Hess, verstorben 18. Juli 2008 
Wer weiss und weiss, dass er weiss, der ist weise - folge inm;

Wer nicht weiss und weiss, dass er nicht weiss, der ist ein Kind - unterweise inn;

Wer weiss und weiss nicht, dass er weiss, der liegt im Schlaf - wecke inn;

Wer nicht weiss und nicht weiss, dass er nicht weiss, der ist ein Narr - meide inn.

Arabisches Sprichwort,

zitiert in Haimes (Haimes, 2004) 


\section{Zusammenfassung}

Experten sind sich einig, dass absolute Sicherheit im Management von Risiken weder technisch erreichbar noch wirtschaftlich tragbar ist. Die Frage ,Wie sicher ist sicher genug?' ist deshalb für das Risikomanagement von zentraler Bedeutung. Als Antwort auf diese Frage entwickelte die Praxis zahlreiche Schutzzielvorschläge. Schutzziele umschreiben das Mass des tolerierbaren Risikos und die Verteilung des Restrisikos in der Gesellschaft.

Die schweizerische Rechtsordnung enthält keine konkreten Angaben zu Schutzzielen. In der Lehre zum technischen Sicherheitsrecht und zum Umweltrecht sind ein ,Dreistufenkonzept' und der sog. ,Zwei-Regel-Ansatz' von zentraler Bedeutung. Regel 1 garantiert die elementaren Sicherheitsbedürfnisse des Einzelnen; Regel 2 sieht eine Reduktion kollektiver Risiken nach Massgabe der Verhältnismässigkeit vor.

Schutzziele als Umschreibung von Mass und Verteilung des tolerierbaren Risikos sind eine Gerechtigkeitsfrage. Als massgebende Aspekte dieser Gerechtigkeit und damit auch als leitende Prinzipien für die Festlegung/Formulierung von Schutzzielen können die Bedürfnisse der Risikobetroffenen und das gesellschaftliche Umfeld (Kontext), der Einfluss der Betroffenen auf das Risiko, die Effizienz sowie die Sozial- und Umweltverträglichkeit risikovermindernder Massnahmen bezeichnet werden.

Neben einer kritischen Beurteilung der gebräuchlichen Schutzzielfestlegungen wird in dieser Publikation ein Vorschlag für ein schutzzielbasiertes Risikomanagementkonzept mit umfassender Berücksichtigung dieser Gerechtigkeitsaspekte im regionalen Risikomanagement unterbreitet. Der Test dieses Konzeptes in Fallbeispielen und Gedanken zu Organisation und Verfahren bei der Festlegung von tolerierbaren Risiken runden die Arbeit ab.

Schlüsselwörter: Schutzziel, Naturgefahr, tolerierbares Risiko, Gerechtigkeit, Fairness, risikobasiertes Recht, öffentliches Risikomanagement, Partizipation 


\section{Abstract}

There is a wide consensus among experts, that absolute safety in risk management is neither technically feasible nor economically affordable. The question 'How safe is safe enough?' plays a crucial role in risk management. To answer this question, risk management practitioners elaborated suggestions of protection objectives. Protection objectives express both the level of tolerable risk and the distribution of residual risk among society.

Swiss legal order provides no accurate indications on protection objectives. In theory on environmental and risk law a 'Three-Step-Concept' and a 'Two-Rules-Approach' are of major importance. Rule \#1 guarantees elementary safety needs of any individual, rule \#2 foresees further reduction of collective risks according to proportionateness of mitigation measures.

Protection objectives as an expression of the level and distribution of tolerable risks are a matter of justice. Social needs, the risk bearers own responsibility and the context of a risk situation as well as efficiency and social and environmental compatibility of risk mitigation measures may be addressed as important aspects of justice and also as guiding principles of protection objectives.

Commonly suggested protection objectives are analyzed in the light of these aspects and a procedure to implement those aspects in regional risk management is suggested. Case studies and some reflections on organizational and procedural aspects on the definition of tolerable risks complete this research.

Keywords: protection objective, natural hazard, tolerable risk, justice, fairness, risk based regulation, public risk management, participation 


\section{Inhaltsverzeichnis}

$1 \quad$ Einleitung 1

1.1 Ausgangslage 1

1.2 Vielfältige und lückenhafte Schutzziellandschaft 2

1.3 Konzept und Abgrenzung 3

1.4 Zielsetzung und Forschungsfragen 3

1.4.1 Zielsetzung 3

1.4.2 Forschungsfragen 4

$1.5 \quad$ Hypothesen und Gliederung 4

1.5.1 Hypothesen 4

1.5.2 Gliederung 5

2 Schäden, Risiken und Schutzziele in Theorie und Praxis $\quad 7$

2.1 Übersicht 7

2.2 Der Begriff des Schadens 8

2.2.1 Was ist ein Schaden? 8

2.2.2 Vorgehen zur Bestimmung eines Schadens 9

2.2.3 Schaden im rechtlichen Sinn 10

2.3 Verletzbare Werte und Schäden 12

2.3.1 Arten von verletzbaren Werten 12

2.3.2 Beeinträchtigung verletzbarer Werte als Schaden 12

$\begin{array}{ll}\text { 2.3.3 Allgemeingültigkeit der Schadensbewertung } & 14\end{array}$

$\begin{array}{ll}\text { 2.3.4 Rechtsgüter und verletzbare Werte } & 15\end{array}$

2.3.5 Bedeutung von verletzbaren Werten und Rechtsgütern 17

2.4 Integrale Schadens- und Risikoindikatoren 20

2.4.1 Indikatoren und Messgrössen 20

2.4.2 Qualität und Eignung von Indikatoren 22

2.4.3 Indikatoren zur vergleichenden Darstellung verletzbarer Werte 23

2.4.4 Monetarisierung von Schäden und Risiken 24

2.4.4.1 Methoden der Monetarisierung 24

2.4.4.2 Menschliches Leben und menschliche Gesundheit 26

2.4.4.3 Ökonomische Werte $\quad 27$

2.4.4.4 Umwelt 29

2.4.4.5 Lebensqualität $\quad 30$

2.4.4.6 Gesellschaftliche Strukturen und Aversion 31

2.5 Tolerierbarkeit von Effekten 31

2.5.1 Begriffe 31

2.5.2 Wahrnehmung, Risikoakzeptanz und Tolerierbarkeit 32

2.5.3 Schutzziele im Risikomanagement 34

2.6 Grenzwerte für tolerierbare Risiken 35

2.6.1 Grenzwerte der Verletzbarkeit 35

2.6.2 Grundlagen zu Grenzwerten des tolerierbaren Risikos 37

2.6.2.1 Gesellschaftlich akzeptierte Risiken 37

2.6.2.2 Zahlungsbereitschaft zur Vermeidung von Risiken 38

2.6.2.3 Berechnung tolerierbarer Risiken 39

2.6.2.4 Abstufung der Grenzwerte $\quad 40$

2.6.3 Grenzwerte für tolerierbare individuelle Todesfallrisiken 41

2.6.3.1 Grenzwerte aus der Literatur $\quad 41$

Josef Th. Hess: Schutzziele im Umgang mit Naturrisiken in der Schweiz, @ vdf Hochschulverlag 2011 
2.6.3.2 Grenzwerte in Schutzzielmatrizes $\quad 44$

2.6.4 Grenzkosten zur Verhinderung von Todesfällen 47

2.6.5 Grenzkosten zur Verhinderung weiterer Schäden 49

2.6.6 Akzeptanzlinien in Wahrscheinlichkeits-Ausmass-Diagrammen $\quad 50$

2.6.6.1 Lage der Akzeptanzlinien $\quad 50$

2.6.6.2 Berechnete tolerierbare Risiken in F/N-Diagrammen 50

2.7 Schutzzielkonzepte 54

2.7.1 Entwicklung von Schutzzielkonzepten 54

2.7.2 Grenzwerte für Versagenswahrscheinlichkeiten 55

2.7.3 Risikogrenzwerte als Schutzziele 56

2.7.4 Tolerierbare Risiken in Wahrscheinlichkeits-Ausmass-Diagrammen 59

$\begin{array}{ll}\text { 2.7.5 Grenzkosten als Schutzziele } & 60\end{array}$

2.7.6 Schutzzielschemen und -matrizes im Management von Naturrisiken 62

2.8 Operationale Aspekte bei Schutzzielen 64

2.8.1 Anforderungen an die operationale Eignung von Schutzzielen 64

2.8.2 Lücken bei den Informationsgrundlagen 65

2.8.3 Operationale Eignung von Schutzzieldarstellungen der Praxis $\quad 65$

2.9 Organisation und Verfahren zur Festlegung tolerierbarer Risiken $\quad 67$

$\begin{array}{ll}\text { 2.9.1 Politische und organisatorische Rahmenbedingungen } & 67\end{array}$

2.9.1.1 Rollen und Verantwortlichkeiten im Risikomanagement $\quad 67$

2.9.1.2 Aufgabenteilung zwischen Bund, Kanton und Gemeinden 69

2.9.1.3 Rahmenbedingungen NFA und effor2 70

2.9.2 Gesellschaftliche Entscheidverfahren 72

2.9.2.1 Arten von Entscheidverfahren und Entscheidungsebenen 72

$\begin{array}{ll}\text { 2.9.2.2 Charakteristiken gesellschaftlicher Entscheidverfahren } & 72\end{array}$

$\begin{array}{ll}\text { 2.9.2.3 Markt-Hierarchie-Paradigma } & 73\end{array}$

$\begin{array}{lll}\text { 2.9.3 Ablauf der Planung } & 74\end{array}$

2.9.4 Beteiligung der Betroffenen in Projekten zur Verminderung von $\begin{array}{ll}\text { Naturrisiken } & 75\end{array}$

2.9.4.1 Mitwirkung im formellen Verwaltungshandeln $\quad 75$

2.9.4.2 Partizipative Projektplanung 76

2.10 Erkenntnisse, Lücken und Handlungsbedarf 79

$\begin{array}{ll}2.10 .1 \text { Erfassung von Schäden und Risiken } & 79\end{array}$

$\begin{array}{ll}2.10 .2 \text { Tolerierbare Effekte } & 81\end{array}$

$\begin{array}{ll}2.10 .3 \text { Schutzziele } & 82\end{array}$

2.10.4 Operabilität 83

2.10.5 Organisation und Verfahren zur Festlegung tolerierbarer Risiken 84

3 Aspekte zur Festlegung der Tolerierbarkeit $\quad 87$

$3.1 \quad$ Übersicht $\quad 87$

3.2 Rechtliche Rahmenbedingungen 87

3.2.1 Schutzziele im Recht $\quad 87$

$\begin{array}{lll}3.2 .2 & \text { Rechtsordnung } & 88\end{array}$

$\begin{array}{lll}3.2 .3 & \text { Rechtsprechung } & 89\end{array}$

$\begin{array}{lll}3.2 .4 & \text { Rechtslehre } & 91\end{array}$

3.2.4.1 Lehre der grundrechtlichen Schutzpflichten 91

3.2.4.2 Schutzziele in der Lehre zum technischen Sicherheitsrecht 92

3.2.4.3 Schutzziele in der Rechtslehre zum Management von Naturrisiken 94

3.2.4.4 Risikobasiertes Recht 96

3.3 Handlungsleitende Prinzipien 96

3.3.1 Gerechtigkeit, Ethik und Schutzziele 96

Josef Th. Hess: Schutzziele im Umgang mit Naturrisiken in der Schweiz, @ vdf Hochschulverlag 2011 
3.3.2 Prinzipien für die Festlegung von Schutzzielen 98

3.3.2.1 Fünf handlungsleitende Prinzipien 98

3.3.2.2 Das Bedürfnisprinzip $\quad 98$

3.3.2.3 Das Einflussprinzip 102

3.3.2.4 Das Kontextprinzip 102

3.3.2.5 Das Effizienzprinzip 103

3.3.2.6 Das Prinzip Vernetzung mit Gesellschaft und Umwelt (Retinität) 104

3.3.3 Schutzzielprinzipien in Schutzzielkonzepten der Praxis 104

$3.4 \quad$ Konzeptionelle Synthese 106

3.4.1 Elemente zur Festlegung von Schutzzielen 106

$\begin{array}{ll}\text { 3.4.2 Wesentliche inhaltliche Elemente } & 107\end{array}$

3.4.3 Wesentliche formelle Elemente 108

3.4.4 Kernelemente eines schutzzielbasierten Risikomanagementkonzeptes

$4 \quad$ Vorschlag eines schutzzielbasierten Managementkonzeptes 111

$\begin{array}{lll}4.1 & \text { Übersicht } & 111\end{array}$

4.2 Schutzzielbasiertes, regionales Managementkonzept 112

4.2.1 Grundsätze 112

4.2.2 Vorgehen 113

4.2.2.1 Überblick 113

4.2.2.2 Ermittlung des Handlungsbedarfs nach Regel 1

4.2.2.3 Priorisierung der Massnahmen nach Regel 2

4.2.3 Die Elemente des Prioritätsindexes 117

4.2.3.1 Bewertung der Objekte und Risikoverminderungsmassnahmen $\quad 117$

4.2.3.2 Der Objektindex 118

4.2.3.3 Der Massnahmenindex 120

4.2.3.4 Der Gefährdungsfaktor 123

4.3 Exkurs 1: Vereinfachte Abschätzung des regionalen Handlungsbedarfs 124

4.4 Exkurs 2: Modifikation der BUWAL-Schutzzielmatrix 126

4.5 Reduktion untolerierbarer Risiken 127

4.5.1 Regionales Risikomanagement 127

4.5.2 Einzelprojekte 128

4.6 Organisation und Verfahren des Risikomanagements 129

4.6.1 Optimale gesellschaftliche Entscheidverfahren 129

4.6.2 Arten der Koordination aufgrund des Markt-Hierarchie-Paradigmas 129

4.6.3 Rollen und Aufgaben im schutzzielbasierten Risikomanagement 131

4.6.4 Einbezug der Betroffenen 132

$5 \quad$ Fallbeispiele 135

$\begin{array}{lll}5.1 & \text { Inhalt und Untersuchungsgebiet } & 135\end{array}$

5.1.1 Inhalt der Fallbeispiele $\quad 135$

5.1.2 Beschreibung der Untersuchungsgebiete 135

5.1.2.1 Engelberg 135

5.1.2.2 Sarnersee und Sarneraa 137

5.1.3 Laufende und geplante Projekte zur Verminderung
von Naturrisiken

5.2 Fallbeispiele 1-3: Engelberg 140

5.2.1 Fallbeispiel 1: Test des Managementkonzeptes 140

5.2.1.1 Grundlagen 140

5.2.1.2 Vorgehen 140

Josef Th. Hess: Schutzziele im Umgang mit Naturrisiken in der Schweiz, ๑ vdf Hochschulverlag 2011 
5.2.1.3 Prioritäten gemäss Managementkonzept $\quad 141$

5.2.1.4 Diskussion der Resultate 144

5.2.1.5 Vergleich mit dem Masterplan 145

5.2.1.6 Methodische Erkenntnisse 145

5.2.2 Fallbeispiel 2: Vereinfachte Abschätzung des Handlungsbedarfs 147

5.2.2.1 Grundlagen und Vorgehen 147

5.2.2.2 Prioritäten aus vereinfachter Abschätzung des Handlungsbedarfes 147

5.2.2.3 Diskussion der Resultate und Vergleich mit dem Masterplan 148

$\begin{array}{lll}\text { 5.2.3 Fallbeispiel 3: Schutzzielmatrizes } & 148\end{array}$

5.2.3.1 Grundlagen und Vorgehen 148

5.2.3.2 Prioritäten gemäss Schutzzielmatrizes $\quad 149$

5.2.3.3 Diskussion der Resultate 151

5.2.3.4 Vergleich mit dem Masterplan $\quad 151$

5.2.4 Vergleich der Resultate zwischen den Verfahren 152

5.3 Fallbeispiel 4: Hochwasserschutz Sarneraatal 153

$\begin{array}{lll}\text { 5.3.1 Ziel des Fallbeispieles } & 153\end{array}$

$\begin{array}{ll}\text { 5.3.2 Politische Rahmenbedingungen } & 153\end{array}$

$\begin{array}{ll}\text { 5.3.3 Variantenevaluation im partizipativen Verfahren } & 154\end{array}$

5.3.4 Durch Verbände und Verwaltung erwirkter Richtungswechsel 157

5.3.5 Demokratische Entscheidungskontrolle I: Politische Entscheidung 157

5.3.6 Demokratische Entscheidungskontrolle II: Nachspiel zum Variantenentscheid 158

5.3.7 Erkenntnisse aus dem Fallbeispiel 158

$6 \quad$ Diskussion und Folgerungen 159

$\begin{array}{lll}6.1 & \text { Schadensbegriff } & 159\end{array}$

6.1.1 Problemstellung 159

6.1.2 Erkenntnisse 159

6.1.3 Würdigung 159

6.2 Festlegung tolerierbarer Risiken 159

$\begin{array}{lll}\text { 6.2.1 Problemstellung } & 159\end{array}$

$\begin{array}{lll}6.2 .2 & \text { Erkenntnisse } & 160\end{array}$

$\begin{array}{ll}6.2 .3 \text { Würdigung } & 160\end{array}$

6.3 Managementkonzept 161

$\begin{array}{ll}\text { 6.3.1 Problemstellung } & 161\end{array}$

$\begin{array}{ll}\text { 6.3.2 Erkenntnisse } & 161\end{array}$

6.3.2.1 Zwei Ebenen, zwei Regeln und fünf Schutzzielprinzipien 161

6.3.2.2 Bestehende Schutzzielkonzepte 162

6.3.2.3 Organisation und Entscheidfindung im Risikomanagement $\quad 162$

$\begin{array}{lr}\text { 6.3.2.4 Fallbeispiele } & 163\end{array}$

$\begin{array}{ll}\text { 6.3.3 Würdigung } & 163\end{array}$

6.4 Offene Fragen und weitere Arbeiten 164

$\begin{array}{ll}\text { 6.4.1 Schäden } & 164\end{array}$

$\begin{array}{ll}\text { 6.4.2 Wirksamkeit von Massnahmen } & 165\end{array}$

6.4.3 Schutzzielprinzipien und Risikomanagementkonzept 165

6.4.4 Risikomanagementstrategien in konkreten Gefahrensituationen 165

$\begin{array}{ll}\text { 6.4.5 Organisation } & 166\end{array}$

$\begin{array}{lll}6.5 & \text { Schlussbemerkung } & 166\end{array}$

$\begin{array}{lr}\text { Verzeichnisse } & 169\end{array}$

$\begin{array}{ll}\text { Anhänge } & 197\end{array}$

Josef Th. Hess: Schutzziele im Umgang mit Naturrisiken in der Schweiz, @ vdf Hochschulverlag 2011 


\section{Einleitung}

\subsection{Ausgangslage}

Naturereignisse wie Lawinen, Überschwemmungen, Rutschungen oder Steinschlag sind in der Schweiz verbreitete Phänomene. Sie gehören zur Entwicklung unserer Landschaft; eine Entwicklung, die über Jahrmillionen unaufhaltsam naturgesetzlichen Abläufen folgt. Wenn Naturereignisse und menschliche Aktivitäten aufeinander treffen, werden sie für den Menschen zum Risiko und damit zum gesellschaftlichen Thema. Der Umgang mit Naturrisiken beschäftigt die Schweizer Bevölkerung seit Jahrhunderten.

Bis weit in die 1990er-Jahre war der Umgang mit Naturgefahren ereignisgesteuert. Die Wertedichte und -verletzbarkeit nahm in den vergangenen Jahrzehnten stark zu. Zunehmend wurde klar, dass sich mit den verfügbaren Mitteln nicht alle Gefahren beseitigen lassen. Die im Jahr 1993 in Kraft gesetzten Bundesgesetze über den Wald (WaG, SR 921.0) und über den Wasserbau (WBG, SR 721.100) beschränken Massnahmen der Öffentlichkeit auf den Schutz vor Naturgefahren bereits auf Menschen und erhebliche Sachwerte. Und die 1997 vom Bundesrat eingesetzte Nationale Plattform Naturgefahren [PLANAT] plädiert in ihrer Strategie für einen Paradigmenwechsel von der Gefahrenabwehr zur Risikokultur (PLANAT, 2002).

Methodisch wurden in der Folge verschiedene Grundlagen für einen risikobasierten Umgang mit Naturgefahren geschaffen. Mit der Arbeit von Hollenstein (Hollenstein, 1997) wurde das im technischen Sicherheitsbereich entwickelte Risikokonzept auf das Management von Naturrisiken übertragen. 1999 publizierte das Bundesamt für Umwelt, Wald und Landschaft [BUWAL, heute BAFU] die an der Professur für forstliches Ingenieurwesen der ETHZ entwickelte Anleitung zur Risikoanalyse bei gravitativen Naturgefahren (Borter, 1999).

Es ist allgemein anerkannt, dass sich ein Risiko nie eliminieren lässt, es sei denn, man verzichte auf das mit diesem Risiko verbundene Verhalten. Da riskantes Verhalten meistens auch Nutzen bringt, ist Verzicht nur selten eine wünschenswerte Option. Vielmehr wird versucht, die Risiken zu vermindern, ohne auf die riskante Tätigkeit zu verzichten. Der Risikoverminderung sind aber Grenzen gesetzt: Vor allem verursachen Sicherheitsmassnahmen Kosten, die mit zunehmendem Sicherheitsanspruch meist überproportional steigen. Oft setzt auch die technische Machbarkeit Grenzen oder Schutzmassnahmen führen zu Zielkonflikten mit anderen gesellschaftlichen Interessen, z. B. dem Schutz der Umwelt. Nicht zuletzt stehen Sicherheitsmassnahmen oder risikominderndes Verhalten auch in Widerspruch zur persönlichen Freiheit.

Solange jemand die Folgen des riskanten Verhaltens selber trägt, ist der Entscheid, welche Risiken jemand eingehen will, persönlich. Allerdings hat die Risikobereitschaft einer Einzelperson sehr schnell Auswirkungen auf die Gesellschaft, z. B. Leid für Angehörige oder volkswirtschaftliche Kosten durch Verlust von Menschenleben, Heilungskosten oder - oft von der Allgemeinheit getragene - Wiederherstellungskosten. Naturereignisse betreffen oft zahlreiche Personen gleichzeitig. Von der Allgemeinheit wird erwartet, dass sie Präventions- beziehungsweise Schadensminderungsmassnahmen ergreift oder bei der Wiederherstellung nach Schäden hilft. Zur Festlegung, wie viel Risiko im Zusammenhang mit einem bestimmten Verhalten tolerierbar ist, ist eine personenübergreifende Festlegung erforderlich. In diesem Sinne vereinbarte tolerierbare Effekte werden als Schutzziele bezeichnet. Die Festlegung von Schutzzielen stellt somit einen unverzichtbaren Bestandteil des Umgangs mit Risiken dar. 


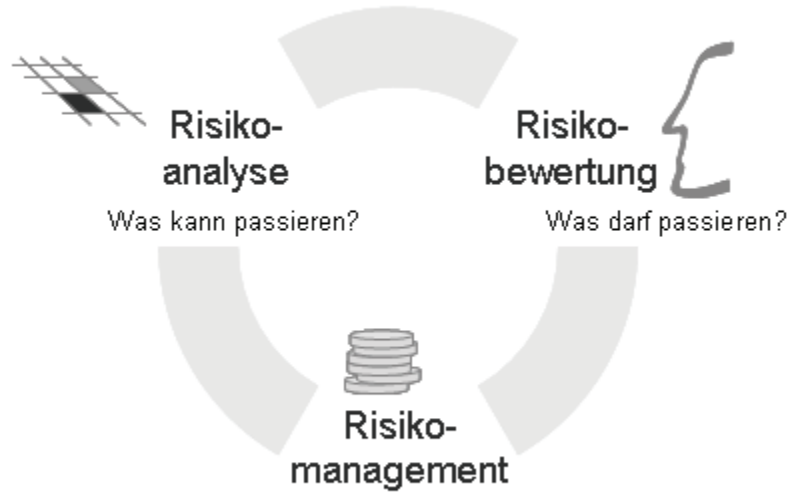

Was können wir tun?

Abbildung 1 Das Risikokonzept (Darstellung des Departementes Umweltwissenschaften [D-UWIS] der ETHZ, Hollenstein, 1997).

Methodischer Rahmen ist das Gedankenmodell des Risikokonzeptes (Abbildung 1). Dieses umfasst drei Elemente: Risikoanalyse (Was kann passieren?), Risikobewertung (Was darf passieren?) und Risikomanagement (Was können wir tun?). Die Differenz zwischen Risiko (Was kann passieren?) und Schutzziel (Was darf passieren?) wird als Schutzdefizit bezeichnet. Die Formulierung dieser Schutzdefizite (Sicherheitslücken) ist Kernaufgabe des Risikomanagements und setzt die Festlegung von Schutzzielen voraus.

Die Formulierung von Schutzzielen dient verschiedenen Zwecken:

1. Schaffung allgemeinverbindlicher Regeln über das Mass an Sicherheit, das bei bestimmten gesellschaftlichen Aktivitäten einzuhalten ist. Diese Zielsetzung birgt grosses Konfliktpotenzial mit der persönlichen Freiheit.

2. Schaffung von Regeln für die Handlungspflicht der Gemeinwesen im Risikomanagement. Schutzziele werden in diesem Zusammenhang als Abgrenzung des Verantwortungsbereiches der öffentlichen Hand zum Verantwortungsbereich des Risikobetroffenen verstanden.

3. Schaffung von Regeln für die Beurteilung risikorelevanter Strukturen und für die Ausübung riskanter Aktivitäten. Diese Zielsetzung betrifft vor allem Planer von Einrichtungen, die einem Risiko ausgesetzt oder mit einem Risiko verbunden sind. Hier geben die Regeln vor, welche Einwirkungen berücksichtigt werden müssen, damit die geforderten Sorgfaltspflichten eingehalten sind.

Die vorliegende Arbeit fokussiert die zweite Zielsetzung. Die Grenze zwischen Verantwortung der Allgemeinheit und der Eigenverantwortung ist im Management von Naturrisiken zentral, da hier die öffentliche Hand eine bedeutende Rolle einnimmt.

\subsection{Vielfältige und lückenhafte Schutzziellandschaft}

Die Praxis des öffentlichen Risikomanagements kennt verschiedene Schutzzielkonzepte (Basler \& Hofmann, 2008). Besonders im Management von Naturrisiken handelt es sich um pragmatische Festlegungen, bei denen in der Regel ein Bezug zu wissenschaftlichen Grundlagen besteht. Trotz der pragmatischen Herleitung sind bekannte Schutzziele Ausdruck eines Grundkonsenses über eine massvolle und gerechte Höhe und Verteilung von tolerierbaren Risiken. Dieser Grundkonsens wird von breiten Kreisen der Fachwelt und auch der Bevölkerung getragen. 
Im regionalen Risikomanagement stehen die Fragen im Zentrum, wie viele Mittel zur Risikominderung bereitzustellen sind und wie begrenzte Mittel in einem bestimmten Gebiet am besten zur Risikominderung eingesetzt werden können. Es gilt demzufolge festzustellen, welche Sicherheitsbedürfnisse abgedeckt werden müssen und welcher Aufwand nötig ist, um diese Sicherheitsbedürfnisse abzudecken. Auf Stufe Einzelprojekt geht es nebst der Beurteilung der zu deckenden Sicherheitsbedürfnisse vor allem um die Evaluation möglichst wirksamer Massnahmenbündel zur Risikoverminderung.

Im praktischen Alltag des Risikomanagements stösst man mit den erwähnten Schutzzielen immer wieder an Grenzen. Häufig werden Massnahmen aufgrund isolierter Einzelplanungen, als Reaktion auf Schadensereignisse oder als Folge politischer Vorstösse umgesetzt. Die Budgetbereitstellung orientiert sich an derartigen Gegebenheiten und an Gewohnheiten. Ob die verfügbaren Ressourcen optimal eingesetzt werden, darf hinterfragt werden. Ebenfalls ist unklar, wie weit die Bedürfnisse der betroffenen Bevölkerung tatsächlich in die Entscheide einfliessen. Das heisst nicht die Schutzziele, sondern planerische, politische oder ökonomische Gegebenheiten bestimmen das Risikomanagement.

\subsection{Konzept und Abgrenzung}

Naturrisiken lassen sich in einer Wirkungskette von Prozess, Einwirkung, Exposition und Effekt beschreiben (Seiler, 1997, Seiler, 2000 und Abbildung 2).
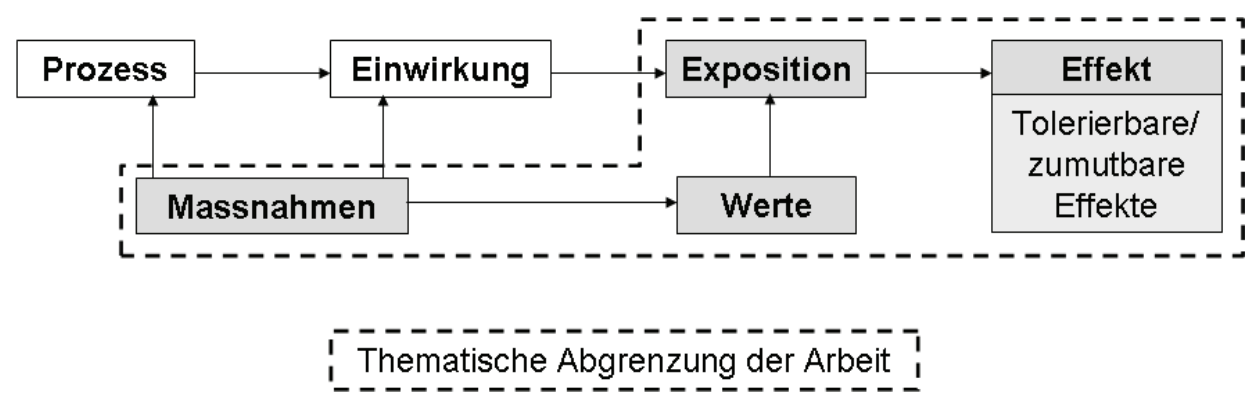

Abbildung 2 Wirkungskette von Naturrisiken (Seiler, 1997; abgeändert).

Prozesse wirken auf die Umwelt. Diese Einwirkungen erzeugen bei entsprechender Exposition Effekte. Die Exposition ist abhängig von den Objekten (,Assets'), die der Gefahr ausgesetzt sind, und deren Wert sowie Verletzlichkeit. Massnahmen können den Prozess oder die Einwirkungen verändern (z. B. technische Verbauungen, Schutzwaldbegründung und -pflege), haben aber auch Einfluss auf die Werte (z. B. raumplanerische Massnahmen) oder deren Verletzlichkeit (z. B. Objektschutzmassnahmen). Die vorliegende Arbeit beschränkt sich auf Expositionen und Effekte. Massnahmen als Teil der Wirkungskette werden in die Überlegungen einbezogen. Nicht Gegenstand der Untersuchungen sind Prozesse und deren Einwirkungen (Abbildung 2).

Naturrisiken stellen ein Teil des Gesamtrisikos einer Gesellschaft dar. Wenn technische und soziale Risiken nicht diskutiert werden, bleibt also ein erheblicher Teil der Risiken für die Gesellschaft unbehandelt.

\subsection{Zielsetzung und Forschungsfragen}

\subsubsection{Zielsetzung}

Angesichts der zentralen Bedeutung von Schutzzielen im Risikomanagement und der diversen und lückenhaften Praxis ist wichtig, 
- dass Schutzziele hinsichtlich ihrer wissenschaftlichen Grundlagen hinterfragt werden und auf dem aktuellen wissenschaftlichen Wissenstand aufbauen;

- dass Schutzziele sowohl auf regionaler Ebene als auch auf Ebene der Einzelprojekte alle relevanten Aspekte in der Bemessung der tolerierbaren Risiken abdecken;

- dass Schutzziele so formuliert sind, dass sie in der Praxis zu Lösungen führen, die die gesellschaftlichen Bedürfnisse optimal abdecken.

Das Dokument ,Vision und Strategie - Sicherheit vor Naturgefahren' (PLANAT, 2002) fordert eine Reihe von Massnahmen zur Verbesserung der Sicherheit vor Naturgefahren. Eine dieser Massnahmen ist die Festlegung klarer einheitlicher Schutzziele.

Für diese Untersuchung werden daher folgende Ziele gesetzt:

1. Herleiten relevanter Grundlagen zur Festlegung und Anwendung von Schutzzielen;

2. Darstellen und Prüfen bekannter Schutzzielkonzepte hinsichtlich inhaltlicher und operationeller Eignung;

3. Entwickeln eines schutzzielbasierten Managementkonzeptes zum Umgang mit Naturrisiken;

4. Erörtern organisatorischer Abläufe und der Entscheidungsfindung, soweit diese für Schutzziele und Risikomanagement grosse Bedeutung haben.

\subsubsection{Forschungsfragen}

Folgende Fragen werden angegangen:

1. Welches sind geeignete Indikatoren und Messgrössen für eine mehrdimensionale Metrik bei Schäden und Schutzzielen?

2. Wie und unter welchen Umständen eignen sich die bekannten Schutzzielumschreibungen für das Management von natürlichen Risiken?

3. Welche Prinzipien sind leitend für die Festlegung von Schutzzielen?

4. Wie lässt sich ein Schutzzielkonzept für das Management von Naturrisiken formulieren, das

- die leitenden Prinzipien berücksichtigt;

- im Vergleich mit anderen Risikobereichen angemessene Sicherheitsstandards definiert;

- im Einklang mit der schweizerischen Rechtsordnung und Rechtspraxis steht;

- hohe Anforderungen an Operabilität und Praxistauglichkeit erfüllt;

- eine demokratische Legitimation und Mitwirkung der Betroffenen ermöglicht und

- zu einem auf die individuellen und gesellschaftlichen Bedürfnisse ausgerichteten, effizienten Handeln führt?

\subsection{Hypothesen und Gliederung}

\subsubsection{Hypothesen}

Folgende Hypothesen werden formuliert:

1. Ein umfassender Schadensbegriff als Grundlage für die Beschreibung von Risiken und Schutzzielen ist formulierbar.

2. Aus der Gerechtigkeitstheorie und Ethik ergeben sich umfassende Prinzipien zur Formulierung von Schutzzielen, die sich in Rechtsordnung, Rechtslehre und Rechtsprechung widerspiegeln und für schweizerische Verhältnisse präzisieren und umsetzen lassen.

3. Es kann ein risikobasiertes Managementkonzept mit den folgenden Elementen und Eigenschaften entwickelt werden: 
(1) umfassender Schadensbegriff;

(2) Schutzziele auf Basis gerechtigkeitstheoretischer und ethischer Prinzipien;

(3) Übereinstimmung mit der schweizerischen Rechtsordnung, Rechtslehre und Rechtspraxis;

(4) Aufbau auf herkömmlichen Instrumenten der Gefahren- und Risikobeurteilung, namentlich Gefahren- und Schadenspotenzialkarten;

(5) Einbezug aller massgeblich Betroffenen.

4. Von der Praxis entwickelte und angewendete Schutzzielkonzepte eignen sich - allenfalls mit Anpassungen - zur Anwendung im Management von Naturrisiken auf regionaler Ebene und in Einzelprojekten.

\subsubsection{Gliederung}

Die Auseinandersetzung mit den Schutzzielen für das Management von Naturrisiken ist in folgende Schritte gegliedert (Abbildung 3):

1. Erfassen verletzbarer Werte aus der Literatur und Rechtsordnung (Kapitel 2.3);

2. Erfassen und Beurteilen von Schadensindikatoren in Literatur und Praxis (Kapitel 2.4);

3. Erfassen tolerierbarer Effekte aus Literatur und Praxis (Kapitel 2.5);

4. Ermitteln von Grenzwerten tolerierbarer Risiken aus Literatur und eigenen Berechnungen (Kapitel 2.6);

5. Darstellen von Schutzzielkonzepten aus der Literatur und Praxis (Kapitel 2.7);

6. Evaluieren von Operabilitätskriterien und Beurteilung bekannter Schutzziele hinsichtlich Einsetzbarkeit (Kapitel 2.8);

7. Darstellen von Organisationsformen und Verfahren zur Festlegung tolerierbarer Risiken anhand der Literatur und Praxis (Kapitel 2.9);

8. Analysieren der Rechtsordnung, der Rechtslehre sowie der Rechtsprechung (Leiturteile des Bundesgerichts und ausgewählter kantonaler Gerichte) hinsichtlich Angaben zu Sicherheitsanforderungen und Herleiten von Konsequenzen für die Schutzziele (Kapitel 3.2);

9. Erfassen handlungsleitender Prinzipien für die Festlegung von Schutzzielen aus der Literatur zur Gerechtigkeitstheorie und Ethik sowie Abschätzen der Bedeutung der leitenden Prinzipien bei bestehenden Schutzzielsystemen (Kapitel 3.3);

10. Vorschlagen eines schutzzielbasierten Managementkonzeptes zur Identifikation von Schutzdefiziten und zur Festlegung und Priorisierung des Handlungsbedarfs einschliesslich grundsätzlicher Überlegungen zur Rolle der wichtigsten Beteiligten und zu Verfahrensfragen (Kapitel 4);

11. Verifizieren und Testen des Managementkonzeptes an Fallbeispielen im Kanton Obwalden (OW) (Kapitel 5);

12. Diskutieren der Ergebnisse (Kapitel 6). 


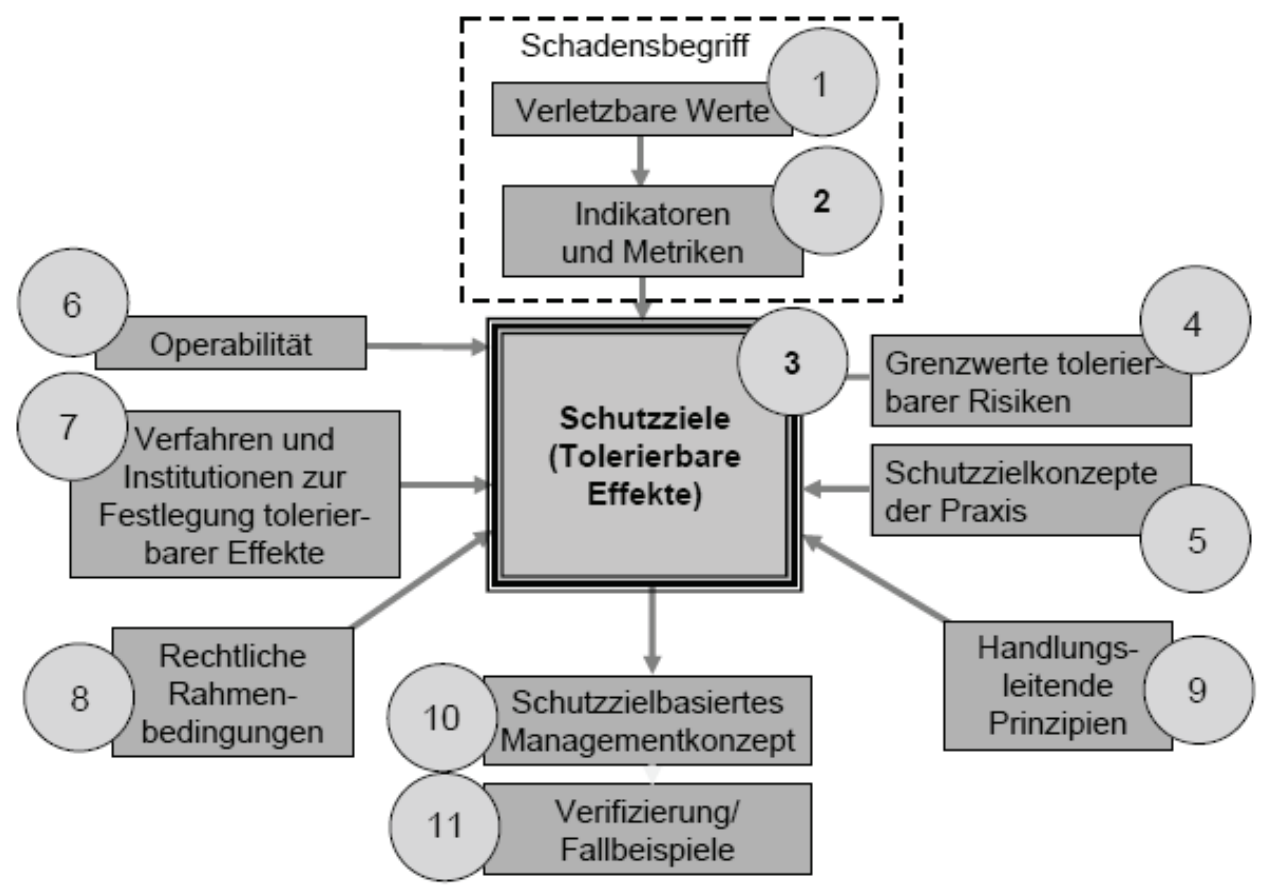

Abbildung 3 Aufbau der Publikation, Nummerierung der Arbeitsschritte. 


\section{Schäden, Risiken und Schutzziele in Theorie und Praxis}

\section{1 Übersicht}

Kapitel 2 fasst den Stand des Wissens zum Schadensbegriff (Kapitel 2.2), zu den verletzbaren Werten (Kapitel 2.3), den Indikatoren zur Darstellung des Schadens (Kapitel 2.4) sowie den Grenzwerten der Verletzbarkeit und des Risikos (Kapitel 2.5 und 2.6) zusammen. Die in der Praxis verbreiteten Schutzzieldarstellungen werden als Übersicht präsentiert (Kapitel 2.7). Aspekte der Operabilität von Schutzzielen werden aufgezeigt und diskutiert (Kapitel 2.8). Abschliessend werden Wissensgrundlagen betreffend Verfahren zur Festlegung von Schutzzielen dargestellt (Kapitel 2.9).

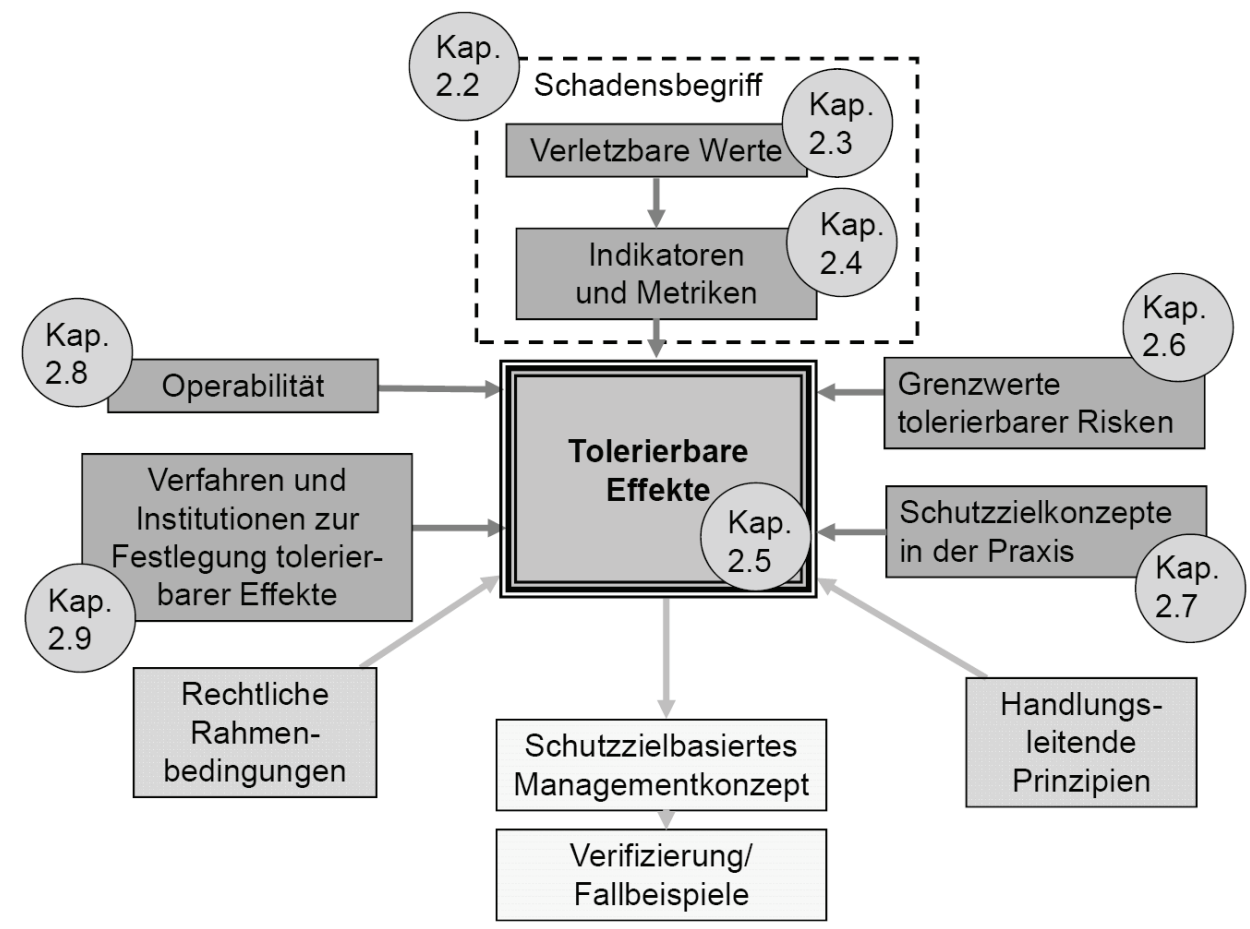

Abbildung 4 Kapitel 2 in der Übersicht.

Risiko- und Schutzzielfragen stellten sich anfänglich im Bereich der technischen Wissenschaften. Aus diesen Disziplinen stammen die ersten Ansätze zur Ermittlung von Risiken sowie Angaben zu deren Tolerierbarkeit. Die - meist als Expertenmeinung definierten Schutzziele haben ihre Wurzeln in der sozialempirischen Forschung. Den Angaben liegen Erhebungen über Risiken, die von der Gesellschaft mehr oder weniger freiwillig und unwidersprochen hingenommen werden, zugrunde. Ferner sind ökonomische und rechtliche Fragen für die Festlegung von Schutzzielen von Bedeutung. In ökonomischer Hinsicht interessieren ein optimales Risikomanagement mit beschränkten Mitteln, Versicherungsaspekte und die Zahlungsbereitschaft zur Risikoverminderung. Aus rechtlicher Sicht steht der Schutz bedeutender Rechtsgüter im Zentrum; aber auch bei Haftungsfragen ist die Tolerierbarkeit von Risiken zentral. 


\subsection{Der Begriff des Schadens}

\subsubsection{Was ist ein Schaden?}

Im Risikomanagement wird häufig die Formel

$$
\text { Risiko = Wahrscheinlichkeit * Auswirkungen [1] }
$$

verwendet (Kaplan, 1981). Die für das Risikomanagement im Sinne dieser Arbeit massgebenden Auswirkungen bestehen in der Verletzung von Werten oder von Rechtsgütern und werden als Schäden bezeichnet. Schutzziele beschreiben tolerierbare Risiken und werden daher mit den gleichen Faktoren dargestellt wie das Risiko.

Der Begriff des Schadens bedarf einer vertieften Erläuterung. Die Literatur zeigt verschiedene Probleme des Schadensbegriffes (u. a. Berg et al., 1994; Greiving, 2002): Schaden im allgemeinen Sinn lässt sich als Zerstörung, Minderung und Beeinträchtigung von konkreten oder abstrakten Werten definieren (Greiving, 2002). Eine sehr detaillierte, über 150 Arten von Schäden umfassende Auflistung wurde im Rahmen des Polyprojektes ,Risiko und Sicherheit' erarbeitet (Berg et al., 1994; Seiler, 2002). Was als Schaden oder Nutzen aufgefasst wird, hängt auch von Wertvorstellungen ab. Da Wertungen subjektiv sind, ist auch der Schadensbegriff subjektiv (Seiler, 1997). Was für eine Person einen Verlust darstellt, bedeutet unter Umständen für jemand anderes einen Gewinn. Somit gibt es grundsätzlich keinen allgemeingültigen Schadensbegriff und folglich auch keinen allgemein gültigen Begriff des Risikos, der eine ,objektive' Risikoanalyse ermöglichen würde (Berg et al., 1994). Es ist eine Frage der Definition, was als Schaden gilt. Diese Festlegung des Schadensbegriffes ist nicht zuletzt auch eine ,Machtfrage' (Berg, 1994; Greiving, 2002). Wer über die Macht verfügt, festzulegen, was ein Schaden sei, kann den Schadensbegriff beeinflussen.

Oft fehlt das Instrumentarium zur Erfassung der Schäden oder es wird - trotz vorhandener Methoden - bewusst auf den häufig erheblichen Aufwand einer umfassenden Schadenserhebung verzichtet. Aus den erwähnten und aus praktischen Gründen werden die als Schäden berücksichtigten Auswirkungen stets nur einen Teil der gesamten Auswirkungen umfassen. Dieser Teil stellt die Schnittmenge der negativ bewerteten und zugleich methodisch erfassbaren Auswirkungen dar (Berg et al., 1994; Abbildung 5).

\section{gesamte Auswirkungen}

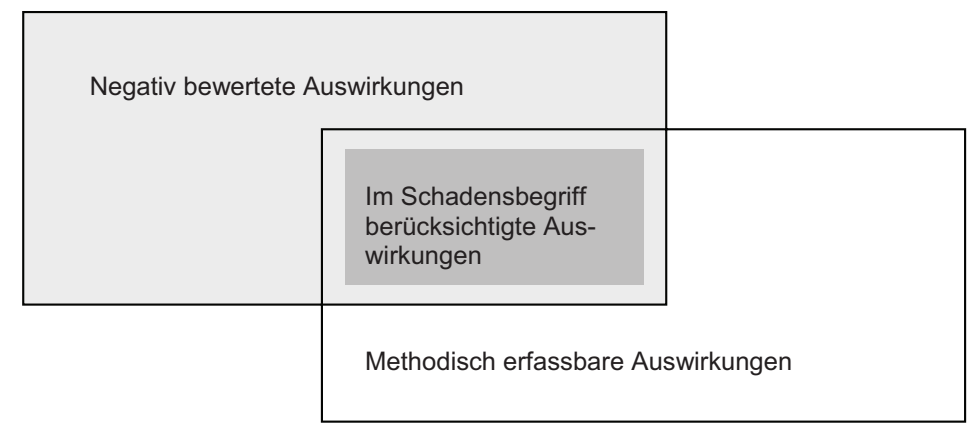

Abbildung 5 Auswirkungen eines Ereignisses und Schadensbegriff (nach Berg et al., 1994). Der operationelle Schadensbegriff bedingt Wissen (i) über die methodische Erfassbarkeit und (ii) über die negative Bewertung der Auswirkung.

Risikobeschreibungen beschränken sich in der Praxis oft auf Schäden, die mit wenig Aufwand zu quantifizieren sind (Tabelle 1). Wesentliche Aspekte des Risikos bleiben damit unberücksichtigt, was mitunter irreführende Resultate ergibt (Seiler, 2002). 
Tabelle $1 \quad$ Verwendete Indikatoren in ausgewählten Berichten über Schadensereignisse und Risiken.

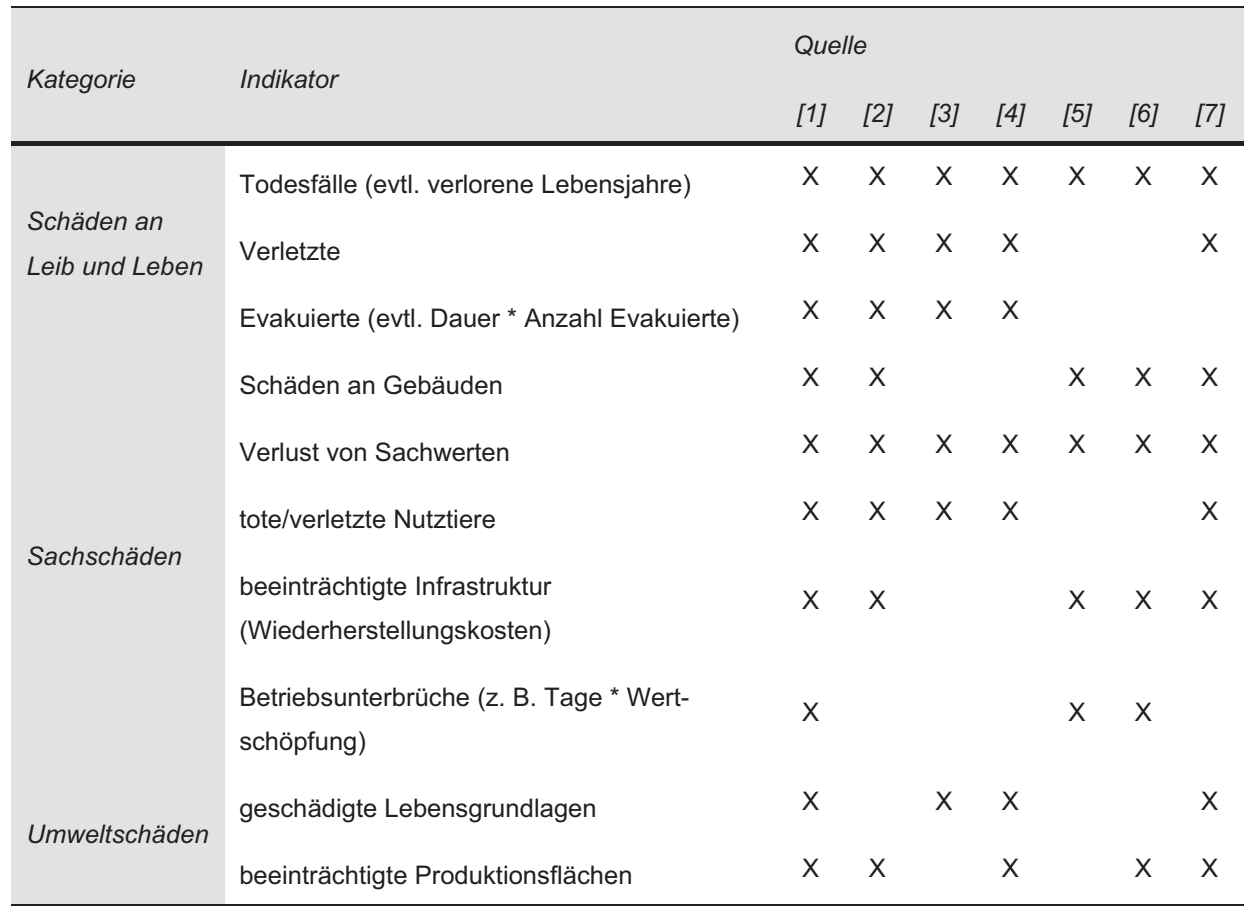

Legende zu den Literaturangaben

[1] Berg et al. (1994)

[2] BUWAL (1999)

[3] BABS (2003)

[4] BUWAL (1991)

[5] Röthlisberger (1998)

[6] SLF (2000); WSL und BUWAL (2001)

[7] PLANAT (2004b)

Sämtliche in Tabelle 1 erwähnten Berichte nennen Todesopfer und Verluste von Sachwerten als Schäden. Schäden an Gebäuden, an Produktionsflächen und an Nutztieren werden als Unterkategorien der Sachschäden explizit ausgewiesen. Kaum erwähnt werden demgegenüber Schäden an gesellschaftlichen Institutionen, ökologische Schäden oder die Beeinträchtigung der Lebensqualität. Daraus ergibt sich als wichtigste Konsequenz für Risikoanalyse und -management, dass der im Einzelfall angewandte Schadensbegriff immer offenzulegen und dessen Verlässlichkeit und Aussagekraft abzuschätzen ist (Berg, 1994).

\subsubsection{Vorgehen zur Bestimmung eines Schadens}

Unabdingbar für jede Schadensbestimmung ist die Abgrenzung des Systems in räumlicher, zeitlicher und sachlicher Hinsicht. Für jede Situation ist zu prüfen, (i) welche Schäden berücksichtigt werden, (ii) für wen die Folgen eines Ereignisses negativ sind und (iii) wie weit die Folgen eines Ereignisses dem Ereignis zuzuordnen sind, das heisst ab wann Folgen ihrerseits als Ereignis gelten. Die letzte Frage ist in der Rraxis oft schwierig zy beantworten. 
Bei der Erfassung indirekter Schäden stellen sich besondere methodische Probleme. Hilfreich könnte der Begriff des adäquaten Kausalzusammenhangs aus der Rechtslehre sein: Folgen, die einem Ereignis nach dem gewöhnlichen Lauf der Dinge und der allgemeinen Lebenserfahrung zugeordnet werden können, wären demnach als Schaden dieses Ereignisses zu betrachten. Die Einführung eines Aversionsfaktors bei Grossschäden wird von einem Teil der Lehre und Praxis als pragmatischer Ansatz zur Berücksichtigung von (indirekten) Folgeschäden begründet.

Nach der Systemabgrenzung können Auswirkungen bewertet werden hinsichtlich (Berg et al., 1994; Abbildung 6):

- Art (positiv/negativ);

- Relevanz;

- methodischer Erfassbarkeit (Vorliegen geeigneter Indikatoren).

Die Art der Auswirkungen eines Ereignisses und deren Relevanz können für jeden Betroffenen unterschiedlich beurteilt werden. Die Art der Auswirkungen hängt - wie erwähnt - von der Interessenslage und der Bewertung durch die betroffene Person ab. Hinsichtlich der Relevanz ist die subjektive Betroffenheit entscheidend. Die methodische Erfassbarkeit eines Schadens ist eine wichtige Voraussetzung für die Operationalisierung des Schadens- und Risikobegriffs.

\subsubsection{Schaden im rechtlichen Sinn}

Als Schaden gilt aus rechtlicher Sicht die Verletzung, also eine materielle oder ideelle Verschlechterung eines Rechtsgutes, die durch ein schädigendes Ereignis entsteht. Rechtsgutverletzungen sind (Seiler, 1997):

- die Verletzung subjektiver Rechte;

- die Verletzung objektivrechtlich geschützter Güter.

Von einer Rechtsgutverletzung ist dann die Rede, wenn ein Schutzgut der öffentlichen Sicherheit und Ordnung erheblich beeinträchtigt ist (Reinhard, 1993). Schaden ist von minderen Beeinträchtigungen wie Belästigung oder Geschmacklosigkeit zu unterscheiden. Letztere stellen keinen Schaden dar und dürfen demzufolge nicht rechtlich oder polizeilich verhindert oder unterbunden werden.

Die Abgrenzung zwischen erheblicher und minderer Beeinträchtigung ist oft nicht einfach und orientiert sich an der Betroffenheit der beeinträchtigten Person. Zu berücksichtigen sind Ort, Zeit und Umstände (z. B. unterschiedliche Beurteilung von Geräuschen tagsüber und nachts). Auch beim rechtlichen Schadensbegriff ergibt sich somit keine intersubjektiv verbindliche Festlegung, was unter Schaden zu verstehen ist. Die Rechtsgüter mit ihrer Verankerung in der Rechtsordnung, die für alle ihr Unterworfenen gilt, sind jedoch allgemeinverbindlich festgelegt. 


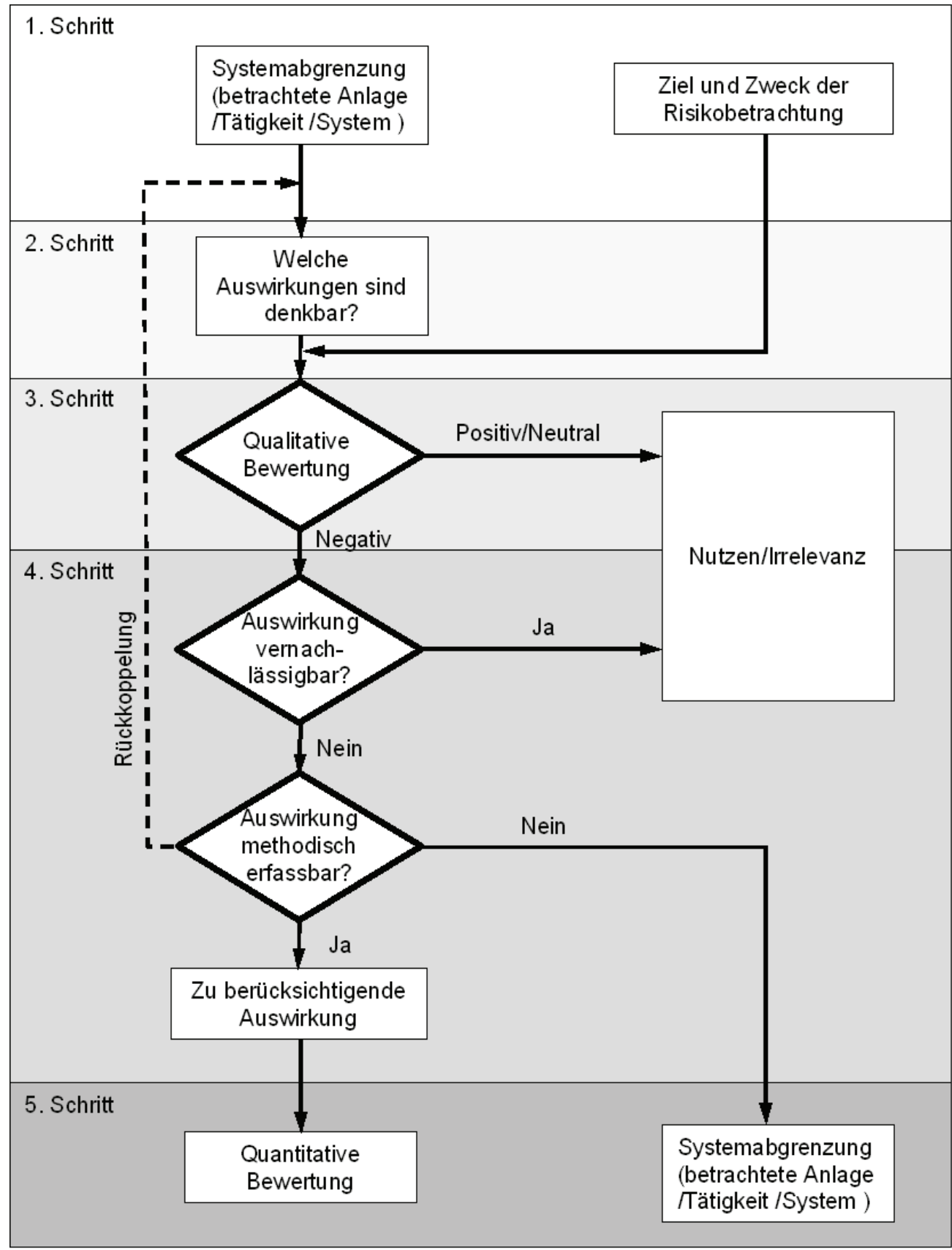

Abbildung 6 Methode zur Abschätzung eines Schadens (Berg, 1994). Entscheidend ist die qualitative Bewertung als negative und relevante Auswirkung sowie die methodische Erfassbarkeit. 


\subsection{Verletzbare Werte und Schäden}

\subsubsection{Arten von verletzbaren Werten}

In der Gesellschaft besteht eine grosse Zahl verletzbarer Werte, die in die folgenden Kategorien zusammengefasst werden können (Berg et al., 1994 und Tabelle 2, erste Spalte):

- Mensch und menschliche Gesundheit,

- Sachgüter,

- gesellschaftliche Strukturen,

- ökologische Werte und

- Lebensqualität.

Für das Management von Naturrisiken sind vor allem die Werte Mensch und menschliche Gesundheit sowie die ökonomischen Werte (Sachgüter) bedeutend. Den Naturwerten kommt eine hohe Bedeutung zu, soweit diese dem Menschen als Lebensgrundlage dienen. Ansonsten wird ihre Bedeutung für das Risikomanagement gering erachtet, da die bei Naturereignissen ablaufenden Prozesse zur natürlichen Dynamik von Lebensräumen gehören und aus Sicht der Natur kein Problem darstellen oder sogar erwünscht sind. Von untergeordneter Bedeutung im Management von Naturrisiken erscheinen die gesellschaftlichen Institutionen, da eine relevante Beeinträchtigung durch Naturereignisse, wie sie in der Schweiz zu erwarten sind, als unwahrscheinlich erachtet wird.

\subsubsection{Beeinträchtigung verletzbarer Werte als Schaden}

Mit dem in Kapitel 2.2.2 und Abbildung 6 dargestellten Verfahren wurde versucht abzuschätzen, inwieweit Beeinträchtigungen verletzbarer Werte als Schäden zu betrachten sind. Dabei wurde für eine Reihe verletzbarer Werte geprüft, ob eine Beeinträchtigung als negativ, relevant und methodisch erfassbar und somit als Schaden bezeichnet werden kann (Tabelle 2). Diese Einschätzung zeigt, dass eine Beeinträchtigung verletzbarer Werte nur in den folgenden Fällen klar als Schaden eingeordnet werden kann:

- ,menschliches Leben' und ,körperliche Integrität',

- die meisten Arten der ,Sachgüter',

- ,öffentliche Infrastruktur',

- 'Tiere', ,Pflanzen', ,Gewässer' und ,Boden', soweit es sich dabei um Lebensgrundlagen des Menschen handelt, und

- ,Kulturdenkmäler'. 
Tabelle 2 Bewertung einer Beeinträchtigung verletzbarer Werte als Schaden.

\begin{tabular}{|c|c|c|c|}
\hline \multirow[t]{2}{*}{ Verletzbarer Wert } & \multicolumn{3}{|c|}{$\begin{array}{l}\text { Generelle Bewertung einer Beeinträchtigung (durch Natur- } \\
\qquad \begin{array}{c}\text { ereignisse) als Schaden } \\
+=\text {,erfüllt'; - = , nicht erfüllt' }\end{array}\end{array}$} \\
\hline & $\begin{array}{c}\text { Negative Auswir- } \\
\text { kungen }\end{array}$ & Relevanz & $\begin{array}{l}\text { Methodische } \\
\text { Erfassbarkeit }\end{array}$ \\
\hline \multicolumn{4}{|l|}{ Mensch und menschliche Gesundheit } \\
\hline - körperliche Integrität & + & + & + \\
\hline - psychische Integrität & + & + & $+/-$ \\
\hline \multicolumn{4}{|l|}{ Sachgüter (ökonomische Werte) } \\
\hline - Gebäude & + & + & + \\
\hline - Wohnraum & + & + & + \\
\hline - Sachwerte, Vermögen & + & + & + \\
\hline - Infrastruktur & + & + & + \\
\hline - wirtschaftliche Tätigkeit & + & + & $+/-$ \\
\hline - Konkurrenzfähigkeit, Image & + & + & $+/-$ \\
\hline - Nutztiere, Nutzpflanzen & + & + & + \\
\hline \multicolumn{4}{|l|}{ Gesellschaftliche Strukturen } \\
\hline - soziale Strukturen & + & - & - \\
\hline - formelle Institutionen & + & - & + \\
\hline - öffentliche Infrastrukturen & + & + & + \\
\hline - Rechtsordnung & + & - & $+/-$ \\
\hline - subjektive Rechte & + & + & $+/-$ \\
\hline - Freiheit & + & - & - \\
\hline - Demokratie & + & - & - \\
\hline - öffentliche Ordnung & + & + & - \\
\hline \multicolumn{4}{|l|}{ Naturwerte } \\
\hline - Tiere, Tierarten & + & + & + \\
\hline - Pflanzen, Pflanzenarten & + & + & + \\
\hline - Biotope, Lebensräume & - & - & + \\
\hline - Grund- und Oberflächenwasser & + & + & + \\
\hline - Luft & - & - & + \\
\hline - Boden & + & + & + \\
\hline - Gene & - & - & + \\
\hline \multicolumn{4}{|l|}{ Lebensqualität } \\
\hline - psychisches Wohlbefinden & + & + & - \\
\hline - Freiheit & + & + & - \\
\hline - ethische Werte & + & + & - \\
\hline - Erholungsgebiete & + & + & $+/-$ \\
\hline - Landschaft & + & + & $+/-$ \\
\hline - Kulturdenkmäler & + & + & + \\
\hline - Ruhe & + & - & + \\
\hline
\end{tabular}




\subsubsection{Allgemeingültigkeit der Schadensbewertung}

In Kapitel 2.2.1 wurde auf die Subjektivität der Wertungen und damit auf die Subjektivität des Schadens hingewiesen. Diese Subjektivität besteht vermutlich nicht für alle Arten von Schäden in gleichem Mass. Für eine Auswahl von Beeinträchtigungen verletzbarer Werte wurde abgeschätzt, inwieweit eine allgemeingültige Bewertung der Wirkung besteht (Tabelle 3).

Tabelle 3 Beurteilung der Allgemeingültigkeit verschiedener Arten von Schäden.

\begin{tabular}{|c|c|c|}
\hline Art der Beeinträchtigung verletzbarer Werte & $\begin{array}{l}\text { Allgemeingültigkeit } \\
\begin{array}{r}\text { der Auswirkung } \\
\text {,Schaden/Nutzen' } \\
+=\text {,gros }\end{array}\end{array}$ & $\begin{array}{l}\text { Allgemeingültigkeit } \\
\text { der Relevanz } \\
=\text {,gering' }\end{array}$ \\
\hline Verlust von Menschenleben (Todesfälle) & + & + \\
\hline $\begin{array}{l}\text { Schwere, irreversible Beeinträchtigung der menschlichen } \\
\text { Gesundheit }\end{array}$ & + & + \\
\hline $\begin{array}{l}\text { Leichte, reversible Beeinträchtigung der menschlichen } \\
\text { Gesundheit (Verletzungen) }\end{array}$ & + & + \\
\hline Ökonomische Verluste & - & $+/-$ \\
\hline Schäden an gesellschaftlichen Institutionen & + & - \\
\hline Beeinträchtigung natürlicher Lebensgrundlagen & + & + \\
\hline Ökologische Schäden irreversibel & + & + \\
\hline Ökologische Schäden reversibel & + & - \\
\hline Beeinträchtigung der Lebensqualität & + & + \\
\hline
\end{tabular}

Durch Naturereignisse bewirkte Todesfälle, Gesundheitsschäden, Schäden an Institutionen, Umwelt und an der Lebensqualität werden von Betroffenen als negativ, als Schaden empfunden. Hinsichtlich ökonomischer Schäden gilt dies nicht für alle Betroffenen. Wie bereits erwähnt können Einzelne in der Folge eines Ereignisses durchaus Nutzen generieren mit der Wiederherstellung beziehungsweise dem Ersatz beschädigter Güter oder mit der Bereitstellung von Massnahmen zur Vermeidung künftiger Schäden. Ökonomischer Nutzen entsteht auch in anderen Regionen, wenn sich wirtschaftliche Aktivitäten aus von Schäden betroffenen Gebieten dorthin verlagern. 
Die Relevanz von Beeinträchtigungen wird von den Betroffenen für einzelne Arten von verletzbaren Werten unterschiedlich wahrgenommen. Schäden an Leib und Leben, ökonomische Verluste, Beeinträchtigungen von Lebensgrundlagen und Lebensqualität allerdings dürften von allen Betroffenen einhellig als relevant beurteilt werden. Bei (reversiblen) ökologischen Schäden oder Schäden an gesellschaftlichen Institutionen hängt die Einschätzung der Relevanz von der Werthaltung der Betroffenen ab. Bei ökonomischen Verlusten entscheiden die persönlichen Verhältnisse des Betroffenen über die subjektive Relevanz des Schadens. Bei Beeinträchtigung der Gesundheit oder der Umwelt hängen die Wahrnehmung des Schadens oder die Relevanz erheblich davon ab, ob die Beeinträchtigungen reversibel/reparabel sind oder nicht.

Aus diesen Darlegungen und Kapitel 2.3.2 kann abgeleitet werden, dass die Beeinträchtigungen der Werte ,menschliches Leben' und ,körperliche Integrität', ,öffentliche Infrastruktur' sowie ,Tiere', ,Pflanzen', ,Gewässer' und ,Boden' als Lebensgrundlagen des Menschen immer und einheitlich als Schaden bezeichnet werden. Auch die Beeinträchtigung von Sachgütern gilt in den meisten Bewertungen als Schaden.

\subsubsection{Rechtsgüter und verletzbare Werte}

Verletzbare Werte aus rechtlicher Sicht sind die Rechtsgüter. Der Begriff Rechtsgut (auch Schutzgut) bezeichnet das rechtlich geschützte Interesse einzelner Menschen (,Individualrechtsgüter', z. B. Menschenwürde, körperliche Unversehrtheit (Leib und Leben), Eigentum, Ehre, sexuelle Selbstbestimmung u.v.a.) und der Gesellschaft (,Universalrechtsgüter') (Wikipedia, 2006). Die Rechtsgüter ergeben sich aus der Gesamtheit der Rechtsordnung. In mehr als einem Dutzend verwaltungsrechtlicher Gesetze sind auf Bundesebene etwa 50 Schutzgüter bezeichnet (Seiler, 1997; Tabelle 4). Dies sind beispielsweise technikrechtliche Gesetze wie das Rohrleitungsgesetz (RLG SR 746.1) oder das Strahlenschutzgesetz (StSG SR 814.50). Nebst den Rechtsgütern des Einzelnen gehören insbesondere auch Umweltgüter und soziale Werte (z. B. wirtschaftliche, soziale und politische Strukturen) zu den Rechtsgütern (Seiler, 1997). Auffallend häufig sind Rechtsgüter aus den Bereichen Umwelt und Lebensqualität in den Gesetzen erwähnt.

Die Polizeigüter stellen eine Teilmenge der Rechtsgüter dar. Sie umfassen unter anderem die öffentliche Sicherheit und Ordnung (Richli, 2004b; Häfelin, 2002), d. h. die Unverletzlichkeit der objektiven Rechtsordnung, der Einrichtungen des Staates und der Rechtsgüter des Einzelnen wie Leib und Leben, Eigentum und Vermögen. Den Polizeigütern kommt im Management von Naturrisiken grosse Bedeutung zu. Weitere Polizeigüter sind öffentliche Gesundheit, Sittlichkeit, Ehre, Freiheit, Treu und Glauben im Geschäftsverkehr sowie Ruhe und Sicherheit. 
Tabelle 4 Rechtsgüter in ausgewählten Bundesgesetzen (Seiler 1997, abgeändert).

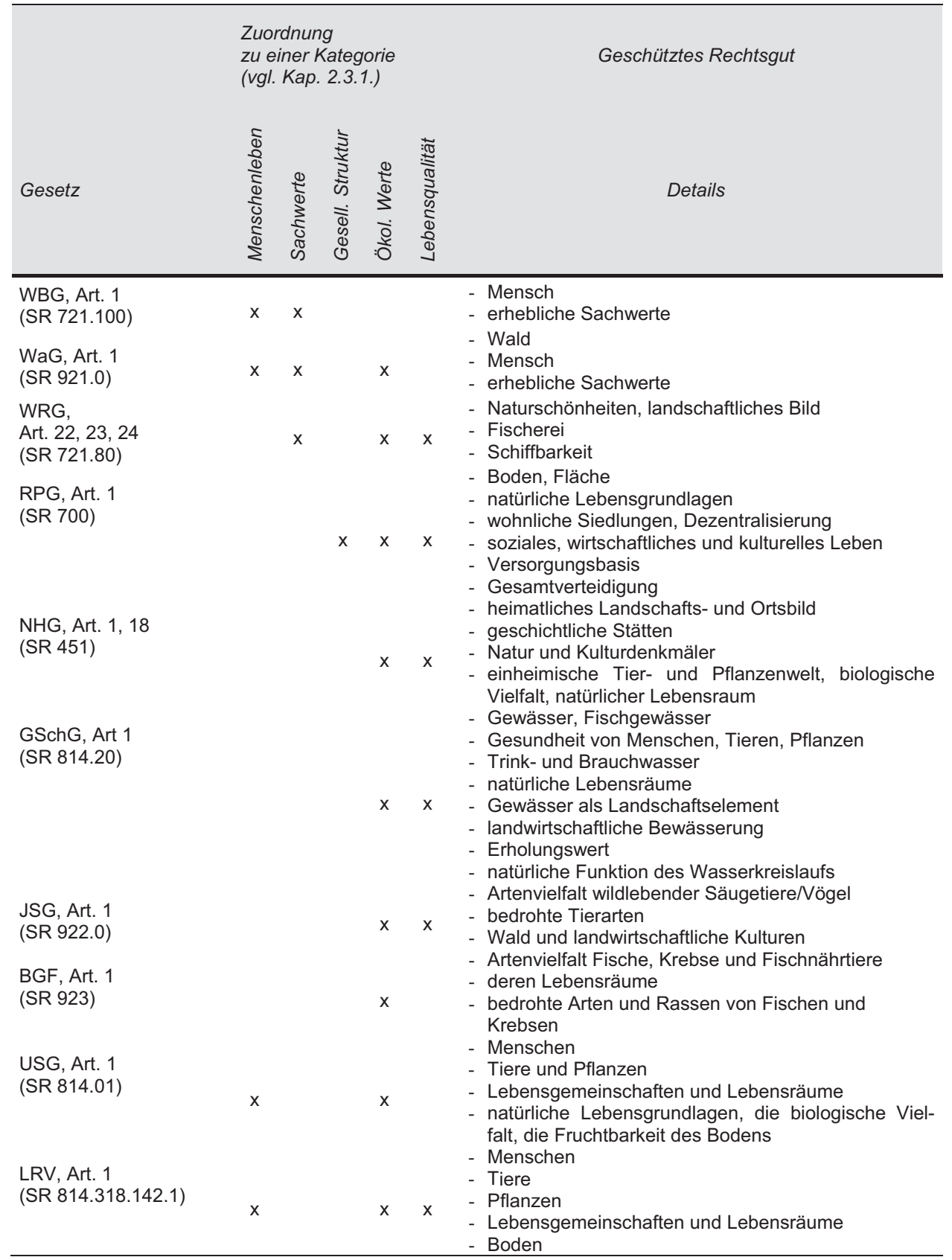

Es bestehen Parallelen zwischen verletzbaren Werten und Rechts- oder Polizeigütern (Tabelle 5). 
Tabelle 5 Die Gegenüberstellung der Konzepte ,verletzbare Werte' und ,Rechtsgüter' zeigt eine weitgehende Deckung.

\begin{tabular}{ll}
\hline Verletzbarer Wert & Geschütztes Rechtsgut \\
\hline Menschenleben & Leib und Leben \\
menschliche Gesundheit & körperliche Unversehrtheit, Gesundheit \\
Ökonomische Werte & Eigentum und Vermögen, Treu und Glauben im Geschäftsverkehr \\
gesellschaftliche Strukturen & Rechtsordnung, Einrichtungen des Staates, Versorgungsbasis, Landes- \\
& verteidigung \\
Ökologische Werte & Tiere, Pflanzen, Gewässer, Naturschönheiten \\
Lebensqualität & Freiheit, Sittlichkeit, Ehre \\
\hline
\end{tabular}

\subsubsection{Bedeutung von verletzbaren Werten und Rechtsgütern}

Ob bestimmten Arten von verletzbaren Werten und Rechtsgütern höhere Bedeutung zukommt als anderen, lässt sich am ehesten anhand der Rechtsordnung abschätzen. So geniessen bestimmte Rechtsgüter im Haftpflichtrecht als absolut geschützte Rechtsgüter eine Sonderstellung (Leib, Leben, Freiheit, Persönlichkeit und Eigentum). Bei diesen Rechtsgütern begründet allein schon deren Verletzung eine Widerrechtlichkeit (Erfolgsunrecht). Demgegenüber sind Vermögensbeeinträchtigungen nur widerrechtlich, wenn eine Rechtsnorm verletzt wird, die dem Schutz dieses Rechtsguts dient (Verhaltensunrecht).

Hochrangige Rechtsgüter sind insbesondere die durch das Verfassungsrecht geschützten Güter (Seiler, 1997):

- fundamentale Staatsziele und Rechtsprinzipien (sozialer Friede usw.),

- Grundrechte (Recht auf Leben und Gesundheit, Recht auf Freiheit und Sicherheit, Menschenwürde, Recht auf Privatleben, Familie, Ehre, Niederlassungsfreiheit, Recht auf Eigentum, Meinungs- und Informationsfreiheit, Versammlungsfreiheit),

- kollektive Rechte (Recht auf Selbstbestimmung, Minderheitenrechte) sowie

- Schutzziele und Aufgaben, welche sich aus Bestimmungen zum gesetzlichen Schutz eines Objektes ergeben.

Strafbestimmungen im Strafgesetzbuch (StGB, SR 311.0) und in Spezialgesetzen ahnden die Verletzung verschiedener Rechtsgüter in unterschiedlich hohem Mass, woraus sich Hinweise auf eine unterschiedliche Gewichtung ableiten lassen (Tabelle 6). Vorsätzliche Rechtsgutverletzungen wurden dabei in einer ersten Betrachtung nicht berücksichtigt, da diese Voraussetzung im Umgang mit Naturrisiken kaum gegeben ist.

Das Strafgesetzbuch sieht als Strafmass für Delikte, welche Rechtsgüter fahrlässig beeinträchtigen, einheitlich Geldstrafen oder Freiheitsstrafen bis drei Jahre vor. Eine Differenzierung nach Art der betroffenen Rechtsgüter ist nicht möglich. Ob bei der Bemessung der Strafen im Einzelfall Unterschiede bestehen, welche eine Gewichtung der betroffenen Rechtsgüter erlauben würden, bleibt offen. 
Vergleiche von Strafbestimmungen in Spezialgesetzen zeigen, dass Delikte, die Leib und Leben beeinträchtigen, strenger geahndet werden als Delikte, die ausschliesslich Umweltgüter betreffen (Tabelle 6).

Tabelle 6 Strafmass für Rechtsgutverletzungen gemäss Strafbestimmungen in ausgewählten Spezialgesetzen und dem Strafgesetzbuch (StGB).

\begin{tabular}{|c|c|c|c|c|c|c|c|c|}
\hline Betroffenes Rechtsgut & \multicolumn{2}{|c|}{ Regelung } & \multicolumn{6}{|c|}{ Höchststrafe } \\
\hline Delikt & $\begin{array}{l}\mathbb{N} \\
\mathbb{N} \\
\mathbb{N} \\
0\end{array}$ & 离 & $\begin{array}{l}0 \\
\frac{0}{\sigma} \\
\stackrel{\omega}{\omega} \\
\frac{0}{d} \\
0\end{array}$ & 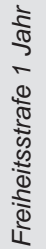 & 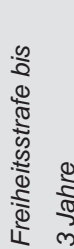 & 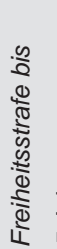 & & 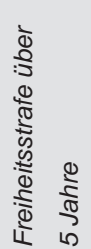 \\
\hline
\end{tabular}

\section{Leib und Leben}

\begin{tabular}{ll|l|l|l|l|l}
\hline Fahrl. Vergehen gegen USG USG 60 & & & & & \\
\hline Fahrl. Gewässerverunreinigung GSchG 70 & & & & & \\
\hline & & & & & & \\
\hline
\end{tabular}

Umwelt

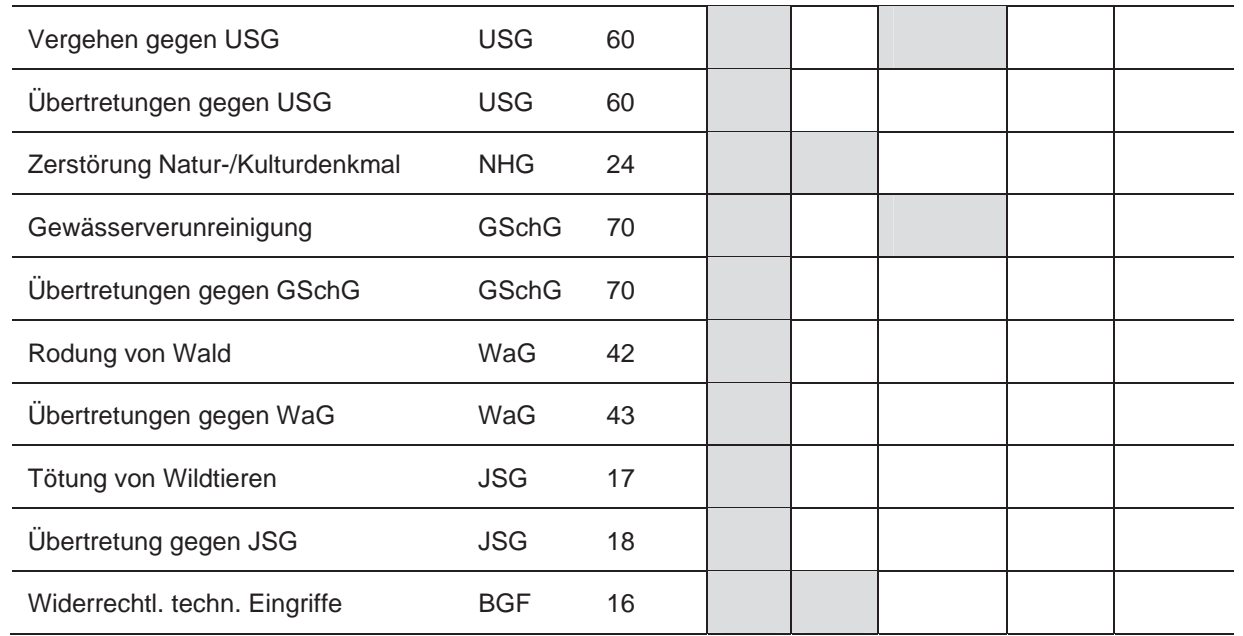

Fortsetzung Tabelle nächste Seite. 


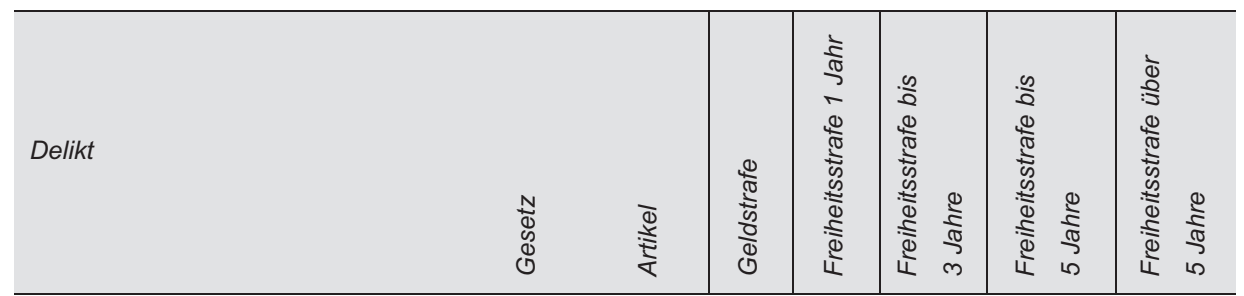

Fahrlässige Delikte nach Strafgesetzbuch (StGB) gegen

Leib und Leben Eigentum und Vermögen, Öffentliche Einrichtungen, Lebensqualität, Umwelt

\begin{tabular}{ll|l|l|l|l|l}
\hline $\begin{array}{l}\text { Alle fahrlässigen Delikte } \\
\text { nach StGB }\end{array}$ StGB 117 & & & & & & \\
\hline
\end{tabular}

Vorsätzliche Delikte nach StGB gegen Leib und Leben

Nach StGB

Artikel 111-118, 127-129, 230bis, 234, 264

Vorsätzliche Delikte nach StGB gegen körperliche Integrität und Gesundheit

Nach StGB

Artikel 122-126, 140, 183, 187-190, 195, 231, 234

Vorsätzliche Delikte nach StGB gegen Eigentum und Vermögen

Nach StGB

Artikel 138-143, 232, 233

Vorsätzliche Delikte nach StGB gegen öffentliche Einrichtungen

Nach StGB

Artikel 237-239, 265-270

Vorsätzliche Delikte nach StGB gegen die Lebensqualität

Nach StGB

Artikel 173-186, 197-198, 234, 264, 260, 261

Vorsätzliche Delikte nach StGB gegen die Umwelt

Nach StGB

Artikel 233, 234

Mögliche Strafmasse

Werden auch vorsätzliche Delikte betrachtet (untere Hälfte von Tabelle 6), ergeben sich ebenfalls geringe Unterschiede zwischen den verschiedenen Arten von betroffenen Rechtsgütern. Die Strafmasse sind eher geprägt vom Verschulden und der Gesinnung des Täters, der Art und Weise der Tatausübung und dem Erfolg der Tat. Höchststrafen werden für Delik- 
te, die Tod oder schwere Körperverletzungen zur Folge haben, ausgesprochen. Die Bestrafung von Verletzungen anderer Rechtsgüter wird häufig daran gemessen, ob die Verletzung dieser Rechtsgüter Todesfälle oder schwere Körperverletzungen zur Folge haben könnten. Strafverschärfend wirkt eine hohe Zahl Betroffener. Aus diesem Grund werden Delikte gegen staatliche Einrichtungen streng bestraft. Ebenfalls vergleichsweise hohe Strafen sind für Vermögensdelikte vorgesehen; besonders, wenn diese gewerbs- oder bandenmässig begangen werden. Beeinträchtigungen der Lebensqualität werden oft nur auf Antrag und mit geringeren Strafen geahndet.

Bestimmungen zur Bestrafung vorsätzlicher Verletzung von Umweltgütern fehlen im Strafgesetzbuch weitgehend (Ausnahme Art. 230bis, Abs. 1, Bst. b). Sie sind in den Strafnormen der Spezialgesetze enthalten. Das Strafmass reicht nicht über Gefängnisstrafen hinaus.

Höchsten Stellenwert unter den Rechtsgütern geniesst zusammenfassend - beurteilt am Strafmass - das Leben des Menschen, gefolgt von der körperlichen Integrität. Gemessen am Strafmass ebenfalls hoch bewertet sind Eigentum und Vermögen sowie öffentliche Einrichtungen des Staates. Aufgrund der Strafandrohungen haben die Rechtsgüter Lebensqualität und Umwelt in der Rechtsordnung eine geringe Bedeutung.

\subsection{Integrale Schadens- und Risikoindikatoren}

\subsubsection{Indikatoren und Messgrössen}

Die Beschreibung von Schäden und Risiken erfolgt mit Indikatoren. Sachrisiken werden beispielsweise in Geldbeträgen pro Jahr angegeben. Personenrisiken werden zumeist in der Dimension von Betroffenen pro Jahr dargestellt (z. B. Anzahl Todesfälle pro Jahr). Im Vordergrund stehen dabei physische Schäden wie Todesfälle oder Verletzungen. Als psychische Beeinträchtigung gilt beispielsweise der ,Alarmfaktor', definiert als Dauer einer Alarmoder Angstsituation multipliziert mit der Anzahl der betroffenen Personen (BUWAL, 1991). Für die Darstellung von weiteren Beeinträchtigungen der Persönlichkeit und von Umweltrisiken existieren spezifische Indikatoren. Beispiele solcher Indizes für Umweltschäden sind Fläche geschädigter Lebensgrundlagen, Einschränkung der ökologischen Qualität, tote (Gross-) Tiere, Fläche eines geschädigten Ökosystems, kontaminierte Bodenfläche oder kontaminierte (oberirdische oder unterirdische) Wasserfläche (Beroggi, 1994; BABS, 2003; BUWAL, 1991; Egli, 2000; Blong, 2003; Scheringer, 1994; Robinson, 2003; Duvernay, 2005). Die Fachliteratur kennt Sets von mehr als 70 Indikatoren mit gegen 200 quantifizierbaren Messgrössen (Berg et al., 1994; Tabelle 7). 
Tabelle 7 Indikatoren und Messgrössen zur Darstellung der Beeinträchtigung verletzbarer Werte.

\begin{tabular}{|c|c|c|}
\hline Kategorie & Art des Schadens/Indikator/Messgrösse & Quelle \\
\hline \multirow{8}{*}{ Leib und Leben } & - Anzahl Todesopfer & div. \\
\hline & - Anzahl verlorene Lebensjahre & [1] [2] [3] \\
\hline & - YLL (Years Lost Life) & [4] \\
\hline & - Geldwerte für Todesfallschäden & [5] \\
\hline & - Anzahl Verletzte & [6] \\
\hline & - QALY (Quality-Adjusted Life-Years) & u. a. [4] \\
\hline & - DALY (Disability-Adjusted Life-Years) & u. a. [4] \\
\hline & - Geldwerte für Verletzungen/Gesundheitsschäden & [5] \\
\hline \multirow{3}{*}{ Sachgüter } & - Geldwerte für Sachschäden & div. \\
\hline & - getötete Grosstiere & [7] \\
\hline & - DI (Damage Index) & [8] \\
\hline \multirow{5}{*}{$\begin{array}{l}\text { Ökologische } \\
\text { Werte }\end{array}$} & - Fläche beschädigter Lebensgrundlagen & [6] \\
\hline & - Fläche mit eingeschränkter ökologischer Qualität & [6] \\
\hline & - Fläche eines geschädigten Ökosystems & [7] \\
\hline & - Fläche von kontaminiertem Boden & [7] \\
\hline & - Fläche von kontaminiertem Wasser & [7] \\
\hline \multirow{5}{*}{ Lebensqualität } & - Anzahl Evakuierte (Dauer der Evakuation) & [6] \\
\hline & - Anzahl Unterstützungsbedürftige & [6] \\
\hline & - Alarmfaktor & [7] \\
\hline & - LQI (Life Quality Index) & [9] [10] \\
\hline & - Geldwerte für Abfindungen & [5] \\
\hline
\end{tabular}

Legende zu den Quellen

[1] Merz, H. A., Schneider Th., Bohnenblust H. (1995)

[2] Seiler, H. (2000)

[3] Egli, M. (2000)

[4] Proske (2004)

[5] Leonardo-Productions-AG (2006)

[6] BABS (2003)

[7] BUWAL (1991)

[8] Blong (2003)

[9] Pandey \& Nathwani (2003)

[10] Pandey et al. (2006) 
Der Index YLL (Years Lost Life) beschreibt die Differenz zwischen mittlerer Lebenserwartung mit einem bestimmten Risiko und ohne dieses Risiko.

Der QALY (Quality-Adjusted Life-Years) umschreibt die ,gesundheitsangepassten' Lebensjahre als Aufrechnung der Lebenszeit multipliziert mit einem Wert für den Gesundheitszustand. Komplementär dazu stellt der DALY (Disability-Adjusted Life-Years) die Differenz zwischen mittlerer Lebenserwartung bei voller Gesundheit und dem QALY dar.

Der Alarmfaktor umschreibt die Dauer einer Alarmsituation oder eines Angstzustandes multipliziert mit der Anzahl betroffener Personen.

Zur Darstellung der Lebensqualität gibt es über 20 Lebensqualitätsparameter und daraus gebildete Indizes. Ein Beispiel ist der HDI (Human Development Index) der UNO, der auf Lebenserwartung, Schreib- und Lesefähigkeit und Pro-Kopf-Einkommen basiert.

Der LQI (Life Quality Index) wird aufgrund des realen Bruttoinlandproduktes pro Person (G) und der Lebenserwartung (E) nach der Formel LQI $=C{ }^{*} \mathrm{G}^{\mathrm{q}}{ }^{*} \mathrm{E}$ berechnet (Pandey, 2003; Pandey, 2006). C ist dabei eine von der jeweiligen Volkswirtschaft abhängige Konstante. Der Exponent q bezeichnet die Elastizität des Konsumnutzens und ergibt sich aus dem Verhältnis von Arbeitszeit und Freizeit. Für die wirtschaftlich entwickelten Länder liegt q zwischen 0.15 und 0.2 .

Die in der Literatur gebräuchlichen Messgrössen weisen unterschiedliche Bezugs- und Masseinheiten auf, die bei Vergleichen zu beachten sind, z. B.:

- für ein ,durchschnittliches' Mitglied der Gesellschaft;

- für ein ,durchschnittliches' Mitglied einer bestimmten Personengruppe;

- pro Zeiteinheit (meist pro Jahr);

- pro Zeit der Gefahrenexposition;

- pro gefahrenem Kilometer;

- pro Fall;

- pro Fläche;

- als Teil der totalen Todesfallwahrscheinlichkeit.

Gewisse Schadensindikatoren nehmen Stellvertreterfunktion für weitere Schäden ein, so z. B. die Anzahl Todesfälle für die Gesamtheit der Personenschäden. Dies erfordert zuverlässige Informationen zur Beziehung zwischen den Stellvertretern und den durch sie vertretenen Schäden (Seiler, 1997).

\subsubsection{Qualität und Eignung von Indikatoren}

Geeignete Indikatoren müssen folgende Kriterien erfüllen (Hahn et al., 2003):

- Stichhaltigkeit (,validity'): Misst der Indikator das zentrale Schlüsselelement?

- Zuverlässigkeit (,reliability'): Ist die Messung über längere Zeit konsistent?

- Sensitivität (,sensitivity'): Reagiert der Indikator auf Veränderungen des Schlüsselelementes?

- Verfügbarkeit (,availability'): Ist die Messung/Informationsbeschaffung einfach?

- Objektivität (,objectivity'): Können Messdaten unter sich ändernden Verhältnissen reproduziert werden?

Die bekannten und verbreitet angewendeten Indikatoren erfüllen diese Eignungskriterien nur teilweise (Tabelle 8). 
Tabelle 8 Beurteilung einiger Indikatoren nach Eignungskriterien.

\begin{tabular}{|c|c|c|c|c|c|c|}
\hline \multirow[t]{2}{*}{ Kategorie } & \multirow[t]{2}{*}{ Indikator } & \multirow[t]{2}{*}{ 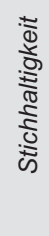 } & 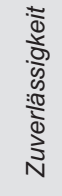 & 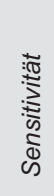 & 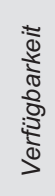 & $\begin{array}{l}: \frac{\pi}{5} \\
: \frac{\pi}{0} \\
\frac{\pi}{0} \\
0\end{array}$ \\
\hline & & & \multicolumn{4}{|c|}{$+=$,erfüllt'; - = ,nicht erfüllt' } \\
\hline \multirow{2}{*}{ Leib und Leben } & Anzahl Todesfälle & + & + & + & + & + \\
\hline & Anzahl Verletzte & $+/-$ & + & + & + & $+/-$ \\
\hline Sachwerte & Geldeinheit & + & + & + & + & + \\
\hline \multirow{2}{*}{ Umweltqualität } & geschädigte Lebensgrundlage & $+/-$ & $+/-$ & + & $+/-$ & $+/-$ \\
\hline & geschädigte ökologische Qualität & $+/-$ & $+/-$ & $+/-$ & $+/-$ & $+/-$ \\
\hline \multirow{3}{*}{ Lebensqualität } & Anzahl Evakuierte & $+/-$ & + & + & $+/-$ & $+/-$ \\
\hline & ,Alarmfaktor' & $+/-$ & $+/-$ & + & $+/-$ & $+1-$ \\
\hline & Anzahl Unterstützungsbedürftige & $+/-$ & $+/-$ & + & $+/-$ & $+/-$ \\
\hline
\end{tabular}

Es gibt nur wenige durchwegs geeignete Indikatoren. Geeignet sind nach dieser gutachtlichen Beurteilung Zahlenangaben zu Todesfällen sowie Geldangaben zu Sachschäden.

\subsubsection{Indikatoren zur vergleichenden Darstellung verletzbarer Werte}

Eine wichtige Voraussetzung für gesamtheitliches und effizientes Management von Risiken ist die Vergleichbarkeit verschiedener Arten von Schäden. Häufig werden für diese Vergleiche Geldwerte herangezogen (Monetarisierung).

Mögliche Varianten solcher Vergleiche (Seiler, 1997):

- Monetarisierung: z. B. Quantifizierung von Personenschäden anhand des auf sozialwissenschaftlichen Grundlagen basierenden ,Value of Societal/Statistical Life' (VOSL) (z. B. Guenther, 1997; Viscusi, 1998; Krupnick, 2002; Persson, 2001; Johansson, 2002; Perreira, 2002);

- Haftpflichtrechtliche Bewertung;

- Wertigkeit der Rechtsgüter, beurteilt anhand der Positionierung der Rechtsnorm, der Höhe der Strafandrohung oder der Wertungshierarchien, die induktiv aus Rechtsnormen hergeleitet werden können;

- Pragmatische Herleitungen in der Praxis: z. B. der Störfallwert gemäss Vollzugshilfen zur Störfallverordnung (BUWAL, 1991).

Das ,Handbuch I zur Störfallverordnung', Anhang G (BUWAL, 1991; BUWAL, 2001) schlägt zur vergleichenden Darstellung verschiedener Arten von Schäden einen Störfallwert vor. Damit lassen sich Schäden wie Todesfälle und Sachschäden, aber auch Verletzungen, Beeinträchtigungen der Lebensqualität durch Evakuierungen oder Alarmierungen (Angstzustände) sowie ökologische Schäden durch Beeinträchtigung von Ökosystemen, Boden und Grundwasser untereinander vergleichen (Tabelle 9). 
Tabelle 9 Der Störwert als Metrik für den Vergleich verschiedener Arten von Schäden (BUWAL, 1991).

\begin{tabular}{|c|c|c|c|c|c|c|}
\hline \multirow[t]{2}{*}{ Indikator } & \multirow{2}{*}{$\begin{array}{l}\text { Masseinheit } \\
\text { Störfallwert }\end{array}$} & \multicolumn{5}{|c|}{ Störfallwert und Schadensausmass } \\
\hline & & 0.02 & 0.32 & 0.60 & 0.89 & 1.0 \\
\hline Todesopfer & $\mathrm{N}$ & 1 & 10 & 100 & 1 '000 & 2'200 \\
\hline Verletzte & $\mathrm{N}$ & 11 & 90 & 800 & $7^{\prime} 000$ & $200^{\prime} 000$ \\
\hline Evakuierte & $\mathrm{N}$ & 4 & 80 & $1 ' 000$ & $14^{\prime} 000$ & $300^{\prime} 000$ \\
\hline Alarmierte & $N * d$ & $2^{\prime} 000$ & $80^{\prime} 000$ & 5 Mio. & 200 Mio. & 750 Mio. \\
\hline Tote Tiere & $\mathrm{N}$ & 40 & 600 & $8^{\prime} 000$ & $1200^{\prime} 000$ & 350,000 \\
\hline Geschädigtes & $\mathrm{km}^{2}$ & 0.15 & 4 & 100 & 3000 & $10^{\prime} 000$ \\
\hline \multicolumn{7}{|l|}{ Ökosystem } \\
\hline Boden & $\mathrm{km}^{2}$ & 0.08 & 2 & 40 & 1 '000 & 3200 \\
\hline Geschädigtes & $\mathrm{km}^{2}$ & 0.08 & 2 & 40 & $11^{\prime} 000$ & $3{ }^{\prime} 200$ \\
\hline \multicolumn{7}{|l|}{ Grundwasser } \\
\hline Sachwerte & Mio. CHF & 5 & 40 & 400 & 4'000 & $10^{\prime} 000$ \\
\hline
\end{tabular}

Die Festlegung der Schadensausmasse für die einzelnen Schadensindikatoren erfolgte pragmatisch nach für schweizerische Verhältnisse typischen Systemgrössen (z. B. Grösse einer Siedlung, eines geografischen Raumes, eines Sees, einer Tierherde usw.).

\subsubsection{Monetarisierung von Schäden und Risiken}

\subsubsection{Methoden der Monetarisierung}

Die bedeutendste Methode zur vergleichbaren Darstellung von Schäden ist - wie erwähnt die Monetarisierung. Dieses Vorgehen wird seit Längerem in verschiedenen Lebensbereichen (v. a. Ökonomie und Recht) angewendet. Die Rechtspraxis besitzt beispielsweise im Haftpflicht- und Versicherungsrecht über grosse Erfahrung in der geldwerten Bemessung verschiedenartiger Schäden, vor allem von Sach- und Personenschäden sowie Beeinträchtigungen der Lebensqualität. Auch bei der Anwendung des Indikators Geldeinheit stellen sich oft erhebliche methodische Probleme, beispielsweise durch die begrenzte Stichhaltigkeit und die schwierige Beschaffung von Informationen (,availability', Tabelle 10). 
Tabelle 10 Der Indikator Geldeinheit eignet sich, mit Einschränkungen, zur Beurteilung verschiedener Kategorien verletzbarer Werte.

\begin{tabular}{|c|c|c|c|c|c|}
\hline Kategorie & 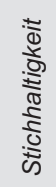 & 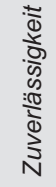 & 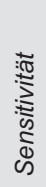 & 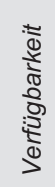 & $\begin{array}{l}: \frac{\pi}{0} \\
: \frac{\pi}{0} \\
.0 \\
0 \\
0\end{array}$ \\
\hline Menschliches Leben & $+/-$ & + & + & $+/-$ & + \\
\hline Menschliche Gesundheit & $+/-$ & + & + & $+/-$ & + \\
\hline Sachwerte & + & + & + & + & + \\
\hline Umweltqualität & $+/-$ & + & + & $+/-$ & + \\
\hline Lebensqualität & $+/-$ & $+/-$ & + & $+/-$ & + \\
\hline
\end{tabular}

In der Ökonomie wurden zahlreiche Methoden entwickelt, um die verschiedenen Arten von verletzbaren Werten und Schäden in Geldwerten darzustellen (z. B. Wilhelm, 1997).

Schäden und ,verhinderte Schäden' können grundsätzlich über Kosten oder Nutzen bewertet werden. Idealerweise erfolgt eine Bewertung zu Marktpreisen. Solche sind jedoch in vielen Situationen nicht verfügbar, sodass auf sogenannte Schattenpreise zurückgegriffen werden muss (Abbildung 7). Als Schattenpreise werden Preise bezeichnet, die als Folge fehlender Marktpreise mit einem alternativen Bewertungsverfahren ermittelt werden. Bedeutend ist dabei die Schadensmethode, nach der der Nutzen eines Guts oder einer Leistung mindestens so hoch anzusetzen ist wie die Kosten zur Reparatur der Schäden, die bei Nichtbereitstellung der Leistung entstehen würden (Wilhelm, 1997).

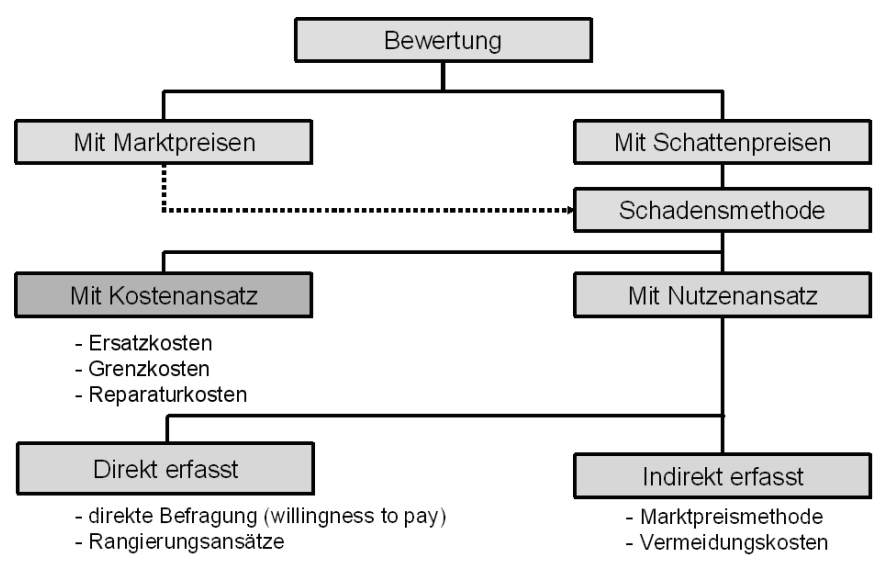

Abbildung 7 Monetäre Bewertung von Schäden. Als Ersatz für fehlende Marktpreise müssen alternative Bewertungen mit Schattenpreisen vorgenommen werden (Wilhelm, 2007).

Für die Ermittlung der ,Schattenpreise' stehen verschiedene Methoden zur Verfügung (Tabelle 11). 
Tabelle 11 Arten von Schäden und Bewertungsmethoden (Wilhelm, 2007).

\begin{tabular}{lll}
\hline Bereich & Indikator & Bewertungsmethoden (Bsp.) \\
\hline Leib und Leben & Todesfälle & Grenzkosten, Humankapital \\
& Verletzte & Heilungskosten \\
& Evakuierte & Zeitkosten \\
Sachwerte & Gebäude & Versicherungswerte \\
Wirtschaftsaktivität & Fahrhabe & Marktwert \\
& Betriebsausfall & Opportunitätskostenansatz \\
Natürliche Ressourcen & Unterbruch Strasse & Zahlungsbereitschaft \\
& Wald & Waldwertschätzung \\
Sozialgefüge & Schutzgebiet & Reisekostenmethode \\
\hline
\end{tabular}

Begriffe in Tabelle 11

Mit dem Grenzkostenansatz wird ein verletzbarer Wert nach der Höhe der Kosten bemessen, die eine Gesellschaft maximal zu investieren bereit ist, um einen Schaden an diesem Wert (z. B. Todesfall) zu verhindern.

Unter Opportunitätskosten versteht man den entgangenen Nutzen einer alternativen, theoretisch nächst besten Mittelverwendung.

Mit der Reisekostenmethode wird der Wert eines Gutes nach dem Aufwand (Reisekosten) bewertet, den die Nutzer auf sich nehmen, um dieses Gut zu nutzen (Wilhelm, 1997).

Um den Wert des öffentlichen Gutes zu ermitteln, werden mit dem Vermeidungskostenansatz die privaten Massnahmen bewertet, die bei Nichtbereitstellung eines öffentlichen Gutes getätigt werden (Wilhelm, 1997).

\subsubsection{Menschliches Leben und menschliche Gesundheit}

Die wichtigsten zu bewertenden Kostenelemente im Zusammenhang mit Beeinträchtigungen von Leben und Gesundheit sind die Leistungspotenzialverluste - etwa der Verlust der Arbeitsleistung - und die ,menschlichen Kosten'. Unter ,menschlichen Kosten' werden Geldbeträge verstanden, die „ausgegeben worden sind oder hätten ausgegeben werden müssen, um das Risiko des Verlustes an Lebenserwartung, des physischen und psychischen Leidens des Opfers, der Verminderung der Lebensqualität, einer bleibenden äusserlichen Verunstaltung sowie des psychischen Leidens der Freunde und Verwandten des Opfers zu mindern bzw. dafür Schmerzensgeld zu zahlen“ (Wilhelm, 1997). Daneben bestehen weitere Kostenelemente wie medizinische Kosten, Verwaltungskosten und sonstige ökonomische Kosten (Wilhelm, 1997).

\section{a) Menschenleben}

Nebst der Zahlungsbereitschaft zur Vermeidung von Todesfällen sind der Humankapitalansatz und der Marktansatz die hauptsächlichen Bewertungsverfahren im Zusammenhang mit Todesfallrisiken von Menschen (Wilhelm, 1997). Die Zahlungsbereitschaft (Vermeidungskostenansatz) umfasst die tatsächlich getätigten Aufwendungen oder die durch Umfragen erhobenen Zahlungsbereitschaft zur Rettung eines Menschenlebens. Sie variiert sehr stark (CHF 
0.6 bis CHF 140 Mio. pro gerettetes Menschenleben, siehe auch Kapitel 2.6.2.2). Generell sind diese Angaben sehr stark von der Volkswirtschaft abhängig: ,Reiche' Volkswirtschaften vermögen bedeutend mehr für Sicherheit aufzuwenden als ,arme' (Schneider, 1996). Der Humankapitalansatz zieht zukünftige Einkommen, die mit Todesfällen verloren gehen, zur Bewertung heran. Aufgrund statistischer Zahlen der Schweizer Bevölkerung ergibt die Bewertung nach dem Humankapitalansatz etwa CHF 1.2 Mio. für ein Menschenleben (Wilhelm, 1997). Guenther (1997) beziffert den sozialen Wert eines Menschenlebens (value of a societal life) zwischen USD 600'000 und USD 7'000'000, mit einem Betrag von USD 4'000'000 als ,konservativen' Durchschnitt (USD zum Wert im Jahr 1990).

Der Marktansatz schliesslich geht davon aus, dass Menschen freiwillig höhere Risiken eingehen, falls sie dafür besser entlöhnt werden. Aus dem Marktansatz (Bereitschaft gegen Lohnerhöhung von x CHF ein y höheres Todesfallrisiko einzugehen) ergeben sich Beträge von CHF 5-15 Mio. pro Menschenleben. Die Ergebnisse wurden aus Vergleichen zwischen Lohn- und Risikodifferenzen ermittelt und streuen zwischen USD 100'000 und USD 15.6 Mio. bei Befragungen sowie von USD 600'000 bis USD 16.2 Mio. bei Untersuchungen des tatsächlichen Verhaltens (Viscusi, 1998; Sommer, 2001).

\section{b) Menschliche Gesundheit}

Zur Bewertung der menschlichen Gesundheit werden Statistiken zu Kosten physischer Personenschäden herangezogen, seltener auch Untersuchungen zur Zahlungsbereitschaft. So basieren die Angaben in KATARISK (BABS, 2003a) auf Statistiken zum Strassen- und Schienenverkehr (ARE, 2002). Die sozialen Kosten für Personenschäden bei Verkehrsunfällen beliefen sich im Jahre 1998 auf CHF 12.3 Mia. (597 Todesopfer, 100'380 Verletzte). Daraus leitet die KATARISK-Studie Grenzkosten von 0.6 bis 20 Mio. CHF für die Verhinderung von Todesfällen und CHF 4'000 bis CHF 300'000 für Verletzte und Kranke ab (BABS, 2003a). Wilhelm (1997) gibt für verletzte Personen aufgrund von Zahlen der Schweizer Unfallversicherungsanstalt SUVA und einem Faktor zur Abschätzung der wirtschaftlichen Folgekosten einen Bewertungsansatz von CHF 14'100 pro Fall einer Personenverletzung infolge Lawinen an.

In der Studie von Perreira (2002) erklärten sich die Befragten bereit, USD 0.7 bis 1.4 Mio. zu bezahlen, um Invalidität in der zweiten Lebenshälfte zu vermeiden oder ungefähr USD 47'000 bis USD 95'000 für jedes vermiedene Jahr von Invalidität nach dem 62. Lebensjahr.

\subsubsection{3 Ökonomische Werte}

\section{a) Sachwerte}

Sachwerte werden in der Regel nach dem Marktwert oder den Erstellungs- bzw. den Beschaffungskosten bemessen. Die KATARISK-Studie zieht die Wiederherstellungs- oder Wiederbeschaffungskosten als Grundlage bei (BABS, 2003a). Geeignete Hinweise ergeben auch die Versicherungswerte. Im Zusammenhang mit der Entwicklung des Risikoberechnungstools ,EconoMe' wurden Werte für verschiedene Arten von Objekten evaluiert (BAFU, 2007b). Der Wert einer Wohneinheit wird beispielsweise zwischen CHF 550'000 und 650'000 angesetzt. Bei Gebäuden sind die Struktur- und Inhaltswerte zu berücksichtigen. Der Gebäudeinhalt hat einen Wert zwischen 15 und 20\% des jeweiligen Wertes von Wohngebäuden. Bei Gewerbe- und Industriebauten kann dieser sogar ein Mehrfaches des Gebäudewertes betragen (Wilhelm, 1997). Für Vieh nennt Wilhelm (1997) einen Wert von CHF 2'300 pro Grossvieheinheit, basierend auf dem Fleischwert. Weitere Angaben zu Nutztieren finden sich auch in Borter (1999). Nicht generell bezifferbar sind in der Regel die immateriellen Werte (Archive, Kulturgüter). 


\section{b) Wirtschaftliche Aktivität}

Neben den unmittelbaren direkten Wertverlusten stellen die indirekten Folgen in der Regel einen erheblichen Teil der Konsequenzen eines Ereignisses dar. Im Wesentlichen bestehen solche indirekten Folgen in einer Verminderung der Wirtschaftsaktivität. Wie bereits erwähnt können Naturereignisse auch neue Wertschöpfung ermöglichen (Wilhelm, 1997). Auch bewirken sie manchmal eine Verlagerung der Wertschöpfung an andere Orte, sodass Produktionsausfälle im Schadensgebiet erhöhte Wertschöpfung in einem anderen Gebiet auslösen.

Für Produktions-, Ertrags- und Mietzinsausfälle wurden nach verschiedenen Naturereignissen Werte zwischen 15 und 56\% des primären Sachschadens ermittelt (Tabelle 12).

Tabelle 12 Betriebliche Folgeschäden von Naturereignissen sind im Vergleich zu den direkten Schäden von sehr unterschiedlicher Bedeutung.

\begin{tabular}{|c|c|c|c|c|c|}
\hline Ereignis & Jahr & $\begin{array}{l}\text { Sachschäden } \\
\text { in Mio. CHF }\end{array}$ & $\begin{array}{l}\text { betriebl. Folgeschä- } \\
\text { den in Mio. CHF }\end{array}$ & Anteil & Quelle \\
\hline Hochwasser Brig-Glis & 1994 & & & $18 \%$ & [1] \\
\hline Lawinenwinter & 1999 & 440 & 245 & $56 \%$ & [2] \\
\hline Lawinenwinter, nur Bergbahnen & 1999 & 17 & 78 & $459 \%$ & [3] \\
\hline Orkan Lothar & 1999 & 730 & 250 & $34 \%$ & [2] \\
\hline Orkan Lothar, nur Bergbahnen & 1999 & 8 & 39 & $488 \%$ & [3] \\
\hline Hochwasser (Privatversicherer) & 2005 & 1335 & 200 & $15 \%$ & [4] \\
\hline
\end{tabular}

Legende zu den Literaturangaben:

[1] Wilhelm (1997) [2] Bründl \& Hegg (2001) [3] Nöthiger (2001) [4] BWG (2005)

Für einzelne Branchen können die indirekten Schäden ausserordentlich hohe Bedeutung erlangen, wie eine Untersuchung über die Auswirkungen des Lawinenwinters und des Orkans Lothar 1999 auf die Bergbahn- und Skiliftunternehmen der Schweiz zeigt (Nöthiger, 2001, Tabelle 12 und Tabelle 13). Die indirekten Schäden infolge Mindereinnahmen betrugen hier im Mittel etwa das Fünffache der unmittelbaren Sachschäden.

Tabelle 13 Die hochgerechneten finanziellen Folgen des Lawinenwinters 1999 und des Orkans Lothar 1999 für die Bergbahn- und Skiliftunternehmen der Schweiz (ohne Tessin).

\begin{tabular}{|c|c|c|c|c|c|c|}
\hline & \multicolumn{2}{|c|}{ Lawinenwinter 1999} & \multicolumn{2}{|c|}{ Orkan Lothar 26.12.1999 } & \multicolumn{2}{|c|}{ Beide Ereignisse zusammen } \\
\hline & 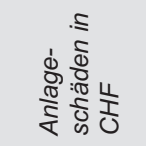 & 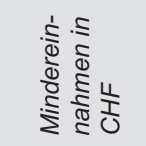 & 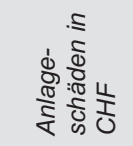 & 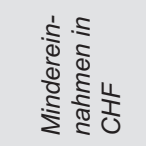 & 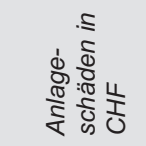 & 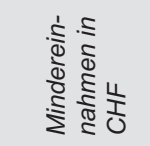 \\
\hline$B E$ & 9'562'000 & $10^{\prime} 422^{\prime} 000$ & 1'107'000 & 6'282'000 & $10^{\prime} 669^{\prime} 000$ & $16^{\prime} 704^{\prime} 000$ \\
\hline$G R$ & 3'331'000 & $25^{\prime} 755^{\prime} 000$ & 0 & 7’693'000 & 3'331'000 & 33'448'000 \\
\hline VS & 2'993'000 & 23’480’000 & $1^{\prime} 607^{\prime} 000$ & $13^{\prime} 737^{\prime} 000$ & $4^{\prime} 6000^{\prime} 000$ & $37^{\prime} 217^{\prime} 000$ \\
\hline$Z S$ & $870 ’ 000$ & 8'348'000 & $2^{\prime} 626^{\prime} 000$ & $77^{\prime} 740 \prime 000$ & 3'496'000 & $16^{\prime} 088^{\prime} 000$ \\
\hline $\mathrm{CH}$ & 17’393’000 & 77'806'000 & 7’595'000 & $39^{\prime} 279^{\prime} 000$ & 24'988'000 & $117^{\prime} 085^{\prime} 000$ \\
\hline
\end{tabular}

$\mathrm{BE}=\mathrm{Kt}$. Bern, GR = Kt. Graubünden, VS = Kt. Wallis, ZS = Zentralschweiz (LU/NW/OW/SZ/UR), $\mathrm{CH}=$ Schweiz (ohne Tessin). 
c) Interventionsmassnahmen

Bei Naturereignissen entstehen Interventions- und Rettungskosten. Beispielsweise leisteten die Feuerwehren zur Bewältigung der Unwetterschäden 2005 im Kanton Obwalden 28'242 Stunden oder ca. 3'200 Einsatztage, die mit CHF 850'000 abgegolten wurden. Dies entspricht lediglich $0.2 \%$ der Gesamtschadenssumme von CHF 400 Mio. Gleichzeitig können dem Interventions- und Rettungsbereich etwa 3'000 Einsatztage Militärhilfe und mindestens 800 Einsatztage ziviler Einsatzkräfte zugerechnet werden (Amt für Militär und Bevölkerungsschutz OW, 2006). Beobachtungen in Einzelsituationen weisen auf eine hohe Effizienz von Interventionsmassnahmen hin. Der Stellenwert von Interventionsmassnahmen im Risikomanagement ist in letzter Zeit angestiegen. Es ist daher denkbar, dass künftig höhere Interventionskosten anfallen, aber die Schadensumme geringer ausfällt.

\subsubsection{Umwelt}

Ökologische Schäden umfassen einerseits Beeinträchtigungen der Natur als Lebensgrundlage des Menschen, anderseits Beeinträchtigungen der Natur an sich. Verschiedentlich wird dargelegt, dass der mit Umweltschäden im Zusammenhang stehende ökonomische Schaden als unterster Wert zu betrachten sei, da die Umwelt auch um ihrer selbst willen geschützt werden müsse und nicht nur soweit sie dem Menschen dienlich sei (Seiler, 1995d). Angaben zur Quantifizierung von Werten und Leistungen der Umwelt bestehen vor allem soweit, als die Umweltgüter eine Bedeutung als Lebens- und Wirtschaftsgrundlage aufweisen (KATARISK-Studie [BABS, 2003a] und Abbildung 8). Umweltleistungen können unterteilt werden in die Bereitstellung von Verbrauchsgütern (z. B. Brennstoffe) und Kapitalgütern (z. B. Boden). Aus diesen Umweltleistungen resultieren die folgenden Umweltnutzungen (Ott et al., 2005):

- Nutzung der Umwelt als Produktionsinput durch die Lieferung von erneuerbaren (Pflanzen, Tiere, Wind, Sonne usw.) und nichterneuerbaren Ressourcen (Erdöl, Mineralien usw.);

- Nutzung der Umwelt als Aufnahmemedium für Reststoffe (Emissionen) wie Abfälle, Abgase, Abwasser, Radioaktivität usw.;

- Nutzung der Umwelt als Standort und Lebensraum für menschliche Aktivitäten (Landwirtschaft, Industrie, Wohnen, Freizeit);

- Nutzung der Umwelt als öffentliches Konsumgut. Die Erhaltung wertvoller Landschaften und der Schutz von vom Aussterben bedrohter Tierarten kann die Lebensqualität erhalten oder erhöhen.

Für die Ermittlung von Wald- und Flurschäden werden oft Wiederherstellungskosten nach Schadensereignissen herangezogen (BABS, 2003a; Abbildung 8). Auch die Angaben von Wilhelm (1997) basieren auf Zahlen zu Schadensereignissen und ergeben für Lawinenschäden in Wäldern einen Bewertungsansatz von CHF 37'000/ha. 


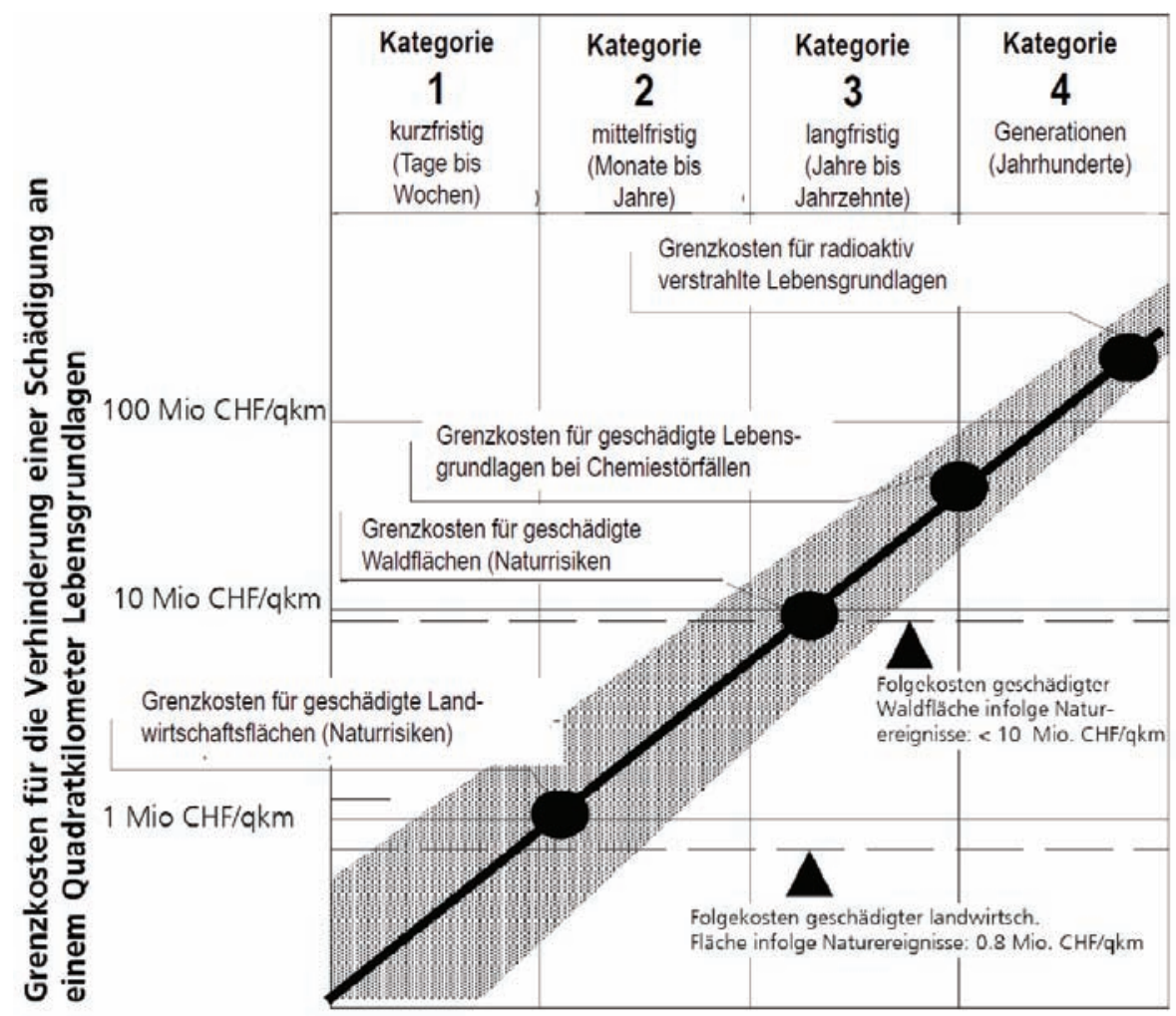

Abbildung 8 Für die Bewertung von Umweltschäden ist vor allem die Dauer der Beeinträchtigung massgebend (BABS, 2003a).

\subsubsection{Lebensqualität}

Angaben zu Kosten oder Vermeidungskosten (Grenzkosten) bei Beeinträchtigung der Lebensqualität bestehen nur lückenhaft. Für Unterstützungsbedürftige schätzt Wilhelm (1997) eine Zahl von CHF 5'000 pro Person und Ereignis. Die KATARISK-Studie errechnet unter Beizug der Richtlinien für die Bemessung der Sozialhilfe sowie aufgrund von Erfahrungszahlen der Fürsorgeämter und Fürsorgestatistiken Grenzkosten pro Unterstützungsbedürftigen von CHF 10'000 bis CHF 100'000 (pflegeintensive langfristig Unterstützungsbedürftige; BABS, 2003).

Bei der Festlegung der Grenzkosten für den Schadensindikator ,Evakuierte' greift die KATARISK-Studie auf die Annahmen der Schweizer Bundesbahnen (SBB) zurück (SBB, 1993). Dabei wurde ein Betrag von CHF 10'000 pro verhinderte Evakuierung einer Person als Grenzkostenbetrag eingesetzt (BABS, 2003).

Der in der Literatur verwendete Life Quality Index (LQI) beschreibt die Abhängigkeit zwischen Bruttoinlandsprodukt und Lebenserwartung (Pandey, 2003; Pandey, 2006). Im Sinne der in Kapitel 2.3.1. und 2.3.2. angeführten verletzbaren Werte und Indikatoren ist dieser Index zur Wertberechnung von Lebensqualität nicht anwendbar. 


\subsubsection{Gesellschaftliche Strukturen und Aversion}

Unbekannt sind monetäre Angaben zur Bewertung gesellschaftlicher Strukturen oder zur Bemessung von Schäden an gesellschaftlichen Strukturen. In schweizerischen Verhältnissen sind relevante Beeinträchtigungen gesellschaftlicher Strukturen am ehesten bei ausserordentlich grossen oder besonders schlimm bewerteten Ereignissen denkbar.

In vielen Risikobeurteilungen wird für Grossereignisse ein Aversionsfaktor verwendet (Kröger, 1996). Aversion ist ein empirisch feststellbares und theoretisch begründbares Phänomen, Ereignisse mit grossem Schadensausmass stärker zu gewichten, als es aufgrund des zugehörigen Schadenserwartungswertes angezeigt wäre (BAFU, 2007). Neuere Forschungsarbeiten setzen die Aversion nicht nur in Verbindung mit dem Ausmass eines Ereignisses, sondern auch mit einem Referenzrisiko (Plattner, 2005). Dies begründet sich darin, dass Ereignisse mit einem ungewohnt grossen Schadensausmass zu einer überproportionalen Reaktion der betroffenen Gemeinschaft führen und überproportional stark auf Ablehnung stossen. Die Aversion steht oft auch für die nicht direkt erfassbaren Nebenfolgen oder Konsequenzen eines Schadensereignisses (Kapitel 2.4.4.3). Diese umfassen zu einem erheblichen Teil Beeinträchtigungen der Gesellschaft und der Lebensqualität. Darunter fallen beispielsweise (Bohnenblust und Schneider, 1984; Bohnenblust und Troxler, 1987; Schneider, 2006; Plattner, 2005):

- Schäden, die durch das Zusammenbrechen betroffener Systeme entstehen;

- Schäden von Folgeereignissen eines Grossereignisses;

- Kosten der Untersuchung und Kosten, die durch den Erlass neuer Gesetze im Nachgang eines Ereignisses entstehen;

- der Verlust an Vertrauen in die zuständigen Behörden und Stellen;

- die Überschätzung des Risikos durch grosse Schadensereignisse und die daraus folgenden ,zu teuren' Schutzmassnahmen;

- der ,Signalwert' eines Grossereignisses, der darin besteht, dass in der Gesellschaft das Bedürfnis entsteht, dass solche Ereignisse nie mehr passieren dürfen;

- Beeinträchtigung der zukünftigen Entwicklung einer Organisation, einer Industrie (z. B. Tourismus) oder eines Unternehmens (z. B. durch den Verlust an Marktanteilen) und

- der Aufwand, der zur Wiederherstellung des ursprünglichen Systemzustandes nötig ist.

Die Aversionsfaktoren können zur Darstellung der Schäden und Risiken für gesellschaftliche Institutionen und des Risikos einer Beeinträchtigung der Lebensqualität herangezogen werden.

\subsection{Tolerierbarkeit von Effekten}

\subsubsection{Begriffe}

Das akzeptierte Risiko wird definiert als Risiko, das ein Individuum oder eine Gruppe bereit ist, aufgrund eigener Entscheidung und als Resultat ihrer alltäglich-intuitiven Risikobewertung ohne Widerspruch einzugehen (informelle Bewertung). Diese Bereitschaft lässt sich aus Aussagen (expressed preferencies) oder dem Verhalten der betroffenen Individuen (revealed preferencies) herleiten (Tabelle 14). Aussagen und tatsächliches Verhalten weichen oft erheblich voneinander ab. In der Literatur werden Unterschiede von bis zu Faktor 1000 zwischen dem als tolerierbar ausgedrückten (expressed preferencies) und dem tatsächlich akzeptierten Risiko (revealed preferencies) ausgewiesen (Ko, 2003; Finlay \& Fell, 1997). 
Das tolerierbare Risiko (approved preferencies) basiert auf Normen oder empirischen Überlegungen (formelle Bewertung). Die Tolerierbarkeit des Risikos wird definiert, indem entweder aufgrund normativer Kriterien ein Risiko als erlaubt oder zulässig bezeichnet wird oder indem ein Risiko festgelegt wird, für das die Aussicht besteht, dass es für Individuen und/oder Gruppen tolerierbar ist (Plattner, 2005). Es wird im Idealfall in einem politischen Meinungsbildungsprozess festgelegt, an dem sich neben den Experten auch Interessensgruppen und politische Entscheidungsträger beteiligen (Heinimann, 2002).

Tabelle 14 Übersicht über die verwendeten Begriffe zur Bewertung von Risiken.

\begin{tabular}{|c|c|c|c|}
\hline & \multicolumn{2}{|l|}{ Informelle Bewertung } & Formelle Bewertung \\
\hline Bewertung durch & \multirow{2}{*}{\multicolumn{2}{|c|}{ Individuen, Gesellschaft }} & Staat, staatliche Stellen \\
\hline Fachausdruck deutsch & & & tolerierbares Risiko \\
\hline Fachausdruck englisch & \multirow{2}{*}{\multicolumn{2}{|c|}{ 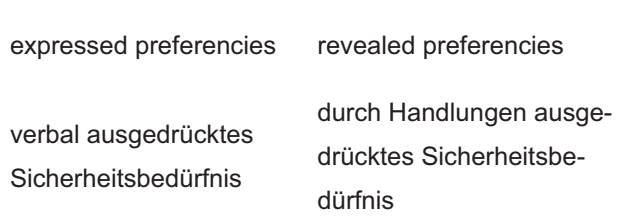 }} & approved preferencies \\
\hline Umschreibung & & & $\begin{array}{l}\text { in Regeln festgelegte } \\
\text { Sicherheitsstandards, } \\
\text {,Schutzziele' }\end{array}$ \\
\hline Basis der Bewertung & \multicolumn{2}{|c|}{ Risikowahrnehmung } & $\begin{array}{l}\text { Gesetze (ökonomische } \\
\text { Prinzipien) }\end{array}$ \\
\hline
\end{tabular}

Es ist anzunehmen, dass die formelle Bewertung durch die informelle Bewertung beeinflusst wird. Dies dürfte vor allem dann der Fall sein, wenn Schutzziele nicht aufgrund von Risikostatistiken, sondern aufgrund des Risikoempfindens der Bevölkerung festgelegt werden.

Eine detaillierte Darstellung wichtiger Begriffe zu Naturgefahren und Risiken befindet sich in Anhang I.

\subsubsection{Wahrnehmung, Risikoakzeptanz und Tolerierbarkeit}

Starr (1969) untersuchte in den 1970er Jahren tatsächlich akzeptierte Risiken aus Unfallstatistiken und verglich diese Risiken mit dem damit verbundenen, tatsächlichen oder wahrgenommenen Nutzen für den Risikobetroffenen. Die Untersuchung ergab folgende grundlegende Erkenntnisse:

- Das mit einer Aktivität eingegangene Risiko steigt exponenziell in Abhängigkeit zu dem damit erzielten Einkommen.

- Freiwillige' Risiken dürfen 1000-mal grösser sein als ,unfreiwillige' Risiken.

- Das tolerierbare Risiko für freiwillige Aktivitäten entspricht etwa der mittleren Sterbewahrscheinlichkeit infolge Krankheit.

- Die Tolerierbarkeit des Risikos ist etwa proportional zur dritten Potenz des (realen oder imaginären) Nutzens (Zahlungsbereitschaft für diese Aktivität).

- Das tolerierbare Risiko steigt mit dem Bewusstsein des Nutzens (dargestellt durch die Zahl der Betreiber einer Aktivität, der Werbung für eine Aktivität und der Nützlichkeit einer Aktivität).

- Das akzeptierte Risiko ist umgekehrt proportional zur Zahl der involvierten Personen.

- Am Beispiel der Atomenergie vertritt Starr (1969) die Auffassung, dass betriebswirtschaftliche Überlegungen zu einem Sicherheitsstandard führen, der deutlich über der öffentlichen Akzeptabilität liegt.

Josef Th. Hess: Schutzziele im Umgang mit Naturrisiken in der Schweiz, @ vdf Hochschulverlag 2011 
Auch später befassten sich Forscher wiederholt mit den Zusammenhängen zwischen wahrgenommenem und akzeptiertem Risiko (Litai et al., 1983: Slovic, 1987). Gewisse Kriterien (Freiwilligkeit, Ernsthaftigkeit, Ursprung, Wirkungseintritt, Exposition, Kontrollierbarkeit, Vertrautheit, Nutzen, Notwendigkeit, Reparierbarkeit, Fairness der Risikoübernahme) führen dazu, dass objektiv gleiche Risiken unterschiedlich wahrgenommen und damit auch unterschiedlich akzeptiert werden. Litai (1983) verwendete zum Vergleich verschiedener Risikobewertungen sogenannte Konversionsfaktoren.

Tabelle 15 Die Art des Risikos beeinflusst die Wahrnehmung und Bewertung entscheidend: Risikokonversionsfaktoren (RCF, Litai et al., 1983).

\begin{tabular}{cc}
\hline Risikokonversion & Wert des Konversionsfaktors \\
\hline natürlich/,man-made' & 20 \\
gewöhnlich/katastrophal & 30 \\
freiwillig/unfreiwillig & 100 \\
verspätet/sofort & $30(11)$ \\
kontrollierbar/unkontrollierbar & $5-10$ \\
alt/neu & 10 \\
nötig/unnötig & $1(7)$ \\
regelmässig/gelegentlich & 1 \\
\hline
\end{tabular}

Der Zusammenhang zwischen wahrgenommenem und akzeptiertem Risiko überträgt sich zumindest bezüglich einzelner Faktoren - auch auf das tolerierbare Risiko. Was besser akzeptiert ist, gilt auch als besser tolerierbar. Die Risikobewertung und damit die Festlegung von Schutzzielen steht unter dem Einfluss von Wahrnehmung, Nutzen, Aversion, gesellschaftlichem Kontext und Werthaltungen (Abbildung 9).

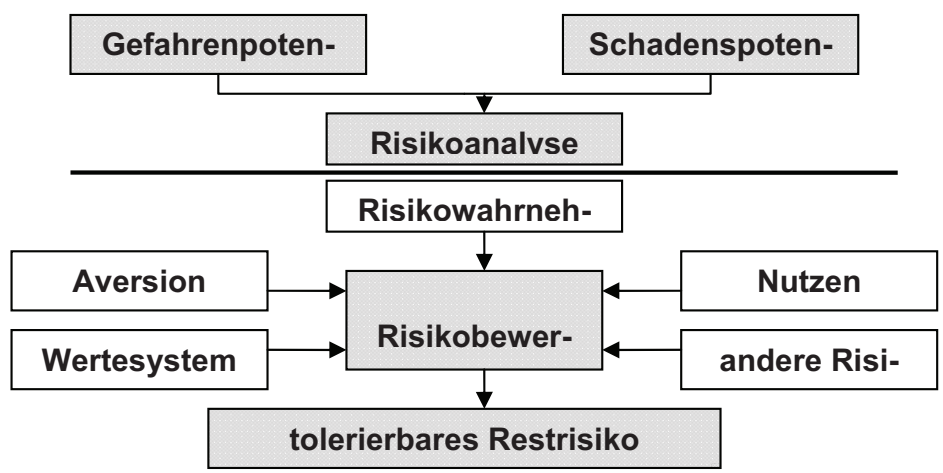

Abbildung 9 Schutzziele und ihre Einflussfaktoren nach Heinimann (2002).

Naturrisiken werden deutlich besser akzeptiert als menschengemachte Risiken (Litai et al., 1983; Tabelle 15). Auch Seiler (1997) vertritt die Ansicht, dass bezüglich Naturgefahren ,etwas höhere' Risiken akzeptiert werden als bezüglich technischer Risiken. Gemäss UntersuJosef Th. Hess: Schutzziele im Umgang mit Naturrisiken in der Schweiz, @ vdf Hochschulverlag 2011 
chungen von Viscusi (1995) werden Naturrisiken allerdings überschätzt, vor allem im Vergleich zu sozialen Risiken (Abbildung 10).

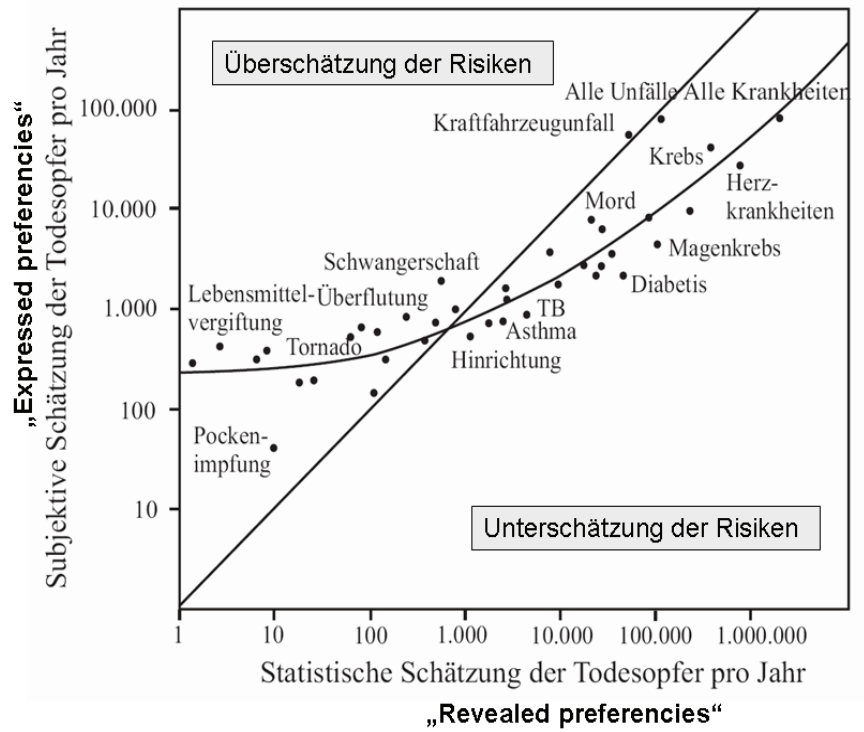

Abbildung 10 Subjektive Wahrnehmung und statistische Schätzung von Todesopfern pro Jahr weichen voneinander ab (Viscusi, 1995 gemäss Darstellung von Proske, 2004).

\subsubsection{Schutzziele im Risikomanagement}

Die Schutzziele stellen eine normative Festlegung dar (Seiler, 1995f; Heinimann, 2002), die sich in der Regel auf eine bestimmte Situation begrenzt (Tabelle 14). Die Festlegung erfolgt meist (hierarchisch) durch Experten oder Verwaltungsbehörden. Eine demokratische Legitimation durch die Risikobetroffenen fehlt häufig. Dennoch besteht meist eine breite Akzeptanz unter den beteiligten Akteuren.

Mit der Entwicklung der Nukleartechnologie wurde die Diskussion über tolerierbare Risiken lanciert und die ersten Grenzwerte wurden festgelegt. Farmer (1967) führte im Umgang mit Kernkraftrisiken eine sogenannte Boundary-Line zwischen akzeptierbaren und nicht akzeptierbaren Risiken ein. Diese Festlegung setzte eine probabilistische Risikoanalyse voraus. Farmer setzte den Ankerpunkt seiner Toleranzlinie für ein einzelnes Kernkraftwerk bei einer Wahrscheinlichkeit von 1.5 * $10^{-4}$ für eine Freisetzung von $10^{4}$ Curies $I^{131}$ fest. Bei einer solchen Freisetzung wird mit 33 Todesfällen (Schilddrüsenkrebs) gerechnet. Die Steigung der Toleranzlinie definierte Farmer völlig subjektiv mit -1.5 im doppelt logarithmischen Raum und führte damit eine überproportionale Gewichtung grosser Schäden ein. 


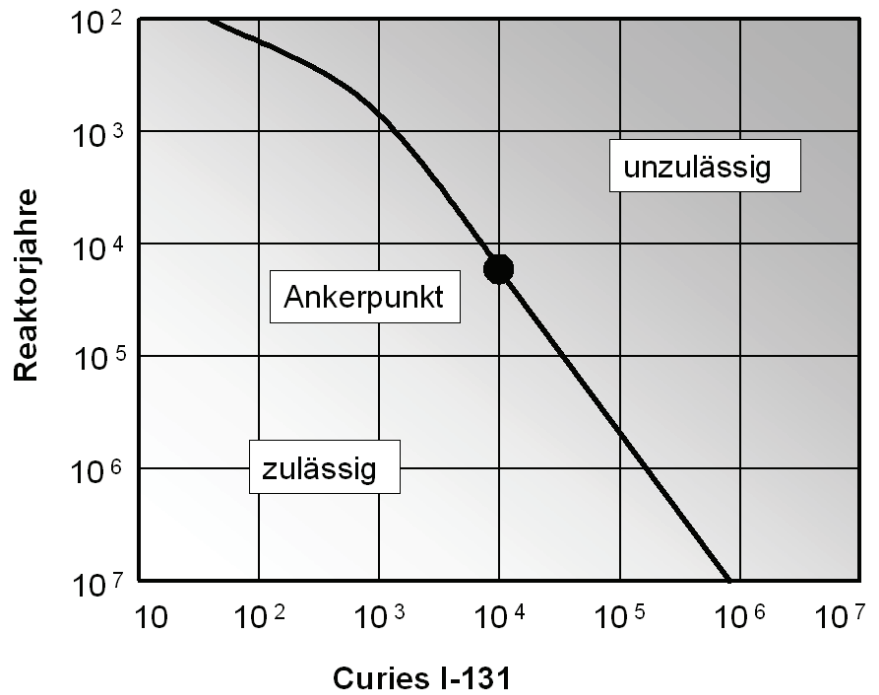

Abbildung 11 ,Farmer-Kurve' zur Abgrenzung zulässiger und unzulässiger Freisetzung von radioaktivem I $^{-131}$ (Farmer, 1967).

\subsection{Grenzwerte für tolerierbare Risiken}

\subsubsection{Grenzwerte der Verletzbarkeit}

In der Schweiz werden seit den 1980er-Jahren bei der Gefahrenkartierung und der Festlegung der künftigen Raumnutzung verschiedene Stufen (Gefahrenstufen) hinsichtlich Wirkungen von Naturprozessen auf Menschen (und Tiere) sowie auf Gebäude unterschieden (BFF, 1984). Massgebend sind Häufigkeit und Intensität eines Gefahrenprozesses (Abbildung 12).

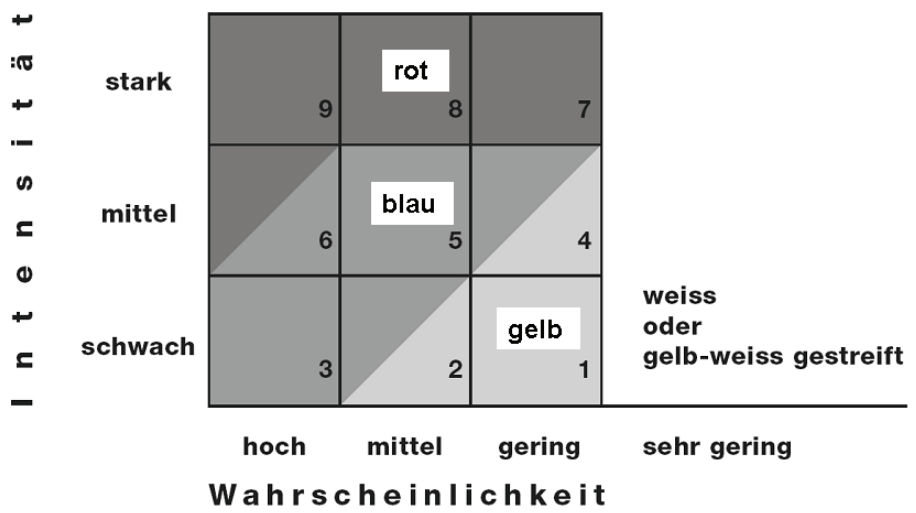

Abbildung 12 Gefahrenstufen im Intensitäts-/Wahrscheinlichkeitsdiagramm für Naturprozesse (BWW, BUWAL, BRP, 1997). 
Tabelle 16 Intensitäten gefährlicher Prozesse (Heinimann et al., 1998).

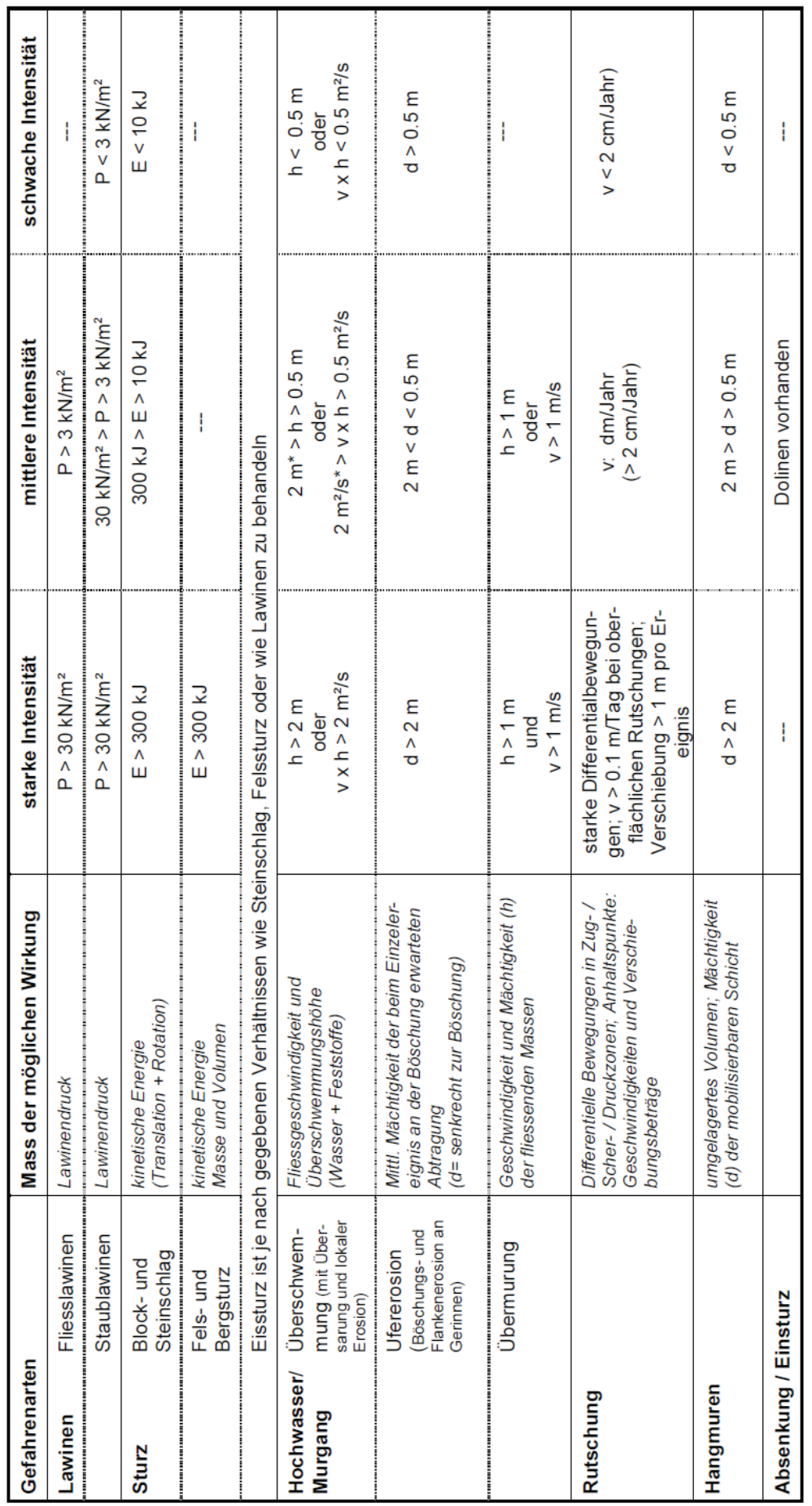


Die Angaben zur Häufigkeit und Intensität erfolgen in je drei Stufen (BWW, BUWAL, BRP, 1997). Bei der Häufigkeit wird unterschieden zwischen 0-30-jährlichen Ereignissen (hohe Wahrscheinlichkeit), 30-100-jährlichen Ereignissen (mittlere Wahrscheinlichkeit) und 100300-jährlichen Ereignissen (geringe Wahrscheinlichkeit). Die Intensitäten sind mit Wertebereichen für physikalische Phänomene verbunden (Heinimann et al., 1998; Tabelle 16):

- Druckwirkung (Lawine);

- kinetische Energie (Sturzprozess);

- Fliessgeschwindigkeit und Fliesshöhe sowie Überschwemmungshöhe und Ablagerungshöhe (Hochwasserprozess und Übermurung).

Es werden folgende Stufen unterschieden:

- Starke Intensität: Menschen und Tiere sind auch innerhalb von Gebäuden gefährdet; mit erheblichen Schäden an Gebäuden bis zur plötzlichen Gebäudezerstörung ist zu rechnen.

- Mittlere Intensität: Menschen und Tiere sind ausserhalb von Gebäuden stark, innerhalb von Gebäuden jedoch kaum gefährdet; Schäden an Gebäuden sind möglich.

- Schwache Intensität: Menschen und Tiere sind innerhalb und ausserhalb von Gebäuden kaum gefährdet; mit Sachschäden in Gebäuden (z. B. Kellerräume) muss gerechnet werden.

Diese Wahrscheinlichkeits- und Intensitätsstufen stellen Grenzwerte der Verletzbarkeit dar.

\subsubsection{Grundlagen zu Grenzwerten des tolerierbaren Risikos}

\subsubsection{Gesellschaftlich akzeptierte Risiken}

Die Festlegung der Höhe des tolerierbaren Risikos basiert auf sozialempirischer Forschung (Starr, 1969; Litai, 1983) und Erhebungen zu gesellschaftlich widerspruchslos hingenommenen Risiken (Schneider, 1996). Die Gesellschaft kennt beispielsweise eine ganze Reihe offensichtlich akzeptierter, individueller Todesfallrisiken (Tabelle 17).

Tabelle 17 Widerspruchslos hingenommene mittlere individuelle Todesfallrisiken (Schneider, 1996).

\begin{tabular}{clll}
\hline $\begin{array}{l}\text { Individuelles Todesfallri- } \\
\text { siko pro Jahr }\end{array}$ & $\begin{array}{l}\text { Todesfallrisiko über alle } \\
\text { Ursachen }\end{array}$ & Berufsrisiken & Diverse Risiken \\
\hline $1 * 10^{-5}$ & & $\begin{array}{l}\text { Brand in Gebäude, Berg- } \\
\text { wandern }\end{array}$ \\
$5 * 10^{-5}$ & & $\begin{array}{l}\text { Arbeiten im Büro } \\
\text { Arbeiten in chemischer } \\
\text { Industrie }\end{array}$ & $\begin{array}{l}\text { Autofahren } \\
10 \text { '000 km als Fussgän- } \\
\text { ger zurücklegen, Delta- }\end{array}$ \\
\hline fliegen
\end{tabular}


Einige Autoren postulieren, dass ein Risiko tolerierbar sei, wenn es das Todesfallrisiko um maximal 1\% verglichen mit allen anderen Todesfallursachen erhöht (Ale, 1991; Vrijling, 1995 und 1998; Morgan, 1997). Grundlage der Betrachtung ist dabei die Altersklasse mit der geringsten Sterblichkeitsrate, die der 10-14-Jährigen, mit einer sich aus der Summe aller Ursachen ergebenden Todesfallwahrscheinlichkeit von $10^{-4}$ bis $10^{-3}$ (Abbildung 13). Flueler und Seiler (2003) leiten auf ähnliche Weise eine maximal tolerierbare Todesfallwahrscheinlichkeit bei unfreiwilligen Risiken von $10^{-5}$ pro Jahr ab.

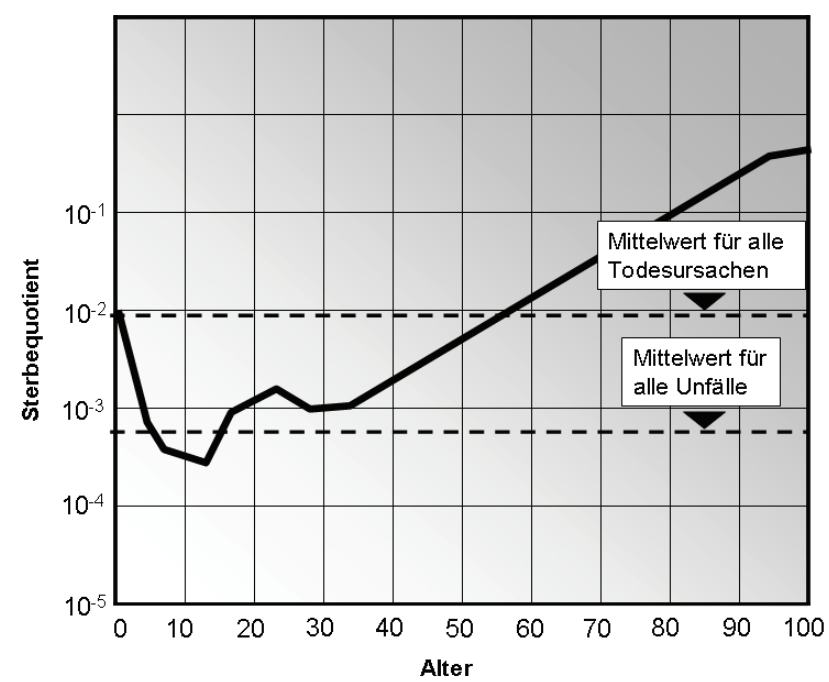

Abbildung 13 Sterbequotient nach Alter (Bundesamt für Statistik, 2000). Tolerierbare Risiken sollen einen Anteil von wenigen Prozent des tiefsten Sterbequotienten nicht übersteigen.

\subsubsection{Zahlungsbereitschaft zur Vermeidung von Risiken}

Eine weitere Grundlage zur Festlegung tolerierbarer Risiken sind die in grosser Zahl vorhandenen Studien über die Zahlungsbereitschaft für risikovermindernde Massnahmen (u. a. Stiefel, 1985; Viscusi, 1994; Tengs, 1995; Schneider, 1996; Wood, 1997; Dini, 1998; Gerdtham, 2002; Kishimoto, 2003). Die Zahlungsbereitschaft (Vermeidungskostenansatz) entspricht dem Betrag, den die Gesellschaft zur Vermeidung von Krankheiten und Unfällen und damit verbundenen Todesfallrisiken auszugeben bereit ist. Aus diesen Untersuchungen lassen sich Angaben über Grenzkosten zur Verhinderung von Todesfällen ableiten (Tabelle 18). 
Tabelle 18 Literaturangaben zur Zahlungsbereitschaft (Grenzkosten/Vermeidungskosten) für die Rettung eines Menschenlebens.

\begin{tabular}{lcccl}
\hline Währung & $\begin{array}{c}\text { Unterer } \\
\text { Wert }\end{array}$ & $\begin{array}{c}\text { Oberer } \\
\text { Wert }\end{array}$ & Quelle/Autor & Bemerkungen \\
\hline Mio. USD & ${ }^{*} 0.9$ & $\left.{ }^{*}\right) 140$ & {$[1]$} & Statistik tatsächlicher Kosten \\
Mio. USD & 0.6 & 4 & {$[2]$} & Konsumentenverhalten \\
Mio. USD & & 2.6 & {$[3]$} & Aufwendungen Verkehrssicherheit \\
Mio. CAD & 1.2 & 3.8 & {$[4]$} & Tatsächliche Zahlungsbereitschaft \\
Mio. USD & & 12 & {$[5]$} & Befragung \\
\hline
\end{tabular}

*) Umgerechnet aus Angaben zu Grenzkosten pro gerettetes Lebensjahr; unterer Wert gilt für medizinische, oberer Wert für Umweltschutzmassnahmen.

Legende zu den Literaturangaben

[1] Tengs \& Adams et al. (1995)

[2] Viscusi (1998)

[3] Persson \& Norinder et al. (2001)

[4] Krupnick \& Alberini et al. (2002)

[5] Perreira \& Sloan (2002)

Dabei fällt eine sehr grosse Divergenz zwischen den für verschiedene Risikosituationen ermittelten Grenzkosten auf. Tengs et al. (1995) untersuchten mehr als 500 Massnahmen zur Verhinderung von vorzeitigen Todesfällen. Die Studie ermittelte eine Spanne zwischen USD 19 '000 pro gewonnenem Lebensjahr für medizinische Massnahmen und USD 2'800'000 für Umweltschutzmassnahmen. Die Ergebnisse solcher Studien waren oft Anlass für Kritik an der Effizienz bestimmter Massnahmen, vor allem jener, die sich aus den Bestimmungen zum Umweltschutz ergeben (Dini, 1998; Lutter, 1999; Subramanian, 2000; Gerdtham, 2002; Kishimoto, 2003).

\subsubsection{Berechnung tolerierbarer Risiken}

Einen Ansatz zur Berechnung von Grenzwerten des tolerierbaren Risikos liefert Whittaker (1986). Er setzt das akzeptierbare Risiko mit der allgemeinen Veränderung der Lebenserwartung in Verbindung. Das akzeptierbare Risiko wird dargestellt als Quotient der allgemeinen jährlichen Zunahme der Lebenserwartung über der aufsummierten Lebenserwartung einer Person. Dieser Ansatz erfüllt die Pareto-Regel. Ein einzelnes Individuum wird durch die Auferlegung eines Risikos nicht schlechter gestellt, die Gesellschaft jedoch profitiert vom mit dem Risiko verbundenen Nutzen. Die Werte für das akzeptierbare Risiko variieren mit dem Alter (und der damit vorhandenen Lebenserwartung) der Person sowie der mittleren Lebenserwartung und der allgemeinen Zunahme der Lebenserwartung in der Gesellschaft, in der die Person lebt.

Aus der Formel zum Lebensqualitätsindex [LQI] lässt sich ebenfalls eine Angabe zur Zahlungsbereitschaft für lebensverlängernde Massnahmen ableiten (Pandey, 2003; Pandey, 2006; Kapitel 2.4.1). Für einen konstanten LQI bei bekanntem Ausgangsbruttoinlandsprodukt [G] und bekannter Lebenserwartung $[E]$ lässt sich unter Annahme eines q errechnen, um wie viel sich das Bruttoinlandsprodukt bei einer bestimmten angestrebten Veränderung der Lebenserwartung ändert. Daraus ergibt sich ein Wert für die Zahlungsbereitschaft für diese Veränderung der Lebenserwartung. 
Tolerierbare Risiken können auch aufgrund der ,zulässigen' Intensitäten gemäss Schutzzielmatrizes errechnet werden (Kapitel 2.6.3.2). Dabei ergeben sich für verschiedene Aktivitäten oder Arten von Raumnutzung Werte für tolerierbare Objektrisiken und tolerierbare individuelle Risiken.

Die Höhe der tolerierbaren Risiken pro Gefahrenquelle lässt sich ebenso aus Akzeptanzlinien in Wahrscheinlichkeits-Ausmass-Diagrammen (F/N-Diagramme) berechnen. Berechnungen dazu siehe Kapitel 2.6.6.2.

\subsubsection{Abstufung der Grenzwerte}

Die Höhe des tolerierbaren Risikos variiert gemäss anerkannter Praxis insbesondere nach dem Grad der Freiwilligkeit (Schneider, 1984; Stiefel, 1985; Schneider, 1996; Bohnenblust \& Slovic, 1998). Freiwilligkeit, aber auch Beherrschbarkeit, persönlicher Nutzen, Zurechenbarkeit oder Langfristigkeit eines Risikos haben einen Einfluss auf die Höhe der Schwellenwerte (Griffiths, 1994; Seiler, 1995a, 1995c, 1995e, 2000 und 2002; Tansel, 1995; Subramanian, 2000; Frederick, 2003). Für freiwillige Risiken wird eine Todesfallwahrscheinlichkeit etwa in der Grössenordnung der natürlichen Sterbewahrscheinlichkeit, also $10^{-2}$ bis $10^{-3}$ pro Jahr, angegeben. Für vollkommen unfreiwillig eingegangene Risiken liegen die angegebenen Werte etwa tausendmal tiefer, zwischen $10^{-5}$ und $10^{-6}$ pro Jahr (Merz et al., 1995; Vrijling, 1995 und 1998; Stallen, 1996). Die UK Health and Safety Executive legt den Toleranzwert für unfreiwillige Risiken bei $3 * 10^{-7}$ pro Jahr fest (Cassidy, 1996).

Die Freiwilligkeit ist in erster Linie Ausdruck der vorhandenen Handlungsoptionen. Entscheidend ist ferner der direkte Nutzen, den der Risikobetroffene aus einer Handlung zieht. Vrijling (1995 und 1998) und Stallen (1996) versuchen, die Risikokategorien durch Einführung eines ,Policy-Factors' genauer zu umschreiben (Tabelle 19).

Tabelle 19 ,Policy-Factor' zur Klassierung von Risikokategorien (Vrijling, 1998). Freiwilligkeit und direkter Nutzen beeinflussen die Höhe des tolerierbaren Risikos.

\begin{tabular}{clll}
\hline \multicolumn{3}{l}{ Wert des ,Policy-Factors' als Funktion von Freiwilligkeit und Nutzen } \\
Policy-Factor & Freiwilligkeit & Direkter Nutzen & Beispiel \\
\hline $10^{2}$ & total freiwillig & ja & Bergsteigen \\
$10^{1}$ & freiwillig & ja & Motorradfahren \\
$10^{0}$ & neutral & ja & Autofahren \\
$10^{-1}$ & unfreiwillig & teilweise & Arbeit in einer Fabrik \\
$10^{-2}$ & unfreiwillig & nein & Wohnen bei einer Flüssiggastankanlage \\
\hline
\end{tabular}

Schneider (1996) führte zur Kategorisierung der Risiken nebst der ,Freiwilligkeit' auch die ,Möglichkeit der Einflussnahme' ein (Abbildung 14). 

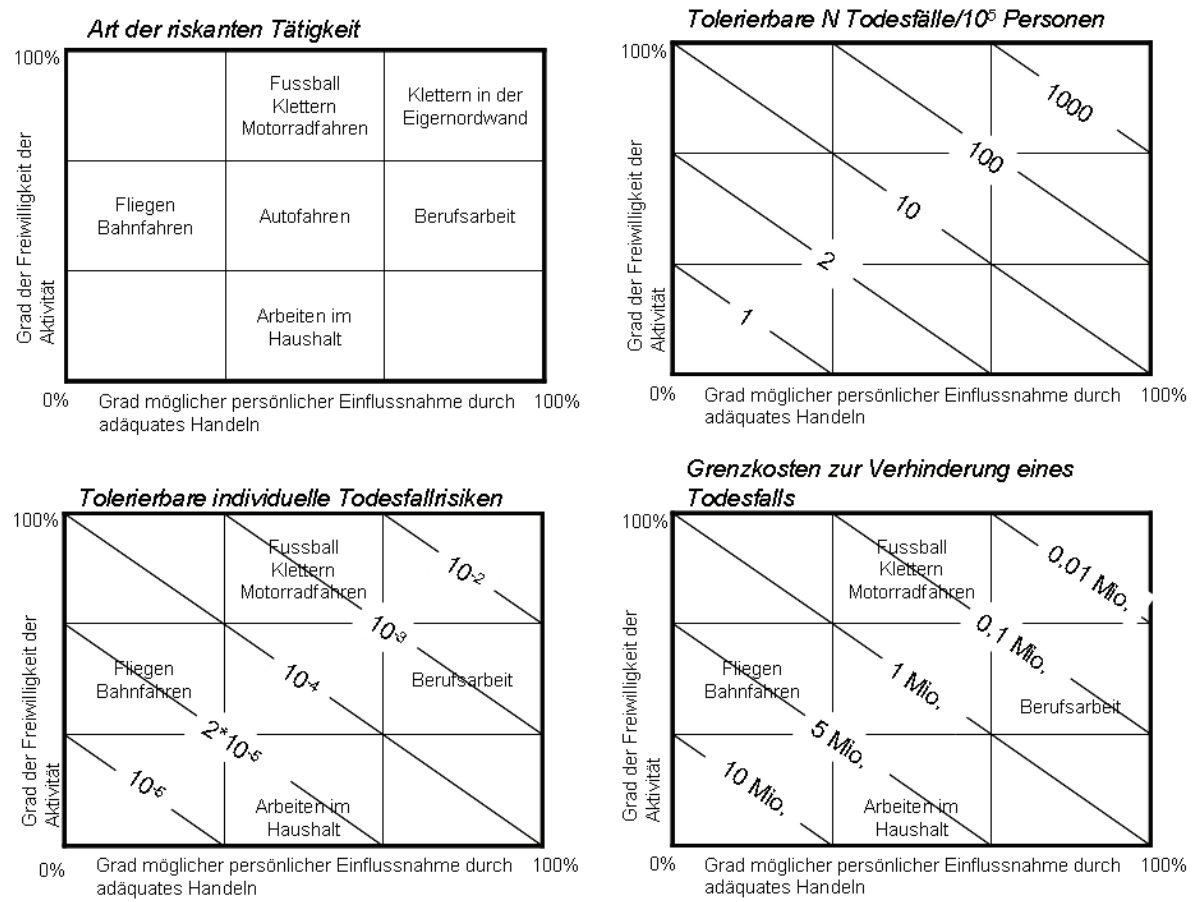

Abbildung 14 Risikokategorien nach Grad der Freiwilligkeit und Möglichkeit der Einflussnahme. Tolerierbare individuelle Todesfallrisiken und Grenzkosten zur Verhinderung von Todesfällen stehen in einem umgekehrt proportionalen Verhältnis (Schneider, 1996).

Wie bereits dargelegt liegt der Unterschied zwischen unfreiwilligen und freiwilligen tolerierbaren Todesfallrisiken in einem Verhältnis von 1:1000. (Die Diagonalenwerte in Abbildung 14 oben rechts entsprechen tolerierbaren Todesfallrisiken pro 100'000 Personen und einem Jahr). Damit ergeben sich Werte für ein tolerierbares individuelles Todesfallrisiko von $10^{-2}$ und $10^{-5}$ pro Jahr (Abbildung 14, unten links).

Einzelne Autoren widersetzen sich dem Konzept einer generellen Abstufung von Risiken nach dem Grad der Freiwilligkeit (Ramsberg, 2002). Eine Reduktion der Aufwendungen zur Verhinderung von Todesfällen bei freiwilligen Risiken sei nur gegeben, wenn der Risikobetroffene

- alle relevanten Informationen über das Risiko, den Nutzen und die Alternativen besitzt;

- die Fähigkeit und Erfahrung hat, diese Informationen zu verarbeiten und

- für sein eingegangenes Risiko kompensiert wurde.

\subsubsection{Grenzwerte für tolerierbare individuelle Todesfallrisiken}

\subsubsection{Grenzwerte aus der Literatur}

Zulässige individuelle Todesfallrisiken werden als Grenzwerte angegeben. Zu den frühesten derartigen Festlegungen gehören jene von Starr (1981), der eine tolerierbare obere Grenze von $10^{-4}$ pro Jahr für kernkraftwerkbedingte Todesfallrisiken angab. Im gleichen Zusammenhang nannte er einen Zielwert von $10^{-8}$ pro Jahr. Diese Werte würden gesellschaftlich akzeptiert und berücksichtigen einen Spielraum für Unsicherheiten. 
In den meisten Literaturangaben liegen tolerierbare Todesfallwahrscheinlichkeiten zwischen $10^{-6}$ und $10^{-3}$ pro Jahr (Merz et al., 1995; Bohnenblust \& Slovic, 1998; Seiler, 2000; Tabelle 20). Die Angaben zur Höhe des tolerierbaren Risikos variieren stark (Faktor 1'000 bis 10'000) und hängen von der Art der Risiken (freiwillig/selbstbestimmt oder unfreiwillig/fremdbestimmt) und vom Kontext ab (Merz et al., 1995). Für Risiken am Arbeitsplatz gilt verbreitet ein Wert von $10^{-4}$ (Wilhelm, 1999). Dieser wurde auch für naturgefahrenbedingte Berufsrisiken von Strassendiensten angegeben (Margreth, 2003). Insgesamt herrscht in der Literatur ein breiter Konsens darüber, dass das unfreiwillig eingegangene tolerierbare individuelle Todesfallrisiko den Wert von $10^{-5}$ bis $10^{-6}$ nicht übersteigen sollte. Im Leitfaden Risikokonzept Lawinen der PLANAT wird allerdings ein höherer Wert, nämlich $10^{-4}$ bis $10^{-3}$ vorgeschlagen (Bründl M. et al., 2006). Die tolerierbare Todesfallwahrscheinlichkeit ist bei Naturrisiken höher angesetzt als bei technischen Risiken - anhand der untersuchten Beispiele etwa im Verhältnis 2:1 (oberer Wert) bis 70:1 (unterer Wert) (Abbildung 15).

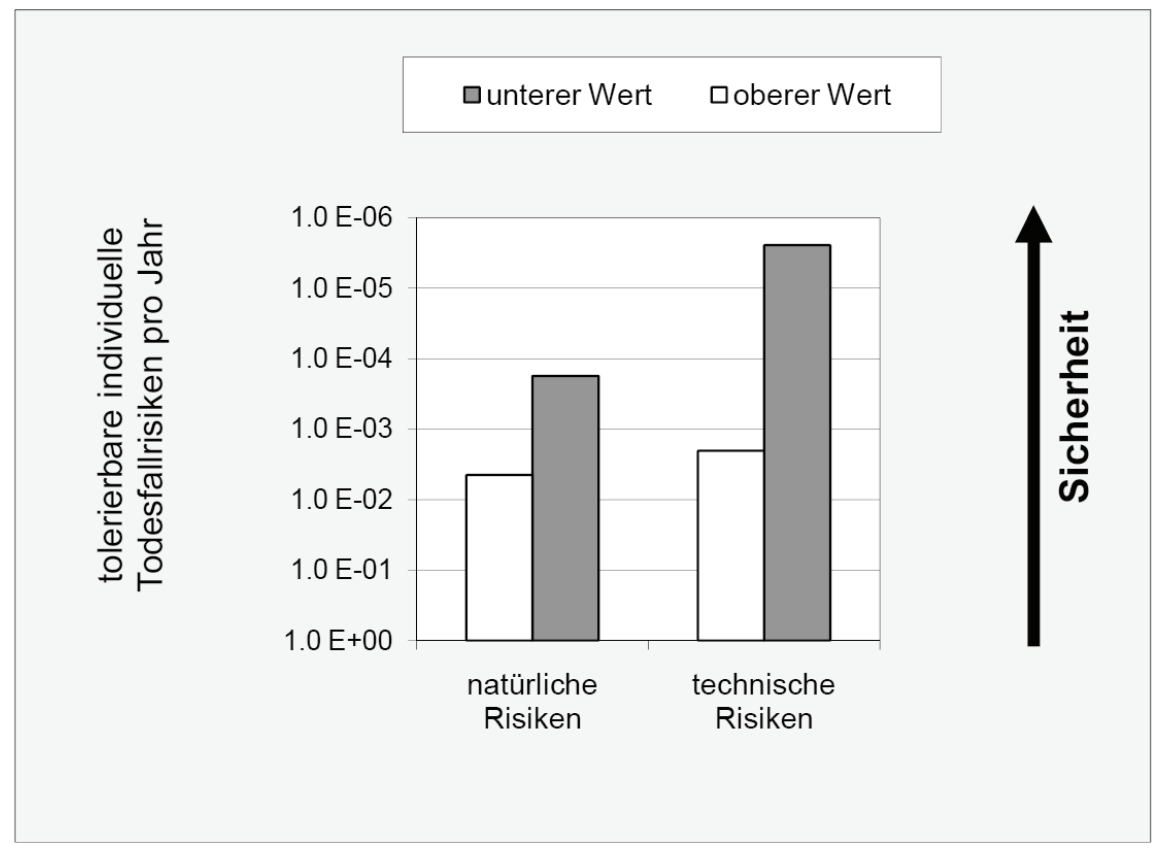

Abbildung 15 Tolerierbare individuelle Todesfallrisiken mit natürlicher und technischer Ursache gemäss Angaben aus der Literatur. Für technische Risiken sind deutlich höhere Sicherheiten gefordert.

Aus einer Zusammenstellung der Grenzwerte für tolerierbare individuelle Todesfallrisiken wird deutlich, dass der Wert in den letzten 25 Jahren etwa konstant blieb (Tabelle 20). 
Tabelle 20 Grenzwerte für tolerierbare individuelle Todesfallwahrscheinlichkeiten.

\begin{tabular}{|c|c|c|c|c|}
\hline \multirow[t]{2}{*}{ Anwendungsbereich } & \multirow[t]{2}{*}{$\begin{array}{l}\text { Bezeichnung des } \\
\text { Schutzzieles/Autor }\end{array}$} & \multirow[t]{2}{*}{ Jahr } & \multicolumn{2}{|c|}{$\begin{array}{l}\text { Höhe des Grenzwertes } \\
\text { (Todesfallwahrscheinlichkeit/Jahr) }\end{array}$} \\
\hline & & & untere Grenze & obere Grenze \\
\hline Kernkraftwerke & Starr & 1981 & $10^{-8}$ & $10^{-4}$ \\
\hline Berufsrisiken WSUME & Bienz, 2000 & 1991 & $10^{-4}$ & $10^{-4}$ \\
\hline Drittrisiken WSUME & Bienz, 2000 & 1991 & $10^{-5}$ & $10^{-5}$ \\
\hline Hangrutsche bestehende Risiken & Fell & 1994 & $10^{-3}$ & $10^{-2}$ \\
\hline Berufsrisiken & Paté-Cornell & 1994 & $10^{-6}$ & $10^{-4}$ bis $10^{-3}$ \\
\hline Gesellschaftliche Risiken & Paté-Cornell & 1994 & $10^{-8}$ bis $10^{-7}$ & $10^{-6}$ bis $10^{-5}$ \\
\hline Technische Risiken allgemein & Merz et al. & 1995 & $10^{-6}$ & $10^{-2}$ \\
\hline Industriebetriebe, Pipelines & Cassidy & 1996 & $3 * 10^{-7}$ & $10^{-5}$ \\
\hline Bauwesen & Schneider & 1996 & $10^{-5}$ & $10^{-2}$ \\
\hline Naturrisiken allgemein & Borter & 1999 & $10^{-6}$ & $10^{-2}$ \\
\hline Berufsrisiken Naturgefahren & Wilhelm & 1999 & $10^{-4}$ & $10^{-4}$ \\
\hline Berufsrisiken & Paté-Cornell & 2002 & $10^{-6}$ & $10^{-3}$ \\
\hline Gesellschaftliche Risiken & Paté-Cornell & 2002 & $10^{-7}$ bis $10^{-6}$ & $10^{-4}$ \\
\hline Hangrutsche bestehende Risiken & Ko & 2003 & $10^{-5}$ & $10^{-4}$ \\
\hline Hangrutsche neue Risiken & Ko & 2003 & $10^{-6}$ & $10^{-5}$ \\
\hline Fracht-/Personenzüge & Ko & 2003 & $10^{-6}$ & $10^{-6}$ \\
\hline Erdbebengef. bestehende Gebäude & SIA & 2004 & $10^{-5}$ & $10^{-5}$ \\
\hline Naturrisiken allgemein & PLANAT & 2004 & $4 * 10^{-6}$ & $10^{-2}$ \\
\hline Kernkraftwerke & INSAG & 2006 & $10^{-6}$ & $10^{-6}$ \\
\hline Naturrisiken allgemein & PLANAT Leitfaden & 2006 & $2 * 10^{-4}$ & $10^{-3}$ \\
\hline
\end{tabular}




\subsubsection{Grenzwerte in Schutzzielmatrizes}

Schutzzielmatrizes stellen dar, welche Intensität eines Gefahrenprozesses bei einer bestimmten Jährlichkeit für eine bestimmte Art der Raumnutzung (Objektkategorie) zulässig ist. Dieses Schutzzielkonzept wird im Management von Naturrisiken häufig angewendet (Kapitel 2.7.6). Mit der Methode gemäss Anhang II (Risiko als Integral im F/N-Diagramm mit linearer Skalierung) wurde berechnet, welche individuellen Risiken sich aus den Schutzzielen gemäss der Schutzzielmatrix nach BUWAL Umweltmaterialien 107 ergeben (Borter, 1999). Die Objektarten wurden einer der vier Risikokategorien (,Freiwilligkeitsklassen') zugeordnet (Schneider, 1984; Schneider, 1996; Anhang III, 3. Spalte). Anhand der tolerierbaren Intensitäten gemäss Schutzzielmatrix (Borter, 1999) wurden die kollektiven tolerierbaren Risiken pro Objektkategorie für eine normierte Fläche von einer Are berechnet. Aus den in BUWAL Umweltmaterialien (Borter, 1999) enthaltenen Angaben zur Anzahl gefährdeter Personen wurde die Anzahl betroffener Individuen (Anhang III, 4. Spalte) abgeschätzt, sodass sich mittels Division der Objektrisiken durch die Zahl betroffener Individuen das individuelle Risiko für jede Objektkategorie berechnen lässt. Es wurde von einer mittleren, ,typischen' Anzahl betroffener Individuen ausgegangen. Im Einzelfall kann die Zahl betroffener Individuen erheblich variieren, was entsprechende Auswirkungen auf das Berechnungsergebnis zur Folge hat.

Tabelle 21 Tolerierbares individuelles Risiko gemäss Schutzzielmatrix nach Borter (1999) im Vergleich zu den tolerierbaren individuellen Todesfallrisiken nach PLANAT (2004).

\begin{tabular}{|c|c|c|c|}
\hline Objektkategorie & Risikokategorie ${ }^{*}$ & $\begin{array}{l}\text { Tolerierbares individuelles } \\
\text { Todesfallrisiko gem. Be- } \\
\text { rechnung anhand Schutz- } \\
\text { zielmatrix }\end{array}$ & $\begin{array}{l}\text { Tolerierbares individuelles } \\
\text { Todesfallrisiko gem. An- } \\
\text { gabe in PLANAT (2004b) }\end{array}$ \\
\hline
\end{tabular}

\section{Lawinenrisiken}

\begin{tabular}{llll}
\hline Ein-/Zweifamilienhaus & 4 & $7.29 * 10^{-4}$ & $3 * 10^{-5}$ bis $4 * 10^{-6}$ \\
Mehrfamilienhaus & 4 & $4.36 * 10^{-7}$ & $3 * 10^{-5} \mathrm{bis} 4 * 10^{-6}$ \\
Stall & 3 & $4.12 * 10^{-4}$ & $2 * 10^{-4} \mathrm{bis} 3 * 10^{-5}$ \\
Weidestall & 2 & $9.12 * 10^{-2}$ & $10^{-3}$ bis $2 * 10^{-4}$ \\
Eisenbahn (Benutzer) & 4 & $8.39 * 10^{-9}$ & $3 * 10^{-5}$ bis $4 * 10^{-6}$ \\
Gewerbebau & 3 & $1.54 * 10^{-6}$ & $2 * 10^{-4}$ bis $3 * 10^{-5}$ \\
Autobahn (Benutzer) & 3 & $2.78 * 10^{-9}$ & $2 * 10^{-4}$ bis $3 * 10^{-5}$ \\
Gemeindestrasse (Benutzer) & 3 & $7.74 * 10^{-6}$ & $2 * 10^{-4}$ bis $3 * 10^{-5}$ \\
Wanderweg & 1 & $4.18 * 10^{-7}$ & $10^{-2}$ bis $10^{-3}$
\end{tabular}

Fortsetzung Tabelle nächste Seite 


\section{Überschwemmungsrisiken}

\begin{tabular}{|c|c|c|c|}
\hline Ein-/Zweifamilienhaus & 4 & $4.62 * 10^{-10}$ & $3 * 10^{-5}$ bis $4 * 10^{-6}$ \\
\hline Mehrfamilienhaus & 4 & $8.58 * 10^{-8}$ & $3 * 10^{-5}$ bis $4 * 10^{-6}$ \\
\hline Stall & 3 & $1.92 * 10^{-10}$ & $2 * 10^{-4}$ bis $3 * 10^{-5}$ \\
\hline Weidestall & 2 & $1.30 * 10^{-9}$ & $10^{-3}$ bis $2 * 10^{-4}$ \\
\hline Eisenbahn (Benutzer) & 4 & $2.95 * 10^{-8}$ & $3 * 10^{-5}$ bis $4 * 10^{-6}$ \\
\hline Gewerbebau & 3 & $8.58 * 10^{-8}$ & $2 * 10^{-4}$ bis $3 * 10^{-5}$ \\
\hline Autobahn (Benutzer) & 3 & $3.06 * 10^{-11}$ & $2 * 10^{-4}$ bis $3 * 10^{-5}$ \\
\hline Gemeindestrasse (Benutzer) & 3 & $6.01 * 10^{-8}$ & $2 * 10^{-4}$ bis $3 * 10^{-5}$ \\
\hline Wanderweg & 1 & $4.95 * 10^{-9}$ & $10^{-2}$ bis $10^{-3}$ \\
\hline \multicolumn{4}{|l|}{ Sturzrisiken } \\
\hline Ein-/Zweifamilienhaus & 4 & $4.62 * 10^{-6}$ & $3 * 10^{-5}$ bis $4 * 10^{-6}$ \\
\hline Mehrfamilienhaus & 4 & $1.36 * 10^{-7}$ & $3 * 10^{-5}$ bis $4 * 10^{-6}$ \\
\hline Stall & 3 & $1.92 * 10^{-5}$ & $2 * 10^{-4}$ bis $3 * 10^{-5}$ \\
\hline Weidestall & 2 & $1.60 * 10^{-4}$ & $10^{-3}$ bis $2 * 10^{-4}$ \\
\hline Eisenbahn (Benutzer) & 4 & $7.50 * 10^{-9}$ & $3 * 10^{-5}$ bis $4 * 10^{-6}$ \\
\hline Gewerbebau & 3 & $2.94 * 10^{-7}$ & $2 * 10^{-4}$ bis $3 * 10^{-5}$ \\
\hline Autobahn (Benutzer) & 3 & $1.75 * 10^{-9}$ & $2 * 10^{-4}$ bis $3 * 10^{-5}$ \\
\hline Gemeindestrasse (Benutzer) & 3 & $5.18 * 10^{-6}$ & $2 * 10^{-4}$ bis $3 * 10^{-5}$ \\
\hline Wanderweg & 1 & $4.17 * 10^{-7}$ & $10^{-2}$ bis $10^{-3}$ \\
\hline \multicolumn{4}{|l|}{ Murgangrisiken } \\
\hline Ein-/Zweifamilienhaus & 4 & $3.23 * 10^{-4}$ & $3 * 10^{-5}$ bis $4 * 10^{-6}$ \\
\hline Mehrfamilienhaus & 4 & $3.59 * 10^{-6}$ & $3 * 10^{-5}$ bis $4 * 10^{-6}$ \\
\hline Stall & 3 & $1.35 * 10^{-4}$ & $2 * 10^{-4}$ bis $3 * 10^{-5}$ \\
\hline Weidestall & 2 & $9.23 * 10^{-3}$ & $10^{-3}$ bis $2 * 10^{-4}$ \\
\hline Eisenbahn (Benutzer) & 4 & $2.95 * 10^{-8}$ & $3 * 10^{-5}$ bis $4 * 10^{-6}$ \\
\hline Gewerbebau & 3 & $1.54 * 10^{-6}$ & $2 * 10^{-4}$ bis $3 * 10^{-5}$ \\
\hline Autobahn (Benutzer) & 3 & $9.38 * 10^{-9}$ & $2 * 10^{-4}$ bis $3 * 10^{-5}$ \\
\hline Gemeindestrasse (Benutzer) & 3 & $3.05 * 10^{-5}$ & $2 * 10^{-4}$ bis $3 * 10^{-5}$ \\
\hline Wanderweg & 1 & $2.05 * 10^{-6}$ & $10^{-2}$ bis $10^{-3}$ \\
\hline
\end{tabular}

*) 1 = freiwillig, 4 = unfreiwillig

Schutzziel gem. PLANAT 2004b verletzt. 


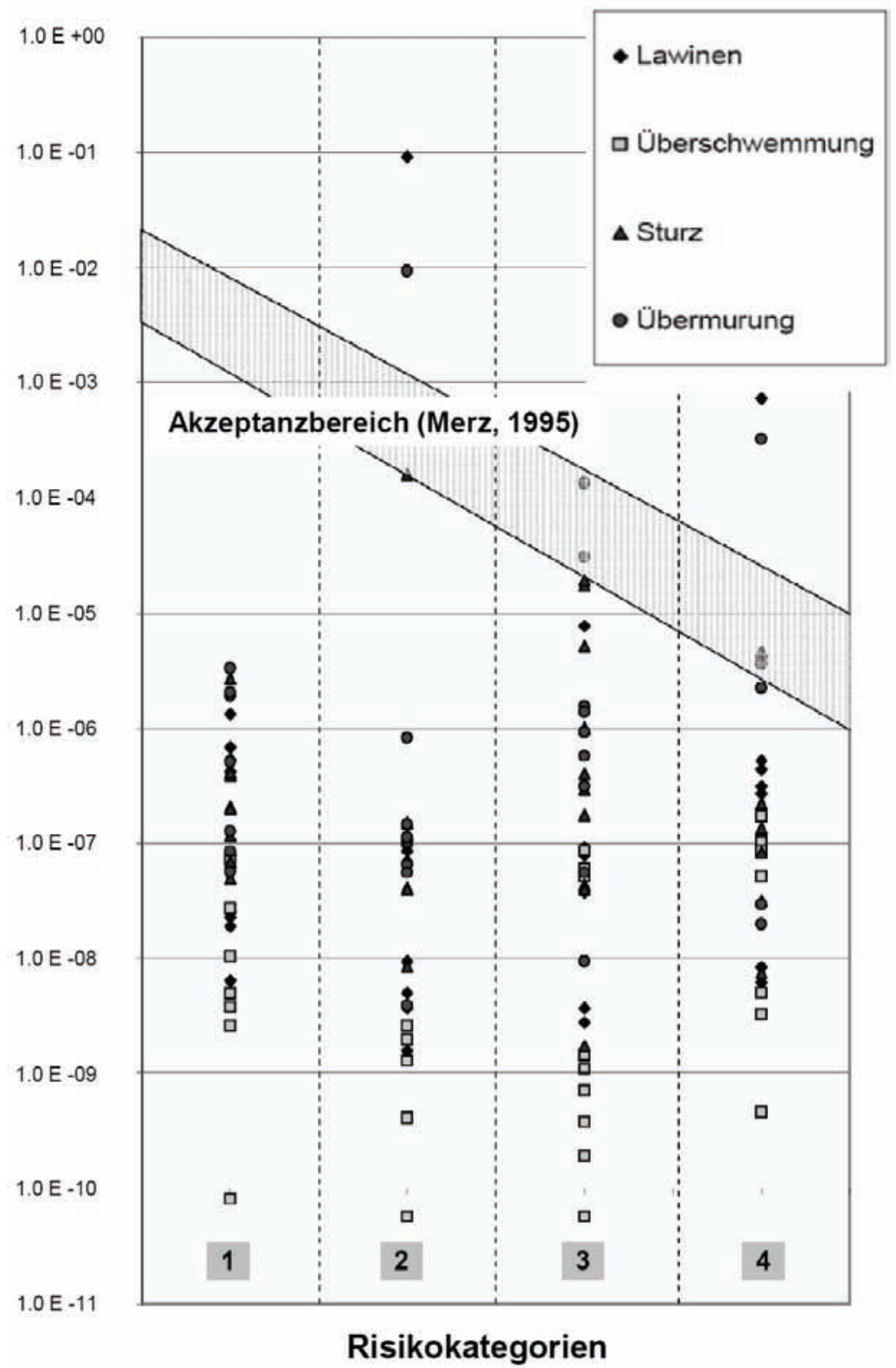

Abbildung 16 Tolerierbare individuelle Todesfallrisiken einzelner Objektkategorien gemäss Schutzzielmatrix (Borter, 1999) (vgl. Tabelle 21) im Vergleich zu tolerierbaren individuellen Todesfallrisiken aus der Literatur (Merz et al., 1995, schraffierter Bereich). Es werden vier Risikokategorien zwischen freiwillig eingegangenen, beeinflussbaren (Kategorie [1]) und unfreiwillig eingegangenen, unbeeinflussbaren (Kategorie [4]) Risiken unterschieden. 
Der Vergleich zwischen den berechneten tolerierbaren individuellen Risiken und den Grenzwerten, die in der Literatur für die jeweilige Risikokategorie angegeben sind, zeigte bei einigen Objektarten erhebliche Unterschiede (Abbildung 16, Tabelle 21 und Anhang IV). Bei Lawinen, Sturzprozessen und Murgängen liegen die errechneten Risiken für einzelne Objektarten deutlich über der oberen Akzeptanzlinie. Demgegenüber liegen die berechneten individuellen Todesfallrisiken bei Überschwemmungen, für viele Objektarten auch bei den übrigen untersuchten Gefahrenprozessen, teilweise weit unterhalb der unteren Akzeptanzlinie. Die detaillierten Resultate dieser Vergleichsrechnungen sind in Anhang IV grafisch dargestellt.

Insgesamt zeigt der Vergleich zwischen den berechneten Grenzwerten und den Angaben in der Literatur, dass die Anwendung der Schutzzielmatrix selten zur Überschreitung, häufig aber zur Unterschreitung tolerierbarer individueller Risiken führt. Aus dem Vergleich wird zudem deutlich, dass die Schutzzielmatrix nicht nur auf die unmittelbaren individuellen Todesfallrisiken ausgerichtet ist, sondern in ganz bedeutendem Mass andere Schäden, wie z. B. Sachschäden und indirekte Schäden, berücksichtigt. So ergibt sich aus der Schutzzielmatrix z. B. für Verkehrsträger ein deutlich höherer Schutz als dies aufgrund der tolerierbaren individuellen Risiken erforderlich wäre. Ebenso sieht die Schutzzielmatrix einen wesentlich höheren Schutz der Individuen in zusammenhängenden Siedlungsgebieten im Vergleich zu Individuen in Einzelgebäuden vor. Eine mögliche Erklärung ist die höhere Gewichtung von Grossschäden durch die Schutzzielmatrix.

\subsubsection{Grenzkosten zur Verhinderung von Todesfällen}

Die meisten Angaben für Grenzkosten zur Verhinderung von Todesfällen liegen zwischen CHF 0.5 bis 2 Mio. für freiwillige und CHF 5 bis 20 Mio. für unfreiwillige Risiken (Merz et al., 1995; Borter, 1999; PLANAT, 2004b). Für Arbeitsplatzrisiken wurden die Grenzkosten gemäss den meisten Literaturangaben bei CHF 4 Mio. festgelegt (Seiler, 2000; Bienz, 2000). Neuere Literatur empfiehlt, den Grenzkostenansatz im Management von Naturrisiken im Siedlungsraum oder an Verkehrsachsen generell bei CHF 5 Mio. pro verhindertem Todesfall festzulegen (Bründl et al., 2006). 
Tabelle 22 Grenzkosten zur Verhinderung von Todesfällen.

\begin{tabular}{|c|c|c|c|c|}
\hline \multirow[t]{2}{*}{$\begin{array}{l}\text { Bezeichnung des } \\
\text { Schutzzieles/Autor }\end{array}$} & \multirow[t]{2}{*}{ Jahr } & \multicolumn{2}{|c|}{$\begin{array}{l}\text { Höhe der Grenzkosten } \\
\text { (Mio. CHF/verh. Todesfall) }\end{array}$} & \multirow[t]{2}{*}{ Anwendungsbereich } \\
\hline & & Untere Grenze & Obere Grenze & \\
\hline Seiler, 2000 & 1988 & 1 & 15 & Nicht-Berufsunfälle \\
\hline VBS & 1991 & 4 & 8 & Berufsrisiken WSUME \\
\hline VBS & 1991 & 10 & 20 & Drittrisiken WSUME \\
\hline Merz et al. & 1995 & 0.5 & 2 & technische Risiken, freiwillig \\
\hline Merz et al. & 1995 & 5 & 20 & technische Risiken, unfreiwillig \\
\hline Schneider & 1996 & 0.01 & 10 & Risiken allg., Bauwesen \\
\hline Borter & 1999 & 0.5 & 2 & natürl. Risiken allg., freiwillig \\
\hline Borter & 1999 & 5 & 20 & natürl. Risiken allg., unfreiwillig \\
\hline SIA & 2004 & 10 & 10 & Erdbebengefahr, best. Gebäude \\
\hline PLANAT & 2004 & 1 & 2 & natürl. Risiken allg., freiwillig \\
\hline PLANAT & 2004 & 10 & 20 & natürl. Risiken allg., unfreiwillig \\
\hline PLANAT Leitfaden & 2006 & 5 & 5 & Naturrisiken Siedlung/Verkehr \\
\hline
\end{tabular}

Ähnlich wie bei den Grenzwerten für individuelle Todesfallrisiken sind auch die Grenzkosten zur Verhinderung eines Todesfalls nach Risikokategorien (Kapitel 2.6.1.4) abgestuft.

Schneider (1996) verbindet die beiden Konzepte und hält zwischen den Werten für tolerierbare individuelle Todesfallrisiken und jenen für tolerierbare Grenzkosten eine umgekehrt proportionale Abhängigkeit fest. Dabei werden im entsprechenden Diagramm (Abbildung 14, unten links) Grenzwerte von $10^{-5}$ für tolerierbare unfreiwillige Todesfallrisiken und von $10^{-2}$ für entsprechende freiwillige Risiken eingesetzt. Die Grenzkosten werden auf CHF 10 Mio. pro verhindertem Todesfall bei völlig unfreiwilligen und CHF 10'000 pro verhindertem Todesfall bei völlig freiwilligen Risiken angesetzt (Abbildung 14, unten rechts). Bei Verwendung dieser Werte ergibt das Produkt aus tolerierbarem Todesfallrisiko pro Jahr und den für die entsprechende Aktivität veranschlagten Grenzkosten an jeder Stelle des Diagramms konstant einen Betrag von CHF 100 pro Jahr.

Vereinzelt werden Grenzkostenwerte oder Kostenwirksamkeiten angegeben, die zur Erreichung eines tolerierbaren individuellen Risikos einzuhalten sind: Gemäss der Wegleitung zur Verbesserung der Erdbebensicherheit bestehender Gebäude gilt eine Erdbebensicherungsmassnahme als noch zumutbar, wenn die Kosten unter 100 Mio. CHF pro gerettetes Menschenleben liegen (Duvernay, 2005). Dieser Betrag umfasst alle Aufwendungen mit dem Ziel, das individuelle Todesfallrisiko für eine bestimmte (höchstbetroffene) Person auf unter $10^{-5}$ pro Jahr zu reduzieren. Diese Festlegung der zumutbaren Kosten ist bemerkenswert. Es handelt sich nämlich um eine Vorgabe von Maximalkosten für die Reduktion individueller Todesfallrisiken. Der Wert wurde für individuelle Risiken um Faktor 10 höher angesetzt als für kollektive Risiken. 
Der PLANAT ,Synthesebericht - Strategie zur Umsetzung des Bundesratsbeschlusses vom 20. August 2003' empfiehlt in Fällen, in denen sich die Einhaltung des individuellen Risikos als sehr aufwendig erweist, die Grenzkosten für das individuelle Risiko beim zwei- bis maximal dreifachen Wert der Grenzkosten für das kollektive Risiko anzusetzen (PLANAT, 2004b).

\subsubsection{Grenzkosten zur Verhinderung weiterer Schäden}

In der Literatur sind vereinzelte Angaben zu Grenzkosten für andere Schadensindikatoren wie Verletzte, Evakuierte oder geschädigte Lebensgrundlagen zu finden. Die wenigen in der Literatur bekannten Angaben zu Umweltschäden bewerten diese aus der Optik der Umwelt als Lebensgrundlage der Menschen (BABS, 2003a; Wilhelm, 1997).

Tabelle 23 Grenzkosten zur Verhinderung verschiedener Arten von Schäden aus KATARISK (BABS, 2003a).

\begin{tabular}{lll}
\hline Schadensindikator & Definition & Grenzkosten \\
\hline $\begin{array}{l}\text { Physisch Geschädigte } \\
(P)\end{array}$ & $\begin{array}{l}\text { Todesopfer } \\
\text { Verletzte } \\
\text { Mittelwert für Todesopfer, Verletzte, Kranke }\end{array}$ & $\begin{array}{l}\text { CHF 0,6-20 Mio./P } \\
\text { CHF 4'000-300'000/P } \\
\text { CHF 1-2 Mio./P }\end{array}$ \\
Evakuierte (Evak.) & Mittelwert für Evakuierte & CHF 10'000/Evak. \\
Unterstützungsbedürf- & kurzfristig mit hoher Selbständigkeit & CHF 10'000/Unt. \\
tige (Unt.) & langfristig pflegeintensiv & CHF 100'000 /Unt. \\
Lebensgrundlagen & landwirtschaftliche Nutzfläche (mittelfristige Schädigung) & CHF 1 Mio./km² \\
& Wald (langfristige Schädigung) & CHF 10 Mio./km² \\
& langfristig geschädigte Lebensgrundlagen infolge chemi- & CHF 50 Mio./km² \\
& scher Störfälle & \\
& langfristig geschädigte Lebensgrundlagen infolge KKW- & CHF 200 Mio./km² \\
& Störfälle & \\
\hline \multirow{2}{*}{ Sachschäden } & & CHF 1/CHF \\
\hline
\end{tabular}

Die Grenzkosten zur Vermeidung von Schäden an Lebensgrundlagen basieren auf Berechnungen des Aufwandes einer Dekontamination und sind daher für die Belange des Managements von Naturrisiken vermutlich hoch angesetzt. Naturgefahren haben in der Regel Räumungskosten und kurzzeitige Produktionsausfälle zur Folge, eine Dekontamination ist kaum erforderlich. Bei Sachschäden legt die KATARISK-Studie als Grenzkosten maximal einen Franken pro verhindertem Franken Schaden fest (BABS, 2003a). In der Rechtsprechung wurde dieser Grundsatz untermauert durch die ,Learned Hand Rule', nach welcher Sicherheitsmassnahmen solange getroffen werden müssen, als der Erwartungswert des dadurch vermiedenen Schadens grösser ist als die Kosten für seine Vermeidung (Mathis, 2004). Bemerkenswert ist im Vergleich dazu der PLANAT-Schutzzielvorschlag 2004 (Tabelle 28), wonach die Grenzkosten für unfreiwillige Sachrisiken bis zum vierfachen Wert des vermiedenen Schadens angesetzt sind (PLANAT, 2004b). Diese Festlegung trägt dem Umstand Rechnung, dass bei Risiken mit geringer Freiwilligkeit und Selbstbestimmung die mit den Risiken verbundenen indirekten Kosten und die Pflicht zur Risikoverminderung schwerer wiegen (Th. Schneider, Mitverfasser des Syntheseberichtes, schriftliche Auskunft 11.7.2006). Neuere Vorschläge plädieren für maximale Grenzkosten von $1 \mathrm{CHF/CHF} \mathrm{(Bründl} \mathrm{et} \mathrm{al.,}$ 2006). 


\subsubsection{Akzeptanzlinien in Wahrscheinlichkeits-Ausmass-Diagrammen}

\subsubsection{Lage der Akzeptanzlinien}

Wahrscheinlichkeits-Ausmass-Diagramme (F/N-Diagramme) werden in der Beurteilung und Bewertung technischer Risiken häufig verwendet (Kapitel 2.7). In einem Punktdiagramm werden Risikoszenarien nach Häufigkeiten (Abszisse) und nach Konsequenzen (Ordinate) dargestellt. Die Aggregierung der Szenarien zum Gesamtrisiko erfolgt durch mathematische Integration der Fläche, die durch die Punkte der verschiedenen Szenarien und die Achsen des Diagramms begrenzt ist (Anhang II). Die Tolerierbarkeit von Risiken wird (ähnlich wie bei Farmer's ,Boundary-Line', Kapitel 2.5.3) durch Diagonalen (sog. Akzeptanzlinien) im Diagrammfeld dargestellt (Abbildung 22). Über die Akzeptanzlinie hinausreichende Szenarien gelten als nicht tolerierbar. Ein Vergleich der Akzeptanzlinien in verschiedenen F/NDiagrammen zeigt erhebliche Unterschiede zwischen den betrachteten Ländern und/oder Behörden sowie zwischen der Art der betrachteten Risiken. In Tabelle 24 sind die Eckwerte der Akzeptanzlinien aus der vergleichenden Zusammenstellung in einem Bericht von Ball (1999) dargestellt. Einige Akzeptanzlinien stellen dar, dass es ein Mass an Schädigungen gibt, das unabhängig von der Eintretenswahrscheinlichkeit als untragbar zu bezeichnen ist. Das Handbuch I zur Störfallverordnung gibt dieses maximal zulässige Schadensausmass mit ca. 2'200 Todesfällen (innert 30 Tagen als direkte Folge des Störfalles) und CHF 10 Mia. Sachschaden an (BUWAL, 1991). Andere Akzeptanzlinien legen diesen Wert bei 1'000 Todesopfern fest. Im Handbuch zur Störfallverordnung ist ein Schadensmass beschrieben, das ungeachtet der Eintretenswahrscheinlichkeit zulässig ist: Ein Ereignis mit zwei Todesfällen liegt in jedem Fall im akzeptablen Bereich (Abbildung 22).

\subsubsection{Berechnete tolerierbare Risiken in F/N-Diagrammen}

Nach dem Verfahren in Anhang II (Risiko als Integral im F/N-Diagramm mit log-logSkalierung) wurden tolerierbare Objektrisiken für die verschiedenen Akzeptanzlinien berechnet (Abbildung 17). Die errechneten tolerierbaren Risiken zeigen eine grosse Streubreite auf. Die Todesfallrisiken liegen oft um 2-3 Zehnerpotenzen auseinander (Ball, 1999).

Hohe Sicherheitsanforderungen ergeben sich aus den Akzeptanzlinien in F/N-Diagrammen bei Staudämmen und Gefahrengutbetrieben. Um bis zu Faktor 10 tiefer ist die geforderte Sicherheiten bei Gefahrenguttransporten, Störfällen allgemein oder Kernanlagen. Unberücksichtigt bleibt bei diesen Darstellungen - wie bei den Darstellungen von Akzeptanzlinien in F/N-Diagrammen generell - die mutmassliche Grösse des Wirkungsgebiets. Störfälle in Kernanlagen betreffen im Fall einer Freisetzung von Radioaktivität ein sehr grosses Wirkungsgebiet, auf das sich das tolerierbare Risiko verteilt. Pro Flächeneinheit sind deshalb die tolerierbaren Risiken im Fall von Kernanlagen sehr gering. 
Tabelle 24 Eckwerte von Akzeptanzlinien in F/N-Diagrammen: Die Akzeptanzlinien in bekannten F/NDiagrammen (Ball, 1999; BUWAL, 1991) zeigen bedeutende Unterschiede in Lage und Neigung.

\begin{tabular}{|c|c|c|c|c|c|c|}
\hline \multirow[t]{2}{*}{ Name der Kurve } & \multirow[b]{2}{*}{ 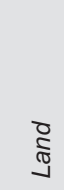 } & \multicolumn{4}{|c|}{ Ankerpunkt } & \multirow{2}{*}{$\begin{array}{l}\text { Bemerkungen/Besonderheiten/minimales } \\
\text { oder maximales Schadensausmass (SA) }\end{array}$} \\
\hline & & 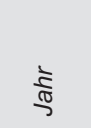 & 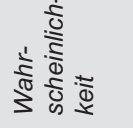 & 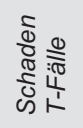 & 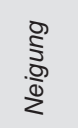 & \\
\hline Farmer & UK & 1967 & $1.5 * 10^{-4}$ & $\geq 33$ & -1.5 & Kernreaktor \\
\hline Groningen & $\mathrm{NL}$ & 1978 & $1 * 10^{-4}$ & $\geq 10$ & $-1 /-2$ & $\begin{array}{l}\left.\text { Gefahrengut; Obergrenze ALARA }{ }^{\star \star}\right) \text {; } \\
\text { max. SA: } 1000 \text { Todesfälle [Tf.] }\end{array}$ \\
\hline Groningen & $\mathrm{NL}$ & 1978 & $1 * 10^{-8}$ & $\geq 10$ & $-1 /-2$ & $\begin{array}{l}\text { Gefahrengut; Untergrenze ALARA; } \\
\text { max. SA: } 10 \mathrm{Tf} .\end{array}$ \\
\hline Kinchin & UK & 1982 & ${ }^{*} 8 * 10^{-5}$ & $\geq 10$ & -1 & Kernreaktor, Sofort-Tf. \\
\hline Kinchin & UK & 1982 & ${ }^{*} 3 * 10^{-6}$ & $\geq 10$ & -1 & Kernreaktor, Todesfallspätfolgen \\
\hline Hong Kong & $\mathrm{HK}$ & 1988 & $1 * 10^{-4}$ & $\geq 10$ & -1 & $\begin{array}{l}\text { neuer Gefahrengutbetrieb; } \\
\text { max. SA: } 1000 \text { Tf. }\end{array}$ \\
\hline Netherlands & $\mathrm{NL}$ & $1980 s$ & $1 * 10^{-5}$ & $\geq 10$ & -2 & $\begin{array}{l}\text { best. Gefahrengutbetrieb; Obergrenze } \\
\text { ALARA; max. SA: } 1000 \text { Tf. }\end{array}$ \\
\hline Netherlands & $\mathrm{NL}$ & 1980s & $1 * 10^{-7}$ & $\geq 10$ & -2 & $\begin{array}{l}\text { best. Gefahrengutbetrieb; Untergrenze } \\
\text { ALARA; max. SA: } 100 \mathrm{Tf} \text {. }\end{array}$ \\
\hline ACDS & UK & 1991 & $2 * 10^{-4}$ & $\geq 500$ & -1 & $\begin{array}{l}\text { Gefahrengutstrecke; Obergrenze ALARA; } \\
\text { max. SA: } 10 \text { '000 Tf. }\end{array}$ \\
\hline ACDS & UK & 1991 & $1 * 10^{-5}$ & $\geq 10$ & -1 & $\begin{array}{l}\text { Gefahrengutstrecke; Untergrenze ALARA; } \\
\text { max. SA: } 100 \mathrm{Tf} .\end{array}$ \\
\hline Offshore & UK & 1991 & ${ }^{*} 7 * 10^{-3}$ & $\geq 10$ & -1 & $\begin{array}{l}\text { Offshore-Installation mit } 150 \text { Betroffenen; } \\
\text { Obergrenze ALARA; max. SA: 1'000 Tf. }\end{array}$ \\
\hline Offshore & UK & 1991 & ${ }^{*} 5^{*} 10^{-6}$ & $\geq 10$ & -1.3 & $\begin{array}{l}\text { Offshore-Installation mit } 150 \text { Betroffenen; } \\
\text { Untergrenze ALARA; max. SA: 1'000 Tf. }\end{array}$ \\
\hline Störfall-VO & $\mathrm{CH}$ & 1991 & $\left.{ }^{*}\right) 1 * 10^{-6}$ & $\geq 30$ & -2 & $\begin{array}{l}\text { div. Risiken; Obergrenze ALARA; min. SA: } 9 \\
\text { Tf.; max. SA: ca. 2'200 Tf. }\end{array}$ \\
\hline Störfall-VO & $\mathrm{CH}$ & 1991 & ${ }^{*} 1 * 10^{-8}$ & $\geq 30$ & -2 & $\begin{array}{l}\text { div. Risiken; Untergrenze ALARA; min. SA: } \\
\text { ca. } 2 \text { Tf.; max. SA: } 500 \text { Tf. }\end{array}$ \\
\hline HK DG-Trsp. & $\mathrm{HK}$ & 1997 & $1 * 10^{-4}$ & $\geq 10$ & -1 & Flüssiggastransporte; max. 1'000 Tf. \\
\hline HK DG-Trsp. & HK & 1997 & *) $1 * 10^{-3}$ & $\geq 10$ & -1 & $\begin{array}{l}\text { Chlorgastransporte; Obergrenze ALARA; } \\
\text { max. SA: 1'000 Tf. }\end{array}$ \\
\hline HK DG-Trsp. & $\mathrm{HK}$ & 1997 & *) $1 * 10^{-5}$ & $\geq 10$ & -1 & $\begin{array}{l}\text { Chlorgastransporte; Untergrenze ALARA; } \\
\text { max. SA: 1'000 Tf. }\end{array}$ \\
\hline Netherlands & $\mathrm{NL}$ & 1996 & $1 * 10^{-5}$ & $\geq 10$ & -2 & $\begin{array}{l}\text { best. Gefahrengutbetrieb; Obergrenze ALA- } \\
\text { RA; keine Untergrenze ALARA; max. SA: } \\
1000 \text { Tf. }\end{array}$ \\
\hline Netherlands & $\mathrm{NL}$ & 1996 & $1 * 10^{-4}$ & $\geq 10$ & -2 & $\begin{array}{l}\text { Gefahrenguttransporte pro km; Obergrenze } \\
\text { ALARA; keine Untergrenze ALARA; max. } \\
\text { SA: } 1000 \mathrm{Tf} .\end{array}$ \\
\hline Störfall-VO & $\mathrm{CH}$ & 2001 & *) $1 * 10^{-5}$ & $\geq 10$ & -2 & $\begin{array}{l}\text { Gefahrenguttransporte pro } 100 \text { m; Ober- } \\
\text { grenze ALARA; min. SA: } 10 \mathrm{Tf} .\end{array}$ \\
\hline Störfall-VO & $\mathrm{CH}$ & 2001 & *) $1 * 10^{-7}$ & $\geq 10$ & -2 & $\begin{array}{l}\text { Gefahrenguttransporte pro } 100 \text { m; Unter- } \\
\text { grenze ALARA; min. SA: } 10 \mathrm{Tf} .\end{array}$ \\
\hline
\end{tabular}




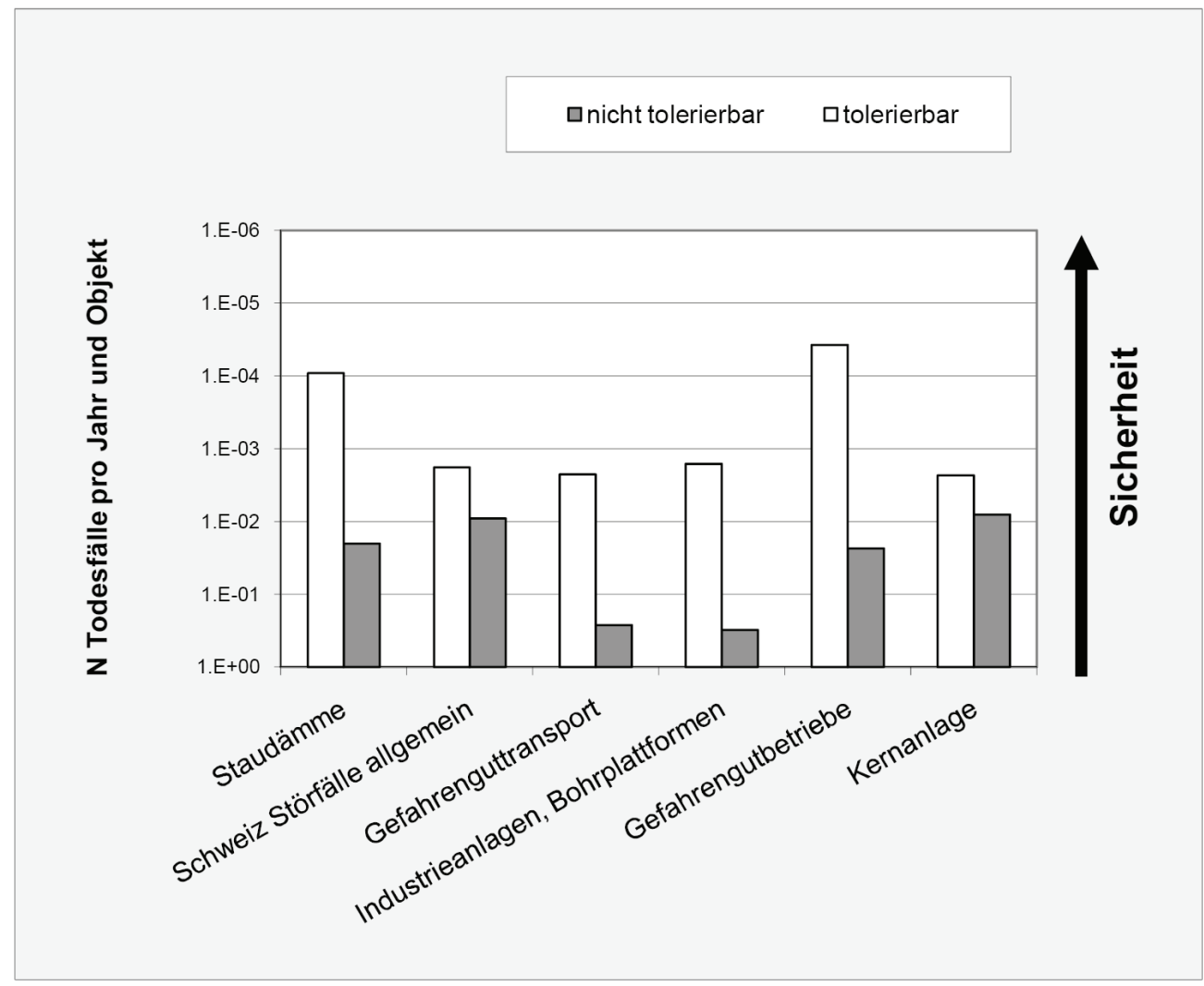

Abbildung 17 Die tolerierbaren Todesfallobjektrisiken für verschiedene Typen gefährlicher Anlagen zeigen Abweichungen bis zu Faktor $10^{2}$ (eigene Berechnungen).

Interessanterweise sind die tolerierbaren Risiken im Lauf der Zeit eher gestiegen. So wurde in den 1970er- und 1980er-Jahren das tolerierbare Risiko für Gefahrengutbetriebe in den Niederlanden noch 10-mal geringer (Sicherheitsanforderungen 10-mal höher) angesetzt als Mitte der 90er-Jahre für solche Betriebe in Hongkong, Grossbritannien und der Schweiz (Abbildung 18). 


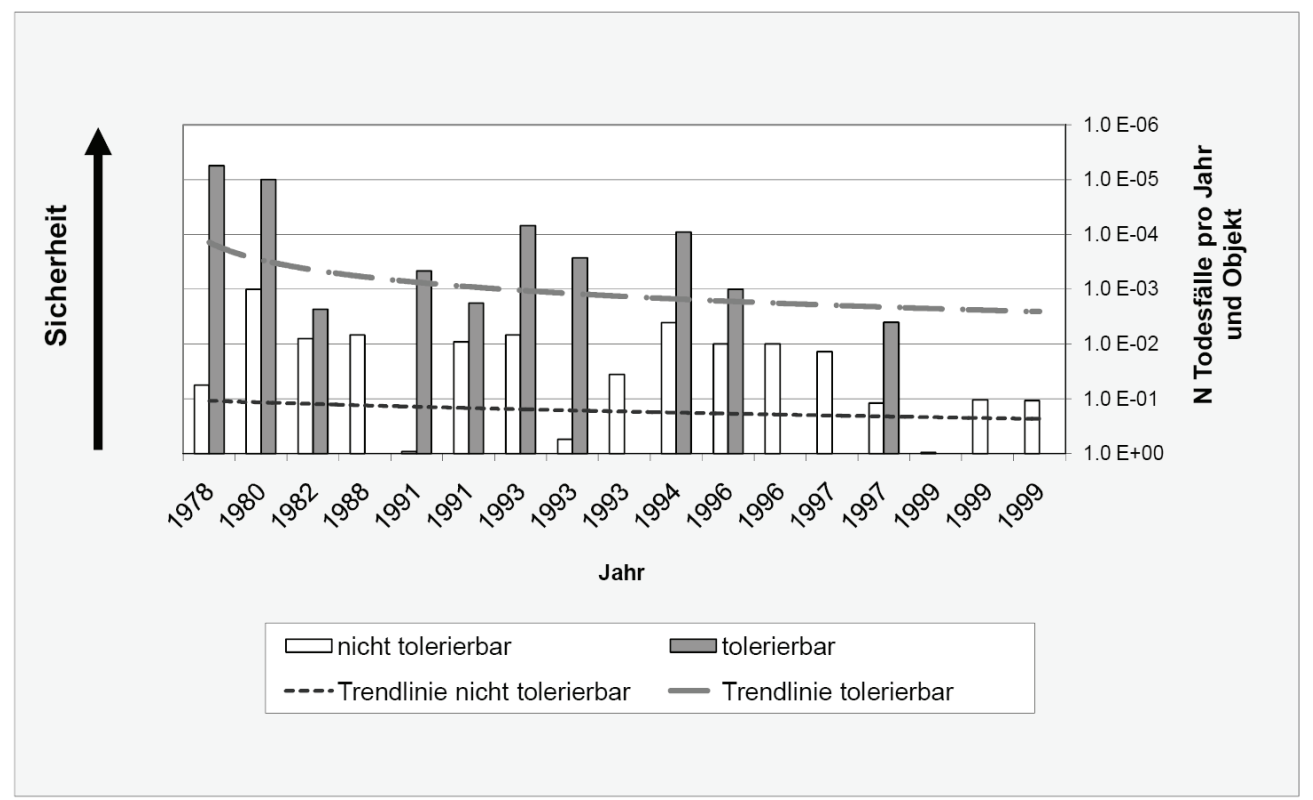

Abbildung 18 Entwicklung der tolerierbaren Todesfallobjektrisiken zwischen 1978 und 2000. In diesen 20 Jahren reduzierten sich die Sicherheitsstandards etwa um Faktor 10 (eigene Berechnungen).

Für einen Vergleich zwischen technischen Risiken und Naturrisiken wurden tolerierbare Risiken für 61 Sturz-, Lawinen- und Wildbachgefahrengebiete in der Gemeinde Engelberg (Kanton $\mathrm{OW}$ ) den obigen Berechnungen von technischen Objektrisiken gegenüber gestellt. Die Berechnung der tolerierbaren Risiken erfolgte mittels der Schadenserwartungswerte und der tolerierbaren Intensitäten anhand der Schutzzielmatrix (Borter, 1999) für 22 Sturzgebiete, 15 Lawinengebiete und 20 Gefahrengebiete mit Wildbach- und Hangprozessen (Tabelle 25).

Tabelle 25 Berechnung tolerierbarer kollektiver Risiken pro Gefahrenquelle für Naturgefahren in Engelberg (detaillierte Resultate Anhang V).

\begin{tabular}{|c|c|c|c|}
\hline Art der Gefahr & $\begin{array}{l}\text { Anzahl berücksichtigte } \\
\text { Gefahrenquellen }\end{array}$ & $\begin{array}{l}\text { Mittlere Grösse der } \\
\text { Gefahrenquelle }\end{array}$ & $\begin{array}{l}\text { Tolerierbares Risiko pro } \\
\text { Gefahrenquelle }\end{array}$ \\
\hline Einheit & $N$ & Are & Todesfälle pro Jahr \\
\hline Sturz & 22 & 936.82 & 0.022978 \\
\hline Lawine & 15 & 2'819.97 & 0.332730 \\
\hline Wildbach/Hangprozesse & 20 & 3'109.05 & 0.014486 \\
\hline Mittel aller Gefahren & 57 & 2'194.57 & 0.101512 \\
\hline
\end{tabular}


Zwischen den einzelnen Gefahrenquellen bestehen erhebliche Unterschiede in der Grössenordnung von vier Magnituden. Besonders hoch sind die tolerierbaren Risiken in Lawinengebieten, geringer in Gebieten mit Wildbächen und bei Hanginstabilitäten. Die mittlere Grösse pro Gefahrenquelle beträgt 22ha (entspricht einem kreisförmigen Wirkungsgebiet von 360m Durchmesser).

Der Vergleich mit internationalen Standards für technische Risiken und mit tolerierbaren Risiken gemäss Störfallverordnung in der Schweiz zeigt, dass die untersuchten natürlichen Gefahrenquellen ein deutlich, nämlich etwa zehn- bis hundertfach höheres tolerierbares Kollektivrisiko aufweisen (Abbildung 19).

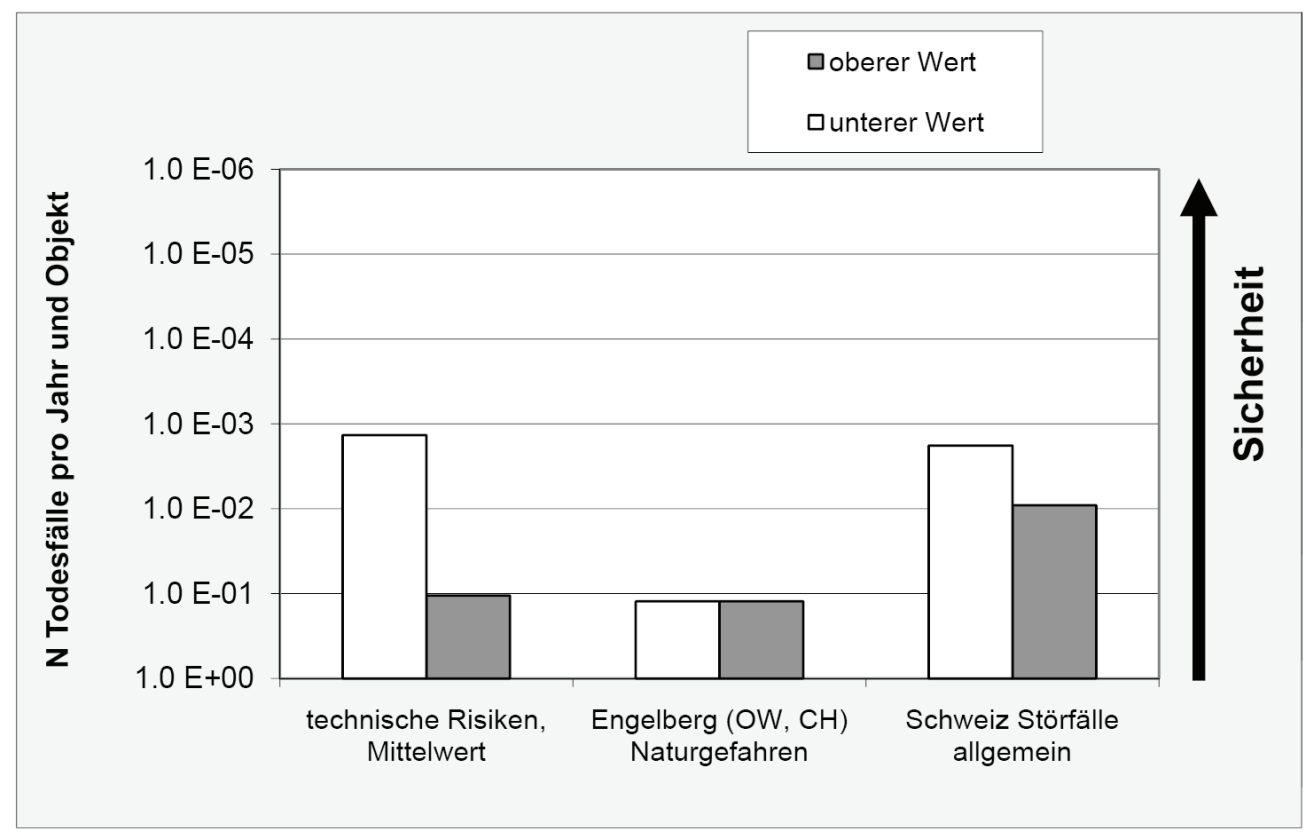

Abbildung 19 Tolerierbare Todesfallobjektrisiken für verschiedene Risikoarten. Für die Naturgefahrengebiete ergeben sich etwa 10-mal tiefere Sicherheitsstandards (eigene Berechnungen).

\subsection{Schutzzielkonzepte}

\subsubsection{Entwicklung von Schutzzielkonzepten}

Der praktische Umgang mit gefährlichen Prozessen kannte implizit schon immer Schutzziele, da nie alle denkbaren Massnahmen auch realisierbar waren. Obwohl dem klassischen Umgang mit Naturgefahren der Makel anlastet, der Gefahrenabwehr statt der Risikokultur verpflichtet zu sein, wurde bereits in vergangenen Jahrzehnten abgewogen, ob und welche Massnahmen ergriffen werden sollen. Unbestritten ist, dass in den letzten zehn Jahren ökonomische, ökologische und technologische Schranken des Umgangs mit Risiken zunehmend ins Bewusstsein rückten. Wesentlich ist dabei die Erkenntnis, dass jede gesellschaftliche Tätigkeit Nutzen bringt, aber auch Risiken birgt. Im öffentlichen Risikomanagement stellen sich auch Fragen der Rechtsgleichheit und Verhältnismässigkeit im Umgang mit Risiken. Diese Entwicklung verlangt zunehmend explizite Formulierungen tolerierbarer Risiken. Zuerst erfolgte dies im Bereich technischer Risiken. 
Erste Festlegungen erfolgten in Form von Grenzwerten für die tolerierbaren Wahrscheinlichkeiten unerwünschter Vorgänge (z. B. Versagen eines Bauwerkes). Zunehmend wurden auch Auswirkungen unerwünschter Vorgänge in die Überlegungen einbezogen, was zu einer Abstufung der Grenzwerte führte. Die unter Kapitel 2.5.3 erwähnte Einführung von Boundary-Lines (Farmer, 1967) stellte einen bedeutenden Entwicklungsschritt dar. Dieser Ansatz wurde weiterentwickelt zur Darstellung tolerierbarer Risiken in WahrscheinlichkeitsAusmass-Diagrammen (F/N-Diagramme). Diese jahrzehntealte Darstellung ist heute noch verbreitet.

Ein weiterer Schritt war der Einbezug von Effizienz- und Verhältnismässigkeitsüberlegungen. Das Mass des tolerierbaren Risikos wurde korreliert mit dem Nutzen der riskanten Tätigkeit und mit den Kosten der Risikoverminderung. In vielen F/N-Diagrammen wird diesem Umstand Rechnung getragen, indem zwischen tolerierbaren und untolerierbaren Risiken ein Zwischenbereich (ALARA- (,as low as reasonably achievable') oder ALARP- (,as low as reasonably possible') Bereich) eingefügt wird, in dem Risiken nach Massgabe der Verhältnismässigkeit zu reduzieren sind.

Gegen den Ansatz der Darstellung von tolerierbaren Risiken in WahrscheinlichkeitsAusmass-Diagrammen erwuchs in den 1990er-Jahren Kritik in dreierlei Hinsicht:

1. Die Darstellung tolerierbarer Risiken in F/N-Diagrammen berücksichtigt keine einheitliche und oft keine ausdrücklich definierte Systemgrösse. Dies wurde später für einzelne Risikosituationen (Verkehrswege, Rohrleitungen) korrigiert.

2. Die Akzeptanzlinien in F/N-Diagrammen berücksichtigen das gesamte Risikoprofil insofern ungenügend, als damit die Tolerierbarkeit einzelner Szenarien beurteilt wird, ohne gleichzeitig die übrigen Szenarien einzubeziehen.

3. Auch mit dem in neueren Ansätzen eingeführten ALARA-Bereich bleiben wirtschaftliche Überlegungen (Nutzen des Risikos, Kosten risikovermindernder Massnahmen) nur teilweise berücksichtigt.

Als Antwort auf diese Kritik wurde der sogenannte Grenzkostenansatz zur Darstellung tolerierbarer kollektiver Risiken entwickelt. Risikovermindernde Massnahmen sollten bis zu dem Punkt ergriffen werden, bei dem die Kosten für einen zusätzlichen Sicherheitsgewinn ein bestimmtes Mass überschreiten.

Weitgehend losgelöst von den Entwicklungen im Umgang mit technischen Risiken wurden für den Umgang mit Naturrisiken qualitative Schutzzielmatrizes entwickelt, zuerst für den Hochwasserschutz, später für den Umgang mit allen Naturgefahren. In diese Matrizes fliessen Nutzenüberlegungen und teilweise auch die Kosten risikovermindernder Massnahmen implizit ein. In den letzten Jahren wurde versucht, die für technische Risiken gebräuchlichen Grenzkosten- und Grenzwertdarstellungen zu übernehmen.

\subsubsection{Grenzwerte für Versagenswahrscheinlichkeiten}

Schutzziele wurden in den 1970er-Jahren im Bauwesen und beispielsweise in der Nukleartechnologie noch bis heute als ,Grenzwerte für Versagenswahrscheinlichkeiten' definiert (Proske, 2004). Vor allem ältere Festlegungen lassen das mit einem Versagen verbundene Schadensausmass unberücksichtigt und bilden somit nicht oder nur indirekt ein tolerierbares Risiko ab. In späteren Darstellungen erfolgte eine qualitative Berücksichtigung des Schadensausmasses durch Abstufung der Werte nach Gebäudeart (JCSS, 1976), nach Anzahl der gefährdeten Personen (CEB, 1976; CIRIA, 1977), dem Nutzungszeitraum (CIRIA, 1977), der möglichen Vorankündigung des Versagens, der Beanspruchung des Bauwerkes (Allen, 1991) sowie von Kostenüberlegungen (Schueremans, 2001). Ein Beispiel sind Angaben zu Grenzzuständen der Gebrauchstauglichkeit und der Tragsicherheit im Probabilistic Model Code part 1 - Basis of Design (JCSS, 2001), auf den auch die SIA-Norm 260 (Grundlagen der Projektierung von Tragwerken; SIA, 2003) abstützt (Tabelle 26).

Josef Th. Hess: Schutzziele im Umgang mit Naturrisiken in der Schweiz, @ vdf Hochschulverlag 2011 
Tabelle 26 Zielgrössen für maximale Wahrscheinlichkeiten einer Nichteinhaltung der Gebrauchstauglichkeit oder einer Überschreitung der Tragsicherheit (Wahrscheinlichkeit pro Jahr; JCSS, 2001).

\begin{tabular}{|c|c|c|c|c|}
\hline Grenzzustand der & Gebrauchstauglichkeit & & Tragsicherheit & \\
\hline Folge eines Versagens & & Gering & Mittel & Hoch \\
\hline Umschreibung & & $\begin{array}{l}\text { kleines Todes- } \\
\text { fallrisiko, geringe } \\
\text { ökonomische } \\
\text { Konsequenzen }\end{array}$ & $\begin{array}{l}\text { mittleres To- } \\
\text { desfallrisiko, } \\
\text { erhebliche } \\
\text { ökonomische } \\
\text { Konsequenzen }\end{array}$ & $\begin{array}{l}\text { hohes Todes- } \\
\text { fallrisiko, gros- } \\
\text { se ökonomi- } \\
\text { sche Konse- } \\
\text { quenzen }\end{array}$ \\
\hline Beispiele & & $\begin{array}{l}\text { landwirtsch. } \\
\text { Anlage }\end{array}$ & $\begin{array}{l}\text { Büro-, Indus- } \\
\text { trie-, Wohn- } \\
\text { gebäude }\end{array}$ & $\begin{array}{l}\text { Spitäler, wich- } \\
\text { tige Brücken }\end{array}$ \\
\hline $\begin{array}{l}\text { Relative Kosten von } \\
\text { Sicherheitsmassnahmen }\end{array}$ & \multicolumn{4}{|c|}{ Wahrscheinlichkeit pro Jahr } \\
\hline Hoch & $10^{-1}$ & $10^{-3}$ & $5 * 10^{-4}$ & $10^{-4}$ \\
\hline Normal & $5 * 10^{-2}$ & $10^{-4}$ & $10^{-5}$ & $5 * 10^{-6}$ \\
\hline Gering & $10^{-2}$ & $10^{-5}$ & $5 * 10^{-6}$ & $10^{-6}$ \\
\hline
\end{tabular}

Die Nuklearindustrie kennt das System der Zielversagenswahrscheinlichkeit (Zielwerte der International Nuclear Safety Advisory Group INSAG, 2006) zur maximal tolerierbaren Häufigkeit von Kernschmelzen CDF (,core damage frequency') oder der tolerierbaren Häufigkeit einer Freisetzung von Radioaktivität. Sinngemäss ähnliche Kriterien werden auch in anderen Industriebereichen, z. B. der chemischen Industrie angewendet (Gibson, 1980).

Tabelle 27 Zielwerte der International Nuclear Safety Advisory Group (INSAG, 2006).

\begin{tabular}{lcc}
\hline Ereignis & Bestehende Anlagen & Neue Anlagen \\
\hline Häufigkeit einer Kernschmelze CDF & $10^{-4}$ & $10^{-5}$ \\
Freisetzung von Radioaktivität & $10^{-5}$ & $10^{-6}$ \\
Individuelles Todesfallrisiko & $10^{-6}$ & $10^{-6}$ \\
\hline
\end{tabular}

\subsubsection{Risikogrenzwerte als Schutzziele}

Grenzwerte werden meist zur Beschreibung tolerierbarer individueller Risiken angewendet (Merz, 1995; Bohnenblust \& Slovic, 1998; Borter, 1999). Seit Mitte der 1990er-Jahre erscheinen solche Grenzwerte sowohl in internationalen als auch in schweizerischen Publikationen (Abbildung 20). 


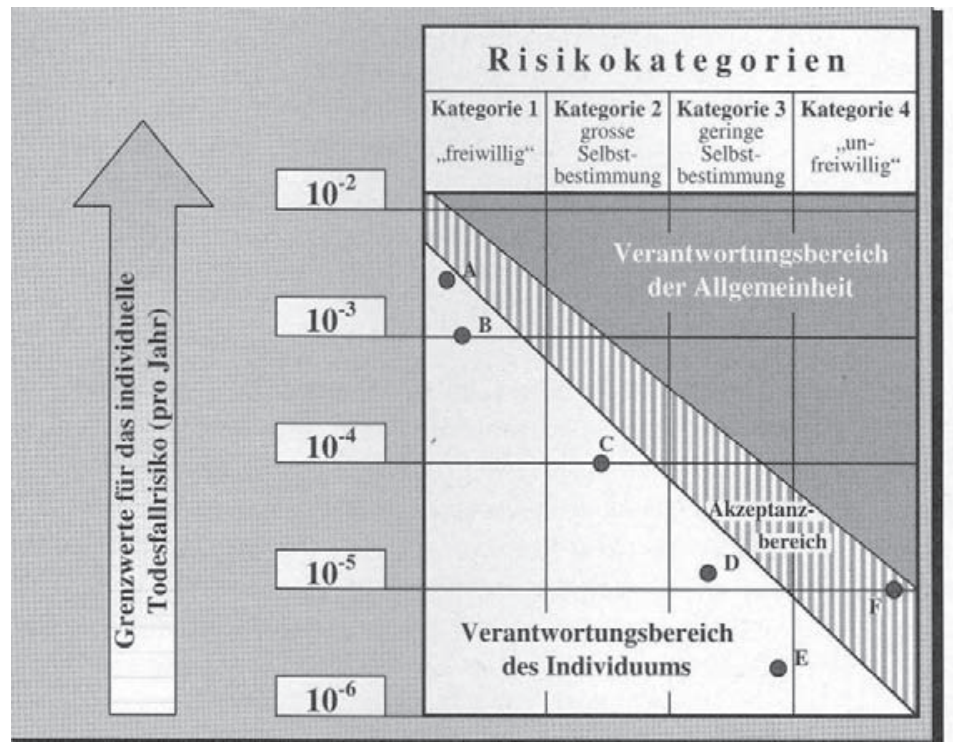

\begin{tabular}{ll}
\multicolumn{2}{l}{ Legende } \\
\hline A 20 Zigaretten/Tag \\
rauchen \\
B Deltafliegen (Hobby) \\
C 10 '000 km Autobahn \\
D tahren \\
Autofahren (als \\
E Eifahrer) \\
F Brand in Gebäude \\
\hline
\end{tabular}

Abbildung 20 Schutzziele als Grenzwerte für das tolerierbare individuelle Todesfallrisiko (Merz et. al, 1995). Die Grenzwerte für ,freiwillige' Risiken und ,unfreiwillige' Risiken unterscheiden sich um einen Faktor 1:1'000 bis $1: 10$ '000.

Grenzwerte für tolerierbare kollektive Risiken zu formulieren ist untypisch. Dies würde die Festlegung einer einheitlichen Bezugsgrösse (z. B. Flächeneinheit) voraussetzen. Abgesehen von den in Kapitel 2.7.3 dargestellten, auf ein Objekt bezogenen Zielversagenswahrscheinlichkeiten sind deshalb Grenzwerte für kollektive Risiken wenig gebräuchlich.

Ein erwähnenswertes Beispiel für solche Grenzwerte im Bereich von Naturrisiken ist die isländische ,Regulation on hazard zoning due to snow- and landslides, classification and utilisation of hazard zones and preparation of provisional hazard zoning' (Iceland, 2000; Bell, 2006). Die Regelung umschreibt tolerierbare kollektive Todesfallrisiken pro Gebäude für verschiedene Arten von Raumnutzungen (Wohngebiete, Industriegebiete, Erholungsgebiete). 


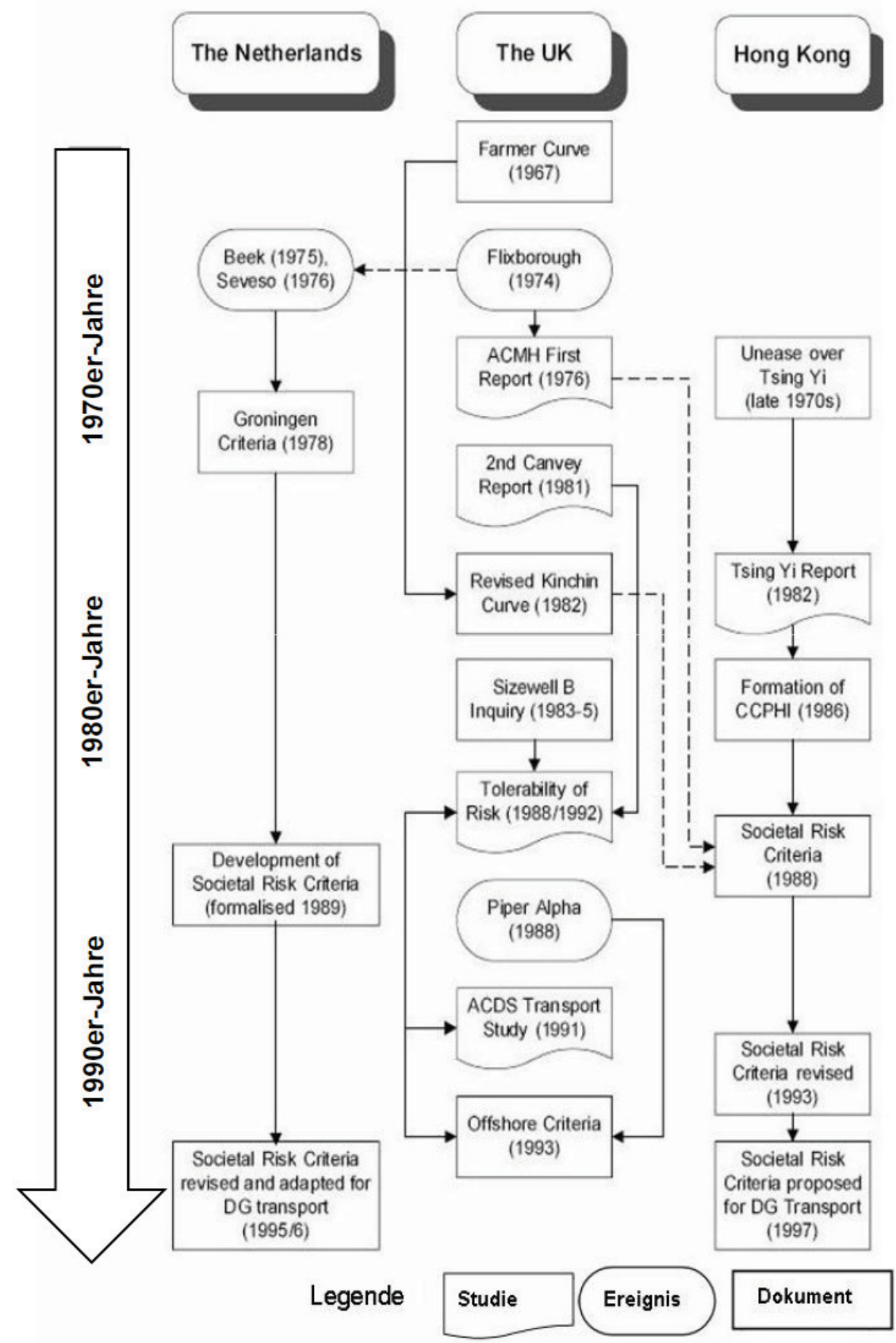

Abbildung 21 Zeitliche Entwicklung der Schutzzielkonzepte (Ball, 1999). Meist führten Unfallereignisse, oft aber auch bedeutende Studien zur Festlegung neuer Schutzziele (Societal Risk Criteria). 


\subsubsection{Tolerierbare Risiken in Wahrscheinlichkeits-Ausmass-Diagrammen}

Seit der erwähnten Einführung der ,Boundary-Line' von Farmer (Kapitel 2.5.3) fanden ,Wahrscheinlichkeits-Ausmass-Diagramme' (F/N-Diagramme) zunehmende Anwendung und Verbreitung bei der Darstellung tolerierbarer technischer Risiken (Societal Risk Criteria). Zuerst wurden F/N-Diagramme (und andere Schutzziele für technische Risiken) für dicht besiedelte und industrialisierte Gebiete, wie Grossbritannien, Niederlande oder Hongkong entwickelt (Ball \& Floyd, 1999). Häufig lösten grössere Unfälle oder bedeutende Studienberichte die Festlegung neuer Schutzziele aus (Abbildung 21). Die bekannteste derartige Darstellung von Schutzzielen in der Schweiz ist im Handbuch zur Störfallverordnung enthalten (BUWAL, 1991 und 2001; Abbildung 22). Charakteristisch in dieser und in den meisten anderen bekannten Schutzzieldarstellungen in F/N-Diagrammen ist der sogenannte ALARA- oder ALARP-Bereich zwischen den Bereichen mit tolerierbaren und nicht tolerierbaren Risiken. In diesem Bereich sind Massnahmen hinsichtlich ihrer Verhältnismässigkeit (z. B. KostenNutzen-Verhältnis) zu prüfen und zu treffen.

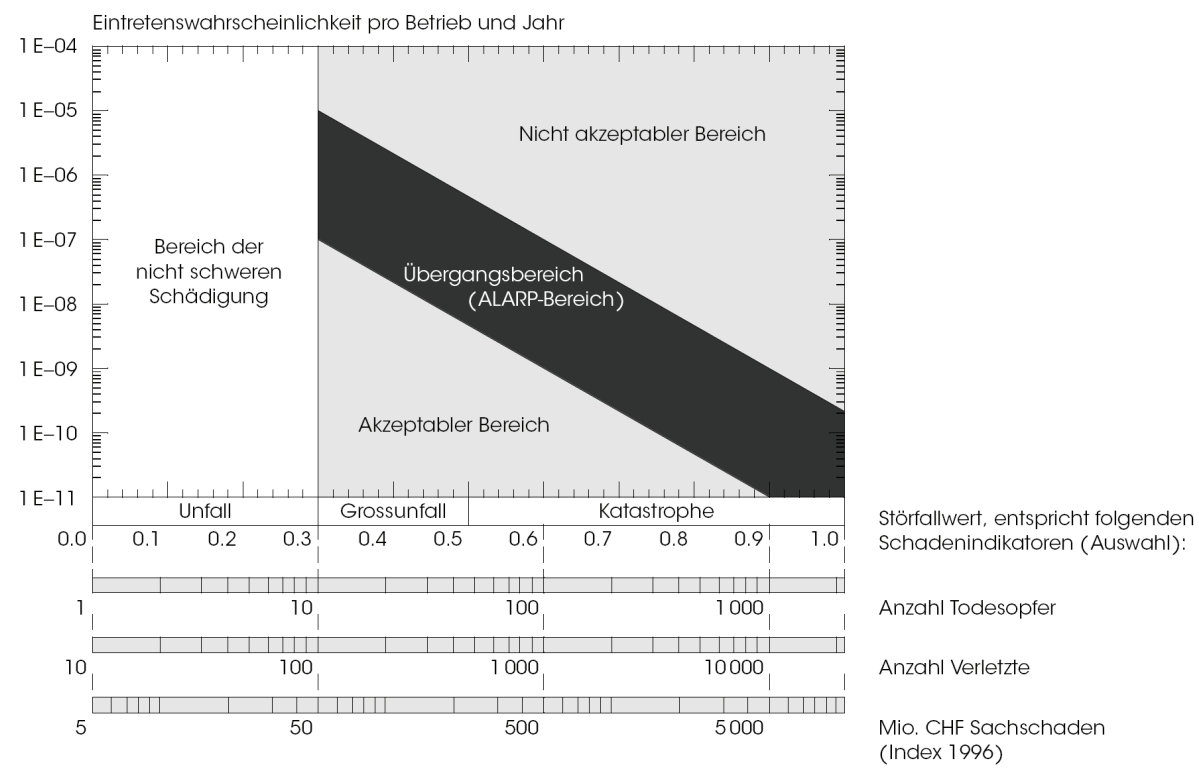

Abbildung 22 F/N-Diagramm der schweizerischen Störfallverordnung (BUWAL, 1991).

Eine Besonderheit des F/N-Diagramms zur schweizerischen Störfallverordnung ist die Berücksichtigung verschiedener Indikatoren. Nebst der Anzahl Todesopfer, auf deren Darstellung sich die meisten anderen F/N-Diagramme beschränken, sind weitere Beeinträchtigungen von Leib und Leben sowie Sach- und Umweltschäden erfasst. Für Umweltschäden sind die Wrights Criteria als ähnliche Illustration von Schutzzielen bekannt (Wright, 1993, zit. in Robinson, 2003).

Die Akzeptanzlinien in F/N-Diagrammen beziehen sich jeweils auf eine Risikoquelle, z. B. einen der Störfallverordnung unterstehenden Betrieb. Die Ausdehnung des Wirkungsgebietes der Risikoquelle oder die Grösse des betrachteten Systems sind nicht normiert. Eine Ausnahme bilden die Richtlinien für Verkehrswege der Beurteilungskriterien zur Störfallverordnung, welche zur Beurteilung die Länge einer Verkehrsachse auf 100m, jene einer Rohrleitung auf $300 \mathrm{~m}$ normieren. 
Im Management von Naturrisiken sind F/N-Diagramme als Schutzzieldarstellungen nicht bekannt. Nach vergleichbaren Prinzipien aufgebaut sind aber die Schutzziele für die Beurteilung der Erdbebensicherheit bestehender Gebäude (Duvernay, 2005; Abbildung 23).

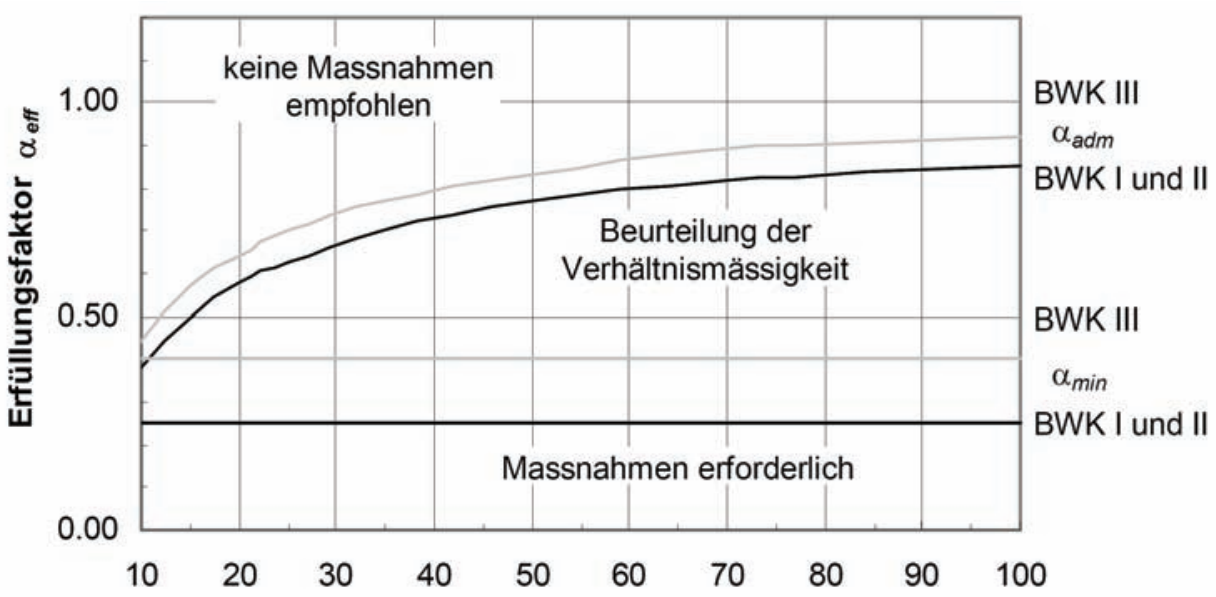

Der Erfüllungsfaktor $\alpha_{\text {eff }}$ stellt eine numerische Aussage über das Mass dar, in welchem ein bestehendes Tragwerk die rechnerischen Anforderungen an Neubauten gemäss geltender Norm erfüllt (SIA 260 f., 2004).

Ein Erfüllungsfaktor $\alpha$ von 0.25 entspricht dem individuellen Todesfallrisiko/Jahr einer dauernd anwesenden Person von $10^{-5}$, ein Erfüllungsfaktor von 0.40 einem solchen von $5^{*} 10^{-6} \cdot \alpha_{\min }$ beschreibt den einzuhaltenden, $\alpha_{\text {adm }}$ den mit verhältnismässigen Mitteln anzustrebenden Erfüllungsfaktor.

Die Bauwerksklassen (BWK) sind eine schematische Charakterisierung von Gebäuden gemäss Norm SIA 261. Die Klassierung berücksichtigt eine ähnliche Gefährdung von Personen, die Bedeutung des Bauwerkes für die Allgemeinheit und die Gefährdung der Umwelt infolge der Beschädigung des Bauwerkes (SIA, 2004). Beispiele: BWK I: Wohnbauten; BWK II: Einkaufszentren; BWK III: Akutspitäler).

Abbildung 23 Schutzziele für die Erdbebensicherheit bestehender Gebäude (Duvernay, 2005).

\subsubsection{Grenzkosten als Schutzziele}

Alternativ zu den F/N-Diagrammen fand in den 1990er-Jahren die Anwendung von Grenzkosten zur Darstellung tolerierbarer kollektiver Risiken Verbreitung in der Fachliteratur (Merz, 1995; Bohnenblust \& Slovic, 1998; Borter, 1999). Weiter entwickelt wurde dieser Ansatz im Rahmen des Polyprojekts ,Risiko und Sicherheit technischer Systeme' an der ETH Zürich (Kröger, 1996).

Tolerierbare kollektive Risiken werden als Grenzkosten zur Vermeidung eines Schadens, beispielsweise eines Todesfalls, formuliert (Abbildung 24). Dieser Ansatz erlaubt es, Systeme unterschiedlicher Grösse und Beschaffenheit miteinander zu vergleichen. Die Darstellung von Schutzzielen als Grenzkosten setzt nebst der Kenntnis des Risikos auch Kenntnisse (i) der (optimalen) Risikoverminderungsmassnahmen, (ii) deren Kosten und (iii) deren Wirksamkeit voraus. 


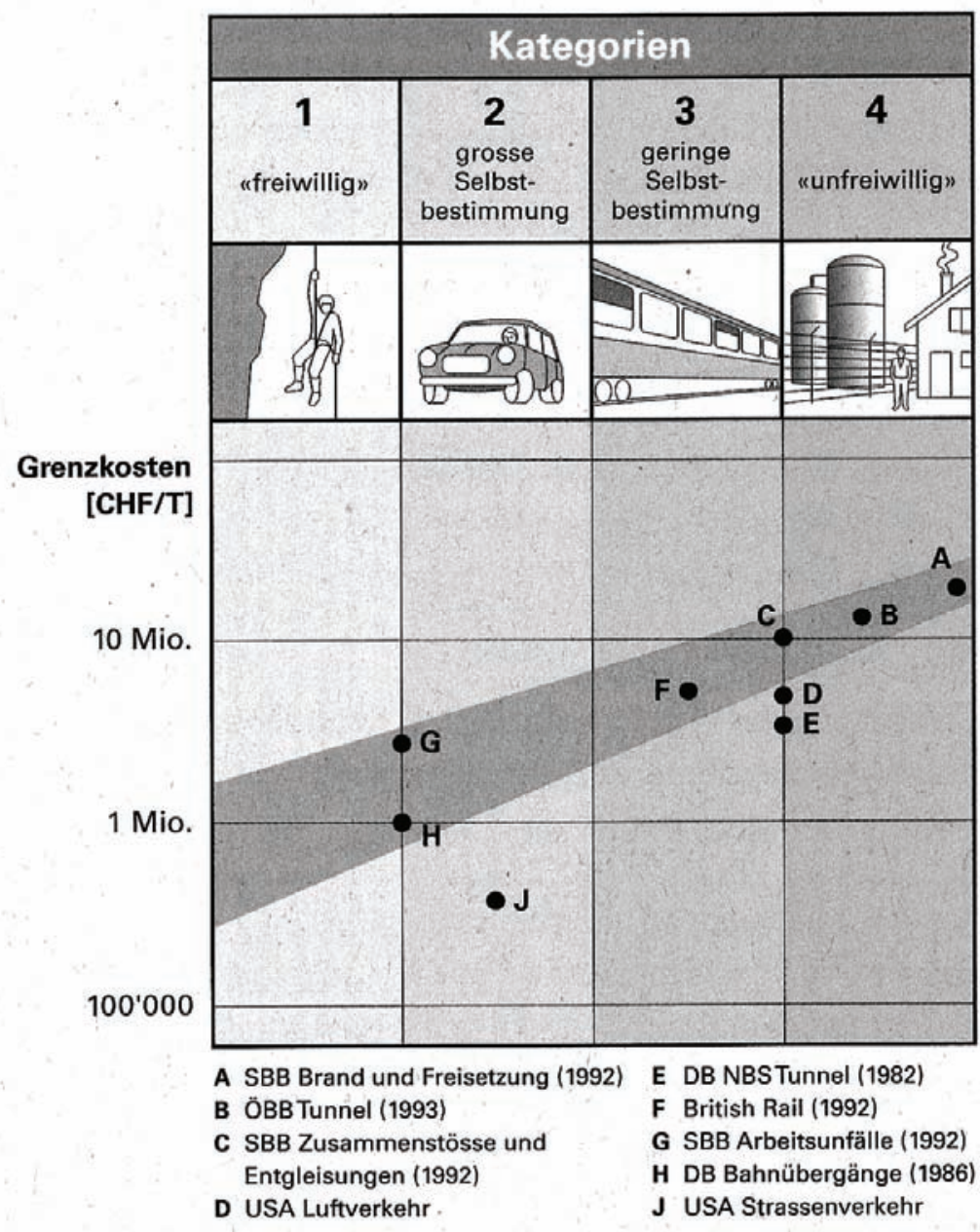

Abbildung 24 Grenzkosten zur Verhinderung eines Todesfalls [T] aus KATARISK (BABS, 2003).

Die Anwendung grenzkostenbasierter Schutzziele führt nicht für alle betroffenen Individuen zu gleich hohen Restrisiken. In der Literatur wird daher gleichzeitig mit der Anwendung des Grenzkostenansatzes die Einhaltung von Grenzwerten des tolerierbaren individuellen Risikos gefordert (Seiler, 2000; Flueler \& Seiler, 2003; ,Zwei-Regel-Ansatz', Kapitel 3.2.4.4). In letzter Zeit fanden Grenzkostenansätze zur Beschreibung tolerierbarer kollektiver Risiken auch im Umgang mit Naturrisiken Eingang in die Praxis (PLANAT, 2004b; Borter, 1999; Duvernay, 2005). Diese Ansätze kombinieren die zwei Bedingungen der Einhaltung von Grenzkosten einerseits und von Grenzwerten für individuelle Todesfallrisiken anderseits (Tabelle 28). 
Tabelle 28 Schutzziele für individuelle und kollektive Todesfall- und Sachrisiken (PLANAT, 2004b).

\begin{tabular}{lcll}
\hline Risikokategorie & $\begin{array}{l}\text { Individuelles Todesfallrisiko } \\
\text { Tolerierbare Todesfallwahr- } \\
\text { scheinlichkeit eines Indivi- } \\
\text { duums (x pro Jahr) }\end{array}$ & $\begin{array}{l}\text { Kollektives Todesfall- } \\
\text { risiko } \\
\text { Grenzkosten zur Rettung } \\
\text { eines Menschenlebens } \\
\text { (Mio. CHF) }\end{array}$ & $\begin{array}{l}\text { Sachrisiken } \\
\text { Grenzkosten zur Verhinde- } \\
\text { rung eines Sachschadens } \\
\text { (x CHF pro 1 CHF Scha- } \\
\text { denskosten) }\end{array}$ \\
\hline 1 freiwillig & $10^{-2}-10^{-3}$ & $1-2$ & 1 \\
2 & $10^{-3}-2 * 10^{-4}$ & $2-5$ & 1 \\
3 & $2 * 10^{-4}-3 * 10^{-5}$ & $5-10$ & 3 \\
4 unfreiwillig & $3 * 10^{-5}-4 * 10^{-6}$ & $10-20$ & 4 \\
\hline
\end{tabular}

\subsubsection{Schutzzielschemen und -matrizes im Management von Naturrisiken}

Mit der Inkraftsetzung des neuen Waldgesetzes (WaG, SR 921.0) und des Wasserbaugesetzes (WBG, SR 721.100) wurden die staatlichen Massnahmen und Pflichten ausdrücklich auf den Schutz von Menschen und erheblichen Sachwerten beschränkt. Dies erforderte Vollzugshilfen, die eine systematische und nachvollziehbare Abgrenzung von zulässigen und unzulässigen Risiken ermöglichen.

Das Kreisschreiben Nr. 20 des Bundesamtes für Umwelt, Wald und Landschaft (BUWAL,1994) umschreibt die Ermittlung der Risiken und Schutzziele anhand einer Matrix. Nebst der Sicht der zuständigen Behörde ist dabei die Mitwirkung der Bevölkerung entscheidend. Das Restrisiko ist zusammen mit der Schutzzieldefinition von Fall zu Fall zu bestimmen. Das Ziel besteht darin, mittlere und grosse Risiken zu kleinen Risiken zu reduzieren (Abbildung 25).

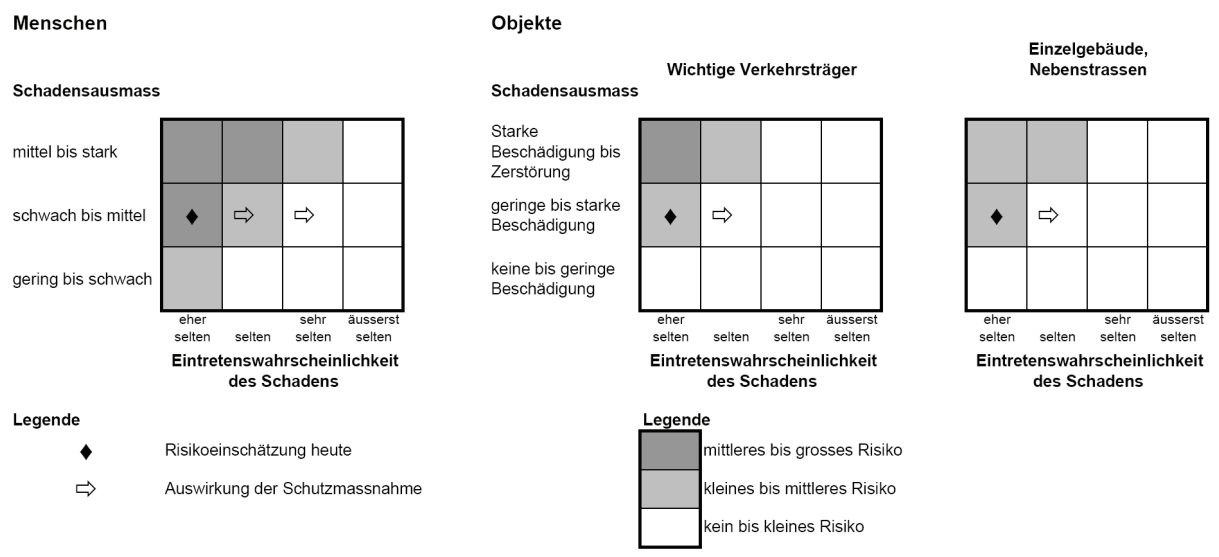

Abbildung 25 Schutzzieldarstellung aus: Anleitungsentwurf zur Durchführung einer Risikoanalyse (BUWAL, Eidg. Forstdirektion. Beilage zu KREISSCHREIBEN. Nr. 20, 1994). Die Darstellung enthält keine eigentlichen Schutzziele, sondern Hinweise auf möglichen Handlungsbedarf. 
Dieser Ansatz wurde Anfang der 1990er-Jahre zur Projektbeurteilung angewendet, aber nicht weiterentwickelt. Im Management von Naturrisiken hat sich die Darstellung von Schutzzielen als qualitative und semiquantitative Schemen und Matrizes durchgesetzt. Nach dem Hochwasser 1987 wurde im Kanton Uri eine erste solche Schutzzielmatrix für den Hochwasserschutz entwickelt (Uri, 1992; Abbildung 26).

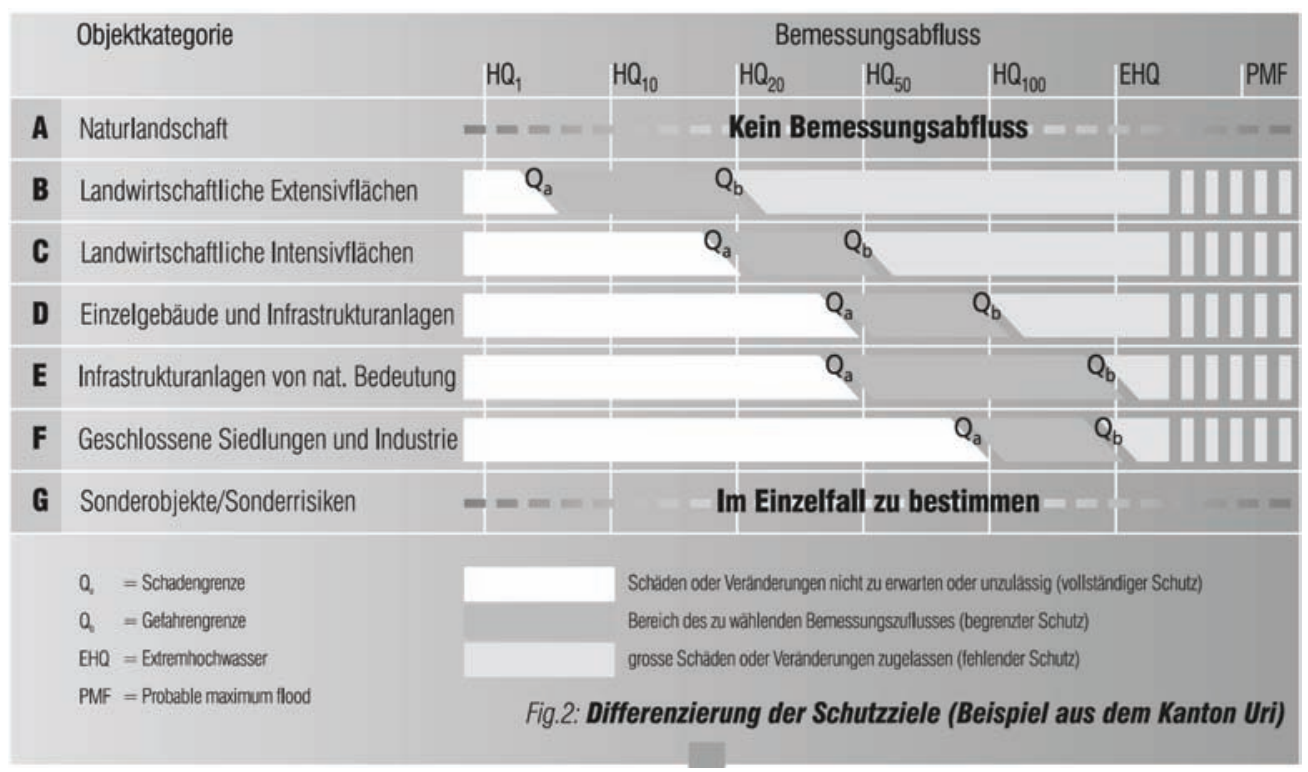

Abbildung 26 Schutzzielmatrix Hochwasserschutz Uri (Anforderungen an den Hochwasserschutz. Bundesamt für Wasserwirtschaft, 1995).

Einzelne Kantone entwickelten dieses Schema weiter zu detaillierteren Schutzzielmatrizes (Kanton Bern, 2003; Kanton Luzern, 2003; Gefahrenkommission Graubünden, 2003). Eine differenziertere und generell anwendbare Schutzzielmatrix für alle Naturgefahren wird in der Schweiz seit 1999 verbreitet angewendet (Borter, 1999). Sie wurde zwischenzeitlich von verschiedenen Kantonen als Standard übernommen (z. B. Kt. Glarus, 2001; Abbildung 27). 
Kanton Glarus

Richtlinien zum Schutz vor Naturgefahren: Anhang (genohmigt vom Regierungsrat am 27. Marz 2001)

Schutzziele gemäss Art. 13 Abs. 3

\begin{tabular}{|c|c|}
\hline Intensitäten & $0:$ Intensitat Null \\
\hline
\end{tabular}

2: Mittiere Intensitat 3: Starke Intensitat

\begin{tabular}{|c|c|c|c|}
\hline Vollstandiger Schutz & \multicolumn{3}{|c|}{ Schutz bis schwache Intensitaten } \\
\hline Schutz bis mittere Intensitaten & \multicolumn{3}{|c|}{ Keineriei Schutz } \\
\hline \multirow[b]{3}{*}{ Naturwerte } & \multicolumn{3}{|c|}{ Zulässige Gefährdung } \\
\hline & \multicolumn{3}{|c|}{ Wiederkehrperiode } \\
\hline & $\begin{array}{c}1-30 \\
\text { (hautig) }\end{array}$ & $\begin{array}{l}30-100 \\
\text { (selten) }\end{array}$ & $\begin{array}{c}100-300 \\
\text { (sehr selten) }\end{array}$ \\
\hline Odland, Naturlandschaften & 3 & 3 & 3 \\
\hline Alpweiden & 2 & 3 & 3 \\
\hline $\begin{array}{l}\text { Wald mit Schutzfunktion (Waidbau B } \\
\text { und } \mathrm{C} \text { ), landwirtschaftich genutztes } \\
\text { Land }\end{array}$ & 2 & 2 & 3 \\
\hline & 1 & 1 & 2 \\
\hline & 0 & 1 & 2 \\
\hline & 0 & 1 & 1 \\
\hline Quellenareal & Festlegung & ise & \\
\hline
\end{tabular}

Abbildung 27 Schutzzielmatrix für gravitative Naturgefahren (Kanton Glarus, 2001).

\subsection{Operationale Aspekte bei Schutzzielen}

\subsubsection{Anforderungen an die operationale Eignung von Schutzzielen}

Naturgefahrenprozesse erstrecken sich oft über grössere Gebiete mit zahlreichen Betroffenen und mit unterschiedlichen Interessen. Ebenso entfalten Massnahmen ihre Wirkung oft über grössere Gebiete. Die operationale Eignung von Schutzzielen darf sich daher nicht auf eine einzelne Risikosituation (Einzelprojekt) beschränken, sondern misst sich am Anspruch der Einsetzbarkeit für das regionale Risikomanagement. Im Risikomanagement werden somit die zwei Ebenen Region und Einzelprojekt/Einzelobjekt unterschieden (Tabelle 29).

Tabelle 29 Ebenen des Risikomanagements.

\begin{tabular}{lll}
\hline Ebene & Zentrale Fragestellung & Typische Instrumente \\
\hline Region & $\begin{array}{l}\text { Ermittlung des Handlungs- } \\
\text { bedarfs } \\
\text { Priorisierung der Massnahmen }\end{array}$ & $\begin{array}{l}\text { Risikoanalyse (Stufe 1 nach Borter, 1999), } \\
\text { Schutzdefizitkarte }\end{array}$ \\
Einzelprojekt/ & Evaluation der optimalen & $\begin{array}{l}\text { Kosten-Nutzen-Analyse (in Verbindung mit Risi- } \\
\text { Einzelobjekt }\end{array}$ \\
& Massnahme & $\begin{array}{l}\text { koanalyse Stufe 2 od. 3 nach Borter, 1999), } \\
\text { Grenzkostenbetrachtung }\end{array}$ \\
\hline
\end{tabular}


Kriterien für eine gute operationale Eignung sind die Berücksichtigung aller relevanten Risiken, die Beachtung aller massgeblichen Aspekte, die Messbarkeit und Vergleichbarkeit der tolerierbaren Risiken sowie die Anbindung der Schutzziele an grossräumig vorhandene oder einfach zu beschaffende Informationen.

\subsubsection{Lücken bei den Informationsgrundlagen}

Informationen zur Raumnutzung liegen weitgehend grossräumig vor (Zonenpläne, Schadenspotenzialkarten). In vielen Gebieten sind heute auch Angaben zur Gefährdungslage vorhanden (Gefahrenkarten). Allerdings sind Gefahren hinsichtlich Intensität und Eintretenswahrscheinlichkeit oft ungenügend erkannt und erkennbar. Ebenso sind die durch Gefahrenprozesse bewirkten Effekte ungenügend erforscht und quantifiziert. Beispielsweise bestehen erhebliche Datenlücken zum prozess- und intensitätsspezifischen Ausmass von Personenund Sachschäden. Auf weitere bedeutende Lücken der Messbarkeit und Vergleichbarkeit von tolerierbaren Risiken wurde bereits hingewiesen (Kapitel 2.4): Die Messbarkeit eines Schadens und damit verbunden die Berechenbarkeit eines Risikos ist in vielen Fällen, namentlich bei der Beeinträchtigung von gesellschaftlichen Institutionen, ökologischen Werten und der Lebensqualität, überhaupt nicht gegeben. Schliesslich fehlen allgemeine Angaben zur Wirksamkeit von Risikoverminderungsmassnahmen. Die Wirksamkeit von Massnahmen ist allgemein lückenhaft dokumentiert und hängt ausserdem stark von örtlichen Gegebenheiten $a b$.

\subsubsection{Operationale Eignung von Schutzzieldarstellungen der Praxis}

Von Interesse ist die Frage, wie weit die heute im Management von Naturrisiken bekannten Schutzziele sich angesichts der erwähnten Anforderungen für das regionale Risikomanagement eignen. Schutzziele lassen sich nach zwei Grundkonzepten gliedern (Abbildung 28). Einerseits sind sie als Grenzwerte für tolerierbare Risiken formuliert, die - ungeachtet des hierfür erforderlichen Aufwandes - anzustreben sind (wirkungsorientierte Schutzziele). Diesem Grundkonzept entsprechen Grenzwertkriterien oder Schutzzielmatrizes. Anderseits sind Schutzziele als Grenzkosten formuliert, bis zu deren Erreichen - ungeachtet des sich daraus ergebenden Restrisikos - Massnahmen zu treffen sind. Die Anwendung des Grenzkostenkriteriums ermöglicht die Maximierung des Gesamtnutzens (effizienzorientierte Schutzziele). 


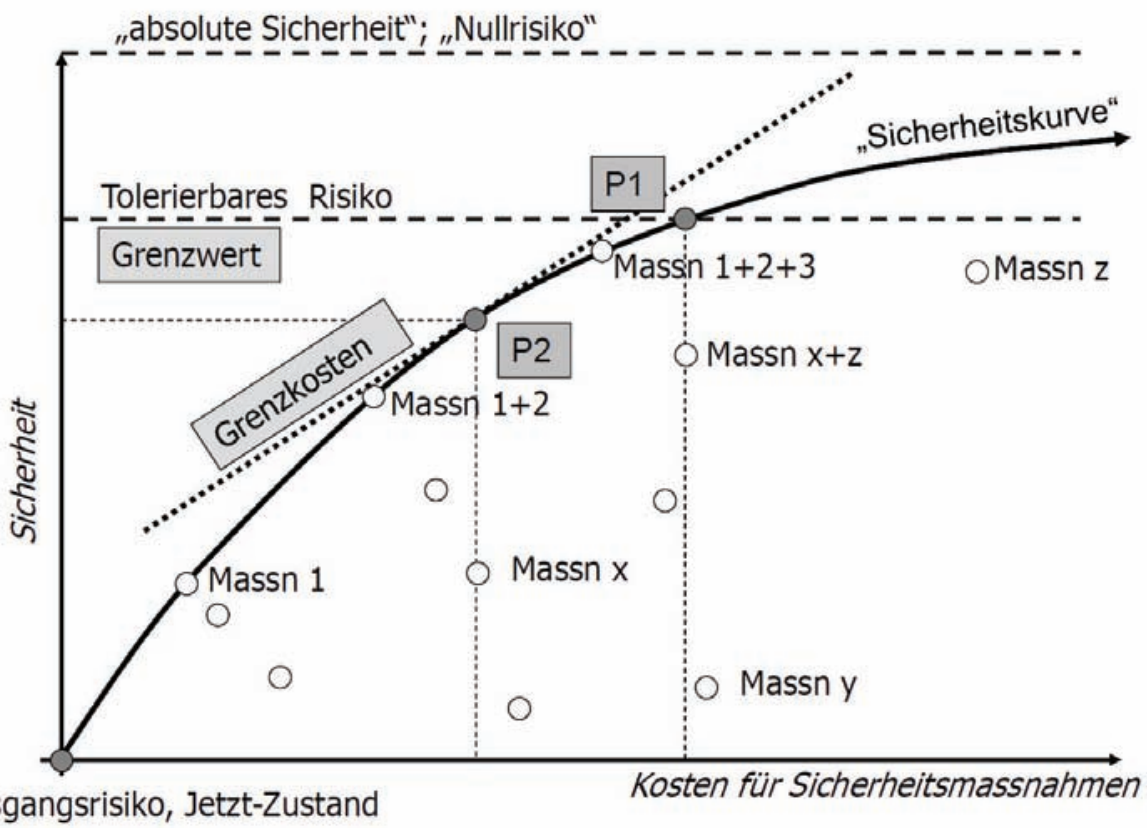

Abbildung 28 Grenzkosten und Grenzwerte als Schutzziele. Die ,Sicherheitskurve' stellt die Verbindungslinie zwischen den kostenwirksamsten Massnahmen (-kombinationen) für jeden Sicherheitsgrad dar (linke oberste Punkte im Diagramm). Gemäss dem Grenzwertkriterium werden Massnahmen ergriffen, bis der Grenzwert des tolerierbaren Risikos erreicht ist (P1). Nach dem Grenzkostenkriterium werden Massnahmen bis zu dem Punkt (P2) ergriffen, an dem die Steigung der ,Sicherheitskurve' der Vorgabe für Grenzkosten entspricht.

Schutzziele lassen sich auch hinsichtlich der Eignung für Einzelprojekte oder für Regionen klassieren (Abbildung 29). Für die Anwendung von Grenzwerten und Schutzzielmatrizes sind Angaben zum vorhandenen Gefahrenpotenzial und den verletzbaren Werten erforderlich. Wo Gefahren- und Schadenspotenzialkarten vorliegen, sind grenzwertbasierte Schutzziele und Schutzzielmatrizes grossräumig einsetzbar. Dank ihrer direkten Anbindung an Gefahrenkarten und Schadenspotenzialkarten eignen sich Schutzzielmatrizes besonders gut für das regionale Risikomanagement.

Die Anwendung von auf Grenzkosten basierenden Schutzzielen erfordert nicht nur Wissen über die Risiken, sondern auch über die Optionen der Risikoverminderung und deren Wirksamkeit. Im regionalen Risikomanagement ist meist eine grosse Zahl von Risikostellen gleichzeitig zu betrachten (z. B. zahlreiche Wildbäche, Lawinenzüge oder Steinschlagquellen). Zur Ermittlung der ,Sicherheitskurve' ist für jede dieser Risikostellen eine erhebliche Anzahl von Varianten an Risikoverminderungsmassnahmen zu eruieren und hinsichtlich Kosten und Wirksamkeit zu analysieren. Erst wenn diese Informationen über alle denkbaren Varianten(-kombinationen) zu allen Risikostellen vorliegen, sind die Voraussetzungen für die Anwendung grenzkostenbasierter Schutzziele gegeben. In der Realität ist dies nur für räumlich eng begrenzte Fragestellungen (Einzelprojekte) möglich. Das Grenzkostenkriterium ist daher im regionalen Risikomanagement kaum anwendbar. 


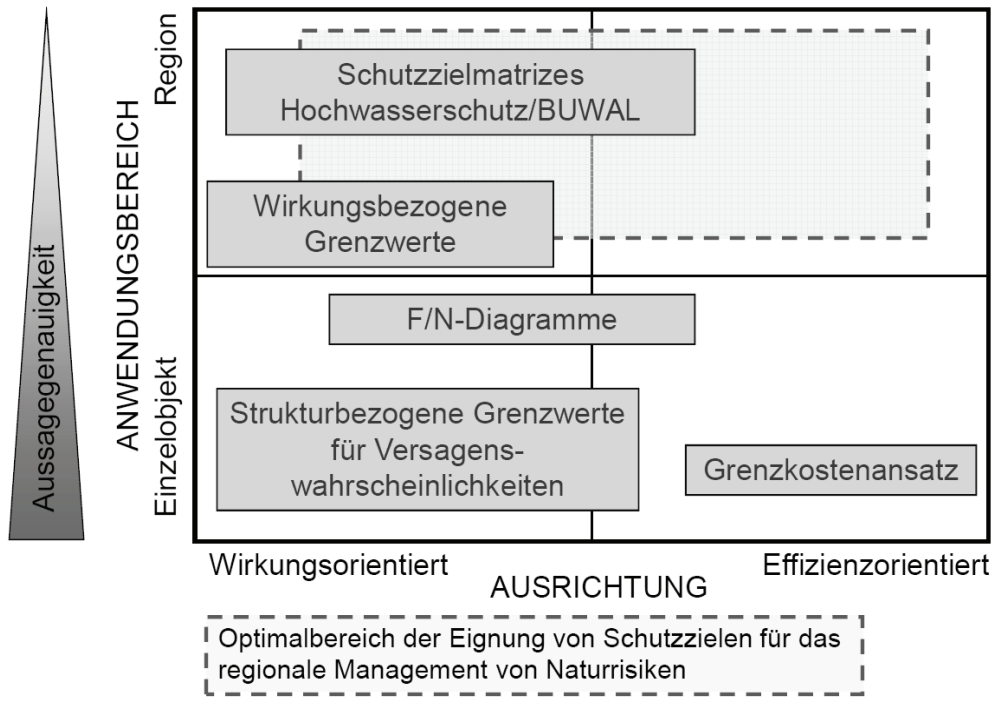

Abbildung 29 Gliederung der Schutzzieldarstellungen hinsichtlich ihrer Ausrichtung (wirkungs- oder effizienzorientiert) und ihrem Anwendungsbereich (Einzelobjekt oder Region). Regional einsetzbare und zugleich effizienzorientierte Schutzzieldarstellungen fehlen.

Die im Bereich technischer Risiken häufig angewendeten F/N-Diagramme kombinieren die Vor- und Nachteile der grenzwert- und grenzkostenbasierten Schutzziele. Für den nicht tolerierbaren Bereich gelten die obigen Feststellungen zu Grenzwerten, im Übergangsbereich die Ausführungen zu Grenzkosten (Kapitel 2.8.3). Die Anwendung dieser Schutzziele erfordert viele Detailinformationen und ist daher für regionales Risikomanagement mit grossem Aufwand verbunden.

\subsection{Organisation und Verfahren zur Festlegung tolerierbarer Risiken}

\subsubsection{Politische und organisatorische Rahmenbedingungen}

2.9.1.1 Rollen und Verantwortlichkeiten im Risikomanagement

Das Management von Naturrisiken in der Schweiz wird heute von zahlreichen Beteiligten des öffentlichen und privaten Sektors auf unterschiedliche Weise getragen (Tabelle 30). 
Tabelle 30 Akteure und typische Aufgaben im Management von Naturrisiken.

\begin{tabular}{|c|c|}
\hline Akteur & (Typische) Aktivitäten, wahrgenommene Aufgaben \\
\hline Staat/Gemeinwesen & $\begin{array}{l}\text { erkennt und bewertet Risiken } \\
\text { bezieht Steuern von Risikobetroffenen und Drittpersonen zur Finanzierung } \\
\text { von Risikoverminderungsmassnahmen } \\
\text { warnt und ergreift polizeiliche Massnahmen bei Gefahr } \\
\text { ergreift Schadensbegrenzungsmassnahmen bei Ereignissen } \\
\text { ergreift Präventionsmassnahmen oder unterstützt diese mit Beitrags- } \\
\text { leistungen } \\
\text { leistet Hilfe in Schadensfällen } \\
\text { erlässt Regeln zur Raumnutzung }\end{array}$ \\
\hline Risikobetroffener & $\begin{array}{l}\text { bezieht Sicherheitsleistungen von Staat und Drittpersonen } \\
\text { leistet Beitrag an Risikoverminderung } \\
\text { leistet Beitrag an Schadensbehebung } \\
\text { bezahlt Versicherungsprämien } \\
\text { duldet Schutzmassnahmen auf seinem Grundstück } \\
\text { duldet Nutzungsbeschränkungen auf seinem Grundstück }\end{array}$ \\
\hline $\begin{array}{l}\text { Trägerschaft } \\
\text { der Massnahme/ } \\
\text { Bauherrschaft }\end{array}$ & $\begin{array}{l}\text { bezieht Sicherheitsleitungen von Staat und Drittpersonen } \\
\text { führt Massnahmen aus } \\
\text { leistet Beitrag an Finanzierung }\end{array}$ \\
\hline Experte & $\begin{array}{l}\text { beurteilt Risiken } \\
\text { plant Massnahmen im Auftrag des Staates oder des Risikobetroffenen }\end{array}$ \\
\hline Hilfswerk & $\begin{array}{l}\text { bezieht Spenden von Drittpersonen und Risikobetroffenen } \\
\text { leistet Hilfe in Schadensfällen }\end{array}$ \\
\hline Versicherung & $\begin{array}{l}\text { bezieht Risikoprämien von Risikobetroffenen und Drittpersonen } \\
\text { leistet Beiträge an Schadensbehebung } \\
\text { verteilt Risiken in Raum und Zeit }\end{array}$ \\
\hline Drittperson & $\begin{array}{l}\text { finanziert Massnahmen über Steuergelder mit } \\
\text { duldet Schutzmassnahmen auf ihrem Grundstück }\end{array}$ \\
\hline
\end{tabular}

Alle Beteiligten leisten in einer bestimmten Weise Beiträge an das Risikomanagement und sind bezüglich ihrer eigenen betroffenen Werte auch Nachfrager von Sicherheitsleistungen. Gemessen an den Finanzströmen wird etwas mehr als die Hälfte der Aufwendungen (56\%) durch Private getragen, welche - als Betroffene und Nichtbetroffene - Versicherungsprämien leisten und auch in bedeutendem Masse Präventions- und Interventionsmassnahmen finanzieren (Abbildung 30). Die verbleibenden Aufwendungen werden hauptsächlich durch die Gemeinwesen getragen, wobei die Aufwendungen etwa zu gleichen Teilen auf Bund, Kanton und Gemeinden entfallen (PLANAT, 2004a). 


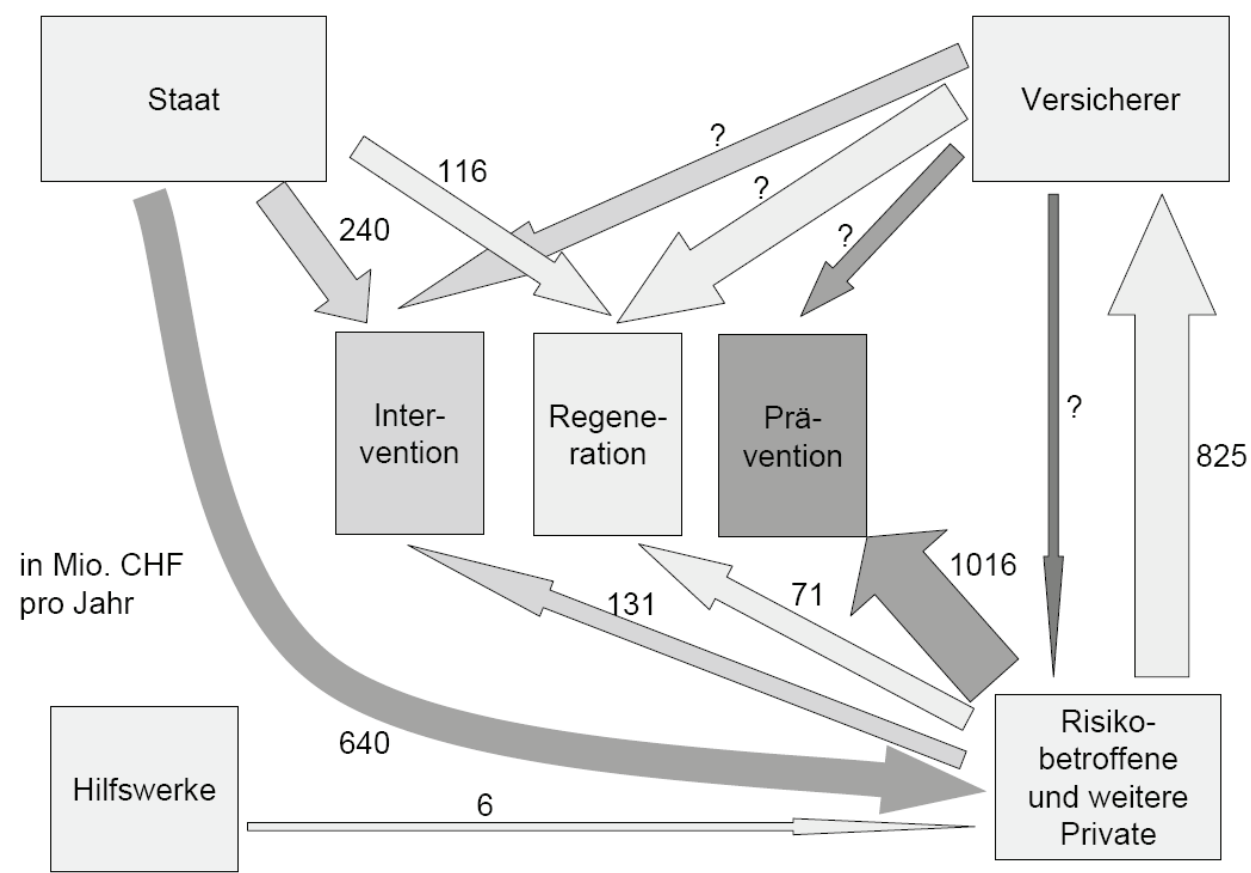

Abbildung 30 Finanzflüsse im Management von Naturrisiken der Schweiz (Zahlen 2004, in Mio. CHF). Etwas mehr als die Hälfte der Aufwendungen wird durch Private in Form von Versicherungsprämien sowie Beiträgen an Prävention, Regeneration und Intervention getragen (Planat, 2004a).

\subsubsection{Aufgabenteilung zwischen Bund, Kanton und Gemeinden}

Dem Bund fallen im Management von Naturrisiken vor allem Grundsatz- und Strategieaufgaben zu. Artikel 76 und 77 der Bundesverfassung (SR 101) übertragen dem Bund eine Aufsichtspflicht und die Kompetenz, Grundsätze festzulegen. Der Bund schöpft diese Kompetenz in unterschiedlichem Masse aus. Weitreichend ist sein Einfluss im Bereich der Prävention: Bei vielen Projekten bestimmt der Bund - als massgeblicher Mitfinanzierer - die Gestaltung einzelner Massnahmen mit.

Die Aufgabenteilung zwischen Kanton und Gemeinden ist föderalistisch heterogen geregelt. In den meisten Kantonen liegt die eigentliche Verantwortung für das Management von Naturrisiken bei den Gemeinden (z. B. Art. 7 und 16 des Wasserbaugesetzes des Kantons Obwalden WBG, GDB 740.1). Die kantonale Verantwortlichkeit beschränkt sich auf ausserordentliche Lagen, in denen die Mittel einzelner Gemeinden nicht mehr ausreichen.

Bund und Kantone verstehen ihre Aufgabe subsidiär (Tabelle 31). Die formelle Hauptverantwortung liegt meist bei den Gemeinden. Jedoch sind die zur Erfüllung der Aufgaben erforderlichen fachlichen Ressourcen und Kompetenzen oft beim Bund und den Kantonen angesiedelt. 
Tabelle 31 Heutige Aufgabenteilung Bund, Kanton, Gemeinde.

\begin{tabular}{|c|c|}
\hline Akteur & (Typische) Aktivitäten, wahrgenommene Aufgaben \\
\hline Bund & $\begin{array}{l}\text { stellt methodische Grundlagen bereit } \\
\text { erlässt Grundsätze und Richtlinien zu Projekten } \\
\text { begleitet Projektplanungen fachlich } \\
\text { leistet Beiträge an Projekte } \\
\text { beeinflusst Entscheide zu (Präventions-)Massnahmen } \\
\text { prüft Projekte und Abrechnungen im Detail } \\
\text { erstellt und verbreitet Warnungen in Gefahrensituationen } \\
\text { leistet Hilfe bei Katastrophen }\end{array}$ \\
\hline Kanton & $\begin{array}{l}\text { verfeinert und vollzieht die Grundsätze und Richtlinien } \\
\text { erstellt Gefahrenkarten und Risikoanalysen } \\
\text { prüft und genehmigt (Gefahren-)Zonenplanungen } \\
\text { begleitet Projektplanungen fachlich } \\
\text { leistet Beiträge an Projekte } \\
\text { beeinflusst Entscheide zu Massnahmen } \\
\text { prüft Projekte und Abrechnungen } \\
\text { verbreitet Warnungen } \\
\text { unterstützt die Gemeinden in ausserordentlichen Lagen }\end{array}$ \\
\hline Gemeinde & $\begin{array}{l}\text { ist formell hauptverantwortlich für die Sicherheit vor Naturgefahren } \\
\text { plant Projekte } \\
\text { leistet Beiträge an Projekte } \\
\text { führt Massnahmen aus } \\
\text { ergreift Notmassnahmen in ausserordentlichen Lagen } \\
\text { erstellt (Gefahren-)Zonenpläne }\end{array}$ \\
\hline
\end{tabular}

\subsubsection{Rahmenbedingungen NFA und effor2}

Am 1.1.2008 trat die Neugestaltung des Finanzausgleichs und der Aufgaben zwischen Bund und Kantonen (NFA) in Kraft. Die NFA regelt die Beziehung zwischen Bund und Kantonen auch im Management von Naturrisiken neu.

Für die staatliche Förderung des Risikomanagements setzt die NFA den folgenden Rahmen (Staehelin, 2003; Haering, 2002):

- Das Management von Naturrisiken ist eine Verbundaufgabe. Die Kantone und der Bund beteiligen sich gemeinsam an den Kosten der Leistungserbringung.

- Die Finanzierungsbeiträge des Bundes sind nicht von der Finanzkraft der einzelnen Kantone abhängig. Die unterschiedliche Finanzkraft wird durch den Ressourcenausgleich der NFA berücksichtigt.

- Für Zusatzlasten aufgrund der geografisch-topografischen Situation erhalten die Kantone einen geografisch-topografischen Lastenausgleich (GLA), der innen zweckungebunden zur Verfügung steht.

- Die Höhe des Bundesbeitrags hängt nicht von den Kosten einzelner Massnahmen ab.

Parallel zur NFA wird das Projekt effor2 umgesetzt. Dessen wesentliche Elemente sind (Staehelin, 2003):

Josef Th. Hess: Schutzziele im Umgang mit Naturrisiken in der Schweiz, ๑ vdf Hochschulverlag 2011 
- strategische Zielvorgaben des Bundes;

- Subventionierung von Ergebnissen statt von Massnahmen und Kosten;

- Abgeltung durch einheitliche Pauschalen;

- Förderung nach Prioritäten und Effizienzkriterien;

- Globalisierung der Beiträge durch Subventionierung mehrjähriger, wirkungsorientierter Programme;

- Delegation der operativen Programmverantwortung an die Kantone sowie

- Vereinheitlichung der Förderung nach Wald- und Wasserbaugesetzgebung.

Im Rahmen des präventiven Managements von Naturrisiken subventioniert der Bund die Produkte ,Schutzbauten' und ,Schutzwald'. Schutzbauten nach Waldgesetz und solche nach Wasserbaugesetz werden in einer separaten Programmvereinbarung abgedeckt.

Für diese Produkte wurden bis Ende 2006 Ziele, Indikatoren, Vorgaben und Rahmenbedingungen in Zusammenarbeit zwischen Bund und den Kantonen entwickelt. Parallel dazu wird das nötige Instrumentarium aufgebaut (Controlling usw.). Im Jahr 2007 fanden die Verhandlungen mit den Kantonen zum Abschluss der Programmvereinbarungen statt (BAFU, 2007a).

Die Programmvereinbarung ,Schutzbauten' umfasst ein sogenanntes Grundangebot (Einzelmassnahmen (Verbauungen, Messstellen, Warndienste) mit Kosten CHF <1 Mio.) und einen Programmteil Gefahrengrundlagen (Erarbeitung und Nachführung von Gefahrengrundlagen; Tabelle 32). Die Programmvereinbarungen ,Schutzwald' umfassen Massnahmen zur Schutzwaldbehandlung und zur Sicherstellung der Infrastruktur für die Schutzwaldbehandlung (BAFU, 2007c).

Tabelle 32 Elemente des Vierjahresprogramms der Programmvereinbarung ,Schutzbauten' (BAFU, 2007a).

\begin{tabular}{|c|c|c|c|c|}
\hline Element & $\begin{array}{l}\text { Gesetzliche } \\
\text { Grundlage }\end{array}$ & Inhalt & Rechtsform & Bundesbeitrag \\
\hline $\begin{array}{l}\text { Grundangebot } \\
\text { technischer } \\
\text { Schutz vor } \\
\text { Naturgefahren }\end{array}$ & $\begin{array}{l}\text { WBG, Art. } 6 \\
\text { WaG, Art. } 36\end{array}$ & $\begin{array}{l}\text { - Projekte mit Kosten < } 1 \text { Mio. CHF } \\
\text { - Periodische Instandstellung, } \\
\text { Ersatz bestehender Schutzbauten } \\
<1 \text { Mio. CHF } \\
\text { - Messstellen, Warndienste }\end{array}$ & $\begin{array}{l}\text { Programm- } \\
\text { vereinbarung }\end{array}$ & $\begin{array}{l}\text { Globalbeitrag max. } \\
35 \% \text { der } \\
\text { anrechenbaren } \\
\text { Kosten }\end{array}$ \\
\hline $\begin{array}{l}\text { Gefahren- } \\
\text { grundlagen }\end{array}$ & $\begin{array}{l}\text { WBG, Art. } 6 \\
\text { WaG, Art. } 36\end{array}$ & $\begin{array}{l}\text { Grundlagen für das Risikomanagement } \\
\text { (Kataster, Gefahrenkarten, Risiko- } \\
\text { beurteilungen, Konzepte etc.) }\end{array}$ & $\begin{array}{l}\text { Programm- } \\
\text { vereinbarung }\end{array}$ & $\begin{array}{l}\text { Globalbeitrag } \\
50 \% \text { der } \\
\text { anrechenbaren } \\
\text { Kosten }\end{array}$ \\
\hline \multicolumn{5}{|c|}{$\begin{array}{l}\text { Einzelprojekte sind nicht Bestandteil der Programmvereinbarung. Sie werden wie bis anhing einzeln verfügt } \\
\text { gemäss der dafür reservierten Mittel. }\end{array}$} \\
\hline Einzelprojekte & $\begin{array}{l}\text { WBG, Art. } 6 \\
\text { WaG, Art. } 36\end{array}$ & $\begin{array}{l}\text { Einzelprojekt mit Kosten } \\
>1 \text { Mio. CHF } \\
\text { (Neubauten, periodische } \\
\text { Instandstellungen) }\end{array}$ & Verfügung & $\begin{array}{l}\text { 35-45\% der Kosten in } \\
\text { Abhängigkeit von der } \\
\text { Wirksamkeit } \\
\text { Bei erheblicher } \\
\text { Belastung der } \\
\text { Kantone kann der } \\
\text { Bund seinen Beitrag } \\
\text { auf höchstens } 65 \%\end{array}$ \\
\hline
\end{tabular}

Die NFA-Bestimmungen sehen eine strategische Rolle des Bundes und eine operative Rolle von Kantonen und Gemeinden vor. Die Ausführung der Massnahmen erfolgt meist durch die 
Gemeinde oder Trägerschaften auf Gemeindeebene. Bund und Kanton fördern die Projekte finanziell.

Die vorgesehene Umsetzung der NFA-Grundsätze relativiert die Trennung zwischen strategischem (Bundesaufgabe) und operativem Risikomanagement (Kantons-/Gemeindeaufgabe) allerdings stark. Auf Stufe Kanton ist weiterhin ein Engagement im operativen Bereich üblich, da die Kantone meist über ein wesentlich grösseres Wissen im Management von Naturrisiken verfügen. Nach wie vor übernimmt auch der Bund operative Mitverantwortung für Einzelprojekte. Projekte mit Kosten von mehr als einer Million CHF werden durch den Bund im Detail technisch beurteilt.

Eine strategische Rolle nimmt der Bund durch eine differenzierte Förderung von Projekten wahr. Die Abstufung der Förderung liegt in der Spannweite von 10\% der Beiträge und erfolgt aufgrund eines Ratings der Leistungen der Beitragsempfänger im integralen Risikomanagement einerseits und der Qualität der geplanten Massnahmen anderseits (BAFU, 2007a). Von Mehrleistungen des Bundes können beispielsweise Kantone profitieren, die Gefahrenkarten erstellt und planerisch umgesetzt haben. Ebenso werden Projekte höher gefördert, die sich durch besondere technische und ökologische Qualität auszeichnen oder in hohem Masse partizipativ geplant wurden.

\subsubsection{Gesellschaftliche Entscheidverfahren}

\subsubsection{Arten von Entscheidverfahren und Entscheidungsebenen}

Schutzziele sind einerseits eine Frage der Höhe des tolerierbaren Risikos, anderseits eine Frage der Verteilung der Restrisiken. Die Höhe des tolerierbaren Risikos erfordert Entscheide darüber, wie viele Mittel zur Risikominderung bereitgestellt werden (Allokation). Gleichzeitig sind Entscheide zur ,gerechten' Verteilung der Restrisiken erforderlich (Distribution). Solche gesellschaftlichen Entscheide können auf verschiedene Weise herbeigeführt werden (Frey, 2002):

- durch Preismechanismus;

- durch Demokratie;

- durch wirtschaftliche Interessengruppen und

- durch Bürokratie und Verwaltung.

Von grosser Bedeutung ist dabei, ob die Entscheide auf der Ebene des gesellschaftlichen Grundkonsenses oder auf der Ebene laufender gesellschaftlicher Prozesse gefällt werden. Auf der Ebene des Grundkonsenses befinden sich die betroffenen Entscheidungsträger im Ungewissen darüber, wie sie persönlich von einem Entscheid betroffen sein werden. Wenn Abmachungen in diesem Zustand der Ungewissheit für alle von Vorteil sind, können sie meist freiwillig und einstimmig getroffen werden. Auf dieser Ebene ergeben sich ,objektivierte', von kurzfristigen und partikularen Interessen unbeeinflusste Regelungen gesellschaftlicher Fragen. Im laufenden gesellschaftlichen Prozess dagegen sind derartige Regelungen nicht möglich. Der Zustand der Ungewissheit besteht nicht. Die Entscheidträger wissen, wie sie ganz persönlich von einem Entscheid betroffen sind. Kurzfristige und partikulare Interessen prägen den Entscheid.

\subsubsection{Charakteristiken gesellschaftlicher Entscheidverfahren}

Sämtliche gesellschaftlichen Entscheidverfahren haben Vor- und Nachteile. Preismechanismus und Demokratie scheinen die grössten Vorteile zu bieten, während Entscheide unter dem Regime der Verwaltung und (wirtschaftlicher) Interessengruppen oft zu ineffizienten Lösungen führen (Frey, 2002). Der Preismechanismus führt grundsätzlich zu einer effizienten Allokation sowie zu Fortschritt und Veränderung. Einschränkungen für dieses Entscheidverfahren sind unvollständige Märkte und - gerade im Umgang mit Naturrisiken relevant das Vorliegen öffentlicher Güter und Externalitäten.

Josef Th. Hess: Schutzziele im Umgang mit Naturrisiken in der Schweiz, @ vdf Hochschulverlag 2011 
Unter den demokratischen Entscheidungsmechanismen führen vor allem direktdemokratische Verfahren zu effizienten Lösungen. Vereinzelt ergeben sich in demokratischen Verfahren nicht eindeutige Entscheide. Beim Vorliegen öffentlicher Güter verleiten demokratische Verfahren zudem zu strategischem Verhalten.

Ein grosses Problem des Entscheidverfahrens unter dem Regime wirtschaftlicher Interessengruppen liegt darin, dass nur ein Teil der Bevölkerung in Interessengruppen organisiert ist. Schlecht organisiert sind insbesondere Konsumenten und Steuerzahler. Durch Interessensgruppen geprägte Entscheide sind nicht selten ineffizient, insbesondere infolge gegenseitiger strategischer Unterstützung (,Stimmentausch') und infolge des Strebens nach Einkommensgenerierung durch Umverteilung anstelle von wirtschaftlicher Produktion (,rentseeking society').

Bürokratisch-hierarchische Strukturen haben sowohl in der Verwaltung als auch in der Privatwirtschaft grosse Bedeutung bei der Umsetzung von Entscheiden. Ihr Nutzen liegt in der Einsparung von Transaktionskosten. Die bürokratischen Strukturen bieten wenig Anreize, sich volkswirtschaftlich optimal zu verhalten, und bewirken oft ineffiziente Lösungen. Die Kontrolle der Verwaltung im laufenden gesellschaftlichen Prozess ist schwierig.

\subsubsection{Markt-Hierarchie-Paradigma}

Nach Williamson (1975) hängt die optimale Koordinationsform zwischen den Akteuren von den Transaktionskosten ab (Markt-Hierarchie-Paradigma). Je tiefer die Transaktionskosten, desto effizienter ist der Markt, je höher diese sind, desto eher ist eine hierarchische Koordination zu bevorzugen. Die Wahl der effizientesten Organisationsform hängt nebst den Transaktionskosten von der Spezifität der Güter ab, in die investiert wird. Hochgradig spezifisch sind Investitionen, wenn die Werte, in die investiert wird, nur für den einen Verwendungszweck optimal genutzt werden können. Ebenso ist eine hierarchische Koordinationsform bei sehr grosser Unsicherheit das kostengünstigste institutionelle Arrangement (Abbildung 31).

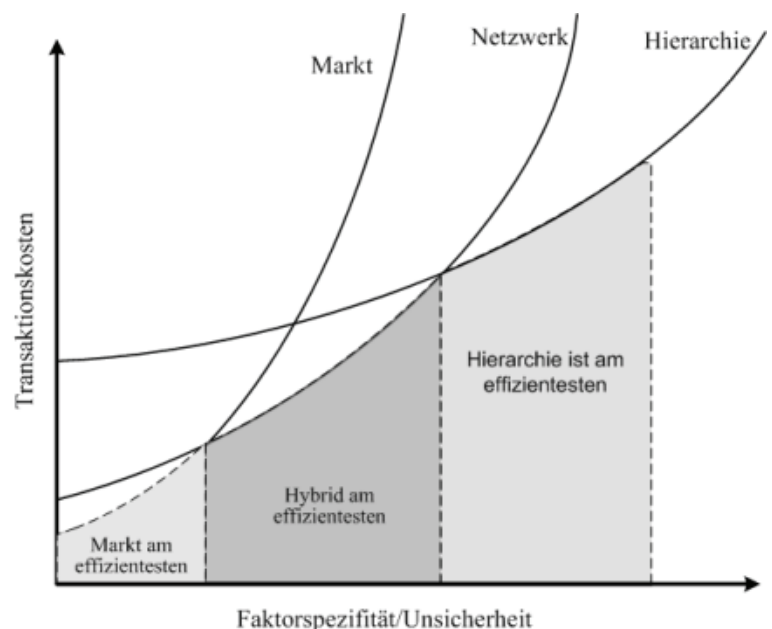

Abbildung 31 Die Effizienz der Koordinationsform zwischen gesellschaftlichen Akteuren hängt von Transaktionskosten, der Faktorspezifität und der Unsicherheit ab (Williamson, 1975).

Neben ökonomischen und rechtlichen Aspekten der Koordination der Marktteilnehmer sind soziale Kontrollmechanismen von grosser Bedeutung. Dazu gehören Vertrauen, Kultur und Reputation (Wikipedia, 2006). Vertrauen verkürzt die Verhandlungsdauer, senkt die Kontrollkosten und ist somit effizienzsteigernd. Unter einer Kultur werden Gemeinsamkeiten in PräJosef Th. Hess: Schutzziele im Umgang mit Naturrisiken in der Schweiz, @ vdf Hochschulverlag 2011 
ferenzen, Werten, Zielen und Kompetenzen verstanden, die die Koordinationskosten minimieren können. Bei längerfristigen Beziehungen kann es in sehr einheitlichen Kulturen aber auch zu neuen Koordinationskosten aufgrund von Abhängigkeiten und opportunistischem Verhalten kommen. Gute Reputation verringert die Informations- und Verhandlungskosten.

Wie in Kapitel 2.9.1 dargelegt, ist das Management von Naturrisiken sehr stark geprägt durch hierarchische Strukturen und Entscheidverfahren. Häufig sind von Entscheiden über Risikoverminderungsmassnahmen zahlreiche Personen(-gruppen) betroffen, deren Interessen zu koordinieren sind. Dies generiert hohen Aufwand, der als Transaktionskosten zur Bereitstellung von Sicherheitsmassnahmen bezeichnet werden kann.

\subsubsection{Ablauf der Planung}

Am Anfang des heute üblichen Planungsablaufes im Risikomanagement auf regionaler Ebene steht idealerweise die Ermittlung von Schutzdefiziten aufgrund der Gefahrenkarte und der Schadenspotenzialkarte (Abbildung 32).

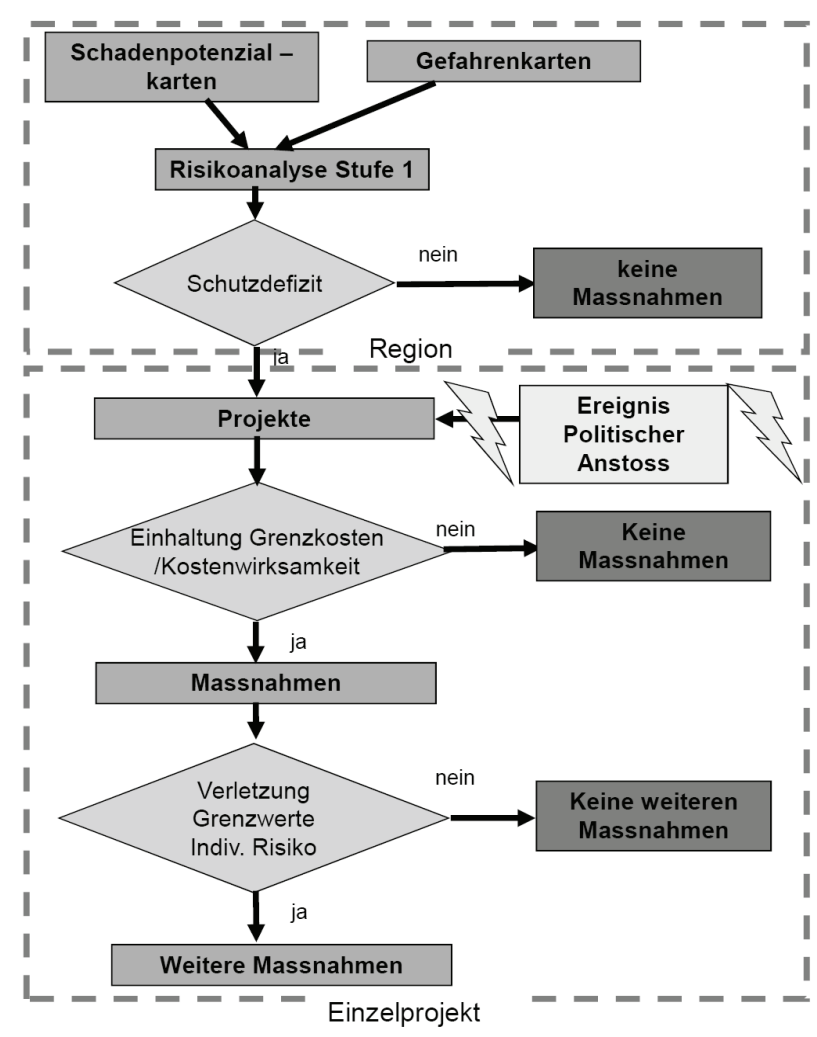

Abbildung 32 Ablauf des Managements von Naturrisiken. Eine Risikoanalyse auf regionaler Ebene (Stufe 1 nach Borter, 1999) zeigt Schutzdefizite (Handlungsbedarf) und Prioritäten auf. Aus diesem Handlungsbedarf, oft aber auch aufgrund von Ereignissen und politischen Anstössen ergeben sich Projekte, die hinsichtlich wirtschaftlicher Kriterien und Erfüllung von Sicherheitsanforderungen zu prüfen sind. 


\subsubsection{Beteiligung der Betroffenen in Projekten zur Verminderung von Naturrisiken}

\subsubsection{Mitwirkung im formellen Verwaltungshandeln}

Wie dargelegt ist das Management von Naturrisiken in der Schweiz stark durch die Verwaltung geprägt. Das schweizerische Verwaltungsrecht kennt eine Reihe von rechtlichen Handlungsformen (Richli, 2004a), die in unterschiedlichem Mass Mitwirkungsmöglichkeiten für die von Entscheiden Betroffenen bieten (Tabelle 33). Das öffentliche österreichische Recht ist ähnlich gestaltet (Weber, 2005). Diese Handlungsformen finden auch im Management von Naturrisiken Anwendung.

Tabelle 33 Handlungsformen des schweizerischen Verwaltungsrechts (Richli, 2004a).

$\begin{array}{lc}\text { Realakte } & \text { Rechtsakte } \\ \text { Taterfolg } & \text { Rechte und Pflichten } \\ & \\ & \text { Öffentlich-rechtlich }\end{array}$

nach innen

Verwaltungs- Dienstbefehl verordnung Rechtsver- Plan Verfügung
ordnung
Privatrechtlich

Wenn sich aus dem öffentlichen Handeln Konflikte mit den Interessen Betroffener ergeben, stehen diesen in vielen Fällen formelle Einsprache- und Beschwerdemöglichkeiten zur Wahrung ihrer Rechte offen. Pläne werden öffentlich aufgelegt mit der Möglichkeit zur Einsprache. Verfügungen können mit Beschwerden angefochten werden. Diese formellen Mitwirkungsmöglichkeiten dienen meist der Bereinigung von Konflikten zwischen öffentlichem Handeln und Privatinteressen. Bei verwaltungsrechtlichen Verträgen können die Vertragsparteien - staatliche Stellen und Privatpersonen - die Bedingungen partnerschaftlich aushandeln.

Im Risikomanagement erweisen sich diese klassischen Instrumente des Verwaltungsrechts angesichts der Komplexität der Fragestellungen, des häufig lückenhaften Wissens und des grossen öffentlichen Interesses oft als ungenügend (Seiler, 1997). In den letzten Jahrzehnten wurden daher Verfahren geschaffen, die der besseren Integration und Koordination der verschiedenen Interessen dienen:

- Risikoermittlung als Basis für eine transparente Darstellung der Entscheidgrundlagen und eine rechtliche Beurteilung;

- $\quad$ standardisierte und anerkannte Qualitätssicherungsverfahren (QS);

- Umweltverträglichkeitsprüfung mit der Möglichkeit zur öffentlichen Einsichtnahme;

- Kooperationsprinzip: Vereinbarung zwischen Staat und Privaten über durch die Privaten zu erreichendes Ziel;

- informaler Rechtsstaat, informationspolitische Massnahmen zur Bewusstseinsbildung sowie

- Mediation, Konfliktregelungsverfahren: Aushandeln von Standards durch Ausgleich unterschiedlicher Interessen unter der Leitung des Staates. 


\subsubsection{Partizipative Projektplanung}

Die Bedeutung einer partizipativen Projektplanung und -umsetzung ist heute allgemein anerkannt. Die Festlegung von Schutz- und Projektzielen stellt nicht eine reine Expertenangelegenheit dar (Heinimann, 2002). Je nach Grösse und Komplexität des Projektes erfolgt in vielen Projekten ein mehr oder weniger expliziter Einbezug der Stakeholder, beispielsweise im Rahmen von Projektsteuergruppen, Workshops, Hearings usw. Diese informelle Mitwirkung ermöglicht, wenn auch unter starker Einflussnahme der Experten, eine Projektgestaltung durch die Beteiligten (Abbildung 33).

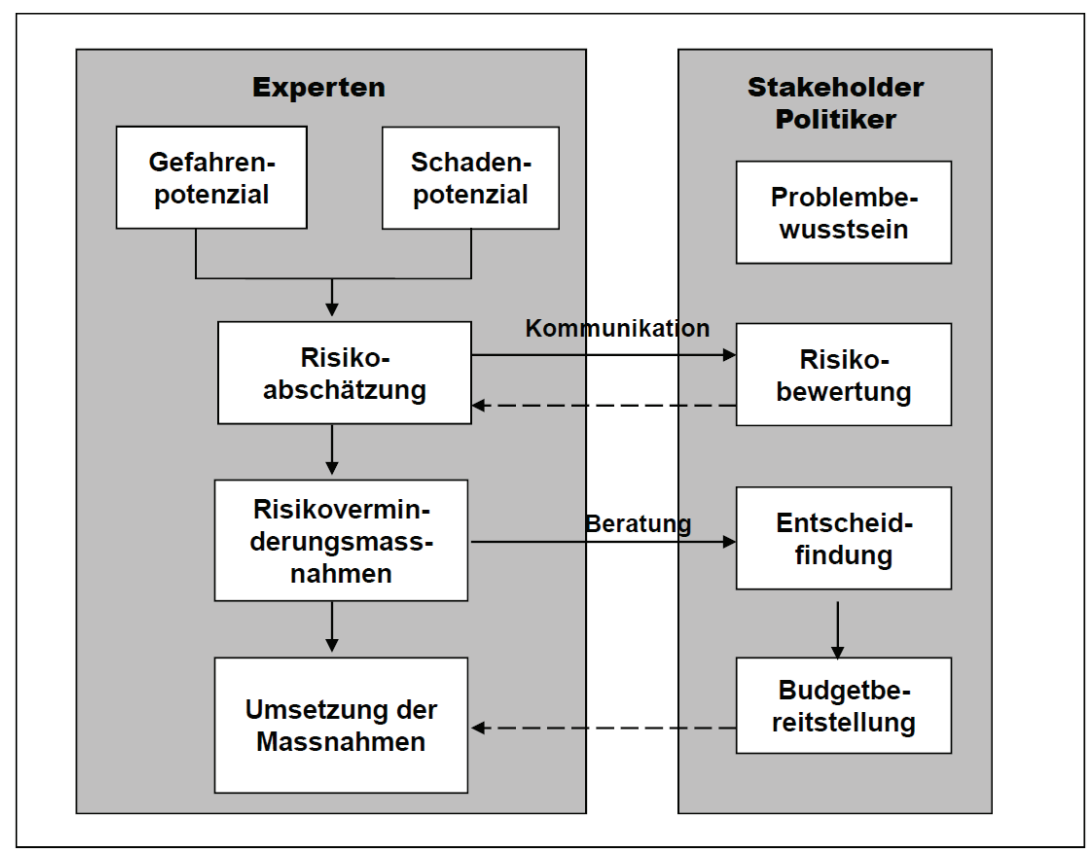

Abbildung 33 Zusammenarbeit von Experten und Laien (Heinimann, 2002).

Die Mitwirkungsverfahren der Stakeholder in der Projektplanung wurden in den letzten Jahren weiterentwickelt (Hostmann et al., 2005). Betroffene und Akteure werden identifiziert und in geeigneter Weise einbezogen. Die Art der Partizipation wird differenziert nach dem Mass der Betroffenheit und den Möglichkeiten der Einflussnahme der Akteure (Tabelle 34 und Abbildung 34). Die Akteure in den Quadranten A, B und D in Abbildung 34 können das Projekt stark beeinflussen oder sind stark betroffen. Je nach Betroffenheit oder Einflussmöglichkeiten kann der Einbezug der Betroffenen auf den zwei Ebenen regionales Risikomanagement und Einzelprojekt differenziert erfolgen. 
Tabelle 34 Klassifizierung der Akteure (eigene Darstellung in Anlehnung an Hostmann et al., 2005).

\begin{tabular}{|c|c|c|c|}
\hline Gruppe & Strategie & $\begin{array}{l}\text { Beispiele von Akteuren } \\
\text { Stufe regionales } \\
\text { Risikomanagement }\end{array}$ & Stufe Einzelprojekt \\
\hline$A$ & $\begin{array}{l}\text { Diese Akteure sind vom Nutzen des Projektes zu } \\
\text { überzeugen. }\end{array}$ & Interessenverbände & $\begin{array}{l}\text { Bund, Kanton, } \\
\text { Interessenverbände }\end{array}$ \\
\hline$B$ & $\begin{array}{l}\text { Diese Akteure sind für den Projekterfolg ent- } \\
\text { scheidend und besonders frühzeitig einzubezie- } \\
\text { hen. }\end{array}$ & Industrie und Gewerbe & $\begin{array}{l}\text { Grundeigentümer, } \\
\text { Unternehmungen }\end{array}$ \\
\hline C & $\begin{array}{l}\text { Diese Akteure können evtl. vernachlässigt wer- } \\
\text { den. }\end{array}$ & & Erholungsnutzer \\
\hline$D$ & $\begin{array}{l}\text { Diese Akteure sind genügend einzubeziehen zur } \\
\text { Vermeidung von Konflikten und Projektverzöge- } \\
\text { rungen (Rechtsmittel). }\end{array}$ & $\begin{array}{l}\text { Grundeigentümer, } \\
\text { Industrie und Gewerbe }\end{array}$ & Unternehmungen \\
\hline
\end{tabular}

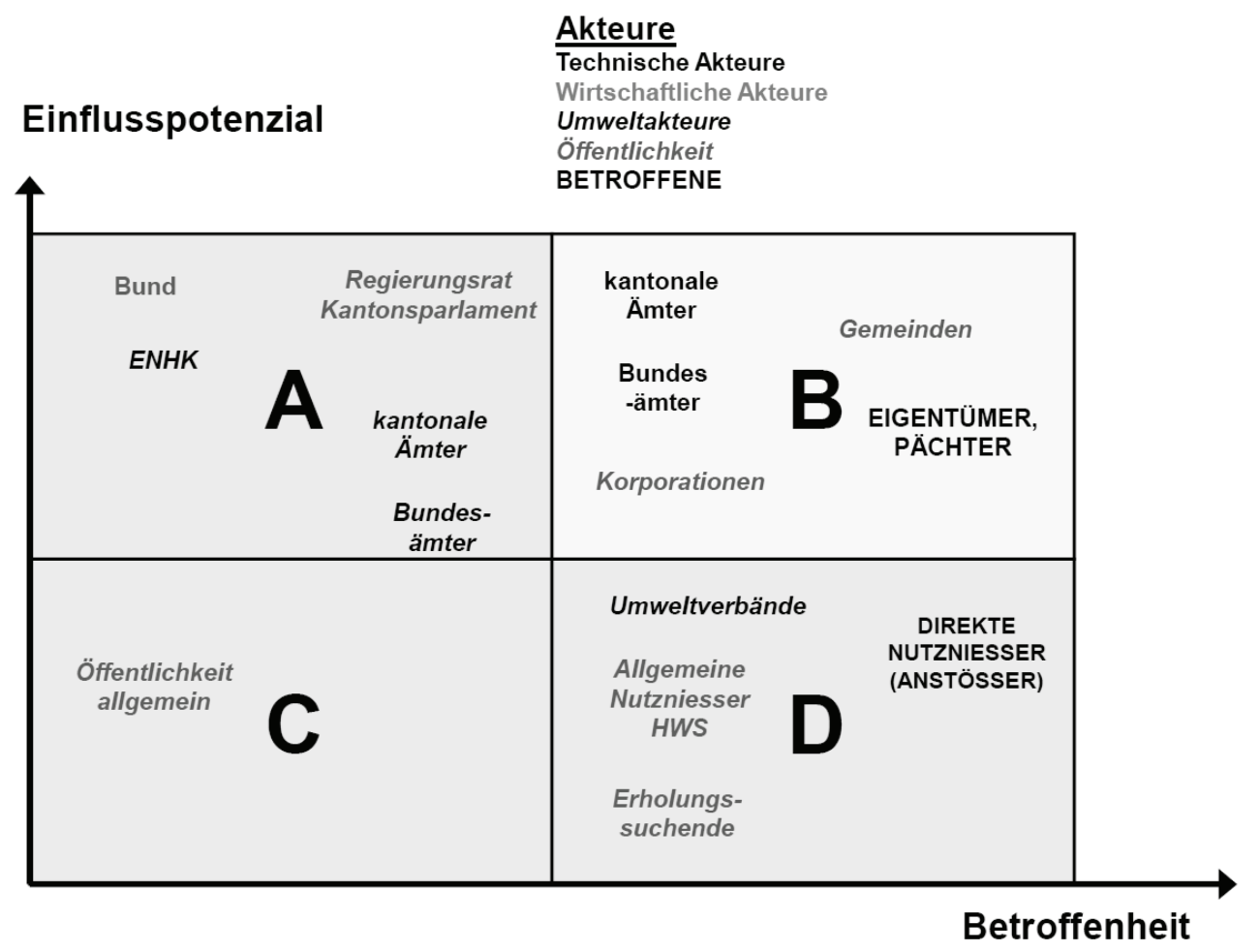

Abbildung 34 Einfluss-Betroffenheits-Matrix (Hostmann, 2005). Akteure beispielhaft dargestellt $($ ENHK $=$ Eidg. Natur- und Heimatschutzkommission, HWS = Hochwasserschutz).

Der Einbezug der Akteure erfordert auf den Ebenen regionales Risikomanagement und Einzelprojekt an die verschiedenen Projektphasen angepasste Formen der Mitwirkung (Tabelle 35). 
Tabelle 35 Themen und Formen des Einbezugs von Akteuren (eigene Darstellung in Anlehnung an Hostmann et al., 2005).

\begin{tabular}{|c|c|c|c|c|c|c|}
\hline \multirow{2}{*}{$\begin{array}{l}\text { Ebene } \\
\text { Projekt- } \\
\text { phasen }\end{array}$} & \multirow{2}{*}{$\begin{array}{l}\text { Region } \\
\text { Strategische } \\
\text { Planung }\end{array}$} & \multicolumn{5}{|c|}{ Einzelprojekt } \\
\hline & & Vorstudie & $\begin{array}{l}\text { Projektierung } \\
\text { (Vorprojekt) }\end{array}$ & $\begin{array}{l}\text { Projektierung } \\
\text { (Bauprojekt) }\end{array}$ & $\begin{array}{l}\text { Realisie- } \\
\text { rung }\end{array}$ & Nutzung \\
\hline $\begin{array}{l}\text { Kern- } \\
\text { themen }\end{array}$ & $\begin{array}{l}\text { Identifikation } \\
\text { Akteure und } \\
\text { Ziele, Ziel- } \\
\text { gewichtung }\end{array}$ & $\begin{array}{l}\text { Varianten- } \\
\text { evaluation, } \\
\text {-diskussion, } \\
\text {-entscheid }\end{array}$ & $\begin{array}{l}\text { Projekt- } \\
\text { optimierung }\end{array}$ & $\begin{array}{l}\text { Verminderung } \\
\text { Interessen- } \\
\text { konflikte }\end{array}$ & $\begin{array}{l}\text { Minimie- } \\
\text { rung Be- } \\
\text { einträchti- } \\
\text { gungen }\end{array}$ & $\begin{array}{l}\text { Erfolgs- } \\
\text { kontrolle }\end{array}$ \\
\hline \multicolumn{7}{|l|}{ Akteure } \\
\hline $\begin{array}{l}\text { Direkt Be- } \\
\text { troffene, } \\
\text { org. Interes- } \\
\text { sengruppen }\end{array}$ & $\begin{array}{l}\text { persönliche } \\
\text { Gespräche }\end{array}$ & $\begin{array}{l}\text { persönliche } \\
\text { Gespräche, } \\
\text { Arbeits- } \\
\text { gruppen }\end{array}$ & $\begin{array}{l}\text { Arbeits- } \\
\text { gruppen }\end{array}$ & $\begin{array}{l}\text { Arbeits- } \\
\text { gruppen }\end{array}$ & & $\begin{array}{l}\text { Arbeits- } \\
\text { gruppen }\end{array}$ \\
\hline $\begin{array}{l}\text { Mittelbar } \\
\text { betroffene } \\
\text { Bevölkerung }\end{array}$ & Workshop & $\begin{array}{l}\text { Umfrage, } \\
\text { Orientie- } \\
\text { rungsvers., } \\
\text { Exkursion, } \\
\text { Vertretung } \\
\text { in Arbeits- } \\
\text { gruppen }\end{array}$ & $\begin{array}{l}\text { Information, } \\
\text { (Orientie- } \\
\text { rungsvers.), } \\
\text { Vertretung in } \\
\text { Arbeits- } \\
\text { gruppen }\end{array}$ & $\begin{array}{l}\text { Vertretung in } \\
\text { Arbeits- } \\
\text { gruppen }\end{array}$ & $\begin{array}{l}\text { Arbeits- } \\
\text { gruppen, } \\
\text { freiwillige } \\
\text { Mitarbeit }\end{array}$ & $\begin{array}{l}\text { Lehrpfad, } \\
\text { Kurse, } \\
\text { Schau- } \\
\text { tafeln }\end{array}$ \\
\hline $\begin{array}{l}\text { Fachstellen } \\
\text { der Verwal- } \\
\text { tung }\end{array}$ & $\begin{array}{l}\text { persönliche } \\
\text { Kontakte }\end{array}$ & $\begin{array}{l}\text { Begleit- } \\
\text { gruppe }\end{array}$ & Konsultation & & & \\
\hline $\begin{array}{l}\text { allgemein } \\
\text { (alle } \\
\text { Akteure) }\end{array}$ & $\begin{array}{l}\text { Information, } \\
\text { (Orientie- } \\
\text { rungsvers.) }\end{array}$ & $\begin{array}{l}\text { Information } \\
\text { (Orientie- } \\
\text { rungsvers.) }\end{array}$ & $\begin{array}{l}\text { Information } \\
\text { (Orientie- } \\
\text { rungsvers.) }\end{array}$ & $\begin{array}{l}\text { Information } \\
\text { (Orientie- } \\
\text { rungsvers.) }\end{array}$ & $\begin{array}{l}\text { Informa- } \\
\text { tion, Bau- } \\
\text { stellenbe- } \\
\text { sichtigung }\end{array}$ & $\begin{array}{l}\text { Informa- } \\
\text { tion }\end{array}$ \\
\hline
\end{tabular}

Mit Inkrafttreten der Neugestaltung des Finanzausgleichs und der Aufgaben zwischen dem Bund und den Kantonen (NFA) am 1.1.2008 ist eine Aufwertung der Mitwirkungsmöglichkeiten in zweierlei Hinsicht vorgesehen (BAFU, 2007a; BAFU, 2007c):

- Die Mittelflüsse zwischen Bund und Kantonen werden nicht mehr verfügt, sondern im Rahmen von verwaltungsrechtlichen Verträgen zwischen Bund und Kanton vereinbart.

- Der Umfang der Bundesförderung für Einzelprojekte (Bundesbeitragssatz) hängt unter anderem auch vom Umfang und der Qualität des partizipativen Planungsprozesses ab (Tabelle 36).

Tabelle 36 Kriterien zur Beurteilung des partizipativen Planungsprozesses (BAFU, 2007a).

\begin{tabular}{lc}
\hline Kriterien zur Beurteilung des partizipativen Planungsprozesses & Punkte $^{*}$ \\
\hline Akteursanalyse zu Beginn des Projektes & $1 / 0$ \\
$\begin{array}{l}\text { Umfassende Information der Bevölkerung vor dem Auflageverfahren über Defi- } \\
\text { zite des Ist-Zustandes, Ziele und Massnahmen des Projektes }\end{array}$ & $1 / 0$ \\
$\begin{array}{l}\text { Zieldefinition unter Einbezug der Akteure } \\
\text { Diskussion Massnahmenvarianten und Handlungsspielraum mit Akteuren, die }\end{array}$ & $1 / 0$ \\
$\begin{array}{l}\text { grosse Betroffenheit und grosses Einflusspotenzial aufweisen } \\
\text { Total }\end{array}$ & $1 / 0$ \\
\hline * $1=\mathrm{JA}, 0=\mathrm{NEIN}$ & Max. 4 \\
\hline
\end{tabular}




\subsection{Erkenntnisse, Lücken und Handlungsbedarf}

\subsubsection{Erfassung von Schäden und Risiken}

Die Darlegungen zum Schadensbegriff und den verletzbaren Werten in Kapitel 2.2 bis 2.4 zeigen verschiedene Lücken und Handlungsbedarf zu Schutzzielen im Umgang mit Naturrisiken auf (Tabelle 37).

Tabelle 37 Erkenntnisse, Lücken und Handlungsbedarf zu Schäden und Risiken.

\begin{tabular}{|c|c|c|c|c|}
\hline Kap. & Thema & Erkenntnisse & Lücken & Handlungsbedarf \\
\hline \multirow[t]{2}{*}{2.2} & \multirow[t]{2}{*}{ Schadensbegriff } & $\begin{array}{l}\text { Ein Schaden ist ein } \\
\text { Effekt, der } \\
\text { - negativ bewertet } \\
\text { - relevant und } \\
\text { - erfassbar ist. }\end{array}$ & \multirow[t]{3}{*}{$\begin{array}{l}\text { Fehlen einer Praxis zum } \\
\text { expliziten Umgang mit } \\
\text { einer differenzierten und } \\
\text { subjektiven Bewertung } \\
\text { von Wert- und Rechts- } \\
\text { gutverletzungen. }\end{array}$} & \multirow{2}{*}{$\begin{array}{l}\text { Treffen von plausiblen } \\
\text { Annahmen zur allge- } \\
\text { meingültigen Bewertung } \\
\text { und Beurteilung der Re- } \\
\text { levanz von Schäden für } \\
\text { das regionale Manage- } \\
\text { ment. }\end{array}$} \\
\hline & & $\begin{array}{l}\text { Erfordernis der Allge- } \\
\text { meingültigkeit }\end{array}$ & & \\
\hline \multirow[t]{2}{*}{2.3} & \multirow[t]{2}{*}{ Verletzbare Werte } & $\begin{array}{l}\text { Verletzbare Werte/ } \\
\text { Rechtsgüter sind } \\
\text { - Leib und Leben, } \\
\text { - Sachwerte, } \\
\text { - gesellsch. Strukturen, } \\
\text { - Naturwerte und } \\
\text { - Lebensqualität. }\end{array}$ & & \\
\hline & & $\begin{array}{l}\text { Hohe Bewertung von } \\
\text { Leib und Leben, Sach- } \\
\text { werte, gesellsch. Struktu- } \\
\text { ren; tiefere Bewertung } \\
\text { von Naturwerten und } \\
\text { Lebensqualität. }\end{array}$ & $\begin{array}{l}\text { Keine klare und allge- } \\
\text { meinverbindliche Hierar- } \\
\text { chie unter den verletzba- } \\
\text { ren Werten. }\end{array}$ & $\begin{array}{l}\text { Vorschlag zur Festlegung } \\
\text { einer allgemeingültigen } \\
\text { Hierarchie unter den } \\
\text { verletzbaren Werten. }\end{array}$ \\
\hline \multirow[t]{4}{*}{2.4} & \multirow[t]{4}{*}{$\begin{array}{l}\text { Indikatoren und } \\
\text { Metriken }\end{array}$} & $\begin{array}{l}\text { Zahlreiche Indikatoren } \\
\text { und Metriken }\end{array}$ & \multirow{3}{*}{$\begin{array}{l}\text { Indikatoren zur umfas- } \\
\text { senden und vergleichen- } \\
\text { den Darstellung aller } \\
\text { verletzbaren Werte und } \\
\text { Schäden fehlen. }\end{array}$} & \multirow{3}{*}{$\begin{array}{l}\text { Vorschlag zu einer um- } \\
\text { fassenden und verglei- } \\
\text { chenden Darstellung } \\
\text { verletzbarer Werte mittels } \\
\text { eines Index. }\end{array}$} \\
\hline & & $\begin{array}{l}\text { Vergleichbarkeit der } \\
\text { Indikatoren gering }\end{array}$ & & \\
\hline & & $\begin{array}{l}\text { Monetarisierung begrenzt } \\
\text { anwendbar }\end{array}$ & & \\
\hline & & $\begin{array}{l}\text { Anzahl Todesfälle und } \\
\text { Geldeinheiten für Sach- } \\
\text { werte als einzige geeig- } \\
\text { nete Indikatoren. } \\
\text { Einzelne Arten von ver- } \\
\text { letzbaren Werten können } \\
\text { nicht mit Indikatoren und } \\
\text { Metriken beschrieben } \\
\text { werden. }\end{array}$ & $\begin{array}{l}\text { Indikatoren mit hoher } \\
\text { Eignung nur für wenig } \\
\text { Arten verletzbarer Werte. } \\
\text { Fehlende Indikatoren zur } \\
\text { Beschreibung der ver- } \\
\text { letzbaren Werte } \\
\text { - gesellsch. Strukturen, } \\
\text { - Naturwerte und } \\
\text { - Lebensqualität. }\end{array}$ & $\begin{array}{l}\text { Entwicklung geeigneter } \\
\text { Indikatoren zur ver- } \\
\text { gleichbaren Beschrei- } \\
\text { bung von Beeinträchti- } \\
\text { gungen gesellschaftlicher } \\
\text { Strukturen, Naturwerten } \\
\text { und Lebensqualität. }\end{array}$ \\
\hline
\end{tabular}

Im Rahmen der vorliegenden Publikation abgedeckter Handlungsbedarf.

Das Risiko wird als Produkt von Eintretenswahrscheinlichkeit und Schäden dargestellt. Als Schaden gilt die Beeinträchtigung eines Wertes oder eines Rechtsguts (Kapitel 2.2). 
Damit eine Beeinträchtigung von Werten als Schaden verstanden wird, muss sie

- negativ bewertet,

- relevant und

- methodisch erfassbar sein.

Das regionale Risikomanagement befasst sich oft gleichzeitig mit verschiedenen Arten und Quellen von Risiken sowie mit einer grossen Zahl von Betroffenen. Diese Ausgangslage ist für das Management von Naturrisiken typisch und erfordert, dass die negative Bewertung und die Beurteilung der Relevanz von Beeinträchtigungen allgemeingültig sind. Allgemeingültig negativ und relevant beurteilt werden Beeinträchtigungen nur bei einzelnen Arten von Werten, insbesondere Leib und Leben oder Naturwerten als Lebensgrundlage. Eine Beeinträchtigung anderer Werte wird dagegen meist differenziert und subjektiv beurteilt (Kapitel 2.3.3).

Die verletzbaren Werte lassen sich - kongruent mit den Rechtsgütern - in die fünf Gruppen Leib und Leben, Sachwerte, gesellschaftliche Strukturen, Naturwerte und Lebensqualität einteilen (Kapitel 2.3). Aus den strafrechtlichen Folgen von Rechtsgutverletzungen kann geschlossen werden, dass den verletzbaren Werten Leib und Leben, Vermögen und gesellschaftliche Strukturen eine höhere, den Umweltwerten und der Lebensqualität eine geringere Bedeutung zufällt (Kapitel 2.3.5).

Zur Beschreibung verletzbarer Werte und Schäden bieten sich zahlreiche Indikatoren an (Kapitel 2.4). Die Qualität und Eignung der Indikatoren ist unterschiedlich, im Allgemeinen jedoch ungenügend (Kapitel 2.4.2). Besonders oft sind Stichhaltigkeit, Verfügbarkeit und Objektivität der Indikatoren nicht gegeben. Als umfassend geeignet erweisen sich die Indikatoren ,Anzahl Todesfälle' und ,Geldwerte'. Diese Indikatoren werden in der Praxis am meisten verwendet. Oft begrenzen sich Risikoanalysen und Risikomanagement auf diese beiden Indikatoren. Zur Erfassung mehrerer Arten verletzbarer Werte, namentlich gesellschaftliche Strukturen, Naturwerte oder Lebensqualität, fehlen Indikatoren vollständig. Die Indikatoren sind in der Regel nicht untereinander vergleichbar. Werden verschiedene Risiken gleichzeitig und vergleichend betrachtet, ist eine vergleichbare Darstellung von Schäden und Risiken notwendig. Die Monetarisierung ist die für Vergleichszwecke am weitesten entwickelte Methode, jedoch ist auch diese nicht umfassend anwendbar (Kapitel 2.4.4).

Bei der Darstellung von Schäden und Risiken ergeben sich folgende wesentliche Lücken (Tabelle 37):

1. Im regionalen Risikomanagement besteht kein Ansatz zum expliziten Umgang mit einer subjektiven Bewertung von Wert- und Rechtsgutverletzungen.

2. Eine allgemein verbindliche Hierarchie unter den verletzbaren Werten fehlt.

3. Indikatoren zur umfassenden und vergleichenden Darstellung aller verletzbaren Werte und Schäden fehlen. Für einzelne Arten von verletzbaren Werten, namentlich gesellschaftliche Strukturen, Naturwerte und Lebensqualität, sind keine valablen Indikatoren bekannt.

4. Indikatoren mit hoher Eignung bestehen nur für Todesfälle und Sachschäden.

Zur Schliessung dieser Lücken besteht folgender Handlungsbedarf (Tabelle 37):

1. Es sind plausible Annahmen zur allgemeingültigen Bewertung und Beurteilung der Relevanz von Schäden für das regionale Risikomanagement zu treffen. Diesem Anspruch wird im Rahmen dieser Arbeit durch Typisierung von verletzbaren Werten Rechnung getragen (Objektindex, Kapitel 4.2.3.2).

2. Unterschiedliche Bedeutung verletzbarer Werte ist durch eine allgemeingültige Hierarchie auszudrücken. Zur Erfüllung dieses Anspruchs werden verletzbare Werte und Rechtsgüter mit der Hierarchie menschlicher Bedürfnisse korreliert (Kapitel 3.3.2.1). Die Operationalisierung erfolgt durch eine Typisierung der verletzbaren Werte (Objektindex, Kapitel 4.2.3.2).

Josef Th. Hess: Schutzziele im Umgang mit Naturrisiken in der Schweiz, @ vdf Hochschulverlag 2011 
3. Es ist eine Metrik für eine umfassende und vergleichbare Darstellung verletzbarer Werte zu entwickeln. Dies wird mit dem bereits erwähnten Objektindex erfüllt (Kapitel 4.2.3.2).

4. Es sind geeignete Indikatoren zur umfassenden und vergleichbaren Beschreibung aller relevanten verletzbaren Werte zu entwickeln. Diesem Anspruch wird im Rahmen der vorliegenden Publikation nicht Rechnung getragen. Die Indikatoren mit hoher Eignung werden in begrenztem Mass im Vorschlag eines schutzzielbasierten Risikomanagementkonzeptes eingesetzt (Kapitel 4.2.2.2).

\subsubsection{Tolerierbare Effekte}

Zum Thema tolerierbare Effekte bestehen zahlreiche wissenschaftliche Untersuchungen. Sie zeigen den Einfluss von Wahrnehmung, Nutzen und Wertesystemen auf (Kapitel 2.5). Grenzwerte wurden für einzelne Arten von Risiken und Indikatoren empirisch und mittels Berechnungen hergeleitet (Kapitel 2.6). Die Untersuchungen erfolgten vor allem für technische Risiken. Im Management von Naturrisiken werden diese Erkenntnisse nicht explizit angewendet. Angaben zu Grenzwerten im Umgang mit Naturrisiken wurden durch eigene Berechnungen anhand verschiedener Schutzzieldarstellungen ergänzt (Kapitel 2.6.2.2 und 2.6.5.2).

Tabelle 38 Erkenntnisse, Lücken und Handlungsbedarf zu tolerierbaren Effekten und Grenzwerten.

\begin{tabular}{|c|c|c|c|c|}
\hline Kap. & Thema & Erkenntnisse & Lücken & Handlungsbedarf \\
\hline 2.5 & $\begin{array}{l}\text { Tolerierbare } \\
\text { Effekte }\end{array}$ & $\begin{array}{l}\text { Wahrnehmung, Nutzen, } \\
\text { Wertesystem und andere } \\
\text { Risiken sind Einflussfak- } \\
\text { toren bei der Festlegung } \\
\text { und Abstufung tolerierba- } \\
\text { rer Effekte. }\end{array}$ & $\begin{array}{l}\text { Keine explizite Berück- } \\
\text { sichtigung der Einfluss- } \\
\text { faktoren im Management } \\
\text { von Naturrisiken. }\end{array}$ & $\begin{array}{l}\text { Konzept zur expliziten } \\
\text { Berücksichtigung von } \\
\text { allen relevanten Ein- } \\
\text { flussfaktoren. }\end{array}$ \\
\hline 2.6 . & $\begin{array}{l}\text { Grenzwerte } \\
\text { tolerierbarer } \\
\text { Risiken }\end{array}$ & $\begin{array}{l}\text { Grenzwerte für einzelne } \\
\text { Risikoarten und Indikato- } \\
\text { ren ergeben sich aus } \\
\text { empirischen Untersu- } \\
\text { chungen und Berechnun- } \\
\text { gen. } \\
\text { Werte für tolerierbare } \\
\text { Risiken liegen bei Natur- } \\
\text { risiken deutlich höher als } \\
\text { bei anderen Risiken. }\end{array}$ & $\begin{array}{l}\text { Fehlende Angaben zu } \\
\text { Grenzwerten für viele } \\
\text { Arten von Risiken. }\end{array}$ & $\begin{array}{l}\text { Herleitung von } \\
\text { Grenzwerten zu Risi- } \\
\text { ken für gesellschaftli- } \\
\text { che Strukturen, Na- } \\
\text { turwerte und Lebens- } \\
\text { qualität. } \\
\text { Berücksichtigung } \\
\text { bekannter Grenzwer- } \\
\text { te in einem schutz- } \\
\text { zielbasierten Konzept } \\
\text { zum Management } \\
\text { von Naturrisiken. }\end{array}$ \\
\hline & & $\begin{array}{l}\text { Aus Schutzzielmatrizes } \\
\text { berechnete Grenzwerte } \\
\text { weichen teilweise von } \\
\text { Grenzwerten in Literatur- } \\
\text { angaben ab. }\end{array}$ & $\begin{array}{l}\text { Fehlender Bezug beste- } \\
\text { hender Schutzzielmatri- } \\
\text { zes zu quantitativen Lite- } \\
\text { raturangaben über tole- } \\
\text { rierbare Risiken. }\end{array}$ & $\begin{array}{l}\text { Berücksichtigung von } \\
\text { Risikoberechnungen. } \\
\text { Anpassung beste- } \\
\text { hender Schutzziel- } \\
\text { matrizes. }\end{array}$ \\
\hline
\end{tabular}

Im Rahmen der vorliegenden Publikation abgedeckter Handlungsbedarf. 
Bezüglich tolerierbarer Effekte und Grenzwerte sind für das Management von Naturrisiken folgende Lücken relevant (Tabelle 38):

5. Einflussfaktoren wie Wahrnehmung, Nutzen, Wertesystem und andere Risiken sind bei der Festlegung tolerierbarer Effekte nicht explizit und nachvollziehbar berücksichtigt.

6. Für viele Arten von Risiken fehlen Angaben zu tolerierbaren Effekten und Grenzwerten.

7. Berechnungen tolerierbarer Risiken anhand bestehender Schutzzielmatrizes im Management von Naturrisiken ergeben Unterschiede und Abweichungen zu Grenzwerten in der Literatur.

Zur Schliessung dieser Lücken besteht folgender Handlungsbedarf (Tabelle 38):

5. Alle relevanten Aspekte bei der Bemessung von Schutzzielen und der Festlegung von Prioritäten sind zu berücksichtigen. Diese Thematik wird durch Analyse und Herleitung rechtlicher, gerechtigkeitstheoretischer und ethischer Aspekte zur Festlegung der Tolerierbarkeit abgehandelt (Kapitel 3). Die Operationalisierung erfolgt im Rahmen eines schutzzielbasierten Konzeptes zum Management von Naturrisiken (Kapitel 4, insbesondere Objektindex, Kapitel 4.2.3.2, und Massnahmenindex, Kapitel 4.2.3.3).

6. Fehlende Grenzwerte sind herzuleiten. Dieser Aspekt wird in der vorliegenden Publikation nicht berücksichtigt. Die bekannten Grenzwerte werden in ein Konzept zum Management von Naturrisiken integriert (Kapitel 4, insbesondere 4.2.2.2).

7. Risikoberechnungen sind im Management von Naturrisiken zu berücksichtigen. Mit der Entwicklung eines Gefährdungsfaktors im Managementkonzept wird diese Lücke geschlossen (Kapitel 4.2.3.4). Bestehende Schutzzielmatrizes sind aufgrund von Berechnungen zu tolerierbaren Risiken zu modifizieren (Kapitel 4.4).

\subsubsection{Schutzziele}

Die pragmatisch und isoliert für einzelne Sicherheitsfragen entwickelten Schutzzielsysteme sind kaum vergleichbar. Sie basieren auf verschiedenen Indikatoren, verschiedenen Bezugsgrössen, verschiedenen Methoden usw. Viele Schutzzielkonzepte sind prozess- oder risikospezifisch.

Tabelle 39 Erkenntnisse, Lücken und Handlungsbedarf zu Schutzzielen.

\begin{tabular}{lllll}
\hline Kap. & Thema & Erkenntnisse & Lücken & Handlungsbedarf \\
\hline 2.7. & Schutzzielkonzepte & In der Praxis ist eine & Fehlen von Homogenität & Entwicklung eines konsi- \\
& grosse Zahl verschiede- & und Konsistenz zwischen & stenten, schutzzielbasier- \\
& ner Schutzzielkonzepte & den Schutzzielkonzepten. & ten Risikomanagement- \\
& vorhanden. & & konzeptes. \\
& Die Festlegung der Si- & Fehlen einer wissen- & Analyse von Grundlagen \\
& cherheitsstandards im & schaftlichen Basis für die & und Prinzipien zur Fest- \\
& Management von Natur- & Festlegung und Differen- & legung und Differenzie- \\
& risiken erfolgt pragma- & zierung von Sicherheits- & rung von Schutzzielen. \\
& tisch. & standards. & Umsetzung dieser Prinzi- \\
& & & pien in einem Risikoma- \\
& & & nagementkonzept.
\end{tabular}

Im Rahmen der vorliegenden Publikation abgedeckter Handlungsbedarf.

Bezüglich der Schutzziele sind für das Management von Naturrisiken folgende Lücken relevant (Tabelle 39): 
8. Die verschiedenen Schutzzielkonzepte sind inhomogen und inkonsistent.

9. Eine nachvollziehbare und explizite Basis zur Festlegung der Schutzziele fehlt.

Zur Schliessung dieser Lücken besteht folgender Handlungsbedarf (Tabelle 39):

10. Ein konsistentes Risikomanagementkonzept ist zu entwickeln. In Kapitel 4 wird ein entsprechendes Konzept vorgeschlagen.

11. Vgl. Handlungsbedarf Nr. 5.

\subsubsection{Operabilität}

Die Operabilität ist grundsätzlich begrenzt durch bereits erwähnte Lücken bei der Erfassung von Schäden und Risiken (Kapitel 2.4). Hinzu kommen lückenhafte Datengrundlagen zu Effekten von Gefahrenprozessen wie gefahrenprozess- und intensitätsspezifische Ausmasse von Personen- oder Sachschäden. Ferner sind allgemeingültige Informationen zur Wirksamkeit von Massnahmen lückenhaft (Kapitel 2.8.2). Dies führt dazu, dass effizienzorientierte Schutzzielkonzepte auf regionaler Ebene fehlen (Kapitel 2.8.3).

Tabelle 40 Erkenntnisse, Lücken und Handlungsbedarf zur Operabilität.

\begin{tabular}{|c|c|c|c|c|}
\hline Kap. & Thema & Erkenntnisse & Lücken & Handlungsbedarf \\
\hline \multirow[t]{3}{*}{2.8} & $\begin{array}{l}\text { Operationale } \\
\text { Aspekte }\end{array}$ & $\begin{array}{l}\text { Die Datengrundlagen zu } \\
\text { Effekten von Gefahren- } \\
\text { prozessen sind lücken- } \\
\text { haft. }\end{array}$ & $\begin{array}{l}\text { Ungenügende Kenntnisse } \\
\text { über von Gefahrenpro- } \\
\text { zessen bewirkte Effekte. }\end{array}$ & $\begin{array}{l}\text { Untersuchungen zum } \\
\text { gefahrenprozess- } \\
\text { und intensitätsspezi- } \\
\text { fischen Ausmass von } \\
\text { Personen- und } \\
\text { Sachschäden. }\end{array}$ \\
\hline & & $\begin{array}{l}\text { Die Informationen zur } \\
\text { Wirksamkeit von Mass- } \\
\text { nahmen sind ungenau } \\
\text { und unvollständig. }\end{array}$ & $\begin{array}{l}\text { Ungenügende Kenntnisse } \\
\text { zur Wirksamkeit von } \\
\text { Risikoverminderungs- } \\
\text { massnahmen. }\end{array}$ & $\begin{array}{l}\text { Analyse der Wirk- } \\
\text { samkeit von Risiko- } \\
\text { verminderungsmass- } \\
\text { nahmen. }\end{array}$ \\
\hline & & $\begin{array}{l}\text { Effizienzorientierte } \\
\text { Schutzziele erfordern } \\
\text { viele einzelfallspezifi- } \\
\text { sche Detailinformatio- } \\
\text { nen. }\end{array}$ & $\begin{array}{l}\text { Fehlen von effizienzorien- } \\
\text { tierten und zugleich regi- } \\
\text { onal einsetzbaren } \\
\text { Schutzzielkonzepten. }\end{array}$ & $\begin{array}{l}\text { Vorschlag zu einer } \\
\text { allgemeingültigen } \\
\text { Typisierung der } \\
\text { Wirksamkeit von } \\
\text { Massnahmen. }\end{array}$ \\
\hline
\end{tabular}

Im Rahmen der vorliegenden Publikation abgedeckter Handlungsbedarf.

Bezüglich operationaler Aspekte bestehen im Management von Naturrisiken vor allem folgende Lücken (Tabelle 40):

12. Die durch Gefahrenprozesse bewirkten Schäden sind nur lückenhaft bekannt. Insbesondere fehlen Informationen über den Einfluss von Art und Intensität der Prozesse und über den Einfluss der Beschaffenheit des betroffenen Schadenspotenzials.

13. Die Wirksamkeit und die Erfolgsfaktoren von Risikoverminderungsmassnahmen sind zu wenig bekannt.

14. Auf Effizienz ausgerichtete Schutzzielkonzepte, die regional einsetzbar sind, fehlen. 
Zur Schliessung dieser Lücken besteht folgender Handlungsbedarf (Tabelle 40):

15. Zur Verbesserung der Risikoabschätzung, aber auch zur Anwendung von Schutzzielen sind vertiefte Kenntnisse über das spezifische Schadensausmass erforderlich, das durch Gefahrenprozesse an bestimmten Risikoobjekten hervorgerufen wird.

16. Die Wirksamkeit und deren Einflussfaktoren sind für alle wichtigen Risikoverminderungsmassnahmen vertieft zu analysieren

17. Es ist ein Vorschlag für effizienzorientierte und regional einsetzbare Schutzzielkonzepte zu entwickeln. Hierzu ist vor allem eine allgemeingültige Typisierung der Wirksamkeit von Massnahmen erforderlich. Diese Zielsetzung wird mit der Entwicklung eines schutzzielbasierten Konzeptes angegangen (sog. Massnahmenindex, Kapitel 4.2.3.3).

\subsubsection{Organisation und Verfahren zur Festlegung tolerierbarer Risiken}

Finanziell wird das Management von Naturrisiken in der Schweiz zu etwas mehr als der Hälfte von Privaten, im Übrigen vom Staat getragen. Das Engagement der Privaten besteht wiederum fast zur Hälfte in der Leistung von Versicherungsprämien. Zahlreiche staatliche Stellen und Versicherungsgesellschaften sind im Risikomanagement tätig. Deren Aktivitäten sind wenig koordiniert und inputgesteuert. Am weitesten entwickelt ist die Koordination im Bereich der staatlichen Präventionsmassnahmen. In diesem Bereich bestehen einheitliche Schutzziele (Kapitel 2.9.1). Strukturen und Entscheidverfahren sind bürokratisch geprägt, da Risikoverminderungsmassnahmen meist öffentliche Güter darstellen und erheblichen Koordinationsaufwand erfordern (Kapitel 2.9.2). Zur Entscheidungsfindung über Risikoverminderungsmassnahmen bestehen hoch entwickelte Verfahren. Diese haben sich im praktischen Risikomanagement nicht etabliert (Kapitel 2.9.3 und 2.9.4).

Tabelle 41 Erkenntnisse, Lücken und Handlungsbedarf zu Organisation und Verfahren bei der Festlegung von Schutzzielen im Management von Naturrisiken.

\begin{tabular}{|c|c|c|c|c|}
\hline Kap. & Thema & Erkenntnisse & Lücken & Handlungsbedarf \\
\hline 2.9 . & $\begin{array}{l}\text { Organisati- } \\
\text { onsstrukturen } \\
\text { und Verfahren } \\
\text { zur Festle- } \\
\text { gung tolerier- } \\
\text { barer Risiken }\end{array}$ & $\begin{array}{l}\text { Die Trennung des stra- } \\
\text { tegischen (Bund, Kanto- } \\
\text { ne) vom operativen } \\
\text { (Gemeinden, Private) } \\
\text { Risikomanagement ist } \\
\text { wenig entwickelt. } \\
\text { Die Finanzierung von } \\
\text { Risikoverminderungs- } \\
\text { massnahmen erfolgt je } \\
\text { zur Hälfte durch Private } \\
\text { und den Staat. Die Fi- } \\
\text { nanzierung erfolgt input- } \\
\text { orientiert. }\end{array}$ & $\begin{array}{l}\text { Fehlen konsistenter } \\
\text { Strategien und einer } \\
\text { klaren Trennung } \\
\text { zwischen strategi- } \\
\text { schem und operati- } \\
\text { vem Risikomana- } \\
\text { gement. } \\
\text { Fehlende Koordina- } \\
\text { tion der Risikover- } \\
\text { minderungsmass- } \\
\text { nahmen. } \\
\text { Inputorientierte } \\
\text { Förderung von Risi- } \\
\text { koverminderungs- } \\
\text { massnahmen. }\end{array}$ & $\begin{array}{l}\text { Entwicklung klarer, koordi- } \\
\text { nierter Strategien im Risi- } \\
\text { komanagement mit konse- } \\
\text { quenter Trennung zwi- } \\
\text { schen strategischem und } \\
\text { operativem Handeln. }\end{array}$ \\
\hline
\end{tabular}




\begin{tabular}{lll}
\hline $\begin{array}{l}\text { Infolge hohem Koordina- } \\
\text { tionsbedarf haben sich } \\
\text { bürokratische Strukturen } \\
\text { etabliert. }\end{array}$ & $\begin{array}{l}\text { Fehlende Kontroll- } \\
\text { mechanismen zur } \\
\text { Überwindung büro- } \\
\text { kratischer Ineffi- } \\
\text { zienz. }\end{array}$ \\
$\begin{array}{lll}\text { Es bestehen gut entwi- } \\
\text { ckelte Methoden für } \\
\text { partizipative Entscheid- } \\
\text { verfahren. }\end{array}$ & $\begin{array}{l}\text { Ungenügende An- } \\
\text { wendung der Metho- } \\
\text { von Naturrisiken. }\end{array}$ & \begin{tabular}{l} 
Applikation der Verfahren \\
in einem schutzzielbasier- \\
\hline
\end{tabular}
\end{tabular}

Im Rahmen der vorliegenden Publikation abgedeckter Handlungsbedarf.

Bezüglich Organisation und Verfahren sind für das Management von Naturrisiken folgende Lücken relevant (Tabelle 41):

18. Über verschiedene Akteure und Handlungsbereiche hinweg konsistente und koordinierte Strategien des Managements von Naturrisiken fehlen.

19. Outputorientierte Steuerung und Förderung von Risikoverminderungsmassnahmen fehlen.

20. Verfahren zur partizipativen Entscheidungsfindung sind wenig etabliert.

Zur Schliessung der Lücken besteht folgender Handlungsbedarf (Tabelle 41):

21. Eine klare, über verschiedene Akteure und Handlungsbereiche hinweg koordinierte Strategie ist zu entwickeln

22. Methoden für eine konsequent outputorientierte Steuerung und Förderung des Risikomanagements sind zu entwickeln. Diese Zielsetzung setzt im Wesentlichen die Abdeckung des in Ziffer 10 und 11 umschriebenen Handlungsbedarfs voraus.

23. Vorhandene Methoden der partizipativen Entscheidfindung im Risikomanagement sind konsequent umzusetzen. 


\section{Aspekte zur Festlegung der Tolerierbarkeit}

\section{1 Übersicht}

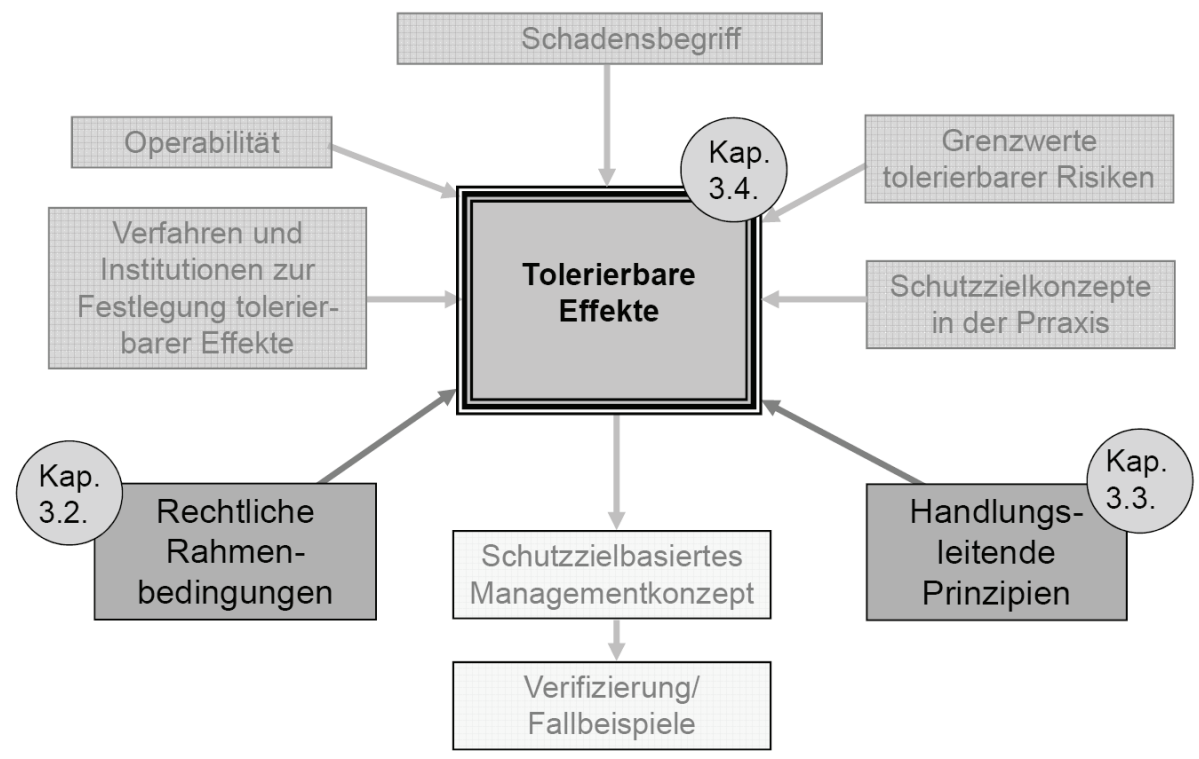

Abbildung 35 Kapitel 3 in der Übersicht.

In Kapitel 3 werden Rechtsordnung, Rechtsprechung und Rechtslehre hinsichtlich Aussagen zur Festlegung von Schutzzielen analysiert (Kapitel 3.2). Weiter werden gerechtigkeitstheoretische Prinzipien zur Bereitstellung und Verteilung von Gütern aufgezeigt und hinsichtlich des Gutes ,Sicherheit' analysiert (Kapitel 3.3). Abschliessend werden die aufgezeigten Aspekte in einer Synthese zusammengefasst (Kapitel 3.4).

\subsection{Rechtliche Rahmenbedingungen}

\subsubsection{Schutzziele im Recht}

Das Recht regelt die Beziehungen zwischen den Mitgliedern der Gesellschaft und zwischen diesen und dem Staat. In den Beziehungen der Mitglieder einer Gesellschaft untereinander können Schutzziele insofern eine Rolle spielen, als dass sie festlegen, in welchem Umfang jemand Drittpersonen gefährden darf. Der Staat nimmt seine Rolle wahr, indem er als Gesetzgeber festlegt oder in der Rechtsprechung beurteilt, ob und bis zu welchem Mass solche gegenseitigen Gefährdungen zulässig sind. Diese Sichtweise ist vor allem im technischen Sicherheitsrecht, im Haftpflicht- und im Strafrecht zentral.

Eine Naturgefahr stellt nach dem allgemeinen Verständnis eine höhere Gewalt dar, die nicht oder zumindest nicht direkt durch menschliches Handeln geschaffen wurde. Wer sich einer Naturgefahr aussetzt und diese damit zum Risiko werden lässt, gefährdet in erster Linie sich selbst. Selbstgefährdung bedarf grundsätzlich keiner rechtlichen Regelung. Allerdings besteht gerade im Umgang mit Naturgefahren die Erwartung an den Staat, für Sicherheit zu sorgen, da Naturgefahren oft wichtige Rechtsgüter betreffen und für die einzelnen Betroffenen oft nicht erkenn- und berechenbar sind. 
Die Schutzziele im Sinne dieser Publikation werden daher als Grenze zwischen dem Verantwortungsbereich der Allgemeinheit und dem Verantwortungsbereich des Individuums verstanden. Sie umschreiben, ob und in welchem Umfang einerseits eine Handlungspflicht der Allgemeinheit besteht und wie weit anderseits eine risikobetroffene Person selber für die Verminderung von Risiken zu sorgen hat. Grundsätzlich bestehen Zweifel, ob und wie weit es möglich ist, allgemeingültige Schutzziele zu definieren und festzulegen (Seiler, 1995a und 1995b; Abrahamsen, 2008).

\subsubsection{Rechtsordnung}

Obwohl für das Management von Naturrisiken von zentraler Bedeutung, sind weder in der Verfassung noch in Gesetzen oder Verordnungen des Bundes und der Kantone Schutzziele formuliert. Schutzzielrelevant sind im weitesten Sinne Artikel 19 des schweizerischen Waldgesetzes (WaG, SR 921.0) und Artikel 1 des schweizerischen Wasserbaugesetzes (WBG, SR 721.100). Darin werden die staatlichen Pflichten und Massnahmen auf den Schutz von Menschen und erheblichen Sachwerten begrenzt. Auf der Regelungsstufe von Ausführungsbestimmungen, Richtlinien, Empfehlungen und Weisungen des Bundes und der Kantone liegen detaillierte Aussagen zu Schutzzielen vor.

Gemäss der Bundesverfassung ist Sicherheit ein Staatszweck (BV Art. 2, SR 101) und eine Staatsaufgabe (BV Art. 57). Die Verfassung garantiert verschiedene Grundrechte für das Individuum (insbesondere Leben, Freiheit, körperliche Unversehrtheit) und dessen Handlungsmöglichkeiten (Niederlassungsfreiheit, Eigentumsgarantie, Wirtschaftsfreiheit; BV Art. 7 bis 36). Ferner sollen gewisse soziale Standards (Sozialziele) angestrebt werden (BV Art. 41). Aus den Grundrechten lassen sich - im Gegensatz zu den Sozialzielen - unmittelbare Ansprüche auf staatliche Leistungen (Massnahmen zum Schutz der Grundrechte) ableiten. Neben Aufgaben und Verantwortlichkeiten der Allgemeinheit betont die Bundesverfassung auch eine Eigenverantwortung des Individuums für sich und für die Gesellschaft (BV Art. 6). Ferner richten sich der Umfang und die Gestaltung der staatlichen Aufgaben und Verantwortlichkeiten - somit auch der Umgang der Allgemeinheit mit Naturrisiken - nach den rechtsstaatlichen Prinzipien der Gesetzmässigkeit, der Wahrung öffentlicher Interessen, der Verhältnismässigkeit, der Rechtsgleichheit sowie Treu und Glauben (BV Art. 5 und 8).

Unter den bundesgesetzlichen Regelungen fällt Art. 19 des Waldgesetzes (WaG, SR 921.0) auf, der der Allgemeinheit eine eigentliche Pflicht zum präventiven Handeln zuweist. Als Schutzgut gemäss den formellen Bundesgesetzen und Verordnungen gelten Menschen und erhebliche Sachwerte. Aber auch die natürliche Umwelt und die Lebensgrundlagen werden zu den geschützten Rechtsgütern gezählt (Kapitel 2.3.4 und Tabelle 4).

Verschiedene Gesetze, namentlich das Waldgesetz und das Wasserbaugesetz (WBG, SR 721.100), verpflichten den Staat, Beiträge an Massnahmen zum Schutz der Menschen und erheblicher Sachwerte vor Naturgefahren zu leisten. Durch die Allgemeinheit zu tragende Präventionsmassnahmen sind auf die Gefahrenprozesse Lawine, Rutschung, Erosion, Steinschlag und Hochwasser begrenzt. Eine entsprechende Regelung fehlt beispielsweise für Erdbeben. Die staatlichen Leistungen setzen in der Regel Eigenleistungen der Betroffenen voraus (WaG, Art. 35 Abs. 2). Sie beschränken sich auf bestehende, zur Zeit ihrer Entstehung für den Betroffenen nicht erkennbare Risiken. Wenn die Gefährlichkeit eines Gebietes bekannt ist, können Massnahmen zur Verminderung von Risiken, die dort nach Erkennen der Gefahr geschaffen wurden, nicht mehr mit öffentlichen Beiträgen unterstützt werden (WaV Art. 42 Abs. 4, SR 921.01; WBV Art. 1 Abs. 2, SR 721.100.1).

Zur Bewältigung ausserordentlicher Lagen sind dem Bund und den Kantonen im Bundesgesetz über den Bevölkerungsschutz und den Zivilschutz weitreichende Aufgaben zum Schutz der Bevölkerung und ihrer Lebensgrundlagen übertragen. Diese umfassen Information, Warnung, Alarmierung sowie die Erteilung von Verhaltensanweisungen (BZG Art. 4 bis 6, SR 520.1). Ausdrückliche und konkrete Schutzziele sind dazu nicht formuliert. 
Die Wiederherstellung nach Schäden ist meist Sache der Betroffenen. Für Personenschäden sieht die Bundesgesetzgebung in den Gesetzen zur Kranken- und Unfallversicherung eine Versicherungspflicht vor (KVG Art. 1a, SR 832.10; UVG Art. 1a, SR 832.20).

Detaillierte und rechtswirksame Schutzziele sind auf Bundesebene in Weisungen und Anleitungen festgelegt (Borter, 1999; BWG, 2001; Kapitel 2.7.6., Abbildung 26 und Abbildung 27. Implizit sind Schutzziele auch in Regelungen zur Raumnutzung in Gefahrengebieten (BFF, 1984; BWW, BUWAL, BRP, 1997) enthalten.

In den kantonalen Rechtsnormen ist die Zuordnung der Verantwortlichkeit in der präventiven Risikominderung heterogen geregelt. Sie reicht von umfassender Verantwortlichkeit des Staates bis zu vollständiger Eigenverantwortung der Betroffenen. Faktisch ergibt sich eine Vereinheitlichung, da im Fall der Verantwortlichkeit des Gemeinwesens die Betroffenen zumeist zu Eigenleistungen herangezogen werden. Im Fall der Verantwortlichkeit von Betroffenen erfolgen wiederum Abgeltungen der Öffentlichkeit. Schutzziele sind im kantonalen Recht - wenn überhaupt - in starker Anlehnung an die Bundesregeln formuliert oder direkt von den Bundesregeln übernommen. Der Interventionsbereich ist auch im kantonalen Recht - wie schon in der Bundesgesetzgebung - durch ein hohes Mass an staatlicher Verantwortlichkeit geprägt.

Im Bereich der Wiederherstellung kennt das Recht in einigen Kantonen im Vergleich zur Bundesgesetzgebung weitergehende Regelungen. Die Wiederherstellung fällt in die Verantwortlichkeit der Betroffenen, ebenso die Deckung und Behebung von nicht versicherbaren Elementarschäden. Die kantonalen Gesetze formulieren nahezu flächendeckend eine Versicherungspflicht, mindestens für Gebäude und teilweise für Fahrhabe. Für nicht versicherbare Elementarschäden bestehen in einzelnen Kantonen Hilfsfonds oder Elementarschadenskassen, die zumeist nicht dem Staat, sondern einer öffentlich-rechtlichen Anstalt (Sachversicherung) angegliedert sind. Direkte staatliche Hilfe für den Wiederaufbau ist in einigen kantonalen Gesetzen vorgesehen, jedoch nur als Nothilfe bei Erschöpfung der übrigen Mittel.

Nebst den gesetzlichen Vorgaben sind Normen und Merkblätter technischer Vereinigungen, etwa die SIA-Tragwerksnormen (SIA 261, 2003a und 2003b), schutzzielrelevant. In diesem Zusammenhang ist das Merkblatt 2018 ,Überprüfung bestehender Gebäude bezüglich Erdbeben' (SIA, 2004) erwähnenswert, das nach Bedeutung der Gebäude und Gebrauchsdauer abgestufte Schutzziele sowie Sicherheitsgrenzwerte und -grenzkosten enthält (Kapitel 2.7.4, Abbildung 23).

Detaillierte Ergebnisse einer Analyse von Rechtsnormen des Bundes und mehrerer Kantone finden sich im Anhang VI.

\subsubsection{Rechtsprechung}

Hinsichtlich der Schutzziele interessiert die Rechtsprechung zur Frage, ob und in welchem Umfang eine Handlungspflicht zur Verminderung oder Beseitigung eines Risikos besteht. Typischerweise stellt sich diese Frage bei Haftungs- oder in Strafrechtsfällen, d. h. nach erfolgtem Schadenseintritt. Vereinzelt wird die Frage auch in der gerichtlichen Auseinandersetzung mit Bewilligungs- und Planungsfragen angesprochen.

Im Umgang mit Naturrisiken fehlen Haftungsnormen. Zumeist ist der Staat Träger einer Handlungspflicht im Umgang mit Naturrisiken. Für hoheitliches Verhalten des Staats oder seiner Beamten und Angestellten besteht eine öffentlich-rechtliche Staatshaftung (Seiler, 1997). Von besonderem Interesse und Bedeutung in der Rechtsprechung zu Schutzzielen bei Naturrisiken sind daher Staatshaftungsfälle.

Das Haftpflichtrecht hat eine andere Zielsetzung und Optik hat als das präventive Recht. Vor allem der im Haftpflicht- und insbesondere im Strafrecht wichtige Gesichtspunkt der Vorhersehbarkeit im Einzelfall hat im Präventionsrecht nicht die gleiche Bedeutung. Im Präventions- 
recht kann nicht massgebend sein, ob eine bestimmte Person subjektiv die Gefahr voraussieht, sondern ob eine solche nach weitgehend objektiver Betrachtung besteht. Die haftpflichtrechtliche Rechtsprechung kann nur mit Vorsicht herangezogen werden, um induktiv zu erschliessen, was nach rechtlicher Auffassung als zulässig gelten kann.

Aus der Analyse einer Reihe von Urteilen des Bundesgerichts und kantonaler Gerichte ergeben sich keine ausdrücklichen und allgemeingültigen Hinweise dazu, welche Risiken tolerierbar sind. Die Rechtsprechung anerkennt nebst den Pflichten eines Sicherheitsverantwortlichen auch eine Eigenverantwortung des Betroffenen. Die Eigenverantwortung wird unterstrichen durch das Erfordernis einer durchschnittlich üblichen Sorgfalt des Betroffenen sowie durch die Berücksichtigung des Selbstverschuldens in Haftungsfragen. Höhere Anforderungen an den Sicherheitsverantwortlichen bestehen, wenn Risiken für die Betroffenen schwer erkennbar und schwer vermeidbar sind (EIm, Signalisation Skipiste, BGE 115 IV 189, E. 3b; Melchsee-Frutt, Gefahren auf Nebenflächen, BGE 122 IV 193 E. 2a; Grotte de la Baume de Bournois, Sturz in Höhleneingang, VGE 21240 E. 2e; Thun, Hochwasser, VGE 21657 E. 3.4) oder die Betroffenen besonders schutzbedürftig sind (Königsfelden, spitalärztliche Tätigkeit, BGE 120 lb 411, E. 4b).

Die Voraussetzung einer Handlungspflicht ist eine Norm, die den Sicherheitsverantwortlichen zum Handeln verpflichtet. Es besteht keine allgemeine Rechtspflicht, im Interesse anderer tätig zu werden (Thun, Hochwasser, VGE 21657 E. 3.3; VGE 21240 E. 2d). Wo besondere gesetzliche Normen fehlen, kann auf Regeln privater oder halbprivater Vereinigungen abgestellt werden, sofern diese allgemein anerkannt sind (Rupperswil, Reitunfall, BGE 127 IV 62, E. 2d; Elm, Lawine auf Skipiste, BGE 117 IV 415, E. 5a).

Der Umfang der Schutzpflicht wird durch die faktisch begrenzten Mittel des Staates beschränkt (Liestal, Schiesslärm, BGE 126 II 300 E. 5c). Nicht jedes Risiko begründet eine Handlungspflicht (Frasnacht, Verkehrsunfall, BGE 80 IV 130, E. 1). Die Rechtsprechung hält - wie die Rechtsordnung - im Grundsatz fest, dass Sicherheitsmassnahmen wirtschaftlich und technisch vernünftig und finanziell zumutbar sein müssen (Mörel, vereiste Strasse, BGE 98 II 40, E. 1; Genf-Cointrin, Baustellenunfall, BGE 90 IV 8, E. 2; Churwalden, Bäume am Pistenrand, BGE 121 III 358 E. 4a; Otelfingen, überlaufendes Gewässer, BGE 91 II 474 E. 5; Grotte de la Baume de Bournois, Sturz in Höhleneingang, VGE 21240 E. 2e; Thun, Hochwasser, VGE 21657 E. 3.4). Die Rechtsprechung weicht allerdings in verschiedenen Fällen von diesem Grundsatz ab, indem sie:

- der Einhaltung reglementarischer Sicherheitsmassnahmen - ungeachtet einer wirtschaftlichen Beurteilung - vorrangige Bedeutung zumisst;

- bei konkreter Vorhersehbarkeit eines (an sich sehr seltenen) Ereignisses hohe Massstäbe an Sicherheitsmassnahmen setzt (Bern, Zumutbarkeit einer Sitzwache BGE 4P.244/2005 E. 4; Seiler, 2006);

- die Anforderungen an Schutzmassnahmen von der Schutzbedürftigkeit der Betroffenen abhängig macht (Grotte de la Baume de Bournois, Sturz in Höhleneingang, VGE 21240 E. 2e; Thun, Hochwasser, VGE 21657 E. 3.4; Königsfelden, spitalärztliche Tätigkeit, BGE 120 lb 411, E. 4b) sowie

- allgemeine Risikoüberlegungen implizit, teilweise sogar explizit ablehnt (Bern, Zumutbarkeit einer Sitzwache BGE 4P.244/2005 E. 4; Zimmermann, 2006).

Während Rechtsordnung und Verwaltung möglichst objektiv vergleichbare Standards für tolerierbare Risiken anstreben, rückt die Rechtsprechung eher die besonderen Umstände und Verhältnisse des Einzelfalles ins Zentrum. Eine detaillierte Analyse von Urteilen des Bundesgerichtes und kantonaler Gerichte ist im Anhang VII angefügt. 


\subsubsection{Rechtslehre}

\subsubsection{Lehre der grundrechtlichen Schutzpflichten}

Die Lehre der grundrechtlichen Schutzpflicht befasst sich mit der Pflicht des Staats, den Bürger vor Eingriffen in verfassungsrechtlich geschützte Rechtsgüter durch Mitbürger oder durch Gefahrenquellen zu schützen (Sobotich, 2000; BBI 1997 I 191). Solche Gefahrenquellen sind auch Naturkräfte (Egli, 2002). Der Staat hat gemäss dieser Lehre nicht nur Eingriffe in die Grundrechte der Bürger zu unterlassen, sondern ist zu einem positiven Tun verpflichtet. Er soll dafür sorgen, dass die Grundrechte besser geschützt werden (Massnahmen zum Schutz des Lebens und der Gesundheit sowie der Persönlichkeitsrechte). Nach der Mehrheit der Lehre entspricht der grundrechtlichen Schutzpflicht des Staats auch ein subjektiver Anspruch des Einzelnen auf Schutz.

Ob und in welchem Mass der Staat die Grundrechte schützen muss, hängt von der Art des betroffenen Rechts und der Schwere der Beeinträchtigung ab. Während Gefahren (im rechtlichen Sinn) abgewehrt werden müssen, müssen Risiken nur im Rahmen der Vorsorge und nur soweit zumutbar reduziert werden (Seiler, 2001). Die Grenze zwischen rechtlich erheblicher Gefahr und irrelevanter Belästigung kann dabei nicht überzeugend gezogen werden (Egli, 2002). Staatliche Intervention ist umso dringender angezeigt, je unmittelbarer und konkreter die Gefahr ist (Müller, 1999).

Entscheidend ist zudem die Art der Gefahrenquelle. Bei Naturgefahren setzen die Prognosemöglichkeit von Ereignissen und die Realisierbarkeit von Schutzmassnahmen dem staatlichen Handeln Grenzen. Weiter ist die Schutzpflicht beeinflusst vom tatsächlich Möglichen, insbesondere von den faktisch begrenzten Mitteln des Staats (Egli, 2002). Es darf nichts gefordert werden, das dem Staat tatsächlich unmöglich ist.

Geht die Gefährdung vom Grundrechtsträger aus (Selbstgefährdung), sind der Handlungspflicht des Staats ebenfalls Grenzen gesetzt (Egli, 2002), da die Freiheitsrechte des Grundrechtsträgers zu wahren sind. In diesem Zusammenhang stellt sich die Frage der Ermächtigung oder Verpflichtung zu polizeilichem Handeln. Nach klassischem Polizeirecht gilt das Opportunitätsprinzip. Dieser Grundsatz bedeutet, dass die Behörden zu polizeilichem Handeln nur ermächtigt, jedoch nicht verpflichtet sind. In neuerer Zeit wird das polizeiliche Handeln immer mehr zur Pflicht; einerseits aufgrund ausdrücklicher Gesetzesnormen, anderseits wegen der wachsenden Ohnmacht der Einzelnen gegenüber den modernen Gefahren. Die Behörden verfügen im Umgang mit der Frage, ob und wie sie polizeilich handeln sollen, oft über einen weiten Beurteilungsspielraum. Dabei haben sie die Art des Polizeigutes, die Schwere der Gefahr, die verfügbaren Mittel und die Umstände des Einzelfalls zu berücksichtigen (Häfelin et al., 2006; Rz 2445-2449).

Adressat der grundrechtlichen Schutzpflicht ist der Staat in allen seinen Erscheinungsformen. Die Umsetzung obliegt primär der Gesetzgebung (Egli, 2002; Seiler, 2001). Diese muss in erster Linie die Grenze zwischen einer unerlaubten Gefährdung und einem hinzunehmenden Restrisiko definieren (Egli, 2002).

Zusammenfassend lässt sich festhalten, dass die Lehre der grundrechtlichen Schutzpflicht eine Handlungspflicht des Staates zum Schutz der Grundrechte - auch gegen Naturgefahren - bejaht. Bei der Entscheidung, auf welche Weise die Schutzpflichten wahrgenommen werden, kommt der zuständigen staatlichen Behörde ein grosser Einschätzungs-, Wertungs- und Gestaltungsspielraum zu. Dabei werden die Art des betroffenen Rechtsguts, die Schwere der Beeinträchtigung, die staatlichen Möglichkeiten und Mittel und allfällige, durch das staatliche Handeln ausgelöste Konflikte mit anderen Grundrechten berücksichtigt. Ein Teil der Lehre erachtet die Grundrechtstheorie für die Festlegung einer staatlichen Schutzpflicht als unergiebig, da sich daraus, im Vergleich zum Straf- und Polizeirecht, nichts Neues ergäbe (Seiler, 2001). 


\subsubsection{Schutzziele in der Lehre zum technischen Sicherheitsrecht}

Beim technischen Sicherheitsrecht stellt sich die Frage nach der staatlichen Handlungspflicht etwas anders: In der Schutzpflicht steht in der Regel nicht der Staat, sondern der Betreiber einer riskanten Tätigkeit. Dennoch spielt der Staat eine zentrale Rolle: Er schreibt als Gesetzgeber vor, welche Sicherheitsmassnahmen zu treffen oder allenfalls sogar, welche Risiken zulässig sind. Als Exekutive prüft der Staat riskante Anlagen, erteilt Bewilligungen und Zulassungen oder nimmt Einfluss auf die Entwicklungsplanung. Zudem urteilt die Judikative nach Ereignissen im Verwaltungsgerichtsverfahren oder in Haftungs- und Strafrechtsfällen. Der Staat befasst sich im Bereich der technischen Risiken nicht als handelnder Schutzpflichtiger, sondern als Regulator und Kontrollinstanz mit Schutzzielen.

Das technische Sicherheitsrecht ist im Allgemeinen geprägt von zahlreichen Bestimmungen mit genauen Vorgaben und Verhaltensanweisungen. Diese Regeln sind nicht risikobasiert hergeleitet, untereinander wenig kohärent und historisch gewachsen. Sie halten fest, was nach Erfahrung und guter Praxis sinnvollerweise zu unternehmen ist, um die Sicherheit zu verbessern. Die Schutzziele als Mass des tolerierbaren Risikos ergeben sich implizit aus der Anwendung dieser Regeln.

Ausdrücklich erörtert wird die Frage des tolerierbaren Risikos in der Anschlussgesetzgebung und in der Literatur zu Art. 10 (Katastrophenschutz) des Umweltschutzgesetzes (USG, SR 814.01). Gemäss Art. 3 der Störfallverordnung (StFV, SR 814.012) müssen zuerst allgemeine Sicherheitsmassnahmen getroffen werden, die nach dem Stand der Sicherheitstechnik verfügbar, nach der Erfahrung ergänzt und wirtschaftlich tragbar sind. Wenn schwere Schädigungen nicht ausgeschlossen sind, müssen besondere Sicherheitsmassnahmen, vor allem Dokumentations- und Informationspflichten, wahrgenommen werden (Art. 6 StFV). Wird das Risiko als nicht tragbar beurteilt, sind zusätzliche Sicherheitsmassnahmen notwendig, die geeignet und erforderlich sind, um das Risiko auf ein tragbares Mass zu vermindern (Art. 8 StFV; Seiler, 2001).

Art. 10 USG bezweckt zwar Schutz, aber nicht absoluten Schutz vor schweren Schädigungen (Leimbacher und Saladin, 1990). Wird der Schaden als schwer, jedoch nicht als besonders schwer bewertet, so kann er tragbar sein je nach Gewichtung, die ihm im Verhältnis zu entgegenstehenden Interessen zukommt. Es findet eine Interessenabwägung zwischen den Interessen des Risikobetroffenen und den Interessen an einer riskanten Tätigkeit statt. Ereignisse, die besonders schwere Schäden verursachen, sind unbedingt zu vermeiden wenn nötig durch das Verbot einer Anlage. Die Eintretenswahrscheinlichkeit der Schädigung ist bei besonders schweren Schädigungen irrelevant. Als besonders schwer gelten Schäden, wenn sie die ganze Schweiz oder wesentliche Teile davon betreffen oder wenn sie nachhaltig wirken können.

Die Annahme einer maximal zulässigen oberen Schadensgrenze ist in Lehre und Praxis umstritten (Seiler, 1997). Das Umweltschutzgesetz und die Störfallverordnung enthalten keine ausdrücklichen maximalen Schadensobergrenzen. Das Handbuch I zur Störfallverordnung (BUWAL, 1991) hält es für möglich, dass es ein Mass an Schädigungen gibt, die durch die privaten und öffentlichen Interessen nicht mehr aufgewogen werden können und unabhängig von der Eintretenswahrscheinlichkeit als untragbar zu bezeichnen sind. Im Anhang des Handbuchs (BUWAL, 1991, Anhang G, Fig. G.4) ist eine solche Grenze bei Störfallwert 1.0 gezogen. Dies entspricht ca. 2'200 Todesfällen (innert 30 Tagen als direkte Folge des Störfalles) und CHF 10 Mia. Sachschaden (Kapitel 2.4.3, Tabelle 9). In den Beurteilungskriterien (BUWAL, 1996) wurde die Frage zur Schadensobergrenze offengelassen: Ausmassachse und Akzeptanzlinie enden bei einem Störfallwert 1.0, da bei den in der Schweiz vorhandenen Betrieben nicht mit Ereignissen mit grösserem Schadensausmass zu rechnen sei. 
Grundsätzlich sind die Kantone, denen der Vollzug der Störfallverordnung obliegt, berechtigt, eine Schadensobergrenze festzulegen. Es werden - ceteris paribus - umso grössere Katastrophenpotenziale akzeptiert, je höher die Entscheidungsebene ist (Seiler, 1995b).

Wird ein Risiko als tragbar beurteilt, so wird mit dieser Feststellung der Anspruch verbunden, dass es von allen Betroffenen in Kauf genommen und getragen werden muss (Trösch, 1992). Getragen werden muss nur das Risiko, nicht aber die Gefahr oder gar ein allfälliger Schaden. Im Anhang des Handbuchs zur Störfallverordnung (BUWAL, 1991, Anhang G, Fig. G.4) ist die Grenze eines in jedem Fall tragbaren Risikos bei Störfallwert 0.1, in den Beurteilungskriterien II (BUWAL 2001) gar bei 0.3 angesetzt. Dies entspricht ca. 2 resp. 10 Todesfällen (innert 30 Tagen als direkte Folge des Störfalles) und ca. CHF 8 resp. 40 Mio. Sachschaden. Diese Werte des auf jeden Fall tolerierbaren Schadens (2 Todesfälle, CHF 8 Mio. Sachschaden) sind sehr hoch im Vergleich zur üblichen Werthaltung im Umgang mit Naturgefahren. Die Werte für das maximal tolerierbare Risiko (ca. 2000 Todesfälle, CHF 10 Mia. Sachschaden) sind dagegen im Vergleich zu den schlimmsten derzeit in Betracht gezogenen Naturgefahrenszenarien (Erdbeben Magnitude 6.9 (vergleichbar Basel 1356), Wiederkehrdauer ca. 1000 Jahre, 1'500 Todesopfer, CHF 60 Mia. Sachschaden) tief.

Die Regelung des Katastrophenschutzes im Umweltschutzgesetz und in der Störfallverordnung umschreibt das in vielen technischen Sicherheitsregeln typische Dreistufenkonzept (Seiler, 1997; Tabelle 42). Das Konzept umfasst einen Bereich,

- in dem Sicherheitsmassnahmen zwingend und keine Abwägung erlaubt sind;

- in dem über die Anwendung von Sicherheitsmassnahmen aufgrund einer Verhältnismässigkeitsprüfung entschieden wird;

- $\quad$ in dem keine Sicherheitsmassnahmen vorzusehen sind.

Tabelle 42 Das Dreistufenkonzept im technischen Sicherheitsrecht (Seiler, 1997) unterscheidet drei Bereiche der Pflicht zur Ergreifung von Sicherheitsmassnahmen: einen Bereich mit absoluter Handlungspflicht, einen Bereich mit Pflicht zu verhältnismässigen Handlungen und einen Bereich ohne Handlungspflicht.

\begin{tabular}{|c|c|c|c|}
\hline Stufe & \multicolumn{3}{|c|}{ Regelungstechnik } \\
\hline & Generalklauseln & Stand der Technik usw. & Grenzwerte \\
\hline $\begin{array}{l}\text { 1. Stufe } \\
\text { Absoluter Bereich }\end{array}$ & Gefahrenabwehr & $\begin{array}{l}\text { Anerkannte Regeln der } \\
\text { Technik; nach der Erfah- } \\
\text { rung geboten }\end{array}$ & $\begin{array}{l}\text { Einhaltung der Grenz- und } \\
\text { Alarmwerte }\end{array}$ \\
\hline $\begin{array}{l}\text { 2. Stufe } \\
\text { Relativer Bereich: zusätzliche } \\
\text { Sicherheitsmassnahmen, } \\
\text { soweit wirtschaftlich vertretbar }\end{array}$ & $\begin{array}{l}\text { Gefahrenvorsorge, } \\
\text { Risikovorsorge }\end{array}$ & $\begin{array}{l}\text { Stand von Wissenschaft } \\
\text { und Technik; Stand der } \\
\text { Technik }\end{array}$ & $\begin{array}{l}\text { ALARA-Bereich, vorsorgli- } \\
\text { che Begrenzung, Pla- } \\
\text { nungswerte, Zielwerte }\end{array}$ \\
\hline $\begin{array}{l}\text { 3. Stufe } \\
\text { Keine zusätzlichen Sicher- } \\
\text { heitsmassnahmen mehr er- } \\
\text { forderlich }\end{array}$ & Sicherheit & Erlaubtes Risiko & Unschädlichkeit \\
\hline
\end{tabular}

Das Dreistufenkonzept wurde als F/N-Diagramm mit Akzeptanzlinien im Handbuch zur Störfallverordnung (BUWAL, 1991, Abbildung 22) umgesetzt. 


\subsubsection{Schutzziele in der Rechtslehre zum Management von Naturrisiken}

Rechtlich besteht - wie bereits erwähnt - ein Unterschied zwischen natürlichen und technischen Risiken (Seiler, 1995d; Seiler, 2002). Bei letzteren kann in der Regel ein Verursacher ausgemacht und nach dem Verursacherprinzip oder - im Schadensfall - nach haftpflichtrechtlichen Grundsätzen belangt werden. Da Naturgefahren hochrangige Rechtsgüter betreffen können, ist unbestritten, dass eine Verpflichtung des Staates zum Schutz der Bevölkerung besteht (Kapitel 3.2.4.1, u. a. Hepperle, 1995; Lüthi, 2004; Bütler, 2006). Diese Verpflichtung besteht auch aufgrund von Rechtsnormen, insbesondere Art. 19 des Waldgesetzes (WaG, SR 921.0).

Im Umgang mit Naturgefahren gelten generell tiefere Anforderungen an die Schutzpflicht als bei Risiken, die die in Anspruch genommene Stelle selber gesetzt hat (Jaun, 2006). Der Sorgfaltsmassstab ist von Beginn an auf das Mögliche und Zumutbare begrenzt. Das Gemeinwesen hat lediglich vorzukehren, was von inm bei umsichtigem und gewissenhaftem Handeln, im Rahmen der ihm zur Verfügung stehenden personellen, logistischen und finanziellen Ressourcen und der zeitlichen Verhältnisse vernünftigerweise erwartet werden kann (Bütler, 2006). Es sind nur (allenfalls vorsorgliche) Schutzmassnahmen zu ergreifen, die in sachlicher, räumlicher, zeitlicher und personeller Hinsicht effektiv erforderlich sind und in einem vernünftigen Verhältnis zum Schutzinteresse der gefährdeten Bevölkerung stehen. Eine Pflicht zur Schadensabwehr besteht nur insoweit, als die Gefahr den Verantwortlichen bekannt oder für sie bei zumutbarer Sorgfalt erkennbar ist. Den zuständigen Organen und Personen kommt ein beträchtliches Ermessen in der Beurteilung der Situation zu. Die Schutzerwartung der Bevölkerung findet ihre Grenze in der Möglichkeit zur Eigenvorsorge. In erster Linie ist jedermann selber für den Schutz seines Hab und Guts verantwortlich.

Gestützt auf die gesetzlichen Vorgaben und auf die Gefahreninformationen haben die Sicherheitsbehörden Schutzziele und -massnahmen festzulegen (Bütler, 2006). Angesichts der zahlreichen und ausgedehnten Gefahrengebiete und der beschränkten Mittel sind die Kosten der Massnahmen und die ohne Massnahmen möglicherweise eintretenden Schäden im Sinne einer Kosten-Nutzen-Analyse gegeneinander abzuwägen. Dabei sind nach dem Grundsatz der Verhältnismässigkeit die finanziellen, personellen und technischen Ressourcen der zu Schutzmassnahmen verpflichteten Gemeinwesen zu berücksichtigen. Der Schutz von Bewohnern und Besuchern abgelegener Täler darf allerdings nicht rein aus Kostengründen vernachlässigt werden (Bütler, 2006).

Je dringlicher und schwerwiegender eine Gefährdungssituation erscheint, desto eher kann von den Sicherheitsbehörden erwartet werden, dass sie handeln und zumutbare Schutzmassnahmen ergreifen. Zu handeln ist in erkennbaren, aussergewöhnlichen Situationen, insbesondere nach Warnungen durch Experten. Dank vertieften Kenntnissen und hochstehenden Beobachtungsmethoden stellen Naturereignisse heute nur noch in seltenen Fällen höhere Gewalt dar. Keine Handlungspflicht besteht lediglich bei ausserordentlichen Ereignissen, die auch durch Spezialisten trotz sorgfältiger Beurteilung nicht vorhersehbar waren (Bütler, 2006).

Von der in konkreten Gefährdungslagen sowie während oder nach Ereignissen erhöhten Handlungspflicht bestehen Parallelen zur in der Gesundheitsökonomie bekannten ,Rule of Rescue' (Sommer, 2001). Diese Regel besagt, dass unwirtschaftliche Massnahmen aus Gründen der Mittelknappheit sehr wohl unterlassen werden, solange es sich um Risiken im ,statistischen' Sinn handelt. Im Fall von konkret erkennbaren Gefährdungen oder nach Eintritt einer Katastrophe oder Notlage werden eben diese Massnahmen ungeachtet wirtschaftlicher Überlegungen ergriffen. Die ,Rule of Rescue' wird auch in der Rechtslehre und Rechtsprechung stark unterstützt. Möglicherweise führt dieses Verhaltensmuster zur Zurückhaltung bei privater Prävention im Wissen um grosse öffentliche Bereitschaft zu Intervention/Wiederherstellung während oder nach einem Ereignis (Godschalk, 1999). 
Für zahlreiche bekannte Situationen im Umgang mit Naturgefahren haben sich aufgrund der einschlägigen Praxis und von Normen sowie als Folge der Rechtsprechung bestimmte Verhaltensregeln entwickelt (Bütler, 2006). Auch wenn sich daraus keine allgemein anwendbaren Schutzziele ableiten lassen, sind diese Verhaltensregeln bezüglich der Schutzerwartung vor Naturgefahren interessant (Tabelle 43).

Tabelle 43 Typische Sorgfaltspflichten und Massnahmen im Umgang mit naturgefahrenbedingten Risikosituationen (nach Bütler, 2006).

\begin{tabular}{l|l|l|l|l}
\hline Risikosituation & \multicolumn{3}{c}{ Typische Massnahme } \\
Schadenspotenzial & Hinweis & Warnung & $\begin{array}{l}\text { Technische } \\
\text { Absperrung }\end{array}$ & $\begin{array}{l}\text { Organisatori- } \\
\text { sche Sperrung }\end{array}$ \\
\end{tabular}

Bestehende Infrastrukturanlage

Geplante Infrastrukturanlagen

Werke im

Gefahrengebiet

Strassen

Bergwege

Bergbahn mit grosser Förderkapazität und unerfahrenem Publikum

Bergbahn mit Tourengebiet ohne markierte Pisten

Skipisten

Freerider-Gebiete

Gletscherskipisten

Baustellen

Eintritt eines Schadensereignisses

Vorhandene Gefahrenhinweise
Bauliche Massnahmen soweit zumutbar, vor allem in Siedlungsnähe und bei viel benutzten Bauten und Anlagen

Keine Bewilligung/evtl. Auflagen

Verkehrssicherungspflicht, entfällt bei unaufmerksamem oder sorglosem, den Umständen nicht angepassten Verhalten der Benutzer

\section{Zeitweilige Sperrung, bei akuter Gefahr}

Bei wiederholter, schwerer Gefährdung
Warntafel an der Bergstation

Personell, technisch und finanziell zumutbare Gefahrenabwehr, Gewährleistung Mindeststandard; Schutz vor schwer erkennbaren und auch bei Vorsicht unvermeidbaren Gefahren

Seil oder Wimpelschnur an der Ausfahrt, Warnung mit Tafel/Warnleuchte

Beidseitige Begrenzung, Absturzsicherung oberhalb von Spalten, Hinweis auf Gletschergefahren in jedem Fall

Ausbildung, Ausrüstung, Überwachung, Einstellen der Arbeiten bei akuter Gefahr

Massnahmen des Gemeinwesens zu Nothilfe und Schadensbegrenzung in jedem Fall

Information der geeigneten und zuständigen Behörden 


\subsubsection{Risikobasiertes Recht}

Im Vergleich zur kontinentaleuropäischen Rechtsordnung ist das angloamerikanische Rechtssystem eher utilitaristisch ausgerichtet (Egli, 2000). Deshalb haben ergebnisorientierte Regeln, bezeichnet als ,Risk Based Regulation' (RBR), ,Performance Based Regulation' (PBR) oder ,Goal Based Regulation' (GBR) in den Vereinigten Staaten von Amerika oder Grossbritannien bereits längere Tradition. Auslöser dieser Regelungsart ist ein zunehmendes Kostenbewusstsein in Wirtschaft und Politik sowie die Tendenz zunehmender gesetzlicher Regelungen und die steigende Zahl von Haftungsfällen (Flueler \& Seiler, 2003). Der Ansatz des risikobasierten Rechts ist in der Schweiz noch wenig verbreitet.

Vom traditionellen Sicherheitsrecht mit den oft detaillierten Handlungsvorschriften zur Risikominderung unterscheidet sich das risikobasierte Recht dadurch, dass es sich auf die Vorgabe von Schutzzielen beschränkt und die Wahl der Massnahmen zur Erreichung der Schutzziele den Rechtsadressaten überlässt (Seiler, 2000).

Im Rahmen des Nationalen Forschungsprojektes Nr. 1113-52163.97 ,Risikobasiertes Recht: wie viel Sicherheit wollen wir?' entstand ein Entwurf eines risikobasierten Sicherheitsgesetzes (Seiler, 2000). Der Entwurf enthält folgende für ein schutzzielbasiertes Risikomanagementkonzept relevante Inhalte:

- Definition der in Betracht fallenden verletzbaren Werte und Schäden (Anzahl Todesfälle, Anzahl verlorene Lebensjahre, Anzahl bleibend körperlich Geschädigte und Invaliditätsgrad, Sach- und Vermögensschäden, Umweltschäden);

- Regel zur Berücksichtigung künftiger Schäden;

- Gewichtung der Schäden nach Freiwilligkeit und Zurechenbarkeit sowie versicherbarer/versicherter Schäden;

- Bewertung von Todesfällen, verlorenen Lebensjahren und körperlicher Verletzung;

- Schutzziele für individuelle Risiken (Grenzwerte);

- Schutzziele für kollektive Risiken (,Learned Hand Rule': Kostenreduktion insoweit, als die Kosten der risikoreduzierenden Massnahmen tiefer sind als der Erwartungswert der dadurch vermiedenen Schäden);

- Regelung der Behandlung von Grossschäden;

- Regel zur Berücksichtigung der Kosten risikomindernder Massnahmen.

In der Literatur zum risikobasierten Recht wurde aus dem Dreistufenkonzept des technischen Sicherheitsrechts ein ,Zwei-Regel-Ansatz' abgeleitet. Zur Festlegung des tolerierbaren Risikos sind nach diesem Ansatz zwei Bedingungen einzuhalten (Seiler, 2000; Flueler \& Seiler, 2003):

- Regel 1:

Begrenzung des maximalen individuellen Risikos (Grenzwert für das maximal akzeptierbare Todesfallrisiko).

- Regel 2:

Begrenzung des Kollektivrisikos (z. B. durch ein Grenzkostenkriterium).

Dem Schutz individueller Rechte wird der Vorrang gegenüber einer gesamtökonomischen Nutzenmaximierung eingeräumt.

\subsection{Handlungsleitende Prinzipien}

\subsubsection{Gerechtigkeit, Ethik und Schutzziele}

Schutzziele sollen das kollektive Risiko der Gesellschaft auf ein tolerierbares Mass reduzieren und gleichzeitig vermeiden, dass das Restrisiko für ein einzelnes Individuum untragbar Josef Th. Hess: Schutzziele im Umgang mit Naturrisiken in der Schweiz, @ vdf Hochschulverlag 2011 
wird. Die Tolerierbarkeit von Risiken ist somit einerseits eine Frage der Höhe des Risikos und anderseits eine Frage der Risikoverteilung in der Gesellschaft (Seiler, 1996a; Flueler \& Seiler, 2003). Wie hohe Risiken auf welche Art in einer Gesellschaft verteilt werden, ist eine Frage der Gerechtigkeit. Die Frage der Gerechtigkeit ist in jedem politischen und gesellschaftlichen System ihrerseits eine Grundfrage. Damit ist sie Gegenstand zahlreicher ethischer, philosophischer, soziologischer, ökonomischer, politologischer und juristischer Auseinandersetzungen, die die menschlichen Kulturen seit Jahrtausenden beschäftigen (u. a. Schulze, 1981; Sommer, 2001; Konow, 2003; Mathis, 2004). Verschiedene Gesellschaften und Zeitalter entwickelten ganz unterschiedliche Vorstellungen von Gerechtigkeit. Diese reichten von egalitärer Gleichbehandlung jedes Individuums bis zu utilitaristischer, gesamtökonomischer Maximierung des Nutzens.

Mit philosophisch-ethischen Aspekten befasste sich auch die damals junge Literatur der Risikowissenschaften in den frühen 1980er-Jahren (Schulze, 1981). Vier verschiedene Ansätze für den Umgang mit (unkompensierten) Risiken wurden beschrieben:

- Utilitarismus: Risiken sind tolerierbar, wenn der zusätzliche Nutzen für den Risikoverursacher die zusätzlichen Kosten des Risikobelasteten übersteigen (,Güter- und ÜbelAbwägung').

- Egalitarismus: Risiken sind tolerierbar, wenn sie zu einer Besserstellung des Schlechtergestellten führen.

- Elitarismus: Risiken sind tolerierbar, wenn sie zu einer Besserstellung des Bessergestellten führen.

- Libertarismus (,verfassungsmässige' Haltung): Risiken dürfen in keinem Fall zu einer Schlechterstellung eines Individuums führen.

W. Schulze und A. Kneese (Schulze, 1981) plädierten schon damals für einen Einbezug ethischer Aspekte in die (risikobezogene) Nutzen-Kosten-Analyse: "[...] Ethical systems which emphasize the good of the whole, such as utilitarism, are shown to differ sharply in decision outcomes from those which emphasize the rights of the individual, such as libertarianism. It is suggested that benefit-cost analysis should be broadened to include alternative weightings of benefits and costs consistent with a variety of ethical views."

In einer neueren Darstellung werden die Gerechtigkeitstheorien in drei Grundrichtungen gegliedert (Konow, 2003):

- Gerechtigkeit nach dem Prinzip von ,Gleichheit und Bedürfnis' (,equality and need'): Güter sollen auf alle Mitglieder der Gesellschaft gleich verteilt werden. Diese Theorie ist vor allem auf das Wohl der schlechtestgestellten Mitglieder einer Gesellschaft ausgerichtet.

- Gerechtigkeit nach dem Prinzip von ,Nutzen und Effizienz' (,utilitarian and welfare economics'): Bei der Güterverteilung werden das Ergebnis und die Folgen ins Zentrum gerückt, wobei die Maximierung des Gesamtnutzens das Ziel ist.

- Gerechtigkeit nach dem Prinzip von ,Gleichmässigkeit, Verdienst und Zurechenbarkeit' (,equity, desert and attribution'): Die Güterverteilung wird vom Beitrag und der persönlichen Verantwortung des Einzelnen abhängig gemacht.

Als Teil der dritten Grundrichtung oder als eigenständige Ausrichtung gilt die Theorie nach dem Prinzip des Kontextes. Die Kontexttheorien besagen, dass Güterverteilungen je nach dem geschichtlichen Zusammenhang, den Beteiligten, der Art des Gutes, Gewohnheiten oder der Prozedur der Verteilung unterschiedlich gerecht empfunden werden. Die verschiedenen Prinzipien werden oft gleichzeitig und vermischt angewendet. Dem Prinzip der Gleichmässigkeit kommt dabei eine führende Bedeutung zu. Güterzuteilungen und damit sinngemäss die Verteilung von Risiken werden als besonders fair empfunden, wenn sie in direktem Zusammenhang mit Faktoren stehen, die beeinflussbar sind. Beeinflussbar sind persönliche Beiträge und Entscheidungen (,effort, choice'), nicht beeinflussbar sind Herkunft 
(,birth'), Pech (,brute luck') und risikoirrelevante Entscheidungen (,choices that do not affect productivity'; Konow, 2003).

Die erwähnten Theorien zielen in erster Linie auf gerechte Behandlung von Einzelpersonen in der Gesellschaft (Personalität). Damit wird ein wesentlicher Aspekt der Umweltethik ausgeblendet, nämlich die Vernetzung der Einzelperson mit der Gesellschaft und der Umwelt (Retinität). Gemäss dem 1994 verfassten Umweltgutachten des Rates von Sachverständigen für Umweltfragen (RSU, 1994) stellen die Personalität und die Retinität das umweltethische Fundament dar. Das anthropozentrisch begründete Prinzip der Personalität sichert eine Sonderstellung des Menschen, der Mensch darf damit jedoch nicht gleichzeitig zum einzigen Inhalt der sich daraus ergebenden moralischen Forderungen gemacht werden. Allerdings kann sich der Mensch nur entfalten durch Umgestaltung der Natur. Ein per se konfliktfreies Verhältnis zwischen Ökologie und Ökonomie kann es somit nicht geben.

In der Diskussion über den Umgang mit der Umwelt ist die Frage zentral, ob Umwelt- bzw. Naturgüter per se geschützt werden sollen oder nur soweit sie dem Menschen nützen (Seiler, 1997). Die Übersicht über das positive Recht (z. B. Umweltschutzrecht, Gewässerschutzrecht, Natur- und Heimatschutzrecht, Strafrecht) zeigt, dass Gesetze zwar traditionell menschliche Interessen schützen, dass es aber auch zahlreiche rechtliche Normen gibt, welche die Umwelt (z. B. Boden, Luft, Wasser, Tiere) unabhängig von oder sogar im Gegensatz zu menschlichen Interessen schützen (Kapitel 2.3.4). Der Naturwirklichkeit kommt Eigenbedeutung zu (Seiler, 1997).

Die Antwort auf die Frage nach der ,richtigen Theorie' für ein gerechtes Mass und eine gerechte Verteilung von Risiken ist letztlich ein Ausdruck des (politischen) Standpunkts und fällt je nach Staatsordnung und Zeitalter anders aus. Für eine bestimmte Region oder ein Projekt ist die passende Antwort optimalerweise in einem demokratischen oder partizipativen Entscheidungsprozess zu suchen (Kapitel 2.9 und 4.6; Hall, 2000).

\subsubsection{Prinzipien für die Festlegung von Schutzzielen}

\subsubsection{Fünf handlungsleitende Prinzipien}

Aus den dargelegten Theorien ergeben sich fünf Prinzipien für die Festlegung von Schutzzielen, die das Handeln im Management von Naturrisiken leiten können:

- Bedürfnis: Schutzziele sollen von der Art und Bedeutung verletzbarer Werte (Rechtsgüter), namentlich Leib, Leben und Gesundheit, Vermögen, Einrichtungen des öffentlichen Lebens, Lebensqualität und Umwelt, abhängen;

- Einfluss: Schutzziele sollen dem Mass der Selbstbestimmung (Freiwilligkeit) und dem Nutzen für die risikobetroffene Person Rechnung tragen;

- Kontext: Schutzziele sollen die persönlichen Umstände des Betroffenen, namentlich Aspekte der Standortgebundenheit und der Verlustaversion berücksichtigen;

- Effizienz: Schutzziele sollen von der Effizienz risikomindernder Massnahmen abhängen;

- Vernetzung: Schutzziele sollen sich an der Umwelt- und Sozialverträglichkeit risikomindernder Massnahmen orientieren.

\subsubsection{Das Bedürfnisprinzip}

Das Bedürfnisprinzip ist auf die menschlichen Bedürfnisse und deren Bedeutung ausgerichtet. Eine anschauliche Darstellung zur Bedeutung der Bedürfnisse ist die Maslow'sche Pyramide (Maslow, 1970), die die menschlichen Bedürfnisse in fünf Ebenen gliedert: 
a. Selbstverwirklichung: Individualität, Talententfaltung, Altruismus, Güte, Kunst und Philosophie.

b. Soziale Anerkennung: Status, Macht, Karriere, sportliche Siege, Auszeichnungen, Statussymbole und Rangerfolge.

c. Soziale Beziehungen: Freundeskreis, Partnerschaft, Liebe, Nächstenliebe, Kommunikation und Fürsorge.

d. Sicherheit: Wohnung, gesicherter Erwerb, Gesetze, Versicherungen, Gesundheit, Ordnung, Religion und Lebensplanung.

e. Körperliche Grundbedürfnisse: Atmung, Wärme, Trinken, Essen, Schlaf und Fortpflanzung.

Für das Risikomanagement ist das Bedürfnisprinzip zentral: Bei der Formulierung von Schutzzielen stellt sich die Frage, welche menschlichen Bedürfnisse in welchem Mass schützenswert sind. Als Versuch einer Antwort wurden die verletzbaren Werte, resp. die geschützten Rechtsgüter, gutachtlich und anhand der erwähnten Stichworte einer oder mehreren Bedürfnisstufen nach Maslow zugeordnet (Tabelle 44).

Tabelle 44 Geschützte Rechtsgüter und deren Zuordnung zu einer Bedürfnisstufe nach Maslow.

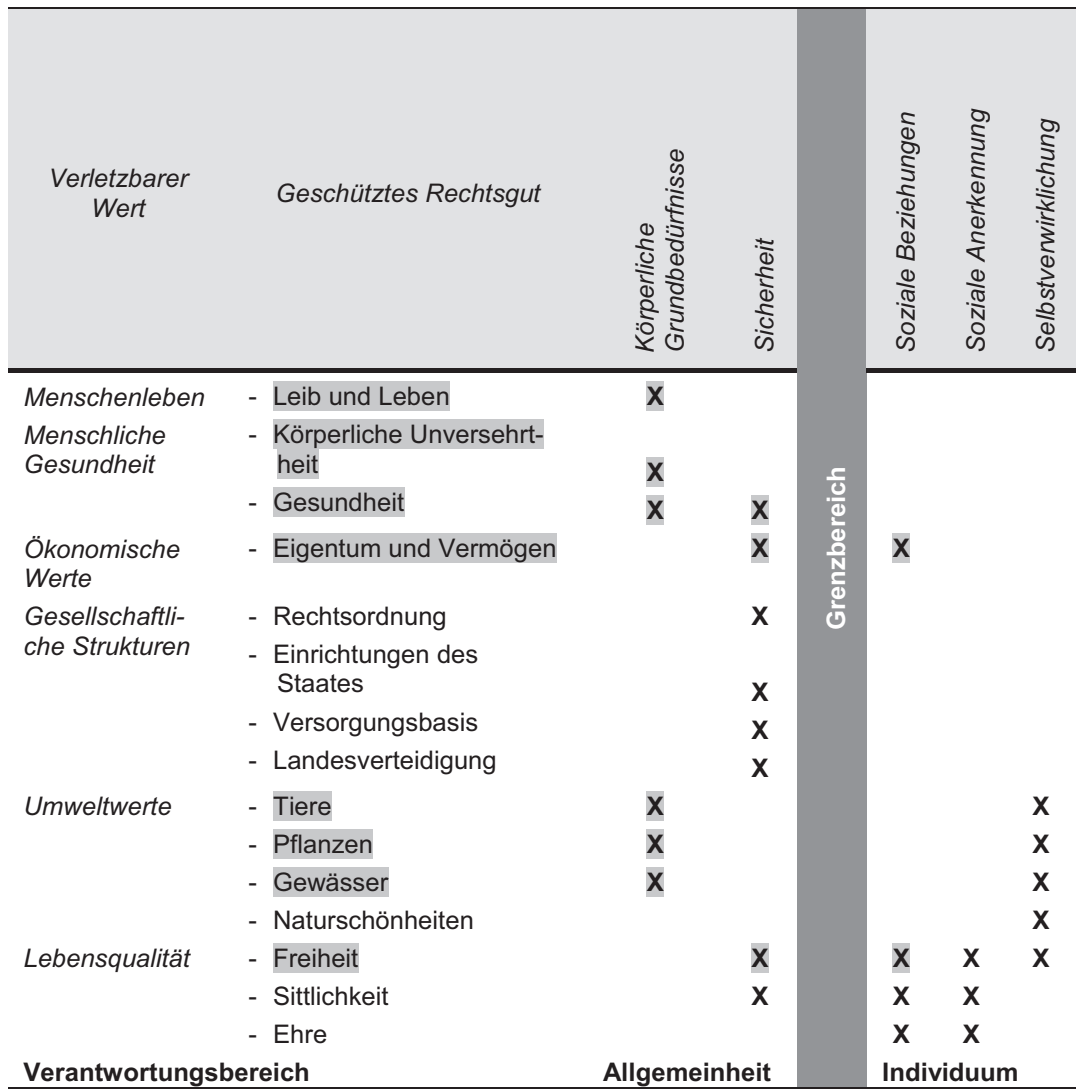

$X \quad$ Zuordnung eines Rechtsgutes zu einer Bedürfnisstufe

X Bedeutendes Rechtsgut beim Management von Naturrisiken (Kapitel 2.3.2) 
Diese Darstellung ermöglicht eine differenzierte Betrachtung der geschützten Rechtsgüter hinsichtlich ihrer Bedeutung. Viele der im Management von Naturrisiken als relevant erachteten Rechtsgüter können in der Maslow'schen Bedürfnispyramide den Stufen ,körperliche Grundbedürfnisse' und ,Sicherheit' zugeordnet werden. Naturwerte werden den Grundbedürfnissen zugeordnet, soweit sie dem Menschen als Lebensgrundlage dienen.

Pragmatisch kann anhand dieser Zuordnung die Grenze zwischen Verantwortlichkeit der Allgemeinheit und dem Verantwortungsbereich des Individuums zwischen die Bedürfnisstufen ,Soziale Beziehungen' und ,Sicherheit' gelegt werden (,Grenzbereich' in Tabelle 44).

Bei der Umsetzung des Bedürfnisprinzips können Schutzziele für einzelne verletzbare Werte quantitativ festgelegt werden. Namentlich gilt dies für den Schutz des Lebens sowie von Sachwerten, die grundlegende materielle Bedürfnisse abdecken. Für Ziele zum Schutz von Leib und Leben wird auf die quantitativen Angaben zu tolerierbaren individuellen Todesfallrisiken zurückgegriffen (Kapitel 2.6.2.1).

Damit die materiellen Bedürfnisse auf den Stufen ,körperliche Grundbedürfnisse' und ,Sicherheit' abgedeckt sind, muss das Existenzminimum nachhaltig gewährleistet sein. Zur Definition des Existenzminimums wird die Terminologie der Sozialhilfe (SKOS, 2005) und der Schuldbetreibungspraxis (Betreibungsämter, 2001) herangezogen. In der Sozialhilfepraxis wird unterschieden zwischen dem absoluten und dem sozialen Existenzminimum und der materiellen Grundsicherung (Abbildung 36).

Die materielle Grundsicherung umfasst die Wohnkosten, die Kosten für die medizinische Grundversorgung und den Grundbedarf für den Lebensunterhalt. Massgebend für die Höhe des Grundbedarfs für den Lebensunterhalt ist das Konsumverhalten des untersten Einkommensdezils, d. h. der einkommensschwächsten zehn Prozent der Schweizer Haushalte. Die medizinische Grundversorgung umfasst die Leistungen der obligatorischen Grundversicherung gemäss Krankenversicherungsgesetzgebung. In der Regel etwa 15\% tiefer liegt der Wert für das absolute Existenzminimum, welches dem verfassungsmässigen Recht auf Hilfe in Notlagen (Art. 12 BV, SR 101) entspricht (SKOS, 2005).

Für die Bemessung des sozialen Existenzminimums werden zusätzlich zur materiellen Grundsicherung die Kosten für die Teilnahme am sozialen und kulturellen Leben hinzugerechnet (SKOS, 2005). Etwa in dieser Höhe liegt auch der Wert des betreibungsrechtlichen Existenzminimums (Betreibungsämter, 2001). Das betreibungsrechtliche Existenzminimum (Notbedarf) umfasst einen monatlichen Grundbetrag für den Lebensunterhalt inkl. Kulturelles, Wohnkosten, Sozial- und obligatorische Versicherungsbeiträge, unumgängliche Berufsauslagen, Unterstützungsbeiträge, Beiträge an Schulung der Kinder sowie weitere unumgängliche Kosten.

Aufgrund von Zahlenangaben in Weisungen und Richtlinien (Vernunft Schweiz, 2007; Betreibungsämter, 2001) wurden folgende Angaben zum Existenzminimum abgeschätzt (jährlicher Bedarf pro Kopf; Berechnungsgrundlage ist eine vierköpfige Familie):

- materielle Grundsicherung

CHF 12'300,

- absolutes Existenzminimum

CHF 10'500,

- betreibungsrechtliches Existenzminimum CHF 14'100.

Für alle Arten von verletzbaren Werten, ausser Leib und Leben und Sachwerten, ist die Anwendung des Bedürfnisprinzips bei der Schutzzielfestlegung infolge fehlender Indikatoren oder ungenügender Daten nur qualitativ möglich. Die Schutzzielfestlegung erfolgt durch eine 


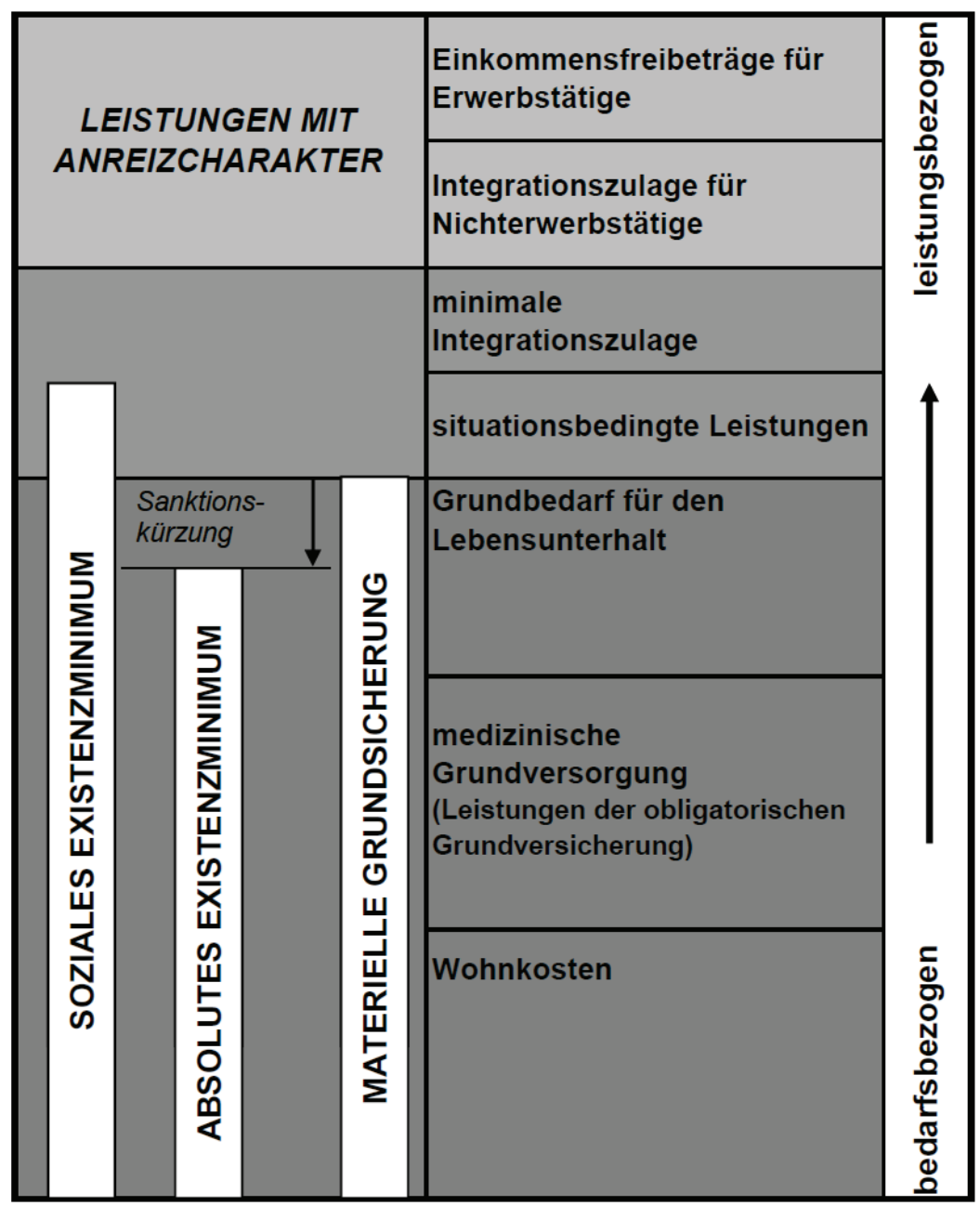

Abbildung 36 Unterstützungsbedürftigkeit gemäss Richtlinien für die Ausgestaltung und Bemessung der Sozialhilfe (SKOS, 2005, S. A. 6-3).

Bewertung der verschiedenen Arten von risikoexponierten Objekten. Dabei wird pro Objektart abgeschätzt, welche Arten von verletzbaren Werten oder geschützten Rechtsgütern beeinträchtigt werden, wenn die entsprechenden Objekte durch Naturereignisse betroffen sind. Anschliessend wird beurteilt, welche Bedeutung den betroffenen verletzbaren Werten in der Bedürfnispyramide zukommt. Diese Bewertung erfolgt typisierend für Risikosituationen und Risikoobjekte. Das Ergebnis ist Bestandteil des Objektindexes (Kapitel 4.2.3.2). 


\subsubsection{Das Einflussprinzip}

Das Einflussprinzip drückt die Möglichkeiten der Beeinflussung von Risiken durch das Verhalten der risikobetroffenen Person aus. Im Risikomanagement ist das Einflussprinzip bereits etabliert durch die in der Literatur und Praxis verbreitete Klassierung der Risiken in Risikokategorien (Kapitel 2.6.1.4). Diese Risikokategorien werden oft auch als Freiwilligkeitsklassen bezeichnet. Die Zuordnung von Risiken in Risikokategorien erfolgt nach verschiedenen Kriterien (Schneider, 1984), namentlich Kenntnis, Vermeidbarkeit und Beeinflussbarkeit des Risikos sowie Nutzenempfindung bei der riskanten Tätigkeit (Fischhoff, 1978; Abbildung 37).

\section{Bewertungsfaktoren}

- Kenntnis

- Vermeidbarkeit

- Beeinflussbarkeit

- Nutzenempfindung
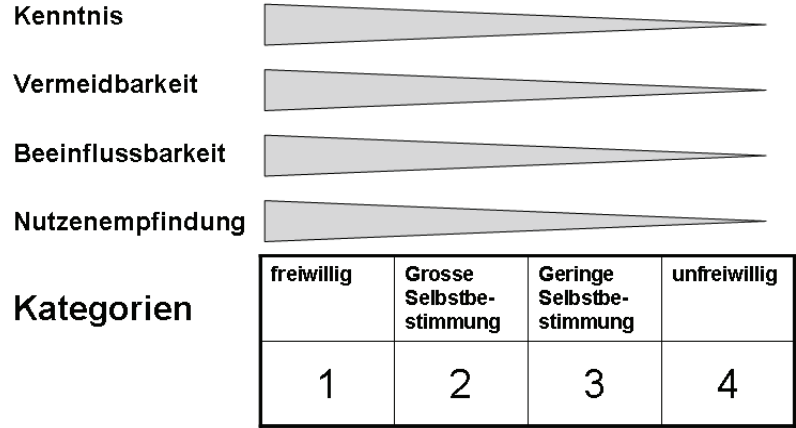

Abbildung 37 Risikokategorien (Schneider, 1984).

Für die Umsetzung des Prinzips ,Einfluss' bei der Schutzzielfestlegung werden die Kriterien Selbstbestimmung und persönlicher Nutzen beim Eingehen des Risikos qualitativ berücksichtigt. Bezüglich dieser Kriterien werden - wie beim Bedürfnisprinzip - Risikosituationen und risikoexponierte Objekte typisierend bewertet. Das Ergebnis ist ebenfalls Bestandteil des Objektindexes (Kapitel 4.2.3.2).

\subsubsection{Das Kontextprinzip}

Das Kontextprinzip ist auf die spezifischen Umstände einer Risikosituation ausgerichtet. Spezifische Umstände können die Vorgeschichte, bestehende Gewohnheiten, die involvierten Personen oder die Prozedur der Risikoverteilung sein. Ein wichtiger Aspekt ist die Orientierung des Gerechtigkeitsempfindens und der Entscheidungen an einem Referenzpunkt. Referenzpunkte sind Werte, an die sich die Beteiligten angepasst haben oder auf die sie sich mit Vereinbarungen oder gegenseitigem Einverständnis geeinigt haben. Veränderungen, also Verluste oder Gewinne, werden anhand dieses Referenzpunkts beurteilt (Kahnemann \& Tversky, 1979; Plattner, 2005). Negative Abweichungen zum Referenzpunkt (Verluste) werden dabei stärker gewichtet als positive (Verlustaversion; Abbildung 38). 


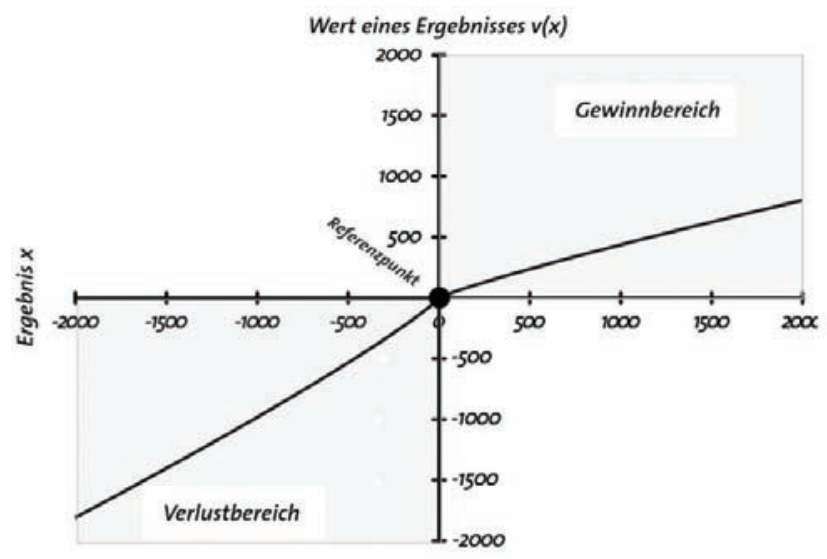

Abbildung 38 Gewinn und Verlust in Abhängigkeit vom Referenzpunkt (Kahnemann und Tversky, 1979).

Ein für das Management von Naturrisiken relevanter Ausdruck der Orientierung an einem Referenzpunkt ist der im Baurecht verbreitet angewandte Bestandesschutz (Bestandesgarantie). Der Bestandesschutz garantiert die Erhaltung von Bauten, die nach geltendem Recht nicht mehr bewilligungsfähig wären, sofern sie während der Dauer ihres bisherigen Bestandes einmal bewilligungsfähig waren. Verfassungsrechtlich gründet der Bestandesschutz auf dem Vertrauensschutz (Art. 9 BV) und der Eigentumsgarantie (Art. 26 BV). Der Bestandesschutz gilt für die Lebensdauer des geschützten Objekts und lässt lediglich Instandsetzungs-, Unterhalts- und Modernisierungsmassnahmen am Objekt zu, ohne dieses wesentlich zu verändern. Massnahmen des Risikomanagements werden durch den Bestandesschutz erheblich beeinflusst.

Ein weiteres wichtiges Kontextelement ist der Standort eines risikoexponierten Objekts. Für das Management von Naturrisiken ist der Aspekt der Standortgebundenheit relevant. Die Standortgebundenheit wird gemäss bundesgerichtlicher Rechtsprechung nur bejaht, wenn eine Baute aus technischen oder betriebswirtschaftlichen Gründen oder wegen der Bodenbeschaffenheit auf einen Standort (ausserhalb der Bauzone) angewiesen ist (Aargau, Standortgebundenheit, BGE 114 lb 317, E. 4a). An diese Erfordernisse sind strenge Anforderungen zu stellen. Dabei beurteilen sich die Voraussetzungen nach objektiven Massstäben und es kann weder auf die subjektiven Vorstellungen und Wünsche des Einzelnen noch auf die persönliche Zweckmässigkeit oder Bequemlichkeit ankommen.

Für die Implementierung des Prinzips ,Kontext' bei der Schutzzielfestlegung werden die Kriterien Standortgebundenheit und Besitzstandswahrung/Bestandesgarantie qualitativ berücksichtigt. Bezüglich dieser Kriterien können Risikosituationen und risikoexponierte Objekte zumindest teilweise typisierend bewertet werden. Auch dieses Bewertungsergebnis ist Bestandteil des Objektindexes (Kapitel 4.2.3.2).

\subsubsection{Das Effizienzprinzip}

Das Effizienzprinzip ist auf die Maximierung des Nutzens (Sicherheit) ausgerichtet und lässt sich operationalisieren anhand der Kostenwirksamkeit von Massnahmen oder der Grenzkosten, bis zu denen Risikoverminderungsmassnahmen getroffen werden. Optimale Effizienz in einer Region wird bei einem bestimmten Mitteleinsatz erreicht, wenn bei allen involvierten Teilsystemen (z. B. allen Gefahrenstellen eines Kantons) bis zu den gleichen Grenzkosten investiert wird, d. h. die Tangente an die Sicherheitskurve aller Systeme eine identische Neigung aufweist (Kapitel 2.8.3, Abbildung 28). 
Für die Implementierung des Effizienzprinzips bei der Schutzzielfestlegung werden die Kriterien Wirksamkeit und Kosten berücksichtigt. Bezüglich dieser Kriterien können Risikoverminderungsmassnahmen typisierend bewertet werden, wobei die Wirksamkeit von der Eignung der Massnahmen abhängt. Die Eignung muss fallweise bestimmt werden und hängt ihrerseits von gefahrenspezifischen und örtlichen Gegebenheiten ab. Art, Intensität und Häufigkeit des Gefahrenprozesses, die Vorhersehbarkeit eines Einzelereignisses, die Grösse und der Verbauungsgrad eines Einzugsgebiets oder die Art und Ausdehnung der gefährdeten Objekte beeinflussen, welche Massnahmen sich für ein bestimmtes Gefahrengebiet besonders eignen. Aufgrund fehlender Daten und aufgrund der zahlreichen fallweisen Besonderheiten kann diese Bewertung auf regionaler Ebene nur qualitativ erfolgen. Das Ergebnis ist Bestandteil des Massnahmenindexes (Kapitel 4.2.3.3).

\subsubsection{Das Prinzip Vernetzung mit Gesellschaft und Umwelt (Retinität)}

Das Prinzip ,Vernetzung' ist auf die Belange der Gesellschaft und Umwelt im Risikomanagement ausgerichtet. Bezüglich der Einbettung des Risikomanagements in Gesellschaft und Umwelt sind drei Bereiche zu beachten (RSU, 1994).

Ein erster Bereich deckt die Verantwortung des Menschen für sich selbst ab: Das Handeln muss individualverträglich sein. Diesem Aspekt dienen die erörterten Prinzipien Bedürfnis, Einfluss, Kontext und Effizienz.

Ein zweiter Bereich umfasst die Verantwortung des Menschen für seine soziale Mitwelt: Das Handeln muss sozialverträglich sein und zwar

- sowohl für die eigene soziale Gruppe als auch für die ganze Menschheit;

- hinsichtlich der direkten als auch der indirekten Folgen;

- $\quad$ hinsichtlich der ökonomischen Zumutbarkeit.

Diesem Aspekt dienen die Prinzipien Effizienz und Vernetzung.

Der dritte Bereich betrifft die Verantwortung des Menschen für seine natürliche Umwelt: Handeln muss sowohl für die Erhaltung der Natur an sich als auch als Lebensgrundlage des Menschen umweltverträglich sein. Diesem Aspekt dient das Prinzip Vernetzung.

Naturprozesse als Ursache von Naturrisiken sind Teil der Natur und ihrer Dynamik. Die Einflussnahme auf Naturprozesse steht somit zumeist im Konflikt mit einer möglichst unbeeinträchtigten Natur. Dies rechtfertigt die in der heutigen Praxis übliche Forderung nach ,ökologischer Kompensation', wenn technische Massnahmen zur Verminderung von Naturrisiken ergriffen werden.

Die Eindämmung technischer Risiken steht dagegen im Einklang mit den Interessen der Natur. Wenn sich technische Risiken realisieren, beeinträchtigt dies meist gleichzeitig den Menschen und die Natur. Gleiches gilt auch, wenn technische Störfälle durch Naturereignisse induziert werden.

Für die Umsetzung des Prinzips ,Vernetzung' bei der Schutzzielfestlegung werden die Kriterien Sozialverträglichkeit (Partizipation, Beeinträchtigung von Rechten und Interessen, Ressourcenschutz) und Umweltverträglichkeit (Auswirkungen auf Artenvielfalt, Landschaftsbild, Boden, Landressourcen und Belastungen) berücksichtigt. Die Risikoverminderungsmassnahmen können bezüglich dieser Kriterien typisierend bewertet werden. Mangels Daten und Indikatoren muss diese Bewertung qualitativ erfolgen. Das Ergebnis fliesst ebenfalls in den Massnahmenindex ein (Kapitel 4.2.3.3).

\subsubsection{Schutzzielprinzipien in Schutzzielkonzepten der Praxis}

Gemäss einer gutachtlichen Einschätzung berücksichtigen alle Schutzziele der Praxis die Schutzzielprinzipien ganz oder teilweise (Tabelle 45). 
Tabelle 45 Gerechtigkeitsprinzipien in Schutzzielkonzepten der Praxis.

\begin{tabular}{|c|c|c|c|c|c|c|}
\hline \multicolumn{2}{|c|}{ Prinzip Schutzzieldarstellung } & Bedürfnis & Einfluss & Kontext & Effizienz & Vernetzung \\
\hline \multicolumn{2}{|c|}{$\begin{array}{l}\text { Grenzwerte für Versagens- } \\
\text { wahrscheinlichkeiten }\end{array}$} & + & - & $+/-$ & $+/-$ & - \\
\hline \multirow{3}{*}{ 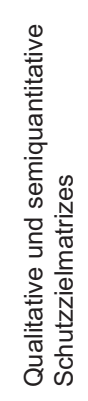 } & $\begin{array}{l}\text { Schutzziele Kreis- } \\
\text { schreiben Nr. } 20\end{array}$ & + & $+/-$ & $+/-$ & $+/-$ & - \\
\hline & $\begin{array}{l}\text { Schutzziele } \\
\text { Hochwasser- } \\
\text { schutz an Fliess- } \\
\text { gewässern }\end{array}$ & + & $+/-$ & $+/-$ & $+/-$ & $+/-$ \\
\hline & $\begin{array}{l}\text { Schutzziele BU- } \\
\text { WAL 107/1 }\end{array}$ & + & $+/-$ & $+/-$ & $+/-$ & $+/-$ \\
\hline \multirow{4}{*}{ 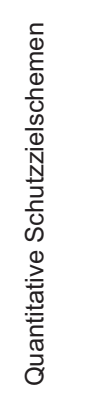 } & F/N-Diagramme *) & + & - & $+/-$ & $+/-$ & $+/-$ \\
\hline & $\underset{* *)}{\mathrm{F} / \mathrm{N}-\text { Diagramme }}$ & $+/-$ & - & - & + & $+/-$ \\
\hline & $\begin{array}{l}\text { Schutzziele Erd- } \\
\text { beben an beste- } \\
\text { henden Gebäu- } \\
\text { den }{ }^{*} \text { ) }\end{array}$ & + & - & $+/-$ & $+/-$ & - \\
\hline & do. $\left.{ }^{* *}\right)$ & $+/-$ & - & $+/-$ & + & - \\
\hline \multirow{2}{*}{ 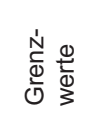 } & $\begin{array}{l}\text { Individuelles } \\
\text { Risiko }\end{array}$ & + & + & - & - & - \\
\hline & Kollektives Risiko & + & $+/-$ & $+/-$ & - & - \\
\hline \multicolumn{2}{|c|}{ Grenzkosten } & $+/-$ & + & - & + & - \\
\hline
\end{tabular}

${ }^{*}$ Bereich absoluter Schutzziele (intolerabel, Massnahmen zwingend erforderlich).

${ }^{\star *}$ Bereich mit Verhältnismässigkeitsprüfung (ALARA).

$+\quad$ dominierendes Prinzip - unbedeutendes Prinzip

+/- mitbestimmendes Prinzip

In besonderem Masse bedürfnisorientiert sind alle grenzwertbasierten Schutzzieldarstellungen. Ein Handlungsbedarf ergibt sich allerdings nur bei Vorliegen eines Schutzdefizits. Die Höhe des Schutzdefizits entscheidet über die Priorität und den Umfang der zu ergreifenden Massnahmen. Der Aufwand, der für Massnahmen zur Einhaltung eines Grenzwerts erforderlich ist, bleibt unbeachtet. Grenzwertangaben zum tolerierbaren Risiko blenden den Aspekt der Effizienz aus.

Bei den im Management von Naturrisiken verbreitet angewendeten Schutzzielmatrizes steht ebenfalls das Bedürfnisprinzip im Vordergrund. Allerdings erlaubt die Abstufung dieser Schutzziele nach Objektkategorien eine implizite Berücksichtigung von Effizienzaspekten. Sind hohe verletzbare Werte in hohem Mass gefährdet, kann vermutet werden, dass Risikoverminderungsmassnahmen effizient sind.

In grenzkosten- und kosten-nutzen-orientierten Schutzzieldarstellungen steht das Effizienzprinzip im Zentrum. Die Schutzziele favorisieren Massnahmen, mit denen sich besonders effizient Risiken vermindern lassen. Das Bedürfnisprinzip bleibt gesamtökonomisch gewahrt, indem bestmögliche Risikoreduktion für eine gesamte Gesellschaft erreicht wird. Für einzel- 
ne Individuen werden die Sicherheitsbedürfnisse jedoch in unterschiedlichem Mass abgedeckt.

Das Prinzip ,Einfluss' spielt in allen Schutzzieldarstellungen, die die Grenzwerte oder Grenzkosten ausdrücklich nach den Risikokategorien (wie z. B. Freiwilligkeitsklassen) abstufen, eine zentrale Rolle. Indirekt berücksichtigt ist der Grad der Beeinflussbarkeit bei einigen Schutzzielmatrizes durch die Festlegung unterschiedlicher Grenzwerte je nach Art der Objektkategorien.

Das Kontextprinzip kommt sehr untergeordnet zum Ausdruck, teilweise und indirekt in der Auswahl und Festlegung der Objektkategorien sowie bei Schutzzielen, die zwischen bestehenden und neuen Risiken unterscheiden.

Das Vernetzungsprinzip kommt hinsichtlich Umweltverträglichkeit in denjenigen Schutzzielen zum Ausdruck, in denen auch Naturwerte massgebliche verletzbare Werte darstellen. Am ausgeprägtesten ist dies im Handbuch zur Störfallverordnung erkennbar, wobei die naturbezogenen Schutzziele vor allem die Natur als Lebensgrundlage des Menschen betreffen. Indirekt kommt die Vernetzung auch in Schutzzielmatrizes zum Ausdruck. Für Objektkategorien mit hohem Naturwert werden sehr tiefe Schutzanforderungen gestellt, sodass Eingriffe faktisch unterbleiben und die natürlichen Prozesse nicht beeinflusst werden.

\subsection{Konzeptionelle Synthese}

\subsubsection{Elemente zur Festlegung von Schutzzielen}

Aus dem Stand des Wissens (Kapitel 2) und den konzeptionellen Überlegungen (Kapitel 3.2 und 3.3) lassen sich verschiedene inhaltliche und formelle Elemente darstellen, die zur Festlegung der Tolerierbarkeit von Risiken entscheidend sind (Abbildung 39).

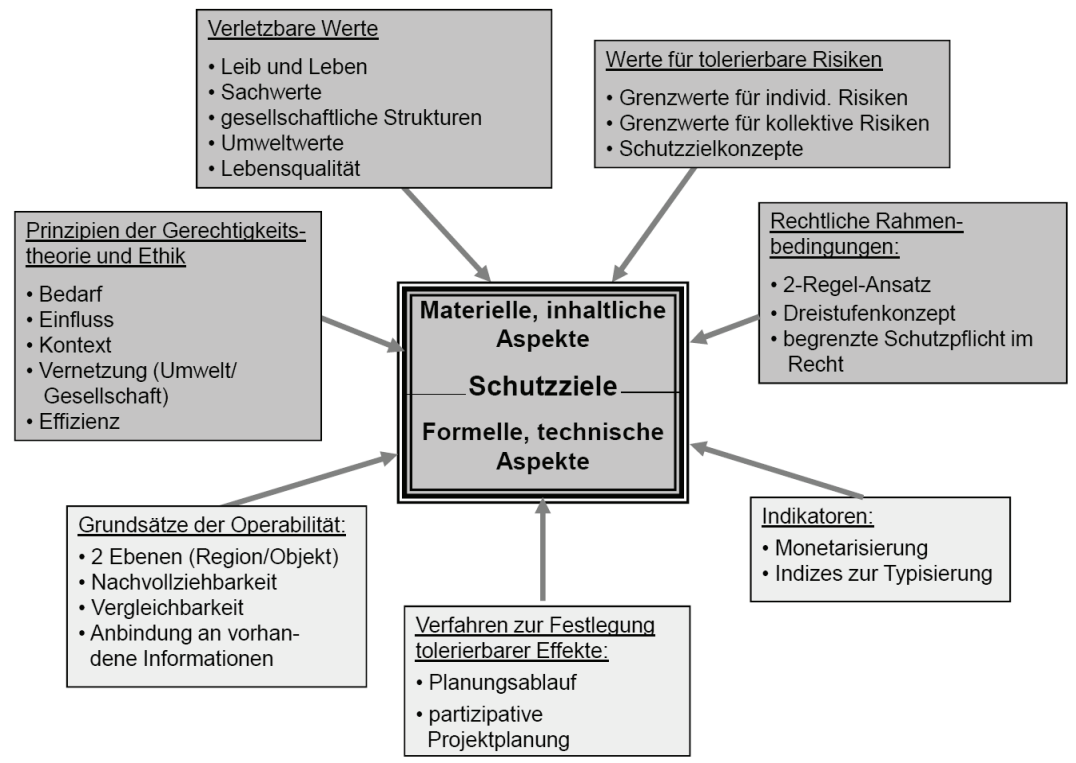

Abbildung 39 Elemente zur Entwicklung eines risikobasierten Managementkonzeptes für Naturrisiken. 


\subsubsection{Wesentliche inhaltliche Elemente}

In Rechtsordnung, Rechtslehre und Rechtsprechung ist unbestritten, dass bei Naturrisiken eine Schutzpflicht besteht, die nicht unbegrenzt ist. Materiell sind die Schutzziele in Empfehlungen (Umweltmaterialien) am konkretesten und im Einklang mit den rechtlichen Rahmenbedingungen geregelt. Diese Schutzziele werden verbreitet angewendet, sind jedoch nicht allgemeinverbindlich.

Die Literatur gibt Werte für das tolerierbare individuelle Todesfallrisiko zwischen $10^{-6} / \mathrm{Jahr}$ und $10^{-2} / \mathrm{Jahr}$ an. Die Abstufung dieser Eckwerte erfolgt nach Freiwilligkeit und direktem Nutzen und liegt somit in der Grössenordnung von 3 bis 4 Magnituden. Allgemeine Eckwerte zu tolerierbaren kollektiven Risiken gibt es nicht, da diese immer von der Grösse und Art des Systems abhängen. Nebst Risiken an Leib und Leben fallen vor allem Risiken für Sachwerte, gesellschaftliche Strukturen, Umweltwerte und Lebensqualität in Betracht.

Aus Theorien der Gerechtigkeit und Ethik können zur Festlegung und Differenzierung der Schutzziele die fünf Prinzipien ,Bedürfnis', ,Einfluss', ,Kontext', ,Effizienz' und ,Vernetzung' herangezogen werden. Das Bedürfnisprinzip, das Einfluss- und das Effizienzprinzip spielen in den bisher von der Praxis entwickelten Schutzzielen eine bedeutende Rolle. Auf regionaler Ebene dominiert das Bedürfnisprinzip. Das Effizienzprinzip kommt vor allem auf Stufe des Einzelprojektes zum Tragen.

Die Rechtslehre - namentlich die Lehre zum risikobasierten Recht - kennt den „Zwei-RegelAnsatz" zur Beschränkung individueller und kollektiver Risiken, der aus dem Dreistufenkonzept des Umweltrechts abgeleitet ist. Nach diesem Ansatz wird der Eliminierung untolerierbarer individueller Risiken (Regel 1) gegenüber der verhältnismässigen Reduktion kollektiver Risiken (Regel 2) der Vorrang eingeräumt.

Im heutigen regionalen Risikomanagement besteht meist ein Dilemma zwischen der Bereitstellung öffentlicher Mittel (Budget) und dem Mittelbedarf zur Erreichung von festgelegten Grenzen tolerierbarer Risiken (Schutzziele). Werden Schutzziele verletzt, muss grundsätzlich gehandelt werden, doch reicht das Budget in der Regel nicht aus, um alle festgestellten Schutzdefizite in kurzer Zeit zu eliminieren.

Der Zwei-Regel-Ansatz kann dieses Dilemma entschärfen. Nur wo ein Handlungsbedarf nach Regel 1 (Grundsicherheitslücken) besteht, hat dies zwingend Auswirkungen auf die Mittelbereitstellung. Eine verantwortliche Behörde muss sich zum Ziel setzen, diese untolerierbaren Risiken innerhalb einer bestimmten Zeit zu eliminieren. Der Handlungsbedarf nach Regel 2 (,ALARP'-Lücken) kann dagegen im Kontext aller übrigen Staatsaufgaben festgelegt werden und hat nur bedingten Einfluss auf die Mittelbereitstellung. Diese hängt von der Zahlungsbereitschaft der Gesellschaft für Risikominderungsmassnahmen ab (Abbildung 40). 
Mittelbereitstellung

(Allokation)
Mittelverteilung

(Distribution)

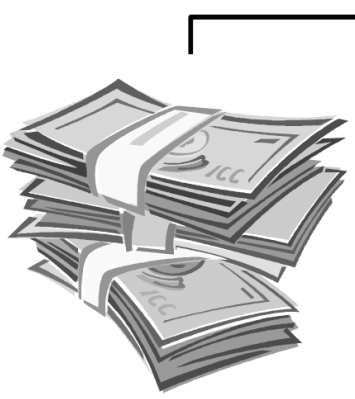

Budget

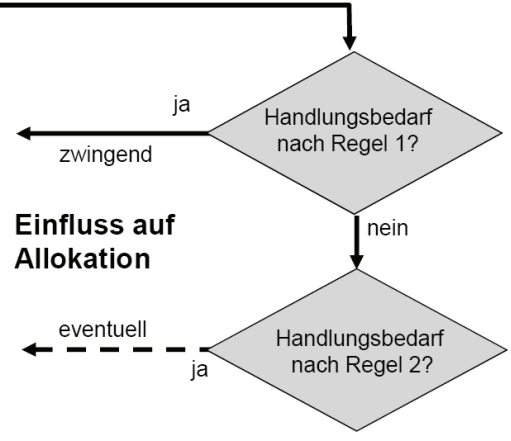

Massnahmenprojekte
Grundsicher-

heitslücken
,ALARP'-

Lücken

Abbildung 40 Zusammenhänge zwischen Allokation, Distribution gemäss Handlungsregeln des Managementkonzeptes. Das Konzept geht davon aus, dass Mittel zur Reduktion untolerierbarer individueller Risiken (Grundsicherheitslücken) zwingend und Mittel zur weiteren Reduktion kollektiver Risiken (,ALARP'-Lücken) bedingt bereitzustellen sind.

Die Schutzziele, die primär für den Präventionsbereich formuliert sind, lassen sich nicht ohne Prüfung für alle Bereiche des Risikomanagements anwenden. Rechtsprechung und Rechtslehre betonen höhere Ansprüche bei konkreten, unmittelbar drohenden Ereignissen.

\subsubsection{Wesentliche formelle Elemente}

Quantitative Aussagen zu Schäden und Risiken sind aufgrund des heutigen Wissensstands nur für Menschenleben und Sachwerte ermittelbar, nicht aber für weitere verletzbare Werte. Quantitative Betrachtungen müssen auf diese Werte und deren Indikatoren begrenzt bleiben.

Da der Schadensbegriff grundsätzlich subjektiv ist, ist aus Gründen der Vergleichbarkeit eine Typisierung, d. h. die Beurteilung eines Schadens bzw. eines Risikos und Schutzziels aus Sicht einer ,typisch betroffenen Person' erforderlich. Dies erfolgt in Form von Indizes (Kapitel 4.2.3.2 und 4.2.3.3).

Für eine gute operationale Eignung sind die Berücksichtigung aller relevanten Risiken, die Beachtung aller massgeblichen Aspekte, die Messbarkeit und die Vergleichbarkeit der tolerierbaren Risiken entscheidend. Die Anbindung der Methoden an grossflächig vorhandene Informationen, namentlich Schadenspotenzialkarten und Gefahrenkarten, ist eine wichtige operationale Voraussetzung für ein schutzzielbasiertes regionales Risikomanagement.

Zur Verbesserung der Akzeptanz des Risikomanagements, zur gegenseitigen Information und zur Berücksichtigung lokaler Bedürfnisse bei der Ermittlung der tolerierbaren Risiken sind partizipative Planungsprozesse von grosser Wichtigkeit.

\subsubsection{Kernelemente eines schutzzielbasierten Risikomanagementkonzeptes}

Aus den Ausführungen ergeben sich Kernelemente für ein schutzzielbasiertes Risikomanagementkonzept (Tabelle 46):

1. Der Zwei-Regel-Ansatz bildet das Grundgerüst des Konzeptes. Damit werden einerseits die elementaren Grundrechte geschützt (Einhaltung von Grenzwerten des individuellen Risikos, Regel 1). Andererseits werden die Risiken nach Massgabe der Verhältnismässigkeit reduziert (Reduktion des kollektiven Risikos, Regel 2). 
2. Die Quantifizierung von Schutzzielen beschränkt sich auf Todesfall- und Sachrisiken.

3. Alle Schutzzielprinzipien sollen möglichst umfassend berücksichtigt werden.

4. Die Umsetzung des Konzeptes, vor allem von Regel 2, erfordert den Einbezug der Betroffenen in hohem Mass.

5. Zur Beurteilung von Einzelprojekten eignen sich bekannte Schutzzielfestlegungen der Praxis, z. B. Konzepte mit Grenzkosten oder Kostenwirksamkeiten sowie Grenzwerte für tolerierbare individuelle Risiken.

Tabelle 46 Kernelemente eines schutzzielbasierten Konzeptes für das Management von Naturrisiken: Risikomanagementebenen und anzuwendende Schutzzielkonzepte.

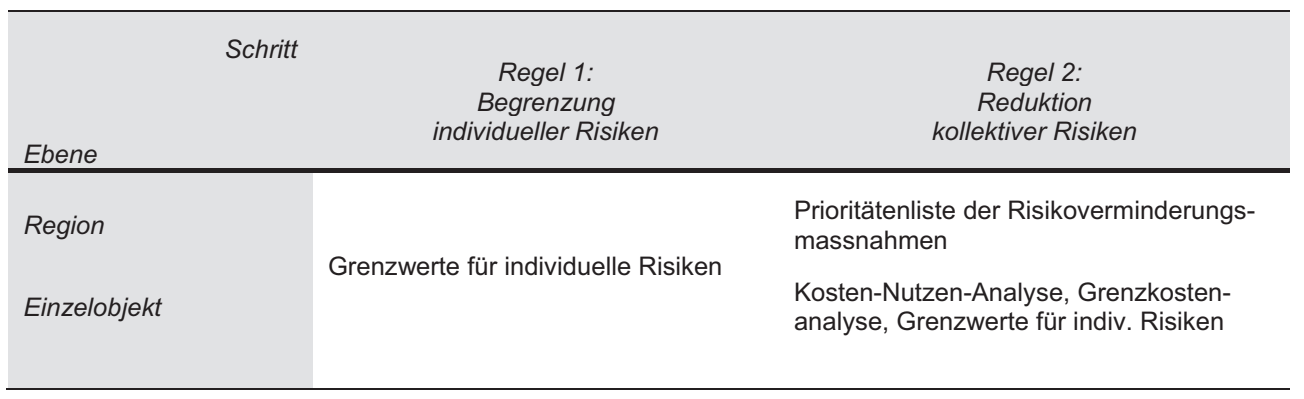




\section{Vorschlag eines schutzzielbasierten Managementkonzeptes}

\section{1 Übersicht}

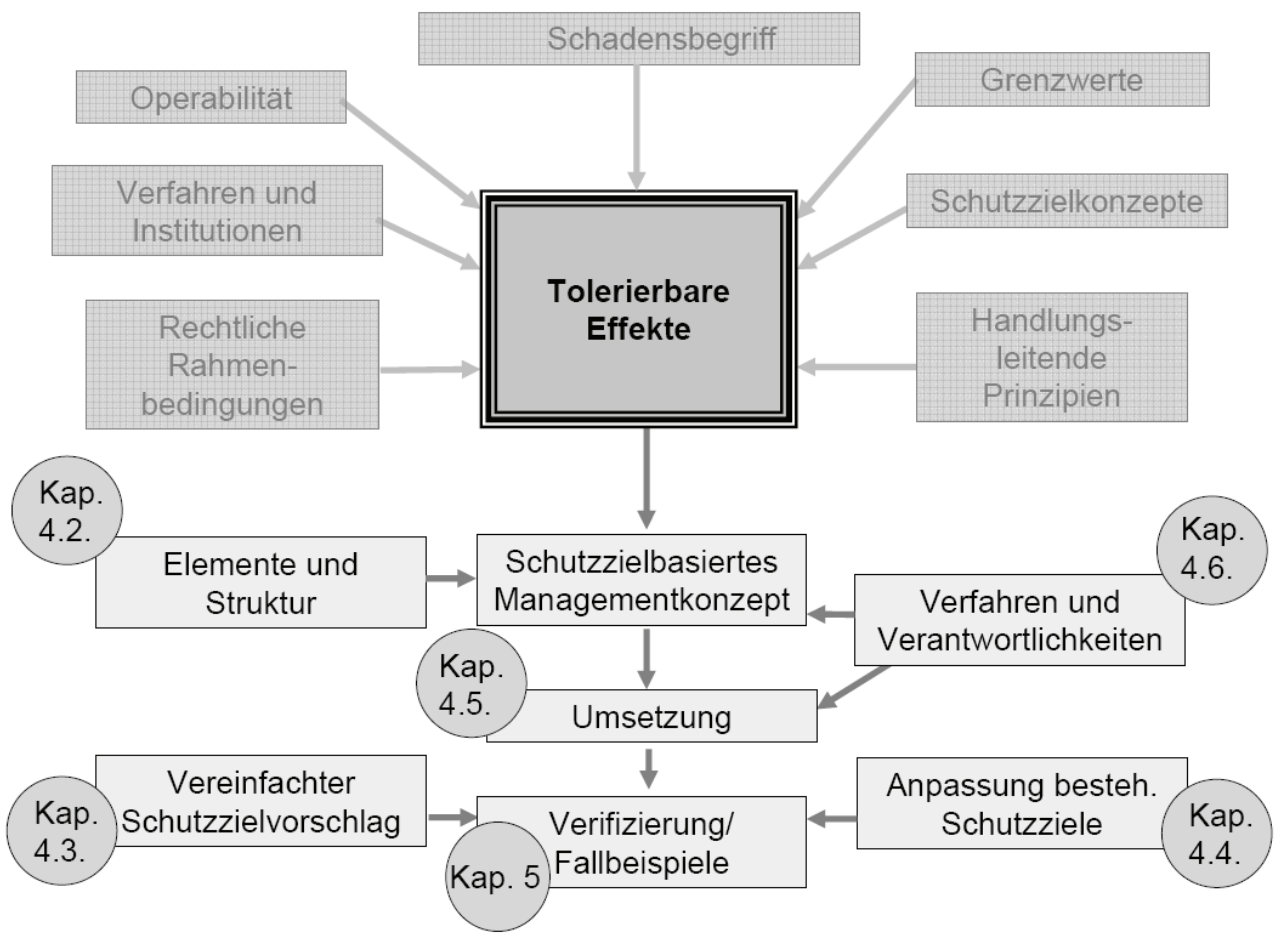

Abbildung 41 Kapitel 4 und 5 in der Übersicht.

Im Zentrum von Kapitel 4 steht die Entwicklung eines schutzzielbasierten Managementkonzeptes. Das Konzept baut auf dem Zwei-Regel-Ansatz auf und deckt das Risikomanagement auf Stufe Region und Einzelprojekt ab. Die fünf Schutzzielprinzipien sollen möglichst umfassend umgesetzt werden (Kapitel 4.2).

Parallel zu diesem Konzept werden zwei Ansätze vorgelegt, wie das heutige (regionale) Management von Naturrisiken weiter entwickelt werden kann. Zum einen wird ein vereinfachter Ansatz vorgeschlagen, um anhand der Arten von risikoexponierten Objekten, der Gefahrenkarte und der Massnahmeneffizienz den Handlungsbedarf zur Risikoverminderung abzuschätzen (Kapitel 4.3). Zum anderen werden die bekannten ,klassischen' Schutzzielmatrizes aufgrund der vorangehend dargestellten Erkenntnisse zu den Schutzzielprinzipien modifiziert (Kapitel 4.4).

Die Umsetzung des Konzeptes auf regionaler Ebene und auf Ebene Einzelprojekt wird erläutert (Kapitel 4.5). Abschliessend werden die Verfahren sowie die Rollen und Verantwortlichkeiten der Akteure und der Einbezug Betroffener im Management von Naturrisiken angesprochen. Entwicklungsmöglichkeiten zur Rollenverteilung und Partizipation im Rahmen eines schutzzielbasierten Managementkonzeptes werden erörtert (Kapitel 4.6). 


\subsection{Schutzzielbasiertes, regionales Managementkonzept}

\subsubsection{Grundsätze}

Das schutzzielbasierte Managementkonzept basiert auf dem Zwei-Regel-Ansatz. Es bezweckt, den Handlungsbedarf und die Priorität von Risikoverminderungsmassnahmen möglichst unter Anwendung aller Schutzzielprinzipien zu ermitteln. Diese umfassende Sichtweise ist - wie im bisherigen Risikomanagement - bei der Anwendung von Regel 1 nicht möglich. Bei diesem Schritt finden nur die Prinzipien ,Bedürfnis', ,Einfluss' und ,Kontext' indirekte Berücksichtigung. In Regel 2 werden dagegen alle Prinzipien umfassend angewendet (Tabelle 47).

Tabelle 47 Berücksichtigung der Schutzzielprinzipien in den Regeln des Managementkonzeptes.

\begin{tabular}{lll}
\hline Schutzzielprinzip & Regel 1 & Regel 2 \\
\hline Bedürfnis & $\begin{array}{l}\text { direkt über Grenzwert } \\
\text { indirekt }^{*} \text { durch Objektindex }\end{array}$ & durch Objektindex \\
Einfluss & indirekt $^{*}$ durch Objektindex & durch Objektindex \\
Kontext & indirekt $^{*}$ durch Objektindex & durch Objektindex \\
Effizienz & -- & durch Massnahmenindex \\
Vernetzung & -- & durch Massnahmenindex
\end{tabular}

$\left.{ }^{\star}\right)$ Der Objektindex beeinflusst die Zuordnung eines Objektes zu einer Risikokategorie (Kapitel 4.2.2.2).

Da Regel 1 nicht alle Schutzzielprinzipien berücksichtigt, soll sie nur in einem - möglichst kleinen - Teil aller Risikosituationen, jenem mit sehr hohen individuellen Risiken, zur Anwendung gelangen. Im vorgeschlagenen Konzept und im Fallbeispiel (Kapitel 5) ist die Anwendung von Regel 1 auf Situationen mit tolerierbaren individuellen Todesfallrisiken von mehr als $10^{-2}$ bis $10^{-4}$ pro Jahr begrenzt (Abbildung 42).

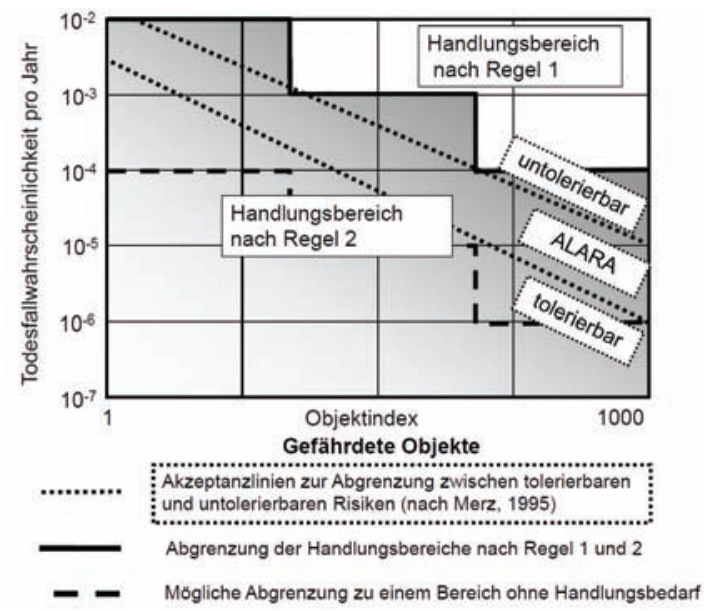

Abbildung 42 Handlungsbereiche nach dem Zwei-Regel-Ansatz (Beispiel Todesfallrisiken). 
Damit wird - unter Annahme der Hypothese, dass der Grenznutzen der Risikoverminderung bei hohen Risiken grösser sei - bezweckt, dass auch die nach Regel 1 evaluierten Massnahmen vermutlich eine hohe Effizienz aufweisen. Zudem soll mit der Wahl hoher Schwellenwerte der Anteil der Projekte, die mit der - das Effizienzprinzip nicht berücksichtigenden Regel 1 evaluiert werden, möglichst gering gehalten werden.

\subsubsection{Vorgehen}

\subsubsection{1 Überblick}

Die Beurteilungseinheit umfasst sowohl für Regel 1 als auch für Regel 2 ein Gefahrengebiet. Zur Anwendung des schutzzielbasierten Managementkonzeptes wird daher das gesamte Untersuchungsgebiet (z. B. eine Gemeinde) in Gefahrengebiete (z. B. Lawinenzüge, Wildbachgebiete) gegliedert. Diese Gliederung ergibt sich aus den naturräumlichen Gegebenheiten und aus der Raumnutzung. Für die Ausscheidung eines Gefahrengebiets gelten folgende Kriterien:

- Identität der Gefahrenquelle: Gefährdung durch die gleiche Gefahrenart mit gleichem Ursprung;

- Homogenität der Raumnutzung: Möglichst homogene Raumnutzung innerhalb des Gebiets;

- Identität des Wirkungsbereichs: Lage im direkten Wirkungsgebiet der gleichen tatsächlich vorhandenen oder potenziell möglichen, grossräumig wirkenden Schutzmassnahmen.

Die Gefahrengebiete weisen naturgegeben eine unterschiedliche Grösse auf. Um eine systematische Bevorzugung grosser Gefahrengebiete zu vermeiden, werden die Resultate der Beurteilung auf eine einheitliche Flächengrösse umgerechnet.

Nach Regel 1 wird in einer ersten Planungsphase der Handlungsbedarf nach hohen bis sehr hohen individuellen Risiken quantitativ ermittelt. Handlungsbedarf ist gegeben, wenn ein gewisses Mass an individuellen Risiken überschritten ist. Dieses Mass ist abgestuft nach Art und Bedeutung der betroffenen Raumnutzung, dargestellt mit dem Objektindex (Kapitel 4.2.3.2). Überschreiten Risiken diese Schwelle, besteht unbedingter Handlungsbedarf (Abbildung 42).

Anschliessend erfolgt die Priorisierung der Projekte nach Regel 2. Im Anwendungsbereich der Regel 2 gibt die Methode keine starren Grenzwerte für tolerierbare Risiken vor. Das Ergebnis dieses Planungsschrittes ist eine Prioritätenliste der zu ergreifenden Massnahmen. Bis zu welcher Priorität Massnahmen ausgeführt werden, ergibt sich aus den verfügbaren Mitteln.

Dieses Konzept ist im Vergleich zum heute üblichen Vorgehen (Kapitel 2.9.3, Abbildung 32) komplexer, bedingt durch das Vorgehen nach zwei Regeln und die klare Trennung zwischen den Planungsebenen Region und Einzelprojekt (Abbildung 43). Bei der Behandlung von Einzelprojekten folgt die Planung und Beurteilung der zu realisierenden Massnahmen dem gleichen Ablauf wie bisher. Hinsichtlich des Umfangs der zu treffenden Massnahmen wird zwischen Regel 1 und Regel 2 differenziert. Nach Regel 1 evaluierte Massnahmen sind bis zu dem Punkt zu treffen, an dem das Mass des tolerierbaren Risikos nach dieser Regel nicht mehr überschritten wird. Nach Regel 2 ermittelte Massnahmen werden in der Reihenfolge der Prioritätenliste nach Massgabe der verfügbaren Mittel ausgeführt. Massnahmenvarianten werden hinsichtlich Kostenwirksamkeit analysiert. Anschliessend erfolgt die Ausführung von Massnahmen bis sowohl die Grenzkostenkriterien als auch allfällige (weitergehende) Schutzziele für individuelle Risiken eingehalten sind (untere gestrichelte Linie Abbildung 42). Als Schutzziele für Grenzkosten und tolerierbare individuelle Risiken können auf Stufe Einzelprojekt die Vorschläge in Tabelle 28 (Kapitel 2.7.5) herangezogen werden. 


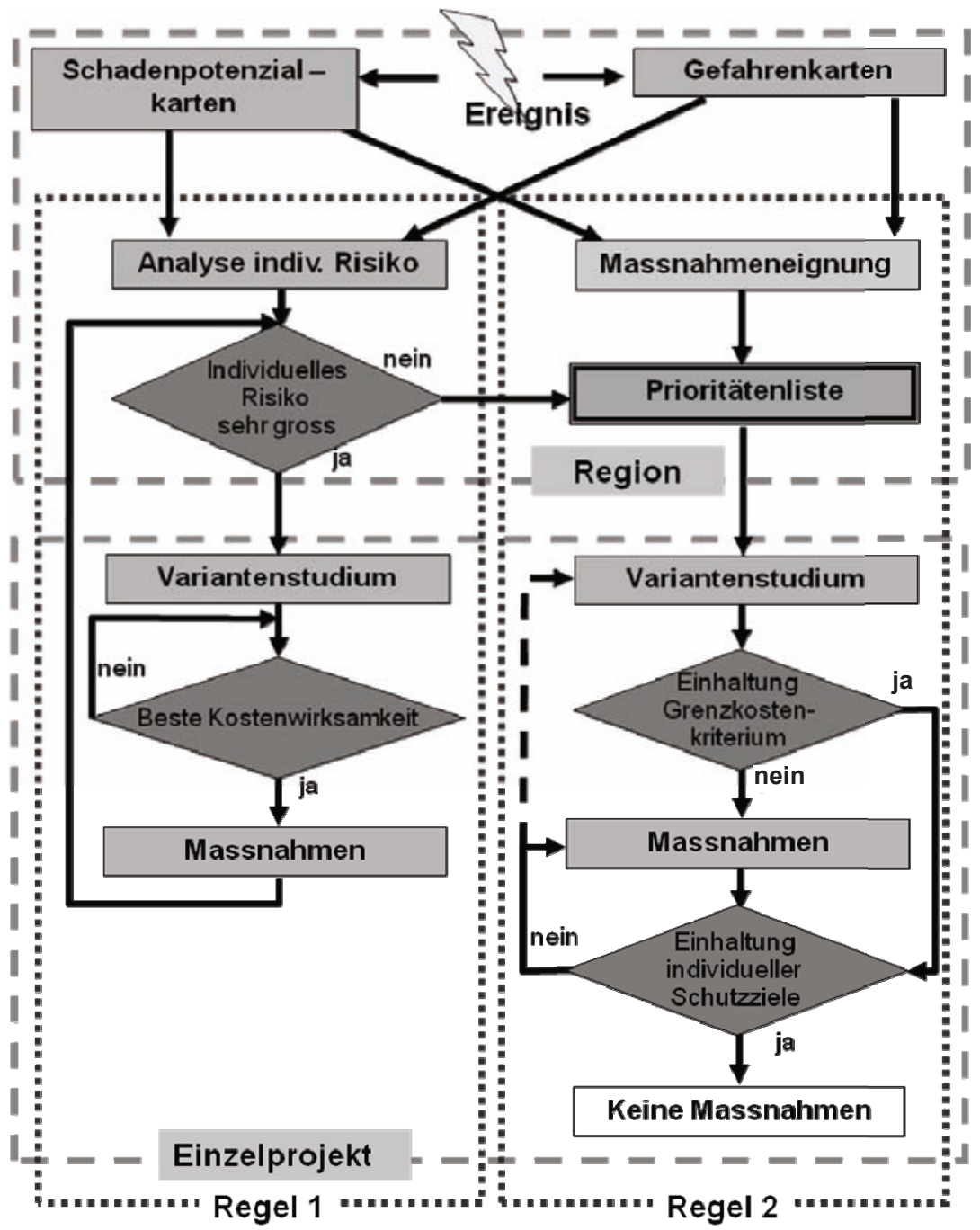

Abbildung 43 Ablauf gemäss schutzzielbasiertem Managementkonzept. Der ,Zwei-Regel-Ansatz' wird auf Ebene Region und auf Ebene Einzelprojekt angewendet.

\subsubsection{Ermittlung des Handlungsbedarfs nach Regel 1}

Zur Ermittlung des Handlungsbedarfs werden die individuellen Risiken für die gesamte zu untersuchende Region quantitativ ermittelt. Die Methode beschränkt sich dabei auf quantifizierbare Indikatoren und Messgrössen, namentlich auf Todesfallrisiken und monetäre Risiken.

Die Arten von Raumnutzung werden nach drei Kategorien differenziert. Für diese gelten abgestufte Werte für das maximal tolerierbare individuelle Todesfall- und Sachrisiko (Abbildung 42 und Tabelle 48). Die Zuordnung zu den Risikokategorien erfolgt aufgrund einer verallgemeinernden Bewertung der Objekte der Schadenspotenzialkarte. Diese Bewertung erfolgt typisierend und berücksichtigt die Schutzzielprinzipien Bedürfnis, Einfluss und Kontext (Objektindex, Kapitel 4.2.3.2).

Josef Th. Hess: Schutzziele im Umgang mit Naturrisiken in der Schweiz, @ vdf Hochschulverlag 2011 
Die Risikosituationen der Kategorie 3 in Tabelle 48 stehen in Verbindung mit der Sicherung menschlicher Grundbedürfnisse und weisen minimale Beeinflussbarkeit sowie hohe Standortgebundenheit auf. Das tolerierbare individuelle Todesfallrisiko soll in dieser Kategorie nicht höher als $10^{-4} / \mathrm{Jahr}$ liegen. Der Wert von $10^{-4} / \mathrm{Jahr}$ entspricht dem Todesfallrisiko aus der Summe aller Ursachen für die Altersklasse mit der geringsten Sterblichkeitsrate, nämlich jene der 10-bis 14-jährigen (Flueler \& Seiler, 2003). Für Risikosituationen der Kategorie 1 besteht dagegen kein Bezug zur Sicherung körperlicher Grundbedürfnisse. Es besteht hohe Beeinflussbarkeit und geringe Standortgebundenheit. Das maximal tolerierbare individuelle Todesfallrisiko wird bei $10^{-2}$ pro Jahr angesetzt. Dieser Wert entspricht verbreiteten Angaben in der Literatur für vollkommen freiwillige Risiken (z. B. Merz et al., 1995) und etwa dem mittleren individuellen Todesfallrisiko aus der Summe aller Ursachen für alle Altersklassen (mittlere natürliche Sterbewahrscheinlichkeit).

Tabelle 48 Schutzziele zum maximal tolerierbaren individuellen Risiko.

\begin{tabular}{cccc}
\hline & \multicolumn{3}{c}{ Schutzziele } \\
$\begin{array}{l}\text { Risikoka- } \\
\text { tegorie }\end{array}$ & $\begin{array}{l}\text { Objektindex (Bedürfnis, } \\
\text { Einfluss und Kontext) }\end{array}$ & $\begin{array}{l}\text { Maximal tolerierbares individuel- } \\
\text { les Todesfallrisiko pro Jahr }\end{array}$ & $\begin{array}{l}\text { Maximal tolerierbares Sachrisiko } \\
\text { pro Individuum in CHF pro Jahr } \\
\text { (Kt. OW) }\end{array}$ \\
\hline 1 & $1-333$ & $10^{-2}$ & CHF 30'000 \\
2 & $333-666$ & $10^{-3}$ & CHF 25'000 \\
3 & $>666$ & $10^{-4}$ & CHF 20'000 \\
\hline
\end{tabular}

Grenzwerte wurden auch für Sachrisiken hergeleitet. Gewählt wurde ein Ansatz, nach welchem Risikokosten (Schadenskosten und Elementarversicherungsprämien) zu vermeiden sind, welche das durchschnittliche Einkommen eines Individuums unter das Einkommen reduzieren, das einem Existenzminimum entspricht. Es wird dabei vom mittleren Volkseinkommen pro Person ausgegangen. Individuelle wirtschaftliche Verhältnisse bleiben aus Gründen der Datenverfügbarkeit, aber auch aus prinzipiellen Gründen eines objektivierenden und rechtsgleichen Risikomanagements unberücksichtigt.

Der Vergleich zwischen dem jährlichen Primäreinkommen von ca. CHF 38'500 pro Kopf (Kt. OW, Zahlen 2004 aus BFS, 2007) und den Einkommensverhältnissen im Fall des Existenzminimums (siehe Kapitel 3.3.2.1) zeigt, dass eine jährliche Risikobelastung von CHF 24'900 bis 28'500 vorliegen muss, um eine Person mit mittlerem Volkseinkommen in den Bereich des Existenzminimums zu versetzen. Dies gilt für Privatpersonen. Für viele Situationen, z. B. Unternehmungen oder öffentliche Anlagen in Risikogebieten, sind keine allgemeingültigen Angaben zu Existenzminima bekannt oder eruierbar, anhand welcher entsprechende Werte für tolerierbare jährliche Risikobelastungen hergeleitet werden könnten. Die Grenzwerte für tolerierbare individuelle Sachrisiken wurden zwischen CHF 20'000 und CHF 30'000 pro Jahr angesetzt (Tabelle 48). Jährliche individuelle Sachrisiken in dieser Höhe dürften nur selten vorliegen. Das nahezu gesamtschweizerisch geltende Obligatorium, Gebäude gegen Elementarschäden zu versichern, reduziert die individuelle Risikobelastung selbst in stark gefährdeten Gebieten erheblich. Dies ist umso mehr der Fall, als die meisten Versicherer ihre Prämiensätze nicht oder nur in geringem Mass auf die Risikosituation abstimmen. Nach eigenen Berechnungen wären oberhalb der erwähnten Werte liegende individuelle Risikobelastungen im Bereich nicht versicherbarer Elementarschäden, z. B. Landwirtschaftsbetriebe mit grossen Flächen in Gebieten mittlerer oder grosser Gefährdung, denkbar. 
Zur Untersuchung individueller Sachrisiken müssen diese einem Individuum zugeordnet werden. Welche Schäden und Risiken welchen Individuen zuzurechnen sind, wäre im Einzelfall anhand der Eigentumsverhältnisse abzuklären, was für grosse Untersuchungsgebiete mit prohibitivem Aufwand verbunden wäre. Für das Konzept und Fallbeispiel wurden Arten von Schadensobjekten herausgegriffen, die typischerweise einzelnen Individuen zugeordnet werden können, wie selbst genutztes Grund- oder Wohneigentum oder Kleingewerbe. Diesen Objektkategorien fällt eine Indikatorfunktion für (unzulässig) hohe Sachrisiken zu. Wie repräsentativ und robust diese Stellvertreter weitere individuelle Sachrisiken (z. B. individuelle Sachrisiken bei öffentlich genutzten Anlagen oder grösseren Industriebetrieben) abbilden, wird hier nicht weiter untersucht.

Im untersuchten Fallbeispiel (Kapitel 5.1.3) wurde davon ausgegangen, dass eine Lebensgemeinschaft von zwei betroffenen Personen 1 ha Wald, 20 ha Weide, 10 ha Wiesland (durchschnittliche Grösse eines Landwirtschaftsbetriebs Kt. OW), 1.6 ha Schutzwald, 1 Einzelgebäude, 1 grossen Stall oder 1 Gewerbebaute besitzt. Daraus wurden anhand der Werte von Tabelle 48 maximal tolerierbare jährliche Sachrisiken für diese Objektkategorien berechnet (Tabelle 49).

Tabelle 49 Jährliche maximal tolerierbare Sachrisiken pro Are, berechnet aufgrund von Annahmen zu typischen Eigentumsverhältnissen (Ausgangslage: Werte Kanton OW).

\begin{tabular}{|c|c|c|c|c|c|}
\hline \multirow[t]{2}{*}{ Objektart } & \multirow[t]{2}{*}{$\begin{array}{l}\text { Risikokategorie } \\
\text { (Tabelle } 48 \text { und } \\
\text { Anhang III) }\end{array}$} & \multirow{2}{*}{$\begin{array}{l}\text { Einem Indivi- } \\
\text { duum typischer- } \\
\text { weise zugeordne- } \\
\text { te Fläche } \\
\text { Are }\end{array}$} & \multicolumn{3}{|c|}{$\begin{array}{l}\text { Jährliche maximal tolerierbare Sach- } \\
\text { risiken pro Are bei einem jährlichen } \\
\text { maximal tolerierbaren individuellen } \\
\text { Sachrisiko von CHF }\end{array}$} \\
\hline & & & $200^{\prime} 000$ & 25,000 & 30,000 \\
\hline Wald & 2 & 50 & -- & 500 & -- \\
\hline Weideland & 2 & $1 ' 000$ & -- & 25 & -- \\
\hline Wiesland & 2 & 500 & -- & 50 & -- \\
\hline Schutzwald (BSF) & 3 & 80 & 250 & -- & -- \\
\hline Dauernd bew. Einzelgebäude & 3 & 0.5 & $400^{\prime} 000$ & -- & -- \\
\hline Zeitw. bew. Einzelgebäude & 3 & 0.3 & $65^{\prime} 000$ & -- & -- \\
\hline Grosse Ökonomiegebäude & 3 & 1 & $200^{\prime} 000$ & -- & -- \\
\hline Einfamilienhauszone & 3 & 1 & $200^{\prime} 000$ & -- & -- \\
\hline Gewerbebaute & 3 & 1 & $200^{\prime} 000$ & -- & -- \\
\hline
\end{tabular}

Mit einer GIS-Analyse [Geographisches Informationssystem] lässt sich feststellen, ob und in welchem Mass in den einzelnen Gefahrengebieten maximal tolerierbare Personen- und Sachrisiken überschritten werden. Auf diese Weise werden die Gefahrengebiete und Stellen mit Handlungsbedarf nach Regel 1 eruiert. Die Gefahrengebiete, in denen Handlungsbedarf ermittelt wurde, lassen sich anschliessend nach dem Ausmass der Überschreitung tolerierbarer Risiken (Höhe der Überschreitung, Grösse der davon betroffenen Flächen) rangieren. 


\subsubsection{Priorisierung der Massnahmen nach Regel 2}

Die Anwendung von Regel 2 bezweckt die Erstellung einer Prioritätenliste von Massnahmen zur Verminderung kollektiver Risiken. Zu diesem Zweck wird ein Prioritätsindex ermittelt. Dieser ist das Produkt aus dem Objektindex (Kapitel 4.2.3.2), dem Massnahmenindex (Kapital 4.2.3.3.) und einem Gefährdungsfaktor (Kapital 4.2.3.4; Formel 2 und Abbildung 44).

$$
\text { Prioritätsindex = Objektindex * Gefärdungsfaktor * Massnahmenindex [2] }
$$

Neben objekt- und massnahmenspezifischen Elementen fliessen ortsspezifische Informationen in die Berechnung des Prioritätsindex ein. Einerseits wird anhand von Eignungskriterien für jedes Gefahrengebiet eine theoretisch optimale Massnahme ermittelt. Anderseits wird zur Berücksichtigung der örtlichen Gefährdung ein Gefährdungsfaktor eingeführt, mit dem dem nach Gefahrenprozess und Gefahrenstufe abgestuften Risiko Rechnung getragen wird.

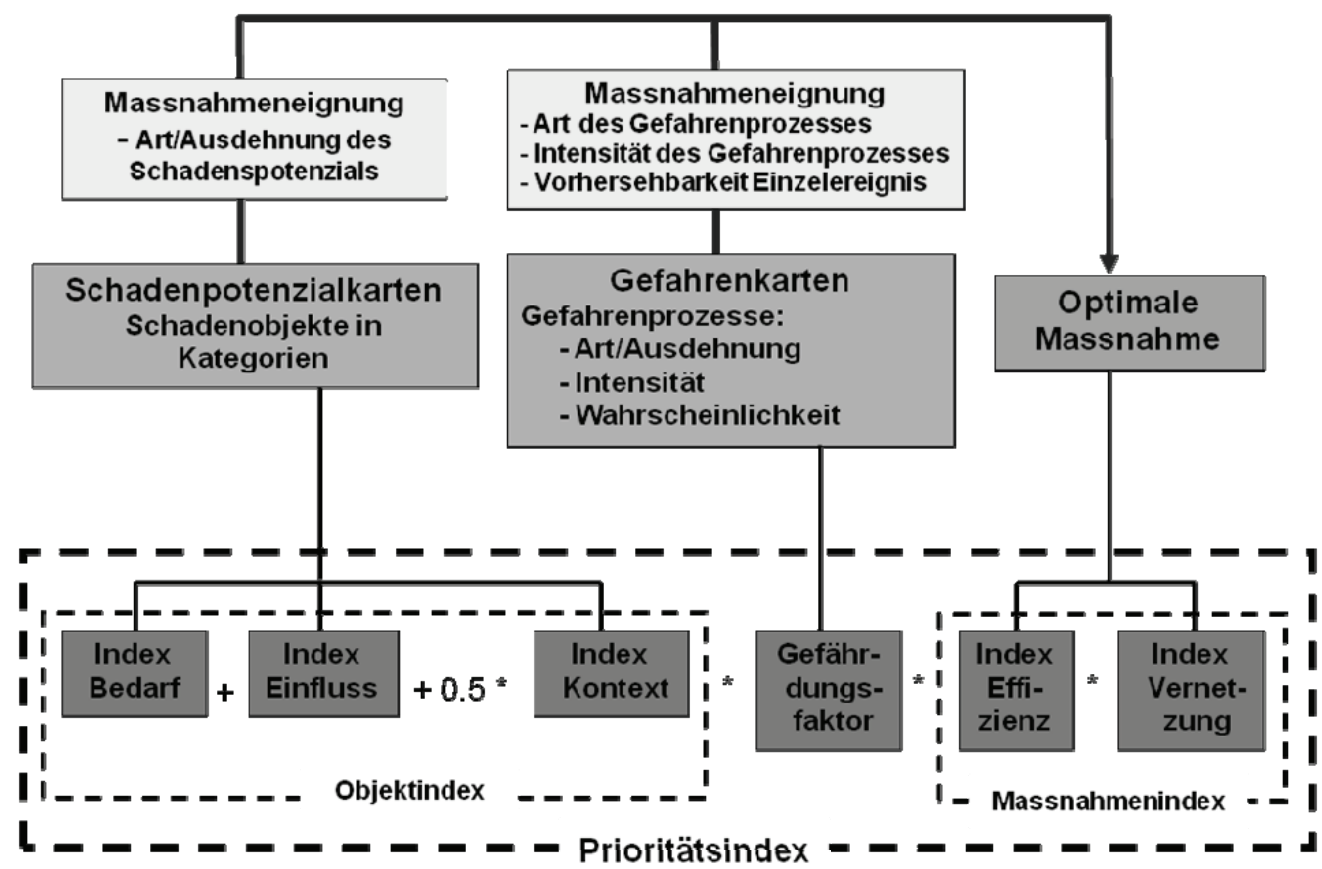

Abbildung 44 Ermittlung eines Prioritätsindexes aufgrund der Schutzzielprinzipien und auf Basis von Schadenspotenzial- und Gefahrenkarten.

\subsubsection{Die Elemente des Prioritätsindexes}

\subsubsection{Bewertung der Objekte und Risikoverminderungsmassnahmen}

Die dem Risiko ausgesetzten Objekte einerseits und die Risikoverminderungsmassnahmen anderseits werden mit den fünf Schutzzielprinzipien in Beziehung gebracht. Objekte und Massnahmen werden aufgrund prinzipienspezifischer Kriterien bewertet (Kapitel 3.3.2 und Tabelle 50). Diese Beurteilung wird verallgemeinernd vorgenommen und erfolgt anhand einer Frageliste (Tabelle 51 und Tabelle 52). 
Tabelle 50 Kriterien zur Bewertung von gefährdeten Objekten und von Risikoverminderungsmassnahmen.

\begin{tabular}{llll}
\hline Bezug & Prinzip & Kriterium \\
\hline Gefährdetes Objekt & Bedürfnis & Art des verletzbaren Wertes \\
& Einfluss & Bedeutung des verletzbaren Wertes \\
& & Selbstbestimmung \\
\hline Risikoverminderungsmassnahme & Kontext & Eigener Nutzen \\
& & Standortgebundenheit \\
& & Besitzstandswahrung \\
& & Wirksamkeit \\
& Vernetzung & Kosten \\
& & Umweltverträglichkeit \\
& & Sozialverträglichkeit \\
\hline
\end{tabular}

Anhand dieser Kriterien wird jedes Objekt resp. jede Massnahme auf einer Skala zwischen 1 und 10 bewertet. Eine solche Bewertung kann grundsätzlich für jede Region spezifisch erfolgen. Die objektbezogene Bewertung wird in einem Objektindex zusammengefasst, der das Mass schutzwürdiger Interessen darstellt (Kapitel 4.2.3.2). Die massnahmenbezogene Bewertung ergibt einen Massnahmenindex, der die Effizienz sowie die Umwelt- und Sozialverträglichkeit einer Massnahme ausdrückt.

\subsubsection{Der Objektindex}

Die Beurteilung der objektspezifischen Kriterien erfolgt gemäss den Fragen in Tabelle 51 für die gleichen Objekte und Objektkategorien, wie sie für die Erstellung von Schadenspotenzialkarten und Risikoanalysen in der Praxis verbreitet angewendet werden (Borter, 1999; Anhang III). Die Bewertung kann durch Betroffene und Vertreter verschiedener gesellschaftlicher Interessen (Laien) erfolgen. Sie sollte möglichst breit in der Bevölkerung abgestützt sein. 
Tabelle 51 Kriterien und Fragen zur Bewertung von gefährdeten Objekten.

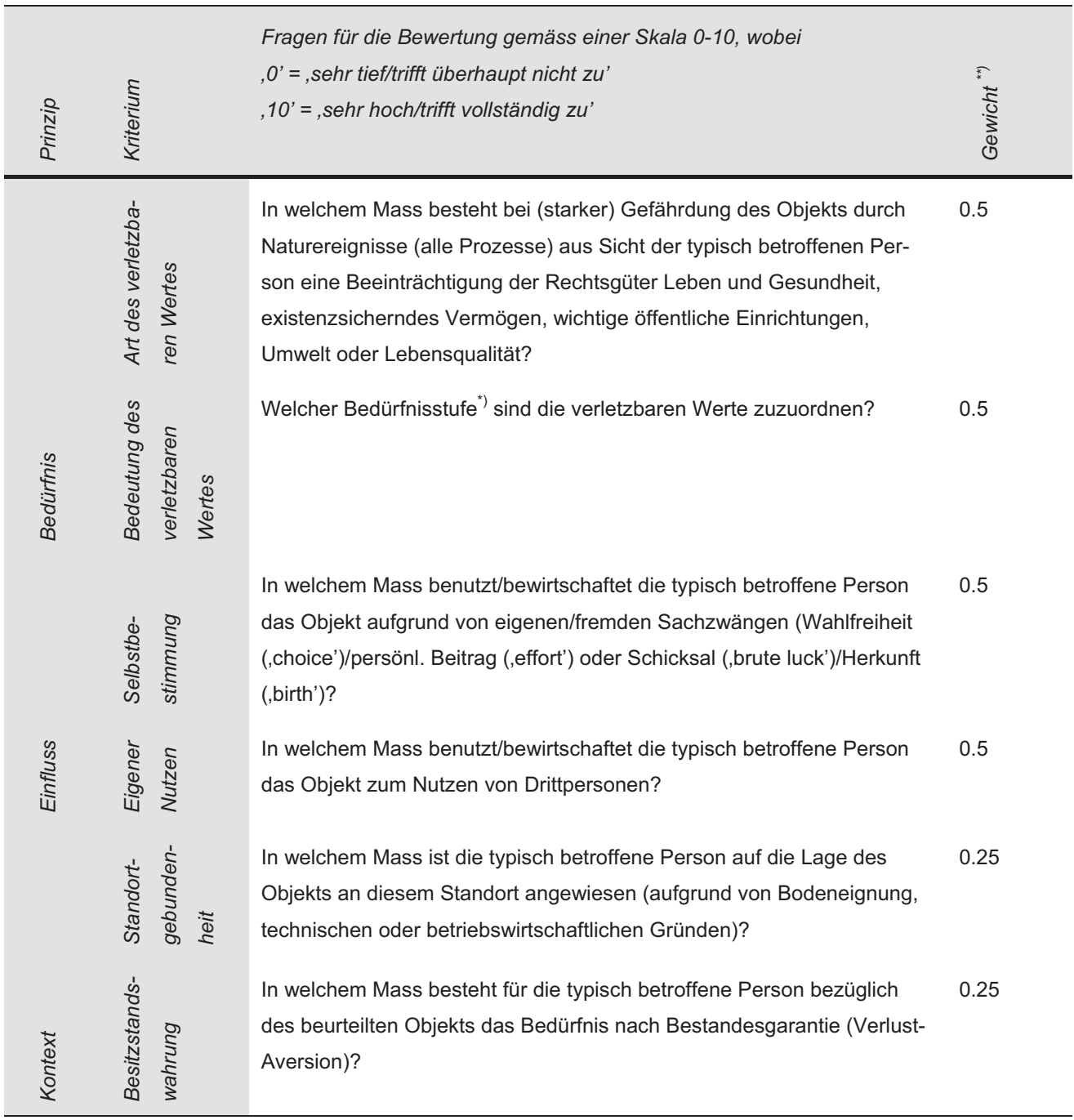

\footnotetext{
$\left.{ }^{*}\right)$ Bedürfnisstufe nach Maslow (Kapitel 3.3.2.1).

${ }^{\star *}$ Für das Fallbeispiel in Kapitel 5 verwendete Gewichtung.
}

Die Kriterien des Kontextprinzips weisen im Vergleich zu anderen Kriterien in höherem Mass einen subjektiven Charakter auf und lassen sich deshalb am wenigsten verallgemeinern. Diese Vermutung bestätigte sich bei der Bewertung im Fallbeispiel (Kapitel 5). Für die Berechung des Objektindexes wurden deshalb die Kontextkriterien nur zur Hälfte gewichtet. Das Ergebnis aus dem Fallbeispiel für die Gemeinde Engelberg (Kapitel 5) ist in Anhang X dargestellt. 
Bei der Ermittlung des Objektindexes stellte sich die Frage, aus welcher Optik die Bewertung erfolgt. Es ist nicht nur die Art eines Objekts an sich massgebend, sondern insbesondere auch, wer von einem Schaden an diesem Objekte betroffen ist, resp. aus wessen Optik dieser Schaden zu messen ist (vgl. Kapitel 2.2.1). Die Bewertung erfolgte deshalb für die meisten Objekte sowohl für den Eigentümer/Betreiber als auch für den Nutzniesser in der Rolle der ,typischerweise betroffenen Person'.

Der Objektindex berechnet sich nach der Formel

$$
\text { Objektindex }=\text { Bedarfsindex }+ \text { Einflussindex }+0.5 * \text { Kontextindex [3] }
$$

Das Ergebnis des nach Formel 3 berechneten Objektindexes wird auf eine Spannweite von 1 bis 1'000 skaliert (1 = tiefster Wert, 1'000 = höchster Wert). Damit wird die empirische Abstufung der Arten von Risiken in der Grössenordnung von 1:1'000 nachgebildet (Schneider, 1996).

\subsubsection{Der Massnahmenindex}

Die Prinzipien ,Effizienz' und ,Vernetzung' beziehen sich auf Massnahmen. Folgende Arten von Massnahmen werden im Rahmen des Konzeptes berücksichtigt:

$\begin{array}{ll}\text { Risikoabklärung } & \text { Ereignisdokumentation/-kataster } \\ & \text { Gefahrenkartierung } \\ & \text { Risikoanalyse } \\ \text { Bewusstseinsbildung } & \text { Ausbildung } \\ \text { Information } & \text { Risikodialog } \\ \text { bauliche Massnahmen } & \text { im Anriss-/Entstehungsgebiet } \\ & \text { im Transitgebiet } \\ & \text { im Ablagerungsgebiet } \\ & \text { am gefährdeten Objekt } \\ \text { biologische Massnahmen } & \text { Ingenieurbiologischer Verbau } \\ & \text { Schutzwaldbegründung/-pflege } \\ \text { planerische Massnahmen } & \text { Ausscheidung Gefahrenzonen } \\ \text { Interventionsmassnahmen } & \text { baupolizeiliche Anordnungen } \\ & \text { Warnung } \\ & \text { Evakuierung/Sperrung } \\ & \text { temp. Objektschutzmassnahmen } \\ & \text { Rettung } \\ & \text { Versicherung } \\ \text { Wiederherstellungsmassnahmen } & \text { Hilfeleistungen }\end{array}$

Der Massnahmenkatalog lässt sich aufgrund der Gegebenheiten in jeder Region anpassen.

Zur Bewertung des Kriteriums Sozialverträglichkeit werden die FSC-Prinzipien (FSC, 2004) und die Grundsätze des BAFU zu Programmvereinbarungen bei der Umsetzung des NFA [Neuer Finanzausgleich] (BAFU, 2007a) herangezogen. Die Beurteilung der Umweltverträglichkeit erfolgt anhand der Kriterien, die bei Umweltverträglichkeitsprüfungen angewendet werden (BUWAL, 1990; grEIE, 2004). 
Die Bewertung der Effizienz erfolgt anhand der Kriterien Wirksamkeit und Kosten. Die Wirksamkeit einer Massnahme kann nicht allgemeingültig bewertet werden, da sie stark von ihrer Eignung im Einzelfall abhängt. Wo sich welche Massnahme am besten eignet, ergibt sich aus verschiedenen gefahren- und gebietsspezifischen Charakteristiken (Tabelle 53).

Die Kriterien des Vernetzungsindexes, die Wirksamkeits- und Kostenkriterien sowie die gebietsspezifischen Charakteristiken werden mittels einer Beurteilung abgeschätzt. Für diese Abschätzung werden Bewertungsfragen formuliert (Tabelle 52 und 53; Resultate Beurteilung Fallbeispiel Kapitel 5 siehe Anhang XI bis XIII). Beurteilende sind - anders als beim Objektindex - Experten, da technische Kenntnisse erforderlich sind.

Tabelle 52 Kriterien und Fragen zur Bewertung von Risikoverminderungsmassnahmen.

\begin{tabular}{|c|c|c|c|}
\hline Prinzip & Kriterium & $\begin{array}{l}\text { Fragen für die Bewertung gemäss einer Skala 0-10, wobei } \\
, 0^{\prime}=\text {, sehr tief/trifft überhaupt nicht zu' } \\
, 10^{\prime}=\text {, sehr hoch/trifft vollständig zu' }\end{array}$ & 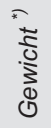 \\
\hline 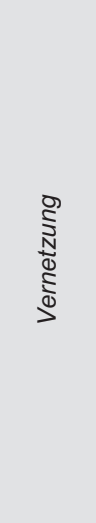 & 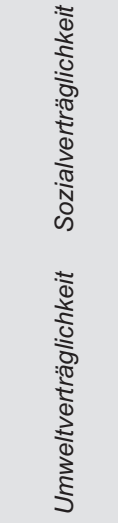 & $\begin{array}{l}\text { In welchem Mass sind Massnahmen hinsichtlich Beeinträchtigung von Grund- } \\
\text { rechten, Nutzung der Landschaft (Erholung, Landwirtschaft usw.), Schutz } \\
\text { spezifischer Interessen und Ressourcenschutz verträglich? } \\
\text { In welchem Mass bieten Massnahmen Möglichkeiten zur Partizipation der } \\
\text { Betroffenen? } \\
\text { In welchem Mass sind Massnahmen hinsichtlich ökolog. Vielfalt, Arten- } \\
\text { schutz/Artenvielfalt, Landschaftsbild/Ortsbild, Boden, } \\
\text { Wald/Landwirtschaftsland, Luftbelastung, Lärm, Erschütterungen verträglich? }\end{array}$ & 0.5 \\
\hline 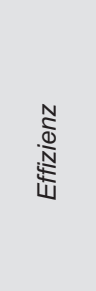 & 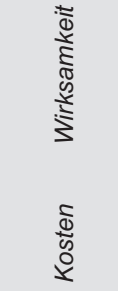 & $\begin{array}{l}\text { Wie wirksam ist die optimal geeignete Massnahme typischerweise? } \\
\text { Wie wirksam ist diese Massnahme als Einzelmassnahme? } \\
\text { In welchem Mass generiert eine Massnahme Kosten für Investitionen, Betrieb } \\
\text { und Unterhalt sowie entgangene Nutzen? }\end{array}$ & 0.5 \\
\hline
\end{tabular}

${ }^{*}$ Für das Fallbeispiel in Kapitel 5 verwendete Gewichtung.

Der Massnahmenindex berechnet sich nach der Formel

$$
\text { Massnahmenindex }=\text { Effizienzindex * Vernetzungsindex [4] }
$$

wobei $\quad$ Effizienzindex $=\frac{W^{*} S y n * L}{C^{*} E Z G}$ [5]

$\begin{array}{lll}\mathrm{W} & = & \text { Index für die Wirksamkeit einer Massnahme } \\ \mathrm{Syn} & = & \text { Synergiefaktor }\left(=, 1^{\prime}, \text { wenn die Massnahme als Einzelmassnahme wirksam ist) }\right. \\ \mathrm{L} & = & \text { Faktor Lebensdauer einer Massnahme } \\ \mathrm{C} & = & \text { Summe der Indexwerte für Massnahmenkosten (Investitionen, Unterhalt, entgangener Nutzen) } \\ \mathrm{EZG}= & \text { Faktor Einzugsgebietsgrösse }\end{array}$


Für jede Kombination von Gebietsmerkmalen (gefahren- und gebietsspezifische Charakteristiken) ergibt sich eine theoretisch am besten geeignete Massnahme, jene mit dem höchsten Massnahmenindex. So erweist sich in einem Lawinenzug (brutaler Prozess mit eher guter Voraussehbarkeit von Einzelereignissen) mit einem grossen Einzugsgebiet und mit zerstreut angeordnetem Schadenspotenzial die Anordnung lokaler Objektschutzmassnahmen als solche mit dem höchsten Massnahmenindex und somit als die optimale Lösung. Jedem Lawinenzug mit diesen Eigenschaften wird dieser Massnahmenindex zugeordnet, welcher wiederum in die Berechnung des Prioritätsindexes einfliesst.

Tabelle 53 Charakterisierung von Gefahrengebieten nach gefahrenspezifischen und örtlichen Merkmalen.

\begin{tabular}{|c|c|c|c|}
\hline Kriterium & Attribut & Beispiel & Bemerkung \\
\hline \multirow{2}{*}{$\begin{array}{l}\text { Art des Gefahrenpro- } \\
\text { zesses }\end{array}$} & brutal & Lawine, Steinschlag, Murgang & \\
\hline & graduell & Hochwasser, Rutschung & Flucht möglich. \\
\hline \multirow{2}{*}{$\begin{array}{l}\text { Intensität des Gefah- } \\
\text { renprozesses }\end{array}$} & hoch & Lawinendrücke $>30$ kN/m² & \\
\hline & $\begin{array}{l}\text { mittel } \\
\text { gering }\end{array}$ & Lawinendrücke $<30 \mathrm{kN} / \mathrm{m}^{2}$ & $\begin{array}{l}\text { Verhältnismässige Objektschutz- } \\
\text { massnahmen möglich. }\end{array}$ \\
\hline \multirow{2}{*}{$\begin{array}{l}\text { Häufigkeit des Gefah- } \\
\text { renprozesses }\end{array}$} & hoch & Jährlichkeit oft < 30 Jahre & \\
\hline & $\begin{array}{l}\text { mittel } \\
\text { selten }\end{array}$ & Jährlichkeit oft <100 Jahre & $\begin{array}{l}\text { Kapitalintensive Massnahmen weni- } \\
\text { ger wirtschaftlich. }\end{array}$ \\
\hline \multirow{2}{*}{$\begin{array}{l}\text { Vorhersehbarkeit } \\
\text { Einzelereignis }\end{array}$} & hoch & Lawine, Hochwasser, Sturm & Frühwarnung möglich. \\
\hline & gering & Sturz, Murgang & \\
\hline \multirow[t]{2}{*}{ Grösse Einzugsgebiet } & sehr gross & $>10 \mathrm{~km}^{2}$ & Flächige Massnahmen im Einzugs- \\
\hline & mittelklein & $<10 \mathrm{~km}^{2}$ & $\begin{array}{l}\text { gebiet werden mit zunehmender } \\
\text { Einzugsgebietsgrösse aufwendiger } \\
\text { und damit weniger wirtschaftlich. }\end{array}$ \\
\hline \multirow{3}{*}{$\begin{array}{l}\text { Anordnung der ge- } \\
\text { fährdeten Objekte }\end{array}$} & punktuell & Einzelgebäude & Je grossflächiger die gefährdeten \\
\hline & flächig & geschlossene Siedlung & $\begin{array}{l}\text { Objekte beschaffen oder angeordnet } \\
\text { sind, desto eher müssen flächig wirk- }\end{array}$ \\
\hline & linear & Verkehrsweg, Leitung & same Massnahmen ergriffen werden. \\
\hline
\end{tabular}

Interessant und bemerkenswert ist, welche Massnahmen aufgrund dieser Charakterisierung der Gefahrengebiete die beste Eignung und damit die höchsten Werte für Massnahmenindizes aufweisen (Tabelle 54).

Als optimale Massnahmen erweisen sich in vielen Fällen bauliche Präventionsmassnahmen am gefährdeten Objekt (lokale Objektschutzmassnahmen). Ebenso sind in vielen Fällen Interventionsmassnahmen (Evakuation, Sperrung sowie Rettung nach Ereignissen) optimal. In einigen Fällen wurde sogar die Hilfeleistung bei der Wiederherstellung nach Ereignissen als beste Massnahme evaluiert. Schutzwaldpflege erweist sich in einem Fall als günstigste Massnahme. Bauliche Präventionsmassnahmen im Einzugs- und Transitgebiet waren in keinem Fall optimale Lösung. Die detaillierten Ergebnisse sind in Anhang XIII dargestellt. 
Tabelle 54 Optimale Massnahme in Abhängigkeit von Merkmalen des Gefahrenprozesses und -gebietes sowie der Anordnung des Schadenspotenzials.

\begin{tabular}{|c|c|c|c|c|c|c|c|}
\hline \multicolumn{3}{|c|}{ Art der Gefährdung } & \multirow{2}{*}{ 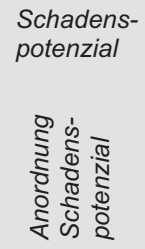 } & \multicolumn{2}{|c|}{ Vorwiegend häufige Ereignisse } & \multicolumn{2}{|c|}{$\begin{array}{l}\text { Vorwiegend seltene } \\
\text { Ereignisse }\end{array}$} \\
\hline 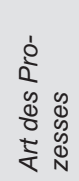 & 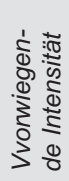 & 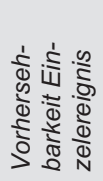 & & $\begin{array}{l}\text { Kleine bis mittlere } \\
\text { Einzugsgebiete }\end{array}$ & $\begin{array}{l}\text { Grosse Einzugs- } \\
\text { gebiete }\end{array}$ & $\begin{array}{l}\text { Kleine bis } \\
\text { mittlere } \\
\text { Einzugs- } \\
\text { gebiete }\end{array}$ & $\begin{array}{l}\text { Grosse } \\
\text { Einzugs- } \\
\text { gebiete }\end{array}$ \\
\hline \multirow{12}{*}{ 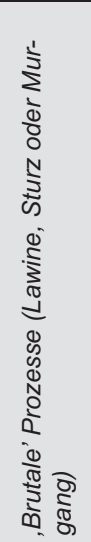 } & \multirow{6}{*}{$\begin{array}{l}\text { के } \\
\text { गे } \\
\text { के }\end{array}$} & \multirow{3}{*}{ కั } & punktuell & \multicolumn{2}{|l|}{ lokaler Objektschutz } & \multirow{6}{*}{ Sperrung/E } & \multirow{6}{*}{ vakuierung } \\
\hline & & & flächig & \multirow{2}{*}{ Hilfeleistungen } & \multirow{2}{*}{$\begin{array}{l}\text { Sperrung/ } \\
\text { Evakuierung }\end{array}$} & & \\
\hline & & & linear & & & & \\
\hline & & \multirow{4}{*}{ 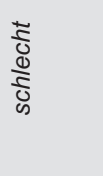 } & punktuell & \multicolumn{2}{|l|}{ lokaler Objektschutz } & & \\
\hline & & & flächig & \multirow{2}{*}{ Hilfeleistungen } & \multirow{2}{*}{$\begin{array}{l}\text { Sperrung/ } \\
\text { Evakuierung }\end{array}$} & & \\
\hline & & & linear & & & & \\
\hline & \multirow{6}{*}{ 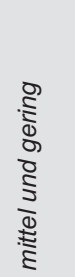 } & & punktuell & lokaler Objektschutz & & & \\
\hline & & \multirow{3}{*}{ కั } & flächig & \multirow{2}{*}{ Sperrung/Evakuierung } & & & \\
\hline & & & linear & & & & \\
\hline & & & punktuell & lokaler Objektschutz & & & \\
\hline & & \multirow[b]{2}{*}{ 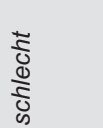 } & flächig & Schutzwaldpflege & \multirow{2}{*}{\multicolumn{3}{|c|}{ Sperrung/Evakuierung }} \\
\hline & & & linear & Sperrung/Evakuierung & & & \\
\hline \multirow{4}{*}{ 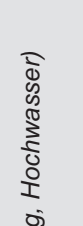 } & \multirow{7}{*}{$\begin{array}{l}\text { के } \\
0 \\
\vdots\end{array}$} & \multirow{4}{*}{ క్ } & punktuell & lokaler Objektschutz & \multirow{2}{*}{$\begin{array}{l}\text { lokaler Objekt- } \\
\text { schutz }\end{array}$} & \multirow{3}{*}{\multicolumn{2}{|c|}{ Sperrung/Evakuierung }} \\
\hline & & & flächig & \multirow{5}{*}{ Hilfeleistungen } & & & \\
\hline & & & linear & & $\begin{array}{l}\text { Sperrung/ } \\
\text { Evakuierung }\end{array}$ & & \\
\hline & & & punktuell & & $\begin{array}{l}\text { lokaler Objekt- } \\
\text { schutz }\end{array}$ & \multicolumn{2}{|l|}{ Rettung } \\
\hline \multirow{8}{*}{ 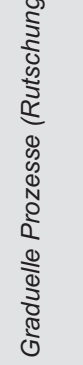 } & & \multirow{3}{*}{$\begin{array}{l}\frac{\tilde{J}}{\delta} \\
\frac{\Phi}{S} \\
\mathscr{S}\end{array}$} & flächig & & Hilfeleistungen & & Sperrung/ \\
\hline & & & linear & & $\begin{array}{l}\text { lokaler Objekt- } \\
\text { schutz }\end{array}$ & tungen & $\begin{array}{l}\text { Evakuie- } \\
\text { rung }\end{array}$ \\
\hline & & & punktuell & lekaler Obiektschutz & & lokaler Obj & itschutz \\
\hline & \multirow{5}{*}{ 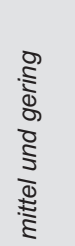 } & \multirow{3}{*}{ కั } & flächig & Iokaler Udjektschutz & & & \\
\hline & & & linear & Sperrung/Evakuierung & & & \\
\hline & & & punktuell & lokaler Objektschutz & & Sperrung/E & akuierung \\
\hline & & ป & flächig & Hilfeleistungen & lokaler Objekt- & & \\
\hline & & 空 & linear & lokaler Objektschutz & $\mathrm{s}$ & & \\
\hline
\end{tabular}

\subsubsection{Der Gefährdungsfaktor}

Der Gefährdungsfaktor drückt die örtliche Gefährdung aus. Er beschreibt das Verhältnis der Risiken, die bei verschiedenen Gefahrenprozessen und Gefahrenstufen für dasselbe Schadenspotenzial bestehen (Tabelle 55). Damit kommt zum Ausdruck, um wie viel höher beispielsweise das Risiko für ein Wohnhaus bei starker Murganggefährdung im Vergleich zum Risiko für ein Wohnhaus bei geringer Überschwemmungsfährdung ist.

Der Gefährdungsfaktor wird anhand von Werten zum spezifischen Schadensausmass pro Gefahrenprozess und Intensitätsstufe berechnet. Die Werte zum spezifischen Schadensausmass für den Prozess Überschwemmung basieren auf der Publikation ,Risikoanalyse bei gravitativen Naturgefahren' (Borter, 1999). Für alle anderen Prozesse wurde auf die 
Angaben in der Tabelle ,Objektparameter' des Risikoberechnungstools ,EconoMe' (BAFU, 2007b) zurückgegriffen. Fehlende Angaben zu spezifischen Todesfallrisiken wurden durch eigene Berechnungen ergänzt. Mit dieser Datenbasis ergibt sich für jede Objektkategorie oder jede Art von Raumnutzung ein spezifischer Gefährdungsfaktor. Zur Vereinfachung werden Vereinheitlichungen vorgeschlagen und im Fallbeispiel (Kapitel 5) angewendet:

- Der Gefährdungsfaktor berücksichtigt nur Sachschäden/Sachrisiken. Die Personenrisiken machen - monetarisiert mit CHF 1.2 Mio. pro Todesfall (Humankapitalansatz) im Untersuchungsgebiet des Fallbeispiels (Gemeinde Engelberg, Kt. OW) weniger als $10 \%$ des Gesamtrisikos aus. Für andere Untersuchungsgebiete ist dieses Verhältnis anhand der tatsächlichen Risikosituation zu überprüfen und über die Berücksichtigung von Personenrisiken zu entscheiden.

- Für jeden Gefahrenprozess wird nur ein Faktor pro Gefahrenstufe (rot, blau, gelb) evaluiert. Das spezifische Risiko pro Gefahrenstufe entspricht vereinfachend dem gewichteten Mittel der Schadenserwartungswerte aller Kombinationen von Intensitäten und Wahrscheinlichkeiten, die der gleichen Gefahrenstufe zugeordnet sind. Statt neun Faktoren pro Gefahrenprozess und Objektkategorie ergeben sich auf diese Weise noch deren drei.

- Objekte mit ähnlichen Gefährdungsfaktoren lassen sich zu Gruppen zusammenfassen. Die Vielfalt spezifischer Gefährdungsfaktoren wird auf einen Faktor für Gebäude (Faktor I), für Kulturland, Freiland und Wald (Faktor II) und Infrastrukturanlagen (Faktor III) reduziert.

Tabelle 55 Risikobasierte Gefährdungsfaktoren pro Gefahrenprozess und Gefahrenstufe.

\begin{tabular}{|c|c|c|c|c|c|}
\hline \multicolumn{6}{|c|}{ Faktor I Gebäude } \\
\hline & Überschwemmung & Lawine & Sturz & Murgang & Rutschung \\
\hline Risiko rot & 11.051 & 20.426 & 1.180 & 22.620 & 20.963 \\
\hline Risiko blau & 6.530 & 1.628 & 0.063 & 2.633 & 16.770 \\
\hline Risiko gelb & 0.716 & 0.019 & 0.001 & 0.007 & 0.021 \\
\hline \multicolumn{6}{|c|}{ Faktor II Kulturland/Freiland/Wald } \\
\hline & Überschwemmung & Lawine & Sturz & Murgang & Rutschung \\
\hline Risiko rot & 0.065 & 0.019 & 0.005 & 0.079 & 0.069 \\
\hline Risiko blau & 0.031 & 0.001 & 0.001 & 0.017 & 0.014 \\
\hline Risiko gelb & 0.002 & 0.001 & 0.001 & 0.001 & 0.001 \\
\hline \multicolumn{6}{|c|}{ Faktor III Verkehrsanlagen/Leitungen/Infrastruktur } \\
\hline & Überschwemmung & Lawine & Sturz & Murgang & Rutschung \\
\hline Risiko rot & 0.108 & 1.916 & 0.111 & 1.976 & 1.720 \\
\hline Risiko blau & 0.050 & 0.294 & 0.013 & 0.340 & 0.172 \\
\hline Risiko gelb & 0.004 & 0.026 & 0.001 & 0.006 & 0.017 \\
\hline
\end{tabular}

Überschwemmungen: Herleitung nach Borter, 1999. übrige Prozesse: Herleitung nach ,EconoMe' (BAFU, 2007b).

\subsection{Exkurs 1: Vereinfachte Abschätzung des regionalen Handlungsbedarfs}

Als Alternative zum dargelegten schutzzielbasierten Managementkonzept und zu den in der Praxis verbreiteten Verfahren zur Ermittlung von Schutzdefiziten wurde ein vereinfachter Ansatz erarbeitet. Dieser basiert auf den in Kategorien gruppierten risikoexponierten Objek- 
ten, der dreistufigen Gefahrenkarte und dem Massnahmenindex (Kapitel 4.2.3.3 und Abbildung 45).

\begin{tabular}{|c|c|c|c|c|c|}
\hline \multirow[b]{2}{*}{ Art des Schadenpotenzials } & \multirow[b]{2}{*}{$\begin{array}{c}\text { Freiwillig- } \\
\text { keits- } \\
\text { kategorie }\end{array}$} & \multicolumn{4}{|c|}{ Gefahrenstufe gemäss Gefahrenkarte } \\
\hline & & rot & blau & gelb & gelb-weiss \\
\hline $\begin{array}{l}\text { Bergwege } \\
\text { Kletterrouten } \\
\text { Naturlandschaften } \\
\text { Ödland } \\
\text { Skitourenrouten } \\
\text { Wanderwege } \\
\text { Skiabfahrtsgelände } \\
\text { Freizeitanlage, Camping } \\
\text { Grünanlage/Parkanlage }\end{array}$ & $\begin{array}{l}1 \\
1 \\
1 \\
1 \\
1 \\
1 \\
1 \\
1 \\
1 \\
\end{array}$ & & & & \\
\hline $\begin{array}{l}\text { Flurwege } \\
\text { unbewohntes Gebäude } \\
\text { Bergbahn, Schiene } \\
\text { Skiliftanlagen } \\
\text { Bergbahn Winter } \\
\text { Bergbahn Sommer } \\
\text { Bauzone unüberbaut }\end{array}$ & $\begin{array}{l}2 \\
2 \\
2 \\
2 \\
2 \\
2 \\
2\end{array}$ & & & & \\
\hline $\begin{array}{l}\text { Weideland } \\
\text { Gemeindestrassen } \\
\text { Agrarwirtschaft allgemein } \\
\text { Ackerland } \\
\text { Wiesland } \\
\text { Schutzwald } \\
\text { Kantonsstrassen } \\
\text { Stall } \\
\text { Gewerbebaute } \\
\text { Autobahn } \\
\text { Gewerbebaute: Anlage } \\
\text { Industriebaute: Anlage }\end{array}$ & $\begin{array}{l}3 \\
3 \\
3 \\
3 \\
3 \\
3 \\
3 \\
3 \\
3 \\
3 \\
3 \\
3\end{array}$ & & & & \\
\hline $\begin{array}{l}\text { Eisenbahn, einspurig } \\
\text { Ein-/Zweifamilienhaus } \\
\text { Mehrfamilienhaus } \\
\text { Doppelspureisenbahn } \\
\text { Siedlungsfläche allgemein } \\
\text { Siedlungskern } \\
\text { Ein-/Zweifamilienhauszone } \\
\text { Mehrfamilienhauszone }\end{array}$ & $\begin{array}{l}4 \\
4 \\
4 \\
4 \\
4 \\
4 \\
4 \\
4\end{array}$ & & & & \\
\hline
\end{tabular}

\section{Legende}

auch wenig effiziente Massnahmen $($ Index < 10) geeignet

Massn. mit hoher und mittl. Effizienz (Index 10-20) geeignet

nur sehr effiziente Massnahmen (Index $>20$ ) geeignet

Abbildung 45 Vereinfachte Abschätzung des Handlungsbedarfs anhand Gefahrenkarte, Objektkategorien und Massnahmeneffizienz (weisse Felder: keine Massnahmen).

Der Massnahmenindex wird bei diesem Verfahren nach der gleichen Methode ermittelt und den einzelnen Gefahrengebieten zugewiesen wie in Kapitel 4.2.3.3 dargestellt. Anhand der Gefahrenstufe und dem Massnahmenindex wird pro Gefahrengebiet der Handlungsbedarf 
abgeschätzt. Das Ergebnis ist eine ,Handlungsbedarfskarte', welche nach einem Dreistufenkonzept aufzeigt,

- wo Massnahmen dringend zu ergreifen sind;

- wo Massnahmen unter geeigneten Voraussetzungen ergriffen werden können und

- wo keine Massnahmen notwendig erscheinen.

Dieser Ansatz ist auf die regionale Abschätzung von Handlungsbedarf und Prioritäten ausgerichtet. Er wird im Fallbeispiel getestet (Kapitel 5.1.3).

\subsection{Exkurs 2: Modifikation der BUWAL-Schutzzielmatrix}

Im praktischen Management von Naturrisiken findet die 1999 publizierte, semiquantitative Schutzzielmatrix (Borter, 1999) aufgrund der direkten Anbindung an Gefahren- und Schadenspotenzialkarten sehr verbreitete Anwendung (Kapitel 2.7.6, Abbildung 27). Wesentliches Merkmal dieser Schutzzielmatrix ist die Rangierung und Klassierung der Objekt- und Raumnutzungsarten in sogenannte Objektkategorien. Diesen Objektkategorien sind für drei Häufigkeitsklassen von Ereignissen (0- bis 30-jährlich, 30- bis 100-jährlich, 100- bis 300-jährlich) maximal tolerierbare Intensitäten zugeordnet. Die in der Schutzzielmatrix pragmatisch und intuitiv gebildeten Objektkategorien sollen basierend auf den Erkenntnissen aus den vorangehenden Kapiteln in zweierlei Hinsicht überprüft werden.

Zum einen ergibt die oben beschriebene Bewertung der Objektarten mittels Objektindex eine - von der klassischen Schutzzielmatrix abweichende - Rangierung der Objekt- und Raumnutzungsarten. Diese führt im Vergleich zur ursprünglichen, ,klassischen' Schutzzielmatrix bei einzelnen Objektarten zur Veränderungen der Objektkategorie. Die Zuordnung in eine höhere Klasse (Objektkategorie) führt zu einem höheren Schutzziel. Die Klassenzugehörigkeit hängt erwartungsgemäss auch davon ab, ob die Bewertung des Objektindexes aus Sicht des Benutzers oder des Eigentümers erfolgte (Tabelle 56, Anhang XIV, 1 und 2).

Zum anderen wurde die Schutzzielmatrix aufgrund der Ergebnisse der Berechnungen in Kapitel 2.6.2.2 verändert (Anhang XIV, 3). Objektarten, bei denen die nach Schutzzielmatrix berechneten tolerierbaren individuellen Todesfallrisiken einen mehr als hundertmal höheren Wert ergaben als die entsprechenden Angaben in der Literatur, wurden der - im Vergleich zur klassischen Schutzzielmatrix - nächst höheren Objektkategorie zugeordnet. Objekte, für die das berechnete individuelle Risiko weniger als ein Hundertstel der Angaben in der Literatur betrug, wurden dagegen der nächst tieferen Kategorie zugeteilt.

Insgesamt wurden somit drei Modifikationen der klassischen Schutzzielmatrix entwickelt und im Fallbeispiel (Kapitel 5.1.4) verifiziert:

- Schutzzielmatrix aufgrund des Objektindexes aus Sicht ,Benutzer eines Objektes';

- Schutzzielmatrix aufgrund des Objektindexes aus Sicht ,Eigentümer eines Objektes';

- Schutzzielmatrix aufgrund der Berechnungen zum individuellen Todesfallrisiko.

Die modifizierten Schutzzielmatrizes sind in den Anhängen XIV, 1 bis 3 dargestellt. In Anhang XIV, 4 wird die Zuordnung der einzelnen Objektarten zu Objektkategorien nach der ,klassischen' und den drei modifizierten Schutzzielmatrizes vergleichend dargestellt. 
Tabelle 56 Unterschiede in der Zuordnung von Objekten zu Objektkategorien zwischen der ,klassischen' Schutzzielmatrix (Borter, 1999) und der Rangierung nach Objektindex.

\begin{tabular}{|c|c|c|}
\hline $\begin{array}{l}\text { Abweichung der Klassenzuge- } \\
\text { hörigkeit im Vergleich zur ,klas- } \\
\text { sischen' Schutzzielmatrix }\end{array}$ & $\begin{array}{l}\text { Betroffene Objektarten } \\
\text { (mit Nr. gemäss Schutzzielmatrix Bort } \\
\text { aus Sicht der } \\
\text { Benutzer }\end{array}$ & Eigentümer \\
\hline $\begin{array}{l}\text { Tiefere Zuordnung um zwei } \\
\text { oder mehr Kategorien }\end{array}$ & $\begin{array}{l}\text { touristische Anlagen (423), } \\
\text { Campingzonen (524), } \\
\text { Grünanlagen (525) }\end{array}$ & Grünanlagen (525) \\
\hline $\begin{array}{l}\text { Tiefere Zuordnung um eine } \\
\text { Kategorie }\end{array}$ & Flurstrassen (321) & Flurstrassen (321) \\
\hline $\begin{array}{l}\text { Höhere Zuordnung um eine } \\
\text { Kategorie }\end{array}$ & $\begin{array}{l}\text { extensive Landwirtschaftsgebiete } \\
(231) \\
\text { Wälder }(231,233) \\
\text { unbewohnte Einzelgebäude (311) } \\
\text { Leitungen von kantonaler Bedeutung } \\
(322) \\
\text { Ökonomiegebäude (4131) }\end{array}$ & $\begin{array}{l}\text { Weideland (231) } \\
\text { Wälder }(232,233) \\
\text { unbewohnte Einzelgebäude (311) } \\
\text { Ökonomiegebäude (4131) }\end{array}$ \\
\hline $\begin{array}{l}\text { Höhere Zuordnung um zwei } \\
\text { oder mehr Kategorien }\end{array}$ & $\begin{array}{l}\text { Leitungen von kommunaler Bedeu- } \\
\text { tung (222) } \\
\text { Schutzwälder (333) } \\
\text { dauernd bewohnte Einzelgebäude } \\
\text { (4111) }\end{array}$ & $\begin{array}{l}\text { Leitungen von kommunaler Bedeu- } \\
\text { tung (222) } \\
\text { Leitungen von kantonaler Bedeu- } \\
\text { tung (322) } \\
\text { Schutzwälder (333) } \\
\text { dauernd bewohnte Einzelgebäude } \\
\text { (4111) } \\
\text { Leitungen von nationaler Bedeu- } \\
\text { tung (422) } \\
\text { touristische Personenförder- } \\
\text { anlagen (423) } \\
\text { Campingzonen (524) }\end{array}$ \\
\hline
\end{tabular}

\subsection{Reduktion untolerierbarer Risiken}

\subsubsection{Regionales Risikomanagement}

Aus der Anwendung der Regel 1 resultiert eine Darstellung der Schutzdefizite sowohl hinsichtlich individueller Personenrisiken als auch - soweit für schweizerische Verhältnisse relevant - hinsichtlich individueller Sachrisiken. Das Ergebnis ist vergleichbar mit der herkömmlichen Darstellung der Schutzdefizite in den Schutzdefizitkarten gemäss ,Risikoanalyse bei gravitativen Naturgefahren', Stufe 1 (Borter, 1999). Die nach Regel 1 ermittelten Resultate sind quantitativ und ermöglichen im Vergleich zu den Schutzdefizitkarten präzisere Aussagen. 
Die Reihenfolge der Massnahmen, die sich aus der Analyse des Handlungsbedarfs nach Regel 1 ergibt, richtet sich nach der Höhe der individuellen Schutzdefizite. Die nach Regel 1 ermittelten Massnahmen haben gegenüber allen anderen Massnahmen Vorrang.

An jeder Gefahrenstelle sind im Minimum die Massnahmen zu realisieren, die mit bester Effizienz und Verträglichkeit das ermittelte Schutzdefizit eliminieren. Es ist keine allgemeingültige Aussage darüber möglich, mit welchen Massnahmen dies am zweckmässigsten erfolgt, ob beispielsweise lokale Objektschutzmassnahmen oder flächig wirksame Massnahmen zu ergreifen sind. Zur Unterstützung der Massnahmenwahl können die im Zusammenhang mit der Ermittlung des Massnahmenindexes (Kapitel 4.2.3.3) gewonnen Erkenntnisse über die Eignung von Massnahmen herangezogen werden.

Aus der Anwendung von Regel 2 resultiert auf regionaler Ebene eine Prioritätenliste und eine Karte der Prioritäten. Für diese Massnahmen gilt eine bedingte Handlungspflicht. Der Umfang der zu ergreifenden Massnahmen richtet sich nach den verfügbaren Mitteln (Budget).

\subsubsection{Einzelprojekte}

Zur Entscheidungsfindung über die Reduktion von untolerierbaren Risiken auf Stufe Einzelprojekt werden grundsätzlich die in der Praxis bereits bekannten Methoden herangezogen. Dabei handelt es sich um die Prüfung der Wirtschaftlichkeit und der Einhaltung von Grenzwerten für individuelle Risiken. Instrumente zur automatisierten Unterstützung dieser Planungsschritte sind in Entwicklung (z. B. ,EconoMe' (BAFU, 2007b) oder RiskPlan2 (BAFU, 2007d)).

Bei den meisten Einzelprojekten erfolgen ein Variantenstudium und eine Variantenbeurteilung nach Kostenwirksamkeitskriterien sowie hinsichtlich genügender Reduktion der individuellen Risiken. Als Schutzziele können beispielsweise die aus dem Umgang mit technischen Risiken übernommenen Vorgaben angewendet werden (Merz, 1995; Kapitel 2.7.5, Tabelle 28).

Mit fortschreitender Entwicklung und Anwendung der Risiko- und Kostenwirksamkeitsbeurteilung und insbesondere mit zunehmender Verbesserung der Datenbasis, vor allem der Schadenserwartungswerte, kann die Qualität der Entscheidfindung und damit das Risikomanagement erheblich verbessert werden.

Für die Reduktion untolerierbarer Risiken auf Stufe Einzelprojekt wird zusammenfassend das Vorgehen in den folgenden Schritten empfohlen:

1. Definition geeigneter, massnahmengerechter Projektperimeter;

2. detaillierte Analyse der Risiken;

3. Evaluation von Massnahmenvarianten; die Massnahmenwahl kann durch den Massnahmenindex unterstützt werden (Kapitel 4.2.3.3);

4. Kostenwirksamkeits- und Grenzkostenanalyse der Massnahmenvarianten, Prüfung der Einhaltung tolerierbarer individueller Risiken;

5. partizipative Entscheidungsfindung unter Beachtung ökonomischer, ökologischer und sozialer Kriterien;

6. wettbewerbsbasierte Umsetzung der Massnahmen;

7. professionelles Controlling. 


\subsection{Organisation und Verfahren des Risikomanagements}

\subsubsection{Optimale gesellschaftliche Entscheidverfahren}

Die Erkenntnisse zu den gesellschaftlichen Entscheidverfahren zeigen, dass preismechanische und direktdemokratische Entscheidverfahren zu favorisieren sind (Kapitel 2.9.2). Preismechanische Entscheidverfahren spielen im Management von Naturrisiken in der Schweiz lediglich im Versicherungsbereich eine Rolle. Im Bereich Prävention und Intervention haben hierarchisch-bürokratische Entscheidverfahren traditionell eine grosse Bedeutung. Diese Entscheidverfahren dominieren deshalb, weil Sicherheit in vielen Fällen ein öffentliches Gut darstellt. Charakteristisch für öffentliche Güter sind die Nichtrivalität (das Gut wird durch die Nutzung nicht, verzehrt') und die Nichtexklusivität (niemand kann von der Nutzung ausgeschlossen werden). Vor allem aus der Nichtexklusivität ergeben sich Probleme mit strategischem Verhalten (Trittbrettfahrerverhalten). Einzelne Betroffene versuchen Sicherheit in Anspruch zu nehmen, ohne etwas dafür zu leisten. Dies führt dazu, dass Sicherheit nicht oder nur durch hierarchische Entscheide bereitgestellt wird.

Ein weiterer Grund für die grosse Bedeutung hierarchischer Entscheidverfahren ist der oft sehr hohe Koordinationsaufwand (Transaktionskosten) für Risikoverminderungsmassnahmen.

\subsubsection{Arten der Koordination aufgrund des Markt-Hierarchie-Paradigmas}

Anhand der Kriterien Transaktionskosten, Faktorspezifität und Unsicherheiten im MarktHierarchie-Paradigma (Kapitel 2.9.2.3) lassen sich Aussagen zur geeigneten Koordinationsform im Management von Naturrisiken ableiten. Wird die Bereitstellung einer Sicherheitsleistung als Transaktion verstanden, können die mit der Vorbereitung und Umsetzung dieser Massnahme verbundenen Kosten (exkl. ,eigentliche' Kosten der Massnahmen wie z. B. Baukosten) als Transaktionskosten bezeichnet werden. Derartige Kosten fallen in zahlreichen Projektschritten an und spielen bei vielen Risikoverminderungsmassnahmen eine bedeutende Rolle (Tabelle 57).

Tabelle 57 Transaktionskosten im Management von Naturrisiken.

\begin{tabular}{ll}
\hline Generell & $\begin{array}{l}\text { Im Management von Naturrisiken (exemplarisch für ein technisches Präventions- } \\
\text { projekt) }\end{array}$ \\
\hline $\begin{array}{l}\text { Suchkosten } \\
\text { Anbahnungskosten }\end{array}$ & $\begin{array}{l}\text { Orientierung der Betroffenen } \\
\text { Informationskosten }\end{array}$ \\
$\begin{array}{l}\text { Gefahrenkartierung und Risikoanalyse } \\
\text { Verhandlungskosten }\end{array}$ & Wirkungsanalyse, Feststellung der Nutzniesser \\
Entscheidungskosten & Ermittlung und Auswahl von Projektvarianten \\
Vereinbarungskosten & Projekt- und Kreditanträge, politische Beratung \\
Abwicklungskosten & Submissionen, Werkverträge \\
Absicherungskosten & Regelung von Dienstbarkeiten, rechtliche Regelungen \\
Durchsetzungskosten & Rechtsmittelverfahren \\
Kontrollkosten & Qualitätskontrolle, Kostenkontrolle \\
Anpassungskosten & Projektänderungen \\
Beendigungskosten & Sicherstellung der Massnahme, Unterhaltsregelung \\
\hline
\end{tabular}


Erfahrungsgemäss sind diese Transaktionskosten umso höher, je grösser der Wirkungsbereich einer Massnahme und die Zahl der Betroffenen sind. Betroffene sind in kollektiven Trägerschaften zu koordinieren.

Typische Beispiele zum Wirkungsbereich von Massnahmen sind:

a. punktuell wirkende Massnahmen

- Objektschutzmassnahmen;

- Galerien;

- örtliche Lawinenauslösung mit ortsgebundenen Anlagen;

b. flächig wirkende Massnahmen

- permanente Stützverbauungen;

- Strassensperrungen/Verkehrsbeschränkungen;

- Lawinenauslösung mit Helikopter.

Die Ortsgebundenheit und die Auslegung der Massnahmen auf die jeweilige Risikosituation führen zu hoher Faktorspezifität.

Im Management von Naturrisiken bestehen erhebliche Unsicherheiten und Wissenslücken. Dies gilt für die Einschätzung der Risiken, besonders aber auch für die Wirksamkeit von Risikoverminderungsmassnahmen. Vor allem Massnahmen mit verzögert eintretender Wirkung, neuere Technologien und Massnahmen mit hohem Einsatz menschlicher Ressourcen bergen grössere Unsicherheiten. Typische Beispiele sind:

- grosse Unsicherheiten

- biologische Massnahmen (Waldpflege);

- Frühwarnung;

- $\quad$ kleine Unsicherheiten

- punktuelle, technische Massnahmen;

- Objektschutzmassnahmen.

Die erheblichen Transaktionskosten, die meist hohe Spezifität der Sicherheitsmassnahmen und die grossen Unsicherheiten sprechen beim Management von Naturrisiken häufig für hierarchische, allenfalls hybride Lösungen (Tabelle 58).

Tabelle 58 Kriterien und Aspekte zur Beurteilung von Koordinationsmöglichkeiten im Management von Naturrisiken.

\begin{tabular}{lll} 
Art der Koordination & Markt & Hierarchie \\
\hline Kriterium & $\begin{array}{l}\text { Tief bei Massnahmen mit sehr } \\
\text { beschränktem Wirkungsgebiet } \\
\text { Transaktionskosten }\end{array}$ & $\begin{array}{l}\text { Hoch bei Massnahmen mit grossem } \\
\text { Wirkungsgebiet (flächige Massnahmen) }\end{array}$ \\
\hline Spezifität & $\begin{array}{l}\text { Hoch, da Massnahmen in der Regel } \\
\text { Unsicherheit }\end{array}$ & $\begin{array}{l}\text { ortsgebunden und auf eine bestimmte } \\
\text { Risikosituation ausgerichtet sind }\end{array}$ \\
\hline ren und Risiken verringert Unsi- & $\begin{array}{l}\text { Risiken und zur Wirksamkeit von Mass- } \\
\text { cherheit }\end{array}$ & $\begin{array}{l}\text { nahmen } \\
\text { nerbreitet grosse Unsicherheiten zu }\end{array}$
\end{tabular}




\subsubsection{Rollen und Aufgaben im schutzzielbasierten Risikomanagement}

Im Management von Naturrisiken werden in absehbarer Zeit bürokratisch-hierarchische Entscheidverfahren weiterhin das dominierende und geeignetste Vorgehen darstellen. Daher ist auf Stufe des gesellschaftlichen Grundkonsenses eine Kontrolle der Verwaltung durch Wettbewerb und direkt demokratische Verfahren vorzusehen. Die Rolle von Steuerzahlern und Konsumenten ist zu stärken. Dies ist durch eine demokratische Festlegung von - heute weitgehend fehlenden - Regeln des Risikomanagements (risikobasierte Gesetze) anzustreben. Dabei handelt es sich insbesondere um Regeln zur Mittelallokation und Mitteldistribution sowie zur Einführung des Wettbewerbs. Kernelement einer Regelung zur Mittelallokation und -distribution muss eine ergebnisorientierte Mittelbereitstellung sein, welche die bisherige aufwandorientierte öffentliche Förderung durch eine koordinierte leistungsorientierte Förderung ersetzt. Zur Steuerung der leistungsorientierten Förderung kann bei Massnahmen nach Regel 1 die erzielte Reduktion von Todesfall- und Sachrisiken, bei Massnahmen nach Regel 2 das Mass der Verminderung des Prioritätsindexes herangezogen werden. Die Entwicklung beider Metriken ist in einem regelmässigen Rating zu verfolgen. Dies ist anspruchsvoll angesichts der noch vielen ungelösten methodischen Fragen.

Ist dieser Grundsatz umgesetzt, ist die Verlagerung der Entscheidkompetenz auf die Betroffenen möglich. Die Aufsicht des Bundes und die Massnahmensteuerung der Kantone kann sich auf das regionale Risikomanagement beschränken, während die Steuerung von Einzelmassnahmen den lokalen Gemeinwesen überlassen bleibt (Tabelle 59). Dabei kann unterschieden werden zwischen Massnahmen nach Regel 1 und Regel 2: Bei Massnahmen nach Regel 1 geht es um die substanzielle Verminderung von existenziellen Risiken. Hier ist ein weiterreichendes Engagement des Staates angezeigt.

Tabelle 59 Vorschlag einer risikomanagementorientierten Aufgabenteilung zwischen Bund, Kantonen und Gemeinden.

\begin{tabular}{|c|c|}
\hline Akteur & Aktivitäten, wahrgenommene Aufgaben \\
\hline Bund & $\begin{array}{l}\text { stellt methodische Grundlagen bereit } \\
\text { entwickelt nationale Strategien } \\
\text { richtet Beiträge nach Leistungseinheiten aus (evtl. begrenzt auf Projekte zur } \\
\text { Förderung nationaler Strategien) } \\
\text { prüft stichprobenartig die Erbringung der Leistungseinheiten }\end{array}$ \\
\hline Kantone & $\begin{array}{l}\text { entwickeln regionale/kantonale Strategien } \\
\text { sind verantwortlich für das regionale Risikomanagement } \\
\text { erstellen Gefahrenkarten und Risikoanalysen } \\
\text { priorisieren Projekte im kantonalen Kontext } \\
\text { beraten in der Entscheidfindung zu Massnahmen } \\
\text { richten Beiträge nach Leistungseinheiten aus } \\
\text { prüfen Erbringung der Leistungseinheiten flächendeckend } \\
\text { unterstützen die Gemeinden in ausserordentlichen Lagen } \\
\text { prüfen und genehmigen (Gefahren-) Zonenplanungen }\end{array}$ \\
\hline Gemeinde/Betroffene & $\begin{array}{l}\text { sind verantwortlich für Sicherheit } \\
\text { planen Projekte } \\
\text { finanzieren Projekte } \\
\text { führen Massnahmen aus } \\
\text { ergreifen Notmassnahmen in ausserordentlichen Lagen } \\
\text { erstellen (Gefahren-) Zonenpläne }\end{array}$ \\
\hline
\end{tabular}


Ein weiterer Fortschritt wäre die gegenseitige Abstimmung, allenfalls sogar Zusammenlegung der oft unkoordinierten Verantwortlichkeiten und Mittelflüsse in den Bereichen Prävention, Intervention und Wiederherstellung. Eine mögliche künftige Organisationsform wäre eine ,Risikomanagementagentur', welche ergebnisorientiert die jeweils effektivsten und effizientesten Verfahren im regionalen und projektbezogenen Risikomanagement anwendet (Abbildung 46). Als Grundlage für organisatorische Verbesserungen ist die Idee einer umfassenden Rechtsgrundlage für alle Naturrisiken (erneut) zu prüfen (UREK-N, 2001).

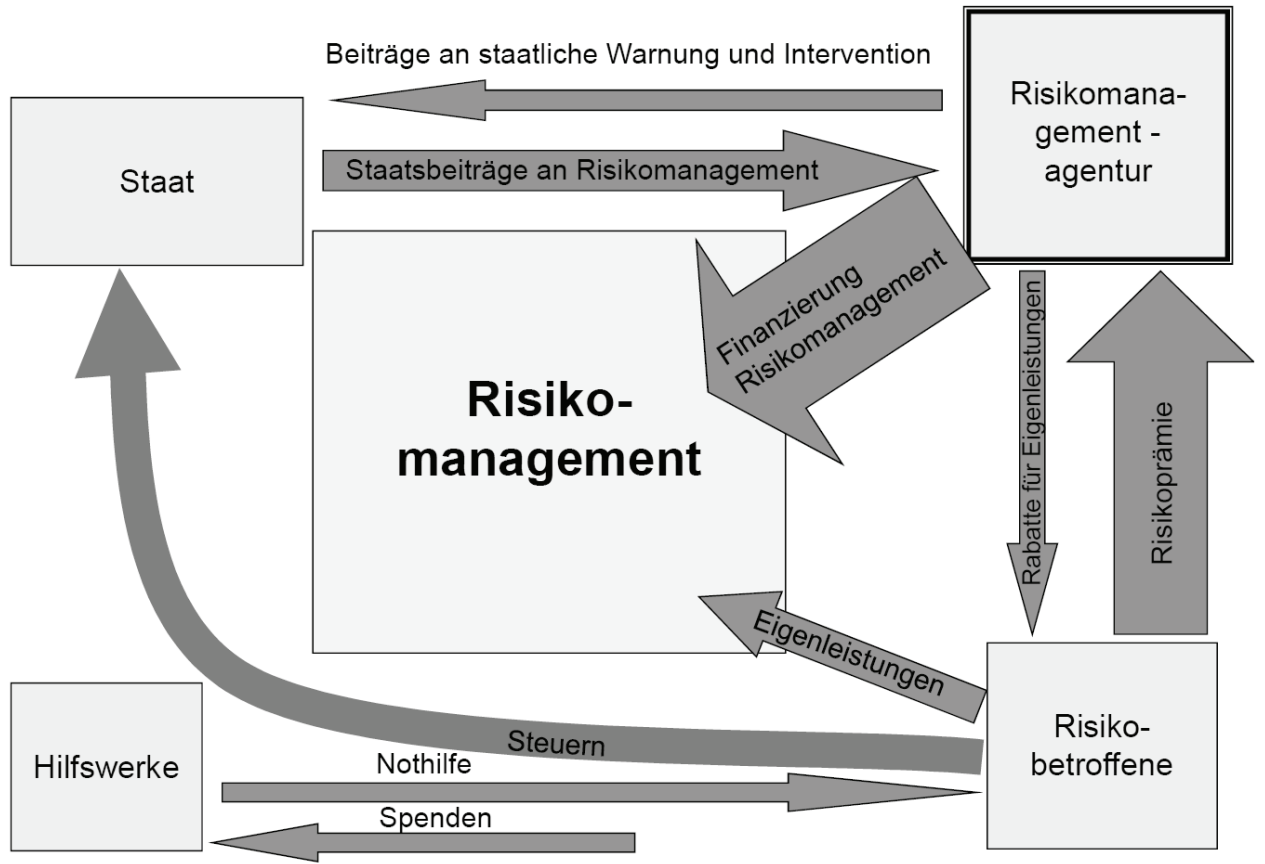

Abbildung 46 Koordination der Verantwortlichkeiten und Finanzflüsse im Management von Naturrisiken durch eine Risikomanagementagentur.

\subsubsection{Einbezug der Betroffenen}

Zur Stärkung der ,Konsumenten' sind die in den letzten Jahren entwickelten Mitwirkungsverfahren flächendeckend umzusetzen (Hostmann et al., 2005). Dies schafft die Voraussetzung, informierte Entscheide zu fällen und die Anliegen der Betroffenen fair zu berücksichtigen. Ausserdem kann die Akzeptanz der Entscheide gefördert werden. Damit sinkt auch die Gefahr, dass Strategien und Entscheide durch mangelnde Kooperation unterlaufen werden. Der Einbezug der Akteure in ein schutzzielbasiertes Risikomanagement ist in verschiedenen Phasen denkbar (Tabelle 60).

Arbeitsgruppen umfassen nebst der fachlich-technischen Projektleitung weitere politische und fachliche Vertreter aus den betroffenen Gebieten. Die Grösse dieser Gremien liegt i. d. R. bei zehn Personen. Workshops sind breiter angelegte Mitwirkungsplattformen, in denen nebst den Mitgliedern der Arbeitsgruppe gezielt ausgewählte Vertretungen der massgeblich betroffenen und einflussstarken Akteure einbezogen sind. Um die Wahrung öffentlicher Interessen sicherzustellen, gehören auch die Fachstellen der Verwaltung zum Kreis der Workshopteilnehmenden. An Workshops nehmen bei komplexeren Projekten über 30 Personen teil (vgl. auch Kapitel 5.3.). 
Tabelle 60 Mitwirkung in den Phasen des Vorschlags zu einem schutzzielbasierten Risikomanagement (eigene Darstellung aufgrund der Ablauforganisation grösserer Integralprojekte im Kanton OW).

\begin{tabular}{|c|c|c|}
\hline Projektphase & $\begin{array}{l}\text { Art der Mitwir- } \\
\text { kung/Beteiligte }\end{array}$ & Kernfragen/Inhalte \\
\hline \multicolumn{3}{|l|}{ Regionales Risikomanagement } \\
\hline \multicolumn{3}{|l|}{ Vorarbeiten: } \\
\hline $\begin{array}{l}\text { Erstellung Gefahrenkarte } \\
\text { Erstellung Schadenspotenzial- } \\
\text { karte }\end{array}$ & Arbeitsgruppe & $\begin{array}{l}\text { Beratung, Informationsbeschaffung, Plausibi- } \\
\text { lisierung, Korreferat }\end{array}$ \\
\hline Erstellung Risikoanalyse & Arbeitsgruppe & $\begin{array}{l}\text { Plausibilisierung, Informationsbeschaffung, } \\
\text { Korreferat }\end{array}$ \\
\hline \multicolumn{3}{|l|}{ Regel 1: } \\
\hline Ermittlung Schutzdefizite & Arbeitsgruppe & Plausibilisierung, Korreferat \\
\hline Prioritätensetzung & Workshop & Prioritätendiskussion \\
\hline & öffentl. Anlass & Information, Diskussion \\
\hline \multicolumn{3}{|l|}{ Regel 2: } \\
\hline $\begin{array}{l}\text { Bewertung Kriterien zu Schutz- } \\
\text { zielprinzipien }\end{array}$ & Arbeitsgruppe & Bewertung Kriterien, Konsensfindung \\
\hline Gewichtung Kriterien & Workshop & Festlegung der Kriteriengewichtung \\
\hline Ermittlung Prioritätsindex & Experten & Berechnung/GIS-Analyse \\
\hline Prioritätenliste & Workshop & Prioritätendiskussion \\
\hline & öffentl. Anlass & Information, Diskussion \\
\hline \multicolumn{3}{|l|}{ Einzelprojekt } \\
\hline Variantenevaluation & Experten, Arbeitsgruppe & Variantensuche, -diskussion \\
\hline Variantenbewertung & Workshop & $\begin{array}{l}\text { Bewertung der Varianten hinsichtlich techni- } \\
\text { scher Eignung, Wirtschaftlichkeit, Ökologie }\end{array}$ \\
\hline Regelung von Rechten & $\begin{array}{l}\text { persönl. Gespräche mit } \\
\text { Betroffenen }\end{array}$ & $\begin{array}{l}\text { Information, Erfassung von Anliegen der } \\
\text { Betroffenen, Diskussion Entschädigungsfra- } \\
\text { gen, Vorverträge }\end{array}$ \\
\hline Vorprojekt & Arbeitsgruppe & $\begin{array}{l}\text { Projektoptimierung hinsichtlich technischer } \\
\text { Eignung, Ökonomie und Ökologie sowie } \\
\text { Beeinträchtigung von Rechten }\end{array}$ \\
\hline Regelung Finanzierung & Behörde & Finanzbeschluss \\
\hline Wahrung öffentl. Interessen & Behörde & Mitberichte \\
\hline Bauprojekt & $\begin{array}{l}\text { Arbeitsgruppe, persönli- } \\
\text { che Gespräche mit Be- } \\
\text { troffenen }\end{array}$ & $\begin{array}{l}\text { Klärung technischer Detailfragen, Verhand- } \\
\text { lungen über Inanspruchnahme von Rechten } \\
\text { und Entschädigungen }\end{array}$ \\
\hline Projektgenehmigung & Auflage, Rechtsmittel & $\begin{array}{l}\text { formelle Mitwirkung (Einsprache), Ausschöp- } \\
\text { fung Rechtsmittel in strittigen Fällen }\end{array}$ \\
\hline Realisation, Betriebsphase & öffentl. Anlässe & Information \\
\hline
\end{tabular}




\section{Fallbeispiele}

\subsection{Inhalt und Untersuchungsgebiet}

\subsubsection{Inhalt der Fallbeispiele}

Im Rahmen von vier Fallbeispielen werden das Konzept und die Methoden (Kapitel 4) verifiziert. Die Fallbeispiele 1-3 befassen sich mit Schutzzielen, der Abschätzung des Handlungsbedarfs und der Priorisierung von Projekten auf regionaler Ebene. Fallbeispiel 4 behandelt den Aspekt der partizipativen Projektplanung. Die Fallbeispiele 1-3 wurden für das Gebiet der Gemeinde Engelberg, Kanton OW durchgeführt. Für das Fallbeispiel 4 wurde das Projekt Hochwasserschutz Sarneraatal herangezogen.

In Fallbeispiel 1 wird die Methode des schutzzielbasierten Managementkonzeptes mit dem ,Zwei-Regel-Ansatz' gemäss Kapitel 4.2 angewendet. Fallbeispiel 2 untersucht die Anwendung des Verfahrens zur vereinfachten regionalen Abschätzung des Handlungsbedarfs anhand der Gefahrenkarte, der Schadenspotenzialkarte und des Massnahmenindexes (Kapitel 4.3).

Mit dem Fallbeispiel 3 wird das Vorgehen bei der Abschätzung des Handlungsbedarfs und die Priorisierung der Projekte (Kapitel 4.4) anhand der bestehenden Schutzzielmatrizes (,Best-Practice' gemäss BUWAL-Methode) aufgezeigt. Dabei werden auch die Einflüsse der vorgeschlagenen Modifikationen dieser ,Best-Practices' betrachtet. Die Ergebnisse, die sich aus dem Einsatz der verschiedenen Methoden ergeben, werden gegenseitig und mit der aktuellen regionalen Planung von Risikoverminderungsmassnahmen in der Gemeinde Engelberg (Masterplan) verglichen. Fragen und Schwierigkeiten, die sich bei der Anwendung der verschiedenen Methoden ergeben, werden erörtert.

Die Fallbeispiele beschränken sich auf die regionale Ebene und enthalten demnach keine Methoden zur Planung und Beurteilung von Einzelprojekten. Für Einzelprojektebene werden praxisübliche Verfahren empfohlen, für die bereits Erfahrungen vorliegen.

Fallbeispiel 4 stellt den Planungs- und Entscheidungsfindungsprozess der Jahre 2006 bis 2008 im Projekt Hochwasserschutz Sarneraatal vor. Bei diesem Projekt wurde die in den Kapiteln 2.9.4.2 und 4.6.4 beschriebene Methodik angewendet. Der Projekt- und Entscheidungsverlauf wird erörtert und methodische Schwierigkeiten werden dargestellt und diskutiert.

\subsubsection{Beschreibung der Untersuchungsgebiete}

\subsubsection{Engelberg}

Engelberg ist eine von sieben Gemeinden des Kantons Obwalden und liegt zuhinterst im Tal der Engelberger Aa. Das Gemeindegebiet erstreckt sich über eine Fläche von $75 \mathrm{~km}^{2}$ zwischen einer Höhe von 569 m ü. M. (Ortsteil Grafenort) und 3’239 m ü. M. (Titlis).

Engelberg zählt 4'000 Einwohner, wovon 8.2\% der Bevölkerung in der Land- und Forstwirtschaft, $15.6 \%$ in Gewerbebetrieben und $76.2 \%$ im Fremdenverkehrs- und Dienstleistungssektor tätig sind. Der Dorfkern befindet sich auf 1'000 m ü. M. am rechtsseitigen Hangfuss im vordersten Abschnitt des Hochtales. Weitere zusammenhängende Siedlungsgebiete liegen in der Mitte des Hochtals (Oberberg) und am Eingang des Horbistals (Neuschwändi, Barmettlen). Das übrige Talgebiet ist zerstreut besiedelt. Die bedeutendsten touristischen Anlagen befinden sich in Dorfnähe beidseitig des Tals. 
Engelberg liegt in den nördlichen Voralpen. Die Geologie ist von Kalkgesteinen dominiert, nur im äussersten Südosten des Gemeindegebiets tritt kristallines Gestein an die Oberfläche. Eine durch den ,Engelberger Bergsturz' vor ca. 10'000 Jahren geschaffene Steilstufe trennt den Ortsteil Grafenort vom Engelberger Hochtal. Dieses ist als flaches, westöstlich orientiertes Trogtal mit einer Breite von 0.5 bis $1 \mathrm{~km}$ ausgebildet. Es erstreckt sich über eine Länge von ca. $8 \mathrm{~km}$ von der Steilstufe bis zur Gemeindegrenze im Surenental. Ca. $2.5 \mathrm{~km}$ oberhalb der Steilstufe mündet rechtsseitig das ca. 3 km lange Horbistal ein.

Die Einhänge des Haupt- und des Seitentals sind bis auf 1'800 m ü. M. mehrheitlich bewaldet. Zwischen der Waldgrenze und den felsigen Gipfelpartien liegen ausgedehnte Steilhänge, aus denen im Winter Lawinen losbrechen. Vor allem der hintere Bereich des Haupttals und das Horbistal liegen im Auslaufgebiet zahlreicher Lawinenzüge. Vereinzelte Steinschlaggebiete bedrohen Teile des Dorfes, einzelne Streusiedlungen und die Zufahrtsstrasse nach Engelberg. Die wichtigsten Gewässer sind die Engelberger Aa, welche das Haupttal durchfliesst, und der Dürrbach, der aus dem Horbistal einmündet. Aus den Talflanken fliessen zahlreiche, meist sehr steile Seitengewässer zu, welche oft auch Murgänge führen. Die Engelberger Aa wurde im 16. Jahrhundert an den südlichen Rand des Hochtals verlegt. Anfang des 20. Jahrhunderts wurden der Dürrbach und vereinzelte kleinere Seitenbäche verbaut. Im Gebiet Rigidal-Brunni wurde zwischen 1985 und 2002 eine grössere Lawinenverbauung zum Schutz der Wohngebiete am Eingang des Horbistals erstellt. Ebenso wurden in den letzten 15 Jahren umfangreiche Steinschlagschutzverbauungen zum Schutz von Teilen des Dorfes (Mühliwald) und der Zufahrtsstrasse nach Engelberg errichtet.

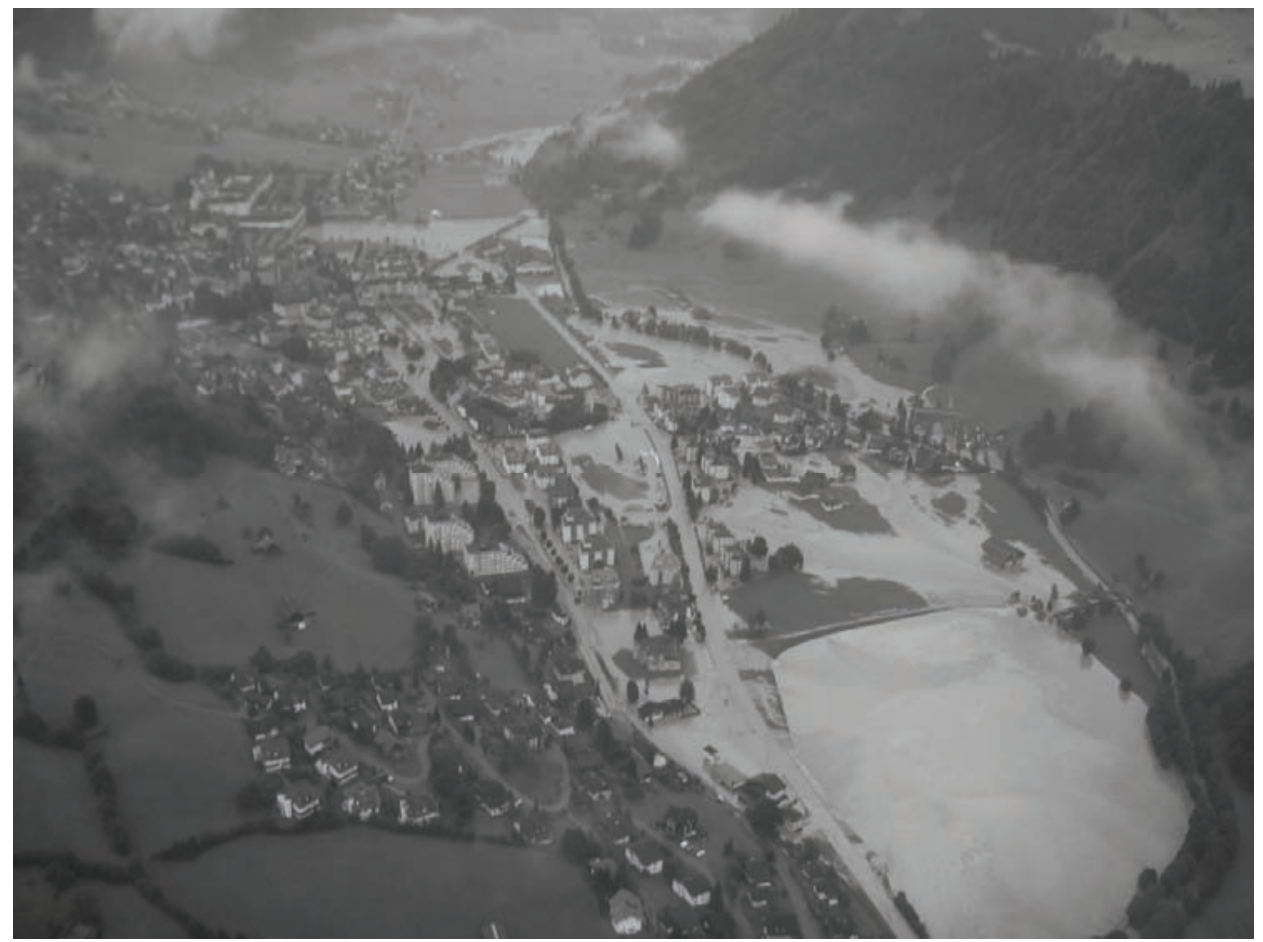

Abbildung 47 Das Engelberger Hochtal beim Hochwasser im August 2005 (Amt für Wald und Raumentwicklung OW).

Engelberg verfügt seit 1975 über einen Lawinenzonenplan. 1995 wurde eine integrale Gefahrenkarte über alle gravitativen Gefahrenprozesse erstellt. Diese Gefahrenkarte wurde im 
Jahr 2001 aufgrund des Lawinenwinters 1999 und der Hochwasserereignisse 1998 und 2000 überprüft und angepasst. Weitere kleinere Anpassungen fanden nach dem Hochwasser 2005 statt. Die Erstellung und Revisionen der Gefahrenkarte erfolgten im Auftrag des Kantons Obwalden durch ein Ingenieurbüro. Dieses wurde fachlich von einer Kommission der Gemeinde und des Kantons begleitet. Die Beurteilung der Gefährdungen erfolgte losgelöst von tatsächlich vorhandenen und geplanten Raumnutzungen. 2003 wurde ein rechtskräftiger Zonenplan erlassen, der die Gefahrenkarte integriert. Neue Bauvorhaben werden anhand der Gefahrenkarte beurteilt und nötigenfalls nicht oder nur mit gefahrengerechten Auflagen bewilligt.

Zur Bewältigung ausserordentlicher Lagen verfügt die Gemeinde Engelberg nebst dem Gemeindeführungsstab und den Wehrdiensten über eine ständige Naturgefahrenkommission. Diese entwickelte sich aus der Lawinenkommission, die bei Lawinengefahr für die Evakuierung gefährdeter Liegenschaften und die Sperrung von Verkehrswegen zuständig ist.

\subsubsection{Sarnersee und Sarneraa}

Der $7.5 \mathrm{~km}^{2}$ grosse Sarnersee liegt im Zentrum des Sarneraatals und weist ein Einzugsgebiet von $267 \mathrm{~km}^{2}$ auf. Er erstreckt sich in Südwest-Nordost-Richtung, ist ungefähr $6 \mathrm{~km}$ lang und hat eine mittlere Breite von $1.3 \mathrm{~km}$. Der mittlere Seespiegel liegt auf $469.40 \mathrm{~m}$ ü. M. Der südwestseitige Zufluss des Sarnersees ist der Dreiwässerkanal, der das Wasser von der Giswiler Laui, von der Aa (aus dem Lungernsee) und von der Kleinen Melchaa führt. Weitere grössere Zuflüsse sind der Steinibach im Westen und die Grosse Melchaa von Osten. Bei Sarnen verlässt die Sarneraa den See.

Am unteren (nördlichen) Ende des Sees liegt der Kern des Hauptorts Sarnen und das Industriegebiet in einer flachen Talebene. Sarnen zählt insgesamt 9'500 Einwohner, wovon 5700 im Dorfkern wohnen. 8\% der Bevölkerung sind in der Land- und Forstwirtschaft, 32\% in Industrie- und Gewerbebetrieben und 60\% im Dienstleistungssektor tätig. 800 Unternehmen beschäftigen über 5'800 Mitarbeitende. Darunter sind auch zwei mittelgrosse Firmen, welche international tätig sind: die SIKA Sarnafil AG (Kunststoffe) und die Leister Process Technologies (Elektrogeräte und Hightechmedizin).

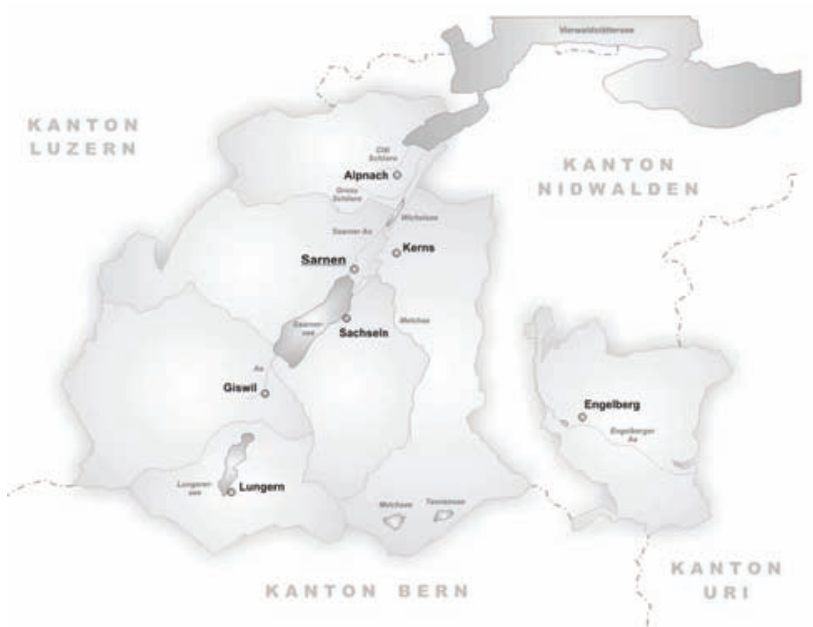

Abbildung 48 Gemeinden des Kantons Obwalden und der Sarnersee mit den wichtigsten Zu- und Abflüssen. 
Wie die Gemeinde Engelberg verfügt auch die Gemeinde Sarnen über eine flächendeckende Gefahrenkarte, welche bei der Beurteilung und Genehmigung neuer Bauvorhaben berücksichtigt wird. Ein Frühwarn- und Interventionsdienst für Massnahmen in ausserordentlichen Lagen befindet sich im Aufbau.

Die hydrologische Situation am Sarnersee veränderte sich im Jahr 1880, als die Grosse Melchaa, die vorher unterhalb des Dorfes in die Sarneraa mündete, in den Sarnersee geleitet wurde. Im Anschluss an diese Korrektion der Melchaa erfolgte in den Jahren 1880 bis 1883 auch ein bescheidener Ausbau der Sarneraa unterhalb des Sees. Seither fanden an der Sarneraa keine grösseren Verbauarbeiten statt. Durch die Umleitung der Melchaa wurde das Dorf Sarnen von der Hochwassergefahr der Melchaa entlastet. Im Gegenzug treten seither der Sarnersee und die Sarneraa häufiger über die Ufer. Besonders Ende des 19. Jahrhunderts und in den letzten zehn Jahren häuften sich Hochwasserereignisse. So richteten Überschwemmungen in den Jahren 1999, 2004 und 2005 grosse Schäden an. Auch in den Jahren 2002 und 2007 trat der See über die Ufer.

Das Hochwasser im August 2005 übertraf am Sarnersee die schlimmsten Szenarien gemäss bisheriger Beurteilung in der Gefahrenkarte. Niederschläge und Abflüsse lagen im gesamten Einzugsgebiet im Bereich eines sehr seltenen Extremereignisses (>HQ 300). So erreichte der Abfluss der Sarneraa einen Spitzenwert von $150 \mathrm{~m}^{3} / \mathrm{s}$. Die bisherige Annahme für ein 300 -jährliches Abflussereignis lag bei $65 \mathrm{~m}^{3} / \mathrm{s}$. Der Pegel des Sarnersees stieg am 23. August auf eine Rekordhöhe von 472.42 m ü. M. und lag zwei Meter über der Schadensgrenze. Innerhalb von nur 36 Stunden war der Seespiegel um 2.20 m angestiegen. Im öffentlichen Bereich führte das Hochwasser vom August 2005 im Kanton Obwalden zu Schäden von CHF 220 Mio. Betroffen waren alle öffentlichen Bereiche: Strassen, Schienennetz der Zentralbahn, Bauten der Verwaltung und der Schulen, Abwasserreinigungsanlage, Stromversorgung, Feuerwehrstützpunkt sowie das Kantonsspital. Im privaten Bereich entstanden Schäden in Höhe von ca. CHF 160 Mio. Insgesamt verursachte das Hochwasser im August 2005 im Kanton Obwalden einen Schaden von rund CHF 380 Mio., wovon nahezu die Hälfte auf die Umgebung des Sarnersees entfällt.

\subsubsection{Laufende und geplante Projekte zur Verminderung von Naturrisiken}

Die Gefahrenkarte und eine quantitative Risikoanalyse für das gesamte Kantonsgebiet von Obwalden liegen vor. Auf Basis der Gefahrenkarte und der Erfahrungen des Unwetters im August 2005 wurde im Herbst 2005 ein Masterplan ,Sicherheit vor Naturgefahren' erstellt. Der Masterplan umfasst zahlreiche der gemäss Gefahrenkarte qualitativ erkannten Risikogebiete, besonders die Schadensgebiete des Hochwassers 2005. Er sieht in allen sieben Gemeinden des Kantons Obwalden Massnahmen bis zum Jahr 2030 vor, unter anderem auch umfangreiche Verbauungen im Gebiet von Engelberg sowie am Sarnersee und der Sarneraa (Tabelle 61). 
Tabelle 61 Auszug aus dem Masterplan ,Sicherheit vor Naturgefahren' für die Gemeinden Engelberg und Sarnen.

\begin{tabular}{|c|c|c|c|c|}
\hline Ort & Art der Massnahme & $\begin{array}{l}\text { Ungefähre Kosten } \\
\text { in CHF }\end{array}$ & Zeitraum & Priorität \\
\hline \multicolumn{5}{|c|}{ Gemeinde Engelberg (alle Projekte gemäss Masterplan) } \\
\hline Engelberg-Nord & Schutzwaldpflege & 2'228'000 & ab 1997 & 5 \\
\hline Engelberg-Süd & Schutzwaldpflege & 273 '250 & ab 1999 & 5 \\
\hline Mittelgrüss/Müliwald & Runsenverbau & 1'300’000 & $2007-2008$ & 1 \\
\hline Mehlbach & Bachverbau & $5^{\prime} 0000^{\prime} 000$ & $2008-2011$ & 2 \\
\hline Fangbach & Geschiebesammler & 2'500'000 & $2011-2013$ & 3 \\
\hline Aa Hochtal, Dorf & Flussverbau & $24^{\prime} 400^{\prime} 000$ & $2009-2018$ & 4 \\
\hline Zelgli & Steinschlagschutz & 1'500'000 & 2012-2014 & 5 \\
\hline Aa Grafenort & Flussverbau & $4^{\prime} 0000^{\prime} 000$ & $2017-2019$ & 6 \\
\hline Dürrbach & Bachverbau & $8000^{\prime} 000$ & $2017-2019$ & 7 \\
\hline Bärenbach & lokale Ufersicherung & $520 \prime 000$ & 2018-2019 & 8 \\
\hline Arnitobel & lokale Ufersicherung & $500 ' 000$ & $2018-2020$ & 9 \\
\hline Aa Hochtal, Oberlauf & lokale Ufersicherung & $600 ' 000$ & $2018-2020$ & 10 \\
\hline Chaltibach & Flussverbau & $4000^{\prime} 000$ & nach 2020 & 11 \\
\hline Erlenbach & Flussverbau & $2000^{\prime} 000$ & nach 2020 & 12 \\
\hline alle Lawinengebiete & Notfallmassnahmen & & Bei Bedarf & $5-25$ \\
\hline Gewässer & Notfallmassnahmen & & Bei Bedarf & 5 \\
\hline \multicolumn{5}{|c|}{ Gemeinde Sarnen (nur Projekt Sarneraa) } \\
\hline $\begin{array}{l}\text { Sarneraa bis } \\
\text { Wichelsee }\end{array}$ & $\begin{array}{l}\text { Flussverbau, } \\
\text { Aufweitung }\end{array}$ & $47^{\prime} 000^{\prime} 000$ & $2008-2013$ & 1 \\
\hline
\end{tabular}

Der Masterplan stellt keine umfassende und systematische Darstellung des Handlungsbedarfs dar. Es handelt sich um eine durch die Verwaltung und die lokalen Behörden mit unvollständigen Informationen erstellte Liste von Massnahmen und Dringlichkeiten. Viele Projekte waren seit Längerem in Vorbereitung (z. B. Verbauung Engelberger Aa) und erlangten nach dem Hochwasserereignis 2005 wesentlich höhere politische Priorität. Entscheidend für die Aufnahme einer Massnahme in den Masterplan ist das Vorhandensein einer öffentlichen oder privaten Trägerschaft zur Verminderung entsprechender Risiken. Deshalb enthält der Masterplan keine Massnahmen für verschiedene Gefahrenquellen, in denen aufgrund von Gefahren- und Schadenspotenzialkarte hohe Risiken vermutet werden, sich bis jetzt aber keine Trägerschaften zu deren Verminderung gebildet haben.

Die laufenden Planungen und Massnahmen in der Gemeinde Engelberg umfassen Projekte zur Minderung von Hochwasser- und Murgangrisiken und ein Steinschlagschutzprojekt im Gebiet Zelgli (Gefahrenquelle Müliwald-Mittelgrüss). Es werden keine Projekte über technische Lawinenschutzmassnahmen geplant oder ausgeführt. Jedoch haben Lawinen ebenso wie die Gewässer hohe Bedeutung im Notfallkonzept der Gemeinde Engelberg. Im Bereich einiger Gefahrengebiete (Eschlenfluewald, Ruchegg-Grüenenwald, Rapperschwendi) werden periodisch Waldpflegearbeiten (Waldpflegeprojekte Engelberg-Nord und Engelberg-Süd) durchgeführt. 
Die Massnahmen zur Verminderung von Naturrisiken sind im Kanton Obwalden grundsätzlich durch private oder öffentliche Nutzniesser zu tragen. Trägerschaften von Hochwasserschutzmassnahmen sind die Einwohnergemeinden. Die Massnahmen sowohl der Einwohnergemeinden als auch privater Nutzniesser werden in bedeutendem Masse durch Beiträge des Bundes und des Kantons unterstützt. Bei den geplanten Massnahmen am Sarnersee und an der Sarneraa liegt die Trägerschaft (ausnahmsweise) beim Kanton Obwalden. Die umliegenden Gemeinden werden nach Massgabe ihres Nutzens (Risikoverminderung) zur Mitfinanzierung herangezogen.

\subsection{Fallbeispiele 1-3: Engelberg}

\subsubsection{Fallbeispiel 1: Test des Managementkonzeptes}

\subsubsection{Grundlagen}

Grundlagen für die Verifizierung des in Kapitel 4 entwickelten Managementkonzeptes bilden die Gefahrenkarte (Stand 2006) und eine Schadenspotenzialkarte (Stand 2007). Die Anwendung des Managementkonzeptes beschränkt sich aufgrund der vorhandenen Datengrundlage auf den Teil des Engelberger Gemeindegebietes mit hohem Schadenspotenzial. Es wurde ein Untersuchungsperimeter ausgeschieden, der das intensiv besiedelte Talgebiet (Haupttal der Engelberger Aa und Horbistal) und einen Teil der seitlichen Einhänge, vor allem entlang der rechtsseitigen Hangflanke mit zahlreichen Streusiedlungen, umfasst.

\subsubsection{Vorgehen}

In einem ersten Schritt wurden die Objektarten der Schadenspotenzialkarte gemäss dem Verfahren in Kapitel 4.2.3.2 mit einem Objektindex bewertet. Die Bewertung erfolgte unabhängig durch drei Personen (Autor, je eine Fachperson aus dem Kanton Obwalden und dem Kanton Bern). Die Einzelbewertungen wurden anschliessend verglichen. Unterschiedliche Bewertungen wurden in einer Diskussion unter den Bewertungspersonen erörtert und abgeglichen.

In einem zweiten Schritt wurden - wiederum unabhängig durch die drei Personen - Massnahmen mit einem Massnahmenindex bewertet. Diese Bewertung ist sowohl für Fallbeispiel 1 (Analyse nach Regel 2) als auch für Fallbeispiel 3 erforderlich. Anhand der Gefahrenkarte wurden in einem nächsten Schritt Gefahrengebiete abgegrenzt. Insgesamt wurden im Untersuchungsgebiet

- 22 Steinschlaggebiete,

- 16 Lawinengebiete und

- 23 Gebiete mit Wildbach- und Hangprozessen

ausgeschieden. Die Gefahrengebiete umfassen die Prozessräume einzelner Gefahrenquellen und weisen eine Grösse zwischen $0.003 \mathrm{~km}^{2}$ und $0.7 \mathrm{~km}^{2}$ auf. Sie stellen die Untersuchungseinheiten bei der Anwendung aller Verfahren der Fallbeispiele 1-3 dar.

Die Gefahrengebiete wurden anschliessend nach den Kriterien in Kapitel 4.2.3.3 (Tabelle 53) charakterisiert. Jedem Gefahrengebiet wurde basierend auf dessen Eigenschaften in einer automatisierten Entscheidungsroutine ein Massnahmenindex zugeordnet. Es handelt sich dabei um den gemäss Kapitel 4.2.3.3 ermittelten Massnahmenindex (auch Anhang XII) für Gefahrengebiete mit der Merkmalkombination des jeweiligen Gebiets. Die örtliche Gefährdung wurde mit einem aufgrund von Gefahrenkarte und Schadenspotenzialkarte berechneten Gefährdungsfaktor nach Kapitel 4.2.3.4 (Tabelle 55) berücksichtigt. 
Zur Anwendung der Regel 1 wurde das Untersuchungsgebiet im Verfahren ,Risikoanalyse bei gravitativen Naturgefahren' (Borter, 1999) hinsichtlich individueller Risiken mit folgenden Schwellen geprüft (Risikoanalyse Stufe 2):

- individuelles Todesfallrisiko $>10^{-2} ;>10^{-3} ;>10^{-4} ;>10^{-5} ;>10^{-6}$ pro Jahr;

- individuelles Sachrisiko > CHF 25 ; > CHF 50; > CHF 250; > CHF 500; > CHF 20'000; > CHF 40'000; > CHF 65'000 pro Are und Jahr für ausgewählte Objektkategorien (Werte gemäss Tabelle 49).

Die Gefahrengebiete wurden anschliessend rangiert nach der Überschreitung der Schwellenwerte des maximalen tolerierbaren Risikos (Kapitel 4.2.2.2 und Tabelle 48). Die Rangierung erfolgt nach dem Ausmass der Risikoüberschreitung und der Grösse der Objektfläche oder -länge, auf denen die Risiken überschritten werden. Die Rangierung wurde sowohl nach dem Gesamtwert der Risikoüberschreitung pro Gefahrengebiet als auch nach dem Gesamtwert pro Flächeneinheit vorgenommen.

Zur Anwendung der Regel 2 wurden das mit dem Objektindex attribuierte Schadenspotenzial, die mit dem Massnahmenindex attribuierten Gefahrengebiete und die durch den Gefährdungsfaktor ausgedrückte Gefährdung mittels geographischem Informationssystem überlagert und anschliessend der Prioritätsindex pro Gefahrenquelle - umgerechnet auf eine Einheitsfläche - ermittelt.

Die 61 Gefahrenquellen wurden anschliessend

a) aufgrund der Überschreitung des tolerierbaren individuellen Risikos nach Tabelle 48 und

b) aufgrund des Prioritätsindexes

nach Dringlichkeit des Handlungsbedarfs rangiert (Prioritäten 1 bis 61).

Die Prioritäten wurden mit den Dringlichkeiten des Masterplans verglichen. Die Prioritäten der Projekte des Masterplans (Tabelle 61, 5. Spalte) wurden aufgrund der Zeithorizonte der geplanten Massnahmen ermittelt. Für Vergleichszwecke wurden auch den Notfallmassnahmen für die Wasser- und Lawinengebiete der Gemeinde Engelberg Dringlichkeiten zugewiesen. Diese Notfallmassnahmen umfassen die Anordnung von Evakuierungen und Sperrungen in ausserordentlichen Gefahrensituationen sowie bauliche Vorkehrungen wie Notbaggerungen, Wasserableitungen oder Hochwassersperren bei Ereignissen. Die Prioritäten der Notfallmassnahmen wurden nach den Alarmstufen des Notfallkonzeptes für die Gemeinde Engelberg zwischen den Werten 5 (häufige Ereignisse oder Gefahrensituationen, wichtige Gefahrenquellen) und 25 (seltene Ereignisse/Gefahrensituationen, weniger bedeutende Gefahrenquellen) festgelegt. Gebiete mit Waldpflegemassnahmen im Rahmen laufender Projekte wurden mit Dringlichkeit 5 belegt. Den Gefahrengebieten, in denen gemäss Masterplan keine Projekte vorgesehen sind oder die nicht im Notfallkonzept aufgeführt sind, wurde zu Vergleichszwecken der Wert ,45’ zugeordnet.

\subsubsection{Prioritäten gemäss Managementkonzept}

Die Priorisierung der Gefahrengebiete aufgrund von Regel 1 ergibt - berechnet nach dem nicht auf eine einheitliche Flächengrösse umgerechneten Ausmass der Überschreitung tolerierbarer Todesfallrisiken pro Gefahrenquelle - hohe Prioritäten für Massnahmen an Wasserund Sturzgefahrenquellen. Massnahmen in Lawinengebieten erscheinen fast ausnahmslos in der zweiten Hälfte der Prioritätenliste (Tabelle 62). 
Tabelle 62 Prioritäten von Massnahmen zur Risikoverminderung gemäss Regel 1, ohne Umrechnung auf einheitliche Flächengrösse.

\begin{tabular}{|c|c|c|c|c|}
\hline Gefahrenquelle & $\begin{array}{l}\text { Gefahren- } \\
\text { prozess [GP] }\end{array}$ & $\begin{array}{l}\text { Projekt/Risikover- } \\
\text { minderungsmassnahme } \\
\text { gem. Masterplan }\end{array}$ & $\begin{array}{l}\text { Priorität gemäss } \\
\text { Managementkon- } \\
\text { zept }\end{array}$ & $\begin{array}{l}\text { Priorität gemäss } \\
\text { Masterplan }\end{array}$ \\
\hline Eschlenfluewald & Sturz & Schutzwaldpflege & 1 & 5 \\
\hline Zelgli & Sturz & Steinschlagschutz & 2 & 5 \\
\hline Ghärst-Obermatt & Wasser & -- & 3 & 45 \\
\hline Ruchegg-Grüenenw. & Sturz & Schutzwaldpflege & 4 & 5 \\
\hline Bord & Wasser & -- & 5 & 45 \\
\hline Rapperschwendi & Sturz & Schutzwaldpflege & 6 & 5 \\
\hline Tellensteinwald & Sturz & -- & 7 & 45 \\
\hline Bärenbach & Wasser & Bachverbau & 8 & 8 \\
\hline Fangbach & Wasser & Geschiebesammler & 9 & 3 \\
\hline Rotigraben & Wasser & -- & 10 & 45 \\
\hline Aa Grafenort & Wasser & Flussverbau & 12 & 6 \\
\hline Arnitobel & Wasser & lokale Ufersicherung & 12 & 9 \\
\hline Chaltibach & Wasser & Flussverbau & 12 & 11 \\
\hline Mittelgrüss/Müliwald & Wasser & Runsenverbau & 16 & 1 \\
\hline Aa Hochtal, Oberlauf & Wasser & lokale Ufersicherung & 24 & 10 \\
\hline Mehlbach & Wasser & Bachverbau & 26 & 2 \\
\hline Aa Hochtal, Dorf & Wasser & Flussverbau & 29 & 4 \\
\hline Erlenbach & Wasser & Flussverbau & 29 & 12 \\
\hline Dürrbach & Wasser & Bachverbau & 58 & 7 \\
\hline
\end{tabular}

Die Darstellung mit Umrechnung der Risikoüberschreitung pro Flächeneinheit führt zu Veränderungen in der Prioritätenliste. Erwartungsgemäss rücken kleinere Gefahrengebiete in der Prioritätenliste nach vorne. Die Lawinengebiete erhalten noch tiefere Prioritäten. Bei den übrigen Gefahrenprozessen verschieben sich die Prioritäten leicht zuungunsten der Wassergefahren und zugunsten der Sturzgefahren (Tabelle 63). 
Tabelle 63 Prioritäten von Massnahmen zur Risikoverminderung gemäss Regel 1, umgerechnet auf ein einheitliches Flächenmass.

\begin{tabular}{|c|c|c|c|c|}
\hline Gefahrenquelle & $\begin{array}{l}\text { Gefahren- } \\
\text { prozess } \\
\text { [GP] }\end{array}$ & $\begin{array}{l}\text { Projekt/Risikover- } \\
\text { minderungsmassnahme } \\
\text { gem. Masterplan }\end{array}$ & $\begin{array}{l}\text { Priorität ge- } \\
\text { mäss Manage- } \\
\text { mentkonzept }\end{array}$ & $\begin{array}{l}\text { Priorität ge- } \\
\text { mäss Master- } \\
\text { plan }\end{array}$ \\
\hline Tellensteinwald & Sturz & -- & 1 & 45 \\
\hline Eschlenfluewald & Sturz & Schutzwaldpflege & 2 & 5 \\
\hline Ruchegg-Grüenenw. & Sturz & Schutzwaldpflege & 3 & 5 \\
\hline Zelgli & Sturz & Steinschlagschutz & 4 & 5 \\
\hline Ghärst-Obermatt & Wasser & -- & 5 & 45 \\
\hline Rapperschwendi & Sturz & Schutzwaldpflege & 6 & 5 \\
\hline Rotigraben & Wasser & -- & 7 & 45 \\
\hline Bord & Wasser & -- & 8 & 45 \\
\hline Fangbach & Wasser & Geschiebesammler & 9 & 3 \\
\hline Bergwaldbach & Wasser & -- & 10 & 45 \\
\hline Bärenbach & Wasser & Bachverbau & 11 & 8 \\
\hline Aa Grafenort & Wasser & Flussverbau & 20 & 6 \\
\hline Arnitobel & Wasser & lokale Ufersicherung & 20 & 9 \\
\hline Chaltibach & Wasser & Flussverbau & 20 & 11 \\
\hline Mittelgrüss/Müliwald & Wasser & Runsenverbau & 21 & 1 \\
\hline Mehlbach & Wasser & Bachverbau & 26 & 2 \\
\hline Aa Hochtal, Oberlauf & Wasser & lokale Ufersicherung & 28 & 10 \\
\hline Aa Hochtal, Dorf & Wasser & Flussverbau & 31 & 4 \\
\hline Erlenbach & Wasser & Flussverbau & 31 & 12 \\
\hline Dürrbach & Wasser & Bachverbau & 59 & 7 \\
\hline
\end{tabular}

Ebenfalls nach Regel 1 wurde das Untersuchungsgebiet hinsichtlich Überschreitung tolerierbarer Sachrisiken analysiert. Diese Analyse ergab in drei Gebieten Überschreitungen auf kleiner Fläche (Engelberger Aa (0.43 ha Weideland); Chüelauigraben (1.68 ha Weideland); Mehlbach (0.02ha Wiesland)).

Die Rangierung des Prioritätsindexes nach Regel 2 führt zu einer stark veränderten Prioritätenliste. Hohe Prioritäten ergeben sich für Massnahmen in Lawinengebieten, mittlere Prioritäten für Gebiete mit Wildbach- und Massenbewegungsgefahren, während Steinschlaggebieten deutlich untergeordnete Bedeutung zufällt (Tabelle 64). 
Tabelle 64 Prioritäten von Massnahmen zur Risikoverminderung gemäss Regel 2.

\begin{tabular}{|c|c|c|c|c|}
\hline Gefahrenquelle & $\begin{array}{l}\text { Gefahrenpro- } \\
\text { zess [GP] }\end{array}$ & $\begin{array}{l}\text { Projekt/Risikover- } \\
\text { minderungsmassnahme } \\
\text { gem. Masterplan }\end{array}$ & $\begin{array}{l}\text { Priorität gemäss } \\
\text { Managementkon- } \\
\text { zept }\end{array}$ & $\begin{array}{l}\text { Priorität ge- } \\
\text { mäss Master- } \\
\text { plan }\end{array}$ \\
\hline Chüelaui & Lawine & Lawinenwarnung & 1 & 5 \\
\hline Schluchen & Lawine & Lawinenwarnung & 2 & 15 \\
\hline Pfaffen & Lawine & Lawinenwarnung & 3 & 5 \\
\hline Titlislawine & Lawine & Lawinenwarnung & 4 & 5 \\
\hline Rigidal & Lawine & Lawinenwarnung & 5 & 15 \\
\hline Seeligraben & Wasser & -- & 6 & 45 \\
\hline Eienwäldli & Lawine & Lawinenwarnung & 7 & 5 \\
\hline Bärenbach & Wasser & lokale Ufersicherung & 8 & 8 \\
\hline Bord & Wasser & -- & 9 & 45 \\
\hline Sitenwald-Bord & Lawine & -- & 10 & 15 \\
\hline Fangbach & Wasser & Geschiebesammler & 12 & 3 \\
\hline Mittelgrüss/Müliwald & Wasser & Runsenverbau & 15 & 1 \\
\hline Mehlbach & Wasser & Bachverbau & 16 & 2 \\
\hline Aa Hochtal, Dorf & Wasser & Flussverbau & 27 & 4 \\
\hline Erlenbach & Wasser & Flussverbau & 27 & 12 \\
\hline Aa Grafenort & Wasser & Flussverbau & 28 & 6 \\
\hline Arnitobel & Wasser & lokale Ufersicherung & 28 & 9 \\
\hline Dürrbach & Wasser & Bachverbau & 29 & 7 \\
\hline Aa Hochtal, Oberlauf & Wasser & lokale Ufersicherung & 33 & 10 \\
\hline Zelgli & Sturz & Steinschlagschutz & 47 & 5 \\
\hline
\end{tabular}

Das schutzzielbasierte Managementkonzept (Kapitel 4) kombiniert Regel 1 und 2, wobei Regel 1 Vorrang zukommt. Daher wurden die Gefahrenquellen zuerst rangiert nach dem Flächen- und Längenmass der gefährdeten Objekte, bei denen individuelle Risiken die tolerierbaren Werte um mehr als einen Faktor 10 überschreiten. Derart hohe individuelle Risiken wurden bei 32 von 61 Gefahrenquellen festgestellt. Anschliessend erfolgte eine Rangierung der Gefahrenquellen, welche Überschreitungen der individuellen Risiken um den Faktor 110 enthalten. Dies trifft bei weiteren 24 Gefahrenquellen zu. Die Prioritäten 1-56 ergeben sich somit aus der Anwendung von Regel 1. Lediglich 5 von 61 Gefahrenquellen waren anschliessend nach Regel 2 zu priorisieren.

\subsubsection{Diskussion der Resultate}

Das Verfahren führt zu einer klaren Aussage zu den Stellen mit grossen Risiken und Handlungsbedarf. Der Analyse der Personenrisiken nach Regel 1 kommt für die Priorisierung der Gefahrengebiete die entscheidende Bedeutung zu. Diese Erkenntnis überrascht, zumal die Schwellenwerte für die maximal tolerierbaren Risiken hoch angesetzt wurden. 
Für die Priorisierung unbedeutend sind die individuellen Sachrisiken. Überschreitungen der tolerierbaren Sachrisiken nach Regel 1 sind selten und führen zu keinen wesentlichen Veränderungen in der Priorisierung. Ebenso ist Regel 2 für die Priorisierung irrelevant. Im Fallbeispiel wurde das Ziel verfehlt, einen überwiegenden Teil der Gefahrengebiete und Massnahmen nach Regel 2 und damit umfassend aufgrund aller Schutzzielprinzipien zu priorisieren (Kapitel 4.2.1). Das Managementkonzept führt insgesamt zu plausiblen Ergebnissen. Mit wenigen Ausnahmen kommt Gefahrengebieten hohe Priorität zu, die erfahrungsgemäss und intuitiv als risikoreich bekannt sind.

Die im Untersuchungsgebiet sehr verbreitete Überschreitung der - an sich hohen Schwellenwerte für Personenrisiken zeigt, dass diese Risiken im Vergleich zum gesamtgesellschaftlichen Kontext sehr hoch sind. Dies weist auf die Notwendigkeit zahlreicher Massnahmen und einer möglicherweise erhöhten Mittelbereitstellung hin. Die Höhe des für Risikoverminderungsmassnahmen bereitzustellenden jährlichen Budgets hängt davon ab, in welchem Zeitraum die überhöhten Risiken eliminiert werden sollen.

\subsubsection{Vergleich mit dem Masterplan}

Die Priorisierung nach Regel 1 (nicht auf eine Einheitsfläche umgerechnet) weist geringe Ähnlichkeiten mit der Priorisierung gemäss Masterplan (Tabelle 61) auf. Nur drei der zwölf Gefahrengebiete mit technischen Verbaumassnahmen gemäss Masterplan erscheinen zwischen Position 1 und 10 der Prioritätenliste nach Regel 1. Innerhalb der ersten zehn Positionen nach Regel 1 befinden sich drei Gefahrenquellen, an denen in den letzten zehn Jahren grössere Steinschlagschutzprojekte abgeschlossen wurden (Eschlenfluewald, RucheggGrüenenwald und Rapperschwendi). Zur Unterstützung der dort errichteten Verbauungen werden noch Waldpflegemassnahmen durchgeführt. Weitere nicht im Masterplan, jedoch in der Prioritätenliste nach Regel 1 zuvorderst erscheinende Gefahrengebiete sind zwar als risikoreich bekannt, es konnten jedoch bis jetzt keine Trägerschaften zur Massnahmenplanung gebildet werden (Tellensteinwald, Rotigraben). Die im Masterplan priorisierten Wasserbauprojekte rangieren nach Regel 1 im Mittelfeld der Prioritätenlisten. Rechnet man die Schutzzielüberschreitung nach Regel 1 auf eine Einheitsfläche um (Tabelle 63), verändert sich dieses Bild nur unwesentlich.

Die Priorisierung nach Regel 2 ergibt im Vergleich zur Prioritätensetzung des Masterplans eine ähnlich geringe Übereinstimmung. Auffallend sind die hohe Priorität der Lawinengebiete und die geringe Priorität der Steinschlaggebiete. So fällt das gemäss Regel 1 prioritäre Steinschlagprojekt Zelgli in den hinteren Teil der Prioritätenliste.

Die Ergebnisse stimmen nur teilweise mit dem Masterplan überein, von dem vermutet wird, er sei Ausdruck des gesellschaftlich gewünschten und als plausibel betrachteten Handlungsbedarfs. Im Vergleich zum Masterplan misst das Managementkonzept den Steinschlagrisiken höhere, den Wasserrisiken tiefere Prioritäten zu.

\subsubsection{Methodische Erkenntnisse}

Das Untersuchungsgebiet ist so zu wählen, dass Entstehungs-, Transit- und Ablagerungsgebiete für alle Gefahrenquellen in ähnlicher Weise berücksichtigt sind. Dies erfordert gute Kenntnisse des Gebiets, insbesondere der Gefahrenprozesse und der Raumnutzung. Die Begrenzung des Untersuchungsgebiets wirkt sich auf die Resultate aus.

Auch die Ausscheidung und Abgrenzung der Gefahrengebiete hat Auswirkungen auf die Resultate. Bei der Abgrenzung sind zu grosse und zu inhomogen besiedelte und genutzte Gebiete zu vermeiden, um die Resultate der Risikoanalyse und Priorisierung nicht zu verwischen. Deshalb wurde beispielsweise der ursprünglich als ein Gefahrengebiet betrachtete 
Prozessraum der Engelberger Aa mit einer Grösse von über $2 \mathrm{~km}^{2}$ in einem zweiten Berechnungsdurchgang in drei Gebiete mit unterschiedlicher Raumnutzung aufgeteilt.

Die Ermittlung des Objektindexes erwies sich als anspruchsvoll. Sie erfolgte im Rahmen des Fallbeispiels unabhängig durch drei Fachpersonen. Die Resultate wiesen zum Teil erhebliche Streuungen auf. Besonders unterschiedlich bewertet wurden die Kriterien des Kontextprinzips, welche in besonderem Masse subjektiven Charakter aufweisen. Das Kontextprinzip wurde daher bei der Ermittlung des Objektindexes nur zur Hälfte gewichtet (Kapitel 4.2.3.2). Die meisten Objekte wurden sowohl aus Sicht des Eigentümers/Betreibers als auch des Nutzniessers als ,typischerweise betroffene Person' beurteilt (Kapitel 4.2.3.2). Für die Berechnung wurde die Bewertung aus Sicht der Nutzniesser berücksichtigt.

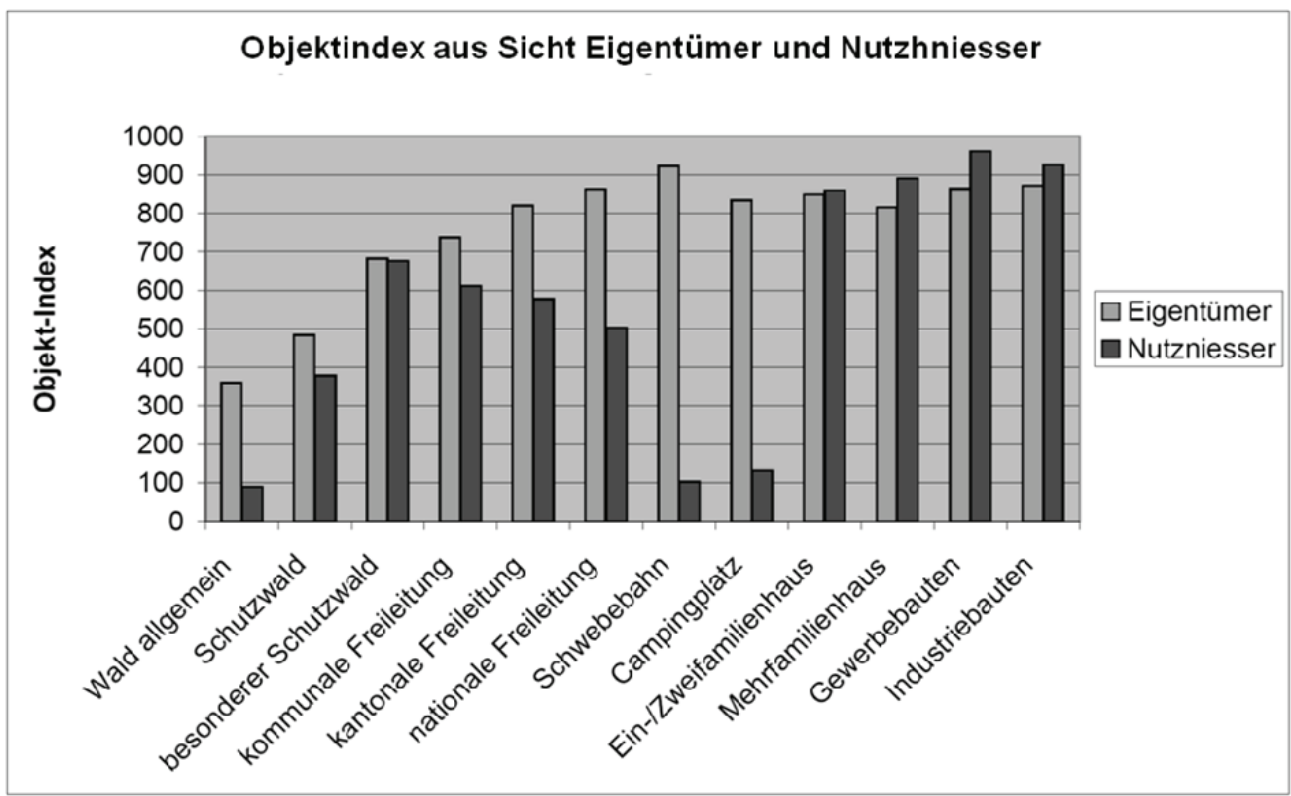

Abbildung 49 Objektindex in Abhängigkeit der risikobetroffenen Person.

Im Bereich Wohnen und Industrie sind die rollenabhängigen Unterschiede gering. Beim Wald werden die Unterschiede mit zunehmender Schutzwaldbedeutung geringer. Grösser werden die Unterschiede bei Leitungen mit zunehmender Grösse des Versorgungsgebiets. Dies ist auf die Annahme zurückzuführen, wonach bei grösseren Leitungen eher Versorgungsalternativen (Redundanzen) bestehen als bei lokalen Leitungen. Ein einzelner Benutzer ist somit vom Ausfall einer lokalen Leitung stärker betroffen. Für die Betreiber steigt dagegen die Betroffenheit mit zunehmender Wichtigkeit der Leitung. Die deutlichsten Unterschiede zwischen Eigentümer und Nutzniesser bestehen bei touristischer Infrastruktur. Hier besteht ein wesentlicher Unterschied, wenn ein Schaden aus Sicht des wirtschaftlich betroffenen Betreibers oder aber des in seiner freiwilligen Freizeitgestaltung eingeschränkten Benutzers betrachtet wird. Aus Sicht des Ersteren kann ein (mit öffentlichen Mitteln) schutzwürdiges Interesse abgeleitet werden. Aus der zweiten Sichtweise lässt sich kaum ein öffentliches Engagement rechtfertigen. Dieser Unterschied drückt sich auch in der langjährigen Diskussion um die Subventionierbarkeit von Massnahmen zum Schutz touristischer Anlagen aus. Artikel 42 der Waldverordnung (WaV, SR 921.01) schloss bisher Massnahmen zum Schutz von touristischen Anlagen kategorisch aus. Die im Rahmen der Anpassung des Verordnungsrechts an die NFA formulierten Art. 2 der Wasserbauverordung (WBV, SR 721.100.1) und Art. 40 der Waldverordnung begrenzen den Ausschluss auf touristische Anlagen, ausserhalb des Sied- 
lungsgebiets'. Dieser Präzisierung ist beizupflichten, zumal der Betrieb einer touristischen Anlage auch als schützenswerte wirtschaftliche Aktivität gelten kann.

Die Bewertung der Massnahmen zur Herleitung der Massnahmenindizes sowie die Charakterisierung der Gefahrengebiete erwiesen sich im Vergleich zum Objektindex als wesentlich einfacher. Hier wiesen die Bewertungen eine wesentlich geringere Streuung auf.

\subsubsection{Fallbeispiel 2: Vereinfachte Abschätzung des Handlungsbedarfs}

\subsubsection{Grundlagen und Vorgehen}

Grundlagen für das Verfahren zur vereinfachten regionalen Abschätzung des Handlungsbedarfs sind die Gefahrenkarte, die Schadenspotenzialkarte und die in Fallbeispiel 1 erwähnten Gefahrengebiete mit ihrem Massnahmenindex. Diese werden in einer GIS-Analyse mit der Schadenspotenzial- und der Gefahrenkarte zu einer Karte des Handlungsbedarfs verschnitten.

\subsubsection{Prioritäten aus vereinfachter Abschätzung des Handlungsbedarfes}

Das Verfahren priorisiert vor allem Lawinen- und Wassergefahrenquellen. Bei der Abschätzung des kumulierten Handlungsbedarfs pro Gefahrengebiet erscheinen die grossen Gefahrengebiete (Engelberger Aa, Titlislawine, Brächgraben) weit oben auf der Prioritätenliste (Tabelle 65).

Tabelle 65 Prioritäten von Massnahmen zur Risikoverminderung gemäss vereinfachter Abschätzung des Handlungsbedarfs.

\begin{tabular}{|c|c|c|c|c|}
\hline Gefahrenquelle & $\begin{array}{l}\text { Gefahren- } \\
\text { prozess } \\
{[G P]}\end{array}$ & $\begin{array}{l}\text { Projekt/Risikover- } \\
\text { minderungsmassnahme } \\
\text { gem. Masterplan }\end{array}$ & $\begin{array}{l}\text { Priorität gemäss } \\
\text { Management- } \\
\text { konzept }\end{array}$ & $\begin{array}{l}\text { Priorität gemäss } \\
\text { Masterplan }\end{array}$ \\
\hline Aa Hochtal, Dorf & Wasser & Flussverbau & 1 & 4 \\
\hline Erlenbach & Wasser & Flussverbau & 1 & 12 \\
\hline Aa Grafenort & Wasser & Flussverbau & 2 & 6 \\
\hline Chaltibach & Wasser & Flussverbau, Renat. & 2 & 11 \\
\hline Arnitobel & Wasser & lokale Ufersicherung & 2 & 9 \\
\hline Titlislawine & Lawine & Lawinenwarnung & 3 & 5 \\
\hline Aa Hochtal, Oberlauf & Wasser & lokale Ufersicherung & 4 & 10 \\
\hline Brächgraben & Lawine & Lawinenwarnung & 5 & 5 \\
\hline Mehlbach & Wasser & Bachverbau & 6 & 2 \\
\hline Eienwäldli & Lawine & Lawinenwarnung & 7 & 5 \\
\hline Bärenbach & Wasser & lokale Ufersicherung & 8 & 8 \\
\hline Schwand & Wasser & -- & 9 & -- \\
\hline Eschlenfluewald & Sturz & ehem. Steinschl.schutzpr. & 10 & 5 \\
\hline Mittelgrüss/Müliwald & Wasser & Runsenverbau & 12 & 1 \\
\hline Fangbach & Wasser & Geschiebesammler & 18 & 3 \\
\hline Dürrbach & Wasser & Bachverbau & 23 & 7 \\
\hline Zelgli & Sturz & Steinschlagschutz & 24 & 5 \\
\hline
\end{tabular}


Die Analyse des Handlungsbedarfs pro Flächeneinheit priorisiert ebenfalls - wenn auch etwas weniger deutlich - die Wassergefahrengebiete. Die tiefste Priorität fällt insgesamt den Steinschlaggebieten zu (Tabelle 66).

Tabelle 66 Prioritäten von Massnahmen zur Risikoverminderung gemäss vereinfachter flächenbereinigter Abschätzung des Handlungsbedarfs.

\begin{tabular}{|c|c|c|c|c|}
\hline Gefahrenquelle & $\begin{array}{l}\text { Gefahren- } \\
\text { prozess } \\
{[G P]}\end{array}$ & $\begin{array}{l}\text { Projekt/Risikover- } \\
\text { minderungsmassnahme } \\
\text { gem. Masterplan }\end{array}$ & $\begin{array}{l}\text { Priorität gemäss } \\
\text { Managementkon- } \\
\text { zept }\end{array}$ & $\begin{array}{l}\text { Priorität gemäss } \\
\text { Masterplan }\end{array}$ \\
\hline Wasserfall & Wasser & -- & 1 & 45 \\
\hline Schuemettlen & Sturz & -- & 2 & 45 \\
\hline Schluchen & Lawine & Lawinenwarnung & 3 & 15 \\
\hline Aa Hochtal, Dorf & Wasser & Flussverbau & 4 & 4 \\
\hline Erlenbach & Wasser & Flussverbau & 4 & 12 \\
\hline Ruchegg-Grüenenw. & Sturz & Schutzwaldpflege & 5 & 5 \\
\hline Fangbach & Wasser & Geschiebesammler & 6 & 3 \\
\hline Bränd-Schwand & Lawine & Lawinenwarnung & 7 & 25 \\
\hline Bärenbach & Wasser & lokale Ufersicherung & 8 & 8 \\
\hline Mittelgrüss/Müliwald & Wasser & Runsenverbau & 9 & 1 \\
\hline Tellensteinwald & Sturz & -- & 10 & 45 \\
\hline Aa Grafenort & Wasser & Flussverbau & 15 & 6 \\
\hline Arnitobel & Wasser & lokale Ufersicherung & 15 & 9 \\
\hline Chaltibach & Wasser & Flussverbau, Renat. & 15 & 11 \\
\hline Mehlbach & Wasser & Bachverbau & 26 & 2 \\
\hline Dürrbach & Wasser & Bachverbau & 31 & 7 \\
\hline Aa Hochtal, Oberlauf & Wasser & lokale Ufersicherung & 34 & 10 \\
\hline Zelgli & Sturz & Steinschlagschutz & 54 & 4 \\
\hline
\end{tabular}

\subsubsection{Diskussion der Resultate und Vergleich mit dem Masterplan}

Die Prioritäten gemäss vereinfachter regionaler Abschätzung des Handlungsbedarfs weisen sowohl hinsichtlich kumuliertem Handlungsbedarf als auch flächenbereinigtem Handlungsbedarf - mit einzelnen Abweichungen (Dürrbach, Fangbach, Zelgli) - eine grosse Übereinstimmung mit der Priorisierung des Masterplans auf. Die meisten Massnahmen des Masterplans befinden sich im ersten Viertel der Prioritätenliste. In der flächenbereinigten Version wird die Prioritätenliste allerdings von drei als nicht besonders risikoreich bekannten Gefahrengebieten angeführt.

\subsubsection{Fallbeispiel 3: Schutzzielmatrizes}

\subsubsection{Grundlagen und Vorgehen}

Das dritte Fallbeispiel umfasst die Erstellung von Schutzdefizitkarten gemäss Vorgehen ,Risikoanalyse bei gravitativen Naturgefahren' (Borter, 1999; Risikoanalyse Stufe 1). Für die Anwendung der Schutzzielmatrizes und die Ermittlung der Schutzdefizite ist eine detailliertere Gefahrenkarte erforderlich, welche die erwarteten Gefahrenprozesse hinsichtlich ihrer Josef Th. Hess: Schutzziele im Umgang mit Naturrisiken in der Schweiz, @ vdf Hochschulverlag 2011 
Häufigkeit und Intensität klassiert (drei Häufigkeitsklassen und drei Intensitätsstufen). Ferner ist eine Schadenspotenzialkarte erforderlich. Nebst der klassischen Schutzzielmatrix (Abbildung 56) wurden modifizierte Schutzzielmatrizes

- aufgrund des Objektindexes aus Sicht ,Benutzer eines Objekts';

- aufgrund des Objektindexes aus Sicht ,Eigentümer eines Objekts' und

- aufgrund der Berechnungen zum individuellen Risiko

angewendet (Anhang XV). Wiederum wurden die Schutzdefizite sowohl nach ihrem gesamten Mass pro Gefahrenquelle als auch umgerechnet pro Flächeneinheit ermittelt.

\subsubsection{Prioritäten gemäss Schutzzielmatrizes}

Bei der Priorisierung der Gefahrenquellen aufgrund der Schutzdefizite ergeben sich im Mittel für alle drei Arten von Gefahrenprozessen (Sturz, Lawine, Wasser) ähnlich hohe Prioritäten. Etwas höhere Prioritäten kommen insgesamt den Lawinengefahrengebieten zu. Grossflächige Gefahrenquellen weisen grosse kumulierte Schutzdefizite und demzufolge höhere Prioritäten auf. Die Wassergefahrengebiete an der Engelberger Aa werden in den meisten Varianten prioritär eingestuft (Tabelle 67). Die Unterschiede zwischen den verschiedenen Varianten der Schutzzielmatrizes sind mit wenigen Ausnahmen (Engelberger Aa im Dorfbereich, Mittelgrüss-Müliwald) gering. Dies gilt auch für die räumliche Verteilung der Schutzdefizite (Abbildung 50 und Abbildung 51).

Tabelle 67 Prioritäten von Risikoverminderungsmassnahmen aufgrund von Schutzdefiziten umgerechnet auf ein einheitliches Flächenmass (GP = Gefahrenprozess, L = Lawine, S = Sturzprozess, W = Wassergefahr).

\begin{tabular}{|c|c|c|c|c|c|c|}
\hline Gefahrenquelle & $G P$ & $\begin{array}{l}\text { Priorität } \\
\text { aufgrund } \\
\text { Schutzdef. } \\
\text { BUWAL-OW }\end{array}$ & $\begin{array}{l}\text { Priorität } \\
\text { gemäss } \\
\text { Schutzdefizit } \\
\text {,Benutzer' }\end{array}$ & $\begin{array}{l}\text { Priorität } \\
\text { gemäss } \\
\text { Schutzdefizit } \\
\text {,Eigentümer' }\end{array}$ & $\begin{array}{l}\text { Priorität } \\
\text { gemäss } \\
\text { Schutzdefizit } \\
\text { ind. Risiken }\end{array}$ & $\begin{array}{l}\text { Priorität } \\
\text { gemäss } \\
\text { Masterplan }\end{array}$ \\
\hline Titlislawine & L & 1 & 7 & 7 & 2 & 5 \\
\hline Aa Grafenort & W & 2 & 2 & 2 & 4 & 6 \\
\hline Chaltibach & W & 2 & 2 & 2 & 4 & 11 \\
\hline Arnitobel & W & 2 & 2 & 2 & 4 & 9 \\
\hline Eschlenfluewald & $S$ & 3 & 1 & 1 & 1 & 5 \\
\hline Rigidal & L & 4 & 5 & 6 & 5 & 15 \\
\hline Ruchegg-Grüenenw. & S & 5 & 4 & 5 & 6 & 5 \\
\hline Bärenbach & W & 6 & 8 & 9 & 9 & 8 \\
\hline Eienwäldli & L & 7 & 10 & 10 & 10 & 5 \\
\hline Zelgli & $S$ & 8 & 9 & 4 & 7 & 5 \\
\hline Aa Hochtal, Dorf & W & 9 & 25 & 27 & 16 & 4 \\
\hline Erlenbach & W & 9 & 25 & 27 & 16 & 12 \\
\hline Fellenrüti-Zelgli & L & 10 & 17 & 17 & 15 & 15 \\
\hline Aa Hochtal, Oberlauf & W & 12 & 15 & 14 & 18 & 10 \\
\hline Tellensteinwald & $S$ & 13 & 3 & 3 & 3 & 45 \\
\hline Mittelgrüss/Müliwald & W & 15 & 29 & 28 & 23 & 1 \\
\hline Rapperschwendi & $S$ & 17 & 6 & 8 & 8 & 5 \\
\hline
\end{tabular}




\begin{tabular}{|c|c|c|c|c|c|c|}
\hline Fangbach & W & 24 & 23 & 23 & 29 & 3 \\
\hline Mehlbach & W & 29 & 45 & 30 & 34 & 2 \\
\hline Dürrbach & W & 30 & 38 & 39 & 35 & 7 \\
\hline
\end{tabular}

Die Betrachtung der Schutzdefizite pro Flächeneinheit verändert erwartungsgemäss die Prioritätenliste zugunsten der Sturzgebiete und zuungunsten der grösseren Wassergefahrengebiete. Vor allem die Gebiete an der Engelberger Aa fallen in mittlere Prioritäten zurück (Tabelle 68).

Tabelle 68 Prioritäten von Risikoverminderungsmassnahmen aufgrund von Schutzdefiziten pro Flächeneinheit (GP = Gefahrenprozess, L = Lawine, $S=$ Sturzprozess, $W=$ Wassergefahr, SD = Schutzdefizit).

\begin{tabular}{|c|c|c|c|c|c|c|}
\hline Gefahrenquelle & $G P$ & $\begin{array}{l}\text { Priorität } \\
\text { aufgrund SD } \\
\text { BUWAL-OW } \\
\text { pro Einheits- } \\
\text { fläche }\end{array}$ & $\begin{array}{l}\text { Priorität } \\
\text { aufgrund SD } \\
\text {,Benutzer' } \\
\text { pro Einheits- } \\
\text { fläche }\end{array}$ & $\begin{array}{l}\text { Priorität } \\
\text { aufgrund } S D \\
\text {,Eigentümer' } \\
\text { pro Einheits- } \\
\text { fläche }\end{array}$ & $\begin{array}{l}\text { Prio. aufgr. } \\
\text { SD ,individ. } \\
\text { Risiken' pro } \\
\text { Einheitsflä- } \\
\text { che }\end{array}$ & $\begin{array}{l}\text { Priorität } \\
\text { gemäss } \\
\text { Masterplan }\end{array}$ \\
\hline Dürrbach & W & 1 & 2 & 2 & 2 & 7 \\
\hline Tellensteinwald & $S$ & 2 & 1 & 1 & 1 & 45 \\
\hline Füren & L & 3 & 18 & 6 & 26 & 15 \\
\hline Ruchegg-Grüenenw. & $S$ & 4 & 6 & 7 & 5 & 5 \\
\hline Schuemettlen & $S$ & 5 & 4 & 4 & 7 & 45 \\
\hline Rigidal & L & 6 & 7 & 9 & 8 & 15 \\
\hline Fellenrüti-Zelgli & L & 7 & 16 & 17 & 13 & 15 \\
\hline Pfaffen & $\mathrm{L}$ & 8 & 9 & 11 & 6 & 5 \\
\hline Eschlenfluewald & $S$ & 9 & 3 & 3 & 3 & 5 \\
\hline Aa in Grafenort & W & 10 & 12 & 13 & 20 & 6 \\
\hline Arnitobel & W & 10 & 12 & 13 & 20 & 9 \\
\hline Chaltibach & W & 10 & 12 & 13 & 20 & 11 \\
\hline Zelgli & $S$ & 11 & 11 & 8 & 9 & 5 \\
\hline Bärenbach & W & 13 & 13 & 14 & 14 & 8 \\
\hline Rapperschwendi & $\mathrm{S}$ & 14 & 5 & 5 & 4 & 5 \\
\hline Mittelgrüss/Müliwald & W & 18 & 46 & 42 & 34 & 1 \\
\hline Dürrenwald & $S$ & 21 & 10 & 12 & 10 & 45 \\
\hline Fangbach & W & 24 & 20 & 21 & 33 & 3 \\
\hline Aa im Hochtal, Dorf & W & 27 & 47 & 51 & 40 & 4 \\
\hline Erlenbach & W & 27 & 47 & 51 & 40 & 12 \\
\hline Aa Hochtal, Oberlauf & W & 29 & 43 & 48 & 43 & 10 \\
\hline Mehlbach & W & 30 & 53 & 35 & 41 & 2 \\
\hline Widerwällhubel & $S$ & 59 & 8 & 10 & 30 & 45 \\
\hline
\end{tabular}




\subsubsection{Diskussion der Resultate}

Die mit den modifizierten Versionen hergeleiteten Schutzdefizite weichen im Allgemeinen wenig von jenen der ,klassischen' Schutzzielmatrix (BUWAL/OW) ab. Ähnliche Priorisierungen ergeben sich aufgrund der modifizierten Matrizes ,Eigentümer' und ,Benutzer'. Zwischen den Resultaten aus diesen beiden Beurteilungen einerseits und den Ergebnissen aus der ,klassischen' Schutzzielmatrix sowie der Schutzzielmatrix aufgrund individueller Risiken anderseits sind die Abweichungen grösser. Bei Sturzgefahrengebieten führen die Beurteilungen nach den Matrizes ,Eigentümer' und ,Benutzer' zu höheren, bei Wassergefahrengebieten zu geringeren Prioritäten als bei der Beurteilung nach der ,klassischen' Matrix.

\subsubsection{Vergleich mit dem Masterplan}

Die aufgrund der Schutzdefizite pro Gefahrenquelle hergeleiteten Prioritäten stimmen in vielen Fällen für alle vier Varianten gut mit den Prioritäten des Masterplanes überein (Tabelle 67). Acht von zwölf Gebieten mit Projekten gemäss Masterplan erscheinen auch im ersten Viertel der Prioritätenliste gemäss Schutzdefiziten. Die Projekte Mittelgrüss, Fangbach, Mehlbach und Dürrbach fallen in dieser Priorisierung überraschend deutlich ins Mittelfeld. Im Vergleich fallen die Prioritäten für Steinschlaggebiete höher und für Lawinen- und Wassergefahrengebiete tiefer aus als nach Masterplan.

Mit der Umrechnung der Schutzdefizite auf eine Einheitsfläche ändert sich die Priorisierung deutlich (Tabelle 68). Nur noch vier der gemäss Masterplan prioritären Projekte erscheinen im ersten Viertel der Prioritätenliste nach Schutzdefiziten. Die übrigen gemäss Masterplan vordringlichen Projekte erhalten mittlere Prioritäten.

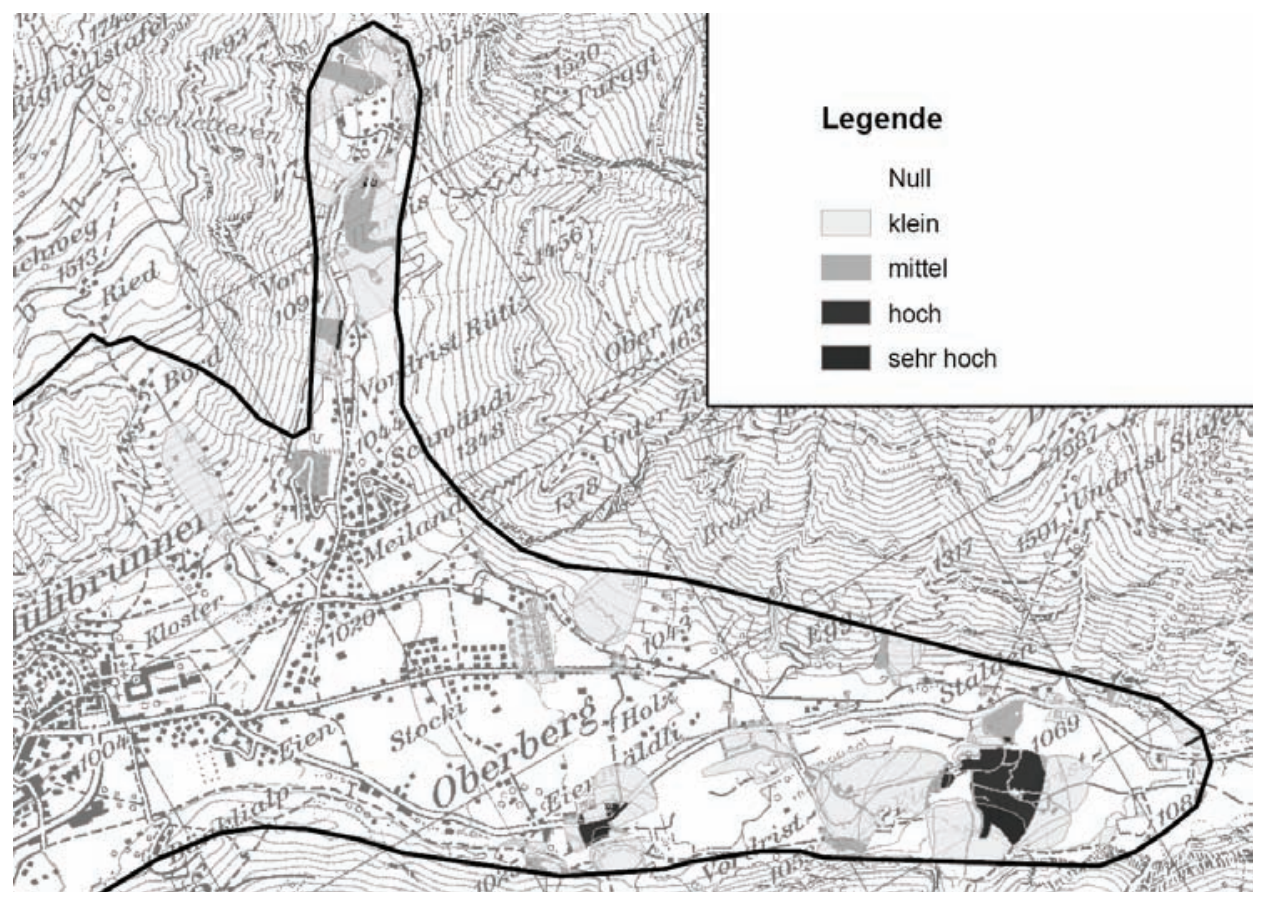

Abbildung 50 Schutzdefizitkarte nach ,klassischer' Schutzzielmatrix, Borter (1999). 


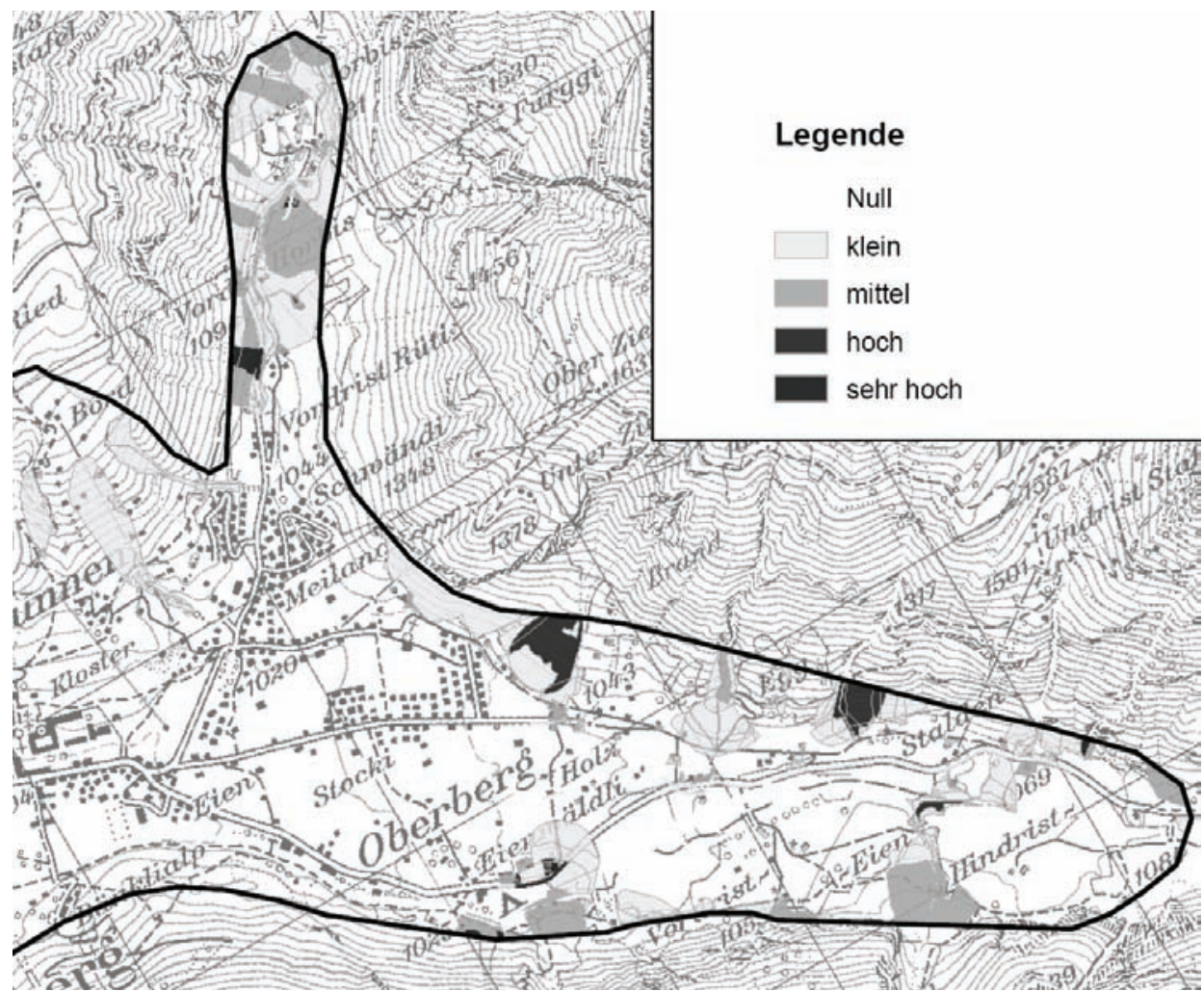

Abbildung 51 Schutzdefizitkarte nach modifizierter Schutzzielmatrix ,Benutzer'.

\subsubsection{Vergleich der Resultate zwischen den Verfahren}

Generell bestehen bei den höchsten Prioritäten von Sturzgefahrengebieten die meisten gegenseitigen Übereinstimmungen zwischen den Resultaten aus den verschiedenen Verfahren. Risikoreiche Sturzgefahrenquellen werden bei allen Verfahren priorisiert. Bei den Lawinen- und Wassergefahrengebieten sind die gegenseitigen Abweichungen im vordersten Bereich der Prioritätenliste deutlicher. Die Resultate liegen näher zusammen, wenn die Priorisierung aufgrund der nicht flächenbereinigten Werte erfolgt. In dieser Version bestehen auch grössere Übereinstimmungen mit den Prioritäten des Masterplans. Dies zeigt, dass die faktische Prioritätensetzung des Masterplans grössere Gefahrengebiete favorisiert und damit durch die Höhe der kollektiven Risiken beeinflusst ist.

Gemittelt über alle Gefahrengebiete und alle Prioritäten liegen die höchsten Prioritäten bei den Wassergefahrengebieten, während die Sturz- und Lawinengebiete etwas tiefer priorisiert werden. Bei den Wassergefahrengebieten sind die Unterschiede zwischen den Mittelwerten, die sich aus den verschiedenen Verfahren ergeben, am geringsten, bei den Lawinengebieten am grössten (Abbildung 52). 

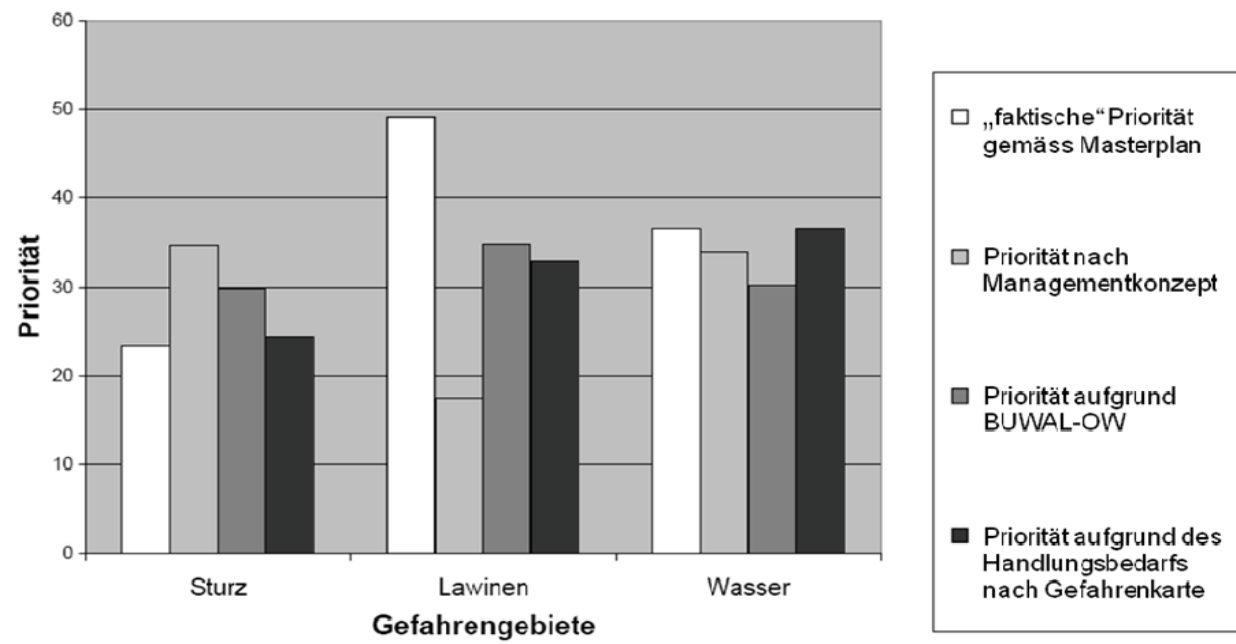

Abbildung 52 Vergleich aller Gefahrenquellen und Prioritäten nach Verfahren (SD = Schutzdefizit).

\subsection{Fallbeispiel 4: Hochwasserschutz Sarneraatal}

\subsubsection{Ziel des Fallbeispieles}

Das Fallbeispiel stellt die Vorgänge der Jahre 2006 bis 2008 bei der Zielfestlegung und -gewichtung sowie der Variantenwahl im Projekt Hochwasserschutz Sarneraatal dar und bezweckt einen Praxistest des in Kapitel 2.9.4.2 und 4.6.2 beschriebenen Mitwirkungsverfahrens. Das Fallbeispiel beschränkt sich auf folgende Schritte in der Planungs- und Entscheidfindungsphase:

- Kontextanalyse;

- Identifikation und Klassifikation der Akteure;

- Zielerhebung durch die Akteure;

- Variantendiskussion mit den Akteuren und

- Variantenentscheid.

Die Massnahmen des Projekts sind mit Ausnahme kleinerer Vorarbeiten nicht realisiert (Stand September 2008).

\subsubsection{Politische Rahmenbedingungen}

Wenige Jahre nach der Einleitung der Grossen Melchaa in den Sarnersee im Jahr 1880 und dem folgenden Ausbau der Sarneraa wurde in verschiedenen politischen Vorstössen auf die Notwendigkeit hingewiesen, die Abflussverhältnisse der Sarneraa im Dorfbereich zu verbessern. Die Projekte kamen nie über die Planungsphase hinaus. Erst nach den grossen Hochwasserereignissen 1999 und 2004 wurden die Bestrebungen um eine Verbesserung der Hochwassersicherheit intensiviert.

Bis zum Sommer 2005 behinderte ein Zuständigkeitskonflikt zwischen Kanton und der Gemeinde Sarnen eine Lösungsfindung. Seit dem Hochwasser vom August 2005 wird unter grossem Zeitdruck an einer Lösung zur Verminderung der Hochwasserrisiken gearbeitet. Die Zuständigkeitsfrage wurde durch die Schaffung eines Spezialgesetzes (GAHS, GDB 740.2) geklärt, das die Trägerschaft dem Kanton zuschreibt, die betroffenen Gemeinden jedoch nach Massgabe ihres Nutzens zur Mitfinanzierung beizieht. 
Die Leitung des Projekts liegt beim Kanton Obwalden. Seit Projektbeginn wird das Projekt durch eine zehnköpfige Projektsteuergruppe begleitet, in der die Gemeinde Sarnen, das Bundesamt für Umwelt sowie kantonale Fachstellen vertreten sind. Eine erweiterte Projektsteuergruppe mit Vertretern der weiteren betroffenen Gemeinden und Anstössern, Fachstellen des Bundes, des Kantons und der Gemeinden sowie der Unterlieger dient dem gegenseitigen Informations- und Meinungsaustausch sowie der Vorberatung von Entscheiden zuhanden der zuständigen politischen Behörde.

\subsubsection{Variantenevaluation im partizipativen Verfahren}

Mit einer Konzeptstudie wurden über zwanzig Varianten zur Verbesserung der Hochwassersicherheit im Sarneraatal geprüft. Folgende drei Varianten wurden zur Weiterbearbeitung empfohlen (Tabelle 69):

- Variante 1 Tieferlegung/Verbreiterung der Sarneraa: Tieferlegung und Verbreiterung der Sarneraa entlang ihrem heutigen Lauf zwischen Seeauslauf und Wichelsee;

- Variante 2 Landenberg: Bau eines Entlastungsstollens vom See durch den Landenberg, Aufweitungen unterhalb der Rückführung des Wassers in die Sarneraa ähnlich wie bei Variante 1, jedoch ohne Tieferlegung im Dorfbereich;

- Variante 3 Bergvariante Ost: Bau eines Entlastungsstollens in der rechten Talflanke zwischen Sarner- und Wichelsee.

Tabelle 69 Vergleichende Zusammenstellung der Varianten zur Verbesserung der Hochwassersicherheit Sarneraatal (Vorstudie).

\begin{tabular}{llccc}
\hline Nr. & Bezeichnung & $\begin{array}{l}\text { Investitionskosten } \\
\text { (Mio. CHF) }\end{array}$ & $\begin{array}{l}\text { Davon Lander- } \\
\text { werb (Mio. CHF) }\end{array}$ & $\begin{array}{l}\text { Betriebs- und Unterhalts- } \\
\text { kosten (in Tausend CHF) }\end{array}$ \\
\hline V1 & $\begin{array}{l}\text { Variante Tieferlegung/ } \\
\text { Verbreiterung }\end{array}$ & 44.6 & 6.1 & 450 \\
V2 & Variante Landenberg & 56.6 & 4.7 & 580 \\
V3 & Variante Ost & 59.1 & 1.3 & 500 \\
\hline
\end{tabular}

${ }^{*}$ Unsicherheit $+/-25 \%$

Zum breiteren Einbezug der Bevölkerung wurde im Frühjahr 2006 eine Workshopgruppe mit Vertretern aus Wirtschaft (9), Umweltschutz (7), Verwaltung (7) und Politik (7) gebildet. Die Auswahl der Mitglieder erfolgte unter Exponenten und Verantwortungsträgern der entsprechenden Gesellschaftsbereiche. Am 10. April 2006 fand ein erster Workshop statt. Dabei wurde ein detaillierter Katalog von Zielen entwickelt, die mit den Hochwasserschutzmassnahmen erreicht werden sollen. Diese Ziele gliedern sich in die drei Gruppen:

- Gesellschaft (Sicherheit, insbes. Hochwasserschutzziele);

- Umwelt (Ökologie, Gewässerraum, Ortsbild, Erholung/Tourismus, Fischerei, Landwirtschaft);

- Wirtschaftlichkeit (Investitions- und Betriebskosten, Kosten/Nutzen, Verfahrens- und Bauzeit). 
Am 29. September 2006 wurde ein zweiter Workshop mit den Beteiligten durchgeführt. Inhalt dieses zweiten Anlasses waren die Gewichtung der Projektziele und - soweit möglich - eine objektivierte Bewertung der drei Varianten hinsichtlich Zielerreichung. Die Gewichtung der Projektziele erfolgte einerseits durch die Workshopgruppe (,Akteure'). Andererseits nahmen auch die Projektleitung und die Projektbearbeiter (,Experten') eine Gewichtung der Ziele vor. Die Resultate zeigen ein einheitliches Bild (Abbildung 53 und Anhang XVI, 1).

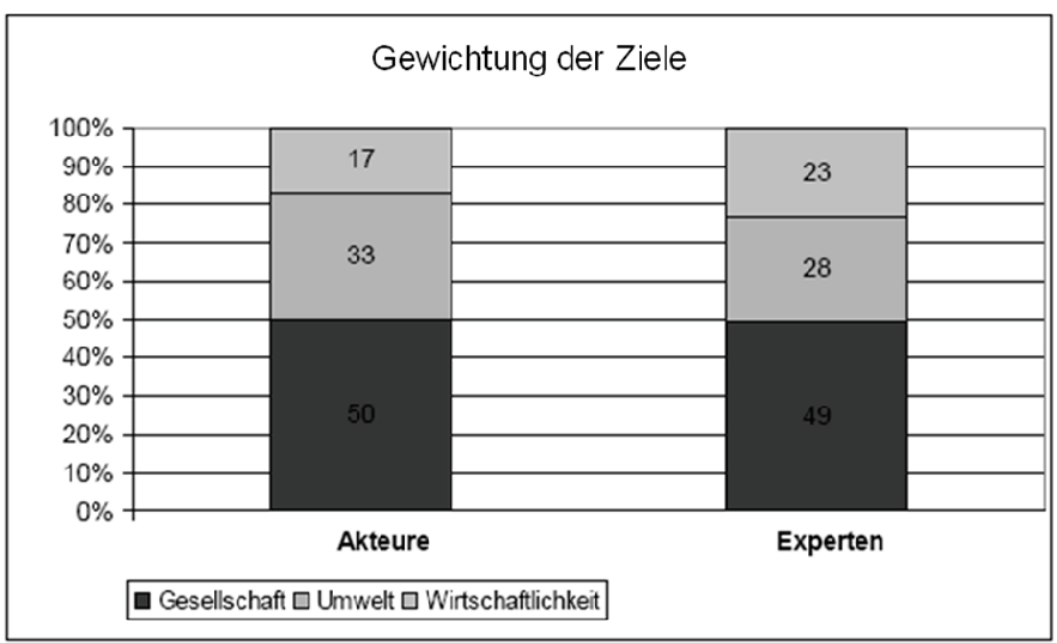

Abbildung 53 Fallbeispiel Sarneraa, Gewichtung der Zielgruppen durch ,Akteure' und ,Experten'.

Die Bewertung der Varianten erfolgte, indem jede Variante hinsichtlich Erfüllung jedes einzelnen Unterzieles mit einem Index von -3 (sehr nachteilige Lösung) bis +3 (sehr vorteilhafte Lösung) taxiert wurde. Die am 29. September 2006 mit der Workshopgruppe durchgeführte Bewertung zeigte kein verwertbares Resultat. Die Bewertung durch die ,Akteure' wurde deshalb für die weitere Projektevaluation nicht berücksichtigt. Die Bewertung der Varianten erfolgte ausschliesslich durch die ,Experten' und zwar in einer Einzelbewertung durch jedes Mitglied der Projektleitung und durch die Projektbearbeiter sowie durch die Bearbeitenden des Vorberichtes zur Umweltverträglichkeit. Diese Resultate wurden anschliessend an einer Bereinigungskonferenz zu einer einheitlichen Bewertung zusammengeführt und detailliert begründet. Die Gewichtung der Ziele und die Bewertung der Varianten bildeten die Grundlage für eine anschliessend durchgeführte Nutzwertanalyse (Tabelle 70). Im Nutzwertvergleich der Zielgruppe ,Gesellschaft' und ,Wirtschaftlichkeit' schnitten die Variante V1 und V3 am besten ab. Die Variante V3 lag in der Zielgruppe ,Umwelt' infolge fehlender ökologischer Aufwertungen an der Sarneraa hinter den Varianten V1 und V2 (Anhang XVI). 
Tabelle 70 Ergebnis der ersten Nutzwertanalyse Vorprojekt Sarneraatal (V1 = Variante Tieferlegung/Verbreiterung; V2 = Variante Entlastungsstollen Landenberg; V3 = Variante Entlastungsstollen Bergvaiante Ost).

\begin{tabular}{lccc}
\hline & \multicolumn{3}{c}{$\begin{array}{c}\text { Bewertung für } \\
\text { V2 }\end{array}$} \\
\hline Gewichtung durch ... & \multicolumn{3}{c}{ Vutzwert } \\
\hline Experten & 0.87 & 0.75 & 0.81 \\
Akteure & 0.95 & 0.87 & 0.95 \\
Rangierung durch ... & & Rang & \\
Experten & 1 & 3 & 2 \\
Akteure & 1 & 3 & 1 \\
$\quad$ Rangpunkte total & 2 & 6 & 3 \\
$\quad$ Rangfolge total & 1 & 3 & 2 \\
\hline
\end{tabular}

Als Nebenergebnis wurde am 2. Workshop vom 29. September 2006 eine Bergvariante Ost mit zusätzlichen ökologischen Aufwertungsmassnahmen an der Sarneraa (V3PLUS) in die Diskussion eingebracht. Diese Variante fand in der Workshopgruppe - ausser bei den Bundesvertretern und Umweltverbänden - breite Zustimmung.

Die Variante 3PLUS wurde ebenfalls in die weitere Evaluation einbezogen. Durch die ökologischen Aufwertungen weist sie im Vergleich zur Bergvariante Ost einen deutlich höheren Nutzwert auf. Allerdings erhöhen sich die Kosten für die Variante 3PLUS infolge zusätzlicher baulicher Massnahmen und Landerwerbskosten um ca. CHF 8 Mio. auf CHF 67.5 Mio.

In einer zweiten Nutzwertanalyse lag nun die Variante 3PLUS auf dem ersten Rang. Dahinter liegen an zweiter Stelle die Variante 1 ,Tieferlegung/Verbreiterung der Sarneraa' und Variante 3 ,Bergvariante Ost' (Tabelle 71). Variante 3PLUS wurde insbesondere favorisiert, weil dadurch weniger private Rechte tangiert würden. Zudem wird das Siedlungsgebiet durch die Bautätigkeit weniger belastet. Es werden mit dieser Variante geringere Widerstände der Anstösser und schnellere Genehmigungsverfahren erwartet. Das Projekt könnte rascher realisiert werden und die Schutzwirkung tritt früher ein. Der Regierungsrat reichte am 12. Dezember 2006 das Projekt zur Stellungnahme beim Bundesamt für Umwelt ein mit dem Antrag, die Variante 3PLUS zu favorisieren.

Tabelle 71 Ergebnis der zweiten Nutzwertanalyse Vorprojekt Sarneraatal.

\begin{tabular}{lcccc}
\hline & \multicolumn{5}{c}{ Bewertung für } \\
& V1 & V2 & V3 & V3+ \\
\hline Gewichtung durch ... & \multicolumn{5}{c}{ Nutzwert } \\
Experten & 0.90 & 0.82 & 0.89 & 0.99 \\
Akteure & 0.96 & 0.89 & 0.99 & 1.11 \\
Rangierung durch ... & 2 & 4 & 2 & 1 \\
Experten & 2 & 4 & 2 & 1 \\
Akteure & 4 & 8 & 4 & 2 \\
$\quad$ Rangpunkte total & 2 & 4 & 2 & 1 \\
$\quad$ Rangfolge total & 2 & \multicolumn{7}{c}{ Rang } \\
\hline
\end{tabular}




\subsubsection{Durch Verbände und Verwaltung erwirkter Richtungswechsel}

Bereits kurz nach dem Beschluss des Regierungsrats mobilisierte ein Umweltverband gegen die Variante 3PLUS und drohte mit der Ergreifung von Rechtsmitteln, da die Variante 3PLUS nicht den Grundsätzen der Wasserbau- und Gewässerschutzgesetzgebung entspreche und ein nationales Schutzgebiet tangiere (NOZ, 2006).

Das Bundesamt für Umwelt nahm am 8. März 2007 Stellung. Aus dieser Stellungnahme geht hervor, dass sowohl für die Variante 3PLUS als auch für die Variante 1 keine absoluten Ausschlusskriterien bestehen. Der Bund bevorzugt jedoch klar die Variante 1 (Tabelle 72). Sie wird als zweckmässig und umweltgerecht beurteilt. Neben der besseren Bewertung aus fachlicher Sicht ist für den Bund entscheidend, dass die Kosten für die Variante 1 deutlich tiefer liegen.

Tabelle 72 An der Stellungnahme beteiligte Bundesstellen und deren Position.

\begin{tabular}{ll}
\hline Stelle & Favorisierte Variante \\
\hline Abteilung Gefahrenprävention BAFU & Variante 1 \\
Abteilung Natur und Landschaft BAFU & Variante 1 \\
Abteilung Artenmanagement BAFU & Variante 1 \\
Abteilung Wasser BAFU & Variante 1 \\
Abteilung Recht BAFU & -- \\
Bundesamt für Kultur (BAK) & -- \\
Eidg. Natur- und Heimatschutzkommission (ENHK) & Variante 1 \\
Eidg. Finanzverwaltung & Variante 1 \\
armasuisse Immobilien, VBS & (Variante 1) \\
Bundesamt für Landwirtschaft & -- \\
\hline
\end{tabular}

Nach Eingang der Stellungnahme des Bundes setzte sich die erweiterte Projektsteuergruppe am 14. März 2007 erneut mit dem Variantenentscheid auseinander. Die Steuergruppe spricht sich nach Beurteilung der neuen Sachlage mehrheitlich für die Realisierung der Variante 1 aus. Einzig die Einwohnergemeinde Sarnen, auf deren Gebiet die Massnahme zur Tieferlegung/Verbreiterung zur Hauptsache erfolgen, favorisiert weiterhin die Realisierung der Variante 3PLUS. Mit Schreiben vom 16. März 2007 beantragte der Einwohnergemeinderat Sarnen die Weiterverfolgung der Variante 3PLUS.

\subsubsection{Demokratische Entscheidungskontrolle I: Politische Entscheidung}

In seinem Bericht vom 20. März 2007 beantragte der Regierungsrat beim Kantonsrat, die Weiterverfolgung der Variante 1 zu genehmigen. Die vorberatende Kommission des Kantonsrats folgte dem Antrag der Regierung einstimmig. Im Vorfeld der Kantonsratsdebatte unternahm eine kantonsrätliche Fraktion politische Anstrengungen, auch die Variante 3PLUS parallel weiter zu verfolgen. Der Kantonsrat lehnte dies aus Kosten- und Zeitgründen ab und stimmte in der Sitzung vom 27. April 2007 mit 39 zu 5 Stimmen dem Antrag des Regierungsrats zu. Gegen diesen Beschluss wurde am 4. Juli ein Referendum ergriffen. Die ablehnende Haltung zu Variante 1 wird vor allem mit der Beeinträchtigung des Ortsbildes von Sarnen durch die Tieferlegung, mit baulichen Risiken für die Grundstücke entlang der Sarneraa und mit den Beeinträchtigungen durch eine Baustelle mitten im Dorfkern von Sarnen begründet. Am 2. Juli 2007 wurde in Sarnen ein Verein ,Interessengemeinschaft Hochwasserschutz Josef Th. Hess: Schutzziele im Umgang mit Naturrisiken in der Schweiz, @ vdf Hochschulverlag 2011 
Sarnen' (IG) mit dem Ziel gegründet, die rasche und sachgerechte Realisierung des Hochwasserschutzes von Sarnen zu fördern. Die IG unterstützte das Referendum öffentlich. Am 25. November stützte das Obwaldner Stimmvolk mit $60 \%$ den Entscheid des Kantonsrats zur Erstellung der Variante Verbreiterung/Tieferlegung. In der Standortsgemeinde Sarnen wurde der Kantonsratsbeschluss mit 53\% der Stimmen abgelehnt.

\subsubsection{Demokratische Entscheidungskontrolle II: Nachspiel zum Varianten- entscheid}

Die IG Hochwasserschutz Sarnen sammelte unterdessen erfolgreich Geldmittel zur Erarbeitung einer Totalunternehmerofferte für eine Stollenlösung ähnlich der ,Bergvariante Ost'. Im Dezember unterzeichnete sie einen Vertrag mit einer Bietergemeinschaft von Bau- und Ingenieurunternehmen zur Erarbeitung eines Alternativprojekts. Dieses Projekt soll dem Kanton im August 2008 unterbreitet werden. Am 17. Januar 2008 reichte ein Baufachmann ein weiteres, auf privater Basis erarbeitetes Projekt mit einer unterirdischen Lösungsvariante ,Kanal im Kanal' für die Hochwasserentlastung ein. Der Regierungsrat trat mit Verweis auf das Ergebnis der Volksabstimmung nicht auf dieses Projekt ein. Am 26. September 2010 kam es zu einer erneuten Volksabstimmung. Ein von der IG Hochwasserschutz Sarnen eingereichtes Volksbegehren, die „Bergvariante Ost“ bis zur Baureife zu planen, wurde mit 60\% der Stimmen angenommen. Die Stimmberechtigten lehnten dabei auch den Gegenvorschlag des Kantonsrates ab, welcher gleichzeitig die weitere Planung am „Hochwasserentlastungsstollen West" (Landenbergstollen) vorsah.

\subsubsection{Erkenntnisse aus dem Fallbeispiel}

Aus diesem Fallbeispiel lassen sich folgende Erkenntnisse zur (partizipativen) Entscheidfindung bei Risikoverminderungsmassnahmen ableiten:

- Die Schutzzieldiskussion überfordert die meisten Teilnehmer eines partizipativen Planungsprozesses und verliert gleichzeitig angesichts der behördlichen Vorgaben und deren finanzieller Relevanz an Bedeutung.

- Bei zeitlicher Nähe zu einem Ereignis überwiegen das Sicherheitsbedürfnis und das Bedürfnis nach raschem Handeln die übrigen Aspekte deutlich.

- Eine möglichst geringe Beeinträchtigung privater Rechte spielt in der Variantenbewertung eine zentrale Rolle.

- Bei weitreichender öffentlicher Finanzierung spielen finanzielle Aspekte eine untergeordnete Rolle (strategisches Verhalten).

- Interessenkonflikte, zu denen im Rahmen der Workshops kein Konsens gefunden werden konnte, werden durch die Akteure ausserhalb der ,offiziellen' Entscheidungsfindungsplattformen gesellschaftlich und politisch thematisiert.

- Die Verwaltung (Bund, Kanton) übt grossen Einfluss auf die Entscheidungsfindung aus.

- Ein partizipativer Entscheidungsprozess stellt hohe Anforderungen an Projektleitung und Beteiligte.

- Workshops erfordern eine intensive Vor- und Nachbereitung. 


\section{Diskussion und Folgerungen}

\subsection{Schadensbegriff}

\subsubsection{Problemstellung}

Die Darstellung von Risiken und Schutzzielen erfordert einen Schadensbegriff. Als Schaden wird in der Literatur übereinstimmend eine Verletzung von Werten oder Schutzgütern bezeichnet. Dieser Schadensbegriff bietet erhebliche methodische Schwierigkeiten. Es bestehen weder Klarheit noch Konsens darüber, bei welchen Werten eine Verletzung als Schaden zu betrachten ist. Für viele dieser Werte fehlen Indikatoren und Metriken, um eine Beeinträchtigung zu beschreiben oder zu bewerten. Schäden lassen sich oft nicht allgemeingültig formulieren. Die Art der Betroffenheit und die Optik, aus der ein Schaden betrachtet wird, beeinflusst die Bewertung entscheidend. Es stellte sich die Frage nach einer umfassenden Darstellung der verletzbaren Werte, die unter den Schadensbegriff zu fassen sind.

\subsubsection{Erkenntnisse}

In der Literatur werden die verletzbaren Werte und Schutzgüter in die fünf Gruppen

- Leib und Leben,

- Sachwerte,

- gesellschaftliche Strukturen,

- Naturwerte und

- Werte der Lebensqualität

gegliedert. Indikatoren und Metriken sind nur für zwei dieser Gruppen, für Leib und Leben sowie für Sachwerte, verfügbar, jedoch auch hier nur lückenhaft.

\subsubsection{Würdigung}

Die vorliegende Arbeit kann die dargelegten methodischen Schwierigkeiten des Schadensund damit verbunden des Risiko- und Schutzzielbegriffes nicht überwinden. Wie bestimmte Arten von Schutzgütern, z. B. gesellschaftliche Strukturen, Naturwerte oder die Lebensqualität, dargestellt und bewertet werden können, bleibt unbeantwortet. Ein umfassender Schadensbegriff konnte nicht hergeleitet werden und erfordert weitere umfassende Forschungsarbeiten.

Der in dieser Arbeit entwickelte Vorschlag für ein schutzzielbasiertes Managementkonzept überbrückt diese Lücke einerseits durch eine Reduktion der Betrachtungen auf die Risikoindikatoren Todesfälle und Sachschäden (Kapitel 4.2.2.2). Anderseits wird die Problematik des Schadensbegriffes umgangen, indem risikoexponierte Objekte typisierend mittels eines Indexes bewertet wurden (Kapitel 4.2.3.2).

\subsection{Festlegung tolerierbarer Risiken}

\subsubsection{Problemstellung}

Ein standardisiertes Verfahren zur Festlegung tolerierbarer Risiken existiert nicht. Die im Management von Naturrisiken gebräuchlichen Schutzziele wurden pragmatisch und ohne erkennbaren Bezug zu wissenschaftlichen Grundlagen entwickelt. Unklar ist die Abstützung dieser Schutzziele in Rechtsordnung, Rechtsprechung und Rechtslehre. Zudem fehlt eine 
demokratische Legitimation der Schutzziele, da diese meist ausschliesslich durch Experten festgelegt und in der Rechtsordnung auf einer sehr tiefen strukturellen Ebene von Richtlinien, Empfehlungen oder Weisungen umschrieben sind.

\subsubsection{Erkenntnisse}

Schutzziele sind einerseits eine Festlegung der Höhe des tolerierbaren Risikos für eine Gesellschaft, anderseits eine Festlegung der Risikoverteilung in dieser Gesellschaft. Besonders der Aspekt der Risikoverteilung verbindet die Schutzzielfrage mit Prinzipien der Gerechtigkeitstheorie und (Umwelt-)Ethik. Verschiedene Kulturen, politische Systeme und Epochen entwickelten unterschiedliche Sichtweisen von Gerechtigkeit. Diese Sichtweisen basieren auf einem oder mehreren der folgenden fünf Prinzipien:

- Bedürfnis: Gerecht ist eine Lösung, die die Bedürfnisse für alle Individuen möglichst gleichwertig abdeckt;

- Einfluss: Gerecht ist eine Lösung, die die Bedürfnisse einzelner Individuen nach Massgabe des Verhaltens abdeckt, mit dem diese selber zur Entstehung und Abdeckung eines Bedürfnisses beigetragen haben;

- Kontext: Gerecht ist eine Lösung, die die Bedürfnisse eines Individuums in einer besonderen Situation abdeckt;

- Effizienz: Gerecht ist eine Lösung, die mit einem bestimmten Mitteleinsatz zu einer maximalen Abdeckung der Summe aller individuellen Bedürfnisse führt;

- Retinität: Gerecht ist eine Lösung, die den Bedürfnissen der Umwelt und der Gesellschaft am besten entspricht.

Die jeweiligen gesellschaftlichen und politischen Wertmassstäbe entscheiden, welche dieser Prinzipien in welchem Mass berücksichtigt werden. Die Prinzipien lassen sich übertragen auf Fragen der Verteilung von Restrisiken oder der Verteilung von Mitteln zur Risikominderung. Sie stellen damit eine geeignete Grundlage zur Formulierung von Schutzzielen dar. Aus diesen Prinzipien ergeben sich unterschiedliche Zielsetzungen. Dies kann zu Zielkonflikten führen. Dies gilt besonders für die Prinzipien Bedürfnis und Effizienz. Die Praxis des Risikomanagements kennt sowohl Schutzziele, bei denen das Bedürfnisprinzip im Vordergrund steht, als auch solche, die sich nach dem Effizienzprinzip orientieren. Dem Bestreben, die Risiken für alle auf ein gleiches tolerierbares Mass zu reduzieren (Bedürfnisprinzip) kann das Bestreben nach einem für die gesamte Gesellschaft optimalen Einsatz beschränkter Mittel (Effizienzprinzip) entgegenstehen.

Das sogenannte ,Dreistufenkonzept' im Umwelt- und Störfallrecht stellt einen materiellen Beitrag des Rechts zur Festlegung von Schutzzielen und zur Lösung dieses Zielkonflikts dar. Die Lehre zum technischen Sicherheitsrecht hat daraus einen ,Zwei-Regel-Ansatz' abgeleitet, nach welchem erstens untolerierbare individuelle Risiken (meist als Grenzwerte definiert) zu beseitigen und zweitens weitere Risikoreduktionsmassnahmen nach Grundsätzen der Wirtschaftlichkeit oder der Verhältnismässigkeit zu ergreifen sind. Dem Schutz individueller Sicherheitsbedürfnisse wird dabei Vorrang gegeben.

\subsubsection{Würdigung}

Eine wesentliche Erkenntnis dieser Arbeit ist die Umschreibung handlungsleitender Prinzipien der Gerechtigkeitstheorie und Ethik als Grundlage für Schutzziele.

Die Herleitung der Schutzzielprinzipien sowie der Kriterien zu deren Bewertung erlaubt kein exakt naturwissenschaftliches Vorgehen im Sinne einer beweisbaren Theorie. Das entwickelte Gedankenmodell ergibt sich aus einer breit abgestützten und anhand eigener Erfahrungen gewichteten Auswertung von Literaturangaben, Praktiken und Grundsätzen. Eine solche 
Auswertung bleibt immer unvollständig. Es kann nicht ausgeschlossen werden, dass im Einzelfall wichtige Aspekte ergänzt werden müssen.

Die handlungsleitenden Prinzipien schaffen die Grundlage für eine strukturierte und nachvollziehbare Festlegung von Schutzzielen. Diese Grundlage erlaubt es, Schutzziele vor dem Hintergrund gerechtigkeitstheoretischer und ethischer Grundsätze sowie der jeweiligen Werte- und Rechtsordnung in einem beliebigen gesellschaftlichen Kontext zu formulieren.

\subsection{Managementkonzept}

\subsubsection{Problemstellung}

Es fehlt besonders auf regionaler Ebene ein konsistentes und umfassendes Managementkonzept für Naturrisiken:

- Das verbreitetste Konzept, die Schutzzielmatrix, ist zwar regional einsetzbar, berücksichtigt aber Effizienzaspekte bei der Risikominderung kaum.

- Ebenso bleiben bei der Verwendung von Grenzwerten des tolerierbaren Risikos (z. B. tolerierbare individuelle Todesfallrisiken) Effizienzaspekte unberücksichtigt.

- Das vereinzelt verwendete Konzept der Grenzkosten als Schutzziele berücksichtigt die Effizienz von Risikoverminderungsmassnahmen, ist aber infolge sehr hoher Anforderungen an verfügbare Daten kaum regional einsetzbar.

Das Management von Naturrisiken sowie die Festlegung und Anwendung von Schutzzielen sind stark beeinflusst von der Art der Entscheidungsprozesse und den Organisationsstrukturen. Entscheidungsprozesse und Organisation im Management von Naturrisiken sind stark durch Verwaltung und Experten geprägt. Das öffentliche Management natürlicher Risiken ist in mehrere Rechtsgrundlagen und Verwaltungseinheiten aufgesplittert. Dies führt zu einem sektoriellen Vollzug mit aufwendiger oder fehlender Koordination. Strategische und operative Aufgaben werden auf den verschiedenen Ebenen des Gemeinwesens vermischt. Die heute bestehende organisatorische Lösung widerspricht dem Grundsatz, wonach dem Bund und den Kantonen strategische, den Gemeinden und Risikobetroffenen operationale Aufgaben zufallen.

Die Steuerung des Risikomanagements erfolgt in vielen Bereichen durch inputorientierte Förderung der öffentlichen Hand. Damit werden Anreize für ökonomisch nicht optimale Massnahmen gesetzt. Demokratische Entscheidverfahren stossen an Grenzen, da Sicherheit oft den Charakter eines öffentlichen Gutes aufweist, was zu strategischem Verhalten verleitet.

\subsubsection{Erkenntnisse}

\subsubsection{Zwei Ebenen, zwei Regeln und fünf Schutzzielprinzipien}

Der aus der Lehre zum technischen Sicherheitsrecht bekannte ,Zwei-Regel-Ansatz' eignet sich als Grundgerüst für ein Konzept zum Management von Naturrisiken. Im Zielkonflikt zwischen individuellen Sicherheitsbedürfnissen und gesamtgesellschaftlicher Effizienz erhalten individuelle Sicherheitsbedürfnisse den Vorrang.

Das Konzept differenziert zwischen regionaler Ebene und Ebene Einzelprojekt. Auf regionaler Ebene können die aus der Gerechtigkeitstheorie übernommenen fünf Prinzipien zur Festlegung tolerierbarer Risiken als Schutzzielprinzipien operationalisiert werden. Sie werden mit verletzbaren Objekten einerseits (Objektindex, Kapitel 4.2.3.2) und mit Risikoverminderungsmassnahmen anderseits (Massnahmenindex, Kapitel 4.2.3.3) in Verbindung gebracht.

Der Objektindex erlaubt eine systematische Bewertung der Risikoobjekte und drückt deren Schutzbedürftigkeit aus. Der Massnahmenindex umschreibt Effizienz und Verträglichkeit von 
Risikoverminderungsmassnahmen. Mit diesen beiden Indizes und der Gefährdung lassen sich Prioritäten auf regionaler Ebene festlegen.

Auf Ebene Einzelprojekt werden die klassischen Schutzzielkonzepte wie Grenzwerte des Risikos oder Grenzkosten für risikovermindernde Massnahmen übernommen. Diese Konzepte werden auf Ebene Einzelprojekt als geeignet erachtet.

Ein Ansatz zur vereinfachten Abschätzung des Handlungsbedarfs ermöglicht aufgrund der Schadenspotenzialkarte, der Gefahrenkarte und der Charakterisierung der Gefahrenquellen eine semiquantitative Abschätzung der Notwendigkeit und Dringlichkeit von Risikoverminderungsmassnahmen.

\subsubsection{Bestehende Schutzzielkonzepte}

Bestehende Schutzzielkonzepte berücksichtigen in der heutigen Form nicht alle Schutzzielprinzipien. Ausserdem ist ihre Anwendbarkeit auf regionaler Ebene teilweise begrenzt. Die Einführung des Objektindexes ermöglicht es, die Schutzzielmatrix zu modifizieren. Die Risikoobjekte können aufgrund der Schutzzielprinzipien Bedürfnis, Einfluss und Kontext bewertet und anhand dieser Bewertung den Objektkategorien der Schutzzielmatrix neu zugeordnet werden.

Berechnungen zeigen, dass tolerierbare individuelle Todesfallrisiken, die sich bei Anwendung der klassischen Schutzzielmatrix (BUWAL/OW) ergeben, in vielen Fällen mit den Literaturangaben über tolerierbare individuelle Todesfall- und Sachrisiken gut übereinstimmen (Kapitel 2.6.1.3).

\subsubsection{Organisation und Entscheidfindung im Risikomanagement}

Die gesellschaftliche Entscheidfindung wird in zwei Ebenen, jene des gesellschaftlichen Grundkonsenses und jene des laufenden gesellschaftlichen Prozesses, gegliedert. Es können vier verschiedene Entscheidverfahren unterschieden werden:

- Markt und Preismechanismus;

- Demokratie;

- wirtschaftliche Interessengruppen sowie

- Bürokratie und Verwaltung.

Diese Verfahren eignen sich für das Management von Naturrisiken in unterschiedlichem Mass. Das Management von Naturrisiken ist weitgehend von bürokratischen, teilweise demokratischen Entscheidverfahren geprägt. Von zunehmender Bedeutung im Management natürlicher Risiken ist der ausdrückliche Einbezug der Bedürfnisse Betroffener. Dazu bestehen weit entwickelte Methoden der partizipativen Planung. Die Lösungsfindung und die Berücksichtigung relevanter gesellschaftlicher Interessen bleiben auch mit der Anwendung dieses Verfahrens anspruchsvoll.

Ein Konsens über eine Lösung ist umso einfacher zu finden, je grösser die Ungewissheit der Beteiligten über ihre persönliche künftige Betroffenheit durch diese Lösung ist. Die Methoden der partizipativen Planung ermöglichen einerseits eine strukturierte Diskussion zur Lösungsfindung. Anderseits führen sie zu verbessertem Informationsstand und damit zu einer Verminderung der Ungewissheit jedes Beteiligten über die persönliche Betroffenheit durch eine Lösung. Nachteile für einzelne Betroffene werden mit besserem Informationsstand offensichtlicher erkennbar. Dies wiederum erschwert die Lösungsfindung. Einzelne Betroffene lassen sich kaum von moralischen Erwägungen leiten, eine Lösung zu akzeptieren, die ihren individuellen Interessen entgegensteht. Sie werden einer Lösung nicht zustimmen, sobald 
sie persönliche Nachteile erkennen. Lösungen können nur noch gefunden werden, wenn auch Vorteile und Kompensationen für Nachteile dargestellt werden können.

\subsubsection{Fallbeispiele}

Fallbeispiele wurden zum schutzzielbasierten Risikomanagementkonzept (Fallbeispiel 1), zu einem Verfahren zur vereinfachten Abschätzung des Handlungsbedarfs (Fallbeispiel 2), zu modifizierten Schutzzielmatrizes (Fallbeispiel 3) und zur partizipativen Entscheidfindung (Fallbeispiel 4) untersucht.

In Fallbeispiel 1 wurden die einzuhaltenden Grenzwerte für das tolerierbare individuelle Risiko (sog. minimale Sicherheitsstandards) nach Regel 1 bewusst sehr hoch angesetzt. Diese hohen Grenzwerte sollen dazu führen, dass möglichst viele Massnahmen nach Regel 2 priorisiert werden, bei welcher alle Schutzzielprinzipien umfassend berücksichtigt werden. Trotz hoher Grenzwerte überraschte das Ergebnis, dass sich der Handlungsbedarf im Fallbeispiel vor allem aus Regel 1 ergab. Als besonders anspruchsvoll erwies sich die Bewertung von Risikoobjekten. Hierfür muss zweckmässigerweise auf das Wissen von Betroffenen (Laien) zurückgegriffen werden, da vor allem gesellschaftliche Umstände und Bedürfnisse zum Ausdruck gelangen sollen. Die Bewertung von Risikoverminderungsmassnahmen und die Feststellung der örtlichen Gefährdung erfordert demgegenüber Expertenwissen.

Das Verfahren in Fallbeispiel 2 umfasst eine semiquantitative Herleitung von Handlungsbedarf und Prioritäten nach den Grundsätzen von Regel 2 des Risikomanagementkonzeptes. Dabei ergaben sich plausible Ergebnisse.

Die in Fallbeispiel 3 untersuchten Varianten der Schutzzielmatrix führten zu geringfügigen Unterschieden zwischen den verschiedenen Versionen. Die Abschätzung des Handlungsbedarfs und der Prioritäten fiel bei allen Varianten der Schutzzielmatrix ähnlich aus.

Fallbeispiel 4 als Darstellung eines realen Entscheidungsprozesses in einem Hochwasserschutzprojekt zeigte die Grenzen der Partizipation im laufenden gesellschaftlichen Prozess auf. Sicherheit als öffentliches Gut und die fehlende Äquivalenz zwischen Nutzniesser und Kostenträger eines Projekts begünstigen strategisches Verhalten und volkswirtschaftlich ineffiziente Entscheide. Sehr bedeutend ist der kommunikative Nutzen des partizipativen Planungsprozesses. Der Einbezug der Betroffenen erhöht die Akzeptanz eines Entscheides deutlich.

\subsubsection{Würdigung}

Das im Rahmen dieser Arbeit entwickelte schutzzielbasierte Konzept darf als Beitrag zur Weiterentwicklung des Managements von Naturrisiken betrachtet werden. Die Verbindung des ,Zwei-Regel-Ansatzes' mit den fünf Schutzzielprinzipien zu einem auf den Ebenen Region und Einzelprojekt anwendbaren Konzept stellt eine Innovation dar, die ihre Tauglichkeit grundsätzlich bewiesen hat. Insbesondere ermöglicht das Schutzzielkonzept auf regionaler Ebene die transparente und umfassende Berücksichtigung aller Schutzzielprinzipien.

Aus der Anwendung des Konzeptes ergibt sich keine abschliessende Vorgabe für die Mittelbereitstellung (Allokation) im Management von Naturrisiken. Direkten Einfluss auf die Allokation für die Verminderung von Risiken hat nur Regel 1. Regel 2 ist auf die optimale Distribution vorhandener Mittel ausgerichtet. Eine absolute Grenze, bis zu welchem Mass Risiken vermindert werden sollen, ergibt sich nach Regel 2 nicht. Die bei der Anwendung von Regel 2 informierte und strukturierte Diskussion über Prioritäten kann durchaus auch Auswirkungen auf die Mittelallokation haben. Noch unbefriedigend ist der Umstand, dass die Herleitung der individuellen Schutzbedürfnisse nach Regel 1 allein auf Todesfall- und Sachrisiken beruht, da Indikatoren, Metriken und Verfahren zur Festlegung weiterer Risiken fehlen. 
Ferner ist die Validierung des Konzeptes ungenügend. Die im Rahmen des Fallbeispiels vorgenommene Bewertung der Objekt- und Massnahmenindizes erfolgte durch Erfahrungswerte und den Beizug weniger Experten. Eine Validierung durch weitere Fallbeispiele und eine breit abgestützte Bewertung unter Laien und Experten fehlen.

Die als Alternative vorgeschlagene Methode zur vereinfachten Abschätzung des Handlungsbedarfs ist vor allem zur regionalen Abschätzung der Notwendigkeit und der Priorität von Risikoverminderungsmassnahmen vielversprechend.

Aus den Untersuchungen zur Variation der Schutzzielmatrix ergaben sich wenig neue Erkenntnisse. Von Interesse und erstmalig ist die Feststellung, dass eine rechnerische Überprüfung der Schutzziele nach Schutzzielmatrix zu Werten für das tolerierbare individuelle Todesfallrisiko führt, welche mit den Angaben in der Literatur gut übereinstimmen. Die Ausführungen im Rahmen dieser Arbeit berühren einige aufgrund des eigenen Erfahrungswissens relevant erscheinende Aspekte der Organisation und der Verfahren im Management von Naturrisiken. Eine vertiefte Einbettung in ökonomische und gesellschaftspolitische Theorien konnte im Rahmen der vorliegenden Arbeit nicht erfolgen. Die Ausführungen zur partizipativen Planung beschränken sich auf die leicht angepasste Anwendung eines für Hochwasserschutzprojekte entwickelten Verfahrens.

\subsection{Offene Fragen und weitere Arbeiten}

\subsubsection{Schäden}

Bedeutende offene Fragen stellen sich nach wie vor bei der Erfassung der Schäden. Trotz verschiedener Untersuchungen in den letzten Jahren bestehen Wissenslücken sogar bei elementaren und vermeintlich einfachen Fragen. Zu erwartende Schäden und die Verletzlichkeit in Abhängigkeit von den Eigenschaften eines Risikoobjekts oder von Art und Intensität der Gefahrenprozesse sind ungenügend bekannt. Diese Wissenslücken sind dringend zu schliessen, um die Risikobeurteilung, aber auch die Beurteilung der Wirksamkeit von Massnahmen zu verbessern.

Unklarheiten bestehen auch bezüglich der korrekten Bewertung von Personenschäden. Die Verwendung der Grenzkosten als Mass für die kardinale Darstellung von Personenrisiken wird von einem Teil der Lehre kritisiert.

Zur Erfassung verschiedener Arten von Schäden bestehen keine oder nur ungenügende methodische Ansätze. Dies betrifft insbesondere die Umweltschäden, aber auch Schäden an gesellschaftlichen Institutionen und Beeinträchtigungen der Lebensqualität.

Erst die Klärung dieser Fragen wird es ermöglichen, verschiedene Arten von Schäden und Risiken vergleichend darzustellen und umfassende quantitative Schutzziele zu formulieren.

Damit lassen sich folgende Schwerpunkte einer künftigen Forschung umschreiben:

1. Verbesserung der Datenlage zur Verletzlichkeit (Vulnerabilität) von Risikoobjekten in Abhängigkeit von

- Art und Eigenschaften des Objekts;

- Art und Intensität der massgeblichen Gefahrenprozesse;

2. Klärung der Monetarisierung von Todesfallrisiken (Grenzkostenansatz? Humankapitalansatz? Andere Ansätze?);

3. Entwicklung von Methoden zur reproduzierbaren und geldwerten Erfassung von Umweltschäden, Schäden an gesellschaftlichen Strukturen und Beeinträchtigungen der Lebensqualität. 


\subsubsection{Wirksamkeit von Massnahmen}

Ungenügend bekannt ist die Wirksamkeit von Massnahmen. Vor allem zur langfristigen Wirksamkeit und Erhaltungsstrategie von technischen und biologischen Präventionsmassnahmen sowie von Vorsorge- und Interventionsmassnahmen fehlt das für ein umfassendes Risikomanagement erforderliche Wissen. Ebenso fehlen Kenntnisse zur Wirksamkeit der zunehmend als wichtig erachteten kommunikativen Risikomanagementmassnahmen wie etwa Information der Risikobetroffenen oder Risikodialog.

Daraus ergibt sich folgender Forschungsbedarf:

4. Entwicklung von Methoden und Verbesserung der Datenlage zur Erfassung der Wirksamkeit von Risikomanagementmassnahmen, insbesondere

- biologische Präventionsmassnahmen;

- Vorsorge- und Interventionsmassnahmen;

- kommunikative Massnahmen sowie

- Kombinationen von Massnahmen.

\subsubsection{Schutzzielprinzipien und Risikomanagementkonzept}

Die in Kapitel 6.3.4 dargelegten Lücken des Konzeptes müssen durch eine breit abgestützte Validierung der Bewertung und eine Sensitivitätsanalyse geschlossen werden. Ferner würden Erkenntnisse aus den in Kapitel 6.4.1 aufgezeigten Forschungsschwerpunkten (vor allem Nr. 3) wesentlich zur Verbesserung des Modells beitragen.

Eine weitere Auseinandersetzung von Forschung und Praxis mit dem im Rahmen dieser Arbeit entwickelten Konzept muss zeigen,

- ob damit die Schutzziel- und Risikomanagementprinzipien umfassend dargestellt sind;

- ob die vorgeschlagene Verknüpfung mit Objektkategorien und Massnahmen zutreffend erfolgte;

- ob die Kriterien zur Berücksichtigung und Operationalisierung der Prinzipien umfassend und richtig gewählt wurden.

Von Interesse ist auch, ob und wie weit sich bei einer breiter abgestützten Bewertung der Risikoobjekte und Massnahmen, die in dieser Arbeit zur Operationalisierung der Schutzzielprinzipien vorgeschlagen wurde, ein Konsens finden lässt.

Zur Klärung dieser Fragen werden folgende Forschungsarbeiten empfohlen:

5. Überprüfung und Ergänzung der im Rahmen dieser Arbeit hergeleiteten Schutzzielprinzipien und Bewertungskriterien;

6. breit abgestützte Bewertung der Schutzzielprinzipien (allgemeingültige Objekt- und Massnahmenindizes);

7. Anwendung des Konzeptes in weiteren Fallbeispielen mit veränderten (insbesondere höheren) Grenzwerten für tolerierbare Risiken nach Regel 1.

\subsubsection{Risikomanagementstrategien in konkreten Gefahrensituationen}

Die praktische Erfahrung zeigt, dass die Auslösung und der Umfang von Risikomanagementmassnahmen in erheblichem Mass ereignisgesteuert sind; ein Umstand, der sich auch im Recht widerspiegelt. Es sind daher Strategien zu entwickeln, um die - auch vom Recht gestützten - höheren Sicherheitsanforderungen in konkret erkennbaren Gefahrensituationen, Notlagen und nach Ereignissen (,Rule of Rescue') mit den allgemein gültigen Schutzzielen abzugleichen. 
Für die künftige Forschung ergibt sich daraus folgendes Thema:

8. Entwicklung von Strategien, die zum Angleichen der Handlungsbereitschaft nach Ereignissen oder in Notlagen mit den allgemein üblichen Schutzzielen führen.

\subsubsection{Organisation}

Zur Verbesserung des Risikomanagements erscheint eine grundlegende Überprüfung der institutionellen Strukturen angezeigt. In weiterführender Forschung ist eine optimale Koordination, evtl. gar Zusammenlegung der Strukturen und Ressourcen im Management natürlicher Risiken zu prüfen.

Es sind Strategien und Regeln zu entwickeln zum Umgang mit Phänomenen wie ,Trittbrettfahrertum' oder ,Tragik der Allmende', die mit dem Charakter der Sicherheit als öffentliches Gut verbundenen sind. Hierbei sind auch die Erkenntnisse ökonomischer Theorien, z. B. der Property-Rights-Theorie, heranzuziehen.

Soweit das Gemeinwesen eine strategische Rolle als Förderer von Risikomanagementmassnahmen einnimmt, ist die Strategie einer strikt outputorientierten Förderung (nach Massgabe des Projektnutzens) zu prüfen, um effiziente Lösungen zu erreichen. Gegen eine konsequent outputorientierte Steuerung sprechen allerdings die bereits erörterten Wissenslücken hinsichtlich Wirksamkeit von Risikoverminderungsmassnahmen (Kapitel 6.4.2, Forschungsschwerpunkt Nr. 4).

Es ergeben sich folgende zusätzliche Schwerpunkte für die künftige Forschung:

9. Entwicklung eines konsequent outputorientierten öffentlichen Fördersystems;

10. Entwicklung von marktwirtschaftlichen Methoden und Instrumenten zur verbesserten Internalisierung von Risikokosten und

11. Entwicklung institutioneller Grundlagen für ein vollständig koordiniertes, ergebnisorientiertes Risikomanagement und ein Entwurf der erforderlichen Rechtsgrundlagen.

\subsection{Schlussbemerkung}

Schutzziele sind eines der drei Hauptelemente des Risikokonzeptes. Sie wurden im Management von Naturrisiken bisher pragmatisch und ohne ausdrücklichen Bezug auf wissenschaftliche und gesellschaftliche Grundlagen festgelegt. Die vorliegende Arbeit zeigt eine Basis zum besseren Verständnis dieser Festlegung auf. Konzeptionelle Vorschläge sollen es erlauben, diese Grundlagen bei der Festlegung künftiger Schutzziele besser zur Geltung zu bringen.

Wie jede Arbeit bringt die vorliegende Untersuchung Erkenntnisse, lässt aber auch Lücken offen. Sie wird den in Kapitel 1.5 formulierten Ansprüchen und Hypothesen in unterschiedlichem Masse gerecht.

Der in Hypothese 1 geforderte umfassende Schadensbegriff konnte aufgrund verschiedener methodischer Schwierigkeiten bei der Bewertung von Schäden und Risiken nicht formuliert werden.

Die Zielsetzung von Hypothese 2 kann die Arbeit weitgehend abdecken. In der Rechtslehre konnte mit dem ,Zwei-Regel-Ansatz' ein überzeugendes Grundgerüst zur Festlegung von Schutzzielen im regionalen Risikomanagement und bei Einzelprojekten gefunden werden. Die individuellen und gesellschaftlichen Anforderungen an Schutzziele lassen sich durch fünf handlungsleitende Prinzipien aus der Gerechtigkeitstheorie und Ethik breit abgestützt umschreiben. 
Ebenfalls ist es gelungen, ein risikobasiertes Managementkonzept zu entwickeln, welches den verschiedenen Ansprüchen von Hypothese 3 gerecht wird.

Für das in der Praxis am meisten verbreitete Konzept der Schutzzielmatrix konnten Anpassungen zur verbesserten Anwendung im Management von Naturrisiken auf regionaler Ebene und in Einzelprojekten vorgeschlagen werden (Hypothese 4).

Offengeblieben sind nebst methodischen Fragen zur Darstellung von Schäden und Risiken vor allem institutionelle Fragen zur Verbesserung des Risikomanagements.

Das Management von Naturrisiken hat sich im vergangenen Jahrzehnt bedeutend entwickelt. Die vorliegende Arbeit zeigt, dass diese Entwicklung noch lange nicht abgeschlossen ist. 


\section{Dank}

Ich bedanke mich bei meiner Frau Luzia und meinen Kindern Karin und José dafür, dass sie mir die Durchführung dieser Arbeit ermöglicht und dabei auf sehr vieles verzichtet haben.

Prof. Dr. H. R. Heinimann hat mit seinen zahlreichen wertvollen Gedanken und Hinweisen die Entwicklung der Arbeit geprägt. Ich bin inm dafür zu grossem Dank verpflichtet, ebenso danke ich den Korreferenten Prof. Dr. H. J. Seiler und Dr. Kurt Hollenstein für ihre Beiträge und die geduldige Durchsicht der Textentwürfe.

Bedanken darf ich mich beim Bundesamt für Umwelt, besonders bei Vizedirektor Andreas Götz für die wohlwollende Unterstützung der Arbeit und bei Dr. Peter Greminger für viele gedankliche Anregungen.

Ein spezieller Dank gehört Dipl.-Geogr. Thomas Wüthrich für die Durchführung der GISAnalysen im Rahmen der Fallbeispiele. Ihm und Dipl.-Forstingenieur Josef Berwert danke ich auch für die Mitarbeit bei der Bewertung der Objekt- und Massnahmenindizes.

Dipl.-Umweltingenieurin Maria Stettler hat mich bei der Durchsicht und Gestaltung des Manuskriptes mit viel Sorgfalt unterstützt. Ich danke Maria Stettler sehr für ihr grosses Engagement. Auch Dipl.-Bauingenieur/Dipl.-Umweltingenieur Viktor Schmidiger, Dipl.-Forstingenieur Roland Christen, Dipl.-Forstingenieur Josef Berwert und Dipl.-Forstingenieur Jürg Stückelberger danke ich für ihre Lektoratsarbeit und ihre Korrekturvorschläge.

Danken möchte ich auch meinem Arbeitgeber, dem Kanton Obwalden, und meinen Mitarbeiterinnen und Mitarbeitern vom Bau- und Raumentwicklungsdepartement Obwalden sowie dem Forstdienst Lungern dafür, dass sie mir durch ihren Einsatz gute Rahmenbedingungen geschaffen haben, diese Arbeit berufsbegleitend durchzuführen. 


\section{Verzeichnisse}

\section{V1. Literatur}

Abrahamsen, E. B., Aven T. (2008). On the consistency of risk acceptance criteria with normative theories for decision-making. Reliability Engineering and System Safety, doi:10.1016/j.ress.2008.03.021.

Ale, B. J. M. (1991). Risk Analysis and Risk Policy in the Netherlands and the Eec. Journal of Loss Prevention in the Process Industries 4(1): 58-64.

Allen, D. E. (1991). Criteria for design safety factors and quality assurance expenditure. Structural Safety and Reliability. Moan T. \& Shinozuka M. Amsterdam. Elsevier: 667678.

ARE (2002). Unfallkosten im Strassen- und Schienenverkehr der Schweiz 1998. Bern. Bundesamt für Raumentwicklung. On www: http://www.are.admin.ch/imperia/ $\mathrm{md} /$ content/are/gesamtvekehr/verkehrskonomie/4.pdf?PHPSESSID=8690efc32ba91 8d9b6b3430fa442c51a (Zugriff 10. Juli 2006).

BABS (2003). KATARISK - Katastrophen und Notlagen in der Schweiz, eine Risikobeurteilung aus Sicht des Bevölkerungsschutzes. Bern. BABS. 83 S.

BABS (2003a). KATARISK - Katastrophen und Notlagen in der Schweiz, eine Risikobeurteilung aus Sicht des Bevölkerungsschutzes. Erläuterung der Methode. Bern. 49 S.

BAFU (2007a). Programmvereinbarungen Schutzbauten und Gefahrengrundlagen. Erläuternder Bericht. Bern. Bundesamt für Umwelt, Wald und Landschaft. $46 \mathrm{~S}$.

BAFU (2007b). EconoMe 1.0. Online Berechnungsprogramm zur Bestimmung der Wirtschaftlichkeit von Schutzmassnahmen gegen Naturgefahren. Bern. Bundesamt für Umwelt, Gefahrenprävention. On www: http://www.grsxnet.ch/EconoMe/index.php (Zugriff 9. Juni 2007).

BAFU (2007c). Erläuterungen zum Programmblatt Schutzwald. Erläuternder Bericht. Bern. Bundesamt für Umwelt. $31 \mathrm{~S}$.

BAFU (2007d). RiskPlan2 - Pragmatisches Risikomanagement. Bern. Bundsamt für Umwelt, BABS. On www: http://www.grsxnet.ch/riskplan2 (Zugriff 24. Nov. 2007).

Ball David J., Floyd P. J. (1999). Societal Risk. London. 96 S.

Basler \& Hofmann (2008). Schutzziele, Einzelprojekt B 2.2 Schlussbericht. Aktionsplan Strategie Naturgefahren Schweiz 2006-2008. Zürich und Zollikofen. Nationale Plattform Naturgefahren. $70 \mathrm{~S}$.

Bell, R., Glade, T. \& Danscheid, M. (2006). Challenges in defining acceptable risk levels. Risk 21 - Coping with Risks due to natural hazards in the 21st century. CENAT. London. Taylor \& Francis/Balkema. 1: 77-87.

Berg, M., Erdmann G., Hofmann, M., Jaggy, M., Scheringer, M., Seiler, H. (1994). Was ist ein Schaden? Zur normativen Dimension des Schadensbegriffes in der Risikowissenschaft. Zürich. vdf Hochschulverlag AG ETH Zürich.

Beroggi, G. (1994). Der Schadensbegriff aus Sicht des Sicherheitsingenieurs. Was ist ein Schaden? Zur normativen Dimension des Schadensbegriffes in der Risikowissenschaft. Zürich. vdf Hochschulverlag AG ETH Zürich: 35-55.

Betreibungs- und Konkursämter (2001). Richtlinien für die Berechnung des betreibungsrechtlichen Existenzminimums (Notbedarf) nach Gesetz Art. 93 SchKG. 231.191: $6 \mathrm{~S}$. 
BFF (1984). Eidg. Richtlinien zur Berücksichtigung der Lawinengefahr bei raumwirksamen Tätigkeiten. Davos. Institut für Schnee- und Lawinenforschung. EDMZ 310.020 d.

BFS (2007). Taschenstatistik der Schweiz 2007. Bern. Bundesamt für Statistik. 36 S.

Bienz, A. F. \& Niederhäuser, F. R. (2000). Sicherheitskonzept für den Umgang mit Munition und Explosivstoffen in Armee und Militärverwaltung. Bern. Stämpfli Verlag AG.

Blong, R. (2003). A new Damage Index. Natural Hazards 30(1): 1-23.

Bohnenblust H. \& Schneider Th. (1984). Risk appraisal - Can it be improved by formal decision models? Uncertainty in risk assessment, risk management and decision making. V.T. Covello. New York/London. Plenum Press: 71-87.

Bohnenblust H. \& Troxler C. (1987). Risk Analysis - Is it a useful tool for the politician in making decisions on avalanche safety? Davos Symposium: Avalanche formation, movement and effects. Davos. International association of hydrological sciences (IAHS).

Bohnenblust, H. \& Slovic, P. (1998). Integrating technical analysis and public values in riskbased decision making. Reliability Engineering \& System Safety 59: 151-159.

Borter, P. (1999). Risikoanalyse bei gravitativen Naturgefahren. Methode/Fallbeispiele und Daten. Umweltmaterialien Naturgefahren Nr. 107/I und II. Bern. Bundesamt für Wald und Landschaft.

Bründl M., Bischof. N. \& Romang H. (2006). Leitfaden Risikokonzept. Umsetzung der Strategie Naturgefahren Schweiz, Einzelprojekt A 1.1. Bern. Nationale Plattform Naturgefahren. $108 \mathrm{~S}$.

Bründl M. \& Hegg C. (2001). Die Naturereignisse der letzten Jahre im Überblick. Birmensdorf/Davos. WSL/SLF. On www: http://www.slf.ch/pdf/forum2001-bruendl-hegg.pdf (Zugriff 10. Juli 2006).

Bütler, M. (2006). Gletscher im Blickfeld des Rechts. Zürich. Rechtswissenschaftliche Fakultät der Universität Zürich. 535 S.

BUWAL (1990). Handbuch Umweltverträglichkeitsprüfung UVP. Richtlinien. Bundesamt für Umwelt, Wald und Landschaft. Bern. On www: http://www.bafu.admin.ch /php/modules/shop/files/pdf/phpMYn6Tg.pdf (Zugriff 1. März 2007).

BUWAL (1991). Handbuch I zur Störfallverordnung, StFV. Richtlinien für Betriebe mit Stoffen, Erzeugnissen oder Sonderabfällen. Bern. Bundesamt für Wald und Landschaft. $74 \mathrm{~S}$.

BUWAL (1996). Beurteilungskriterien I zur Störfallverordnung StFV - Richtlinien für Betriebe mit Stoffen, Erzeugnissen oder Sonderabfällen. Bern. Bundesamt für Wald und Landschaft. $13 \mathrm{~S}$.

BUWAL (1999). StorMe (Ereigniskataster). Bern. Bundesamt für Wald und Landschaft. On www: http://www.bafu.admin.ch/naturgefahren/01922/01926/01927/index.html?lang=de (Zugriff 25. April 2008).

BUWAL (2001). Beurteilungskriterien II zur Störfallverordnung StFV - Richtlinien für Verkehrswege. Bern. Bundesamt für Wald und Landschaft. $11 \mathrm{~S}$.

BUWAL (Hrsg.) (2001). Lothar. Der Orkan 1999. Ereignisanalyse. Birmensdorf, Bern. Eidg. Forschungsanstalt für Wald, Schnee und Landschaft, Bundesamt für Umwelt, Wald und Landschaft. $400 \mathrm{~S}$.

BUWAL (1994). Schutzbauten und -anlagen (431.1), Begründung und Pflege von Wald mit besonderer Schutzfunktion (431.2). KREISSCHREIBEN. Nr. 20. 4 S. 
BUWAL (1994a). Anleitungsentwurf zur Durchführung einer Risikoanalyse. KREISSCHREIBEN. Nr. 20: $7 \mathrm{~S}$.

BWG (2005). Bericht über die Hochwasserereignisse 2005. Biel. Bundesamt für Wasser und Geologie. On www: http://www.uvek.admin.ch/themen/umwelt /00640/00815/index.html?lang=de (Zugriff 11. Juli 2006).

BWG (2006). Schapo http://www.bwg.admin.ch/service/download/d/schapo/schapoah.pdf und http://www.bwg.admin.ch/service/download/d/schapo/schapo.xIt (Zugriff 8. Sept. 2006).

BWG (2001). Hochwasserschutz an Fliessgewässern, Wegleitungen des BWG. Bern. Bundesamt für Wasser und Geologie. $72 \mathrm{~S}$.

BWW (1995). Anforderungen an den Hochwasserschutz. Bern. Bundesamt für Wasserwirtschaft. $4 \mathrm{~S}$.

BWW, BRP, BUWAL (1997). Empfehlungen zur Berücksichtigung der Hochwassergefahren bei raumwirksamen Tätigkeiten. Bern. Bundesamt für Wasserwirtschaft, Bundesamt für Raumplanung, Bundesamt für Umwelt, Wald und Landschaft. $32 \mathrm{~S}$.

Cassidy, K. (1996). Approaches to the risk assessment and control of major industrial chemical and related hazards in the United Kingdom. International Journal of Environment and Pollution 6(4-6): 361-387.

CEB, Comité Euro-International du béton (1976). International System of Unified Standard - Codes of Practice for Structures. Bulletin d'information 116 E Vol. I: Common unified rules for different types of construction and material (3rd Draft, Master Copy).

CIRIA (1977). Rationalisation of Safety and Serviceability factors in Structural Codes. Report No. 63. Construction Industry Research and Information Association. London.

Dini, J. W. (1998). Cost-effectiveness of life-saving interventions. Plating and Surface Finishing 85(9): 72-73.

Duden - Das große Wörterbuch der deutschen Sprache in 10 Bänden. Aktualisierte Online-Ausgabe. Mannheim, Leipzig, Wien, Zürich: Dudenverlag 1999-2004. On www: http://www.xipolis.net/1bd26d6f15c420575bf14ace444b3d486/suche/artikel.php?shor tname=felix\&artikel_id=61431 (Zugriff 18. Dez. 2006).

Duvernay, B. (2005). Beurteilung der Erdbebensicherheit bestehender Gebäude, Konzept und Richtlinien für die Stufe 3. Wegleitungen des Bundesamtes für Wasser und Geologie. Biel. Bundesamt für Wasser und Geologie. $68 \mathrm{~S}$.

Egli, M. (2000). Risk Based Regulation - Impressionen aus dem Ausland. Bern. Stämpfli Verlag AG. $47 \mathrm{~S}$.

Egli, P. (2002). Drittwirkung von Grundrechten - zugleich ein Beitrag zur Dogmatik der grundrechtlichen Schutzpflichten im Schweizer Recht. Zürich. Schulthess Juristische Medien AG. 394 S.

Eurodicautom (2004). European Terminology Database. European Commission. Bruxelles. On www: http://europa.eu.int/eurodicautom/Controller (Zugriff 3. März 2004).

Faber, M. \& Vrouwenvelder, T. (2001). Probabilistic Model Code. JCSS. Zürich, JCSS. On www: www.jcss.ethz.ch (Zugriff 17. Mai 2008).

Fell, R. (1994). Landslide Risk Assessment and Acceptable Risk. Canadian Geotechnical Journal 31(2): 261-272.

Finlay, P. J. \& Fell R. (1997). Landslides: Risk perception and acceptance. Canadian Geotechnical Journal 34(2): 169-188. 
Fischhoff B., Slovic. P., Lichtenstein S., Read S. \& Combs B. (1978). How safe is safe enough? A Psychometric Study of Attitudes Towards Technological Risks and Benefits. Policy Sciences 9: 127-152.

Flueler, T. \& Seiler, H. (2003). Risk-based regulation of technical risks: lessons learnt from case studies in Switzerland. Journal of Risk Research 6(3): 213-231.

Frederick, S. (2003). Measuring intergenerational time preference: Are future lives valued less? Journal of Risk and Uncertainty 26(1): 39-53.

Frey, B. \& Kirchgässner G. (2002). Demokratische Wirtschaftspolitik - Theorie und Anwendung. München, Verlag Franz Vahlen $\mathrm{GmbH}$.

FSC (2004). FSC-Prinzipien und -Kriterien für die Waldbewirtschaftung. F.S.C.-A. Schweiz. Rodersdorf. On www: fsc-schweiz.ch (Zugriff 1. März, 2007).

Gerdtham, U. G. \& Johannesson, M. (2002). Do life-saving regulations save lives? Journal of Risk and Uncertainty 24(3): 231-249.

Gibson, S. B. (1980). Hazard Analysis and Risk Criteria. Chemical Engineering Progress 76(11): 46-50.

Godschalk, D. R., Beatley Th., Berke Ph., Brower D. J. \& Kaiser, E. J. (1999). Natural Hazard mitigation: Recasting Disaster Policy and Planning. Washington D.C.

grEIE (2004). Empfehlungen zum Inhalt von Umweltverträglichkeitsberichten (UVB). Fachgruppe der kantonalen UVP-Verantwortlichen der Westschweiz. Bern, Groupe des Résponsables des Etudes d'Impact de la Suisse occidentale et du Tessin: 21 S.

Greiving, S. (2002). Räumliche Planung und Risiko. München, Gerling Akademie Verlag $\mathrm{GmbH}$.

Griffiths, R. F. (1994). Discounting of Delayed Versus Early Mortality in Societal Risk Criteria. Journal of Loss Prevention in the Process Industries 7(5): 432-438.

Gsponer, G. (2007a). Programmvereinbarungen Schutzbauten und Gefahrengrundlagen. Erläuternder Bericht. Bern. Bundesamt für Umwelt, Wald und Landschaft. $46 \mathrm{~S}$.

Guenther, C. F. \& Thein, C. (1997). Estimated cost of person-Sv exposure. Health Physics 72(2): 204-221.

Haering, B., Gsponer G. \& Koch, P. (2002). effor 2 Konzeptbericht - Wirkungsorientierte Subventionspolitik im Rahmen des Waldgesetzes. Umweltmaterialien Nr. 145. Bern. Bundesamt für Umwelt, Wald und Landschaft. $118 \mathrm{~S}$.

Häfelin, U. \& Müller G. (2002). Allgemeines Verwaltungsrecht. Zürich.

Häfelin, U., Müller G. \& Uhlmann F. (2006). Allgemeines Verwaltungsrecht. Zürich, St. Gallen. Dike Verlag AG.

Hahn, H., Villagran de Leon, Juan C. \& Hidajat, R. (2003). Indicators and other disaster risk management instruments for communities and local governments. Comprehensive Risk Management by Communities and Local Governments. I.-A. D. Bank. Eschborn, Deutsche Gesellschaft für Technische Zusammenarbeit (GTZ) GmbH: 34 S.

Haimes, Y. Y. (2004). Risk modelling, assessment and management. Hoboken, Wiley.

Hall, W. J. \& Wiggins, J. H. (2000). Acceptable Risk: A Need for Periodic Review. Natural Hazards Review 1(3): 180-187.

Heinimann, H. R. (2002). Risk Management - A Framework to Improve Effectiveness and Efficiency of Resource Management. Mountain Watershed Management, Lessons from the Past - Lessons for the Future. Documentation, Nr. 165. Davos. Bundesamt für Umwelt, Wald und Landschaft. $202 \mathrm{~S}$. 
Heinimann, H. R., Hollenstein, K., Kienholz, H., Krummenacher, B., Mani, P. (1998). Methoden zur Analyse und Bewertung von Naturgefahren. Bern. Bundesamt für Wald und Landschaft. $247 \mathrm{~S}$.

Hepperle, E. (1995). Schutz vor Naturgefahren aus planungsrechtlicher Perspektive. Zürich Schweizer Ingenieur und Architekt 113(48): 1112-1116.

Hollenstein, K. (1997). Analyse, Bewertung und Management von Naturrisiken. Professur für forstliches Ingenieurwesen. Zürich. vdf Hochschulverlag AG ETH Zürich. 220 S.

Hostmann, M., Buchecker, M., Ejderyan, O., Geiser, U., Junker, B., Schweizer, St., Truffer, B., Zaugg, M. (2005). Wasserbauprojekte gemeinsam planen - Handbuch für die Partizipation und Entscheidungsfindung bei Wasserbauprojekten. Kastanienbaum. Eawag, Laboratoire de constructions hydrauliques (LCH, EPFL Lausanne), Versuchsanstalt für Wasserbau, Hydrologie und Glaziologie (VAW, ETH Zürich). 48 S.

Iceland (2000). Regulation on hazard zoning due to snow- and landslides, classification and utilisation of hazard zones and preparation of provisional hazard zoning. 505/2000: 18 S.

INSAG (2006). Promoting Safety in Nuclear Installations. Fact sheets. Wien. International Atomic Energy Agency. On www: http://www.iaea.org/Publications/Factsheets/English/safetynuclinstall.pdf (Zugriff 12. Juli, 2006).

Jaun, M. (2006). Verantwortung der Gemeinden und des Kantons im Umgang mit Naturgefahren - Rückgriff der GVB im Falle unterlassener Schutzmassnahmen. Bern. Gebäudeversicherung des Kantons Bern. $16 \mathrm{~S}$.

JCSS (1976). First order reliability concepts for design codes. Joint Committee on Structural Safety. Bulletin d'information: $112 \mathrm{~S}$.

Jenni, H. P. (1993). Vor lauter Bäumen den Wald noch sehen: Ein Wegweiser durch die neue Waldgesetzgebung. BUWAL Schriftenreihe Umwelt Nr. 210. Bern. Bundesamt für Umwelt, Wald und Landschaft. $114 \mathrm{~S}$.

Johansson, P. O. (2002). On the definition and age-dependency of the value of a statistical life. Journal of Risk and Uncertainty 25(3): 251-263.

Kahnemann, D. \& Tversky, A. (1979). Prospect Theory: an analysis of decision under risk. EconoMetrica 47(2): 263-291.

Kanton Glarus (2001). Richtlinien zum Schutz vor Naturgefahren. 4 S. und 1 Anhang.

Kanton Graubünden (2003): Gefahrenkommission. Amt für Wald. Risikomatrix. Chur. 1 S.

Kanton Luzern (2003). Naturgefahren im Kanton Luzern - ein neuer Umgang mit dem Risiko. Luzern. 8 S. On www: http://www.lawa.lu.ch/naturgefahren.pdf (Zugriff 11. Juli 2006).

Kanton Uri (1992). Richtlinie für den Hochwasserschutz. Baudirektion. Altdorf. 17 S.

Kaplan, S. \& Garrick, J.B. (1981). On the quantitative Definition of Risk. Risk Analysis 1(1): $11-27$.

Kishimoto, A., Oka, T. \& Nakanishi, J. (2003). The cost-effectiveness of life-saving interventions in Japan - Do chemical regulations cost too much? Chemosphere 53(4): 291-299.

Ko, C. K., Flentje, P. \& Chowdhury, R. (2003). Quantitative landslide hazard and risk assessment: a case study. Quarterly Journal of Engineering Geology and Hydrogeology 36: 261-272.

Konow, J. (2003). Which is the fairest one of all? A positive analysis of justice theories. Journal of Economic Literature 41(4): 1188-1239. 
Kröger W., Seiler H. \& Gheorghe A., (Hrsg.) (1996). Technik, Risiko, Sicherheit. Abschlussbericht des Polyprojektes Risiko und Sicherheit technischer Systeme der ETHZ 1991-1994. Zürich. vdf Hochschulverlag AG ETH Zürich.

Krupnick, A., Alberini, A., Cropper, M., Simon, N., O'Brien, B., Goeree, R., Heintzelman, M. (2002). Age, health and the willingness to pay for mortality risk reductions: A contingent valuation survey of Ontario residents. Journal of Risk and Uncertainty 24(2): 161-186.

Leimbacher J. \& Saladin P. (1990). Katastrophenschutz: Schutz vor Schädigungen oder Schutz vor Risiken? Schriftenreihe Umwelt, Recht. Bern. Bundesamt für Umwelt, Wald und Landschaft. $73 \mathrm{~S}$.

Leonardo-Productions AG (2006). Leonardo - Das Programm zur Berechnung von Personenschäden. Zürich, Schulthess Juristische Medien AG.

Litai, D., Lanning D. D. \& Rasmussen N. C. (1983). The public perception of risk. The Analysis of actual versus perceived risk. New York/London. V. T. C. et al.: 212-224.

Loat, R. \& Meier, E. (2003). Dictionary of Flood Protection. Biel, Haupt.

Lüthi, R. (2004). Rechtliche Aspekte im Zusammenhang mit der Gefahrenkarte. PLANAT Reihe 4/2004. Biel. PLANAT. 44 S. und 10 Anhänge.

Lutter, R., Morrall, J. F. \& Viscusi, W. K. (1999). The cost-per-life-saved cutoff for safetyenhancing regulations. Economic Inquiry 37(4): 599-608.

Margreth, S., Stoffel, L. \& Wilhelm, C. (2003). Winter opening of high alpine pass roads analysis and case studies from the Swiss Alps. Cold Regions Science and Technology 37(3): 467-482.

Maslow, A. H. (1970). Motivation and personality. New York. Harper \& Row.

Mathis, K. (2004). Effizienz statt Gerechtigkeit? - Auf der Suche nach den philosophischen Grundlagen der ökonomischen Analyse des Rechts. Berlin. Duncker \& Humblot.

Merz, H. A., Schneider, Th. \& Bohnenblust, H. (1995). Bewertung von technischen Risiken. Beiträge zur Strukturierung und zum Stand der Kenntnisse. Modelle zur Bewertung von Todesfallrisiken. Zürich. vdf Hochschulverlag AG ETH Zürich.

Morgan, G. C. (1997). A regulatory perspective on slope hazards and associated risks to life. Landslide risk assessment proceedings of the International Workshop on Landslide Risk Assessment. Honolulu, Hawaii, USA. 19-21 February 1997. D. M. Cruden. Rotterdam. Balkema. XI, 371: 285-295.

Müller, J. P. (1999). Grundrechte in der Schweiz: im Rahmen der Bundesverfassung von 1999, der UNO-Pakte und der EMRK. Bern. Stämpfli Verlag AG.

NAGEF (2002). Naturgefahren bei Fuss- und Wanderwegen. Bern. Arbeitsgruppe Naturgefahren des Kantons. $12 \mathrm{~S}$.

NAGEF (2003). Schutzziele Naturgefahren Kanton Bern. Bern. Arbeitsgruppe Naturgefahren des Kantons. $5 \mathrm{~S}$.

Naturgefahren Schweiz (2008). Die nationale Plattform Naturgefahren. On www: http://www.naturgefahren.ch/index.php?userhash=12619249\&l=d\&navID=154 (Zugriff am 28. Mai 2008).

Nef J. (1987). Haftpflicht und Versicherungsschutz des Bergsteigers. Dissertation Nr. 4631. Zürich.

Neue Obwaldner Zeitung. Ausgabe vom 30. November 2006.

Nidwaldner Sachversicherung (2006). On www: http://www.nsv.ch/ (Zugriff 21. Dez. 2006). 
Niederer \& Pozzi, C. (1992). Engelberger Aa. Generelles Hochwasserschutzprojekt. Kantonsingenieurbüro. Stans. Kanton Nidwalden: 36-40.

Nöthiger, C. J. (2001). Die Auswirkungen der Naturereignisse 1999 auf die Bergbahn- und Skiliftunternehmen der Schweiz. Interne Berichte. Davos. Eidg. Institut für Schnee- u. Lawinenforschung. $38 \mathrm{~S}$.

Nöthiger, C. J. (2003). Naturgefahren und Tourismus in den Alpen. Untersucht am Lawinenwinter 1999 in der Schweiz. Birmensdorf, Davos. Eidg. Forschungsanstalt für Wald, Schnee und Landschaft, Eidg. Institut für Schnee- und Lawinenforschung.

Ott, W., Baur, M., Iten, R. \& Vettori A. (2005). Konsequente Umsetzung des Verursacherprinzips. Umwelt-Materialien Nr. 201. Bern. Bundesamt für Umwelt, Wald und Landschaft. $184 \mathrm{~S}$.

Pandey, M. D. \& Nathwani, J. S. (2003). Canada wide standard for particulate matter and ozone: Cost-benefit analysis using a life quality index. Risk Analysis 23(1): 55-67.

Pandey, M. D., Nathwani, J. S., Lind, N. C. (2006). The derivation and calibration of the lifequality index (LQI) from economic principles. Structural Safety 28(4): 341-360.

Paté-Cornell, E. (1994). Quantitative safety goals for risk management of industrial facilities. Structural Safety 13: 145-157.

Paté-Cornell, E. (2002). Risk and Uncertainty Analysis in Government Safety Decisions. Risk Analysis 22(3): 633-646.

Perreira, K. M. \& Sloan, F. A. (2002). Living healthy and living long: Valuing the nonpecuniary loss from disability and death. Journal of Risk and Uncertainty 24(1): 5-29.

Persson, U., Norinder, A., Hjalte, K., Gralén, K. (2001). The value of a statistical life in transport: Findings from a new contingent valuation study in Sweden. Journal of Risk and Uncertainty 23(2): 121-134.

PLANAT (2004a). Strategie Naturgefahren Schweiz, Teilprojekt A - Gesamtübersicht. Studie zur Umsetzung der Strategie. Nationale Plattform Naturgefahren PLANAT. Zürich. Ernst Basler + Partner AG. 45 S.

PLANAT (2004b). Strategie Naturgefahren Schweiz - Synthesebericht. Umsetzung des BRB vom 20. August 2003. Nationale Plattform Naturgefahren PLANAT. Davos. Eidg. Institut für Schnee- und Lawinenforschung. $79 \mathrm{~S}$.

Plattner, T., Hollenstein, K. \& Heinimann, H. R. (2005). Risikobewertung bei Naturgefahren - Schlussbericht. ETH. Zürich. Nationale Plattform Naturgefahren PLANAT. 85 S.

Proske, D. (2004). Katalog der Risiken - Risiken und ihre Darstellung. Dresden. Eigenverlag Dirk Proske.

Ramsberg, J. (2002). When should expenditure per life saved vary? Journal of Risk Research 5(3): 249-263.

Reinhard, H. (1993). Allgemeines Polizeirecht. Aufgaben, Grundsätze und Handlungen. Bern. Haupt Verlag.

Richli, P. (2004a). Verwaltungsrecht I. Vorlesungsmanuskript. Uni Luzern. 272 S.

Richli, P. (2004b). Verwaltungsrecht II. Vorlesungsmanuskript. Uni Luzern. 324 S.

Robinson R. (2003). R2A (Risk \& Reliability Associates) 4th edition. Melbourne, R2A. On www: http://www.r2a.com.au/publications/publications.html (Zugriff 9. April 2004).

Röthlisberger, G. (1998). Unwetterschäden in der Schweiz. Bericht der WSL Nr. 346. Birmensdorf. Eidg. Forschungsanstalt für Wald, Schnee und Landschaft. 
RSU (1994). Umweltgutachten 1994. Rat von Sachverständigen für Umweltfragen. Stuttgart. Kohlhammer, später Metzler-Poeschel.

SBB (1993). Sicherheitsstandards für unterirdische Verkehrsanlagen der SBB, Schlussbericht Teil 1. Betrachtungen für das SBB-Netz. Zollikon. Ernst Basler+Partner AG.

Scheringer, M., Berg, M. \& Müller-Herold, U. (1994). Jenseits der Schadensfrage: Umweltschutz durch Gefährdungsbegrenzung. Was ist ein Schaden? Zur normativen Dimension des Schadensbegriffes in der Risikowissenschaft. Zürich. vdf Hochschulverlag AG ETH Zürich. Dokumente Nr. 2: 115-146.

Schneider, J. (1996). Sicherheit und Zuverlässigkeit im Bauwesen. Zürich und Stuttgart. vdf Hochschulverlag ETH Zürich und Teubner 1996.

Schneider, T. (1984). Das Risiko-Konzept. Cours postgrade sur la Sécurité du Travail - IV Sicherheitsanalyse. Zürich.

Schneider, T. (2006). Risk Aversion - a delicate issue in risk assessment. Risk 21 - Coping with Risks due to natural hazards in the 21st century. CENAT. London, Taylor \& Francis/Balkema. 1: 59-66.

Schueremans, L. (2001). Probabilistic Evaluation of Structural unreinforced masonry. F. T. W. Katholieke Universiteit Leuven. Departement Burgerlijke Bouwkunde. Heverlee, Belgien.

Schulze W.D. \& Kneese A. V. (1981). Risk in Benefit-Cost Analysis. Risk Analysis 1(1). Society for Risk Analysis, Rutgers University of New Jersey, USA.

Seiler, H. (1995a). Rechtliche und rechtsethische Aspekte der Risikobewertung. Rechtsfragen technischer Risiken, Aufsätze zum Risikorecht. vdf Hochschulverlag AG an der ETH Zürich, Zentralstelle Polyprojekt Risiko und Sicherheit technischer Systeme. Dokument 6: 1-26.

Seiler, H. (1995b). Staats- und verwaltungsrechtliche Fragen der Bewertung technischer Risiken. Rechtsfragen technischer Risiken. Aufsätze zum Risikorecht. Zentralstelle Polyprojekt Risiko und Sicherheit technischer Systeme. Zürich. vdf Hochschulverlag AG ETH Zürich. Dokument Nr. 6: 27-50.

Seiler, H. (1995c). Rechtsgleichheit und Störfallrecht. Rechtsfragen technischer Risiken. Aufsätze zum Risikorecht. Zentralstelle Polyprojekt Risiko und Sicherheit technischer Systeme. Zürich. vdf Hochschulverlag AG ETH Zürich. Dokument Nr. 6: 51-68.

Seiler, H. (1995d). Die rechtliche Behandlung unterschiedlicher und aggregierter Risiken. Rechtsfragen technischer Risiken. Aufsätze zum Risikorecht. Zentralstelle Polyprojekt Risiko und Sicherheit technischer Systeme. Zürich. vdf Hochschulverlag AG ETH Zürich. Dokument Nr. 6: 69-98.

Seiler, H. (1995e). Die Bedeutung von Ungewissheit und Wahrscheinlichkeit im Recht. Rechtsfragen technischer Risiken. Aufsätze zum Risikorecht. Zentralstelle Polyprojekt Risiko und Sicherheit technischer Systeme. Zürich. vdf Hochschulverlag AG ETH Zürich. Dokument Nr. 6: 99-112.

Seiler, H. (1995f). Rechtliche Instrumente zur Koordination risikoreicher Tätigkeiten mit anderen Aktivitäten und Bedürfnissen, dargestellt am Beispiel der Schweiz. Rechtsfragen technischer Risiken. Aufsätze zum Risikorecht. Zentralstelle Polyprojekt Risiko und Sicherheit technischer Systeme. Zürich. vdf Hochschulverlag AG ETH Zürich. Dokument Nr. 6: 113-134.

Seiler, H. (1996a). Aktuelle Tendenzen des Risikorechts. Risikoforschung zwischen Disziplinarität und Interdisziplinarität. G. Banse. Berlin. Ed. Sigma. 1: S. 145-164. 
Seiler, H. (1996b). The legal implementation of regional safety planning: Taking into account the risk based regulation approach. International Journal of Technology Management 12(4): 438-448.

Seiler, H. (1997). Recht und technische Risiken. Grundzüge des technischen Sicherheitsrechts. Zürich. vdf Hochschulverlag AG ETH Zürich.

Seiler, H. (2000). Schweizerischer Nationalfonds zur Förderung der Wissenschaftlichen Forschung. Risikobasiertes Recht. Wie viel Sicherheit wollen wir? Bern. Stämpfli.

Seiler, H. (2001). Erläuterungen zum Katastrophenschutz Art. 10 USG. Kommentar zum Umweltschutzgesetz. Zürich. $67 \mathrm{~S}$.

Seiler, H. (2002). Harmonised Risk Based Regulation - a legal viewpoint. Safety Science 40(1-4): 31-49.

Seiler, H. (2006). Risk based Regulation. Risk 21 - Coping with Risks due to natural hazards in the 21st century. CENAT. London, Taylor \& Francis/Balkema. 1: 25-30.

Seiler, H. (2006). Risiko, Recht und Gerichte: Vom Umgang der Gerichte mit Risiko im Haftpflicht- und Strafrecht. Risikomanagement und Recht. Winterthur, unveröffentlicht.

SIA (2003). Grundlagen der Projektierung von Tragwerken. Swisscodes. Zürich. SIA 260:2003. 43 S.

SIA (2003a). Einwirkungen auf Tragwerke. Swisscodes. Zürich. SIA 261:2003: 114 S.

SIA (2003b). Einwirkungen auf Tragwerke - Ergänzende Festlegungen. Swisscodes. Zürich. SIA 261/1:2003. 32 S.

SIA (2004). Überprüfung bestehender Gebäude bezüglich Erdbeben. Swisscodes. Zürich. SIA Merkblatt 2018. 38 S.

SKOS (2005). Richtlinien für die Ausgestaltung und Bemessung der Sozialhilfe. Bern. Schweizerische Konferenz für Sozialhilfe. 169 S.

SKUS (2006). Richtlinien für Anlage, Betrieb und Unterhalt von Schneesportabfahrten. Bern. $28 \mathrm{~S}$.

SKUS (2006). Richtlinien für Skifahrer und Snowboarder. Bern. 12 S.

SLF (2000). Der Lawinenwinter 1999. Ereignisanalyse. Davos. Eidg. Institut für Schneeund Lawinenforschung. $588 \mathrm{~S}$.

Slovic, P. (1987). Perception of Risk. Science, New Series 236(4799): 280-285.

Sobotich, V. (2000). Staatshaftung aus Kontrolltätigkeit im Baurecht. Zürich. Schulthess Juristische Medien AG.

Sommer, J. H. (2001). Muddling Through Elegantly: Rationierung im Gesundheitswesen. Basel. Schwabe.

Staehelin, E. \& Hefti, Ch. (2003). Effor2 - Teilprojekt Neugestaltung der Bundesbeiträge. Bern. Bundesamt für Umwelt, Wald und Landschaft. $14 \mathrm{~S}$.

Stähelin, S. (1993). Problematik des Begriffs der schweren Schädigung im Störfallrecht. Umweltrecht in der Praxis 7(3): 233-241.

Stallen, P. J. M., Geerts, R., Vrijling, H. K. (1996). Three conceptions of quantified societal risk. Risk Analysis 16(5): 635-644.

Starr, C. (1969). Social Benefit versus Technological Risk. Science 165(3899): 1232-1238. 
Starr, C. (1981). Risk Criteria for Nuclear Power Plants: A Pragmatic Proposal. Risk Analysis 1(2): 113-120.

Stiefel, U. \& Schneider, J. (1985). Was kostet Sicherheit? Schweizer Ingenieur und Architekt 104(47): 1175-1182.

Subramanian, U. \& Cropper, M. (2000). Public choices between life saving programs: The trade off between qualitative factors and lives saved. Journal of Risk and Uncertainty 21(1): 117-149.

Tansel, B. (1995). Natural and Manmade Disasters. Accepting and Managing Risks. Safety Science 20(1): 91-99.

Tengs, T. O., Adams M. E., Pliskin, J. S., Gelb, D., Siegel, J.E., Weinstein, M. C., Graham, J. D. (1995). 500 Lifesaving Interventions and Their Cost-Effectiveness. Risk Analysis 15(3): 369-390.

Trösch, A. (1992). Die Tragbarkeit von Risiken. Umweltrecht in der Praxis 6(4/1): 329-339.

Vernunft Schweiz (2007). Armutsgrenze. Lexikon Vernunft Schweiz. Verein Vernunft Schweiz. On www: http://www.vernunft-schweiz.ch/glossar/458/Existenzminimum+.html (Zugriff 23. Nov. 2007).

Viscusi, W. K. (1994). Mortality Effects of Regulatory Costs and Policy Evaluation Criteria. Rand Journal of Economics 25(1): 94-109.

Viscusi, W. K. (1995). Risk, Regulation and Responsibility - Promoting reason in workplace and product safety regulation. Risk, Regulation and Responsibility: Principle for Australian Risk Policy. Institute of Public Affairs, Perth.

Viscusi, W. K. (1998). Rational risk policy. The 1996 Arne Ryde memorial lectures. Oxford. Clarendon Press.

Vrijling, J. K., Vanhengel, W., Houben, R. J. (1995). A Framework for Risk-Evaluation. Journal of Hazardous Materials 43(3): 245-261.

Vrijling, J. K., Vanhengel, W., Houben, R. J. (1998). Acceptable risk as a basis for design. Reliability Engineering \& System Safety 59(1): 141-150.

Weber, K. (2005). Die rechtlichen Handlungsformen des alpinen Naturgefahrenmanagements. alpS-Forum und Stammtisch. Innsbruck. Zentrum alpS.

Whittaker, J. D. (1986). Evaluation of Acceptable Risk. The Journal of Operational Research Society 37(6 (Jun., 1986)): 541-547.

Wikipedia (2006). Risiko. Wikipedia - die freie Enzyklopädie. W. F. Inc. St. Petersburg, FL 33701-4313, USA. Wikimedia Foundation Inc. On www: http://de.wikipedia.org.

Wilhelm, C. (1997). Wirtschaftlichkeit im Lawinenschutz. Mitteilungen. Davos. Eidg. Institut für Schnee- und Lawinenforschung. $308 \mathrm{~S}$.

Wilhelm, C. (1999). Kostenwirksamkeit von Lawinenschutzmassnahmen an Verkehrsachsen. Bern. Bundesamt für Umwelt, Wald und Landschaft. $110 \mathrm{~S}$.

Williamson, O. E. (1975). Markets and Hierarchies: Analysis and Antitrust Implications. New York. The Free Press.

Wood, A., Palmer, R., Petroff, C. (1997). Comparison and assessment of zero-rise floodplain ordinances. Journal of Water Resources Planning and Management ASCE 123(4): 239-245.

Zimmermann, M. (2006). Urteil des Bundesgerichts 4P.244/2005 vom 6. Februar 2006. Haftung und Versicherung (2006): 133-136. 


\section{V2. Verwendete Rechtsgrundlagen}

\section{Bundesgesetze}

\begin{tabular}{|c|c|}
\hline SR & Voller Titel \\
\hline 0.131 .313 .6 & $\begin{array}{l}\text { Abkommen vom 28. November } 1984 \text { zwischen der Schweize- } \\
\text { rischen Eidgenossenschaft und der Bundesrepublik Deutsch- } \\
\text { land über die gegenseitige Hilfeleistung bei Katastrophen oder } \\
\text { schweren Unglücksfällen }\end{array}$ \\
\hline 0.131 .334 .9 & $\begin{array}{l}\text { Abkommen vom 14. Januar } 1987 \text { zwischen dem Schweizeri- } \\
\text { schen Bundesrat und der Regierung der Französischen Re- } \\
\text { publik über die gegenseitige Hilfeleistung bei Katastrophen } \\
\text { oder schweren Unglücksfällen }\end{array}$ \\
\hline 0.131 .345 .4 & $\begin{array}{l}\text { Abkommen vom 2. Mai } 1995 \text { zwischen der Schweizerischen } \\
\text { Eidgenossenschaft und der Italienischen Republik über die } \\
\text { Zusammenarbeit im Bereich der Risikovorsorge und -vorbeu- } \\
\text { gung und der gegenseitigen Hilfeleistung bei natürlichen oder } \\
\text { durch menschliche Tätigkeit verursachten Katastrophen }\end{array}$ \\
\hline
\end{tabular}

0.131.313.3 Eidgenossenschaft und der Republik Österreich über die gegenseitige Hilfeleistung bei Katastrophen oder schweren Unglücksfällen

Übereinkommen zum Schutz der Alpen (Alpenkonvention)

0.700.1 (mit Anhang) vom 7. November 1991 (Stand am 12. August

Abkürzung 2003)

Protokoll zur Durchführung der Alpenkonvention von $1991 \mathrm{im}$ Bereich Berglandwirtschaft

Protokoll zur Durchführung der Alpenkonvention von $1991 \mathrm{im}$ Bereich Bergwald

Protokoll zur Durchführung der Alpenkonvention von $1991 \mathrm{im}$ Bereich Raumplanung und nachhaltige Entwicklung

ZGB

Bundesgesetz über den Versicherungsvertrag (Versicherungs-

221.220 .1 vertragsgesetz) vom 2. April 1908 (Stand am 27. Dezember 2005)

Schweizerisches Strafgesetzbuch vom 21. Dezember 1937 (Stand am 1. Januar 2008)

OV-UVEK

Bundesgesetz über die Meteorologie und Klimatologie (MetG)

429.1 vom 18. Juni 1999 (Stand am 21. März 2000)

VVG

Verordnung über die Meteorologie und Klimatologie vom 23.

429.11 Februar 2000 (Stand am 21. März 2000)

MetV

Bundesgesetz über den Natur- und Heimatschutz vom 1. Juli

Bundesgesetz über die Armee und die Militärverwaltung (Militärgesetz) vom 3. Februar 1995 (Stand am 13. Juni 2006) 
520.1

520.12

616.1

700

700.1

721.100

721.100 .1

725.11

725.111

725.116 .2

742.101

746.1

814.01

814.012

814.20

814.50

832.10

832.20

910.1

921.0
Bundesgesetz vom 4. Oktober 2002 über den Bevölkerungsschutz und den Zivilschutz (Bevölkerungs- und Zivilschutzgesetz) (Stand am 13. Juni 2006)

Verordnung über die Warnung, Alarmierung und Verbreitung von Verhaltensanweisungen an die Bevölkerung (Alarmierungsverordnung) vom 5. Dezember 2003 (Stand 30. Dezember 2003)

Bundesgesetz über Finanzhilfen und Abgeltung (Subventionsgesetz, SuG) vom Oktober 1990 (Stand am 13. Juni 2006)

SuG

Bundesgesetz über die Raumplanung (Raumplanungsgesetz) vom 22. Juni 1979 (Stand am 22. August 2000)

RPG

Raumplanungsverordnung (RPV) vom 28 Juni 2000

(Stand am 22. August 2000)

Bundesgesetz über den Wasserbau (Wasserbaugesetz) vom 21. Juni 1991 (Stand am 1. Januar 1995)

AV

Verordnung über den Wasserbau (Wasserbauverordnung) vom 2. November 1994 (Stand am 8. Februar 2000)

WBV

Bundesgesetz über die Nationalstrassen vom 8. März 1960 (Stand am 13. Juni 2006)

NSG

Verordnung über die Nationalstrassen vom 18. Dezember 1995 (Stand am 21. Dezember 2004)

NSV

Bundesgesetz über Verwendung der zweckgebundenen Mineralölsteuer vom 22. März 1985 (Stand am 23. November 2004)

MinVG

Eisenbahngesetz vom 20. Dezember 1957 (Stand 1. Mai 2007)

EBG

Bundesgesetz über Rohrleitungsanlagen zur Beförderung flüssiger oder gasförmiger Brenn- oder Treibstoffe (Rohrleitungsgesetz) vom 4. Oktober 1963 (Stand am 13. Juni 2006)

RLG

Bundesgesetz über den Umweltschutz (Umweltschutzgesetz) vom 7. Oktober 1983 (Stand am 1. Januar 2008)

USG

Verordnung über den Schutz vor Störfällen (Störfallverordnung) vom 27. Februar 1991 (Stand am 23. August 2005)

Bundesgesetz über den Schutz der Gewässer (Gewässerschutzgesetz, GSchG) vom 24. Januar 1991 (Stand am 1. Januar 2008)

Strahlenschutzgesetz vom 22. März 1991 (Stand am 28. Dezember 2004)

Bundesgesetz über die Krankenversicherung vom 18. März 1994 (Stand am 27. Dezember 2006)

StFV

USG

StSG

KVG

Bundesgesetz über die Unfallversicherung vom 20. März 1981 (Stand am 13. Juni 2006)

UVG

Bundesgesetz über die Landwirtschaft (Landwirtschaftsgesetz) vom 29. April 1998 (Stand am 26. September 2006)

LWG

Bundesgesetz über den Wald (Waldgesetz, WaG) vom 4. Oktober 1991 (Stand am 1. Januar 2008) 
Bundesgesetz über die Jagd und den Schutz wildlebender

922.0

Säugetiere und Vögel (Jagdgesetz, JSG) vom 20. Juni 1986

(Stand am 1. Januar 2008)

923.0

Bundesgesetz über die Fischerei (BFF) vom 21. Juni 1991 (Stand am 1. Januar 2008)

BGF

Bundesgesetz betreffend die Aufsicht über Versicherungsun-

961.01 ternehmen (Versicherungsaufsichtsgesetz) vom 17. Dezember 2004 (Stand am 13. Juni 2006)

Verordnung über die Beaufsichtigung von privaten Versiche-

961.011 rungsunternehmen (Aufsichtsverordnung) vom 9. November 2005 (Stand am 14. November 2006)

Kantonale Gesetze

\begin{tabular}{|c|c|c|}
\hline Kanton/Nr. & Voller Titel & Abkürzung \\
\hline \multicolumn{3}{|l|}{ Bern } \\
\hline BSG 521.1 & $\begin{array}{l}\text { Kantonales Bevölkerungsschutz- und Zivilschutzgesetz vom } \\
\text { 24. Juni } 2004\end{array}$ & KBZG \\
\hline BSG 751.11 & $\begin{array}{l}\text { Gesetz über Gewässerunterhalt und Wasserbau vom 14. Feb- } \\
\text { ruar } 1989\end{array}$ & WBG \\
\hline BSG 751.111.1 & Wasserbauverordnung vom 15. November 1989 & WBV \\
\hline BSG 873.11 & Gesetz vom 6. Juni 1971 über die Gebäudeversicherung & GVG \\
\hline BSG 873.111 .2 & $\begin{array}{l}\text { Prämientarif der Gebäudeversicherung vom 24. November } \\
2004 \text { (Stand 1. Februar 2005) }\end{array}$ & \\
\hline BSG 910.1 & Kantonales Landwirtschaftsgesetz vom 16. Juni 1997 & KLwG \\
\hline BSG 921.11 & Kantonales Waldgesetz vom 5. Mai 1997 & KWaG \\
\hline BSG 921.111 & Kantonale Waldverordnung vom 29. Oktober 1997 & KWaV \\
\hline
\end{tabular}


SRL $760 \quad$ Wasserbaugesetz vom 30. Januar 1979

Wasserbaugesetz

SRL 945

Kantonales Waldgesetz vom 1. Februar 1999

Waldgesetz

Uri

Urner Rechtsbuch

No. 40.1211

Wasserbaugesetz vom 30. November 1980

WBG

Urner Rechtsbuch Gesetz über die Staatshilfe bei Elementarschäden vom 6. De-

No. 40.1401

zember 1964

Urner Rechtsbuch

No. 40.2111

Kantonale Waldverordnung vom 13. November 1996

KWV

Schwyz

SRSZ 313.110

Kantonale Verordnung zum Bundesgesetz über den Wald vom 21. Oktober 1998

SRSZ $451.100 \quad$ Wasserrechtsgesetz vom 11. September 1973

SRSZ 521.100

Gesetz über den Bevölkerungsschutz und den Zivilschutz vom 16. März 2005

Nidwalden

NG 867.1

Gesetz über die Nidwaldner Gebäude- und Mobiliarversiche-

Sachversicherung vom 27. April 1986

rungsgesetz

NG 867.3

Gesetz über die Vergütung nicht versicherbarer Elementarschäden

Hilfsfondsgesetz

NG 831.1

Einführungsgesetz zum Bundesgesetz über den Wald vom

Kantonales

11. März 1998

Waldgesetz

Obwalden

GDB 101

Verfassung des Kantons Unterwalden ob dem Wald vom 19. Mai 1968

KV

GDB 213.7

Gesetz über die amtliche Schätzung der Grundstücke und das Grundpfandrecht vom 26. Oktober 2006

Schätzungsund Grundpfandgesetz

GDB 710.1

Baugesetz vom 12. Juni 1994

Baugesetz

GDB 720.11

Strassenverordnung vom 14. September 1935

Strassenverordnung 
GDB 740.1 Gesetz über den Wasserbau und die Wassernutzung (Wasserbaugesetz) vom 31. Mai 2001

WBG

GDB 740.11 Wasserbauverordnung vom 31. Mai 2001

WBV

Gesetz über die Regelung der Abflussverhältnisse des Sarner-

GDB 740.2 sees zur Hochwassersicherheit des Sarneraatales vom 31. Mai 2007

GAHS

GDB 930.11

Kantonale Forstverordnung vom 30. Januar 1960

Forstverordnung

Zug

BGS 731.1

Gesetz über die Gewässer vom 25. November 1999

GewG

Basellandschaft

SGS 570

Kantonales Waldgesetz vom 11. Juni 1998

kWaG

Basel-Stadt

SG 911.6

Waldgesetz vom 6. Februar 2000

WaG BS

SG 911.61

Verordnung zum Waldgesetz vom 18. Dezember 2001

WaV BS

Appenzell-Innerrhoden

711

Wasserbaugesetz vom 29. April 2001

WbauG

Appenzell-Ausserrhoden

bGS $931.11 \quad$ Verordnung zum Kantonalen Waldgesetz vom 9. Dezember 1996

kantonale

Waldverordnung

St. Gallen

sGS 734.11

Wasserbaugesetz vom 23. März 1969

Wasserbau-

gesetz

Graubünden

BR 830.1

Gesetz über die Gebäudeversicherung vom 12. April 1970

GebVG

BR 830.200

Verordnung über die Finanzierung der Gebäudeversicherung im Kanton Graubünden vom 7. September 1970 
BR 835.100 Gesetz über die Vergütung nicht versicherbarer Elementarschäden vom 23. September 1984

GVE

BR 920.100

Kantonales Waldgesetz vom 25. Juni 1995

KWAG

Wallis

GS/VS 921.1

Forstgesetz vom 1. Februar 1985

ForstG

\section{V3. Materialienverzeichnis}

- Botschaft über eine neue Bundesverfassung vom 20. November 1996. Bern, Bundeskanzlei, BBI 1997 | 1-642.

- Botschaft vom 6. Mai 1970 über die Ergänzung der Bundesverfassung durch einen Artikel 24septies betreffend den Schutz des Menschen und seiner natürlichen Umwelt gegen schädliche oder lästige Einwirkungen. Bern. Bundeskanzlei, BBI 1970 I 761780.

- Botschaft zu einem Bundesgesetz über die Raumplanung (RPG) vom 27. Februar 1978. Bern. Bundeskanzlei, BBI 1978 I 1006-1045.

- Botschaft zu einem Bundesgesetz über den Umweltschutz (USG) vom 31. Oktober 1979. Bern. Bundeskanzlei, BBI 1979 III 749-853.

- Botschaft zu einem Bundesgesetz über Walderhaltung und Schutz vor Naturereignissen (Waldgesetz, WaG) vom 29. Juni 1988. Bern. Bundeskanzlei, BBI 1988 III 173238.

- Botschaft über ein zweites Paket von Massnahmen zur Neuverteilung der Aufgaben zwischen Bund und Kantonen vom 25. Mai 1988. Bern. Bundeskanzlei, BBI 1988 II 1333-1441.

- Amtliches Bulletin der Bundesversammlung Ständerat (1989). Bern. EDMZ. 99.

- Amtliches Bulletin der Bundesversammlung Nationalrat (1991). Bern. EDMZ. 1/101.

- Amtliches Bulletin der Bundesversammlung Nationalrat (1998N). Bern. EDMZ. Separatdruck Reform der Bundesverfassung: 498 S.

- Amtliches Bulletin der Bundesversammlung Ständerat (1998S). Bern. EDMZ. Separatdruck Reform der Bundesverfassung: $230 \mathrm{~S}$.

- 02.401 - Parlamentarische Initiative Verfassungsartikel über den Schutz vor Naturgefahren (UREK-N, 2001). Bericht zur Ergänzung der Verfassung mit einem Artikel über den Schutz vor Naturgefahren. Subkommission Erdbeben der UREK-N. Vorentwurf. On www: http://www.parlament.ch/ed-pa-berichte-pa-vernehml-02-401naturgefahren.pdf? wobj=11244 (Zugriff: 23.März 2007).

- 00.458 - Parlamentarische Initiative. Erdbeben. Nationale Versicherung für Gebäude. Eingereicht von Walter Hess am 13.12.2000. On www: http://search.parlament.ch/cvgeschaefte?gesch_id=20000458 (Zugriff: 30. März 2007).

- 98.3600 - Motion. Erdbeben. Vorsorgliche Massnahmen. Eingereicht von Simon Epiney am 16.12.1996. On www: http://search.parlament.ch/print/homepage/cvgeschaefte.htm?gesch_id=19983600 (Zugriff: 23. März 2007).

- Vernehmlassungsunterlagen zu den NFA-Ausführungsverordnungen. On www: http://www.efd.admin.ch/00468/index.html?lang=de\&msg-id=12151 (Zugriff: 4. Juni 2007). 


\section{V4. Verwendete Gerichtsurteile}

Bundesgericht

Entscheid-Nr. Stichworte

Entscheiddatum

80 IV $130 \quad$ Frasnacht; Verkehrsunfall; unübersichtliches Gartentor

11. Jun. 1954

90 IV 8

Genf-Cointrin; Baustellenunfall; Betonplatten; Sorgfaltspflicht

10. Mrz. 1964

90 IV 265

Frutigen; Strassenbelag

4. Dez. 1964

91 || 474

Otelfingen; überlaufendes Gewässer

93 || 230

Tarasp; Felskopf; Haftung des Grundeigentümers

14. Juli 1965

98 || 40

100 la 287

Mörel; vereiste Strasse; Strassenunterhaltspflicht

12. Mai 1967

15. Feb. 1972

100 || 134

Küsnacht; Hallenbad; Ausschluss Auswärtiger

102 II 343

Giswil; Wildbachdurchlass an Strasse; Werkeigentümerhaftung

18. Sept. 1974

21. Mai 1974

$108 \mathrm{lb} 167$

Vercorin; Bergstrasse; Sorgfaltspflicht; Verhältnismässigkeit

15. Okt. 1976

$112 \mathrm{lb} 322$

Trient; Rodung; polizeiliche Gründe

3. Feb. 1982

113 IV 68

Liestal; psychiatrische Klinik; Staatshaftung; Suizid

30. Sept. 1986

29. Juli 1987

114 la 245

Zürich; Garantenstellung

20. Apr. 1988

114 lb 317

Aargau; Standortgebundenheit

1. Dez. 1988

115 || 15

Vormund; Haftung gegenüber Dritten

28. Feb. 1989

115 IV 189

Elm; Signalisation Skipiste; genügende Absperrung

8. Nov. 1989

116 || 422

St. Gallen; Plauschbad; Werkeigentümerhaftung

116 IV 182

Täsch; Lawinensicherung Strasse; Verantw. Strassenmeister

116 IV 306

Ballwil; Signalisation Baustelle

19. Sept. 1990

30. Nov. 1990

117 lb 28

Samnaun; Richtstrahlantenne; Bagatellrisiko

11. Dez. 1990

117 IV 58

Kaiseraugst; Betäubungsmittel; erlaubtes Risiko

6. März 1991

117 IV 130

Bauarbeiter; Garantenstellung; unechtes fahrl. Unterlassungsdelikt

31. Jan. 1991

117 IV 415

Elm; Lawine auf Skipiste; Absperrung

27. März 1991

$118 \mathrm{lb} 473$

Listeriose; Information der Öffentlichkeit; Informationspflicht

26. Nov. 1991

118 IV 130

S-Charl; Skitour; Sorgfaltspflicht

11. Nov. 1992

16. Jan. 1992

119 la 28

Genf; Hausbesetzer; grundrechtliche Schutzpflicht

11. Feb. 1993

$119 \mathrm{lb} 334$

Zeuzier; Stausee; Kausalität; Haftung des Grundeigentümers

3. Feb. 1993

119 lb 380

Walzenhausen; Autoeinstellplatz; Verhältnismässigkeit

23. Juni 1993

$120 \mathrm{lb} 411$

Königsfelden; spitalärztliche Tätigkeit

25. Okt. 1994

120 IV 334

Basel-Landschaft; Drogenweitergabe; erlaubtes Risiko

23. Dez. 1994

121 || 378

Mattstätten; Plangenehmigungsverfahren; Verhältnismässigkeit

121 III 358

Churwalden; Bäume am Pistenrand; Verkehrssicherungspflicht

122 III 229

Orny; Nozon; Überschwemmung

122 IV 193

Melchsee-Frutt; Gefahren auf Nebenflächen; Verkehrssich.pflicht

25. Okt. 1995

3. Okt. 1995

23. April 1996

10. Juli 1996 
122 IV 225 Frauenfeld; Aufmerksamkeit im Strassenverkehr; geringes Risiko

4. Juni 1996

123 III 306

Neuenburg; Strandbad; Sorgfaltspflicht; Selbstverschulden

6. Mai 1997

124 II 219 Biel-Pieterlen; Elektrosmog; Bagatellrisiko; Verhältnismässigkeit

1. April 1998

124 IV 114

Teufen; Kollision mit Eisenbahn; geringes Risiko

125 IV 9

Zermatt; Rothornbahn; Sicherheitsdispositiv

30. April 1998

126 | 112

Bern; psych. Universitätsklinik; pol. Generalklausel

1. Dez. 1998

126 || 300

Liestal; Schiesslärm

23. Mai 2000

126 II 522

Zürich Flughafen; Dock Midfield

3. Mai 2000

126 III 113

Thyon-Les Collons; Skiliftmast; Sorgfaltspflicht, Restrisiko

8. Dez. 2000

126 IV 13

Däniken; Schienenkran; Fahrlässigkeit; Gefahrensatz

18. Jan. 2000

127 || 18

Pfäffikon; Chlorgas; Risikoanalyse

10. Nov. 1999

8. Jan. 2001

127 IV 62

Rupperswil; Reitunfall; Sorgfaltspflicht

128 | 327

Graubünden; Polizei VO; Rechtmässigkeit; Verhältnismässigkeit

23. Nov. 2000

129 III 65

Tesserete; vereiste Strasse

130 I 337

Spitalärztliche Tätigkeit; Staatshaftung; Willkür

130 III 193

Glarus; Gefahren ausserhalb der Piste; Verkehrssicherungspflicht

26. Aug. 2002

29. Okt. 2002

19. Okt. 2004

131 || 670

Basel; Messe; SARS; Sofortmassnahmen

23. Dez. 2003

17. Okt. 2005

1P. 88/1995 Disentis; Stützmauer

1A.252/1997 Uster; Gewässerbaulinie

14. April 1998

2P.13/1997 Schwyz; Pflichten des Notars; Staatshaftung

7. Dez. 1998

1P.190/2003 Pura; Hangrutsch

30. Juni 2003

1P.591/ 2003 Morcote; Steinschlag

16. Sept. 2004

1P.323/2004 Evolène; Lawine

16. Aug. 2004

4P.92/2004 Bern; Sitzwache Spital

19. Okt. 2004

4P.244/2005 Bern; Zumutbarkeit einer Sitzwache

6. Feb. 2006

6P.39/2006 Evolène; Lawine

30. Aug. 2006

6P.40/2006 Evolène; Lawine

30. Aug. 2006

Kantonale

Gerichte

Kanton Stichworte

Datum

Entscheid-Nr.

Bern

VGE 21240 Grotte de la Baume de Bournois; Sturz in Höhleneingang

18. Dezember 2002

VGE 21234U Hochwasser; Thun; Verantwortlichkeit Kanton

8. März 2004

VGE 21657U Hochwasser; Thun; Verantwortlichkeit Einwohnergemeinde

8. März 2004 
VGE 22214U Bern; Sitzwache

Wallis

1. StG-K VS Evolène; Lawine; Verantwortlichkeit; Evakuation; Sperrung

11. Januar 2006

\section{V5. Abkürzungen}

$\mathrm{AB}$

Amtliches Bulletin

Abs.

Absatz

aBV

alte Bundesverfassung der Schweizerischen Eidgenossenschaft

Anm. Anmerkung

ARE

Bundesamt für Raumentwicklung

Art.

Artikel

Aufl.

Auflage

BABS

Bundesamt für Bevölkerungsschutz

BAFU

Bundesamt für Umwelt

$\mathrm{BBI}$

Bundesblatt

BGE

Bundesgerichtsentscheid

bGS

bereinigte (systematische) Gesetzessammlung (Kanton Appenzell-Ausserrhoden)

BGS Bereinigte Gesetzessammlung (Kanton Zug)

BR

Bündner Rechtsbuch

BRP

ehemaliges Bundesamt für Raumplanung

BSG

Bernische Systematische Gesetzessammlung

bspw.

beispielsweise

Bst.

Buchstabe

BUWAL ehemaliges Bundesamt für Umwelt, Wald und Landschaft

BWG ehemaliges Bundesamt für Wasser und Geologie

BWW ehemaliges Bundesamt für Wasserwirtschaft

div. diverse

do. ebenso, in gleicher Weise

f., ff. folgende

GDB Gesetzesdatenbank (Kanton Obwalden)

GS Gesetzessammlung des Kantons Glarus

GS/VS Gesetzessammlung des Kantons Wallis

Hrsg. Herausgeber 
i. d. R. in der Regel

Kap. Kapitel

Kt. Kanton

Komm. Kommentar

lit. $\quad$ Litera $=$ Buchstabe

NFA Neugestaltung des Finanzausgleichs und der Aufgabenteilung

NG Nidwaldner Gesetzessammlung

o. ä. oder ähnlich

o. dgl. oder dergleichen

PLANAT Nationale Plattform Naturgefahren

Rz Randziffer

S. Seite $(n)$

SIA Schweizer Ingenieur und Architektenverband

SG Systematische Gesetzessammlung (Kanton Basel-Stadt)

sGS systematische Gesetzessammlung des Kantons St. Gallen

SGS Systematische Gesetzessammlung (Kanton Basel-Landschaft)

sog. sogenannt(e/er/es)

SR Systematische Sammlung des Bundesrechts

SRL Systematische Rechtssammlung des Kantons Luzern

SRSZ Systematische Gesetzsammlung (Kt. Schwyz)

u. a. unter anderem

u. Ä. und Ähnliche

u. dgl. und dergleichen

m./u. E. meines/unseres Erachtens

u. v. a. unter vielen anderen

UNO United Nations Organization, Organisation der Vereinten Nationen

u. U. unter Umständen

UREK-N Kommission für Umwelt, Raumplanung und Energie des Nationalrats

VE Verfassungsentwurf

vgl. vergleiche

vs. versus

WAP Waldprogramm Schweiz

ZBI Schweizerisches Zentralblatt für Staats- und Verwaltungsrecht (bis 1988 Schweiz. Zentralblatt für Staats- und Gemeindeverwaltung) 

Ziff. Ziffer
z. B. zum Beispiel

\section{V6. Abbildungsverzeichnis}

Abbildung 1 Das Risikokonzept (Darstellung des Departementes Umweltwissenschaften [D-UWIS] der ETHZ, Hollenstein, 1997) .................................. 2

Abbildung 2 Wirkungskette von Naturrisiken (Seiler, 1997; abgeändert). ........................3

Abbildung 3 Aufbau der Publikation, Nummerierung der Arbeitsschritte..........................6

Abbildung $4 \quad$ Kapitel 2 in der Übersicht.............................................................. 7

Abbildung 5 Auswirkungen eines Ereignisses und Schadensbegriff (nach Berg et al., 1994). Der operationelle Schadensbegriff bedingt Wissen (i) über die methodische Erfassbarkeit und (ii) über die negative Bewertung der Auswirkung.

Abbildung 6 Methode zur Abschätzung eines Schadens (Berg, 1994). Entscheidend ist die qualitative Bewertung als negative und relevante Auswirkung sowie die methodische Erfassbarkeit.

Abbildung 7 Monetäre Bewertung von Schäden. Als Ersatz für fehlende Marktpreise müssen alternative Bewertungen mit Schattenpreisen vorgenommen werden (Wilhelm, 2007).

Abbildung 8 Für die Bewertung von Umweltschäden ist vor allem die Dauer der Beeinträchtigung massgebend (BABS, 2003a).....

Abbildung 9 Schutzziele und ihre Einflussfaktoren nach Heinimann (2002)......

Abbildung 10 Subjektive Wahrnehmung und statistische Schätzung von Todesopfern pro Jahr weichen voneinander ab (Viscusi, 1995 gemäss Darstellung von Proske, 2004).

Abbildung 11 ,Farmer-Kurve' zur Abgrenzung zulässiger und unzulässiger Freisetzung von radioaktivem I ${ }^{-131}$ (Farmer, 1967).

Abbildung 12 Gefahrenstufen im Intensitäts-/Wahrscheinlichkeitsdiagramm für Naturprozesse (BWW, BUWAL, BRP, 1997).

Abbildung 13 Sterbequotient nach Alter (Bundesamt für Statistik, 2000). Tolerierbare Risiken sollen einen Anteil von wenigen Prozent des tiefsten Sterbequotienten nicht übersteigen.

Abbildung 14 Risikokategorien nach Grad der Freiwilligkeit und Möglichkeit der Einflussnahme. Tolerierbare individuelle Todesfallrisiken und Grenzkosten zur Verhinderung von Todesfällen stehen in einem umgekehrt proportionalen Verhältnis (Schneider, 1996).

Abbildung 15 Tolerierbare individuelle Todesfallrisiken mit natürlicher und technischer Ursache gemäss Angaben aus der Literatur. Für technische Risiken sind deutlich höhere Sicherheiten gefordert.

Abbildung 16 Tolerierbare individuelle Todesfallrisiken einzelner Objektkategorien gemäss Schutzzielmatrix (Borter, 1999) (vgl. Tabelle 21) im Vergleich zu tolerierbaren individuellen Todesfallrisiken aus der Literatur (Merz et al., 1995, schraffierter Bereich). Es werden vier Risikokategorien zwischen freiwillig eingegangenen, beeinflussbaren (Kategorie [1]) und unfreiwillig eingegangenen, unbeeinflussbaren (Kategorie [4]) Risiken unterschieden.. 46 
Abbildung 17 Die tolerierbaren Todesfallobjektrisiken für verschiedene Typen gefährlicher Anlagen zeigen Abweichungen bis zu Faktor $10^{2}$ (eigene

Berechnungen).

Abbildung 18 Entwicklung der tolerierbaren Todesfallobjektrisiken zwischen 1978 und 2000. In diesen 20 Jahren reduzierten sich die Sicherheitsstandards etwa um Faktor 10 (eigene Berechnungen).

Abbildung 19 Tolerierbare Todesfallobjektrisiken für verschiedene Risikoarten. Für die Naturgefahrengebiete ergeben sich etwa 10-mal tiefere Sicherheitsstandards (eigene Berechnungen).

Abbildung 20 Schutzziele als Grenzwerte für das tolerierbare individuelle Todesfallrisiko (Merz et. al, 1995). Die Grenzwerte für ,freiwillige' Risiken und ,unfreiwillige' Risiken unterscheiden sich um einen Faktor 1:1'000 bis 1:10'000 ...57

Abbildung 21 Zeitliche Entwicklung der Schutzzielkonzepte (Ball, 1999). Meist führten Unfallereignisse, oft aber auch bedeutende Studien zur Festlegung neuer Schutzziele (Societal Risk Criteria)......................................................58

Abbildung 22 F/N-Diagramm der schweizerischen Störfallverordnung (BUWAL, 1991)...59

Abbildung 23 Schutzziele für die Erdbebensicherheit bestehender Gebäude (Duvernay, 2005)

Abbildung 24 Grenzkosten zur Verhinderung eines Todesfalls [T] aus KATARISK (BABS, 2003).

Abbildung 25 Schutzzieldarstellung aus: Anleitungsentwurf zur Durchführung einer Risikoanalyse (BUWAL, Eidg. Forstdirektion. Beilage zu KREISSCHREIBEN. Nr. 20, 1994). Die Darstellung enthält keine eigentlichen Schutzziele, sondern Hinweise auf möglichen Handlungsbedarf.

Abbildung 26 Schutzzielmatrix Hochwasserschutz Uri (Anforderungen an den Hochwasserschutz. Bundesamt für Wasserwirtschaft, 1995).

Abbildung 27 Schutzzielmatrix für gravitative Naturgefahren (Kanton Glarus, 2001).

Abbildung 28 Grenzkosten und Grenzwerte als Schutzziele. Die ,Sicherheitskurve' stellt die Verbindungslinie zwischen den kostenwirksamsten Massnahmen (-kombinationen) für jeden Sicherheitsgrad dar (linke oberste Punkte im Diagramm). Gemäss dem Grenzwertkriterium werden Massnahmen ergriffen, bis der Grenzwert des tolerierbaren Risikos erreicht ist (P1). Nach dem Grenzkostenkriterium werden Massnahmen bis zu dem Punkt (P2) ergriffen, an dem die Steigung der ,Sicherheitskurve' der Vorgabe für Grenzkosten entspricht.

Abbildung 29 Gliederung der Schutzzieldarstellungen hinsichtlich ihrer Ausrichtung (wirkungs- oder effizienzorientiert) und ihrem Anwendungsbereich (Einzelobjekt oder Region). Regional einsetzbare und zugleich effizienzorientierte Schutzzieldarstellungen fehlen.

Abbildung 30 Finanzflüsse im Management von Naturrisiken der Schweiz (Zahlen 2004, in Mio. CHF). Etwas mehr als die Hälfte der Aufwendungen wird durch Private in Form von Versicherungsprämien sowie Beiträgen an Prävention, Regeneration und Intervention getragen (Planat, 2004).

Abbildung 31 Die Effizienz der Koordinationsform zwischen gesellschaftlichen Akteuren hängt von Transaktionskosten, der Faktorspezifität und der Unsicherheit ab (Williamson, 1975).

Abbildung 32 Ablauf des Managements von Naturrisiken. Eine Risikoanalyse auf regionaler Ebene (Stufe 1 nach Borter, 1999) zeigt Schutzdefizite (Handlungsbedarf) und Prioritäten auf. Aus diesem Handlungsbedarf, oft

Josef Th. Hess: Schutzziele im Umgang mit Naturrisiken in der Schweiz, @ vdf Hochschulverlag 2011 
aber auch aufgrund von Ereignissen und politischen Anstössen ergeben sich Projekte, die hinsichtlich wirtschaftlicher Kriterien und Erfüllung von Sicherheitsanforderungen zu prüfen sind.

Abbildung 33 Zusammenarbeit von Experten und Laien (Heinimann, 2002). ..................76

Abbildung 34 Einfluss-Betroffenheits-Matrix (Hostmann, 2005). Akteure beispielhaft dargestellt $($ ENHK $=$ Eidg. Natur- und Heimatschutzkommission, HWS = Hochwasserschutz)

Abbildung 35 Kapitel 3 in der Übersicht.

Abbildung 36 Unterstützungsbedürftigkeit gemäss Richtlinien für die Ausgestaltung und Bemessung der Sozialhilfe (SKOS, 2005, S. A. 6-3)

Abbildung 37 Risikokategorien (Schneider, 1984)

Abbildung 38

Gewinn und Verlust in Abhängigkeit vom Referenzpunkt (Kahnemann und Tversky, 1979).

Abbildung 39 Elemente zur Entwicklung eines risikobasierten Managementkonzeptes

für Naturrisiken.

Abbildung 40 Zusammenhänge zwischen Allokation, Distribution gemäss Handlungsregeln des Managementkonzeptes. Das Konzept geht davon aus, dass Mittel zur Reduktion untolerierbarer individueller Risiken (Grundsicherheitslücken) zwingend und Mittel zur weiteren Reduktion kollektiver Risiken (,ALARP'-Lücken) bedingt bereitzustellen sind.

Abbildung 41 Kapitel 4 und 5 in der Übersicht.

Abbildung 42

Handlungsbereiche nach dem Zwei-Regel-Ansatz (Beispiel Todesfallrisiken).

Abbildung 43 Ablauf gemäss schutzzielbasiertem Managementkonzept. Der ,ZweiRegel-Ansatz' wird auf Ebene Region und auf Ebene Einzelprojekt angewendet.

Abbildung 44 Ermittlung eines Prioritätsindexes aufgrund der Schutzzielprinzipien und auf Basis von Schadenspotenzial- und Gefahrenkarten

Abbildung 45 Vereinfachte Abschätzung des Handlungsbedarfs anhand Gefahrenkarte, Objektkategorien und Massnahmeneffizienz (weisse Felder: keine Massnahmen).

Abbildung 46 Koordination der Verantwortlichkeiten und Finanzflüsse im Management von Naturrisiken durch eine Risikomanagementagentur.

Abbildung 47 Das Engelberger Hochtal beim Hochwasser im August 2005 (Amt für Wald und Raumentwicklung $\mathrm{OW}$ ).

Abbildung 48 Gemeinden des Kantons Obwalden und der Sarnersee mit den wichtigsten Zu- und Abflüssen.

Abbildung 49 Objektindex in Abhängigkeit der risikobetroffenen Person. 146

Abbildung 50

Schutzdefizitkarte nach ,klassischer' Schutzzielmatrix ,Borter (1999).

Abbildung 51

Schutzdefizitkarte nach modifizierter Schutzzielmatrix ,Benutzer'

Abbildung 52

Vergleich aller Gefahrenquellen und Prioritäten nach Verfahren (SD = Schutzdefizit).

Abbildung 53 Fallbeispiel Sarneraa, Gewichtung der Zielgruppen durch ,Akteure' und ,Experten'. 


\section{V7. Tabellenverzeichnis}

Tabelle $1 \quad$ Verwendete Indikatoren in ausgewählten Berichten über Schadens-

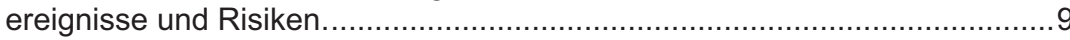

Tabelle 2 Bewertung einer Beeinträchtigung verletzbarer Werte als Schaden. ..........13

Tabelle 3 Beurteilung der Allgemeingültigkeit verschiedener Arten von Schäden. ......14

Tabelle 4 Rechtsgüter in ausgewählten Bundesgesetzen (Seiler 1997, abgeändert).. 16

Tabelle 5 Die Gegenüberstellung der Konzepte ,verletzbare Werte' und ,Rechtsgüter' zeigt eine weitgehende Deckung.

Tabelle 6 Strafmass für Rechtsgutverletzungen gemäss Strafbestimmungen in ausgewählten Spezialgesetzen und dem Strafgesetzbuch (StGB).

Tabelle 7 Indikatoren und Messgrössen zur Darstellung der Beein-

trächtigung verletzbarer Werte.

Tabelle 8

Beurteilung einiger Indikatoren nach Eignungskriterien.

Tabelle 9

Tabelle 10

Der Störwert als Metrik für den Vergleich verschiedener Arten von Schäden (BUWAL, 1991).

Der Indikator Geldeinheit eignet sich, mit Einschränkungen,

zur Beurteilung verschiedener Kategorien verletzbarer Werte.

Tabelle 11

Arten von Schäden und Bewertungsmethoden (Wilhelm, 2007)

Tabelle 12

Betriebliche Folgeschäden von Naturereignissen sind im Vergleich zu den direkten Schäden von sehr unterschiedlicher Bedeutung.

Tabelle 13 Die hochgerechneten finanziellen Folgen des Lawinenwinters 1999 und des Orkans Lothar 1999 für die Bergbahn- und Skiliftunternehmen der Schweiz (ohne Tessin).

Tabelle 14

Übersicht über die verwendeten Begriffe zur Bewertung von Risiken.

Tabelle 15

Die Art des Risikos beeinflusst die Wahrnehmung und Bewertung entscheidend: Risikokonversionsfaktoren (RCF, Litai et al., 1983).

Tabelle 16

Intensitäten gefährlicher Prozesse (Heinimann et al., 1998)

Tabelle 17

Widerspruchslos hingenommene mittlere individuelle Todesfallrisiken

(Schneider, 1996).

Tabelle 18 Literaturangaben zur Zahlungsbereitschaft (Grenzkosten/

Vermeidungskosten) für die Rettung eines Menschenlebens

Tabelle 19 ,Policy-Factor' zur Klassierung von Risikokategorien (Vrijling, 1998).

Freiwilligkeit und direkter Nutzen beeinflussen die Höhe des tolerierbaren

Risikos.

Tabelle 20 Grenzwerte für tolerierbare individuelle Todesfallwahrscheinlichkeiten.

Tabelle 21

Tabelle 22

Tolerierbares individuelles Risiko gemäss Schutzzielmatrix nach Borter (1999) im Vergleich zu den tolerierbaren individuellen Todesfallrisiken nach PLANAT (2004).

Grenzkosten zur Verhinderung von Todesfällen.

Tabelle 23

Grenzkosten zur Verhinderung verschiedener Arten von Schäden aus KATARISK (BABS, 2003a).

Tabelle 24 Eckwerte von Akzeptanzlinien in F/N-Diagrammen: Die Akzeptanzlinien in bekannten F/N-Diagrammen (Ball, 1999; BUWAL, 1991) zeigen

bedeutende Unterschiede in Lage und Neigung.... 
Tabelle 25 Berechnung tolerierbarer kollektiver Risiken pro Gefahrenquelle für Naturgefahren in Engelberg (detaillierte Resultate Anhang V).

Tabelle 26 Zielgrössen für maximale Wahrscheinlichkeiten einer Nichteinhaltung der Gebrauchstauglichkeit oder einer Überschreitung der Tragsicherheit (Wahrscheinlichkeit pro Jahr; JCSS, 2001).

Tabelle 27 Zielwerte der International Nuclear Safety Advisory Group (INSAG, 2006). 56

Tabelle 28 Schutzziele für individuelle und kollektive Todesfall- und Sachrisiken (PLANAT, 2004b). 62

Tabelle 29

Ebenen des Risikomanagements. .64

Tabelle 30

Akteure und typische Aufgaben im Management von Naturrisiken. .68

Tabelle 31 Heutige Aufgabenteilung Bund, Kanton, Gemeinde. .70

Tabelle 32

Tabelle 33 Elemente des Vierjahresprogramms der Programmvereinbarung ,Schutzbauten' (BAFU, 2007a).

Tabelle 34

Tabelle 35

Handlungsformen des schweizerischen Verwaltungsrechts (Richli, 2004a). 75 Klassifizierung der Akteure (eigene Darstellung in Anlehnung an Hostmann et al., 2005).

Themen und Formen des Einbezugs von Akteuren (eigene Darstellung in Anlehnung an Hostmann et al., 2005).

Tabelle 36 Kriterien

Tabelle 37

Erkennt

Tabelle 38

Erkenntnisse, Lücken und Handlungsbedarf zu tolerierbaren Effekten und Grenzwerten.

Tabelle 39

Erkenntnisse, Lücken und Handlungsbedarf zu Schutzzielen

Tabelle 40

Erkenntnisse, Lücken und Handlungsbedarf zur Operabilität.

Tabelle 41

Tabelle 42 Das Dreistufenkonzept im technischen Sicherheitsrecht (Seiler, 1997)

Erkenntnisse, Lücken und Handlungsbedarf zu Organisation und Verfahren bei der Festlegung von Schutzzielen im Management von Naturrisiken. .....84 unterscheidet drei Bereiche der Pflicht zur Ergreifung von Sicherheitsmassnahmen: einen Bereich mit absoluter Handlungspflicht, einen Bereich mit Pflicht zu verhältnismässigen Handlungen und einen Bereich ohne Handlungspflicht.

Tabelle 43 Typische Sorgfaltspflichten und Massnahmen im Umgang mit naturgefahrenbedingten Risikosituationen (nach Bütler, 2006).

Tabelle 44 Geschützte Rechtsgüter und deren Zuordnung zu einer Bedürfnisstufe nach Maslow.

Tabelle 45

Gerechtigkeitsprinzipien in Schutzzielkonzepten der Praxis. 105

Tabelle $46 \quad$ Kernelemente eines schutzzielbasierten Konzeptes für das Management von Naturrisiken: Risikomanagementebenen und anzuwendende Schutzzielkonzepte.

Tabelle 47 Berücksichtigung der Schutzzielprinzipien in den Regeln des schutzzielbasierten Managementkonzeptes.

Tabelle 48 Schutzziele zum maximal tolerierbaren individuellen Risiko.

Tabelle 49 Jährliche maximal tolerierbare Sachrisiken pro Are, berechnet aufgrund von Annahmen zu typischen Eigentumsverhältnissen (Ausgangslage:

Werte Kanton OW).

Josef Th. Hess: Schutzziele im Umgang mit Naturrisiken in der Schweiz, @ vdf Hochschulverlag 2011 
Tabelle $50 \quad$ Kriterien zur Bewertung von gefährdeten Objekten und von Risikoverminderungsmassnahmen. 118

Tabelle $51 \quad$ Kriterien und Fragen zur Bewertung von gefährdeten Objekten

Tabelle 52

Kriterien und Fragen zur Bewertung von Risikoverminderungsmassnahmen.

Tabelle 53 Charakterisierung von Gefahrengebieten nach gefahrenspezifischen und örtlichen Merkmalen.

Tabelle 54 Optimale Massnahme in Abhängigkeit von Merkmalen des

Gefahrenprozesses und -gebietes sowie der Anordnung des

Schadenspotenzials.

Tabelle 55 Risikobasierte Gefährdungsfaktoren pro Gefahrenprozess und

Gefahrenstufe.

Tabelle $56 \quad$ Unterschiede in der Zuordnung von Objekten zu Objektkategorien

zwischen der ,klassischen' Schutzzielmatrix (Borter, 1999) und der

Rangierung nach Objektindex.

Tabelle 57 Transaktionskosten im Management von Naturrisiken.

Tabelle 58 Kriterien und Aspekte zur Beurteilung von Koordinationsmöglichkeiten im Management von Naturrisiken.

Tabelle $59 \quad$ Vorschlag einer risikomanagementorientierten Aufgabenteilung zwischen Bund, Kantonen und Gemeinden.

Tabelle $60 \quad$ Mitwirkung in den Phasen des Vorschlags zu einem schutzzielbasierten Risikomanagement (eigene Darstellung aufgrund der Ablauforganisation grösserer Integralprojekte im Kanton OW).

Tabelle 61 Auszug aus dem Masterplan ,Sicherheit vor Naturgefahren'

für die Gemeinden Engelberg und Sarnen.

Tabelle 62 Prioritäten von Massnahmen zur Risikoverminderung gemäss Regel 1, ohne Umrechnung auf einheitliche Flächengrösse.

Tabelle 63 Prioritäten von Massnahmen zur Risikoverminderung gemäss Regel 1, umgerechnet auf ein einheitliches Flächenmass.

Tabelle 64 Prioritäten von Massnahmen zur Risikoverminderung gemäss Regel 2. ...144

Tabelle 65 Prioritäten von Massnahmen zur Risikoverminderung gemäss vereinfachter Abschätzung des Handlungsbedarfs.

Tabelle 66 Prioritäten von Massnahmen zur Risikoverminderung gemäss vereinfachter flächenbereinigter Abschätzung des Handlungsbedarfs.

Tabelle 67 Prioritäten von Risikoverminderungsmassnahmen aufgrund von Schutzdefiziten umgerechnet auf ein einheitliches Flächenmass (GP = Gefahrenprozess, L = Lawine, $\mathrm{S}=$ Sturzprozess, $\mathrm{W}=$ Wassergefahr).

Tabelle 68 Prioritäten von Risikoverminderungsmassnahmen aufgrund von Schutzdefiziten pro Flächeneinheit (GP = Gefahrenprozess, L = Lawine, $\mathrm{S}=$ Sturzprozess, $\mathrm{W}=$ Wassergefahr, $\mathrm{SD}=$ Schutzdefizit).

Tabelle 69 Vergleichende Zusammenstellung der Varianten zur Verbesserung der Hochwassersicherheit Sarneraatal (Vorstudie).

Tabelle $70 \quad$ Ergebnis der ersten Nutzwertanalyse Vorprojekt Sarneraatal

(V1 = Variante Tieferlegung/Verbreiterung; V2 = Variante Entlastungs-

stollen Landenberg; V3 = Variante Entlastungsstollen Bergvaiante Ost). ..156 
Tabelle $71 \quad$ Ergebnis der zweiten Nutzwertanalyse Vorprojekt Sarneraatal. 156

Tabelle 72 An der Stellungnahme beteiligte Bundesstellen und deren Position. 157 


\section{Anhänge}

\section{Inhaltsverzeichnis}

I Begriffe 199

$\begin{array}{lll}\text { I.I } & \text { Technische Risikobegriffe } & 199\end{array}$

I.II Schutzziele, Sicherheit und Restrisiko $\quad 200$

I.III Risikorelevante Begriffe im Recht 200

II Risikoberechnung - Aggregierung von Szenarien zum Gesamtrisiko 203

III Objektkategorien und Risikokategorien 205

IV Tolerierbare individuelle Todesfallrisiken; Vergleich zwischen Schutzzielen und Grenzwerten

IV.I Berechnete Schutzziele (Matrix) und Grenzwert (1) 206

IV.II Berechnete Schutzziele (Matrix) und Grenzwert (2) 207

IV.III Berechnete Schutzziele (Matrix) und Grenzwert (3) 208

IV.IV Berechnete Schutzziele (Matrix) und Grenzwert (4) 209

V Tolerierbare Risiken pro Gefahrenquelle (Fallbeispiel Engelberg) 210

$\begin{array}{lll}\text { VI } & \text { Schutzzielrelevante Rechtsnormen } & 211\end{array}$

VI.I Schutzzielrelevante Regelungen in der Verfassung

VI.I.I Überblick 211

VI.I.II Zweckartikel der Bundesverfassung 212

$\begin{array}{ll}\text { VI.I.III Rechtsstaatsprinzipien } & 213\end{array}$

VI.I.IV Individuelle und gesellschaftliche Verantwortung (Art. 6 BV, SR 101) 214

VI.I.V Grundrechte (Art. 7 - 36 BV, SR 101) 215

VI.I.VI Sozialziele (Art. 41 BV, SR 101) 217

VI.I.VII Aufgaben und Zuständigkeiten 218

VI.II Regelungen in Bundesgesetzen und Verordnungen 219

VI.II.I Sicherheit vor Naturgefahren als öffentliche Aufgabe 219

VI.II.II Umfang der Verantwortlichkeit der Allgemeinheit 223

VI.III Ausführungsbestimmungen, Richtlinien, Weisungen, Empfehlungen auf Bundesebene $\quad 224$

VI.IV Einschlägige Bestimmungen kantonaler Gesetze 225

VI.IV.I Verantwortlichkeit der Allgemeinheit oder Eigenverantwortung der

Betroffenen? 225

VI.IV.II Umfang der Verantwortlichkeit der Allgemeinheit 229

VI.IV.IIISchutzziele in den Kantonen $\quad 232$

VII Schutzzielrelevante Gerichtsurteile $\quad 233$

VII.I Subjektive Voraussetzungen einer Handlungspflicht 233

VII.I.I Handlungen des Staates bei der Erfüllung öffentlicher Aufgaben 233

VII.I.II Handlungspflicht nach privatrechtlichen Normen 235

VII.II Objektive Voraussetzungen einer Handlungspflicht 236

Josef Th. Hess: Schutzziele im Umgang mit Naturrisiken in der Schweiz, @ vdf Hochschulverlag 2011 
VII.III Anforderungen an den Handlungspflichtigen 241

VII.IV Sorgfaltspflicht der risikobetroffenen Person $\quad 242$

VII.V Adäquater Kausalzusammenhang 243

VIII Beurteilung von Schutzzieldarstellungen 244

VIII.I Nachvollziehbarkeit und Vergleichbarkeit 244

VIII.II Gewährleistung individueller Sicherheit und Wirtschaftlichkeit 245

VIII.III Anforderungen an Informationsgrundlagen 246

VIII.IV Einsatzmöglichkeit für regionales Risikomanagement 247

$\begin{array}{lll}\text { IX } & \text { Systematik der Schutzzielprinzipien } & 248\end{array}$

$\begin{array}{lll}X & \text { Objektkategorien und Schutzzielprinzipien } & 249\end{array}$

XI Massnahmen und Schutzzielprinzipien (Vernetzung) 250

XII Massnahmen und Schutzzielprinzipien 251

XII.I Massnahmeneignung (Eignungskriterien) 251

XII.II Massnahmeneignung (Eignungskriterien) 252

XII.III Massnahmeneignung (Eignungskriterien) 253

XIII Massnahmenindex $\quad 254$

XIII.I Beispiel: Häufige Ereignisse, kleine bis mittlere Einzugsgebiete 254

XIII.II Übersicht 255

XIV Modifizierte Schutzzielmatrix 256

XIV.I Version ,Benutzer' 256

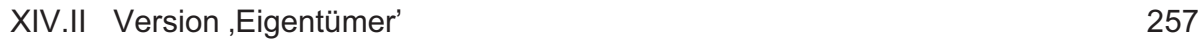

XIV.III Version, individuelles Risiko' 258

XIV.IV Vergleich der Versionen 259

$\begin{array}{lll}\text { XV } & \text { Fallbeispiel Engelberg } & 260\end{array}$

XV.I Projektpriorisierung Managementkonzept nach Gefahrenprozessen 260

XV.II Projektpriorisierung Managementkonzept rangiert 261

$\begin{array}{lll}\text { XVI } & \text { Fallbeispiel Sarnenraa, Bewertung Projektvarianten } & 262\end{array}$

XVI.I Bewertung und Gewichtung durch Experten 262

XVI.II Bewertung durch Experten, Gewichtung durch Akteure 263 


\section{Begriffe}

\section{I.I Technische Risikobegriffe}

\section{Risiko}

Ein Risiko (von arabisch ,rizq', der von Gottes Gnade oder Geschick abhängige Lebensunterhalt) ist die kalkulierte Prognose eines möglichen Schadens bzw. Verlustes im negativen Fall (Gefahr) oder eines möglichen Nutzens bzw. Gewinns im positiven Fall (Chance) (Wikipedia, 2006). Im Umgang mit Naturrisiken ist der Risikobegriff ausschliesslich negativ besetzt und umschreibt die Möglichkeit, dass aus einem Zustand, Umstand oder Vorgang ein Schaden entstehen kann (Loat, 2003).

Ein Risiko ist die Wahrscheinlichkeit des Eintretens eines negativ bewerteten Ereignisses (mathematisch) oder die Wahrscheinlichkeit des Eintretens eines negativen Ereignisses multipliziert mit dem finanziellen Ausmass. Das Gegenwort zu ,Risiko' ist ,Sicherheit' (Wikipedia, 2006).

\section{Individuelles und kollektives Risiko}

Es wird zwischen dem individuellen Risiko und dem kollektiven Risiko unterschieden. Beim individuellen Risiko wird das Risiko aus der Sicht eines (bestimmten) Individuums beurteilt. Als kollektives Risiko wird ein Risiko bezeichnet, das aus der Sicht eines Kollektivs beurteilt wird (Plattner, 2005).

\section{Effektives Risiko}

Das effektive Risiko entspricht dem Produkt aus empirisch ermittelbaren Auswirkungen im Falle eines Ereigniseintritts und empirisch ermittelter Eintretenswahrscheinlichkeit des Ereignisses (Plattner, 2005).

\section{Wahrgenommenes Risiko}

Das wahrgenommene Risiko kann als subjektive Aufnahme des Risikos mit einer subjektiven Verarbeitung und Bewertung von risikobezogenen Informationen definiert werden (NDK, 2001). Das wahrgenommene Risiko beinhaltet somit eine systematische Abweichung vom rational-quantitativen Risikomass (effektives Risiko). In der Ingenieurwissenschaft wird das Risiko gemäss der sogenannten ,Versicherungsformel' als Produkt von Schadenshäufigkeit und Schadensausmass umschrieben:

$$
R(\text { Risiko })=h_{s}(\text { Schadenshäufigkeit) } * S \text { (Schadensausmass) }
$$

Allgemeiner wird Risiko auch als Produkt aus Gefahr und Schadenspotenzial ausgedrückt. Naturrisiken sind Risiken, die im Zusammenhang mit Naturgefahren stehen (Loat, 2003).

\section{Schadenspotenzial, Schaden und Gefahr}

Der Begriff des Schadenspotenzials bezeichnet die Grösse des möglichen Schadens im betrachteten Gefahrengebiet (Loat, 2003).

Unter Schaden wird die negativ bewertete, idealiter zu vermeidende Folge eines Ereignisses verstanden (Loat, 2003; Seiler, 1997).

Der Begriff der Gefahr bezeichnet einen Zustand, Umstand oder Vorgang, aus dem ein Schaden für Mensch, Umwelt oder Sachgüter entstehen kann. 


\section{Naturgefahr}

Als Naturgefahren gelten sämtliche Vorgänge in der Natur, die für Mensch, Umwelt und Sachgüter schädlich sein können (Loat, 2003).

Üblicherweise werden folgende Arten von Gefahren in Betracht gezogen (PLANAT, 2004b):

Hydrologische/meteorologische Naturgefahren

Hochwasser: Überschwemmung, Ufererosion, Übermurung

Unwetter: Gewitter, Hagel, Wintersturm, tropischer Wirbelsturm

Lawine (Schnee- und Eislawine)

Kältewelle

Trockenheit, Hitzewelle, Waldbrand.

Geologische Naturgefahren

Massenbewegungen: Rutschung, Stein-/Blockschlag, Felssturz, Bergsturz, Hangmure

Erdbeben

Vulkanismus

Tsunami.

\section{I.II Schutzziele, Sicherheit und Restrisiko}

Schutzziele beschreiben das Mass der Sicherheit, das mit Schutzmassnahmen erreicht werden soll (Loat, 2003). Sicherheit gilt als Zustand, für den das verbleibende Risiko als akzeptabel eingestuft wird (Eurodicautom, 2004). In der Literatur werden Schutzziele häufig mit tolerierbaren Risiken gleichgesetzt.

Als Restrisiko gilt das Risiko, das nach der Realisierung aller vorgesehenen Schutzmassnahmen noch verbleibt. Nach Heinimann (Heinimann, 1998, zit. nach Schneider, 1994) setzt sich das Restrisiko aus bewusst akzeptiertem Risiko und Risiko aus menschlichen Fehlhandlungen zusammen. Letztere ergeben sich aus

dem objektiven Nichterkennen von Risiken,

dem subjektiven Nichterkennen objektiv bekannter Risiken,

der Vernachlässung von Risiken,

der Anwendung ungeeigneter Massnahmen,

der falschen Anwendung geeigneter Massnahmen.

\section{I.III Risikorelevante Begriffe im Recht}

Unter Risiko im (sicherheits-)rechtlichen Sinne (z. B. Art. 10 USG, SR 814.01) wird „die Möglichkeit der schweren Schädigung verstanden, die bei einem ausserordentlichen Ereignis von einer Anlage ausgehen kann" (Trösch, 1992). Wesentlich ist, dass sich diese Möglichkeit nicht zu einer Gefahr im Rechtssinne verdichtet.

In der Rechtslehre bedeutend ist der Begriff des erlaubten Risikos (Wikipedia, 2006). Ein erlaubtes Risiko ist dann gegeben, wenn eine Gefahr geschaffen wird, diese aber von der Rechtsordnung hingenommen wird, um ein gedeihliches menschliches Zusammenleben überhaupt erst möglich zu machen.

Der (wirkliche oder vermeintliche) Nutzen einer Tätigkeit kann es rechtfertigen, ein gewisses Risiko in Kauf zu nehmen, wenn diese Tätigkeit sonst gar nicht oder nur mit unverhältnismässig hohen materiellen oder anderen Aufwendungen möglich wäre. Die Nutzen-RisikoAbwägung ist dabei im Einzelnen eine schwierige und für jeden Fall neu zu entscheidende Frage (Kaiseraugst, Betäubungsmittel, BGE 117 IV 58 E. 2b; Basel-Landschaft, Abgabe Drogen, BGE 120 IV 334).

Josef Th. Hess: Schutzziele im Umgang mit Naturrisiken in der Schweiz, @ vdf Hochschulverlag 2011 
Die Strafrechtsdogmatik kennt den Begriff des erlaubten Risikos, um Sachverhalte zu beschreiben, die zwar zu einer Schädigung führen, aber trotzdem nicht strafbar sind (Seiler, 1997, S. 117). Die Rechtfertigung kann sich ergeben

- aus einer gesetzlichen Erlaubnis;

- $\quad$ aus der Üblichkeit und Normalität eines bestimmten Verhaltens, der sogenannten Sozialadäquanz.

Eine rechtliche Umschreibung des Restrisikos findet sich im sogenannte Kalkar-Entscheid des Deutschen Bundesverfassungsgerichtes (BVerfGE 49, 89 (143)), wonach gemäss ,praktischer Vernunft' Risiken unterhalb der Schwelle des erlaubten Restrisikos durch Grenzen des menschlichen Erkenntnisvermögens verursacht und daher als sozial-adäquate Last von allen Bürgern zu tragen seien (Seiler, 1996, S. 159ff).

Gefahr als juristischer Terminus technicus bezeichnet eine Sachlage, die bei ungehindertem Ablauf zu einem Schaden führt (Seiler, 1997, S. 44f.), oder die erhöhte Möglichkeit der Schädigung bzw. Verletzung von Rechtsgütern (Nef, 1987, S. 41). Im Polizeirecht bezeichnet Gefahr diejenigen Situationen, welche ein polizeiliches Eingreifen rechtfertigen oder verlangen. Im Zivilrecht gelten diejenigen Situationen als Gefahr, welche einen präventiven Abwehranspruch begründen können. Im Haftpflichtrecht ergibt sich aus dem Gefahrenbegriff das Mass für die verlangte Sorgfaltspflicht. Analoges gilt für das Strafrecht, wobei hier zwischen konkreter und abstrakter Gefährdung unterschieden wird (Reinhard, 1993, S. 110 f.). Die konkrete Gefahr meint eine in der Lebenswirklichkeit vorliegende Sachlage im Einzelfall. Massnahmen der Gefahrenabwehr richten sich immer gegen konkrete Gefahren. Als abstrakte Gefahr wird eine Sachlage bezeichnet, aus der nach allgemeiner Lebenserfahrung in einer Grosszahl der Fälle eine konkrete Gefahr für ein polizeiliches Schutzgut entsteht.

Als rechtlich relevante Gefahr (Egli, 2002, S. 299; Reinhard, 1993, S. 105 f.) gilt eine Sachlage, die bei ungehindertem Ablauf des zu erwartenden Geschehens mit hinreichender Wahrscheinlichkeit zu einem Schaden der öffentlichen Sicherheit und Ordnung führt (polizeirechtlicher Gefahrenbegriff).

Für die hinreichende Wahrscheinlichkeit sind Eintretenswahrscheinlichkeit, voraussichtliches Schadensausmass und Art des bedrohten polizeilichen Schutzgutes massgebend. Hinreichende Wahrscheinlichkeit bedeutet einerseits, dass der Schaden nicht mit Gewissheit eintreten muss (Reinhard, 1993, S. 107 f.). Anderseits genügt nicht jede entfernte Möglichkeit eines Schadens. Je grösser der mögliche Schaden ist und je hochwertiger die gefährdeten Rechtsgüter sind, desto geringere Anforderungen sind an die Wahrscheinlichkeit zu stellen (Variabilität der Wahrscheinlichkeit). Ferner ist für das Erfordernis einer hinreichenden Wahrscheinlichkeit mit einem Schadenseintritt in absehbarer Zeit zu rechnen. Der polizeilichen Gefahr gleichgestellt ist die Anscheinsgefahr (Reinhard, 1993, S. 108 f.) Der Begriff bezeichnet eine Sachlage, welche bei verständiger Würdigung aller erkennbaren Umstände und hinreichender Sachverhaltsaufklärung als gefährlich erschien, obwohl diese Annahme im Nachhinein widerlegt wird.

Zur Begrifflichkeit von Gefahr, Gefahrenverdacht/Gefahrenvorsorge und Sicherheit unterscheidet Seiler (Seiler, 1997, S. 153-156): 
Tabelle 1 Rechtsbegriffe zu Gefahr, Risiko und Sicherheit (Seiler, 1997).

\begin{tabular}{|c|c|c|}
\hline Bezeichnung & Charakterisierung & Rechtsfolge \\
\hline Gefahr & $\begin{array}{l}\text { Relevant erhöhte } \\
\text { Wahrscheinlichkeit eines } \\
\text { Schadens }\end{array}$ & $\begin{array}{l}\text { Gefahrenabwehr (absolut, } \\
\text { zwingend) }\end{array}$ \\
\hline Gefahrenverdacht & $\begin{array}{l}\text { Schädigung ungewiss oder } \\
\text { Wahrscheinlichkeit klein }\end{array}$ & $\begin{array}{l}\text { Gefahren-/Risikovorsorge } \\
\text { (im Rahmen des technisch } \\
\text { Möglichen und wirtschaftlich } \\
\text { Zumutbaren) }\end{array}$ \\
\hline Sicherheit & $\begin{array}{l}\text { Hinreichend kleine } \\
\text { Wahrscheinlichkeit einer } \\
\text { Schädigung }\end{array}$ & $\begin{array}{l}\text { erlaubtes Risiko, keine } \\
\text { zusätzlichen } \\
\text { Sicherheitsmassnahmen } \\
\text { erforderlich }\end{array}$ \\
\hline
\end{tabular}

Sicherheit ist nicht als reziproker Begriff zum Gefahrenbegriff der polizeilichen Generalklausel, sondern als reziproker Begriff zum ,unerlaubten Risiko' zu verstehen (Seiler, 1996, S. 150). Sicherheit muss im doppelten Sinn vorliegen, damit überhaupt von Sicherheit im Rechtssinne gesprochen werden kann: Das Risiko einer Rechtsgutverletzung muss klein und das Wissen darum, dass das Risiko klein ist, muss hinreichend gross und zuverlässig sein (Seiler, 1996).

Als Schaden im rechtlichen Sinn wird die Verletzung eines Rechtsgutes bezeichnet (Seiler, 1997). Ein Schaden ist eine Verletzung (erhebliche Beeinträchtigung) eines Schutzgutes der öffentlichen Sicherheit und Ordnung (Reinhard, 1993). Bei den relevanten Schutzgütern, deren Verletzung einen Schaden darstellen kann, handelt es sich um ,Leib und Leben' von Menschen und allenfalls weitere traditionelle Polizeigüter (Seiler, 1997). Dazuzurechnen sind auch Umweltgüter und soziale Werte (z. B. wirtschaftliche, soziale und politische Strukturen). Im Privatrecht wird der Schaden auch als Vermögensverminderung oder als entgangener Gewinn umschrieben. Eine Störung liegt vor, wenn ein Schaden für die öffentliche Sicherheit und Ordnung bereits eingetreten ist.

Vor allem im Umweltrecht/Störfallrecht hat der Begriff der schweren Schädigung zentrale Bedeutung. Der Begriff ist nicht klar umrissen (Stähelin, 1993). Wesentliche Elemente zur Bestimmung der Schwere sind (Stähelin, 1993):

- die Breite (Flächenwirkung) und Nachhaltigkeit der Schädigung,

- die Wirkung inner- oder ausserhalb des Betriebsareals und

- $\quad$ die Möglichkeit der Bewältigung durch die (ordentlichen) Kräfte.

Die zentrale Wertung wird der Exekutive überlassen (Stähelin, 1993), was aus demokratischen und rechtsstaatlichen Überlegungen nicht unproblematisch ist. Diese Probleme sind durch Verfahren zu kompensieren (Stähelin, 1993).

Als Katastrophe wird ein Unglücksfall grossen aussergewöhnlichen Ausmasses verstanden (Seiler, 2001), wobei oft zusätzlich das Element der Plötzlichkeit genannt wird. Im Sinne der Ereignisbewältigung gilt als Katastrophe ein Ereignis, bei dem die ordentlichen Schutz- und Rettungskräfte überfordert sind und zusätzliche Unterstützung benötigen. Als höhere Gewalt gelten aussergewöhnliche Naturereignisse, welche mit unabwendbarer Gewalt und in unvorhersehbarer Weise hereinbrechen (BGE 111 II 429 ff., 433 f.). 


\section{Risikoberechnung - Aggregierung von Szenarien zum Gesamtrisiko}

Bei der Berechnung von Risiken ist zu berücksichtigen, dass sich das Gesamtrisiko zumeist aus einer Summe von Risiken einzelner Szenarien ergibt. Für die Kumulation dieser beliebigen Zahl von Risikoszenarien zum Gesamtrisiko eines Systems sind in der Analyse von Naturrisiken verschiedene Verfahren gebräuchlich. Gemeinsam ist diesen Verfahren, dass bestimmte Szenarien stellvertretend ermittelt werden. Im Bereich von Naturrisiken sind - basierend auf der Praxis der Gefahrenkartierung - 30-jährliche, 100-jährliche, 300-jährliche und EHQ-Szenarien gebräuchlich. Unterschiede bestehen bei der Aggregierung dieser Szenarien zum Gesamtrisiko. Dies kann mittels einer sog. Risikomatrix erfolgen (Merz, 1995; Borter, 1999). Dieses bei technischen Risiken häufige Verfahren setzt voraus, dass sich das Risikoprofil (z. B. in einer Ereignisbaumanalyse) in diskrete Einzelszenarien aufgliedern lässt, wobei die aufsummierte Wahrscheinlichkeit aller ermittelten Szenarien 1 beträgt.

Bei der Beurteilung von Naturrisiken sind typischerweise nebst den ermittelten stellvertretenden Szenarien (z. B. 30-, 100-, 300-jährliches Ereignis) unendlich viele weitere Szenarien denkbar. In diesen Fällen ist die Aggregierung über ein Wahrscheinlichkeits-AusmassDiagramm (F/N-Diagramm) zweckmässig (Kapitel 2.7.4.). Für F/N-Diagramme sind Darstellungen mit linearer oder mit doppelt logarithmischer Achsenskalierung gebräuchlich. Zur Ermittlung des Gesamtrisikos muss die ,Risikokurve' zwischen den stellvertretenden Szenarien (Punkte mit ermittelter Wahrscheinlichkeit und Schadensausmass im F/N-Diagramm) interpoliert werden. Die Ermittlung des Gesamtrisikos erfolgt durch Berechnung der Fläche (des Integrals) unter der Schadenskurve.

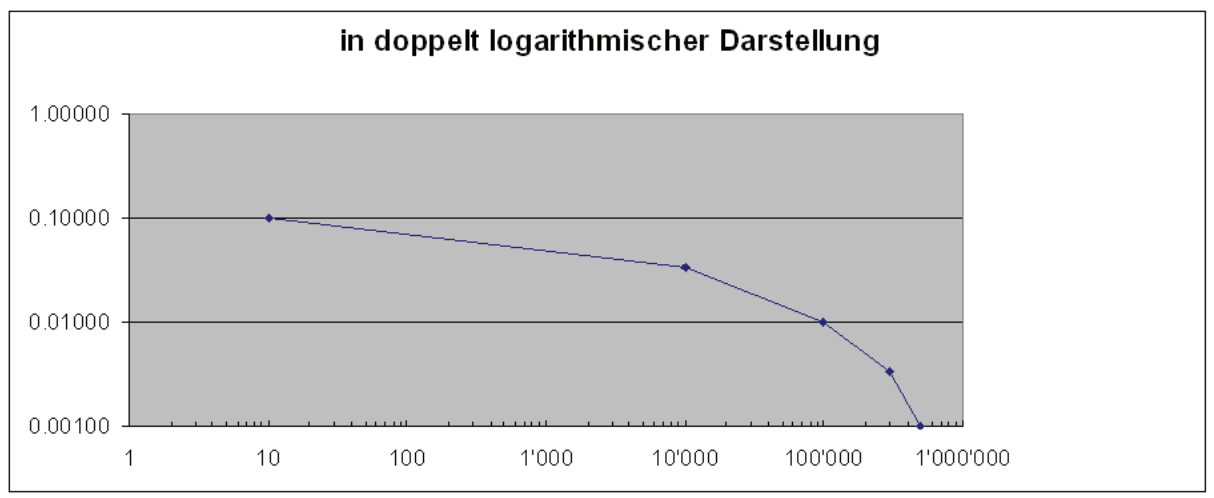

Abbildung 1 Schadenskurve mit doppelt logarithmischer Skalierung. 


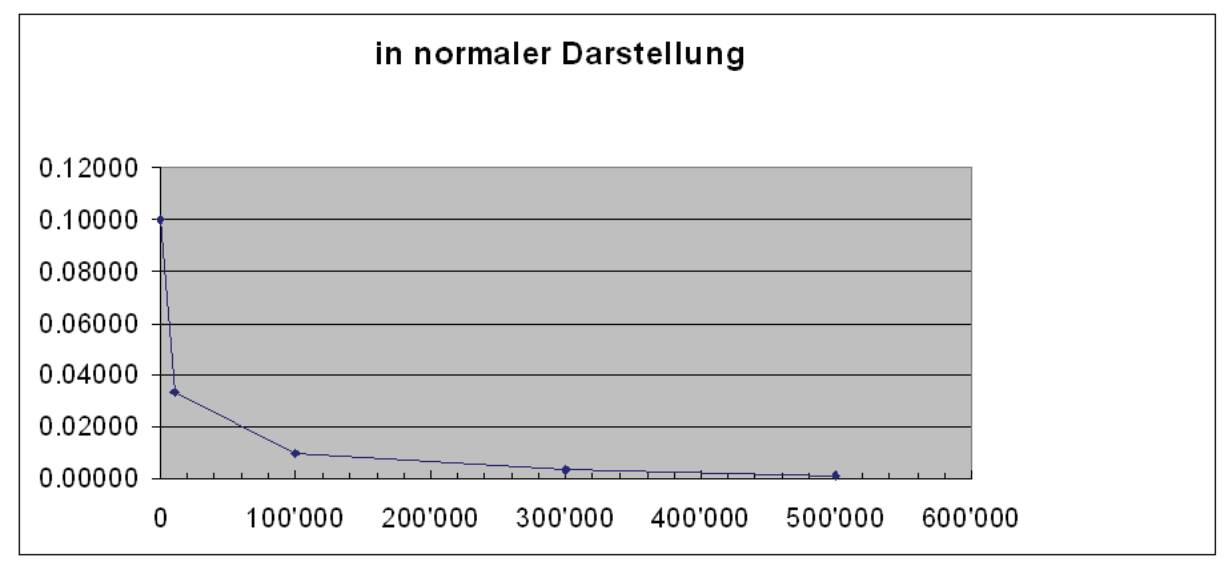

Abbildung 2 Schadenskurve mit linearer Skalierung.

Die Wahl der Interpolation (linear im log-log-Diagramm oder linear im Diagramm mit linearer Skalierung) führt in einigen Fällen zu erheblichen Unterschieden betreffend Gesamtrisiko. Im Rahmen dieser Arbeit durchgeführte Berechnungsbeispiele zu Personenschäden mit sehr kleinen Zahlenwerten für das Schadensausmass (typisch für Personenrisiken) ergaben Unterschiede zwischen den beiden Interpolationsverfahren von bis zu Faktor 50’000.

Für die Wahl des optimalen Interpolationsalgorithmus sind Lage und Verlauf der ,Risikokurve' im W/A-Diagramm entscheidend. Diese Parameter sind nur mit einer aufwendigen Analyse ermittelbar. Nebst der mathematisch-statistischen Richtigkeit spielt vor allem im regionalen Risikomanagement die Einheitlichkeit der Berechnungsmethoden eine Rolle.

Eine auf linearer Interpolation im Diagramm mit linearer Skalierung beruhende Berechnung zur Ermittlung von Risiken und von Kostenwirksamkeiten bei Projekten zur Risikominderung steht heute beim BAFU als Berechnungstool zur Verfügung (BWG, 2006 und BAFU, 2007b). 


\section{Objektkategorien und Risikokategorien}

Tabelle 2 Risikoobjekte, Risikokategorien und Anzahl betroffener Individuen

Objektkategorien gemäss Borter (1999) Bd. I und II

\begin{tabular}{ll}
\hline 1 & Bergwege \\
\hline 1 & Kletterrouten \\
\hline 1 & Skitourenrouten \\
\hline 1 & Naturlandschaften \\
\hline 2.1 & Weideland, Alpweiden \\
\hline
\end{tabular}

2.1 Wanderwege

2.1 Flurwege 2

2.2 Unbewohntes Gebäude (Scheune usw.) 2

2.2 Kommunaler Verkehrsweg, Gemeindestrasse 3

2.2 Landwirtschaftliches Nutzgebiet allgemein 3

2.2 Ackerland

2.2 Wiesland

2.2 Wald mit besonderer Schutzfunktion

2.3 Ein-/Zweifamilienhaus

2.3 Mehrfamilienhaus

2.3 Gewerbebaute

2.3 Stall, Ökonomiegebäude

2.3 Kantonaler Verkehrsweg, Kantonsstrasse

2.3 Eisenbahn, einspurig

2.3 Bergbahnen

2.3 Skiabfahrtsgelände

3.1 Nationaler Verkehrsweg, Autobahn

3.1 Eisenbahn, doppelspurig

$3.1 \quad$ Skiliftanlage

3.1 Bergbahnstation

3.2 Siedlungsfläche allgemein

3.2 Siedlungskern

3.2 Ein-/Zweifamilienhaussiedlung

3.2 Mehrfamilienhaussiedlung

3.2 Gewerbegebiet

3.2 Industriegebiet

3.2 Bauzone unüberbaut

3.2 Freizeit-/Campinganlage

3.2 Grünanlage/Parkanlage

Risiko-
kategorie
$\left.{ }^{*}\right)$

Anzahl möglicherweise betroffener Individuen $^{\star \star}$ )

100

120

$1 \quad 50$

150

320

1200

250

4

$3 \quad 50$

4

34

34

$3 \quad 20$

43

46

36

33

$310^{\prime} 000$

$4 \quad 15^{\prime} 000$

$2 \quad 10^{\prime} 000$

$155^{\prime} 000$

$3 \quad 150$ '000

$4 \quad 100^{\prime} 000$

$2 \quad 25^{\prime} 000$

25000

$4 \quad 5$

$4 \quad 10$

43

$4 \quad 6$

36

310

25

120

1200

*) gemäss Merz et. al, 1995; PLANAT, 2004b (1=freiwillige Risiken, 4=unfreiwillige Risiken).

**) Schätzung der Anzahl verschiedener Individuen, die sich typischerweise während eines Jahren in oder auf einem Objekt (Referenzfläche 1 Are bei flächigen Objekten, Referenzlänge $10 \mathrm{~m}$ bei linienförmigen Objekten) aufhalten. 


\section{Tolerierbare individuelle Todesfallrisiken; Vergleich zwischen Schutzzielen und Grenzwerten}

\section{IV.I Berechnete Schutzziele (Matrix) und Grenzwert (1)}

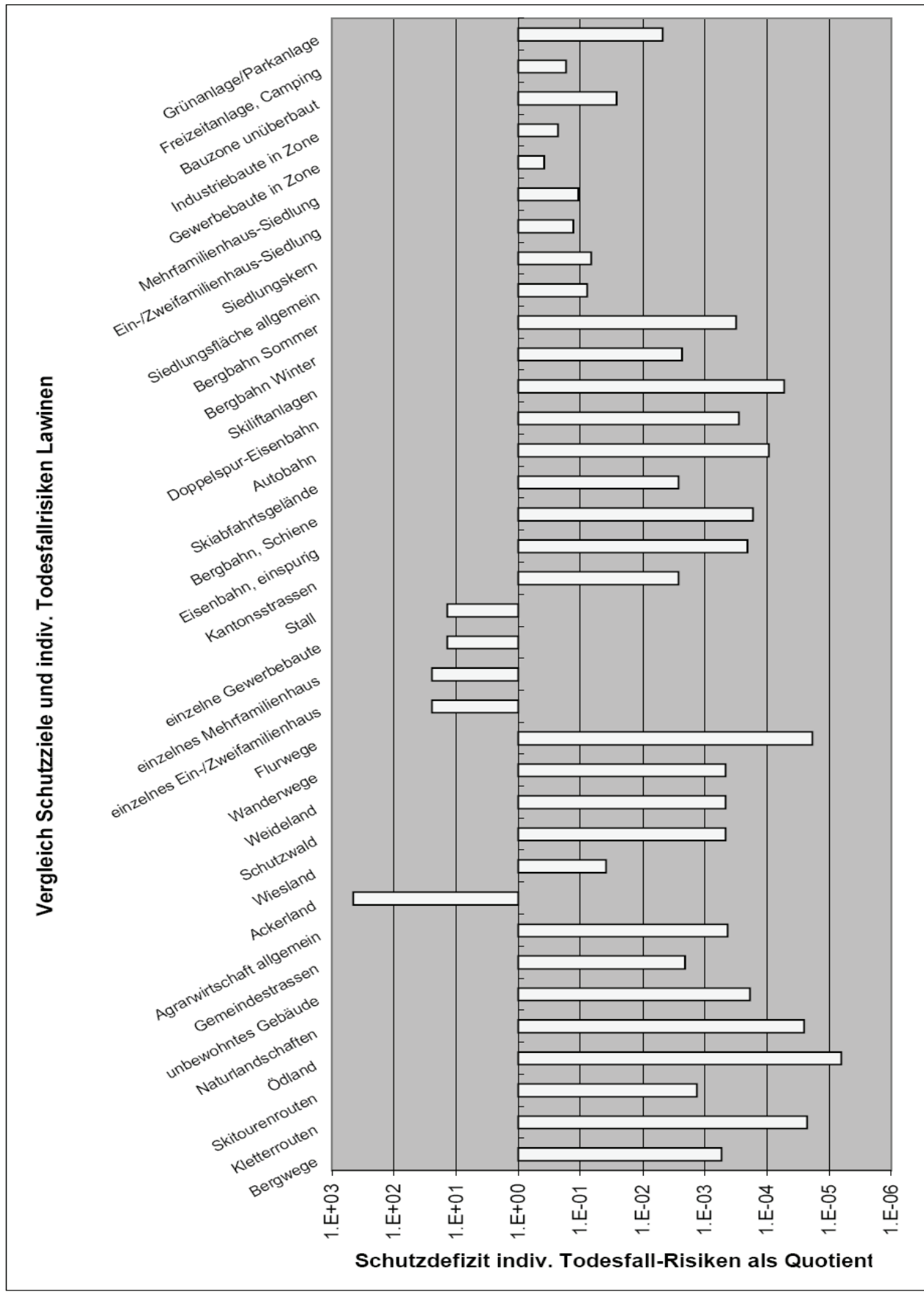




\section{IV.II Berechnete Schutzziele (Matrix) und Grenzwert (2)}

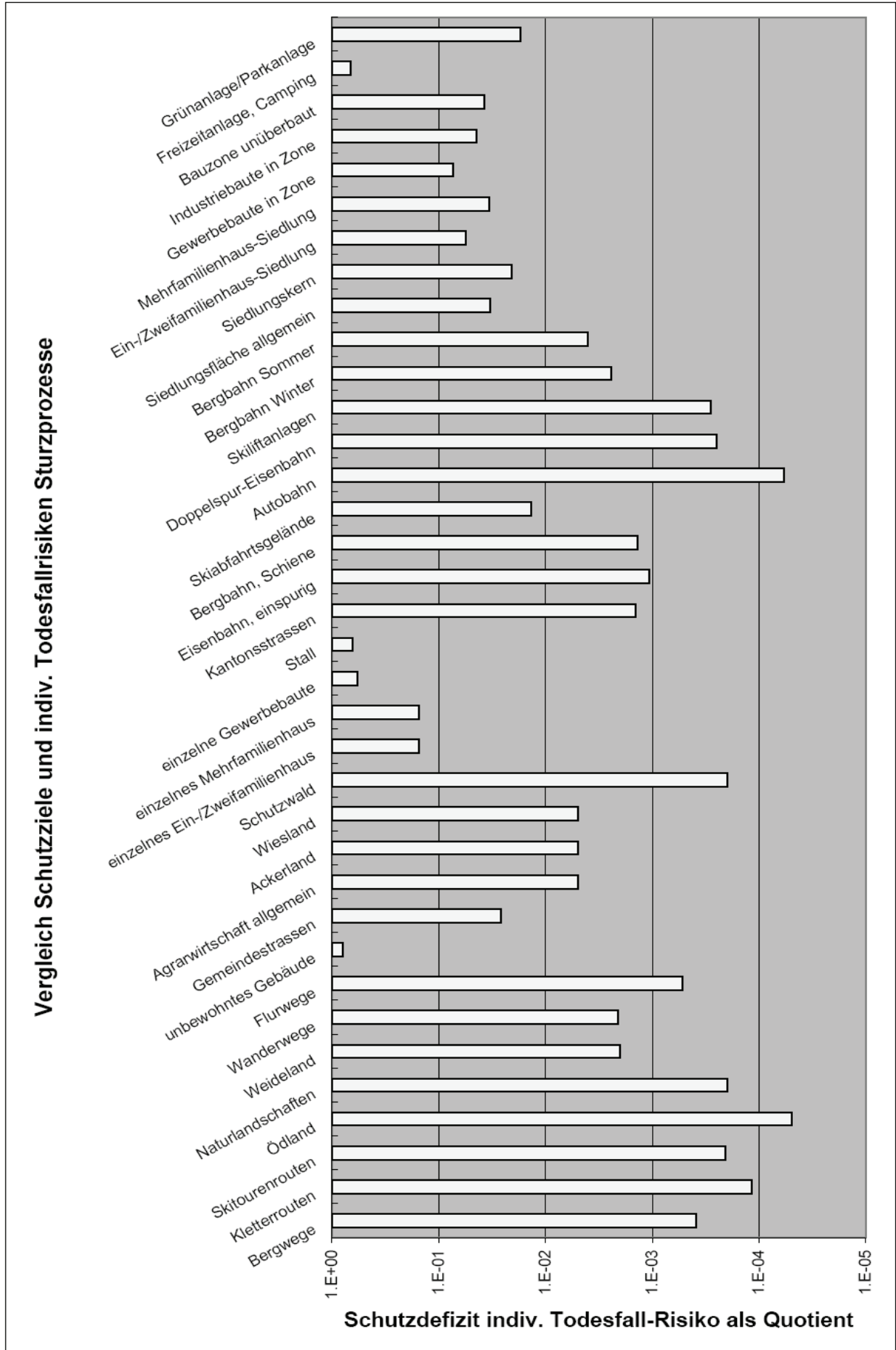




\section{IV.III Berechnete Schutzziele (Matrix) und Grenzwert (3)}

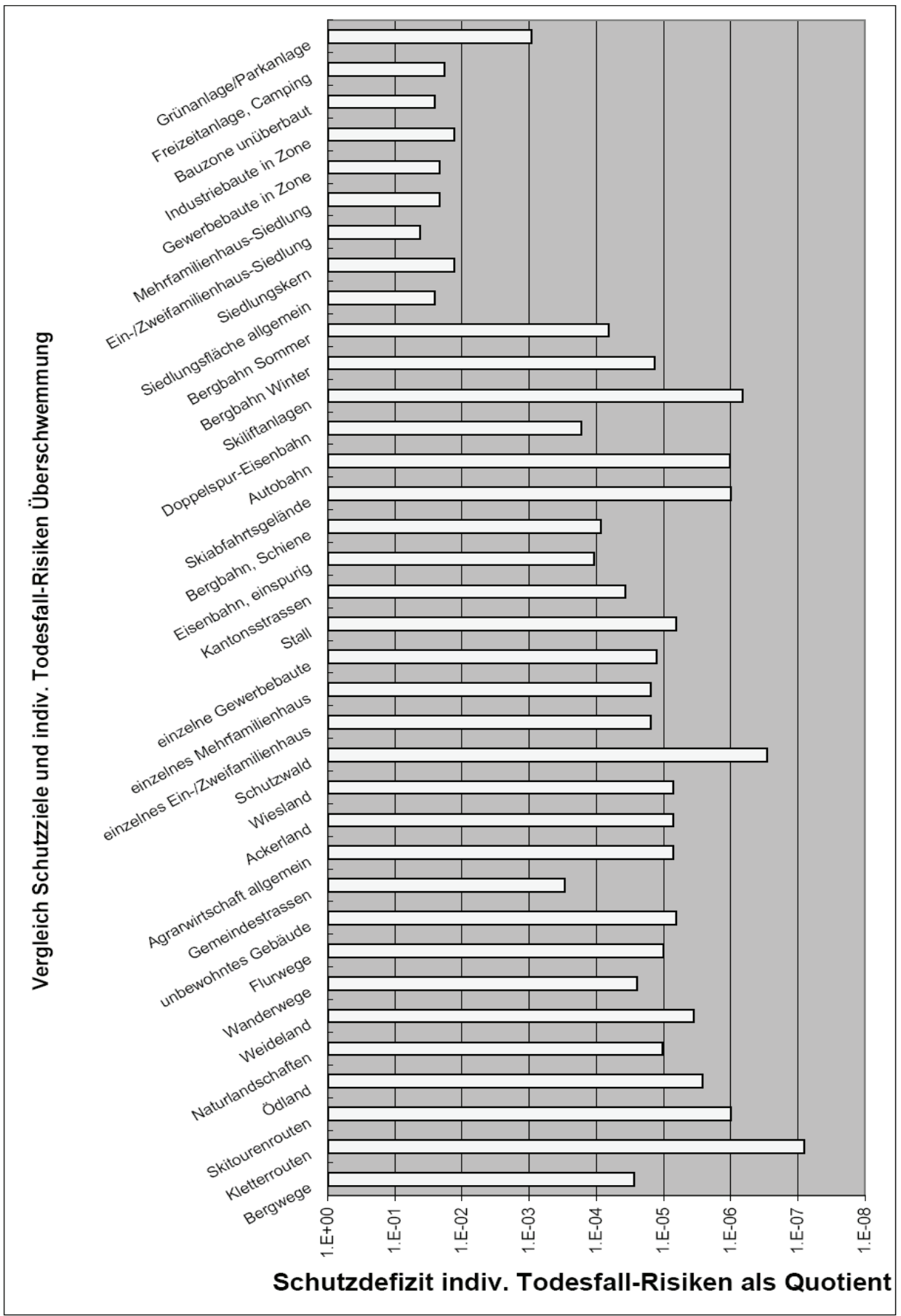




\section{IV.IV Berechnete Schutzziele (Matrix) und Grenzwert (4)}

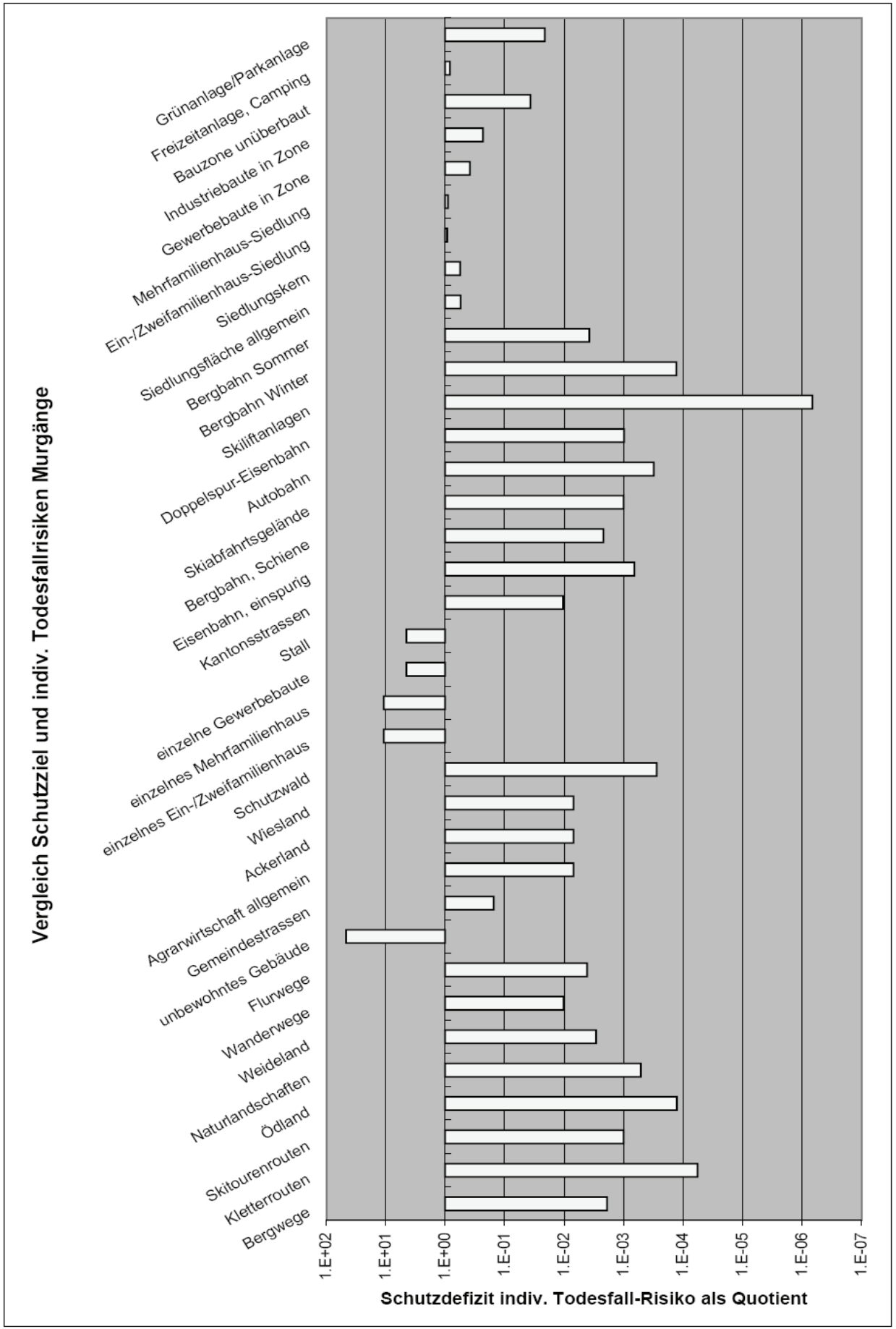




\section{Tolerierbare Risiken pro Gefahrenquelle (Fallbeispiel Engelberg)}

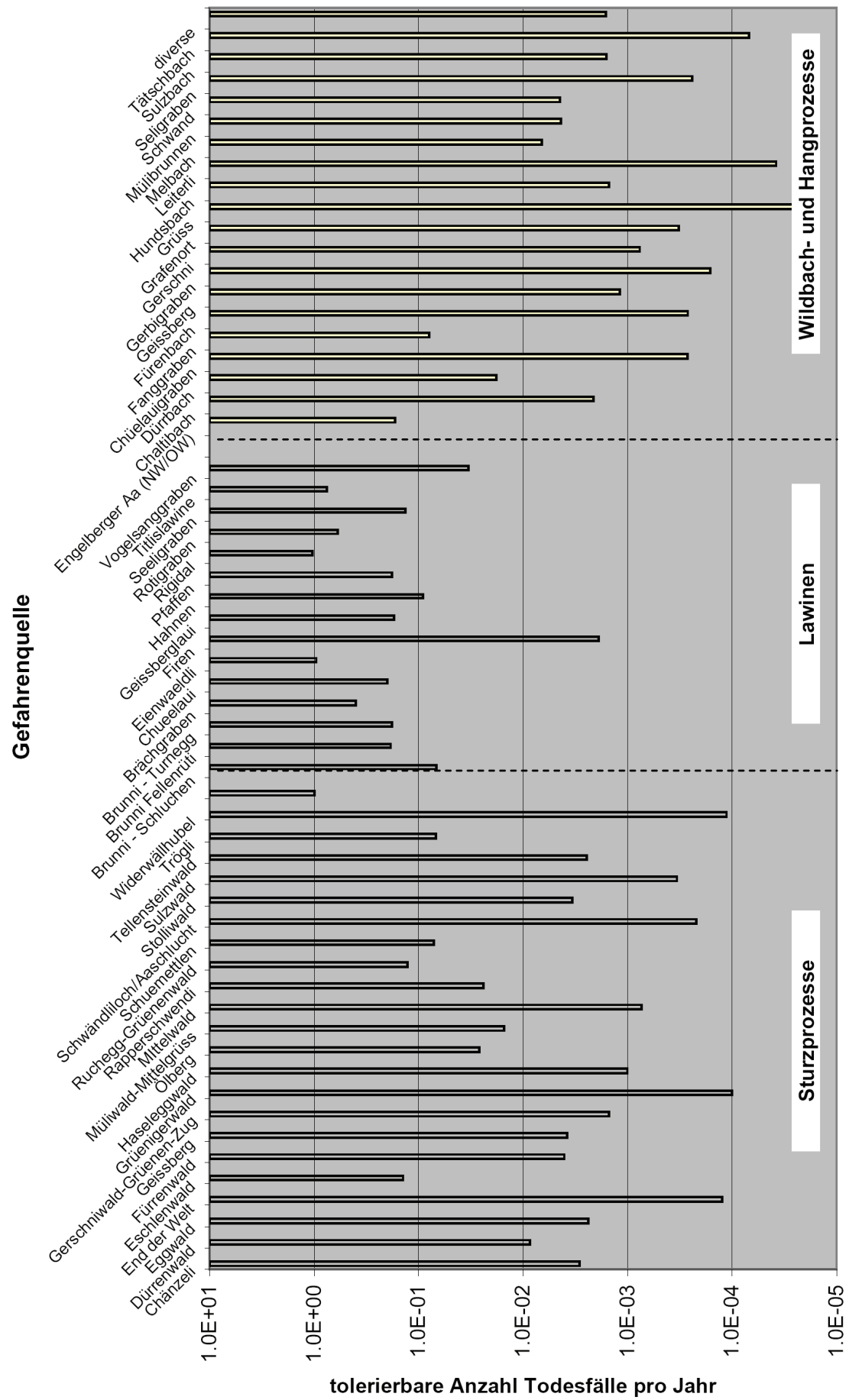




\section{Schutzzielrelevante Rechtsnormen}

\section{VI.I Schutzzielrelevante Regelungen in der Verfassung}

\section{VI.I.I Überblick}

Die schweizerische Bundesverfassung (BV, SR 101) enthält verschiedene für Schutzziele relevante Bestimmungen. Es sind dies insbesondere Art. 2, 5 und 6 der allgemeinen Verfassungsbestimmungen sowie das Kapitel über die Grundrechte (BV Art. 7 bis 36) und die Sozialziele (BV Art. 41). In Bezug auf die vorerwähnten Fragestellungen lassen sich diese Verfassungsbestimmungen folgendermassen darstellen:

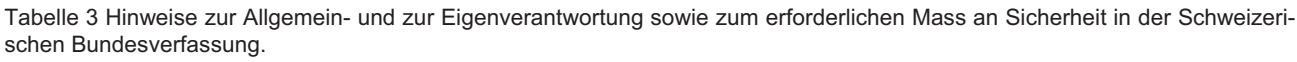

\begin{tabular}{|c|c|c|c|}
\hline $\begin{array}{l}\text { Für Fragestellung } \\
\text { massgebliche } \\
\text { Artikel der } \\
\text { Bundesverfassung }\end{array}$ & $\begin{array}{l}\text { Sicherheit als Aufgabe } \\
\text { der Allgemeinheit }\end{array}$ & $\begin{array}{l}\text { Eigenverantwor- } \\
\text { tung des } \\
\text { Individuums }\end{array}$ & $\begin{array}{l}\text { Hinweise zum } \\
\text { gebotenen/erforder- } \\
\text { lichen Mass an } \\
\text { Sicherheit }\end{array}$ \\
\hline Art. 2 Zweck & $\begin{array}{l}\text { Abs. } 1 \text { Sicherheit } \\
\text { Abs. } 2 \text { Wohlfahrt } \\
\text { Abs. } 4 \text { Natürliche } \\
\text { Lebensgrundlagen und } \\
\text { friedliche Ordnung }\end{array}$ & & $\begin{array}{l}\text { Abs. } 3 \\
\text { Chancengleichheit }\end{array}$ \\
\hline Art. 5 Grundsätze & $\begin{array}{l}\text { Abs. } 1 \text { Gesetzmässigkeit } \\
\text { Abs. } 2 \text { Öffentliches } \\
\text { Interesse }\end{array}$ & & $\begin{array}{l}\text { Abs. } 2 \\
\text { Verhältnismässigkeit } \\
\text { Abs. } 3 \text { Treu und } \\
\text { Glauben }\end{array}$ \\
\hline Art. 6 Verantwortung & & $\begin{array}{l}\text { Eigenverantwort- } \\
\text { ung und } \\
\text { gesellschaftliche } \\
\text { Verantwortung } \\
\text { erforderlich }\end{array}$ & \\
\hline Art. 7 & & & Schutz der \\
\hline Menschenwürde & & & Menschenwürde \\
\hline Art. 8 & & & Abs. 1 \\
\hline Rechtsgleichheit & & & $\begin{array}{l}\text { Rechtsgleichheit } \\
\text { Abs. } 2 \\
\text { Diskriminierungsver- } \\
\text { bot }\end{array}$ \\
\hline $\begin{array}{l}\text { Art. } 9 \text { Treu und } \\
\text { Glauben }\end{array}$ & & & $\begin{array}{l}\text { Treu und Glauben } \\
\text { und Willkürverbot }\end{array}$ \\
\hline $\begin{array}{l}\text { Art. } 10 \text { Leben und } \\
\text { persönliche Freiheit }\end{array}$ & $\begin{array}{l}\text { Abs. } 1 \text { Recht auf Leben } \\
\text { Abs. } 2 \text { Recht auf } \\
\text { persönliche Freiheit, } \\
\text { körperliche und geistige } \\
\text { Unversehrtheit, } \\
\text { Bewegungsfreiheit }\end{array}$ & & $\begin{array}{l}\text { Abs. } 3 \text { Verbot von } \\
\text { Folter und grausamer } \\
\text { oder unmenschlicher } \\
\text { Behandlung }\end{array}$ \\
\hline $\begin{array}{l}\text { Art. } 12 \text { Hilfe in } \\
\text { Notlagen }\end{array}$ & & $\begin{array}{l}\text { Nothilfe setzt } \\
\text { Unmöglichkeit } \\
\text { einer Selbsthilfe } \\
\text { voraus }\end{array}$ & $\begin{array}{l}\text { Hilfe und Betreuung } \\
\text { und Mittel für ein } \\
\text { menschenwürdiges } \\
\text { Dasein }\end{array}$ \\
\hline
\end{tabular}




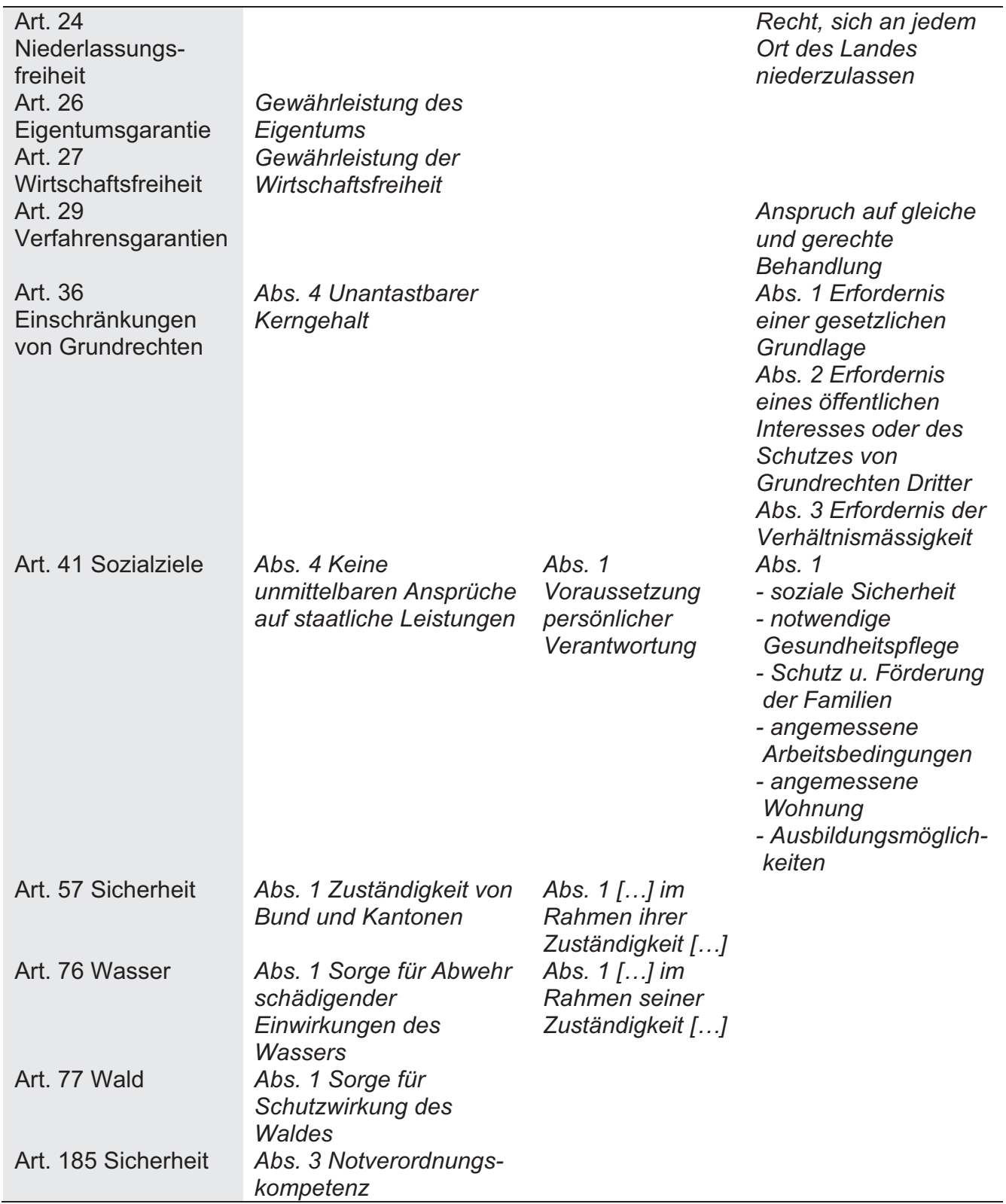

\section{VI.I.II Zweckartikel der Bundesverfassung}

Bereits im Zweckartikel sind generelle Vorstellungen zu Schutzzielen enthalten

BV Art. 2 Zweck

${ }^{1}$ Die Schweizerische Eidgenossenschaft schützt die Freiheit und die Rechte des Volkes und wahrt die Unabhängigkeit und die Sicherheit des Landes.

${ }^{2}$ Sie fördert die gemeinsame Wohlfahrt, die nachhaltige Entwicklung, den inneren Zusammenhalt und die kulturelle Vielfalt des Landes.

Josef Th. Hess: Schutzziele im Umgang mit Naturrisiken in der Schweiz, @ vdf Hochschulverlag 2011 


\footnotetext{
${ }^{3}$ Sie sorgt für eine möglichst grosse Chancengleichheit unter den Bürgerinnen und Bürgern.

${ }^{4}$ Sie setzt sich ein für die dauerhafte Erhaltung der natürlichen Lebensgrundlagen und für eine friedliche und gerechte internationale Ordnung.
}

Artikel 2 orientiert sich an der klassischen Viergliedrigkeit des Staatszwecks: Freiheits-, Sicherheits-, Gemeinschafts- und Wohlfahrtszweck. Grundsätzlich haben alle Bundeszwecke das gleiche Gewicht (BBI 1997 | 126). Für Schutzziele von Bedeutung ist das in Absatz 2 und 4 umschriebene Nachhaltigkeitsprinzip. Bemerkenswert ist der Einschub des im Verfassungsentwurf 1996 noch nicht enthaltenen Absatzes 3 zur möglichst grossen Chancengleichheit. Dieser Passus war in der parlamentarischen Beratung stark umstritten. So wurde vor einer Überforderung des Staates oder mangelnder Justiziabilität gewarnt (AB N, 1998, S. 129 ff., S. 406 ff., S. 462 ff.).

\section{VI.I.III Rechtsstaatsprinzipien}

Für Schutzziele von Bedeutung sind die in der heutigen Verfassung geregelten Grundsätze des rechtsstaatlichen Handelns, auch als Rechtsstaatsprinzipien bezeichnet (Häfelin \& Müller, 2002; Richli, 2004):

- Gesetzmässigkeit,

- öffentliches Interesse,

- Rechtsgleichheit und Willkürverbot,

- Verhältnismässigkeit,

- Vertrauensschutz und

- Rechtssicherheit.

Die einschlägigen Verfassungsbestimmungen lauten:

Art. 5 Grundsätze rechtsstaatlichen Handelns

${ }^{1}$ Grundlage und Schranke staatlichen Handelns ist das Recht.

${ }^{2}$ Staatliches Handeln muss im öffentlichen Interesse liegen und verhältnismässig sein.

${ }^{3}$ Staatliche Organe und Private handeln nach Treu und Glauben.

${ }^{4}$ Bund und Kantone beachten das Völkerrecht.

\section{Gesetzmässigkeit (Art. 5 Abs. 1 BV, SR 101)}

Dieses Prinzip verweist - auch bezüglich der Schutzziele - auf die Rechtsordnung, insbesondere auf die Spezialgesetze, auf die nachstehend unter Kapitel VI.II einzugehen ist.

\section{Öffentliches Interesse (Art. 5 Abs. 2 BV, SR 101)}

Gemäss Botschaft zur Bundesverfassung (BBI 1997 | 133) umfasst das öffentliche Interesse in erster Linie polizeiliche Interessen, d. h. öffentliche Ruhe und Ordnung, Sicherheit, Gesundheit, Sittlichkeit sowie Treu und Glauben im Geschäftsverkehr. Das öffentliche Interesse erstreckt sich ferner auf Anliegen sozialer, kultureller, historischer und wissenschaftlicher Art sowie Anliegen des Umweltschutzes, des haushälterischen Umgangs mit dem Boden, des sparsamen Umgangs mit Energie usw. Es schliesst zudem das Interesse des Staates an seiner Unabhängigkeit, seinem Ruf, seiner Glaubwürdigkeit usw. ein. Das öffentliche Interesse kann sich im Lauf der Zeit ändern; es kann auch in räumlicher Hinsicht variieren. Es ist nicht zuletzt die Bundesverfassung, die darüber Aufschluss gibt, was als öffentliches Interesse anzuerkennen ist. Für Schutzziele im Management von Naturrisiken besonders relevant sind ein Teil dieser polizeilichen Interessen. 


\section{Verhältnismässigkeit (Art. 5 Abs. 2 BV, SR 101)}

Das Verhältnismässigkeitsprinzip enthält drei Elemente (BBI 1997 | 133 f.): Eine staatliche Massnahme muss geeignet sein, das angestrebte Ziel zu erreichen (Geeignetheit). Staatliches Handeln darf in materieller, räumlicher, zeitlicher und persönlicher Hinsicht nicht schärfer einschränken, als dies für die Erreichung des Ziels nötig ist (Erforderlichkeit, ,geringstmöglicher' Eingriff). Schliesslich muss die geeignete und erforderliche Massnahme in einem vernünftigen Verhältnis zum angestrebten Ziel stehen (Verhältnismässigkeit im engeren Sinn oder Verhältnismässigkeit des geforderten Opfers). Das Verhältnismässigkeitsprinzip findet sowohl in der Leistungsverwaltung als auch in der Eingriffsverwaltung Anwendung. In beiden Bereichen richtet sich der Grundsatz sowohl an die rechtsanwendenden Behörden als auch an den Gesetzgeber.

Für Schutzziele im Management von Naturrisiken ist das Verhältnismässigkeitsprinzip in zweierlei Hinsicht relevant. Einerseits sind effizienzorientierte Schutzzieldarstellungen auf dem Verhältnismässigkeitsprinzip begründet. Weiter tragen die in Kapitel 2.6.2.4 dargestellten Risikokategorien dem Verhältnismässigkeitsprinzip Rechnung.

\section{Treu und Glauben (Art. 5 Abs. 3 BV, SR 101)}

Nach dem Grundsatz von Treu und Glauben lassen sich unter Umständen Sicherheitsansprüche aus behördlichen Zusicherungen oder behördlicher Praxis ableiten. Der spezifische grundrechtliche Schutz, den das Bundesgericht aus dem Grundsatz von Treu und Glauben abgeleitet hat (Schutz des berechtigten Vertrauens in behördliche Zusicherungen oder anderes, Erwartungen begründendes Behördenverhalten), wird im Grundrechtskatalog geregelt (Art. 8 VE 96; BBI 1997 | 134). Der Grundsatz von Treu und Glauben ist im Verfahren des Risikomanagements zentral. Vertrauen ist heute als Schlüsselfaktor eines effektiven und effizienten Risikomanagements erkannt.

\section{Rechtsgleichheit und Willkürverbot (Art.8 Abs. 1 und 2 und Art. 9 BV, SR 101)}

Art. 8 Rechtsgleichheit

1 Alle Menschen sind vor dem Gesetz gleich.

2 Niemand darf diskriminiert werden [...].

Diese Bestimmung schützt eine rechtliche Gleichheit. Diese Gleichheit unterscheidet sich von wirtschaftlicher oder sozialer Gleichstellung, die nur Gegenstand eines politischen Programms sein kann (BBI 1997 | 142). Das Willkürverbot ist eine unverzichtbare Grundlage des Rechtsstaates; es sichert dem Einzelnen im Umgang mit den Behörden ein Mindestmass an Gerechtigkeit. Aufgrund dieser Eigenschaft als Mindestgarantie erscheint das Willkürverbot wie ein subsidiäres Verfassungsrecht, das dann angerufen wird, wenn kein anderes Grundrecht oder kein anderes spezifisches Recht geltend gemacht werden kann. Kein öffentliches Interesse, und sei es noch so stark, rechtfertigt eine willkürliche Handlung des Staates (BBI 1997 | 144).

\section{VI.I.IV Individuelle und gesellschaftliche Verantwortung (Art. 6 BV, SR 101)}

Schutzziele grenzen die Verantwortlichkeit des Individuums gegenüber Dritten, namentlich der Allgemeinheit, ab.

\section{Art. 6 Individuelle und gesellschaftliche Verantwortung}

Jede Person nimmt Verantwortung für sich selber wahr und trägt nach ihren Kräften zur Bewältigung der Aufgaben in Staat und Gesellschaft bei.

Artikel 6 der BV war in der ursprünglichen Botschaft vom 20. Nov. 1996 nicht enthalten und wurde erst im Entwurf der Verfassungskommission des Nationalrates vom 21. November 1997 als Art. 3 b eingebracht. Im Parlament wurde das Ansinnen (SR am 20. Jan. 1998, NR 
am 18. März 1998) kontrovers diskutiert (AB 1998S, S. 25ff, S. 152; AB 1998N, S. 137ff., S. 408). Einerseits wurde ein solcher Artikel gefordert, um der drohenden Überforderung des Staates entgegenzutreten, anderseits sprach die mangelhafte Durchsetzbarkeit gegen einen solchen Artikel. Art. 6 der Bundesverfassung hält fest, dass jedes Individuum Verantwortung trägt und sich - auch in Fragen der Sicherheit vor Naturgefahren - nicht ausschliesslich auf Dritte, namentlich die Allgemeinheit, abstützen kann. Wie weit die Verantwortung reicht, die das Individuum wahrzunehmen hat, ist dagegen nicht ausformuliert.

\section{VI.I.V Grundrechte (Art. 7 - 36 BV, SR 101)}

Aus den Grundrechten ergeben sich Ansprüche des Individuums gegenüber dem Staat und Dritten. Gemäss Botschaft zur Schweizerischen Bundesverfassung (BBI 1997 I 191 ff.) ist die Funktion der Grundrechte sowohl defensiv, indem sie den Einfluss des Staates auf die Individuen beschränken, als auch positiv, indem sie den Staat zu einem Tun veranlassen oder inn gar dazu verpflichten (BBI 1997 I 191). Dabei sind die Grundrechte - im Gegensatz zu den im gleichen Titel geregelten Sozialzielen - direkt anwendbar (BBI 1997 I 137). Aus den Grundrechten ergeben sich somit eigentliche Schutzziele. Die Grundrechte fallen nach herrschender Lehre unter den Begriff der öffentlichen Sicherheit und Ordnung (Reinhard, 1993).

Für das Management von Naturrisiken erscheinen folgende Grundrechte als besonders relevant:

Art. 7 Menschenwürde (primäres und subsidiäres Grundrecht (BBI 1997 I 140),

Art. 8 Rechtsgleichheit (rechtliche, nicht aber wirtschaftliche und soziale Gleichheit (BBI 1997 ( 142)),

Art. 9 Schutz vor Willkür und Wahrung von Treu und Glauben,

Art. 10 Recht auf Leben und auf persönliche Freiheit,

Art. 12 Recht auf Hilfe in Notlagen

(Das BGer hat die Befriedigung der elementarsten menschlichen Bedürfnisse (Nahrung, Kleidung, Obdach) als Bedingung eines menschenwürdigen Daseins, das diese Bezeichnung verdient, und der menschlichen Entfaltung, anerkannt. In dem Mass, in dem die Sicherstellung des Existenzminimums diesen erstrangigen Bedürfnissen entspricht, kann das Recht darauf in einer Beschwerde ans Bundesgericht als Beschwerdegrund angeführt werden (BBI 1997 I 149 f.). Im Vergleich zum Verfassungsentwurf 1996 wurde mit mit dem Einschub des Satzteiles „[...] und nicht in der Lage ist, für sich zu sorgen, [...]“ die Bedeutung der eigenen Verantwortung betont.),

Art. 24 Niederlassungsfreiheit,

Art. 26 Eigentumsgarantie

(Der Eigentümer muss bei der Ausübung seines Rechts öffentliche Interessen wie Raumplanung, den Schutz vor Umwelt, Wasser und Wald oder Natur- und Heimatschutz usw. berücksichtigen (BBI 1997 I 173)),

Art. 27 Wirtschaftsfreiheit (Als Einschränkung der Wirtschaftsfreiheit sind grundsätzlich Massnahmen zum Schutz des Lebens und der Gesundheit sowie zum Schutz weiterer sogenannter Polizeigüter (bes. öffentliche Sittlichkeit, Treu und Glauben im Geschäftsverkehr) zulässig. Grundsätzlich zulässig sind auch sozialpolitisch motivierte Massnahmen sowie andere Massnahmen, die nicht primär wirtschaftspolitischen Zwecken dienen (darunter insbesondere Massnahmen der Raumplanung, der Energiepolitik, der Umweltpolitik und der Sprachpolitik; BBI 1997 I 175),

Art. 29 Allgemeine Verfahrensgarantien. 
Art. 35 und Art. 36 BV regeln Geltungsbereich und Einschränkungen von Grundrechten.
Art. 35 Verwirklichung der Grundrechte
${ }^{1}$ Die Grundrechte müssen in der ganzen Rechtsordnung zur Geltung kommen.
${ }^{2}$ Wer staatliche Aufgaben wahrnimmt, ist an die Grundrechte gebunden und verpflichtet, zu ihrer Verwirk- lichung beizutragen.
${ }^{3}$ Die Behörden sorgen dafür, dass die Grundrechte, soweit sie sich dazu eignen, auch unter Privaten wirksam werden.

Dieser Artikel gibt den staatlichen Behörden den allgemeinen Auftrag, für die tatsächliche Verwirklichung der Grundrechte zu sorgen, d. h. alle geeigneten Mittel einzusetzen, um das angestrebte Ziel zu erreichen. Dieser Auftrag schliesst zunächst die Verpflichtung ein, sich jeder Tätigkeit zu enthalten, die den Grundrechten schaden könnte. Dies entspricht der klassischen Funktion der Grundrechte als Abwehrrechte, die dem Staat eine Pflicht zur Zurückhaltung auferlegt. Der Auftrag enthält aber weiter auch die Verpflichtung der staatlichen Behörden, sich so zu verhalten, dass die Grundrechte geschützt und gefördert werden (positive Funktion; BBI 1997 I 192).

Abs. 3 bringt zum Ausdruck, dass die Grundrechte auch eine Wirkung auf die Beziehungen unter den Einzelnen haben. Diese Wirkung kann aber nicht allgemeinverbindlich und starr festgelegt werden. Sie wandelt sich je nach Art des betreffenden Grundrechts und der Umstände. Es ist jedes Mal eine Analyse der konkreten Situation erforderlich, um zu entscheiden, ob die Wirkung unter Privaten möglich ist (BBI 1997 I 193).
Art. 36 Einschränkungen von Grundrechten
${ }^{1}$ Einschränkungen von Grundrechten bedürfen einer gesetzlichen Grundlage. Schwerwiegende Ein- schränkungen müssen im Gesetz selbst vorgesehen sein. Ausgenommen sind Fälle ernster, unmittelbarer und nicht anders abwendbarer Gefahr.
${ }^{2}$ Einschränkungen von Grundrechten müssen durch ein öffentliches Interesse oder durch den Schutz von Grundrechten Dritter gerechtfertigt sein.
${ }^{3}$ Einschränkungen von Grundrechten müssen verhältnismässig sein.
${ }^{4}$ Der Kerngehalt der Grundrechte ist unantastbar.

Die Bestimmung macht klar, dass die von der Verfassung garantierten Grundrechte nicht absolut zu verstehen sind und dass sie eingeschränkt werden können oder gar müssen. Solche Einschränkungen dürfen die Grundrechte allerdings nicht ihres Gehalts entleeren. Daher sind die Voraussetzungen, unter denen eine Einschränkung von Grundrechten zulässig ist, ihrerseits verfassungsrechtlich festgelegt. Ein Grundrechtseingriff ist nur unter den vier in Artikel 36 ausdrücklich formulierten Bedingungen zulässig (BBI 1997 I 194 f).

Der zweite Satz von Abs. 1 nimmt von den Voraussetzungen einer Grundrechtsbeschränkung die allgemeine Polizeiklausel aus, die im Fall einer ernsten, unmittelbaren und nicht anders abwendbaren Gefahr eine ausreichende gesetzliche Grundlage für einen Grundrechtseingriff bietet, aber auch hier müssen die weiteren Voraussetzungen für den Eingriff in ein Grundrecht erfüllt sein (BBI 1997 I 196).

Abs. 4 sieht vor, dass ein Grundrecht nicht derart eingeschränkt werden darf, dass sein Wesensgehalt angetastet wird. Der Wesens- oder Kerngehalt ist derjenige Bereich eines Rechts, der keinen Eingriff duldet: Beispielsweise stellen der Respekt der Menschenwürde und das absolute Folterverbot nach der Rechtsprechung des Bundesgerichts unantastbare Kerngehalte der persönlichen Freiheit dar (BBI 1997 I 196 f). 


\section{VI.I.VI Sozialziele (Art. 41 BV, SR 101)}

Weitere Hinweise zu Schutzzielen ergeben sich aus dem Verfassungsartikel über die Sozialziele.
Art. 41
${ }^{1}$ Bund und Kantone setzen sich in Ergänzung zu persönlicher Verantwortung und privater Initiative dafür ein, dass
- jede Person an der sozialen Sicherheit teilhat;
- $\quad$ jede Person die für ihre Gesundheit notwendige Pflege erhält;
- Familien als Gemeinschaften von Erwachsenen und Kindern geschützt und gefördert werden;
- $\quad$ Erwerbsfähige ihren Lebensunterhalt durch Arbeit zu angemessenen Bedingungen bestreiten können;
- Wohnungssuchende für sich und ihre Familie eine angemessene Wohnung zu tragbaren Bedin- gungen finden können;
- $\quad$ Kinder und Jugendliche sowie Personen im erwerbsfähigen Alter sich nach ihren Fähigkeiten bil- den, aus- und weiterbilden können;
- Kinder und Jugendliche in ihrer Entwicklung zu selbstständigen und sozial verantwortlichen Per- sonen gefördert und in ihrer sozialen, kulturellen und politischen Integration unterstützt werden.

\footnotetext{
${ }^{2}$ Bund und Kantone setzen sich dafür ein, dass jede Person gegen die wirtschaftlichen Folgen von Alter, Invalidität, Krankheit, Unfall, Arbeitslosigkeit, Mutterschaft, Verwaisung und Verwitwung gesichert ist.

${ }^{3}$ Sie streben die Sozialziele im Rahmen ihrer verfassungsmässigen Zuständigkeiten und ihrer verfügbaren Mittel an.

${ }^{4}$ Aus den Sozialzielen können keine unmittelbaren Ansprüche auf staatliche Leistungen abgeleitet werden.
}

Im Gegensatz zu den Grundrechten sind die Sozialziele nicht direkt anwendbar. Somit ergeben sich daraus keine direkten Schutzziele, sondern lediglich gestaltende Elemente möglicher Schutzziele, die zunächst vom Gesetzgeber konkretisiert werden müssen (BBI 1997 I 137). Die Sozialziele richten sich denn auch in erster Linie an den Gesetzgeber.

Es handelt sich beim Artikel 41 (Artikel 33 VE 96) um ein staatspolitisches Grundsatzbekenntnis. Die vorgeschlagene Sozialzielbestimmung macht sichtbar, dass Bund und Kantone nicht nur die Freiheiten und die demokratischen Rechte des Einzelnen respektieren und schützen, sondern auch einen Beitrag zur Daseinsvorsorge leisten, indem sie sich dafür einsetzen, dass grundlegende Bedürfnisse in elementaren Lebenssituationen befriedigt werden können (BBI 1997 I 204).

Die verfassungsrechtliche Verankerung von Sozialzielen ist allerdings nicht ohne normative Bedeutung. Bei einem Zurückfallen unter ein minimales Schutzniveau geriete man zweifellos in Konflikt mit dem Verfassungsartikel (BBI 1997 I 200). Insofern kommt auch dem Artikel über die Sozialziele Schutzzielcharakter zu.

In Abs. 1 des Art. 41 erscheint die bereits in Art. 6 BV festgehaltene Voraussetzung einer persönlichen Verantwortung in der Formulierung, dass die Sozialziele durch Bund und Kantone in Ergänzung zu persönlicher Verantwortung und privater Initiative zu erreichen sind wieder.

Bund und Kantone übernehmen mithin sozialpolitische Verantwortung, nicht aber eine eigentliche Erfolgsgarantie (BBI 1997 I 201). Was die einzelnen Ziele und Zielgruppen betrifft soziale Sicherheit, Gesundheit, Arbeit, Wohnen, Bildung, Jugend, - handelt es sich nicht um zufällig ausgewählte Bereiche. Es sind durchwegs Felder der Sozialpolitik, in denen es um elementare Aspekte menschlichen Daseins in einer modernen hochkomplexen Gesellschaft geht (BBI 1997 I 202). 


\section{VI.I.VII Aufgaben und Zuständigkeiten}

Für das Management von Naturrisiken sind auch weitere Bestimmungen zur Sicherheit wie Art. 57 (Art. 53 VE 96), Art. 61 (Art. 57 VE 96), Art. 76 (Art. 60 VE 96), Art. 77 (Art. 61 VE 96) und Art. 185 (Art. 173 VE 96) der BV relevant.

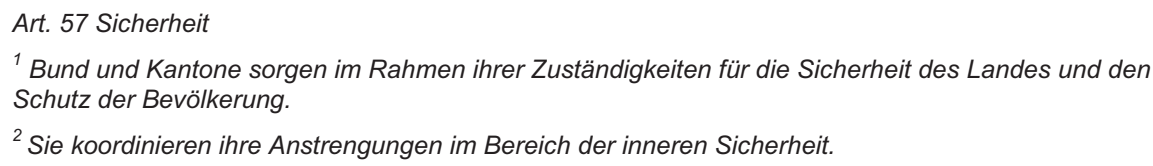

Abs. 1 geht vom Grundsatz der primären Verantwortung der Kantone für die innere Sicherheit auf ihrem Gebiet aus (Polizeihoheit) und bestimmt die Sorge für die Sicherheit des Landes und den Schutz der Bevölkerung als gemeinsame Aufgabe von Bund und Kantonen im Rahmen ihrer jeweiligen Zuständigkeiten (BBI 1997 I 237).

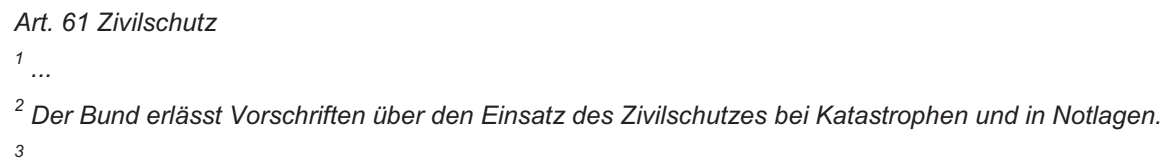

Der Zivilschutz hat den Auftrag, die Bevölkerung im Fall von bewaffneten Konflikten, in Katastrophenfällen und in anderen Notlagen zu schützen, zu retten und zu betreuen (BBI 1997 I 243).

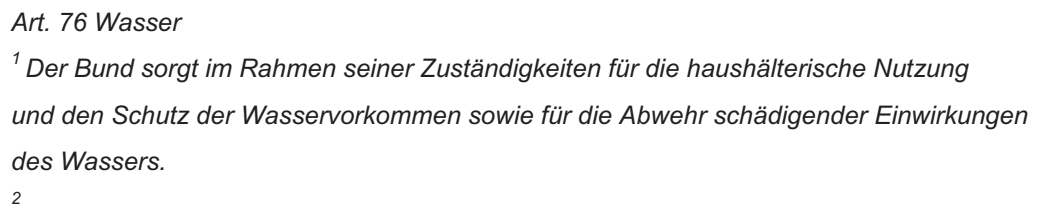

Abs. 1 nennt in Fortführung von Artikel 24 bis Absatz I aBV die drei Ziele des Wasserrechts und verpflichtet den Bund zu deren Verfolgung. Das Ziel der haushälterischen Nutzung der Wasservorkommen bezweckt einen sorgfältigen Umgang mit der natürlichen Ressource Wasser. Bei der Abwehr schädigender Einwirkungen geht es unter anderem um den Hochwasserschutz. Unter die Massnahmen zur Abwehr sind auch präventive Vorkehren zu rechnen, wie etwa Aufforstungen oder Entsiegelung von Böden (BBI 1997 I 250).

\section{Art. 77 Wald}

1 Der Bund sorgt dafür, dass der Wald seine Schutz-, Nutz- und Wohlfahrtsfunktionen

erfüllen kann.

$2 \ldots$

Mit Abs.1 werden die grundlegenden Waldfunktionen, wie sie heute in Artikel 1 des Waldgesetzes (SR 921.0) formuliert sind, auf Verfassungsstufe gehoben. Dies rechtfertigt sich vorab deshalb, weil die Ziele der Walderhaltung sich im Verlauf der letzten Jahrzehnte erheblich verändert und erweitert haben: So bietet der Wald nicht nur Schutz vor schädlichen Naturereignissen (wie Lawinen, Rutschungen, Erosion und Steinschlag). Es kommt ihm auch eine bedeutende Regulierungsfunktion für Atmosphäre, Klima und Wasserhaushalt zu. Ferner wirkt er als Lebensraum für Pflanzen und Tiere, als Erholungsraum für Menschen sowie als Produktionsgrundlage der Holzwirtschaft. Entsprechend ist zum quantitativen Waldschutz (Erhaltung der Waldfläche, Artikel 3 des Waldgesetzes; Schutz vor Kahlschlag; Nachhaltigkeit der Waldnutzung) der qualitative Waldschutz (Bekämpfung der Waldschäden) hinzugekommen (BBI 1997 I 252).

\section{Art. 185 Äussere und innere Sicherheit}




\footnotetext{
${ }^{2}$ Er (der Bundesrat) trifft Massnahmen zur Wahrung der inneren Sicherheit.

${ }^{3}$ Er kann, unmittelbar gestützt auf diesen Artikel, Verordnungen und Verfügungen erlassen, um eingetretenen oder unmittelbar drohenden schweren Störungen der öffentlichen Ordnung oder der inneren oder äusseren Sicherheit zu begegnen. Solche Verordnungen sind zu befristen.
}

Abs. 3 bildet die Grundlage für verfassungsunmittelbare Verordnungen und Verfügungen des Bundesrates im Bereich der äusseren und der inneren Sicherheit, sofern ausserordentliche Umstände ein solches Vorgehen als geboten erscheinen lassen (BBI 1997 | 418). Der Bundesrat kann somit unter gewissen Voraussetzungen ohne entsprechende Grundlage in einem formellen Gesetz gesetzesvertretende und gesetzesergänzende Verordnungen erlassen. Die darin enthaltenen Anordnungen müssen notwendig, zeitlich dringlich, durch überwiegende öffentliche Interessen gerechtfertigt und verhältnismässig sein. Sie dürfen nicht im Widerspruch zu Erlassen der Bundesversammlung stehen und sie müssen die Grundsätze insbesondere der Rechtsgleichheit und von Treu und Glauben respektieren (BBI 1997 | 418).

Es lassen sich aus Art. 57, 61 und 185 BV keine expliziten Angaben zu Schutzzielen ableiten, ebenso wenig wie aus den Verfassungsartikeln, die speziell das Management von Naturrisiken betreffen (Lüthi, 2004), nämlich zu den drei Bereichen Raumplanung (Art. 75 BV, Art. 58 VE 96), Wasser (Art. 76 BV, Art. 60 VE 96) und Wald (Art. 77 BV, Art. 61 VE 96). Die Raumplanung soll gemäss BV Art. 75, Abs. 1 der ,zweckmässigen Nutzung' dienen, woraus sich ableiten lässt, dass planerische Massnahmen auf die Gefahren als Rahmenbedingungen auszurichten sind. Gemäss BV Art. 76 Abs. 1 zum Wasser hat der Bund für die Abwehr schädigender Einwirkungen des Wassers zu sorgen, ohne dass die Erfüllung dieser Aufgabe weiter konkretisiert wird. Ähnlich generell ist die Formulierung in BV Art. 77 Abs. 1, wonach der Bund dafür zu sorgen hat, dass der Wald seine Funktionen - darunter auch jene des Schutzes vor Naturgefahren - erfüllt. Auch in der Botschaft zur neuen Bundesverfassung (BBI 1997 | 1-642) sowie in den Amtlichen Bulletins der Beratung im Ständerat (AB SR, 1998) und im Nationalrat (AB NR, 1998) sind keine differenzierteren Angaben enthalten. Schutzzielfragen werden demzufolge in den Verfassungsbestimmungen über Aufgaben und Zuständigkeiten generell und implizit angesprochen.

\section{VI.II Regelungen in Bundesgesetzen und Verordnungen}

\section{VI.II.I Sicherheit vor Naturgefahren als öffentliche Aufgabe}

Die Sicherheit vor Naturgefahren als öffentliche Aufgabe wird nach den Risikomanagementbereichen Prävention, Intervention bei ausserordentlichen Lagen und Wiederaufbau getrennt betrachtet. Im Präventionsbereich weist Art .19 des Waldgesetzes (WaG, SR 921.0) der Allgemeinheit eine ausdrückliche Verantwortung für die Sicherheit von Menschen und erheblichen Sachwerten vor Naturereignissen zu.

WaG Art. 19

Wo es der Schutz von Menschen oder erheblichen Sachwerten erfordert, sichern die Kantone die Anrissgebiete von Lawinen sowie Rutsch-, Erosions- und Steinschlaggebiete und sorgen für den forstlichen Bachverbau. Für die Massnahmen sind möglichst naturnahe Methoden anzuwenden.

Gemäss Botschaft zum Waldgesetz wird mit diesem Artikel klar dargelegt, dass der Schutz von Menschenleben und erheblichen Sachwerten vor Naturereignissen eine Aufgabe der öffentlichen Hand ist (BBI 1988 III, 199). Dieser Grundsatz gab in der parlamentarischen Beratung keinen Anlass zu Diskussionen (AB NR 1991 II 312, AB SR 1989 III 272).

Aufgrund dieser Bestimmung besteht Handlungsbedarf für den kantonalen Gesetzgeber und die Vollzugsbehörden. Viele Kantone delegieren diese Pflichten im Rahmen ihrer Organisationsautonomie weiter, leisten aber anteilsmässig Förderungsbeiträge an Massnahmen des Risikomanagements. Eine schuldhafte Vernachlässigung von Pflichten, die mit dem Waldgesetz den Kantonen übertragen werden, kann Haftungsfolgen haben (Jenni, 1993). 
Mit Art. 19 des Waldgesetzes vergleichbar verbindliche Formulierungen einer Handlungspflicht fehlen in anderen Gesetzen. Insbesondere lassen die entsprechenden Formulierungen des Wasserbaugesetzes (WBG, SR 721.100) mehr Interpretationsspielraum. Mit Art. 2 des WBG wird den Kantonen die Aufgabe des Hochwasserschutzes zugewiesen.

WBG Art. 2 Zuständigkeit

Der Hochwasserschutz ist Aufgabe der Kantone.

Gemäss Artikel 3 haben sie ausserdem den Hochwasserschutz zu gewährleisten.

Art. 3 Massnahmen

1 Die Kantone gewährleisten den Hochwasserschutz in erster Linie durch den Unterhalt der Gewässer und durch raumplanerische Massnahmen.

$2 \ldots$

Gewährleisten bedeutet: Dafür sorgen, eine Gewähr dafür sein, dass etwas sichergestellt, nicht gefährdet ist (Duden, 2006). Gemäss Darstellung in der Botschaft zum Wasserbaugesetz geht es in Art. 2 vor allem um die Klärung der Aufgabenverteilung zwischen Bund und Kantonen und in Art. 3 um die Darstellung einer Prioritätensetzung unter den Hochwasserschutzmassnahmen (BBI 1988 II 1389). Auch aus den amtlichen Bulletins lässt sich nichts entnehmen, das eine weitergehende Interpretation zuliesse (AB SR 1989 III 291, AB NR 1990 IV 1810).

Die Verantwortung der Allgemeinheit im Management von Naturrisiken wird durch die Pflicht zur Leistung von öffentlichen Beiträgen unterstrichen. Da die waldbaulichen und technischen Massnahmen in der Regel die Möglichkeiten der Privaten und Gemeinden übersteigen, leistet neben den Kantonen der Bund erhebliche Beiträge an die Kosten von Schutzmassnahmen (Art. 36; BBI 1988 III, 199). Förderbeiträge an Risikomanagementmassnahmen werden als Abgeltungen und Anspruchsubventionen verstanden. Die entsprechende Vorschrift ist in Art. 35 des Waldgesetzes (WaG, SR 921.0) festgehalten:

WaG Art. 36 Schutz vor Naturereignissen

${ }^{1}$ Der Bund gewährt [...] Abgeltungen an Massnahmen, die Menschen und erhebliche Sachwerte vor Naturereignissen schützen.

In ähnlicher Weise ist dasselbe in Art. 6 des Wasserbaugesetzes (WBG, SR 721.100) geregelt:

WBG Art. 6 Abgeltungen an wasserbauliche Massnahmen

${ }^{1}$ Der Bund fördert im Rahmen der bewilligten Kredite Massnahmen, die dazu dienen, Menschen und erhebliche Sachwerte vor den Gefahren des Wassers zu schützen.

$[\ldots]$

Die Waldverordnung (WaV, SR 921.01) präzisiert in Art. 39 die Bestimmungen zur Leistung öffentlicher Beiträge. Sie grenzt in Art. 39 Abs. 4 gleichzeitig den Umfang der staatlichen Verpflichtungen ein:

WaV Art. 39 Schutz vor Naturereignissen

[....]

${ }^{4}$ Keine Abgeltungen werden gewährt an:

a. Massnahmen, die zum Schutz von Neubauten in erheblich gefährdeten Gebieten erforderlich sind;

b. Massnahmen zum Schutz touristischer Anlagen, wie Seilbahnen, Skilifte, Skipisten oder Wanderwege, die sich ausserhalb des Siedlungsgebietes befinden.

Eine gleich lautende Einschränkung ist auch in Art. 2 Abs. 4 der Wasserbauverordnung (WBV, SR 721.100.1) formuliert. Diese Bestimmungen untermauern das Prinzip von Treu und Glauben, in dem ausgeschlossen wird, dass Risiken im Vertrauen auf staatliche Unterstützung bei der Risikoverminderung wissentlich geschaffen werden. Sie sind dahingehend bemerkenswert, dass eine bestimmte Art der Raumnutzung - nämlich touristische NutzunJosef Th. Hess: Schutzziele im Umgang mit Naturrisiken in der Schweiz, @ vdf Hochschulverlag 2011 
gen ausserhalb des Siedlungsgebietes - generell von öffentlichen Beiträgen an die Risikominderung ausgenommen wird.

Eine weitere Begrenzung der staatlichen Verantwortlichkeit ergibt sich aus den Pflichten des (risikobetroffenen) Beitragsempfängers in Art. 35 Abs. 1 des Waldgesetzes (WaG, SR 921.0).

\section{WaG Art. 35 Grundsätze \\ ${ }^{1}$ Förderungsbeiträge nach diesem Gesetz werden im Rahmen der bewilligten Kredite und unter der Vo- raussetzung gewährt, dass:}
a. $[\ldots]$
b. $[\ldots]$
c. der Empfänger eine Eigenleistung erbringt, die in einem angemessenen Verhältnis zu seiner wirtschaft- lichen Leistungsfähigkeit, den übrigen Finanzierungsquellen und der ihm zumutbaren Selbsthilfe steht;
d. Dritte, die Nutzniesser oder Schadenverursacher sind, zur Mitfinanzierung herangezogen werden;
e. $[\ldots]$

Umfangreiche Aufgaben zum Schutz der Bevölkerung und ihrer Lebensgrundlagen in ausserordentlichen Lagen werden dem Bund und den Kantonen durch Art. 4 bis 6 des Bundesgesetzes über den Bevölkerungsschutz und den Zivilschutz übertragen (BZG SR 520.1; Intervention):

\section{BZG Art. 4 Führungsorgane}

Die zuständigen Behörden bilden Führungsorgane für die folgenden Aufgabenbereiche:

a. Sicherstellung der Information der Bevölkerung über Gefährdungen, Schutzmöglichkeiten und Schutzmassnahmen;
b. Warnung und Alarmierung sowie Erteilung von Verhaltensanweisungen an die Bevölkerung;
c. Sicherstellung der Führungstätigkeit;
d. Koordination der Vorbereitungen und der Einsätze der Partnerorganisationen;
e. Sicherstellung einer zeit- und lagegerechten Bereitschaft sowie der personellen und materiellen Ver- stärkung des Bevölkerungsschutzes im Hinblick auf bewaffnete Konflikte.

Auch im Hinblick auf die Bewältigung ausserordentlicher Lagen obliegen den betroffenen Individuen Pflichten, so etwa die Pflicht, Anordnungen zu befolgen oder Schutzräume zu erstellen:

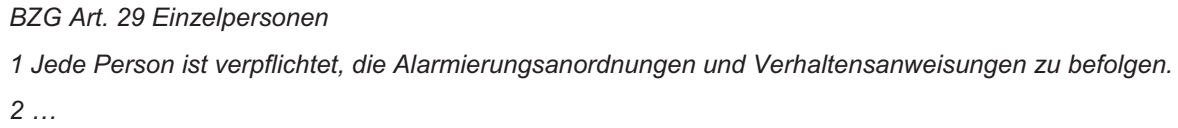

${ }^{1}$ Die Hauseigentümer und -eigentümerinnen haben beim Bau von Wohnhäusern, Heimen und Spitälern Schutzräume zu erstellen, auszurüsten und diese zu unterhalten.

${ }^{2}$ Die Gemeinden haben in Gebieten, in denen zu wenig Schutzplätze vorhanden sind, für ausgerüstete öffentliche Schutzräume zu sorgen.

${ }^{3}$ Die Kantone können Eigentümer und Eigentümerinnen sowie Besitzer und Besitzerinnen unbeweglicher und beweglicher Kulturgüter verpflichten, bauliche Massnahmen zu deren Schutz zu treffen oder zu dulden.

Kaum eigentliche Rechtspflichten des Bundes und der Kantone bestehen aufgrund von Bundesgesetzen für die Katastrophenhilfe und den Wiederaufbau nach Ereignissen. Jedoch sind solche Möglichkeiten in Gesetzen ausdrücklich vorgesehen, beispielsweise in der Verordnung über die militärische Katastrophenhilfe im Inland (VmKI, SR 510.123). 
VmKI Art. 2 Grundsatz

Katastrophenhilfe kann bei einem Ereignis geleistet werden, das so viele Schäden und Ausfälle verursacht, dass die Mittel und Möglichkeiten der betroffenen Gemeinschaft ausgeschöpft sind.

Ferner gelten die bereits im Zusammenhang mit ausserordentlichen Lagen erwähnten Bestimmungen in Art. 4 BZG auch für die Wiederaufbauphase.

Grosse Naturereignisse führen in der Regel auch zu erheblichen Schäden an Verbauungen. Diese können meist im Rahmen der Regelungen zur Förderung der Prävention nach WaG oder nach Art. 6 des Wasserbaugesetzes (WBG, SR 721.100) wiederhergestellt werden.

WBG Art. 6 Abgeltungen an Massnahmen des Hochwasserschutzes

${ }^{1}[\ldots]$

${ }^{2}$ Er (der Bund) leistet Abgeltungen namentlich für:

a. die Erstellung, die Instandstellung und den Ersatz von Schutzbauten und -anlagen;

b. $[\ldots]$

Häufig werden hiezu ausserhalb des ordentlichen Budgets Sonderkredite zur Verfügung gestellt. Die Wasserbau- und die Waldverordnung kennen ferner eine Bestimmung, die auf ausserordentliche Belastungen eines Kantons Rücksicht nimmt.

WBV Art. 2 Abgeltungen an wasserbauliche Massnahmen und WaV Art. 39 Schutz vor Naturereignissen

[...]

${ }^{3}$ Wird ein Kanton durch ausserordentliche Schutzmassnahmen, namentlich nach Unwetterschäden, erheblich belastet, so kann der Beitrag [...] ausnahmsweise auf höchstens 65\% der Kosten der Massnahme erhöht werden.

Im Zusammenhang mit Wiederherstellungen kommt den Versicherungen eine bedeutende Rolle zu. Die Elementarschadensversicherung ist weitgehend kantonal geregelt. Jedoch besteht eine bundesrechtliche Aufsichtspflicht über im Versicherungsgeschäft tätige Firmen, geregelt in Art. 3 des Bundesgesetzes vom 17. Dezember 2004, betreffend die Aufsicht über Versicherungsunternehmen (VAG, SR 961.01). Eine bemerkenswerte Bestimmung im Zusammenhang mit dieser Aufsichtspflicht ist die Vorschrift der kombinierten Feuer- und Elementarschadensversicherung gemäss Art. 33 Versicherungsaufsichtsgesetz (VAG, SR 961.01) und Art. 171 der Verordnung über die Beaufsichtigung von privaten Versicherungsunternehmen (AVO, SR 961.011).

\section{AVO Art. 171 Kombinierte Feuer- und Elementarschadenversicherung \\ ${ }^{1}$ Versicherungsunternehmen, die in der Schweiz gelegene Sachen (Fahrhabe und Gebäude) im Rahmen des Versicherungszweiges B8 gegen Feuer versichern, müssen diese auch zum Vollwert gegen Elemen- tarschäden versichern. \\ ${ }^{2}$ Die Versicherung ersetzt die in der Zerstörung, Beschädigung oder im Abhandenkommen versicherter Sachen bestehenden Elementarschäden.}

Damit wird sichergestellt, dass zusammen mit den - in den meisten Kantonen obligatorischen - Feuerversicherungen auch Elementarschadensversicherungen abgeschlossen werden. Die Aufsichtsverordnung in Art. 173 (AVO, SR 961.011) hält zudem fest, was unter den Begriff Elementarschäden gefasst wird.

AVO Art. 173 Versicherte Elementarschäden

${ }^{1}$ Elementarschäden sind Schäden, die durch Hochwasser, Überschwemmung, Sturm, Hagel, Lawinen, Schneedruck, Felssturz, Steinschlag oder Erdrutsch entstehen.

${ }^{2}$ Als Sturm gilt ein Wind von mindestens $75 \mathrm{~km} / \mathrm{h}$, der in der Umgebung der versicherten Sachen Bäume umwirft oder Gebäude abdeckt.

${ }^{3}$ Keine Elementarschäden sind:

a. Schäden, verursacht durch Bodensenkungen, schlechten Baugrund, fehlerhafte bauliche Konstruktion, mangelhaften Gebäudeunterhalt, Unterlassung von Abwehrmassnahmen, künstliche Erdbewe- 
gungen, Schneerutsch von Dächern, Grundwasser, Ansteigen und Überborden von Gewässern, das sich erfahrungsgemäss in kürzeren oder längeren Zwischenräumen wiederholt;

b. ohne Rücksicht auf ihre Ursache Schäden, die entstehen durch Wasser aus Stauseen oder sonstigen künstlichen Wasseranlagen, Rückstau von Wasser aus der Kanalisation oder Veränderungen der Atomstruktur;

c. Betriebs- und Bewirtschaftungsschäden, mit denen erfahrungsgemäss gerechnet werden muss, wie Schäden bei Hoch- und Tiefbauten, Stollenbauten, bei Gewinnung von Steinen, Kies, Sand oder Lehm;

d. Schäden durch Erschütterungen, welche ihre Ursache im Einsturz künstlich geschaffener Hohlräume haben;

e. Erschütterungen, welche durch tektonische Vorgänge in der Erdkruste ausgelöst werden (Erdbeben) und vulkanische Eruptionen.

Bemerkenswert ist dabei, dass beispielsweise Erdbebenschäden ausgeschlossen sind. Ferner setzt Art. 175 AVO Selbstbehalte und Art. 176 AVO Leistungsbegrenzungen fest, womit wiederum ein Bereich mit Eigenverantwortung definiert ist.

\section{VI.II.II Umfang der Verantwortlichkeit der Allgemeinheit}

Das Wasserbaugesetz (WBG, SR 721.100) bezweckt gemäss Art. 1 Abs. 1

WBG Art. 1

${ }^{1}$ [...] den Schutz von Menschen und erheblichen Sachwerten vor schädlichen Auswirkungen des Wassers, insbesondere vor Überschwemmungen, Erosionen und Feststoffablagerungen (Hochwasserschutz).

Das Waldgesetz (WaG, SR 921.0) enthält eine komplementäre Bestimmung

WaG Art. 1 Zweck

${ }^{2}$ Es (das Gesetz) soll ausserdem dazu beitragen, dass Menschen und erhebliche Sachwerte vor Lawinen, Rutschungen, Erosion und Steinschlag (Naturereignissen) geschützt werden.

Die beiden Gesetze regeln den Umgang mit allen relevanten gravitativen Naturgefahrenprozessen. Im Bereich der Wasserrisiken bestehen Überschneidungen. Massnahmen gegen andere Risiken sind in diesen Gesetzen nicht vorgesehen. Auf sämtliche Risiken bezieht sich dagegen das Bevölkerungs- und Zivilschutzgesetz (BZG SR 520.1). Es gelangt nur bei Notlagen zur Anwendung.

BZG Art. 2 Zweck

Zweck des Bevölkerungsschutzes ist es, die Bevölkerung und ihre Lebensgrundlagen bei Katastrophen und in Notlagen [...] zu schützen [...].

Schutzgüter des Wasserbau- (WBG, SR 721.100) und des Waldgesetzes (WaG, SR 921.0) sind Menschen und erhebliche Sachwerte. Das Wasserbaugesetz schliesst zudem die natürlichen Lebensgrundlagen ein.

WBG Art. 4 Anforderungen

${ }^{1}[\ldots]$

${ }^{2}$ Bei Eingriffen in das Gewässer muss dessen natürlicher Verlauf möglichst beibehalten oder wiederhergestellt werden. Gewässer und Ufer müssen so gestaltet werden, dass:

a. sie einer vielfältigen Tier- und Pflanzenwelt als Lebensraum dienen können;

b. die Wechse/wirkungen zwischen ober- und unterirdischen Gewässern weitgehend erhalten bleiben;

c. eine standortgerechte Ufervegetation gedeihen kann.

$[\ldots]$.

Das Bevölkerungs- und Zivilschutzgesetz (BZG SR 520.1) bezweckt, nebst der Bevölkerung auch deren Lebensgrundlagen zu schützen. 


\section{BZG Art. 2 Zweck}

Zweck des Bevölkerungsschutzes ist es, die Bevölkerung und ihre Lebensgrundlagen [...] zu schützen sowie zur Begrenzung und Bewältigung von Schadenereignissen beizutragen.

In allgemeiner Form begrenzt Art. 1 der Wasserbauverordnung (WBV, SR 721.100.1) die Pflichten der Allgemeinheit:

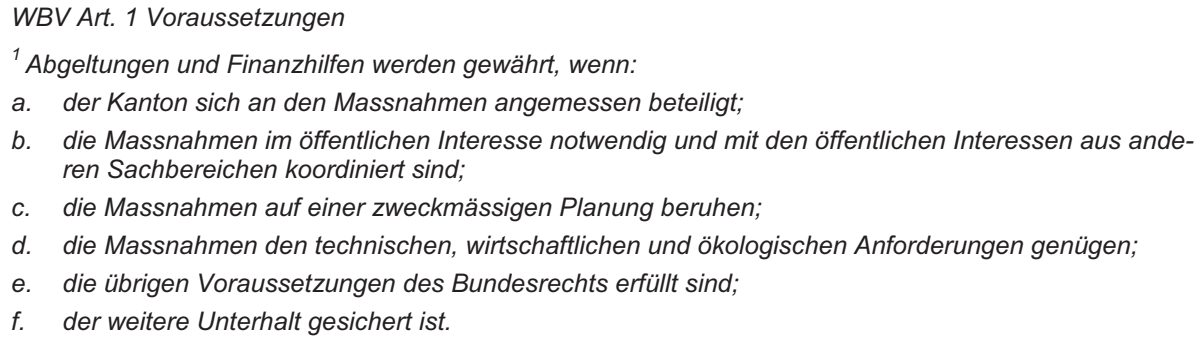

Diese Bedingungen ergeben sich auch aus den allgemeinen Prinzipien des Verwaltungsrechts sowie aus den besonderen Bestimmungen in Art. 10 des Subventionsgesetzes (SuG, SR 616.1).

Interpretationsbedarf besteht hinsichtlich des Begriffes der ,erheblichen Sachwerte' im WaG und im WBG. Dies gilt im Übrigen auch für das Bundesgesetz über die Raumplanung (RPG SR 700), welches den Begriff ,erheblich' in Art. 6 Abs. 2 lit. c ebenfalls enthält (BBI 1978 I 1018). Gemäss Duden bedeutet ,erheblich' beträchtlich, ins Gewicht fallend (Duden, 2006). Weder Gesetz noch Botschaft enthalten genauere Umschreibungen. Beim Wasserbaugesetz werde mit dieser Formulierung die geltende Praxis neu ausdrücklich im Gesetz erwähnt (BBI 1988 II 1389). Die Botschaft zum Waldgesetz (BBI 1988 III, 199) erläutert dazu, dass „Naturereignisse gleichermassen über den menschlichen Lebensraum und über Gebiete, die der Natur überlassen sind, hereinbrechen. Da die Natur vor solchen Ereignissen nicht zu schützen ist, wird klar zum Ausdruck gebracht, dass es nur um den Schutz von Menschenleben und erheblichen Sachwerten geht." Naturbelassene Gebiete sind somit ausdrücklich nicht Gegenstand des Schutzzieles. In gleicher Weise betont der Kommentar zum Waldgesetz (Jenni, 1993), es sei nicht das Ziel, Naturereignisse um jeden Preis zu verhindern. Sie erfüllen in der Natur auch ihre Aufgaben, indem sie beispielsweise verschiedenen Arten neuen Lebensraum verschaffen. Den Verordnungen zum Raumplanungsgesetz, zum Wasserbaugesetz, zum Waldgesetz sowie zum Bevölkerungs- und Zivilschutzgesetz können keine weiteren Präzisierungen zum Umfang der Verantwortlichkeit der Öffentlichkeit und somit zu den Schutzzielen entnommen werden.

\section{VI.II Ausführungsbestimmungen, Richtlinien, Weisungen, Empfehlungen auf Bundesebene}

Zur Präzisierung und operationellen Ausgestaltung der Schutzziele bestehen zahlreiche Ausführungsbestimmungen, Richtlinien, Empfehlungen und Weisungen des Bundes und der Kantone (Kap. 2.7.6.). Erwähnt seien in diesem Zusammenhang:

- Schutzziele für den Hochwasserschutz in folgenden Dokumenten: BWW: Anforderungen an den Hochwasserschutz (BWW, 1995), BWG: Hochwasserschutz an Fliessgewässern (BWG, 2001).

- Schutzziele für alle gravitativen Naturgefahren: BUWAL: Anleitungsentwurf zur Durchführung einer Risikoanalyse (Anhang zum Kreisschreiben Nr. 20, BUWAL, Eidg. Forstdirektion, 1994a), BUWAL: Risikoanalyse bei gravitativen Naturgefahren (Borter, 1999).

- Schutzziele für Erdbebensicherheit (begrenzt auf Bundesbauten): Beurteilung der Erdbebensicherheit bestehender Gebäude, Konzept und Richtlinien für die Stufe 3 (Duvernay, 2005), darin enthalten auch SIA Merkblatt 
2018: Überprüfung bestehender Gebäude bezüglich Erdbeben (SIA, 2004) mit Schutzzielen.

Sämtliche Schutzzielvorschläge kennen eine Abstufung nach Art und Intensität der Raumnutzung. Zum Naturgefahrenmanagement bei touristischen Aktivitäten bestehen verschiedene Richtlinien und Weisungen mit vorwiegend präskriptivem Charakter, die kaum explizite Schutzziele beinhalten, z. B.:

- SKUS-Richtlinien für Anlage, Betrieb und Unterhalt von Schneesportabfahrten (SKUS, 2006),

- SKUS-Richtlinien für Skifahrer und Snowboarder (SKUS, 2006),

- Richtlinie Naturgefahren bei Fuss- und Wanderwegen Kt. Bern - Aufgaben und Verantwortlichkeiten der Gemeinden (NAGEF Bern, 2002).

Längere Praxis besteht bei Weisungen über Schutzziele im Technischen Sicherheitsrecht und im Umweltrecht (Kap. 2.7.). Hier dominieren präskriptive Regelungen ohne explizite Schutzzielformulierungen. Bezüglich ausdrücklicher Schutzziele erwähnenswert sind jedoch das Handbuch zur Störfallverordnung (BUWAL, 1991) oder die ,Weisungen über das Sicherheitskonzept für den Umgang mit Munition und Explosivstoffen in Armee und Militärverwaltung', welche 1991 durch das Departement für Verteidigung, Bevölkerungsschutz und Sport in Kraft gesetzt wurden (Bienz, 2000).

\section{VI.IV Einschlägige Bestimmungen kantonaler Gesetze}

Die Bundesgesetzgebung überträgt Verantwortung und Zuständigkeit für den Umgang mit Naturgefahren zu einem erheblichen Teil an die Kantone. Diese haben ihrerseits Regelungen zum Umgang mit Naturgefahren getroffen. Das kantonale Recht stellt somit den bedeutenderen Teil des Naturgefahrenrechts dar. Die nachfolgenden Ausführungen basieren auf einer Analyse ausgewählter Gesetzeserlasse der Kantone BE, LU, UR, SZ, OW, NW, GL, BS, BL, AR, AI, SG, GR und VS.

\section{VI.IV.I Verantwortlichkeit der Allgemeinheit oder Eigenverantwortung der Betrof- fenen?}

Am umfangreichsten sind die Vorschriften im Bereich der (finanzintensiven) technischen Gefahrenprävention. In einzelnen Kantonen sind vor allem wasserbauliche Präventionsaufgaben vollumfänglich den Gemeinwesen übertragen. So sind nach Art. 11 des Wasserbaugesetzes des Kantons Uri (Urner Rechtsbuch No. 40.1211) Bau und Ausbau öffentlicher Gewässer in der Regel Sache des Kantons. Mehrheitlich Sache öffentlicher Gemeinwesen sind in Uri auch Verbauungen nach Art. 17 der kantonalen Waldverordnung (Urner Rechtsbuch No. 40.2111).
KWV UR Art. 17 Schutz vor Naturereignissen, Schutzmassnahmen
${ }^{1}$ Wo es der Schutz von Menschen oder erheblichen Sachwerten erfordert, sichern der Kanton, die Korpo- rationen und die Gemeinden die betroffenen Gebiete vor Lawinen, Rutschungen, Erosion, Steinschlag und ähnlichen Gefahren.

Auch wenn Gemeinwesen für Präventionsmassnahmen zuständig sind, wie beispielsweise auch im Gewässergesetz des Kantons Zug (BGS 731.1) geregelt, schliesst dies eine Mitbeteiligung der betroffenen Anstösser nicht aus, vgl. § 75 des Gewässergesetzes des Kantons ZG: 


\section{GewG ZG § 75}

Private Gewässer 1. Klasse

a) ausserhalb des Waldes

${ }^{1}$ Die Kosten der wasserbaulichen Massnahmen an privaten Gewässern 1. Klasse ausserhalb des Waldes werden neben den Gemeinden auch den Grundeigentümerinnen und Grundeigentümern des Gewässerraumes sowie den Abwasser einleitenden Gemeinwesen auferlegt.

Gemäss verschiedenen kantonalen Wasserbaugesetzen besteht eine Duldungspflicht, so etwa in den Kantonen Bern (BSG 751.11), Zug (BGS 731.1) oder Obwalden (GDB 740.1):

WBG OW Art. 14 Duldungspflichten

${ }^{1}$ Wer an ein öffentliches oder privates Gewässer anstösst, muss dulden, dass Dritte das Grundstück betreten, befahren oder sonst benutzen, um am Gewässer Sofortmassnahmen zur Gefahrenabwehr, Wasserbau, Unterhalt oder Kontrollen vorzunehmen.

Zahlreiche Regelungen lassen Raum für die Übernahme der Verantwortlichkeit einerseits durch Gemeinwesen oder anderseits durch Einzelne oder Organisationen von Betroffenen, beispielsweise $\S 8$ der kantonalen Verordnung zum Bundesgesetz über den Wald im Kanton SZ (SRSZ 313.110):

${ }^{1}$ Die Ausführung von Massnahmen zum Schutz von Menschen oder erheblichen Sachwerten vor Naturereignissen (Art. 19 WaG) obliegt den Grund- und Werkeigentümern und, soweit es die Verhältnisse erfordern, den betroffenen Gemeinden.

Auch Art. 7 des Obwaldner Wasserbaugesetzes (GDB 740.1) sieht eine primäre Verantwortung der Wuhrgenossenschaften mit subsidiärer Zuständigkeit der Gemeinden vor:

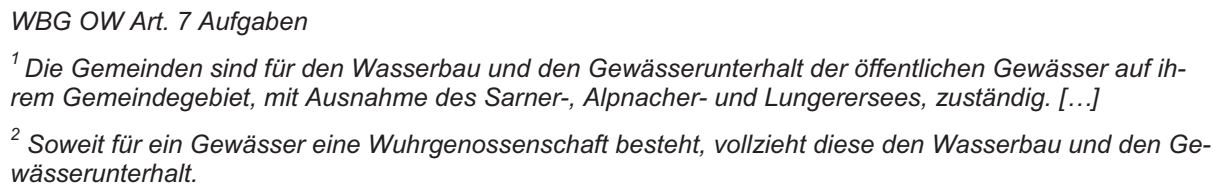

Besonders bevorteilte Dritte werden nach zahlreichen Bestimmungen in die Pflicht genommen. Es handelt sich dabei oft um Betreiber von Anlagen wie Bahnen oder Kraftwerke. So gelangt im Kanton Uri bei überwiegendem Drittinteresse Art. 23 WBG UR (Urner Rechtsbuch No. 40.1211) zur Anwendung:

WBG UR Art. 23 Unterhaltspflicht des Verursachers

${ }^{1}$ Wasserbauliche Schutzanlagen, die im überwiegenden Interesse eines Dritten erstellt worden sind, hat der Verursacher zu unterhalten.

${ }^{2}[\ldots]$.

Art. 31 des bernischen Kantonalen Waldgesetzes (BSG 921.11) überträgt die Verantwortlichkeit der Sicherheit von Anlagen den jeweiligen Betreibern, dies mit Ausnahme der Wanderwege:

\section{KWaG BE Art. 31}

3. Anlagebetreiber

${ }^{1}$ Die Betreiberinnen und Betreiber von Anlagen wie Strassen, Bahnen und anderen Transportanlagen oder Kraftwerken sind dafür verantwortlich, dass vorsorgliche Massnahmen für die Sicherheit der Benützerinnen und Benützer vor Naturereignissen im Sinne von Artikel 28 Absatz 1 getroffen werden.

${ }^{2}$ Die Walderschliessungs- und Wanderwege sind von diesen Massnahmen ausgenommen.

Gleichlautend sind auch die Bestimmungen in Art. 24 des Nidwaldner Einführungsgesetzes zum Bundesgesetz über den Wald (NG 831.1). Auch Art. 26 der Strassenverordnung des Kantons Obwalden (GDB 720.11) rechnet die Verantwortlichkeit für die Strassensicherheit dem Strasseneigentümer zu, auch wenn der Ursprung des Gefahrenprozesses auf einem Nachbargrundstück liegt. 
Nach verschiedenen - meist älteren - Gesetzen obliegt die Verantwortlichkeit für den technischen Gefahrenschutz den Betroffenen oder Organisationen von Betroffenen. Dies gilt beispielsweise in Kantonen, deren Gesetze lediglich Förderungsbestimmungen oder eine Klausel enthalten, wonach Gefahren zu vermindern seien. Beispiele sind etwa die Forstverordnung des Kantons OW (GDB 930.11), das Forstgesetz des Kantons VS (GS/VS 921.1) oder die Waldgesetzgebung der Kantone BL (SGS 570) und BS (SG 911.61). Ausdrücklich formuliert ist die Zuständigkeit der Betroffenen im Wasserrechtsgesetz des Kantons Schwyz (SRSZ 451.100):

\section{Wasserrechtsgesetz SZ}

$\S 45$ 4. Lastenverteilung

a) Grundsatz

Die Ausführung von Massnahmen im Sinne von § 44 dieses Gesetzes und der Unterhalt obliegen bei öffentlichen und privaten Gewässern grundsätzlich den bisher pflichtigen Grundeigentümern oder Belasteten.

\section{$\S 46$ b) Ausdehnung des Pflichtenkreises}

${ }^{1}$ Übersteigen die Aufwendungen für den Unterhalt und die Verbauung von Bächen und Flüssen die Kräfte der Pflichtigen oder stehen sie in keinem Verhältnis zum Wert oder Ertrag der belasteten Grundstücke, so kann der Pflichtenkreis auf weitere Grundeigentümer und die Träger von privaten oder öffentlichen Werken und Anlagen, für welche die Verbauung mittelbar oder unmittelbar von Vorteil oder Interesse ist, ausgedehnt werden.

${ }^{2}$ In den Pflichtenkreis sind insbesondere jene Liegenschaften einzubeziehen, von denen dem zu verbauenden Gewässer Wasser zufliesst.

\section{§ 47 c) Verteilungsgrundsätze}

${ }^{1}$ Bei der Verteilung der Lasten innerhalb des Pflichtenkreises sind der Wert der belasteten Sache, die bestehenden Wuhrpflichten, die Gefahren sowie die Vorteile und Interessen zu berücksichtigen.

${ }^{2}$ Für ein Grundstück, das in mehrere Bachperimeter einbezogen ist und deshalb im Verhältnis zu seinem Wert oder Ertrag unzumutbar belastet wird, ist die Schatzung in jedem Perimeter angemessen, jedoch höchstens auf die Hälfte herabzusetzen.

In der Regel sind auch in Fällen, in denen die Zuständigkeit den Betroffenen übertragen ist, in erheblichem Mass öffentliche Beiträge zur Erfüllung der Aufgaben vorgesehen. Faktisch ergibt sich damit hinsichtlich der Lastenverteilung für die Aufgaben zur Minderung von Naturrisiken eine Annäherung zwischen den verschiedenen Varianten der Zuständigkeit.

Rechtlich besteht jedoch ein bedeutender Unterschied zwischen Fällen, in denen das Gesetz Aufgaben einem Gemeinwesen oder Privaten förmlich überträgt und jenen, in denen das Gesetz nur eine Förderung vorsieht. Nur wenn die Verantwortlichkeit dem Gemeinwesen übertragen ist, können die Massnahmen als staatliches Handeln bezeichnet werden, zu dem die Gemeinwesen in gewisser Hinsicht auch verpflichtet sind.

In fast allen einschlägigen kantonalen Gesetzen ist der raumplanerische Umgang mit Naturgefahren den Gemeinden zugewiesen, so beispielsweise in der Waldverordnung des Kantons Bern (BSG 921.111):
KWaV BE Art. 39
Aufgaben der Gemeinden
1. Vorsorge
${ }^{1}$ Die Gemeinden wachen im Siedlungsgebiet mit Hilfe des Gefahrenkatasters, der Gefahrenhinweiskarte und anderen vorhandenen Grundlagen, Beobachtungen oder Hinweisen über das Auftreten und die Ent- wicklung einer Gefährdung durch Naturereignisse und ordnen die erforderlichen Massnahmen an.
${ }^{2}$ Wo für das Siedlungsgebiet erkennbare Naturgefahren bestehen, errichten sie eine Gefahrenkarte, aus der die Naturgefahren sowie die daraus entstehenden Risiken für Menschen und erhebliche Sachwerte ersichtlich sind.
${ }^{3}$ Sie berücksichtigen die Gefahrenkarten sowie andere Grundlagen gemäss Absatz 1 bei der Nutzungs- planung, bei der Erteilung von Baubewilligungen und allen anderen raumwirksamen Tätigkeiten.

Josef Th. Hess: Schutzziele im Umgang mit Naturrisiken in der Schweiz, @ vdf Hochschulverlag 2011 


\footnotetext{
${ }^{4}$ Sie sorgen dafür, dass neue und bestehende Bauten und Anlagen bezüglich ihres Standortes, der Nutzungsart oder der Bauweise soweit zumutbar den vorhandenen Naturgefahren angepasst werden, gegebenenfalls durch Verlegung an sichere Orte.
}

Die Realisierung von Objektschutzmassnahmen fällt - meist basierend auf baupolizeilichen Bestimmungen (vgl. hiezu Art. 48 des Baugesetzes vom 12. Juni 1994, Kt. OW, BauG, GDB 710.1); in die Pflicht der Betroffenen, indem solche Massnahmen bei Neu- und Umbauten in Form von Bauauflagen verfügt werden. Vereinzelt werden solche Objektschutzmassnahmen neuerdings durch Beiträge kantonaler Gebäudeversicherungen unterstützt. So beteiligte sich die Nidwaldner Sachversicherung NSV gleichzeitig mit der Schadensbehebung nach dem Unwetter 2005 finanziell an präventiven Massnahmen, um künftige Schäden zu verhindern oder zumindest zu vermindern. Wenn die NSV das Präventionskonzept genehmigt hat, leistet sie einen Beitrag von $20 \%$ an die Kosten, im Maximum jedoch CHF 5'000 (Nidwaldner Sachversicherung, 2006).

Im Bereich der Intervention werden, wie durch die Bundesgesetze, durch verschiedene kantonale Gesetze weitreichende Verantwortlichkeiten an die Gemeinwesen, vor allem die Gemeinden übertragen. Beispielsweise hält das Gesetz über den Bevölkerungsschutz und den Zivilschutz des Kantons Schwyz (SRSZ 521.100) fest:

\title{
IVII. Bevölkerungsschutz SZ
}

\section{$\S 4$ 1. Aufgaben des Kantons}

a) Im Allgemeinen

${ }^{1}$ Der Kanton stellt den Schutz der Bevölkerung und ihrer Lebensgrundlagen bei Katastrophen und in Notlagen sowie im Falle bewaffneter Konflikte sicher und trägt zur Begrenzung und Bewältigung von Schadenereignissen bei, soweit die Gemeinden dazu nicht in der Lage sind.

${ }^{2}$ Er unterstützt die Gemeinden bei der Erfüllung ihrer Aufgaben.

${ }^{3}$ Er stellt für Katastrophen, Notlagen und bewaffnete Konflikte die notwendigen Sach- und Personenmittel für die medizinische und psychologische Versorgung der Betroffenen bereit.

\section{$\S 7$ 2. Aufgaben der Gemeinden}

${ }^{1}$ Die Gemeinden schützen die Bevölkerung und ihre Lebensgrundlagen bei Katastrophen, Notlagen und in bewaffneten Konflikten mit den dafür aufgebauten Einsatzkräften. Sie stellen mit einem Führungsstab die Führung und Kommunikation sicher.

Nebst diesen Bestimmungen ergibt sich die Verantwortlichkeit der Allgemeinheit vor allem hinsichtlich Interventionsmassnahmen aus geschriebenen oder ungeschriebenen Polizeigeneralklauseln, stellvertretend für viele Art. 24 der Obwaldner Kantonsverfassung (GDB 101):

\author{
Kantonsverfassung OW Art. 24 A. Schutz der öffentlichen Ordnung \\ Kanton und Gemeinden sorgen für die Aufrechterhaltung der Ruhe, Ordnung, Sicherheit und Sittlichkeit.
}

Im Bereich der Wiederherstellungen gilt der Grundsatz, dass der Geschädigte seinen Schaden selber zu tragen hat (casum sentit dominus) und diesen nur auf Dritte abwälzen kann, wenn dies gesetzlich oder durch einen Vertrag so vorgesehen ist. Die Rechtsordnung ermöglicht allerdings staatliche Hilfeleistungen (kantonales Landwirtschaftsgesetz vom 16. Juni 1997, Kt. Bern, KLwG, BSG 910.1) oder sieht diese in Notlagen sogar verbindlich vor (z. B. der Nothilfefonds gemäss Art. 24 des Gesetzes über die Vergütung nicht versicherbarer Elementarschäden vom 23. September 1984 (BR 835.100) oder das Gesetz über die Staatshilfe bei Elementarschäden des Kantons Uri (Urner Rechtsbuch No. 40.1401)).

\section{Staatshilfe bei Elementarschäden UR Art. 1 Grundsatz}

Wenn ein Kantonseinwohner durch ausserordentliche Naturereignisse auf Kantonsgebiet in Not gerät, hat er Anspruch auf Staatshilfe innert den Grenzen des gegenwärtigen Gesetzes. 


\begin{abstract}
Art. 6 Bedürftigkeitsprinzip
${ }^{1}$ Ein Hilfsanspruch entsteht nur insoweit, als der Anspruchsberechtigte bzw. seine Familiengemeinschaft durch das Schadenereignis in Notlage versetzt wird.

${ }^{2}$ Massgebend für die Beurteilung der Notlage sind: die Höhe des Schadens, die finanzielle Lage des Geschädigten sowie dessen Familien- und Verdienstverhältnisse.
\end{abstract}

Die meisten Elementarschadensversicherungen decken Schäden an Gebäuden und Mobiliar. Schäden an Boden und Kulturen können nicht versichert werden (nicht versicherbare Elementarschäden). Deshalb bestehen in verschiedenen Kantonen gesetzlich geschaffene Fonds, etwa die Elementarschadenskasse des Kantons Graubünden (BR 835.100) oder der Nidwaldner Hilfsfonds als selbständige Anstalt des öffentlichen kantonalen Rechts. Dem Hilfsfondsgesetz (NG 867.3) sind die meisten Grundstücke unterstellt (Art. 1). Grundstücke, die infolge ihres Standortes einer besonderen Gefährdung ausgesetzt sind, können ausgeschlossen werden (Art. 16). Der Hilfsfonds wird unter anderem durch eine jährliche Abgabe geäufnet (Art. 17). Nicht versicherbare Elementarschäden werden bis zu $60 \%$ aus dem Hilfsfonds vergütet (Art. 29). Wo Hilfsfonds oder Elementarschadenskassen fehlen, sind Geschädigte auf freiwillige Leistungen des Fonds für Hilfe bei nicht versicherbaren Elementarschäden oder von Hilfswerken angewiesen. Sowohl die Gesetze, die eine Staatshilfe vorsehen, als auch Regelungen in Hilfsfondsgesetzen setzen eine zumutbare Schadenswehr des Betroffenen voraus, vgl. z. B. Gesetz über die Staatshilfe bei Elementarschäden des Kantons Uri (Urner Rechtsbuch No. 40.1401):

Staatshilfe bei Elementarschäden UR Art. 4 Objektive Voraussetzungen

${ }^{1}$ Die Hilfe wird gewährt bei Elementarschäden an Grundstücken, die hervorgerufen sind durch Naturereignisse, deren sich der Geschädigte nicht oder nicht genügend erwehren konnte [...].

Verschiedene Regelungen sehen vor, dass bei grossen Ereignissen ausserordentliche Beiträge gewährt werden können, so etwa die Wasserbaugesetze der Kantone Uri (Urner Rechtsbuch No. 40.1211), Appenzell-Innerrhoden (Nr. 711) oder St. Gallen (sGS 734.11).

Eine entscheidende Rolle bei der Wiederherstellung spielen die Versicherungen. 17 Kantone verfügen über eine kantonale Gebäudeversicherungsanstalt mit entsprechenden gesetzlichen Regelungen (z. B. Gesetz über die Gebäudeversicherung im Kanton Graubünden vom 12. April 1970, BR 830.1). In sechs Kantonen ist das Gebäudeversicherungswesen privatrechtlich geregelt. Meist besteht auch in diesen Kantonen eine gesetzliche Versicherungspflicht (z. B. geregelt im Schätzungs- und Grundpfandgesetz des Kantons Obwalden (GDB 213.7)).
Art. 21 Versicherungspflicht
1 Der Eigentümer oder die Eigentümerin eines Gebäudes ist verpflichtet, dieses bei einer in der Schweiz konzessionierten Versicherungsgesellschaft mindestens zum Zeitwert gegen Feuer- und Elementarscha- den zu versichern.
${ }^{2}$ Die kantonale Steuerverwaltung kann bei Schätzungen in die Versicherungspolicen Einsicht nehmen.

\title{
VI.IV.II Umfang der Verantwortlichkeit der Allgemeinheit
}

Die Inkraftsetzung des Waldgesetzes (WaG, SR 921.0) und des Wasserbaugesetzes (WBG, SR 721.100) führte zu zahlreichen Revisionen kantonaler Anschlussgesetze. Diese neueren Gesetze folgen in der Umschreibung der Aufgaben meist den Formulierungen der Bundesgesetzgebung in Art. 19 des Waldgesetzes (SR 921.0) oder Art. 1 bis 3 des Wasserbaugesetzes (WBG, SR 721.100). Beispiele hiezu sind die waldrechtlichen Bestimmungen der Kantone Graubünden (BR 920.100), Bern (BSG 921.11), Nidwalden (NG 831.1), Luzern (SRL 945) oder Basel-Stadt (SG 911.6). Festzuhalten ist, dass unter den Massnahmen zur Verminderung von Naturrisiken immer auch Massnahmen zur Erhaltung und Förderung von Schutzwäldern enthalten sind. Als Beispiel diene Art. $23 \mathrm{f}$ der Verordnung zum kantonalen Waldgesetz des Kantons Appenzell-Ausserrhoden (bGS 931.11): 


\section{Schutz vor Naturereignissen}

Kant. Waldverordnung AR Art. 23 Wald mit besonderer Schutzfunktion

${ }^{1}$ Wälder, welche in Gebieten mit drohenden Naturgefahren wegen ihrer Lage massgebend zum Schutz von Menschen oder erheblichen Sachwerten beitragen, werden als Wald mit besonderer Schutzfunktion bezeichnet.

${ }^{2}$ Die kantonale Waldplanung hält ihre Lage fest.

Art. 24 Sicherung von Gefahrengebieten

Wo der Wald keine hinreichende Schutzwirkung mehr zu gewährleisten vermag, sind geeignete Massnahmen zur Förderung und Erhaltung seiner besonderen Schutzfunktion zu treffen.

In einer Reihe von Kantonen bestehen lediglich Förderungsbestimmungen für Massnahmen zur Verminderung von Naturrisiken, so etwa in der Forstverordnung des Kantons Obwalden (GDB 930.11) oder im Forstgesetz des Kantons Wallis (GV/VS 921.1). Einzelne Kantone umschreiben die Aufgaben der Öffentlichkeit zur Sicherheit vor Naturgefahren detaillierter, so etwa Art. 17 der Waldverordnung des Kantons Uri (Urner Rechtsbuch No. 40.2111) und Art. 3 des Wasserbaugesetzes des Kantons Uri (Urner Rechtsbuch No. 40.1211):

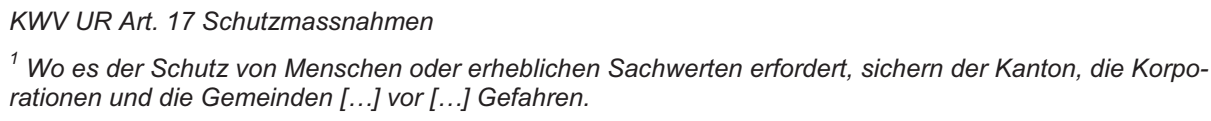

Sie unterstützen dieses Ziel durch eine dauernde Pflege und sinnvolle Nutzung des Waldes. Der erforderliche Aufwand und der zu erwartende Nutzen sind gegeneinander abzuwägen.

${ }^{2}$ Der Regierungsrat sorgt für eine integrale Planung der Schutzmassnahmen. Er richtet einen Frühwarndienst ein.

WBG Art. 3 Grundsatz, Berücksichtigung anderer öffentlicher Interessen

${ }^{1}$ Die Gewässer sind so auszubauen und zu unterhalten, dass Wasser, Geschiebe und Schnee abfliessen können und eine Gefährdung von Bauwerken und genutztem Boden möglichst vermieden werden kann. Dabei sind der erforderliche Aufwand und der zu erwartende Nutzen abzuwägen.

${ }^{2}$ Alle Massnahmen des Wasserbaues und des Gewässerunterhaltes sind auf die Verhältnisse im Einzugsgebiet und dessen Sanierungsmöglichkeit abzustimmen.

${ }^{3}$ Wasserbau und Gewässerunterhalt haben auf andere öffentliche Interessen möglichst Rücksicht zu nehmen.

Auch Art. 41 Abs. 1 der bernischen kantonalen Waldverordnung (BSG 921.111) sieht eine Pflicht zur Ausführung von Massnahmen nur vor, soweit diese zumutbar sind.

Diese Regelungen grenzen die Pflichten der Allgemeinheit ein, indem die Verhältnismässigkeit (Aufwand/Nutzen) und andere öffentliche Interessen zu wahren sind.

Ähnliche Bestimmungen enthält auch Art. 15 des Wasserbaugesetzes des Kantons Bern (BSG 751.11). Dieses Gesetz erwähnt in Art. 7 ausdrücklich die Inkaufnahme einer Gefährdung:

WBG BE Art. 7 Wasserbau

${ }^{1}[\ldots]$

${ }^{2}$ Wird die Gefährdung von Sachwerten in Kauf genommen, sind im Wasserbauplan Überflutungsgebiete zu bezeichnen, sofern
a) keine Menschen gefährdet sind und
b) keine grossen Schäden zu befürchten sind.
[...]

Diese Regelung ist bedeutend im Zusammenhang mit Art. 39 desselben Gesetzes: WBG BE Art. 39 Entschädigung in Überflutungsgebieten

\subsection{Entschädigungen in Überflutungsgebieten}

Josef Th. Hess: Schutzziele im Umgang mit Naturrisiken in der Schweiz, @ vdf Hochschulverlag 2011 


\footnotetext{
${ }^{1}$ Im Überflutungsgebiet gemäss Wasserbauplan hat der Berechtigte Anspruch auf eine angemessene Entschädigung oder auf einen Beitrag an höhere Versicherungsprämien. Der Regierungsrat bestimmt die Ansätze der Entschädigungen und Beiträge.

${ }^{2}$ Der Grosse Rat stellt die erforderlichen Mittel im Voranschlag ein.

${ }^{3}$ Der Regierungsrat hat die Finanzkompetenz zur Ausrichtung der Entschädigung. Die Delegation von Ausgabenbefugnissen nach Finanzhaushaltgesetz bleibt vorbehalten.

${ }^{4}$ Der Regierungsrat kann die Schadenregulierung der Direktion für Bau, Verkehr und Energie oder Dritten übertragen.
}

Art. 35 Der Wasserbauverordnung (BSG 751.111.1) präzisiert Grundsätze zur Bemessung der Entschädigung und Verfahrensfragen:

WBV BE Art. 35 Überflutungsschäden

${ }^{1}$ Die Festsetzung der Ansätze für die angemessene Entschädigung der Überflutungsschäden erfolgt unter Berücksichtigung des Erntewerts der üblichsten Kulturen, der Ernteerschwerungen, der Ersatzkultur und der Wiederherstellung des ursprünglichen Zustandes. Sie erfolgt zusammen mit der Festsetzung der Beiträge an höhere Versicherungsprämien. (Fassung vom 22. 8. 2001)

[...]

Hinsichtlich Aufgaben der Öffentlichkeit ist diese Regelung insofern bemerkenswert, dass sich eine Verantwortlichkeit der Allgemeinheit auch auf Gebiete und Betroffene mit vergleichsweise geringem Schadenspotenzial erstreckt.

Sehr weitgehend ist auch die Formulierung des Wasserbaugesetzes des Kantons St. Gallen (sGS 734.11):

Wasserbaugesetz SG Art. 2 Massnahmen a) Grundsatz

${ }^{1}$ Die Gewässer sind so zu unterhalten und auszubauen, dass das Wasser ungehindert abfliessen und eine Gefährdung von Bauwerken und genutztem Boden vermieden werden kann.

Wasserbaugesetz SG Art. 34. Gewässerausbau Gegenstand

${ }^{1}$ Der Ausbau der Gewässer soll einen hinreichenden Abfluss gewährleisten, Sohle und Ufer sichern sowie einer geregelten Geschiebeführung und dem Schutz der Umgebung vor Überflutung dienen.

${ }^{2}[\ldots]$

Gefährdungen oder Überflutungen sollen demnach vermieden werden. Ähnlich absolut kann die Regelung im Wasserrechtsgesetz des Kantons Schwyz (SRSZ 451.100) verstanden werden:

Wasserrechtsgesetz SZ § 44 3. Sicherungsmassnahmen

${ }^{1}$ Wo die Beibehaltung des natürlichen Zustandes von privaten und öffentlichen Gewässern die Gefahr von Überschwemmungen, Erdrutschen oder andern Schäden für die Allgemeinheit mit sich bringt, sind sie durch Korrektion, Verbauung oder Aufforstung zu sichern.

Jedoch setzen auch hier andere öffentliche Interessen gewisse Grenzen:

${ }^{2}$ Dabei ist dem öffentlichen Interesse an der Erhaltung der natürlichen Wasservorräte und des Gleichgewichts des Wasserhaushaltes sowie dem Schutz des Landschaftsbildes und des Fischbestandes angemessen Rechnung zu tragen.

In Anlehnung an die Bundesgesetzgebung werden in den meisten wasserbaulichen Regelungen auch ökologische Zielsetzungen ausdrücklich erwähnt, so etwa im Berner Wasserbaugesetz, im Obwaldner Wasserbaugesetz, im Luzerner Wasserbaugesetz oder in $\S 43$ des Schwyzer Wasserrechtsgesetzes (SRSZ 451.100).

Wasserrechtsgesetz SZ § 43 2. Erhaltung und Wiederherstellung des natürlichen Zustandes

${ }^{1}$ Behörden und Amtsstellen des Kantons, der Bezirke und Gemeinden sorgen dafür, dass öffentliche und private Gewässer als Lebensräume für einheimische Tier- und Pflanzenarten sowie als Landschaftselemente erhalten und wenn möglich verbessert werden. 
${ }^{2}$ Kanton und Bezirke unterstützen und fördern Massnahmen, die der Revitalisierung eines Fliessgewässers dienen oder einen naturnahen Hochwasserschutz darstellen.

\section{VI.IV.III Schutzziele in den Kantonen}

Auch einzelne Kantone haben schliesslich in den letzten 15 Jahren Schutzzielschemen und -darstellungen entwickelt. Diese Regelungen sind teilweise in enger Anlehnung an die entsprechenden Dokumente des Bundes entstanden. Weisungen, Richtlinien und Reglemente des Bundes und der Kantone entstanden meist koordiniert und inspirierten sich gegenseitig.

Einige bedeutende Beispiele kantonaler Festlegungen zu Schutzzielen sind nachstehend aufgeführt.

- Schutzziele für den Hochwasserschutz in folgenden Dokumenten:

- Kanton Uri: Richtlinie für den Hochwasserschutz (1992)

- Kanton Nidwalden: Schutzziele für die Engelbergeraa (1992)

- Schutzziele für gravitative Naturgefahren:

- Kanton Glarus: Richtlinien zum Schutz vor Naturgefahren (2001)

- Kanton Luzern: Naturgefahren im Kanton Luzern (2003)

- Kanton Graubünden: Risikomatrix (2003)

- Schutzziele für die Erdbebensicherheit: teilweise Verbindlichkeitserklärung der SIA-Normen

Im Gegensatz zu anderen Naturgefahren ist vor allem bei Erdbeben der Verweis auf die anerkannten Regeln der Baukunst, namentlich die SIA-Norm 261, gebräuchlich. 


\section{Schutzzielrelevante Gerichtsurteile}

\section{VII.I Subjektive Voraussetzungen einer Handlungspflicht}

\section{VII.I.I Handlungen des Staates bei der Erfüllung öffentlicher Aufgaben}

\section{Norm als Voraussetzung für eine Handlungspflicht zum Schutz von Risikobetroffenen}

Nach Rechtsprechung und herrschender Lehre liegt der Haftungsnorm des Art. 41 OR wie der Staatshaftung die objektive Widerrechtlichkeitstheorie zugrunde (Thun, Hochwasser, VGE 21657 E. 3.3). Danach ist eine Schadenszufügung widerrechtlich, wenn sie gegen eine allgemeine gesetzliche Pflicht verstösst, indem entweder ein absolutes Recht (wie Leib, Leben, Eigentum, Persönlichkeitsrecht) der geschädigten Person beeinträchtigt (Erfolgsunrecht) oder eine reine Vermögensschädigung durch Verstoss gegen eine einschlägige Schutznorm bewirkt wird (Verhaltensunrecht). Die im objektiven Normverstoss begründete Widerrechtlichkeit entfällt, wenn eine Schädigung reiner Vermögensrechte stattgefunden hat, dabei jedoch keine Verhaltensnorm verletzt worden ist, die nach ihrem Zweck vor derartigen Schädigungen schützen soll (Thun, Hochwasser, VGE 21657 E. 3.3).

Es besteht keine allgemeine Rechtspflicht, im Interesse anderer tätig zu werden (Thun, Hochwasser, VGE 21657 E. 3.3; VGE 21240 E. 2d). Daher hängt die Widerrechtlichkeit bei einer Unterlassung auch dann, wenn absolute Rechte beeinträchtigt worden sind, davon ab, ob eine besondere Rechtspflicht zum Handeln besteht, die sich aus einer Schutznorm ergibt (Thun, Hochwasser, VGE 21657 E. 3.3; Mörel, vereiste Strasse, BGE 98 II 40, E. 1; GenfCointrin, Baustellenunfall, BGE 90 IV 8, E. 1). Voraussetzung für eine (Staats-)Haftung ist, dass eine durch rechtliche Vorschriften oder allgemeine Rechtsgrundsätze begründete Garantenpflicht verletzt worden ist, welche verpflichtet hat, zu handeln, um den Schaden zu verhindern (Thun, Hochwasser, VGE 21657 E. 3.3). Nach allgemeiner Ansicht lassen sich zwei Grundtypen von Garantenstellungen unterscheiden. Die Obhutspflichten umfassen die Garantenstellungen zur Verteidigung bestimmter Rechtsgüter gegenüber unbestimmt vielen Gefahren. Demgegenüber beinhalten die Überwachungspflichten Garantenstellungen zur Überwachung bestimmter Gefahrenquellen zum Schutze unbestimmt vieler Rechtsgüter (Zürich, Garantenstellung, BGE 113 IV 68, E. 5b; Evolène, Lawine, BGE 6P.40/2006 E. 9.2). Im Falle einer Verletzung strafrechtlich geschützter Güter wird dem Garanten ein unechtes Unterlassungsdelikt vorgeworfen. Ein unechtes Unterlassungsdelikt ist gegeben, wenn wenigstens die Herbeiführung des Erfolges durch Tun ausdrücklich mit Strafe bedroht wird. Das heisst, der Beschuldigte hätte durch sein Tun den Erfolg abwenden können. Er ist infolge seiner besonderen Rechtsstellung dazu auch so sehr verpflichtet, dass die Unterlassung der Erfolgsherbeiführung durch aktives Handeln gleichwertig erscheint. Eine derartige Garantenstellung besteht insbesondere für den Täter, der Kraft seiner besonderen Rechtsstellung das Gut vor der diesem drohenden Gefahr hätte schützen müssen (Evolène, Lawine, BGE 6P.40/2006, E. 9.1).

\section{Arten von massgeblichen Normen}

Wo besondere Normen ein bestimmtes Verhalten gebieten, bestimmt sich das Mass der dabei zu beachtenden Sorgfalt in erster Linie nach diesen Vorschriften (Ballwil, Signalisation Baustelle BGE 116 IV 306, E. 1a). Fehlen Vorschriften, kann auf analoge Regeln privater oder halbprivater Vereinigungen, sofern diese allgemein anerkannt sind, abgestellt werden (Rupperswil, Reitunfall, BGE 127 IV 62, E. 2d; Elm, Lawine auf Skipiste, BGE 117 IV 415, E. 5a). Dies schliesst nicht aus, dass der Vorwurf der Fahrlässigkeit auch auf allgemeine Rechtsgrundsätze wie etwa den allgemeinen Gefahrensatz gestützt werden kann (GenfCointrin, Baustellenunfall, BGE 90 IV 8, E. 1; Däniken, Schienenkran, BGE 126 IV 13 E. 7a/bb mit Hinweisen; Rupperswil, Reitunfall, BGE 127 IV 62, E. 2d). Laut diesem allgemei- 
nen Gefahrensatz muss, wer eine Gefahr schafft, die notwendigen Vorkehrungen treffen, um Schäden zu vermeiden.

Eine Handlungspflicht kann sich ferner aus dem allgemeinen Rechtsgrundsatz des Störerprinzips ergeben. Es wird zwischen Verhaltensstörern, Zustandsstörern (Pura, Hangrutsch, 1P.190/2003, E. 4.3) und, in der Lehre umstritten, Zweckveranlassern unterschieden. Verhaltensstörereigenschaften ergeben sich für den Staat vor allem durch Unterlassen, da der Staat in den seltensten Fällen selber aktiv Polizeigüter stört. Unterlassen begründet die Störereigenschaft nur, wenn eine besondere Rechtspflicht zu sicherheits- und ordnungswahrendem Handeln besteht.

Die polizeiliche Generalklausel kann unter gewissen Voraussetzungen fehlende gesetzliche Grundlagen ersetzen. Das Bundesgericht erachtet dies als zulässig, wenn und soweit die öffentliche Ordnung und die Rechtsgüter des Staates oder Privater gegen schwere und unmittelbar drohende Gefahren zu schützen sind, die mit gesetzlichen Mitteln nicht abgewendet werden können. Es muss allerdings ein Zustand der zeitlichen Dringlichkeit vorliegen. Auf die polizeiliche Generalklausel gestützte Anordnungen haben sich gegen den Störer zu richten und sie müssen dem Grundsatz der Verhältnismässigkeit entsprechen (Disentis, Stützmauer, BGE 1P. 88/1995 E. 5c). Verwaltungsorganisatorische Vorschriften, welche bloss die Zuständigkeitsbereiche innerhalb der Verwaltung festlegen, begründen keine Verpflichtung zur Abwehr von Vermögensschäden Privater (Thun, Hochwasser, VGE 21657 E. 4.2.4).

\section{Grundrechtliche Schutzpflicht als Voraussetzung für staatliche Handlungspflicht?}

Nach neuerer Auffassung haben Grundrechte nicht nur eine abwehrende Funktion gegen Beeinträchtigungen durch den Staat, sondern begründen auch eine staatliche Schutzpflicht gegen Gefährdungen, die von Dritten verursacht werden. Der Staat wird nicht nur zu einem Dulden oder Unterlassen, sondern auch zu einem positiven Tun verpflichtet (Liestal, Schiesslärm, BGE 126 II 300 E. 5a). Gemäss der neuesten Auffassung über die Rolle und die Tragweite der Grundrechte besteht diese Eingriffspflicht grundsätzlich auf allgemeine Art und Weise im Zusammenhang mit jedem dieser Rechte, eingeschlossen der Eigentumsgarantie, weil die effektive Möglichkeit, sie friedlich auszuüben, ein Bestandteil der öffentlichen Ordnung ist, deren Sicherung dem Staat zufällt (Genf, Hausbesetzer, BGE 119 la 28, in Pra 82 (1993) Nr. 206, E. 2). Die Polizei ist besonders mit der Aufrechterhaltung von Ruhe und Ordnung beauftragt und muss deshalb handeln, sobald eine Person in der Ausübung eines Grundrechtes behindert oder bedroht ist. Ein Einzelner ist nicht berechtigt, das Eingreifen der staatlichen Gewalt zu fordern, um irgendeines seiner Grundrechte zu schützen. Um die staatliche Gewalt zu beanspruchen, muss qualifiziertes Recht gefährdet sein. Welches Grundrecht auch immer betroffen ist, die Eingriffspflicht hängt in jedem Fall von der Schwere des Eingriffes und von allen Umständen ab, in denen die Polizei handeln muss. Diese Pflicht ist vor allem dann der Zweckmässigkeit des Eingriffs untergeordnet, wenn dieser umfangreiche Mittel benötigt oder wenn daraus Störungen von Ruhe und Ordnung entstehen könnten (Genf, Hausbesetzer, BGE 119 la 28, in Pra 82 (1993) Nr. 206, E. 2; Thun, Hochwasser, VGE 21657 E. 4.1).

Einschränkend hält das BGer fest, dass die grundrechtliche Schutzpflicht ebenso wenig wie das Umweltrecht einen absoluten Schutz gegen jegliche Beeinträchtigung und Risiken gewähren kann (Liestal, Schiesslärm, BGE 126 II 300 E. 5b). Das ergibt sich einerseits aus den faktisch begrenzten Mitteln des Staates, andererseits aber auch daraus, dass ein solch absoluter Schutz unweigerlich dazu führen müsste, dass zahlreiche Tätigkeiten Dritter verboten werden müssten. Dies stünde im Konflikt zu deren ebenfalls verfassungsrechtlich geschützten Betätigungsmöglichkeiten. Auch bei Annahme einer grundrechtlichen Schutzpflicht ist deshalb eine Abwägung zwischen den beteiligten Interessen erforderlich. Dies ist in erster Linie Sache der Gesetzgebung, welche durch Festlegung der unzulässigen bzw. zulässigen Tätigkeiten die Grenze zwischen einer unerlaubten Gefährdung und einem hinzunehmenden Restrisiko definiert. 
Die Frage nach der Tragweite der grundrechtlichen Schutzpflicht ist daher in der Regel gleichbedeutend mit der Frage nach der richtigen Anwendung des einschlägigen Gesetzesrechts. Erweisen sich beispielsweise beanstandete Weisungen als mit dem eidgenössischen Umweltrecht vereinbar, so verletzen sie deshalb nicht das Grundrecht auf körperliche Unversehrtheit, selbst wenn damit nicht jegliches Risiko völlig ausgeschaltet werden kann (Liestal, Schiesslärm, BGE 126 II 300 E. 5c). Das Verwaltungsgericht des Kantons Bern fasst diese Rechtspraxis in seinem Entscheid zur Verantwortlichkeit bei Hochwasser folgendermassen zusammen (Thun, Hochwasser, VGE 21657 E. 4.1): In Lehre und Rechtsprechung wird eine grundrechtlich begründete staatliche Schutzpflicht angenommen, die heute aus Art. 35 der Bundesverfassung abgeleitet wird. Dies bedeutet allerdings nicht, dass der Einzelne Anspruch auf Schutz gegen jegliche Beeinträchtigung grundrechtlich geschützter Güter hat. Der Umfang der Schutzpflicht hängt von der Schwere der drohenden Beeinträchtigung und von den gesamten Umständen ab (Genf, Hausbesetzer, 119_la_28, E. 2 in Pra 82 (1993) Nr. 206). Zudem sind die faktisch begrenzten Mittel des Staates und denkbare negative Auswirkungen der staatlichen Schutzmassnahmen zu berücksichtigen (Elm, Signalisation Skipiste, BGE 115 IV 189, E. 3b; Melchsee-Frutt, Gefahren auf Nebenflächen, BGE 122 IV 193 E. 2a; Grotte de la Baume de Bournois, Sturz in Höhleneingang, VGE 21240 E. 2e; Thun, Hochwasser, VGE 21657 E. 3.4). Ausmass und Umfang der grundrechtlichen Schutzpflicht ergeben sich in erster Linie aus der einschlägigen Gesetzgebung (Liestal, Schiesslärm, BGE 126 II 300 E. 5c).

\section{VII.I.II Handlungspflicht nach privatrechtlichen Normen}

\section{Handlungspflicht des Grundeigentümers}

Die Haftung eines Grundeigentümers gemäss Artikel 679 ZGB setzt eine Eigentumsüberschreitung voraus. Hiezu urteilt das Bundesgericht, dass eine Überschreitung des Eigentumsrechtes nur in einem menschlichen Verhalten liegen kann, das mit der Ausübung der tatsächlichen Herrschaft über das Grundstück, d. h. mit dessen Bewirtschaftung oder sonstigen Benützung, zusammenhängt (Tarasp, Felskopf, BGE 93 II 230 E. 3). Einwirkungen, die ausschliesslich durch Naturereignisse verursacht werden, fallen nicht unter den Begriff der Eigentumsüberschreitung. Ebenso kann mit rein passivem Verhalten die Verantwortlichkeit aus Art. 679 ZGB mangels Zusammenhang mit der Bewirtschaftung oder Benützung des Grundstücks in der Regel nicht begründet werden. Ein Unterlassen kann unter dem Gesichtspunkte von Art. 679 ZGB nur erheblich sein, wenn ein Grundeigentümer die Vorkehrungen nicht trifft, die nötig sind, um zu verhindern, dass infolge von gegenwärtigen oder früheren Bewirtschaftungs- oder Benützungshandlungen Gefahren für die Nachbarn entstehen. Das Bestehenlassen des ausschliesslich durch die Natur geschaffenen Zustandes einer Liegenschaft fällt nicht unter Art. 679 ZGB, weil ein Zusammenhang zwischen diesem Verhalten und der Benützung oder Bewirtschaftung des Grundstücks fehlt. Auch schon in einem früheren Urteil (Otelfingen, überlaufendes Gewässer, BGE 91 II 474 E. 6) hielt das Bundesgericht fest, dass der (Staat als) Grundeigentümer (eines Gewässers), der keine Massnahmen trifft, um den schädlichen Folgen eines Naturereignisses vorzubeugen, nicht aufgrund von Art. 679 ZGB belangt werden kann. Doch ist das Gemeinwesen als Eigentümer öffentlicher Sachen nicht nur insoweit der Haftung nach Art. 679 ZGB unterstellt, als es eine aus dem Privatrecht fliessende Befugnis ausübt, sondern auch, soweit es kraft des ihm zustehenden Hoheitsrechts handelt. Entscheidend ist dabei, ob die übermässigen Einwirkungen auf Nachbargrundstücke bei der Erfüllung öffentlicher Aufgaben unvermeidlich oder nur mit unverhältnismässigen Aufwendungen vermeidbar sind oder nicht (Otelfingen, überlaufendes Gewässer, BGE 91 II 474 E. 5).

\section{Handlungspflicht als Werkeigentümer}

Die Werkeigentümerhaftung gemäss Artikel 58 OR wird der Haftungsform der milden Kausalhaftung zugerechnet. Die Haftung kann ausgeschlossen werden durch den Nachweis, 
dass die erforderliche Sorgfalt aufgewendet wurde. Haftbar wird der Werkeigentümer bei fehlerhafter Anlage und mangelhaftem Unterhalt des Werkes. Haftbar ist der Staat häufig als Eigentümer öffentlicher Sachen wie z. B. Strassen. Dieser Haftung sind allerdings Grenzen gesetzt. So wird in einem Urteil über den Umfang der Strassenunterhaltspflicht (Mörel, vereiste Strasse, BGE 98 II 40, E. 1) nur bei Vernachlässigung elementarer Massnahmen von einem Unterhaltsmangel gesprochen, wenn die Vorschriften des öffentlichen Rechts im einzelnen Fall beobachtet worden seien. Entscheidend für die Werkeigentümerhaftung ist die Zumutbarkeit (z. B. Vercorin, Bergstrasse, BGE 102 II 343, E. 3; Mörel, vereiste Strasse, BGE 98 II 40, E. 2; Giswil, Wildbachdurchlass an Strasse, BGE 100 II 134, E. 4; St. Gallen, Plauschbad, BGE 116 II 422, E. 1). Die aufzuwendenden Kosten müssen in einem vernünftigen Verhältnis stehen zum Schutzinteresse der Benützer des Werkes und zu dessen Zweck. Nicht jede Gefahrenquelle ist ohne Weiteres ein Mangel in der Herstellung oder im Unterhalt; vernünftigerweise kann nicht verlangt werden, dass jedes noch so kleine Risiko ausgeschlossen ist.

Ein Werk ist mangelhaft, wenn es den Benützern bei bestimmungsgemässem Gebrauch keine genügende Sicherheit bietet. Ob ein Mangel vorliegt, ist konkret zu beurteilen (Tesserete, vereiste Strasse, BGE 129 III 65, E. 1.1). Zu berücksichtigen ist eine allgemein zu erwartende, nicht bestimmungsgemässe Benutzung (St. Gallen, Plauschbad, BGE 116 II 422, E. 1).

Besonders strenge Sicherheitsanforderungen sind zu stellen, wenn die Gefährdung einmal bekannt geworden ist (St. Gallen, Plauschbad BGE, 116 II 422, E. 1). Die Duldung einer erkannten Gefahr begründet einen Schuldvorwurf. Bei einem schädigenden Ereignis sind die Haftungsvoraussetzungen nach Art. 41 OR gegeben. Anderseits muss der Eigentümer nicht jeder denkbaren Gefahr vorbeugen, vor der sich jedermann problemlos mit einem Mindestmass an Vorsicht selber schützen kann (Thyon-Les Collons, Skiliftmast, BGE 126 III 113, E. $2 a / c c)$. Er muss nicht damit rechnen, dass eine Person eine Einrichtung in einer bestimmungswidrigen Art und Weise gebraucht.

\section{VII.II Objektive Voraussetzungen einer Handlungspflicht}

\section{Naturereignisse als höhere Gewalt}

Eine Handlungspflicht zum Schutz eines Geschädigten oder Gefährdeten besteht in der Regel nicht, wenn ein Ereignis unvorhersehbar und unvermeidlich, unabhängig vom Verhalten mit unabwendbarer Gewalt eintritt (Frutigen, Strassenbelag, BGE 90 IV 265, E. 2b). Doch übt die Rechtsprechung Zurückhaltung mit einer solchen Sichtweise und betrachtet beispielsweise Gewitter mit wolkenbruchartigen Regenfällen in der warmen Jahreszeit, insbesondere in Berggegenden, nicht als derart aussergewöhnlich, als dass nicht damit gerechnet werden muss (Giswil, Wildbachdurchlass an Strasse, BGE 100 II 134, E. 5; Otelfingen, überlaufendes Gewässer, BGE 91 II 474 E. 8). Rasch abfliessende Hochwasser und grosse Murgänge bei starken Niederschlägen seien eine normale Folge der Lage und der Geologie des Einzugsgebiets. In ausserordentlichen Situationen ist mit ausserordentlichen Ereignissen zu rechnen (Evolène, Lawine, BGE 6P.40/2006 E. 2.3 und E. 6).

\section{Vernachlässigbare Risiken}

Eine Handlungspflicht wird nicht durch jede, nur abstrakte Möglichkeit eines Unfalls ausgelöst (Frasnacht, Verkehrsunfall, BGE 80 IV 130, E. 1). Auch beruht ein glimpflicher Verlauf eines Unfalls nicht notwendigerweise auf einem vom gewöhnlichen Lauf der Dinge abweichenden und daher nicht massgeblichen, glücklichen Zufall (Teufen, Kollision mit Eisenbahn, BGE 124 IV 114, E. 3c). Im Gegenteil, je nach den konkreten Umständen des Einzelfalls kann es dem gewöhnlichen Lauf der Dinge entsprechen. Eine allfällig erhebliche Schädigung würde auf einem vom gewöhnlichen Lauf der Dinge abweichenden und daher nicht massgeblichen, unglücklichen Zufall beruhen. Nach ständiger Rechtsprechung des Bundesge-

Josef Th. Hess: Schutzziele im Umgang mit Naturrisiken in der Schweiz, @ vdf Hochschulverlag 2011 
richts wird im Rahmen der einfachen Kausalhaftung nicht verlangt, dass sämtliche Sicherheitsmassnahmen getroffen werden, die alle denkbaren Risiken vermeiden (Bern, Sitzwache, VGE 22214U, E. 3.3).

\section{Zulässige Belastung im ,Normalbetrieb’ als Kriterium für das Unterlassen von Hand- lungen}

Das Umweltschutzrecht anerkennt eine gewisse Belastung der Gesellschaft im störfallfreien Normalbetrieb von Anlagen. Das Umweltschutzgesetz (USG, SR 814.01) schützt sowohl vor schädlichen wie auch vor lästigen Auswirkungen. Gemäss der Botschaft zum ehemaligen Verfassungsartikel (Art. 24 septies, heute Art. 74 Abs. 1 und 2) sind schädliche Einwirkungen solche, die die physische und psychische Gesundheit des Menschen schädigen. Lästige Einwirkungen kennzeichnen sich dadurch, dass sie die betroffenen Menschen in ihrem Dasein beeinträchtigen, ohne ihnen gesundheitlichen Schaden zuzufügen (BBI 1970 I 762, 776; Zürich Flughafen, BGE 126 || 522, E. 42). Zur Bemessung der erheblichen Störung des Wohlbefindens im Sinne von Art. 15 USG wird in der Regel auf die Ergebnisse soziologischer Erhebungen bzw. darauf abgestellt, wie viele der befragten Personen sich bei einer bestimmten Belastung stark gestört fühlen (Zürich Flughafen, BGE 126 II 522, E. 42). Da die Störung erheblich sein muss, genügt nicht, dass sich wenige Einzelne beeinträchtigt fühlen. Angesichts des Gebotes der Berücksichtigung sensibler Bevölkerungsgruppen (Art. 13 Abs. 2 USG) kann andererseits nicht verlangt werden, dass eine Mehrheit der Bevölkerung stark belästigt sein muss, damit eine Störung erheblich wird. Bei der Festsetzung der Immissionsgrenzwerte in der Lärmschutzverordnung ist deshalb davon ausgegangen worden, dass der Anteil stark Gestörter 25\% (bzw. 15\% bis 25\%) der Betroffenen nicht übersteigen soll (Zürich Flughafen, BGE 126 II 522, E. 42, S. 575).

Nach heute geltendem Umweltrecht sind verschärfte Emissionsbegrenzungen, die unter anderem in Verkehrs- und Betriebsvorschriften bestehen können, unabhängig von der wirtschaftlichen Zumutbarkeit anzuordnen (Art. 11 Abs. 3 in Verbindung mit Art. 12.1 lit. c. USG; Zürich Flughafen, BGE 126 II 522, E. 42). Vorausgesetzt wird nur, dass ein angemessenes Verhältnis zwischen dem Nutzen der Massnahme und der Schwere der damit verbundenen Nachteile besteht. Vorsorgliche Emissionsbegrenzungen sind anzuordnen, soweit dies technisch und betrieblich möglich und wirtschaftlich tragbar ist (Biel-Pieterlen, Elektrosmog, BGE 124 II 219, E. 8b). Im Fall einer Emission von bloss 0.08\% des Grenzwertes besteht grundsätzlich kein Anlass zu weitergehenden Anordnungen im Sinne der Vorsorge (Samnaun, Richtstrahlantenne, BGE 117 lb 28, E. 5b und 6c). Bei derart geringer Umweltbeeinflussung liegt umweltschutzrechtlich ein Bagatellfall vor. Dagegen gelten Emissionen in der Grössenordnung von $20 \%$ des Grenzwertes als beachtlich.

\section{Unzulässige Schäden und Risiken, unbedingte Handlungspflicht}

Ebenso wie sich die Frage stellt nach der Intensität der Schädigung, bei der eine Handlung angezeigt scheint, stellt sich die Frage, ob gewisse Risiken mit erheblichem Schadenspotenzial in jedem Fall unabhängig von der Eintretenswahrscheinlichkeit unzulässig seien. USG und Störfallverordnung enthalten keine ausdrückliche maximale Schadensobergrenze (Pfäffikon, Chlorgas, BGE 127 II 18, E. 5 d/bb). Das Handbuch I zur Störfallverordnung hält es für möglich, dass es ein Mass an Schädigungen gibt, das durch die privaten und öffentlichen Interessen nicht mehr aufgewogen werden kann und unabhängig von der Eintretenswahrscheinlichkeit als untragbar zu bezeichnen ist. Als maximal zulässiger Schaden werden im Handbuch ca. 2'500 Todesfälle (innert 30 Tagen, als direkte Folge des Störfalles) oder 10 Mia. CHF Sachschaden angegeben (BUWAL, 1991, S. 36). Grundsätzlich sind die Kantone, denen der Vollzug der Störfallverordnung obliegt, berechtigt, eine maximale Schadensobergrenze festzulegen (Seiler, 1995a, S. 15). Dabei muss es sich um Schadensausmasse handeln, die Grosskatastrophen darstellen, nicht um Störfallwerte von 0.5 oder 0.6 (ca. 50-100 Tote). Ein Gefährdungspotenzial in dieser Grössenordnung haben sehr viele Anlagen (z. B. Tankstellen, Tanklastwagen, Eisenbahn- oder Strassentunnels, Chemiebetriebe). Würde 
man bei solchen Schadenspotenzialen ungeachtet der Wahrscheinlichkeit Betriebsverbote zulassen, könnte fast jede gewerbliche, industrielle oder kulturelle Tätigkeit gestützt auf die Störfallverordnung verboten werden (Pfäffikon, Chlorgas, BGE 127 II 18, E. 5 d/bb).

\section{Wirksamkeit der Massnahme}

Zum Mass der Handlungspflicht hält die Rechtsprechung fest, dass die Garantin bzw. der Garant nicht alle erdenklichen Sicherheitsvorkehren treffen muss, sondern nur diejenigen, die sich aus besonderen Vorschriften ergeben oder aufgrund allgemeiner Vorsichtsregeln als zweckmässig und vernünftigerweise zumutbar erweisen (Grotte de la Baume de Bournois, Sturz in Höhleneingang, VGE 21240 E. 2e; Thun, Hochwasser, VGE 21657 E. 3.4), um eine drohende Gefahr abzuwehren (vgl. auch Mörel, vereiste Strasse, BGE 98 II 40, E. 1; GenfCointrin, Baustellenunfall, BGE 90 IV 8, E. 2; Churwalden, Bäume am Pistenrand, BGE 121 III 358 E. 4a; Otelfingen, überlaufendes Gewässer, BGE 91 II 474 E. 5).

Sind Sicherheitsmassnahmen denkbar, die nicht reglementarisch vorgesehen sind, muss eine Abwägung der entgegenstehenden Interessen zeigen, was vernünftigerweise verlangt werden konnte; in dieser Hinsicht muss einerseits der Grad der Wirksamkeit der Massnahme, ihre Kosten und ihre Nachteile und anderseits der Grad der Wahrscheinlichkeit der Gefahr und das Ausmass des zu erwartenden Schadens berücksichtigt werden (Thyon-Les Collons, Skiliftmast, BGE 126 III 113, E. 2b).

Nach ständiger Rechtsprechung des Bundesgerichts wird somit im Rahmen der einfachen Kausalhaftung nicht verlangt, dass sämtliche Sicherheitsmassnahmen getroffen werden, die alle denkbaren Risiken vermeiden. So müssen nicht alle denkbaren Massnahmen getroffen werden, um auch seltenen Ereignissen vorzubeugen, obwohl natürlich bekannt ist, dass solche Ereignisse vorkommen können (Bern, Sitzwache, VGE 22214U, E. 3.3). Dies entspricht dem selbstverständlichen und rechtlich gebotenen Umgang mit kleinen Wahrscheinlichkeiten. Dieser Umgang sieht vor, dass eine Massnahme nicht verlangt werden kann, wenn sie im Verhältnis zu den dadurch vermiedenen Risiken finanziell nicht zumutbar ist. Davon wird eine Ausnahme gemacht, wenn ein Ereignis trotz seiner generell kleinen Wahrscheinlichkeit in einem konkreten Fall vorhersehbar ist. Risikoreduzierende Massnahmen sind dann zu treffen, wenn entweder die Eintretenswahrscheinlichkeit genügend hoch ist oder wenn trotz generell kleiner Eintretenswahrscheinlichkeit in einem konkreten Fall voraussehbar ist, dass das Ereignis eintritt. Umgekehrt ausgedrückt darf auf aufwendige risikoreduzierende Massnahmen verzichtet werden, wenn die Eintretenswahrscheinlichkeit sehr gering ist und auch keine konkreten Anzeichen bestehen, dass das Ereignis bevorsteht (Bern, Sitzwache, VGE 22214U, E. 3.3).

Die Ressourcen, die einem Handlungspflichtigen zur Verfügung stehen, sind begrenzt (Bern, Sitzwache, VGE 22214U, E. 5.1). Jede Handlung (im beurteilten Fall: medizinische Leistungen der Spitäler) muss deshalb nicht nur wirksam und zweckmässig, sondern auch wirtschaftlich sein. Je mehr Massnahmen mit einem schlechten Kosten-Wirksamkeitsverhältnis getroffen werden, desto weniger Mittel stehen zur Verfügung, um Massnahmen mit einem guten Kosten-/Wirksamkeitsverhältnis zu treffen. Dies hätte zur Folge, dass mit den zur Verfügung stehenden Ressourcen nicht das Optimum an Leistung erbracht werden kann. Dies ist unzweckmässig, unwirtschaftlich und damit gesetzeswidrig (Bern, Sitzwache, VGE 22214U, E. 5.1). Mit dem Rechtsgleichheitsgebot (Art. 8 Abs. 1 BV) ist es nicht vereinbar, in einigen Fällen sehr hohen Aufwand zu betreiben, um geringfügige Risiken weiter zu reduzieren, wenn in anderen Fällen selbst wesentlich weniger aufwendige Massnahmen nicht getroffen werden (Bern, Sitzwache, VGE 22214U, E. 5.2).

\section{Zumutbarkeit der Massnahme}

Der Handlungspflichtige muss nicht mehr tun, als man von inm angesichts der konkreten Umstände vernünftigerweise erwarten darf (Thyon-Les Collons, Skiliftmast, BGE 126_III_113, E. 2a/aa). Immer wieder werden in der Rechtsprechung auch die wirtschaftliJosef Th. Hess: Schutzziele im Umgang mit Naturrisiken in der Schweiz, @ vdf Hochschulverlag 2011 
chen Verhältnisse des Handlungspflichtigen in die Überlegungen einbezogen: Es können nur Aufwendungen verlangt werden, die wirtschaftlich und technisch zumutbar sind (St. Gallen, Plauschbad, BGE 116 || 422, E. 1). Es ist in jedem Einzelfall zu prüfen, ob der Handlungspflichtige nach den zeitlichen, technischen und finanziellen Gegebenheiten überhaupt in der Lage war, seine Aufgabe zu erfüllen (Mörel, vereiste Strasse, BGE 98 II 40, E. 2). Die Aufwendungen eines Handlungspflichtigen müssen in einem vernünftigen Verhältnis zu seinen Mitteln und zu seinen übrigen Auslagen stehen (Vercorin, Bergstrasse, 102 II 343, E. 1; Giswil, Wildbachdurchlass an Strasse, BGE 100 II 134, E. 4; Mörel, vereiste Strasse, BGE 98 II 40, E. 2; Tesserete, vereiste Strasse BGE 129 III 65, E. 1.1). Konsequenterweise ist ein Unterlassen unrechtmässig, wenn die vorhandenen Mittel ein Handeln erlaubt hätten (Tesserete, vereiste Strasse, BGE 129 III 65, E. 5).

Die finanzielle Zumutbarkeit von Massnahmen kann nur solange bejaht werden, wie der dafür erforderliche Aufwand in einem Rahmen liegt, der auch dann, wenn er verallgemeinert wird, von der (Gesundheits-)Politik bzw. der gesamten Volkswirtschaft finanziert werden kann. Als eine sinnvolle Regel zur Konkretisierung dieses Grundsatzes hat sich seit Jahren bewährt: Sicherheitsmassnahmen müssen solange getroffen werden, wie der Ertragswert des dadurch vermiedenen Schadens grösser ist als die Kosten für seine Vermeidung (,Learned Hand Rule').

Für die als noch zumutbar zu betrachtenden Beträge lassen sich ungefähre Grössenordnungen feststellen: So werden Kosten bis zu CHF 300'000 pro QALY (,Quality-Adjusted Life Year') angenommen. In der allgemeinen Risikodiskussion werden Grenzkostenbeträge von maximal CHF 20 Mio. pro gerettetes Menschenleben oder maximal CHF 500'000 pro gerettetes Menschenlebensjahr diskutiert (Bern, Sitzwache, VGE 22214U, E. 5.2).

In einem konkreten Fall urteilte das Verwaltungsgericht des Kantons Bern, dass Kosten von CHF 1.5 Mia. pro gerettetes Menschenleben oder CHF 150 Mio. pro gerettetes Lebensjahr finanziell nicht zumutbar und die Unterlassung derart teurer Massnahmen damit nicht pflichtwidrig gewesen sei (Bern, Sitzwache, VGE 22214U, E. 5.6). Das Bundesgericht hob diesen Entscheid jedoch auf (Bern, Zumutbarkeit einer Sitzwache BGE 4P.244/2005). In seinen Erwägungen gelangte es zu einer völlig anderen Kosten-Nutzen-Berechnung: Es erachtete Kosten von durchschnittlich CHF 1'680 pro Fall zur Überwachung eines potenziell suizidgefährdeten Patienten als finanziell ohne Weiteres zumutbar (Bern, Zumutbarkeit einer Sitzwache BGE 4P.244/2005 E. 4). Dabei wird die Wahrscheinlichkeit, ob dieser tatsächlich Selbstmord begeht, nicht beachtet. Das Bundesgericht lehnt damit implizit ab, dass die allfälligen Kosten für die schweizerische Volkswirtschaft als Bezugsgrösse für finanzielle Zumutbarkeit herangezogen werden können (Zimmermann, 2006, 136). Vielmehr sei bei der Beurteilung der finanziellen Zumutbarkeit von Sicherheitsmassnahmen ausschliesslich auf den konkret zu beurteilenden Einzelfall abzustellen.

\section{Erlaubtes Risiko}

Der (wirkliche oder vermeintliche) Nutzen einer Tätigkeit kann rechtfertigen, ein gewisses Risiko in Kauf zu nehmen, wenn diese Tätigkeit sonst gar nicht oder nur mit unverhältnismässig hohen materiellen oder anderen Aufwendungen möglich wäre. Die Nutzen-RisikoAbwägung ist im Einzelnen eine schwierige und für jeden Fall neu zu entscheidende Frage (Kaiseraugst, Betäubungsmittel, BGE 117 IV 58 E. 2b; Basel-Landschaft, Abgabe Drogen, BGE 120 IV 334).

\section{Besondere und schwer erkennbare Gefahren}

Zu schützen ist insbesondere vor Gefahren, welche für die zu Schützenden nicht ohne Weiteres erkennbar sind und sich als eigentliche Fallen erweisen (EIm, Signalisation Skipiste, BGE 115 IV 189, E. 3b; Melchsee-Frutt, Gefahren auf Nebenflächen, BGE 122 IV 193 E. 2a; Grotte de la Baume de Bournois, Sturz in Höhleneingang, VGE 21240 E. 2e; Thun, Hochwasser, VGE 21657 E. 3.4). Eine Pflicht besteht bei besonders grossen oder atypischen GeJosef Th. Hess: Schutzziele im Umgang mit Naturrisiken in der Schweiz, @ vdf Hochschulverlag 2011 
fahren (Churwalden, Bäume am Pistenrand, BGE 121 III 358, Glarus; Gefahren ausserhalb der Piste, BGE 130_III_193 E. 2.4.3). Das besondere Risiko kann darin bestehen, dass die Gefahr schwierig zu erkennen oder schwer zu vermeiden ist. Soweit zumutbar ist zu verhindern, dass die Gefahren, welche auch bei vorsichtigem Verhalten des Betroffenen nicht vermieden werden können, nicht zu einer Schädigung führen (Churwalden, Bäume am Pistenrand, BGE 121 III 358, E. 4a; Thyon-Les Collons, Skiliftmast, BGE 126 III 113, E. 2a/aa).

\section{Schutzbedürftigkeit der betroffenen oder gefährdeten Person}

Der Umfang der Schutzpflicht hängt von der Natur des Rechtsverhältnisses und von den Kenntnissen bzw. der Schutzbedürftigkeit der beteiligten Personen ab (Schwyz, Pflichten des Notars BGE 2P.13/1997, in Pra 1999 S. 271 E. 3b/bb). Je hilfsbedürftiger und unselbständiger die zu schützenden Personen sind und je grösser das Risiko ist, desto intensivere Vorsichtsmassnahmen sind erforderlich (Grotte de la Baume de Bournois, Sturz in Höhleneingang, VGE 21240 E. 2e; Thun, Hochwasser, VGE 21657 E. 3.4; Königsfelden, spitalärztliche Tätigkeit, BGE $120 \mathrm{lb}$ 411, E. 4b).

\section{Öffentliche Interessen und Privatinteressen}

Hochwasserschutzmassnahmen haben alle übrigen öffentlichen Interessen - namentlich möglichst naturnahe und öffentlich zugängliche Ufer - sowie die privaten Interessen der betroffenen Eigentümerinnen und Eigentümer in ausreichendem Masse zu berücksichtigen (Uster, Gewässerbaulinie BGE 1A.252/1997, E. 5). Im Fall einer stark gefährdeten (roten) Zone muss die Gefahr - und damit verbunden das öffentliche Interesse an der Sicherheit als so gross beurteilt werden, dass dagegen die rein wirtschaftlich-gewerblichen Ausnützungsinteressen der betroffenen Grundeigentümer nicht aufkommen können (St. Moritz, Gefahrenzone in Rutschhang, BGE 114 la 245, E. 6a; Morcote, Steinschlag, BGE 1P.591/2003, E. 3.5). Vor allem in ausserordentlichen Lagen kommt den öffentlichen Interessen, dem Schutz der Bevölkerung und ihrer Lebensgrundlagen, der Wahrung der Handlungsfreiheit und der Wiederherstellung geordneter Verhältnisse ein hohes Gewicht zu (BZG SR 520.1; KSZG BSG 521.1). Demgegenüber gehören private Vermögenswerte nicht zu den prioritär zu schützenden Objekten, jedenfalls solange sie nicht Lebensgrundlagen darstellen (Thun, Hochwasser, VGE 21657 E. 6.2).

\section{Eigenverantwortung}

In erster Linie ist jedermann selber für den Schutz seines Hab und Guts verantwortlich. Der Eigentümer kann Massnahmen treffen, um drohende Schäden abzuwehren, oder sich versichern. Unterlässt er dies, trägt er eingetretene Schäden grundsätzlich selber und kann nicht den Staat dafür haftbar machen. Dies gilt auch für Schäden durch Naturgefahren: Der Staat ist nicht verpflichtet, sämtliche Gefahren abzuwehren, auch solche, die er nicht selber geschaffen hat (Thun, Hochwasser, VGE 21657 E. 4.1). Eine solche Pflicht, mit entsprechender Schutzwirkung, hätte eine Überforderung der Ansprüche an den Staat zur Folge und ergibt sich aus dem schweizerischen Recht nicht (Listeriose, Information der Öffentlichkeit, BGE 118 lb 473 E. 5d; Thun, Hochwasser, VGE 21657 E. 4.1). Die staatliche Tätigkeit kann die Eigenverantwortung der Privaten nicht aufheben (Thun, Hochwasser, VGE 21657 E. 5.3). Wie erwähnt ist es primär Sache der Eigentümerinnen und Eigentümer, ihr Eigentum vor Hochwasser zu schützen. In zweiter Linie obliegen die Präventionsmassnahmen den Gemeinwesen (Thun, Hochwasser, VGE 21657 E 6.2).

Ein Kanton haftet auch nicht für das allgemeine Lebensrisiko. Die üblichen Risiken sind nicht dem Kanton anzulasten (Grotte de la Baume de Bournois, Sturz in Höhleneingang, VGE 21240 E 5b; Mörel, vereiste Strasse, BGE 98 II 40, E. 4). 


\section{VII.III Anforderungen an die Handlungspflichtigen}

\section{Sorgfaltspflicht der Handlungspflichtigen}

Die Frage der Sorgfaltspflicht spielt in Haftungsfällen eine zentrale Rolle. Sorgfaltswidrig ist eine Handlungsweise dann, wenn der Täter zum Zeitpunkt der Tat aufgrund seiner Kenntnisse und Fähigkeiten die damit bewirkte Gefährdung des Opfers hätte erkennen können und wenn er zugleich die Grenzen des erlaubten Risikos überschritt (Ballwil, Signalisation Baustelle, BGE 116 IV 306, E. 1a; Königsfelden, spitalärztliche Tätigkeit, BGE 120 lb 411 E. 4a; Frauenfeld, Aufmerksamkeit im Strassenverkehr, BGE 122 IV 225, E. 2a; Däniken, Schienenkran, BGE 126 IV 13, E. 7a/bb; Rupperswil, Reitunfall, BGE 127 IV 62, E. 2d). Die zu erwartenden Geschehensabläufe müssen für den konkreten Handlungspflichtigen mindestens in den wesentlichen Zügen voraussehbar sein (Däniken, Schienenkran, BGE 126 IV 13, E. 7a/bb). Daher ist zentral, ob der Handlungspflichtige eine Gefährdung der Rechtsgüter des Betroffenen hätte voraussehen bzw. erkennen können und müssen. Haftbar macht sich insbesondere, wer eine konkret erkennbare Gefahr nicht erfasst, sie fehlerhaft einschätzt oder sie nicht beachtet (Königsfelden, spitalärztliche Tätigkeit, BGE 120 lb 411, E. 4b; Grotte de la Baume de Bournois, Sturz in Höhleneingang, VGE 21240 E. 2e). Strafbar macht sich auch, wer trotz erkannter Gefahr die notwendigen und zumutbaren Massnahmen nicht trifft, um den Eintritt einer Schädigung zu vermeiden (St. Gallen, Plauschbad 116 II 422, E. 1; Grotte de la Baume de Bournois, Sturz in Höhleneingang, VGE 21240 E. 2e; Evolène, Urteil der 1. Strafgerichtskammer des Kantonsgerichts Wallis vom 11. Januar 2006, E. 8.2 und E. 8.3.).

\section{Grenzen der Sorgfaltspflicht}

Ist allerdings den Verantwortlichen eine Gefahr nicht bekannt und bei zumutbarer Sorgfalt nicht erkennbar, so besteht keine Pflicht, sie abzuwehren (Täsch, Lawinensicherung Strasse, BGE 116 IV 182, E.8; Grotte de la Baume de Bournois, Sturz in Höhleneingang, VGE 21240 E. 2e; Thun, Hochwasser, VGE 21657 E. 3.4).

\section{Berücksichtigung der Fähigkeiten des Sorgfaltspflichtigen und der Umstände}

Die Sorgfaltspflicht ruft nach einer strengeren Beurteilung, wenn die Tätigkeit durch eine Fachperson erfolgte (z. B. patentierter Bergführer: S-charl, Skitour, BGE 118 IV 130 E. 3b). Je grösser die konkrete aktuelle Gefahr ist, desto intensiver müssen die erforderlichen Vorsichtsmassnahmen sein (Königsfelden, spitalärztliche Tätigkeit, BGE 120 lb 411, E. 4b). Im Rahmen der als Kausalhaftung ausgestalteten Staatshaftung kommt es für die Beurteilung, ob eine Gefahr erkennbar und Schutzvorkehren geboten gewesen wären, nicht auf die individuellen Fähigkeiten und Kenntnisse der handelnden Staatsangestellten an, sondern darauf, was bei objektivierter Betrachtung aufgrund der gegebenen Umstände vernünftigerweise vom Staat erwartet und verlangt werden kann (Grotte de la Baume de Bournois, Sturz in Höhleneingang VGE 21240 E. 2e; Thun, Hochwasser, VGE 21657 E. 3.4).

Das Mass der Aufmerksamkeit, das vom Handlungspflichtigen verlangt wird, richtet sich nach den gesamten Umständen, den örtlichen Verhältnissen, den voraussehbaren Gefahrenquellen (Frauenfeld, Aufmerksamkeit im Strassenverkehr, BGE 122 IV 225, E. 2b), den Mitteln und der Zeit, die im einzelnen Fall zur Verfügung stehen (Königsfelden, spitalärztliche Tätigkeit, BGE 120 lb 411 E. 4a). Wenn der Handlungspflichtige sein Augenmerk im Wesentlichen auf etwas Bestimmtes zu richten hat, kann ihm für anderes eine geringere Aufmerksamkeit zugebilligt werden (Frauenfeld, Aufmerksamkeit im Strassenverkehr, BGE 122 IV 225, E. 2b). Der Handlungspflichtige hat seine Aufmerksamkeit in erster Linie auf die zu erwartenden Gefahren zu richten und daneben höchstens sekundär auf ungewöhnliche und abwegige Verhaltensweisen. Man kann nicht verlangen, dass jedermann zu jeder Zeit ein Höchstmass an Aufmerksamkeit und Umsicht erbringt (Frauenfeld, Aufmerksamkeit im Strassenverkehr, BGE 122 IV 225, E. 2c). Vor allem wenn Vorschriften fehlen, dürfen keine 
unverhältnismässig hohen Anforderungen gestellt werden (Mattstätten, Plangenehmigungsverfahren, BGE 121 II 378, E. 15d/cc).

Bei der Bestimmung des im Einzelfall anzuwendenden Massstabes des sorgfaltsgemässen Verhaltens kann - soweit verfügbar - auf Bestimmungen zurückgegriffen werden, die der Unfallverhütung und der Sicherheit dienen (Frauenfeld, Aufmerksamkeit im Strassenverkehr, BGE 122 IV 225, E. 2a).

\section{Beurteilungsspielraum und Ermessen der Handlungspflichtigen}

Den zuständigen Stellen und Personen muss ein gewisses Ermessen in der Beurteilung der Situation zugestanden werden (Königsfelden, spitalärztliche Tätigkeit, BGE 120 lb 411 E. 4a; Grotte de la Baume de Bournois, Sturz in Höhleneingang, VGE 21240 E. 2e, Thun, Hochwasser, VGE 21657 E. 3.4), insbesondere in ausserordentlichen Situationen, in denen rasches Handeln erforderlich ist (Thun, Hochwasser VGE 21657 E. 3.4 und E. 4.3). Dem Handlungspflichtigen ist sowohl in der Beurteilung wie in der Bestimmung von Massnahmen nach dem objektiven Wissensstand oftmals ein Entscheidungsspielraum gegeben, der eine Auswahl zwischen zahlreichen Möglichkeiten zulässt. Werden nach Beurteilung verschiedener Gutachter unterschiedliche Entscheide als plausibel betrachtet, so kann nicht gesagt werden, dass die eine davon eine Sorgfaltspflichtverletzung darstellt (Bern, Sitzwache VGE 22214U, E. 4.3.2). Sich für eine der Möglichkeiten zu entscheiden, fällt in das pflichtgemässe Ermessen des Handlungspflichtigen, ohne dass er zur Verantwortung gezogen werden könnte, wenn er bei einer Beurteilung ex post nicht die objektiv beste Lösung gefunden hat (Liestal, psychiatrische Klinik, BGE 112 lb 322 E. 4a; Grotte de la Baume de Bournois, Sturz in Höhleneingang VGE 21240 E. 2e; Thun, Hochwasser VGE 21657 E. 3.4). Die Beurteilung der Behörden muss vom gegenwärtigen Stand der Technik und des Wissens ausgehen (Mattstätten, Plangenehmigungsverfahren, BGE 121 II 378, E. 15d/cc), doch dürfen neue Erkenntnisse vorbehalten werden. Liegen solche vor, so besteht möglicherweise Anlass für eine Wiederaufnahme des Verfahrens (Samnaun, Richtstrahlantenne BGE 117 Ib 28, E. 6d).

\section{VII.IV Sorgfaltspflicht der Risikobetroffenen}

Neben der Sorgfaltspflicht der Handlungspflichtigen bestehen immer auch Sorgfaltspflichten der Geschädigten. Es kann von der geschädigten Person verlangt und erwartet werden, dass sie die den Umständen und ihren Kenntnissen entsprechende, durchschnittlich übliche Sorgfalt beachtet (Grotte de la Baume de Bournois, Sturz in Höhleneingang VGE 21240 E. 2e; Thun, Hochwasser, VGE 21657 E. 3.4; Vercorin, Bergstrasse, BGE 102 II 343, E. 1; Thyon-Les Collons, Skiliftmast, BGE 126 III 113, E. 2d). So kann sich beispielsweise nicht auf die Haftung des Gemeinwesens nach Art. 58 OR berufen, wer bei vereisten Strassen diese Umstände nicht gebührend beachtet und beispielsweise zu schnell fährt (Mörel, vereiste Strasse, BGE 98 II 40, E. 2; Tesserete, vereiste Strasse, BGE 129 III 65, E. 1.1). Ein Strassenbenützer muss sich bewusst sein, dass die Strassen Naturphänomenen ausgesetzt sein können und dass die Benützung der Strasse gefährlich sein kann. Gemäss Lehre und Rechtsprechung hat sich der Verkehr grundsätzlich den Strassenverhältnissen anzupassen. Damit erklärt sich, warum bei Unfällen mit Motorfahrzeugen ausserorts nie eine Haftung des Strasseneigentümers angenommen wurde (Tesserete, vereiste Strasse, BGE 129 III 65, E. 1.1).

Gefahren, die einem Verhalten eigen sind, trägt der Risikobetroffene selbst (z. B. Skiabfahrt: Glarus, Gefahren ausserhalb der Piste, BGE 130 III 193, E 2.5). In verschiedenen Urteilen wird festgehalten, dass, wer eine nicht gekennzeichnete Abfahrt befährt, dies in aller Regel in eigener Verantwortung und auf eigenes Risiko tut (EIm, Signalisation Skipiste BGE 115 IV 189, E 3a). Für die Unterstützung von Verbaumassnahmen mit öffentlichen Mitteln werden aus diesen Gründen die Kosten von Sicherheitsmassnahmen für neue Gebäude (in bekannten Gefahrengebieten) bei der Bemessung des Gesamtbeitrages nicht berücksichtigt (Mendrisio 2A.245/1996 E 6f). 


\section{VII.V Adäquater Kausalzusammenhang}

Zur Frage, ob eine Gefahr erkennbar gewesen wäre, gilt der Massstab der Adäquanz. Im Zusammenhang mit Schutzzielen ist die individuelle Vorhersehbarkeit durch einen Rechtsadressaten allerdings nur beschränkt von Bedeutung. Fragen der individuellen Vorhersehbarkeit stellen sich kaum in der Prävention von Ereignissen mit rein statistischer Eintretenswahrscheinlichkeit, häufig jedoch im Zusammenhang mit der Intervention bei oder der Wiederherstellung nach einem konkreten Ereignis.

Zur Erfüllung des adäquaten Kausalzusammenhanges muss ein Verhalten geeignet sein, nach dem gewöhnlichen Lauf der Dinge und den Erfahrungen des Lebens einen Erfolg wie den eingetretenen herbeizuführen oder mindestens zu begünstigen (S-Charl, Skitour, BGE 118 IV 130, E. 3c; Grotte de la Baume de Bournois, Sturz in Höhleneingang, VGE 21240 E. 2e; Thun, Hochwasser, VGE 21657 E. 3.5). Die Adäquanz ist nur zu verneinen, wenn sehr aussergewöhnliche Umstände, wie das Mitverschulden eines Dritten oder Material- bzw. Konstruktionsfehler, als Mitursachen hinzutreten, mit denen nicht gerechnet werden musste und die derart schwer wiegen, dass sie als wahrscheinlichste und unmittelbarste Ursache des Ergebnisses erscheinen und so alle anderen mitverursachenden Faktoren - namentlich das Verhalten des Angeschuldigten - in den Hintergrund stellen (Rupperswil, Reitunfall, BGE 127 IV 62, E. 2d; Däniken, Schienenkran BGE 126 IV 13, E. 7a/bb).

Der adäquate Kausalzusammenhang wird durch überwiegendes Selbstverschulden des Betroffenen unterbrochen. Dies gilt auch für die Kausalhaftung (Grotte de la Baume de Bournois, Sturz in Höhleneingang, VGE 21240 E. 6b).

Schädigung durch Unterlassung setzt adäquate Kausalität voraus. Das heisst, dass durch ein pflichtgemässes Handeln der Eintritt des Schadens mit Sicherheit oder überwiegender Wahrscheinlichkeit hätte vermieden werden können (Grotte de la Baume de Bournois, Sturz in Höhleneingang VGE 21240 E. 2f; Thun, Hochwasser, VGE 21657, E. 3.5). Es ist zu prüfen, ob das Geschehene bei pflichtgemässem Verhalten ausgeblieben wäre. Dieser hypothetische Kausalzusammenhang setzt zumindest eine hohe Wahrscheinlichkeit voraus (Ballwil, Signalisation Baustelle BGE 116 IV 306, E. 2a; S-Charl, Skitour, BGE 118 IV 130 E. 6a; Thun, Hochwasser, VGE 21657, E. 3.5). Ein (pflichtwidriges) Verhalten ist im natürlichen Sinne kausal, wenn es nicht weggedacht werden kann, ohne dass auch das Eingetretene entfiele: Dieses Verhalten muss nicht alleinige oder unmittelbare Ursache des Erfolgs sein (Ballwil, Signalisation Baustelle, BGE 116 IV 306, E. 2a). Mit dieser ,Conditio-sine-qua-nonFormel' wird ein hypothetischer Kausalverlauf untersucht und geprüft, was beim Weglassen bestimmter Tatsachen geschehen wäre. Ein solchermassen vermuteter natürlicher Kausalverlauf lässt sich nicht mit Gewissheit beweisen. Deshalb genügt es, wenn das Verhalten des Täters mindestens mit einem hohen Grad der Wahrscheinlichkeit oder mit an Sicherheit grenzender Wahrscheinlichkeit Ursache des Ergebnisses bildete. Dem Handlungspflichtigen steht seinerseits der Einwand des rechtmässigen Alternativverhaltens offen, d. h. er kann geltend machen, dass der Schaden auch dann eingetreten wäre, wenn die Verantwortlichen sich pflichtgemäss verhalten hätten (Orny, Überschwemmung, 122 III 229, E. 5a). Im Falle komplexer Naturvorgänge kann sich die adäquate Kausalität auch auf ,aussergewöhnliche' Auswirkungen erstrecken, d. h. auf Auswirkungen, die zwar in den Augen des Laien aussergewöhnlich erscheinen, nicht aber in denjenigen des Experten (Rawyl, Sondierstollen, BGE 119 lb 334, E. 4).

In einzelnen Fällen kann die sog. Risikoerhöhungstheorie angewendet werden (Ballwil, Signalisation Baustelle, BGE 116 IV 306, E. 2a). Nach der Risikoerhöhungstheorie liegt ein Delikt auch dann vor, wenn durch eine Unterlassung die Chance des Erfolgseintritts erhöht wurde. Dies kann insbesondere dann der Fall sein, wenn der Erfolg auch eingetreten wäre, wenn die Pflicht nicht verletzt worden wäre (Seiler, 1997, S. 121). Ob der Täter die Gefahr, die sich verwirklicht hat, steigerte, ist unter Auswertung aller im Zeitpunkt des Urteils, also ex post bekannten Umstände zu ermitteln. 


\section{Beurteilung von Schutzzieldarstellungen}

\section{VIII.I Nachvollziehbarkeit und Vergleichbarkeit}

Die Beschreibung des Risikos und damit der Schutzziele als Umschreibung des tolerierbaren Risikos umfasst mehrere Elemente:

Schutzziel $=$ akzeptiertes Risiko $R_{\text {acc }}$

$\mathbf{R}_{\text {acc }}=f($ Schadensausmass SA, Wahrscheinlichkeit $\mathbf{F})$

wobei

SA $=f($ Prozessart $\mathbf{H}$, Prozessintensität I, Raumnutzung U)

und

$$
\mathbf{U} \quad=\quad \mathbf{f}(\text { Wert } \mathbf{A}, \text { Vulnerabilität } \mathbf{V} \text {, Präsenzwahrscheinlichkeit } \mathbf{P})
$$

Als weitere Elemente zur vergleichenden Beurteilung von Schutzzielen wurde die Berücksichtigung der Systemgrösse S und die Berücksichtigung von Wirtschaftlichkeitskriterien W aufgenommen. Schutzzielen wird Nachvollziehbarkeit und Vergleichbarkeit in dem Masse zugerechnet, als sie die aufgeführten Elemente berücksichtigen.

Tabelle 4 Bewertung bestehender Schutzzieldarstellungen hinsichtlich Nachvollziehbarkeit und Vergleichbarkeit.

\begin{tabular}{|c|c|c|c|c|c|c|c|c|c|}
\hline \multirow{2}{*}{\multicolumn{2}{|c|}{$\frac{\text { Faktor }}{\text { Ansatz }}$}} & $\begin{array}{c}\text { Auftretens- } \\
\text { wahrschein- } \\
\text { lichkeit }\end{array}$ & Prozessart & $\begin{array}{l}\text { Prozess- } \\
\text { intensität }\end{array}$ & Wert & $\begin{array}{l}\text { Verletz- } \\
\text { lichkeit }\end{array}$ & $\begin{array}{c}\text { Präsenz- } \\
\text { wahr- } \\
\text { schein- } \\
\text { lichkeit }\end{array}$ & $\begin{array}{l}\text { System- } \\
\text { grösse }\end{array}$ & \multirow{2}{*}{$\begin{array}{l}\text { Durch- } \\
\text { schnitt }\end{array}$} \\
\hline & & $F$ & $H$ & 1 & $A$ & V & $P$ & $M$ & \\
\hline \multicolumn{2}{|c|}{$\begin{array}{c}\text { Grenzwerte (1) für } \\
\text { Versagenswahrschein- } \\
\text { lichkeiten }\end{array}$} & 1 & 1 & 1 & 1 & 1 & 1 & 0 & 0.9 \\
\hline \multirow{3}{*}{ 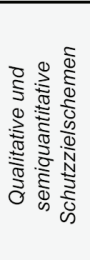 } & $\begin{array}{c}\text { Schutzziele KS } \\
\text { Nr. } 20\end{array}$ & 1 & 1 & 1 & 1 & 1 & 1 & 0 & 0.9 \\
\hline & $\begin{array}{c}\text { Schutzziele } \\
\text { Hochwasser- } \\
\text { schutz an } \\
\text { Fliessge- } \\
\text { wässern } \\
\end{array}$ & 2 & 2 & 1 & 1 & 1 & 1 & 1 & 1.3 \\
\hline & $\begin{array}{l}\text { Schutzziele } \\
\text { BUWAL 107/1 }\end{array}$ & 2 & 2 & 2 & 1 & 1 & 1 & 1 & 1.4 \\
\hline \multirow{4}{*}{ 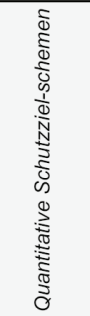 } & $\begin{array}{c}\text { F/N-Diagramme } \\
*)\end{array}$ & 2 & 1 & 2 & 2 & 2 & 2 & 0 & 1.6 \\
\hline & $\begin{array}{c}\text { F/N-Diagramme } \\
* *)\end{array}$ & 2 & 1 & 2 & 2 & 2 & 2 & 0 & 1.6 \\
\hline & $\begin{array}{c}\text { Schutzziele } \\
\text { Erdbeben an } \\
\text { bestehenden } \\
\text { Gebäuden *) }\end{array}$ & 2 & 2 & 2 & 2 & 2 & 1 & 1 & 1.7 \\
\hline & Do. $\left.{ }^{* *}\right)$ & 2 & 2 & 2 & 2 & 2 & 1 & 1 & 1.7 \\
\hline \multirow{2}{*}{ 㐫 } & Indiv. Risiko & 2 & 1 & 2 & 1 & 1 & 2 & 2 & 1.6 \\
\hline & Kollekt. Risiko & 2 & 1 & 2 & 2 & 1 & 2 & 2 & 1.7 \\
\hline \multicolumn{2}{|c|}{ Grenzkosten } & 2 & 1 & 2 & 2 & 2 & 2 & 2 & 1.9 \\
\hline
\end{tabular}

*) Bereich absoluter Schutzziele (intolerabel, Massnahmen zwingend erforderlich).

${ }^{* *}$ ) Bereich mit Verhältnismässigkeitsprüfung (ALARA).

2 Berücksichtigung

1 teilweise Berücksichtigung

0 keine Berücksichtigung 


\section{VIII.II Gewährleistung individueller Sicherheit und Wirtschaftlichkeit}

Schutzzielkonzepte sind in unterschiedlichem Masse auf die Abdeckung unterschiedlicher Sicherheitsbedürfnisse einerseits und auf die Effizienz risikovermindernder Massnahmen anderseits ausgerichtet.

Tabelle 5 Bewertung bestehender Schutzzieldarstellungen hinsichtlich Gewährleistung individueller Sicherheit und Wirtschaftlichkeit.

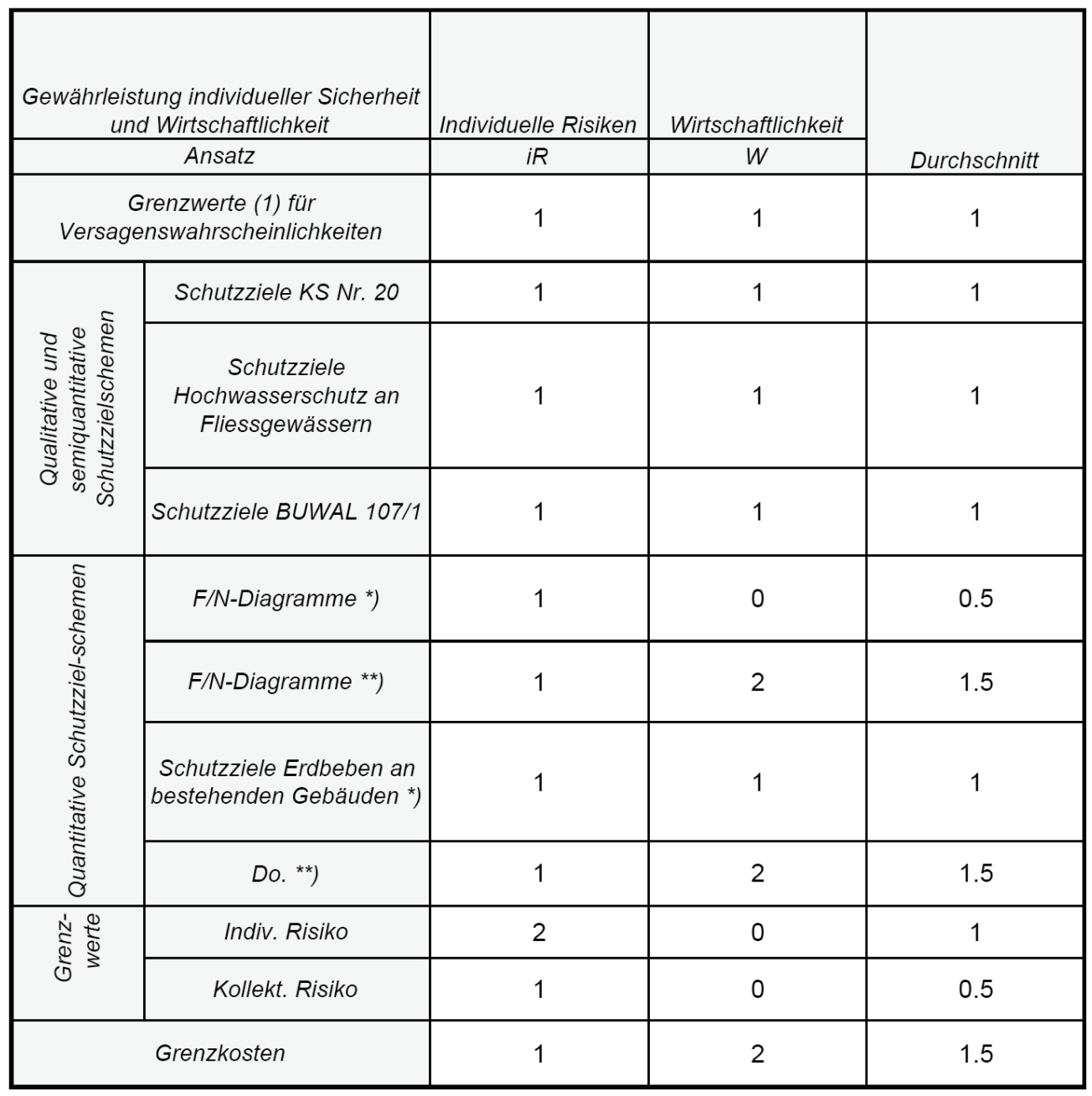

*) Bereich absoluter Schutzziele (intolerabel, Massnahmen zwingend erforderlich).

${ }^{* *}$ ) Bereich mit Verhältnismässigkeitsprüfung (ALARA).

2 Berücksichtigung

1 teilweise Berücksichtigung

0 keine Berücksichtigung

Rein grenzwertbasierte Schutzziele klammern Wirtschaftlichkeit aus. Ein bestimmter Sicherheitsanspruch ist zu erfüllen, ungeachtet der damit verbundenen Kosten. Auch die F/NDiagramme fallen im Bereich der nicht tolerierbaren Risiken in diese Kategorie. Auf der andern Seite stellt bei Grenzkostenansätzen und auch im ALARA-Bereich der F/N-Diagramme die Wirtschaftlichkeit ein zentrales Kriterium dar. 


\section{VIII.III Anforderungen an Informationsgrundlagen}

Die betrachteten Schutzzieldarstellungen wurden ferner hinsichtlich des zu ihrer Anwendung erforderlichen Informationsaufwandes beurteilt:

- Informationsbedarf (als ungünstig wurden Verhältnisse beurteilt, wenn eine aufwendige Erhebung von vielen Parametern am Einzelobjekt notwendig ist);

- Automatisierbarkeit (Einsatz von GIS, Verwendung grossräumig vorhandener Daten möglich);

- Aufbau auf verbreitet vorhandenen Grundlagen (Gefahrenkarten, gängige Verfahren der Risikoanalyse).

Tabelle 6 Beurteilung der Anforderungen an Datengrundlage.

\begin{tabular}{|c|c|c|c|c|c|}
\hline \multicolumn{2}{|c|}{ Eignung betr. Datengrundlage } & \multirow[t]{2}{*}{$\begin{array}{l}\text { Informati- } \\
\text { onsbedarf }\end{array}$} & \multirow[t]{2}{*}{$\begin{array}{c}\text { Automa- } \\
\text { tisierbarkeit }\end{array}$} & \multirow[t]{2}{*}{$\begin{array}{l}\text { Anküpfung } \\
\text { an } \\
\text { vorhandene } \\
\text { Operate }\end{array}$} & \multirow[b]{2}{*}{ Durchschnitt } \\
\hline \multicolumn{2}{|r|}{ Ansatz } & & & & \\
\hline \multicolumn{2}{|c|}{$\begin{array}{c}\text { Grenzwerte (1) für } \\
\text { Versagenswahrschein- } \\
\text { lichkeiten }\end{array}$} & 0 & 0 & 0 & 0.0 \\
\hline \multirow{3}{*}{ 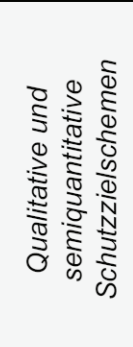 } & $\begin{array}{c}\text { Schutzziele KS } \\
\text { Nr. } 20\end{array}$ & 2 & 2 & 1 & 1.7 \\
\hline & $\begin{array}{c}\text { Schutzziele } \\
\text { Hochwasser- } \\
\text { schutz an Fliess- } \\
\text { gewässern }\end{array}$ & 2 & 2 & 2 & 2.0 \\
\hline & $\begin{array}{l}\text { Schutzziele } \\
\text { BUWAL 107/1 }\end{array}$ & 2 & 2 & 2 & 2.0 \\
\hline \multirow{4}{*}{ 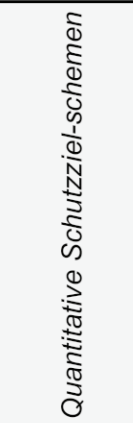 } & $\begin{array}{c}\text { F/N- Diagramme } \\
* \text { *) }\end{array}$ & 0 & 1 & 1 & 0.7 \\
\hline & $\underset{* \pi}{\text { F/N-Diagramme }}$ & 0 & 1 & 1 & 0.7 \\
\hline & $\begin{array}{l}\text { Schutzziele } \\
\text { Erdbeben an } \\
\text { bestehenden } \\
\text { Gebäuden *) }\end{array}$ & 0 & 1 & 0 & 0.3 \\
\hline & Do. $\left.{ }^{* *}\right)$ & 0 & 1 & 0 & 0.3 \\
\hline \multirow{2}{*}{$\begin{array}{ll}\frac{0}{N} \\
\frac{1}{0} \\
\frac{1}{0}\end{array}$} & Indiv. Risiko & 1 & 1 & 1 & 1.0 \\
\hline & Kollekt. Risiko & 1 & 1 & 1 & 1.0 \\
\hline \multicolumn{2}{|c|}{ Grenzkosten } & 0 & 0 & 1 & 0.3 \\
\hline
\end{tabular}

*) Bereich absoluter Schutzziele (intolerabel, Massnahmen zwingend erforderlich).

${ }^{* *}$ ) Bereich mit Verhältnismässigkeitsprüfung (ALARA).

2 günstige Bedingungen

1 teilweise günstige Bedingungen

0 ungünstige Bedingungen

Josef Th. Hess: Schutzziele im Umgang mit Naturrisiken in der Schweiz, @ vdf Hochschulverlag 2011 


\section{VIII.IV Einsatzmöglichkeit für regionales Risikomanagement}

Die betrachteten Schutzzieldarstellungen wurden schliesslich beurteilt hinsichtlich:

- Eignung zur grossräumigen Übersicht der Risikoverteilung und

- Eignung zur grossräumigen Übersicht der Effizienz von Risiko-Management Massnahmen.

Tabelle 7 Beurteilung der Eignung für regionales Risikomanagement.

\begin{tabular}{|c|c|c|c|c|}
\hline \multirow{2}{*}{\multicolumn{2}{|c|}{ Eignung reg. Management }} & \multicolumn{2}{|c|}{ Eignung für Projektpriorisierung } & \multirow[b]{3}{*}{ Durchschnitt } \\
\hline & & \multirow{2}{*}{$\begin{array}{c}\text { Risikover- } \\
\text { teilung }\end{array}$} & \multirow{2}{*}{$\begin{array}{c}\text { Massnahmen- } \\
\text { effizienz }\end{array}$} & \\
\hline & Ansatz & & & \\
\hline \multicolumn{2}{|c|}{$\begin{array}{c}\text { Grenzwerte (1) für } \\
\text { Versagenswahrschein- } \\
\text { lichkeiten }\end{array}$} & 0 & 0 & 0 \\
\hline \multirow{3}{*}{ 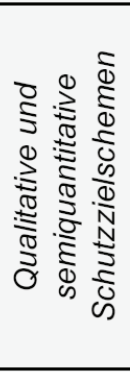 } & $\begin{array}{c}\text { Schutzziele KS } \\
\text { Nr. } 20\end{array}$ & 2 & 1 & 1.5 \\
\hline & $\begin{array}{c}\text { Schutzziele } \\
\text { Hochwasser- } \\
\text { schutz an } \\
\text { Fliessge-wässern }\end{array}$ & 2 & 1 & 1.5 \\
\hline & $\begin{array}{c}\text { Schutzziele } \\
\text { BUWAL 107/1 }\end{array}$ & 2 & 1 & 1.5 \\
\hline \multirow{4}{*}{ 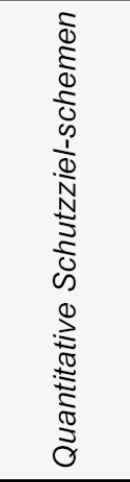 } & $\begin{array}{c}\text { F/N- Diagramme } \\
\left.{ }^{*}\right)\end{array}$ & 0 & 0 & 0 \\
\hline & $\left.\begin{array}{c}\text { F/N- Diagramme } \\
* *\end{array}\right)$ & 0 & 2 & 1 \\
\hline & $\begin{array}{l}\text { Schutzziele } \\
\text { Erdbeben an } \\
\text { bestehenden } \\
\text { Gebäuden *) }\end{array}$ & 0 & 0 & 0 \\
\hline & Do. $\left.{ }^{* *}\right)$ & 0 & 2 & 1 \\
\hline \multirow{2}{*}{$\frac{N}{\frac{1}{d}} \frac{\mathbb{1}}{d}$} & Indiv. Risiko & 1 & 0 & 0.5 \\
\hline & Kollekt. Risiko & 1 & 0 & 0.5 \\
\hline \multicolumn{2}{|c|}{ Grenzkosten } & 0 & 2 & 1 \\
\hline
\end{tabular}

*) Bereich absoluter Schutzziele (intolerabel, Massnahmen zwingend erforderlich)

**) Bereich mit Verhältnismässigkeitsprüfung (ALARA)

2 sehr geeignet

1 teilw. geeignet

0 ungeeignet 


\section{Systematik der Schutzzielprinzipien}

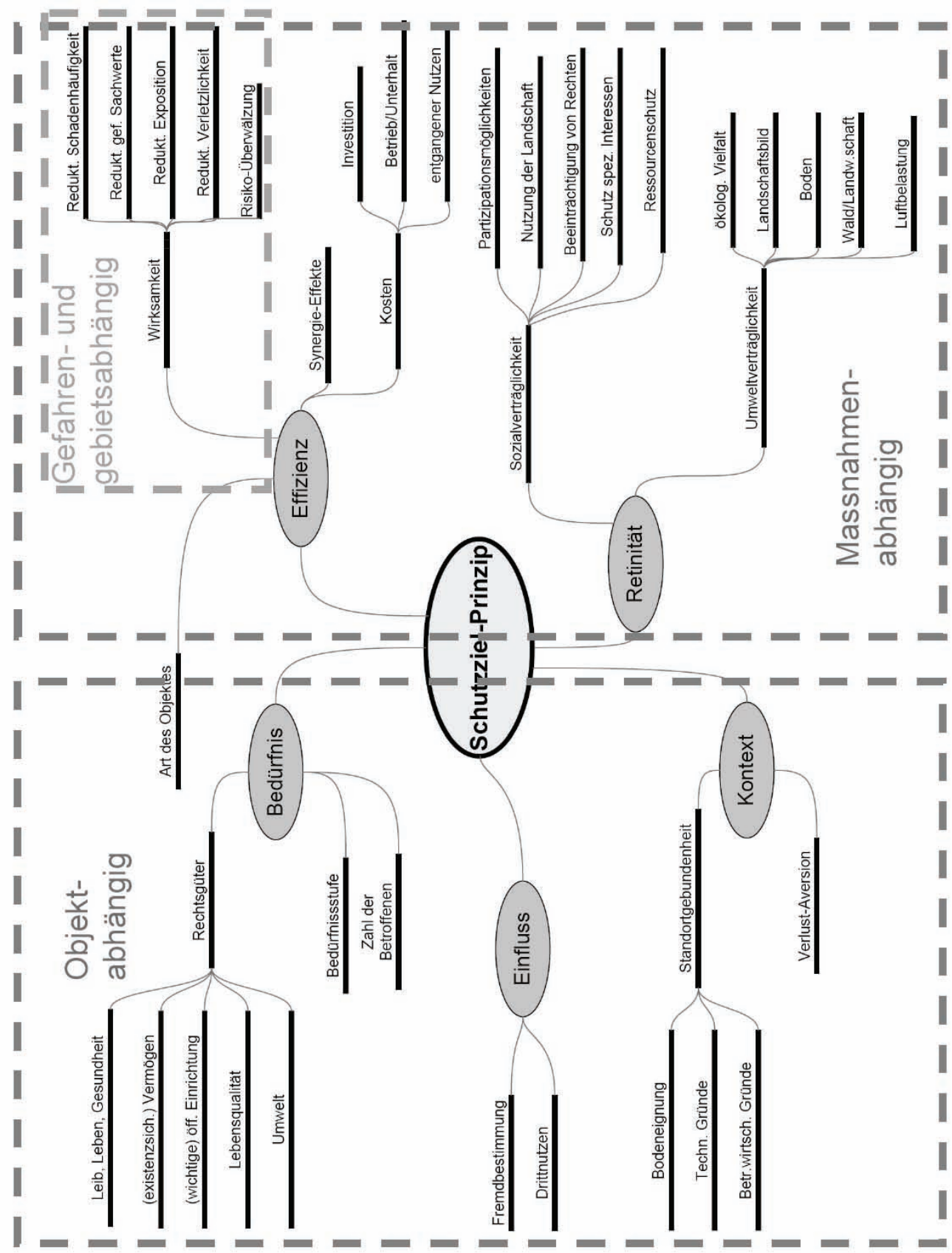




\section{Objektkategorien und Schutzzielprinzipien}
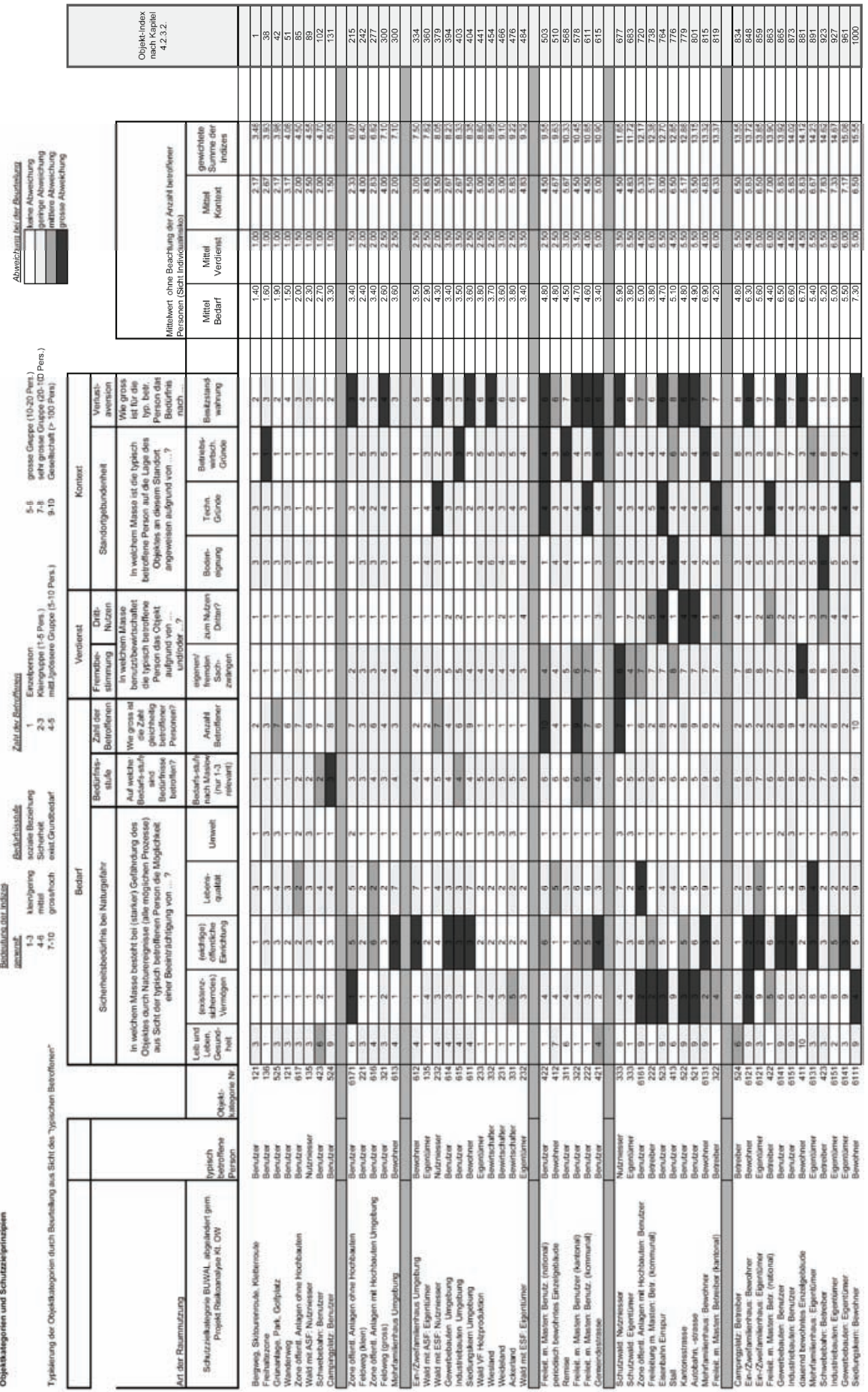


\section{Massnahmen und Schutzzielprinzipien (Vernetzung)}

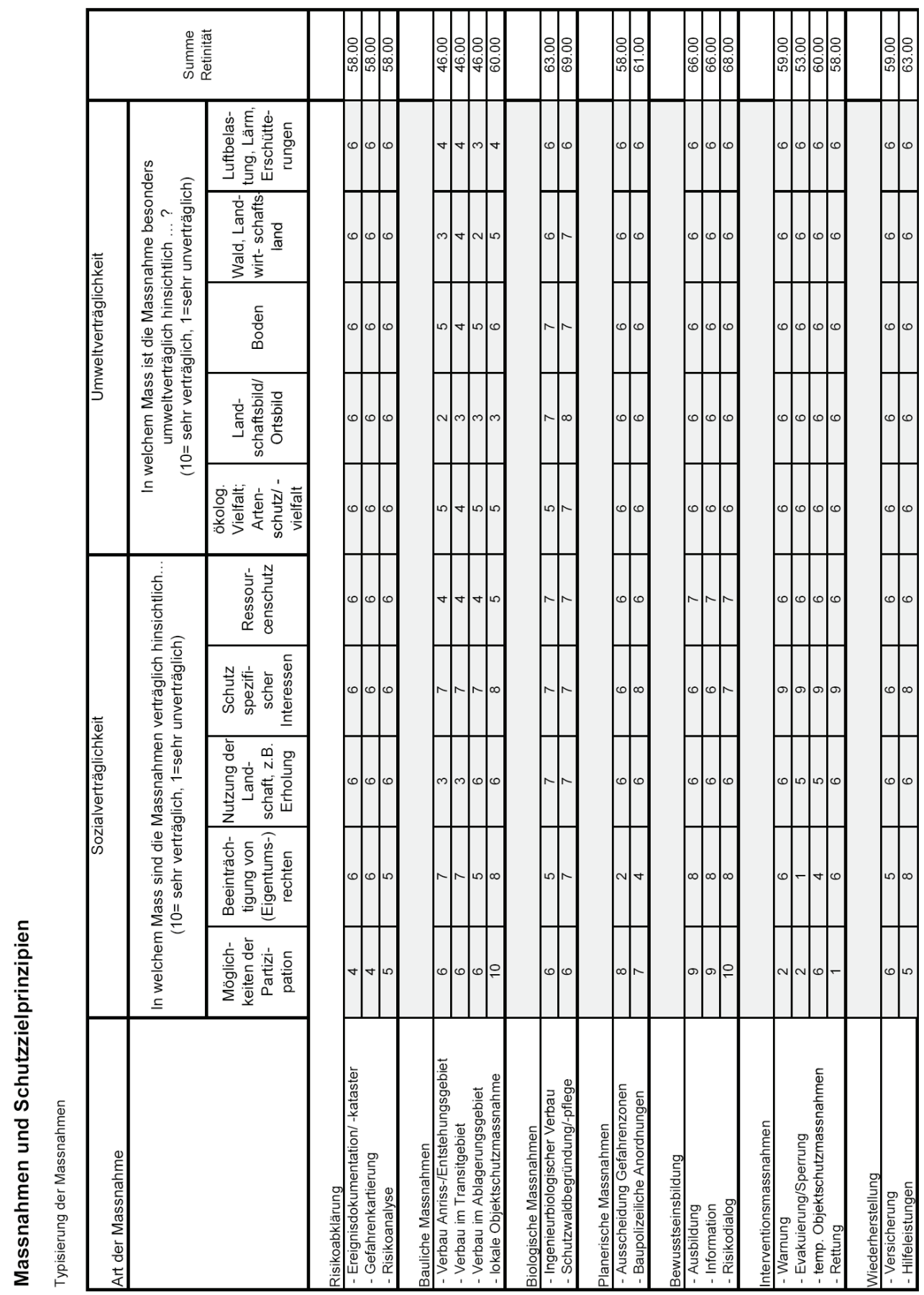




\section{Massnahmen und Schutzzielprinzipien}

\section{XII.I Massnahmeneignung (Eignungskriterien)}

\begin{tabular}{|c|c|c|c|c|c|c|c|c|c|c|c|c|}
\hline \multicolumn{4}{|c|}{ Kommentar zu Massnahmen und (örtlicher) Eignung } & $\begin{array}{c}\text { Abstufung } \\
1-3 \\
4-6 \\
7-10 \\
\end{array}$ & $\begin{array}{l}\text { der Indize } \\
\text { gering } \\
\text { mittel } \\
\text { gross }\end{array}$ & & & & & & & \\
\hline & \multicolumn{12}{|c|}{ Effizienz } \\
\hline Art der Massnahme & \multicolumn{12}{|c|}{ Nutzen: In welchem Mass bieten Massnahmen typischerweise Schutz vor Schäden durch ...? } \\
\hline Charakter des Gefahrenprozesses & \multicolumn{12}{|c|}{... brutale Prozesse wie Lawinen, Sturz, Murgänge ...? } \\
\hline Intensität des Gefahrenprozesses & \multicolumn{6}{|c|}{.... grosser Intensität? } & \multicolumn{6}{|c|}{... mittlerer/geringer Intensität? } \\
\hline lokale Vorhersehbarkeit Ereignis & \multicolumn{3}{|c|}{$\begin{array}{l}\text {... bei eher guter } \\
\text { Vorhersehbarkeit von } \\
\text { Einzelereignissen (Lawinen) }\end{array}$} & \multicolumn{3}{|c|}{$\begin{array}{l}\text {... bei eher schlechter } \\
\text { Vorhersehb. von } \\
\text { Einzelereignissen (übrige) }\end{array}$} & \multicolumn{3}{|c|}{$\begin{array}{l}\text {... bei eher guter } \\
\text { Vorhersehbarkeit von } \\
\text { Einzelereignissen (Lawinen) }\end{array}$} & \multicolumn{3}{|c|}{$\begin{array}{l}\text {.. bei eher schlechter } \\
\text { Vorhersehbarkeit von } \\
\text { Einzelereignissen (übrige) }\end{array}$} \\
\hline Art des Schadenpotenzials & 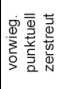 & 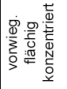 & 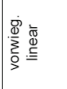 & 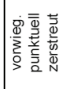 & 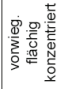 & 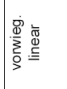 & 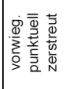 & 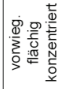 & 畩 & 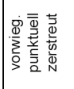 & 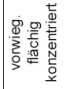 & 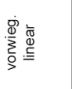 \\
\hline \multicolumn{13}{|l|}{ Risikoabklärung } \\
\hline $\begin{array}{l}\text { - Ereignisclokumentation/-kataster } \\
\text { - Gefahrenkartierung } \\
\text { - Risikoanalyse } \\
\end{array}$ & \multicolumn{12}{|c|}{$\begin{array}{l}\text { Den Gefahrengrundlagen kommt eine Bedeutung als Grundlage zu. Für sich allein bieten sie keinen wirksamen Schutz vor } \\
\text { Schäden. Die Wirksamkeit wird daher als gering beurteilt. }\end{array}$} \\
\hline \multicolumn{13}{|l|}{ Bauliche Massnahmen } \\
\hline - Verbau Anriss-/Entstehungsgebiet & \multicolumn{12}{|c|}{$\begin{array}{l}\text { Den Massnahmen im Anrissgebiet wird bei brutalen Prozessen allgemein eine gute Wirksamkeit zugerechnet. Die Wirksamkeit } \\
\text { wird umso höher eingestuft, je konzentrierter das Schadenpotenzial ist. }\end{array}$} \\
\hline - Verbau im Transitgebiet & \multirow{2}{*}{\multicolumn{12}{|c|}{$\begin{array}{l}\text { Bei den technischen Massnahmen im Transit- und Ablagerungsgebiet wird den technischen Massnahmen bei grosser Intensität } \\
\text { eine eher geringe, bei mittlerer bis geringer Intensität jedoch eine bedeutende Wirksamkeit zugerechnet. Unterschiede ergeben } \\
\text { sich wiederum aus der Konzentration des Schadenpotenzials, aber auch aufgrund der involvierten Prozesse (Lawinen einerseits } \\
\text { und übrige brutale Prozesse anderseits). }\end{array}$}} \\
\hline - Verbau im Ablagerungsgebiet & & & & & & & & & & & & \\
\hline - lokale Objektschutzmassnahmen & \multicolumn{12}{|c|}{$\begin{array}{l}\text { Bei den lokalen Objektschutzmassnahmen gilt das Gleiche wie bei den technischen Massnahmen im Transit- und } \\
\text { Ablagerungsgebiet, jedoch kommt der Konzentration des Schadenpotenzials ausgeprägte Bedeutung zu. } \\
\text { Objektschutzmassnahmen sind v.a. punktuell einsetzbar. }\end{array}$} \\
\hline \multicolumn{13}{|l|}{ Biologische Massnahmen } \\
\hline - Ingenieurbiologischer Verbau & \multicolumn{12}{|c|}{$\begin{array}{l}\text { Ingenieurbiologischen Verbauungen wird bei brutalen Prozessen eine eher geringe Bedeutung zugemessen, jedoch ergeben sich } \\
\text { Unterschiede zwischen den Prozessen (geringe Wirksamkeit bei Lawinen, bessere Wirksamkeit bei übrigen brutalen Prozessen. }\end{array}$} \\
\hline - Schutzwaldbegründung/-pflege & \multicolumn{12}{|c|}{$\begin{array}{l}\text { Dem Schutzwald wird vor allem im Entstehungsgebiet (Lawinen) aber tlw. auch im Transitgebiet (Steinschlag) erhebliche } \\
\text { Wirksamkeit zugemessen. Unterschiedliche Bewertungen erfolgten bezüglich Intensitäten (schlechtere Wirksamkeit bei grosser } \\
\text { Intensität) und Konzentration des Schadenpotenzials. }\end{array}$} \\
\hline \multicolumn{13}{|l|}{ Planerische Massnahmen } \\
\hline - Ausscheidung Gefahrenzonen & \multicolumn{12}{|c|}{$\begin{array}{l}\text { Der Ausscheidung von Gefahrenzonen wurde eine hohe Wirksamkeit zugemessen. Etwas schlechter bewertet wurde dabei die } \\
\text { Wirksamkeit bei linearem Schadenpotenzial ( } \boldsymbol{z} \text {.B. Life Lines, bei deren Anlage Gefahrengebiete meist nicht umgangen werden } \\
\text { können). Eine begrenzte Wirksamkeit ergibt sich, wenn Gefahrenzonen über bereits bebautes Gebiet gelegt werden. }\end{array}$} \\
\hline - Baupolizeiliche Anordnungen & \multicolumn{12}{|c|}{$\begin{array}{l}\text { Für baupolizeiliche Anordnungen (=Bauauflagen für Objektschutz) wurden ähnliche Bewertungen vorgenommen wie für lokale } \\
\text { Objektschutzmassnahmen (siehe oben). Bei flächigem Schadenpotenzial sind organisatorische Auflagen möglich, daher die } \\
\text { leicht höhere Bewertung als bei Objektschutzmassnahmen. Prozessspezifisch besteht eine geringe Wirksamkeit vor allem bei } \\
\text { Rutschungen. }\end{array}$} \\
\hline \multicolumn{13}{|l|}{ Bewusstseinsbildung } \\
\hline \begin{tabular}{|l|} 
- Ausbildung \\
- Information \\
- Risikodialog \\
\end{tabular} & \multicolumn{12}{|c|}{$\begin{array}{l}\text { Den Informations- und Bewusstseinsbildungsmassnahmen per se wird wiederum eine eher geringe Wirksamkeit beigemessen. } \\
\text { Etwas besser bewertet wird die Wirksamkeit bei vorhersehbaren Prozessen sowie bei graduellen Prozessen, bei denen im } \\
\text { Ereignisfall eine grössere Reaktionszeit besteht. }\end{array}$} \\
\hline \multicolumn{13}{|l|}{ Interventionsmassnahmen } \\
\hline - Warnung & $\begin{array}{l}\text { Die Wirks } \\
\text { Gefahren } \\
\text { Wirksamk }\end{array}$ & $\begin{array}{l}\text { amkeit der } \\
\text { rozesses } \\
\text { eit. }\end{array}$ & $\begin{array}{l}\text { Warnung } \\
\text { ab. Bei gra }\end{array}$ & $\begin{array}{l}\text { längt einers } \\
\text { duellen Pro }\end{array}$ & $\begin{array}{l}\text { seits von d } \\
\text { zessen un }\end{array}$ & $\begin{array}{l}\text { i Vorhers } \\
\text { I bessere }\end{array}$ & $\begin{array}{l}\text { hbarkeit de } \\
\text { Vorherseh }\end{array}$ & $\begin{array}{l}\text { r Einzelere } \\
\text { arkeit der }\end{array}$ & $\begin{array}{l}\text { eignisse u } \\
\text { Einzelere }\end{array}$ & $\begin{array}{l}\text { andersei } \\
\text { nisse best }\end{array}$ & $\begin{array}{l}\text { tis vom Ch } \\
\text { teht eine e }\end{array}$ & $\begin{array}{l}\text { rakter des } \\
\text { höhte }\end{array}$ \\
\hline - Evakuierung/Sperrung & $\begin{array}{l}\text { Für die W } \\
\text { Wirksamk } \\
\text { Betroffen } \\
\text { dass unb }\end{array}$ & $\begin{array}{l}\text { iksamkeit } \\
\text { eit aufgrun } \\
\text { selber die } \\
\text { wegliches }\end{array}$ & $\begin{array}{l}\text { von Evaku } \\
\text { d des zwin } \\
\text { richtigen } \\
\text { Schadenp }\end{array}$ & $\begin{array}{l}\text { ierungen ur } \\
\text { genden Ch } \\
\text { Konsequen } \\
\text { otenzial dar }\end{array}$ & $\begin{array}{l}\text { a Sperrun } \\
\text { arakters di } \\
\text { zen ziehen } \\
\text { mit nicht ge }\end{array}$ & $\begin{array}{l}\text { gen gilt gr } \\
\text { eser Mass } \\
\text { muss. So } \\
\text { eschützt w }\end{array}$ & $\begin{array}{l}\text { ndsätzlich } \\
\text { hahmen höl } \\
\text { vohl bei Wa } \\
\text { rden kann. }\end{array}$ & $\begin{array}{l}\text { das Gleich } \\
\text { her eingest } \\
\text { arnungen al }\end{array}$ & $\begin{array}{l}\text { e wie für V } \\
\text { tuft als etw } \\
\text { Is auch be }\end{array}$ & $\begin{array}{l}\text { Jarnungen. } \\
\text { bei Warnu } \\
\text { Evakuierur }\end{array}$ & $\begin{array}{l}\text { Doch wur } \\
\text { ungen, be } \\
\text { ngen ist } z\end{array}$ & $\begin{array}{l}\text { de die } \\
\text { denen der } \\
\text { beachten, }\end{array}$ \\
\hline - temp. Objektschutzmassnahmen & $\begin{array}{l}\text { Die Wirks } \\
\text { mittlerer }\end{array}$ & $\begin{array}{l}\text { amkeit tem } \\
\text { is geringer }\end{array}$ & $\begin{array}{l}\text { porärer O O } \\
\text { Intensität. }\end{array}$ & jektschutzn & nassnahm & beschre & kt sich aut & f Prozesse & mit vorhe & sehbaren $\mathrm{E}$ & inzelereig & issen und \\
\hline Rettung & \begin{tabular}{|l|} 
Mit Rettur \\
Lawinen \\
abnehme
\end{tabular} & $\begin{array}{l}\text { gsmassna } \\
\text { rerden leic } \\
\text { ider Intens }\end{array}$ & $\begin{array}{l}\text { men köni } \\
\text { t günstig } \\
\text { ät des } \mathrm{G}\end{array}$ & $\begin{array}{l}\text { ien teilweis } \\
\text { r beurteilt } \\
\text { fahrenproz }\end{array}$ & $\begin{array}{l}\text { e der Verlu } \\
\text { as die übrį } \\
\text { esses. }\end{array}$ & $\begin{array}{l}\text { t von } \mathrm{Me} \\
\text { on brutal }\end{array}$ & $\begin{array}{l}\text { chenlebe } \\
\text { Prozesse }\end{array}$ & $\begin{array}{l}\text { oder Ges } \\
\text { Auch stei }\end{array}$ & $\begin{array}{l}\text { undheitss } \\
\text { t die Wirl }\end{array}$ & $\begin{array}{l}\text { äden vern } \\
\text { amkeit de }\end{array}$ & $\begin{array}{l}\text { mindert we } \\
\text { I Rettung }\end{array}$ & $\begin{array}{l}\text { den. } \\
\text { nit }\end{array}$ \\
\hline Wiederherstellung & & & & & & & & & & & & \\
\hline - Versicherung & $\begin{array}{l}\text { Versicher } \\
\text { der (nicht }\end{array}$ & $\begin{array}{l}\text { ungen vern } \\
\text { risikogere }\end{array}$ & $\begin{array}{l}\text { רeiden nich } \\
\text { chten) Prar }\end{array}$ & $\begin{array}{l}\text { tt die Ereigr } \\
\text { nienabstufu }\end{array}$ & $\begin{array}{l}\text { hisse und } \\
\text { lng sind } V e\end{array}$ & tie Schäde & $\begin{array}{l}n, \text { mildern a } \\
\text { en für Betro }\end{array}$ & $\begin{array}{l}\text { aber die wir } \\
\text { offene in Ri }\end{array}$ & $\begin{array}{l}\text { tschaftlich } \\
\text { isikogebie }\end{array}$ & $\begin{array}{l}\text { en Folgen. } \\
\text { en wirksam }\end{array}$ & Vor allem & ingesichts \\
\hline - Hilfeleistungen & $\begin{array}{l}\text { Für Hilfel } \\
\text { Ereigniss } \\
\text { (auch we }\end{array}$ & $\begin{array}{l}\text { istungen g } \\
\text {, welche z } \\
\text { in letztere }\end{array}$ & $\begin{array}{l}\text { ilt das Gle } \\
\text { udem viel } \\
\text { Einzelne h }\end{array}$ & $\begin{array}{l}\text { che wie für } \\
\text { Schadenpo } \\
\text { art treffen). }\end{array}$ & $\begin{array}{l}\text { Versicher } \\
\text { tenzial betr }\end{array}$ & $\begin{array}{l}\text { ngen. Die } \\
\text { effen, grö }\end{array}$ & $\begin{array}{l}\text { vorgenomn } \\
\text { sere Hilfele }\end{array}$ & $\begin{array}{l}\text { mene Abstu } \\
\text { eistungskan }\end{array}$ & $\begin{array}{l}\text { fifung berü } \\
\text { mpagnen }\end{array}$ & $\begin{array}{l}\text { ksichtigt, d } \\
\text { uslösen als }\end{array}$ & $\begin{array}{l}\text { lass heftig } \\
\text { kleine } \mathrm{Er}\end{array}$ & $\begin{array}{l}\text { re } \\
\text { ignisse }\end{array}$ \\
\hline
\end{tabular}




\section{XII.II Massnahmeneignung (Eignungskriterien)}

Kommentar zu Massnahmen und (örtlicher) Eignung

Typisierung der Massnahmen
Abstufung der Indizes

$1-3$ gering

4-6 mittel

$7-10$ gross

\begin{tabular}{|c|c|c|c|c|c|c|c|c|c|c|c|c|}
\hline \multicolumn{13}{|c|}{ 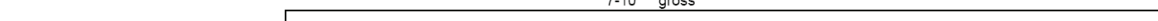 } \\
\hline & \multicolumn{12}{|c|}{ Effizienz } \\
\hline Art der Massnahme & \multicolumn{12}{|c|}{ Nutzen: In welchem Mass bieten Massnahmen typischerweise Schutz vor Schäden durch ...? } \\
\hline Charakter des Gefahrenprozesses & \multicolumn{12}{|c|}{... graduelle Prozesse wie Rutschungen, Hochwasser ...? } \\
\hline Intensität des Gefahrenprozesses & \multicolumn{6}{|c|}{... grosser Intensität? } & \multicolumn{6}{|c|}{... mittlerer/geringer Intensität? } \\
\hline lokale Vorhersehbarkeit Ereignis & \multicolumn{3}{|c|}{$\begin{array}{c}\text {... bei eher guter } \\
\text { Vorhersehbarkeit von } \\
\text { Einzelereignissen (Hochwasser) }\end{array}$} & \multicolumn{3}{|c|}{$\begin{array}{l}\text {... bei eher schlechter } \\
\text { Vorhersehb. von } \\
\text { Einzelereignissen (Rutsch) }\end{array}$} & \multicolumn{3}{|c|}{$\begin{array}{c}\text {... bei eher guter } \\
\text { Vorhersehbarkeit von } \\
\text { Einzelereignissen (Hochwasser) }\end{array}$} & \multicolumn{3}{|c|}{$\begin{array}{l}\text {... bei eher schlechter } \\
\text { Vorhersehb. von } \\
\text { Einzelereignissen (Rutsch }\end{array}$} \\
\hline Art des Schadenpotenzials & 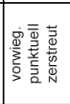 & 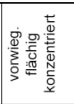 & 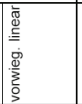 & 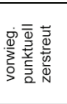 & 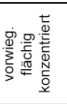 & 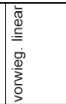 & 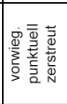 & 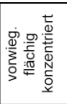 & 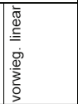 & 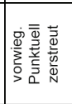 & 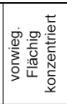 & 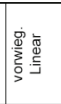 \\
\hline
\end{tabular}

\begin{tabular}{|c|c|}
\hline Risikoabklärung & \\
\hline $\begin{array}{l}\text { - Ereignisdokumentation/ -kataster } \\
\text { - Gefahrenkartierung } \\
\text { - Risikoanalyse }\end{array}$ & $\begin{array}{l}\text { Den Gefahrengrundlagen kommt eine Bedeutung als Grundlage zu. Für sich allein bieten sie keinen wirksamen Schutz vor } \\
\text { Schäden. Die Wirksamkeit wird daher als gering beurteilt. }\end{array}$ \\
\hline - Verbau Anriss-/Entstehungsgebiet & $\begin{array}{l}\text { Die Wirksamkeit baulicher Massnahmen im Einzugsgebiet wird für Hochwasser und Rutschungen als bedeutendste graduelle } \\
\text { Prozesse eher gering beurteilt. }\end{array}$ \\
\hline - Verbau im Transitgebiet & $\begin{array}{l}\text { Die Wirksamkeit wird für Hochwasser einerseits und für Rutschungen anderseits stark unterschiedlich beurteilt. Im Transitbereich } \\
\text { besteht bezüglich Hochwasser vor allem die Möglichkeit des Geschiebe- und Schwemmholzrückhaltes. }\end{array}$ \\
\hline - Verbau im Ablagerungsgebiet & $\begin{array}{l}\text { Die unterschledliche Wirksamkeit für Hochwasser und für Rutschungen gilt auch im Ablagerungsgebiet. Vor allem bel Hochwasser } \\
\text { bestehen wirksame Massnahmen im Ablagerungsgebiet. Eine Abstufung ergibt sich hier aus der Konzentration und Anordnung des } \\
\text { Schadenpotenzials: Ausgedehnte und flächige Objekte sind schwieriger zu schützen. }\end{array}$ \\
\hline - lokale Objektschutzmassnahmen & $\begin{array}{l}\text { Es gelten die gleichen Überlegungen wie bei den butalen Prozessen. Jedoch bestehen bedeutende Unterschiede zwischen den } \\
\text { hauptsächlichen graduellen Prozessen Hochwasser und Rutschungen. Während es gegen Hochwasser (zumindest mittlerer bis } \\
\text { geringer Intensität) adäquate Objektschutzmassnahmen gibt, sind solche gegen Rutschungen kaum auszumachen. }\end{array}$ \\
\hline - Ingenieurbiologischer Verbau & $\begin{array}{l}\text { Die Wirksamkeit ingenieurbiologischer Massnahmen per se wird sowohl gegen Hochwasser als auch gegen Rutschungen eher } \\
\text { gering eingestuft. Etwas bessere Wirksamkeit wird den Massnahmen bei mittlerer und geringer Intensität zugemutet. }\end{array}$ \\
\hline - Schutzwaldbegründung/-pflege & $\begin{array}{l}\text { Dem Schutzwald wird eine geringe bis mittlere Wirksamkeit gegen Hochwasser und bei mittleren und geringeren Intensitäten auch } \\
\text { gegen Rutschungen zugemutet. }\end{array}$ \\
\hline \multicolumn{2}{|l|}{ Planerische Massnahmen } \\
\hline - Ausscheidung Gefahrenzonen & $\begin{array}{l}\text { Der Ausscheidung von Gefahrenzonen wurde eine hohe Wirksamkeit zugemessen. Etwas schlechter bewertet wurde dabel die } \\
\text { Wirksamkeit bei linearem Schadenpotenzial (z.B. Life Lines, bei deren Anlage Gefahrengebiete meist nicht umgangen werden } \\
\text { können). Eine begrenzte Wirksamkeit ergibt sich, wenn Gefahrenzonen über bereits bebautes Gebiet gelegt werden. }\end{array}$ \\
\hline - Baupolizeiliche Anordnungen & $\begin{array}{l}\text { Für baupolizelliche Anordnungen (=Bauauflagen für Objektschutz) wurden ähnliche Bewertungen vorgenommen wie für lokale } \\
\text { Objektschutzmassnahmen (siehe oben). Bei flächigem Schadenpotenzial sind organisatorische Auflagen möglich, daher die leicht } \\
\text { höhere Bewertung als bei Objektschutzmassnahmen. Prozessspezifisch besteht eine geringe Wirksamkeit vor allem bei } \\
\text { Rutschungen. }\end{array}$ \\
\hline -Warnung & $\begin{array}{l}\text { Die Wirksamkeit der Warnung hängt einerseits von der Vorhersehbarkeit der Einzelereignisse und anderseits vom Charakter des } \\
\text { Gefahrenprozesses ab. Bei graduellen Prozessen und besserer Vorhersehbarkeit der Einzelereignisse besteht eine erhöhte } \\
\text { Wirksamkeit. }\end{array}$ \\
\hline - Evakuierung/Sperrung & $\begin{array}{l}\text { Für die Wirksamkeit von Evakuierungen und Sperrungen gilt grundsätzlich das Gleiche wie für Warnungen. Doch wurde die } \\
\text { Wirksamkeit aufgrund des zwingenden Charakters dieser Massnahmen höher eingestuft als etwa bei Warnungen, bei denen der } \\
\text { Betroffene selber die richtigen Konsequenzen ziehen muss. Sowohl bei Warnungen als auch bei Evakuierungen ist zu beachten, } \\
\text { dass unbewegliches Schadenpotenzial damit nicht geschützt werden kann. }\end{array}$ \\
\hline - temp. Objektschutzmassnahmen & $\begin{array}{l}\text { Vor allem bei Hochwassern geringer bis mittlerer Intensität wird Objektschutzmassnahmen eine bedeutende Wirksamkeit } \\
\text { zugerechnet. Gegen Hochwasser grosser Intensität und gegen Rutschungen sind dagegen kaum wirksame temporäre } \\
\text { Objektschutzmassnahmen vorhanden. }\end{array}$ \\
\hline Rettung & $\begin{array}{l}\text { Bei graduellen Prozessen wird die Wirksamkeit der Rettung höher eingestuft als bei den brutalen Prozessen, da aufgrund der } \\
\text { längeren Reaktionszeit eine gewisse Möglichkeit der Selbstrettung besteht und - nebst Leib und Leben - in gewissem Umfang auch } \\
\text { bewegliche Sachwerte in Sicherheit gebracht werden können. }\end{array}$ \\
\hline \multicolumn{2}{|l|}{ Wiederherstellung } \\
\hline - Versicherung & $\begin{array}{l}\text { Versicherungen vermeiden nicht die Ereignisse und die Schäden, mildern aber die wirtschaftlichen Folgen. Vor allem angesichts der } \\
\text { (nicht risikogerechten) Prämienabstufung sind Versicherungen für Betroffene in Risikogebieten wirksam. }\end{array}$ \\
\hline - Hilfeleistungen & $\begin{array}{l}\text { Für Hilfeleistungen gilt das Gleiche wie für Versicherungen. Die vorgenommene Abstufung berücksichtigt, dass heftigere Ereignisse, } \\
\text { welche zudem viel Schadenpotenzial betreffen, grössere Hilfeleistungskampagnen auslösen als kleine Ereignisse (auch wenn } \\
\text { letztere Einzelne hart treffen). }\end{array}$ \\
\hline
\end{tabular}




\section{XII.III Massnahmeneignung (Eignungskriterien)}

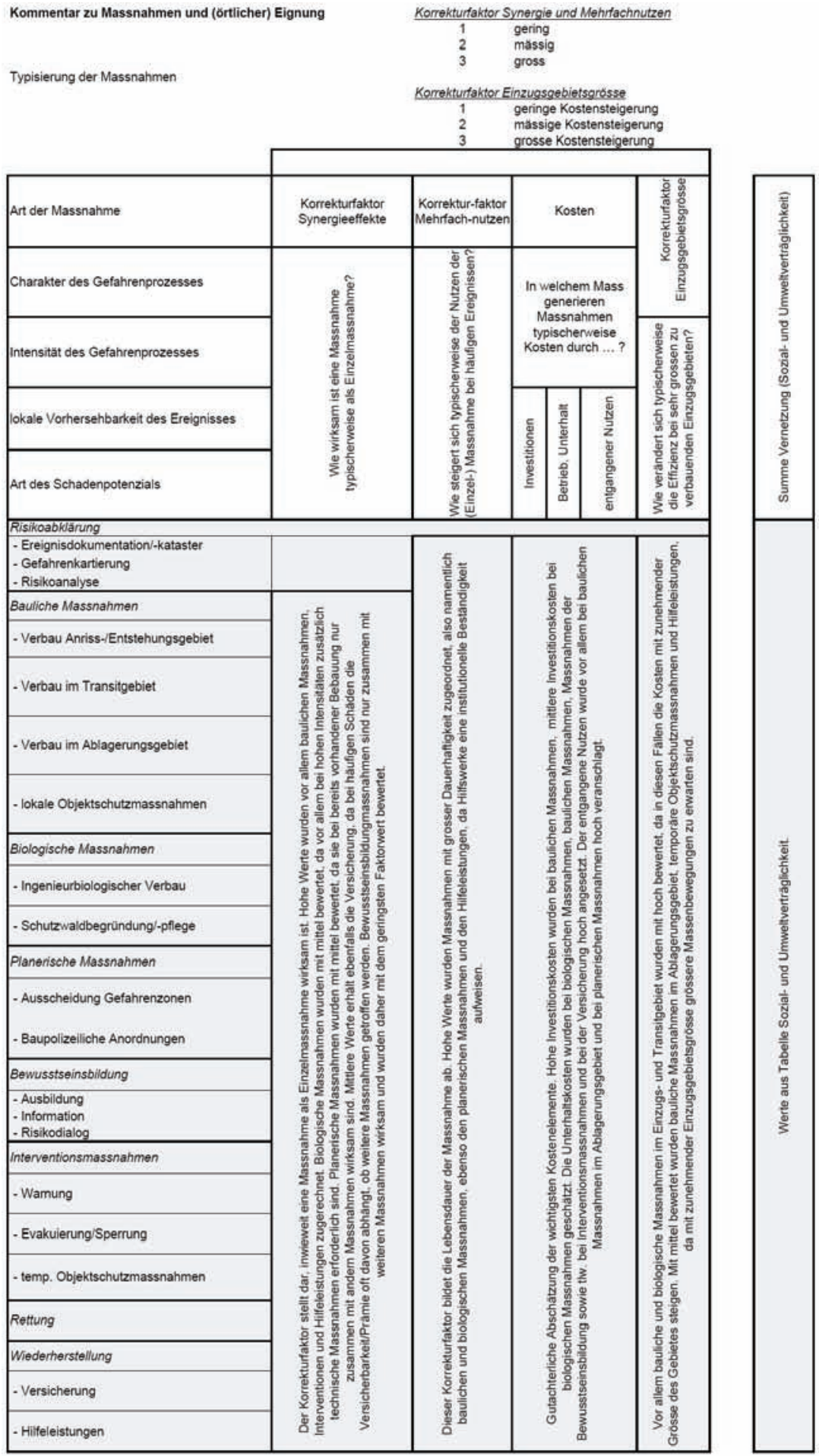




\section{Massnahmenindex}

\section{XIII.I Beispiel: Häufige Ereignisse, kleine bis mittlere Einzugsgebiete}

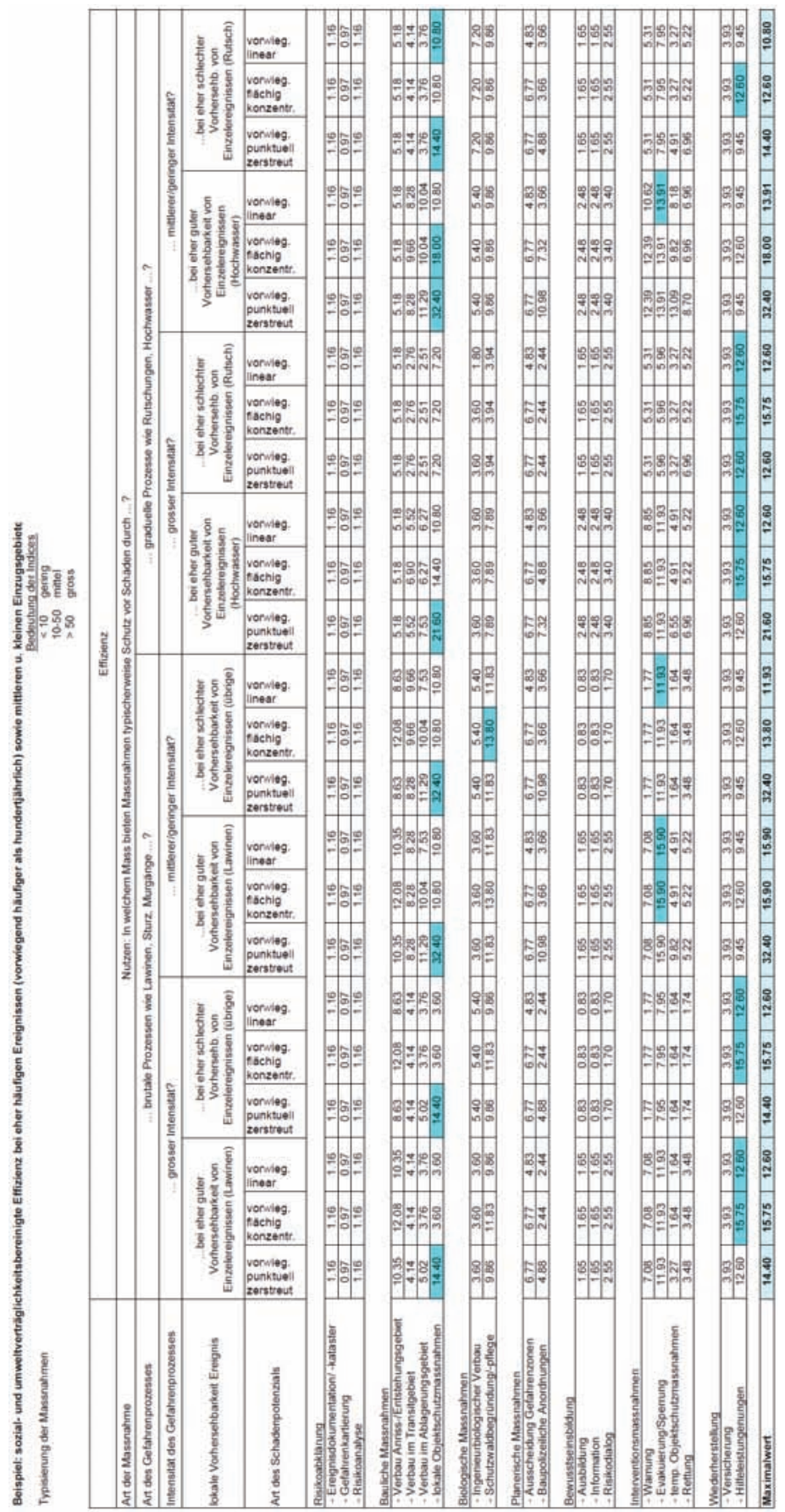




\section{XIII.II Übersicht}

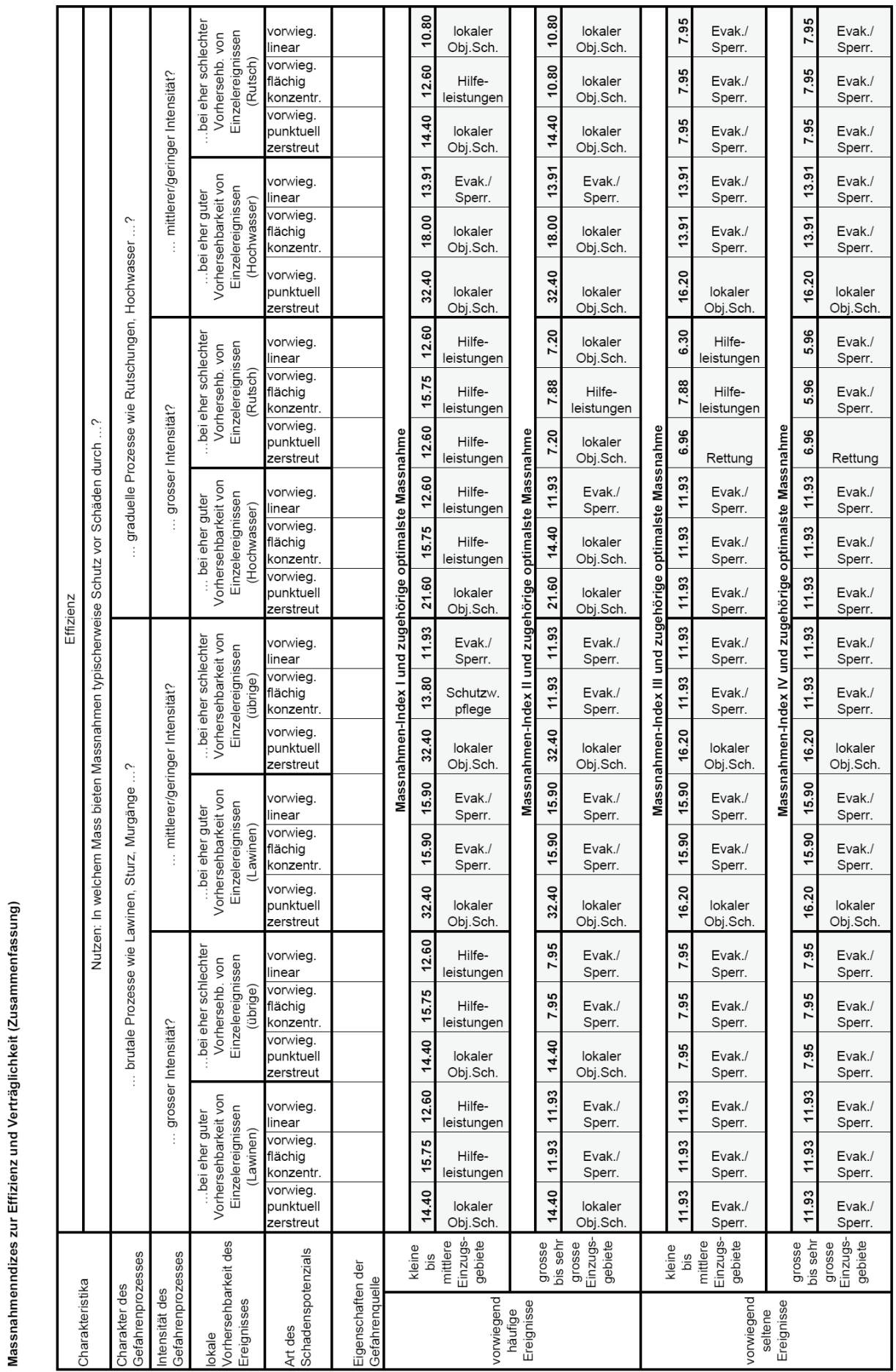




\section{Modifizierte Schutzzielmatrix}

\section{XIV.I Version ,Benutzer'}

Fallbelsplel Engelberg, Kanton Obwalden

modifizierte Objektkategorieeintellung 1 aufgrund Objektindex aus Sicht Benutzer

\begin{tabular}{|c|l|l|l|}
\hline \multicolumn{2}{|c|}{ zulässige Intensităten gem. Schutzzielen } \\
\hline 0 & keine Intensitat & 2 & mittlere Intensitat \\
& geringe Intensitat & 3 & hohe intensitat \\
\hline
\end{tabular}

\begin{tabular}{|c|c|c|c|c|c|c|c|c|c|}
\hline & \multirow{2}{*}{\multicolumn{2}{|c|}{$\begin{array}{l}\text { Sachwerte } \\
(-1 \cdot)\end{array}$}} & \multirow{2}{*}{\multicolumn{2}{|c|}{\begin{tabular}{|l} 
Infrastruktur \\
$(-2)$.
\end{tabular}}} & \multirow{2}{*}{\multicolumn{2}{|c|}{\begin{tabular}{|l} 
Naturwerte \\
$(3)$.
\end{tabular}}} & \multicolumn{3}{|c|}{ Schutzziel } \\
\hline & & & & & & & $30 \%$. & $100 \%$ & $300-4$ \\
\hline \multirow[t]{6}{*}{1} & & $\begin{array}{l}\text { Standortgebundone } \\
\text { Bauten, exkd. } \\
\text { Sondernsiken }\end{array}$ & 121 & $\begin{array}{l}\text { Woge 6. Klosse (LK 6. Klasse) } \\
\text { gemass Landeskarte, z. B. } \\
\text { Wanderwege, Skipisten und } \\
\text { Lopen. } \\
\text { Maschinerwege (LK 5. Klasse), } \\
\text { Ski- und Berglourenrouten } \\
\text { (gemass SAC-Karten usw.). }\end{array}$ & 131 & Naturtandschaften & 3 & 3 & 3 \\
\hline & & & 423 & $\begin{array}{l}\text { Tounistische } \\
\text { Personenbeforderungs-anlagen } \\
\text { (Benutzer) }\end{array}$ & 132 & Bachgerinne & 3 & 3 & 3 \\
\hline & & & 524 & Campingzonen (Benutzen) & 133 & Gewasser & 3 & 3 & 3 \\
\hline & & & 525 & $\begin{array}{l}\text { Grünanlagen, Golplatze und } \\
\text { Pake (BZ) }\end{array}$ & 134 & Odland & 3 & 3 & 3 \\
\hline & & & 617 & $\begin{array}{l}\text { Umgebung Zone offentl. Anlege } \\
\text { ohne Hochbauton }\end{array}$ & 135 & $\begin{array}{l}\text { Wald mit allgemener } \\
\text { Schueftunition (exkl. } \\
\text { Vorrangfunktion } \\
\text { Holzproduation, } \\
\text { Nutzniesser) }\end{array}$ & 3 & 3 & 3 \\
\hline & & & & & 136 & $\begin{array}{l}\text { Freihate- } \\
\text { Reservezone }\end{array}$ & 3 & 3 & 3 \\
\hline 2 & गा1 & 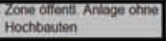 & 221 & $\begin{array}{l}\text { Kleine Wald und Furstrasse (LK } \\
\text { 4. Klasse) }\end{array}$ & & & 2 & 3 & 3 \\
\hline & & & 321 & $\begin{array}{l}\text { Grosse Wala und Furstrasso (IIK) } \\
\text { 3. Klasse) }\end{array}$ & & & 2 & 3 & 3 \\
\hline & & & 613 & & & & 2 & 3 & 3 \\
\hline & & & 616 & $\begin{array}{l}\text { Umgobung Zone offentl. Anloge } \\
\text { mat Hochbouten }\end{array}$ & & & 2 & 3 & 3. \\
\hline \multirow[t]{5}{*}{3} & & & $6 \pi 1$ & Umpebung Dord-Kemzonen & 231 & $\begin{array}{l}\text { Extansiv bewrisch } \\
\text { Landwirtschafts land } \\
\text { (Weide-(Abliachen) }\end{array}$ & 1 & 2 & 3 \\
\hline & & & 612 & $\begin{array}{l}\text { Umgebung Eintamilienhaus: } \\
\text { Zweifanilienhouszonen }\end{array}$ & 232 & $\begin{array}{l}\text { Waid mit erhohter } \\
\text { Schutzfunition } \\
\text { (Nutznesser) }\end{array}$ & 1 & 2 & 3 \\
\hline & & & 814 & Umgobung Gowerbezonen & & $\begin{array}{l}\text { Rccorboutland } \\
\text { (Fruchidolge-flachen) }\end{array}$ & 1 & 2 & 3 \\
\hline & & & 615 & $\begin{array}{l}\text { Umgebung industrie- und } \\
\text { Gewerbezonen }\end{array}$ & 332 & $\begin{array}{l}\text { Intensiv bewritsch. } \\
\text { Landwintschatts-land }\end{array}$ & 1 & 2 & 3 \\
\hline & & & & & 233 & Holzprodustions-waik & 1 & 2 & 3 \\
\hline \multirow[t]{5}{*}{4} & 311 & $\begin{array}{l}\text { abripe nicht bewohnte } \\
\text { Einzolgoboudo (Wiphimion } \\
\text { Kleinstalle, Remisen, } \\
\text { Gadeh etc) }\end{array}$ & 222 & $\begin{array}{l}\text { Lehungen von kommunaler } \\
\text { Bedeutung (Benutzor) }\end{array}$ & & & 1 & 1 & 2 \\
\hline & 4121 & $\begin{array}{l}\text { periodisch bewohrite } \\
\text { Einzelgebdude }\end{array}$ & 322 & $\begin{array}{l}\text { Letungen von kantonoler } \\
\text { Bedeutung (Benutzer) }\end{array}$ & & & 1 & 1 & 2 \\
\hline & & & 412 & $\begin{array}{l}\text { Umgebung periodisch bewohnter } \\
\text { Einzelgeboue }\end{array}$ & & & 1 & 1 & 2 \\
\hline & & & & & & & 1 & 1 & 2 \\
\hline & & & 422 & $\begin{array}{l}\text { Leltungen von nationaler } \\
\text { Bedeutung (Benutzer) }\end{array}$ & & & 1 & 1 & 2 \\
\hline \multirow[t]{4}{*}{5} & & grosse Okonomingebaudit & 473 & $\begin{array}{l}\text { Umigebung grosser } \\
\text { Okonomiegebnude }\end{array}$ & 383 & $\begin{array}{l}\text { Wald mit besonderer } \\
\text { Schutatunition } \\
\text { (Nutzriessen) }\end{array}$ & 0 & 1 & 2 \\
\hline & 6131 & $\begin{array}{l}\text { Mohrfamilionhauszzonen } \\
\text { (Bewohner) }\end{array}$ & 521 & Auntobahn-strasso (Benutzerf) & & & 0 & 1 & 2 \\
\hline & 6161 & $\begin{array}{l}\text { Zone offentl. Anlage mit } \\
\text { Hochoution }\end{array}$ & 522 & $\begin{array}{l}\text { national bedeutende } \\
\text { Vektehrswege (LK 1. Klasse) } \\
\text { (Benutber) }\end{array}$ & & & 0 & 1 & 2 \\
\hline & & & 523 & Bahnstrecken und Tunnelportale & & & 0 & 1 & 2 \\
\hline \multirow[t]{5}{*}{6} & 4111 & $\begin{array}{l}\text { desuend bewohnto } \\
\text { Einzelgebevode }\end{array}$ & $4 \pi 1$ & $\begin{array}{l}\text { Umgorung douernd beworintar } \\
\text { Einzelgeboude }\end{array}$ & & & 0 & 0 & 1 \\
\hline & 611 & $\begin{array}{l}\text { Dor-Kemzonen } \\
\text { (Bowohner) }\end{array}$ & & & & & 0 & 0 & 1 \\
\hline & 6121 & $\begin{array}{l}\text { Eintambienhaus-1 } \\
\text { Zwerfamilienhouszonen }\end{array}$ & & & & & 0 & 0 & 1 \\
\hline & 6141 & $\begin{array}{l}\text { Gewerbebauten } \\
\text { (Benutzer) }\end{array}$ & & & & & 0 & 0 & 1 \\
\hline & 6151 & $\begin{array}{l}\text { Industriebouten } \\
\text { (Benutzer) }\end{array}$ & & & & & 0 & 0 & 1 \\
\hline
\end{tabular}

Verschiebungen autgrund Bewertung Objekandex 


\section{XIV.II Version ,Eigentümer’}

Fallbeispiel Engelberg, Kanton Obwalden modifizierte Objektkategorieeinteilung 2 aufgrund Objektindex aus Sicht Eigentümer

\begin{tabular}{|c|c|c|c|}
\hline \multicolumn{4}{|c|}{ zulassige Intensitaten gem. Schutzzielen } \\
\hline 0 & keine Intensităt & $\overline{2}$ & mittlere Intensitat \\
\hline$T$ & geringe Intensităt & $\sqrt{3}$ & hohe Intensität \\
\hline
\end{tabular}

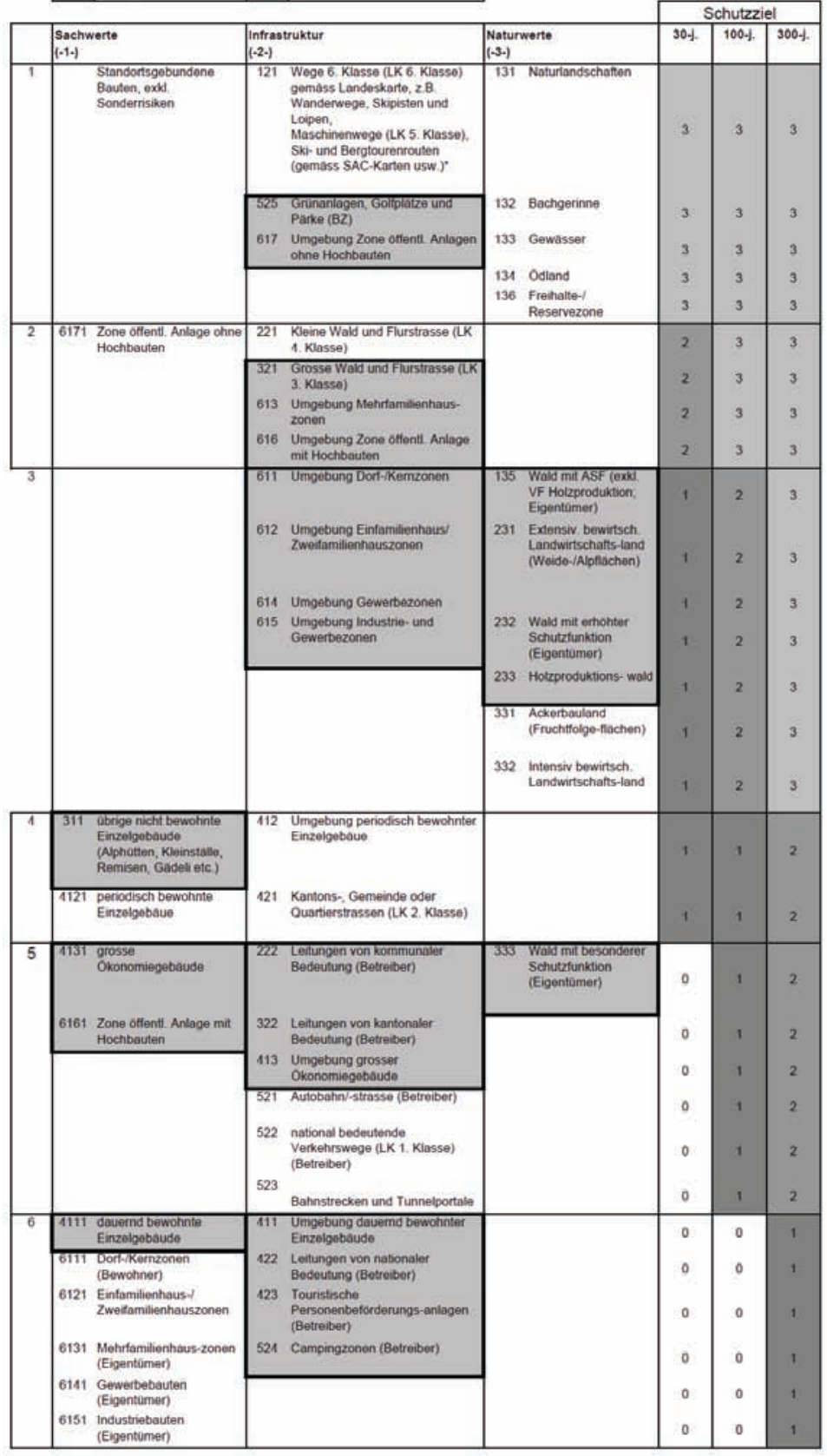

Verschiebungen autgrund Bewertung Obiektindex 


\section{XIV.III Version ,individuelles Risiko'}

Fallbeispiel Engelberg, Kanton Obwalden modifizierte Objektkategorieeinteilung 3 aufgrund individueller Risiken

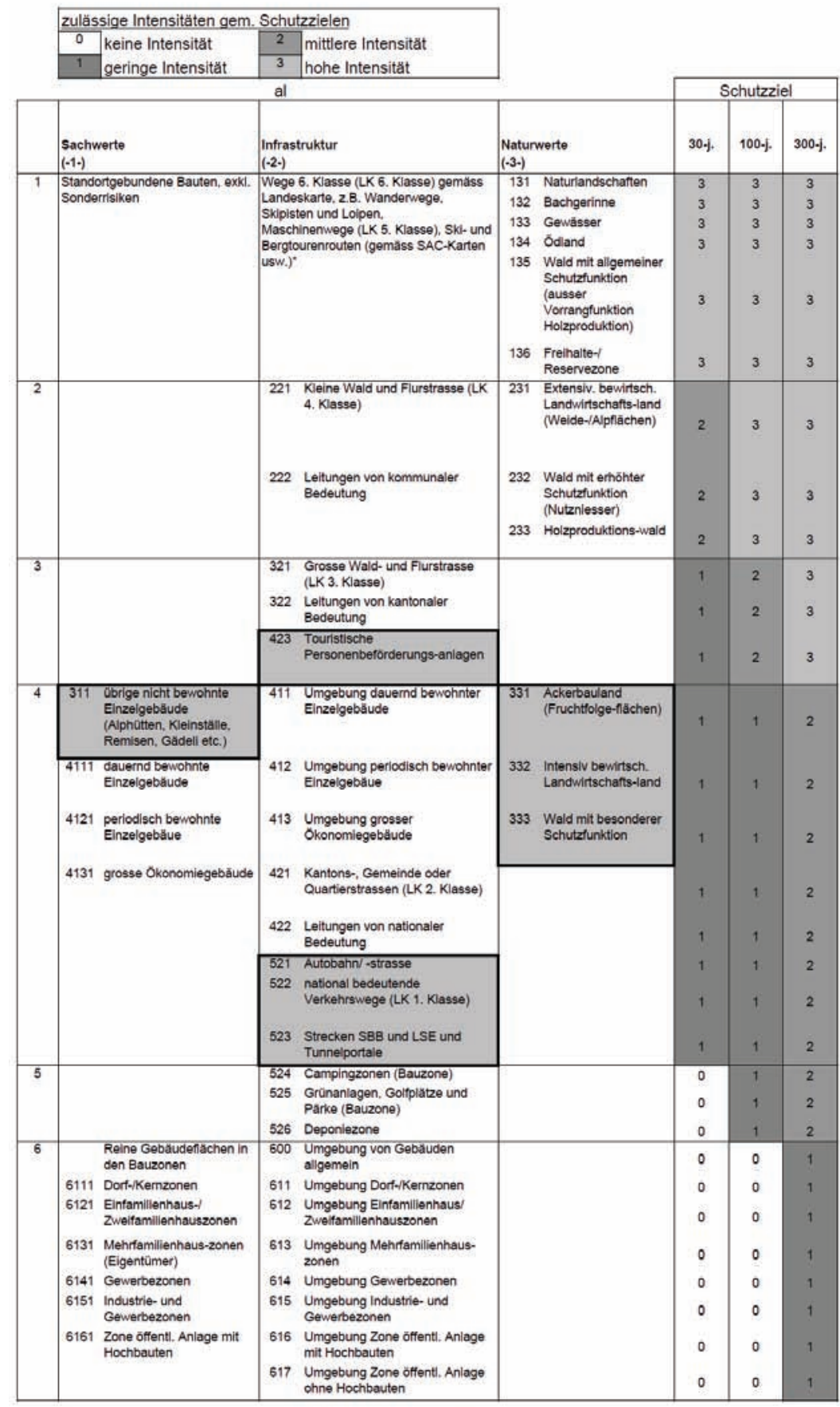

Verschiebungen aufgrund individueller Risiken 


\section{XIV.IV Vergleich der Versionen}

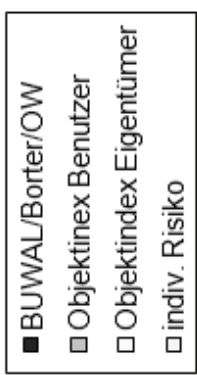

엄
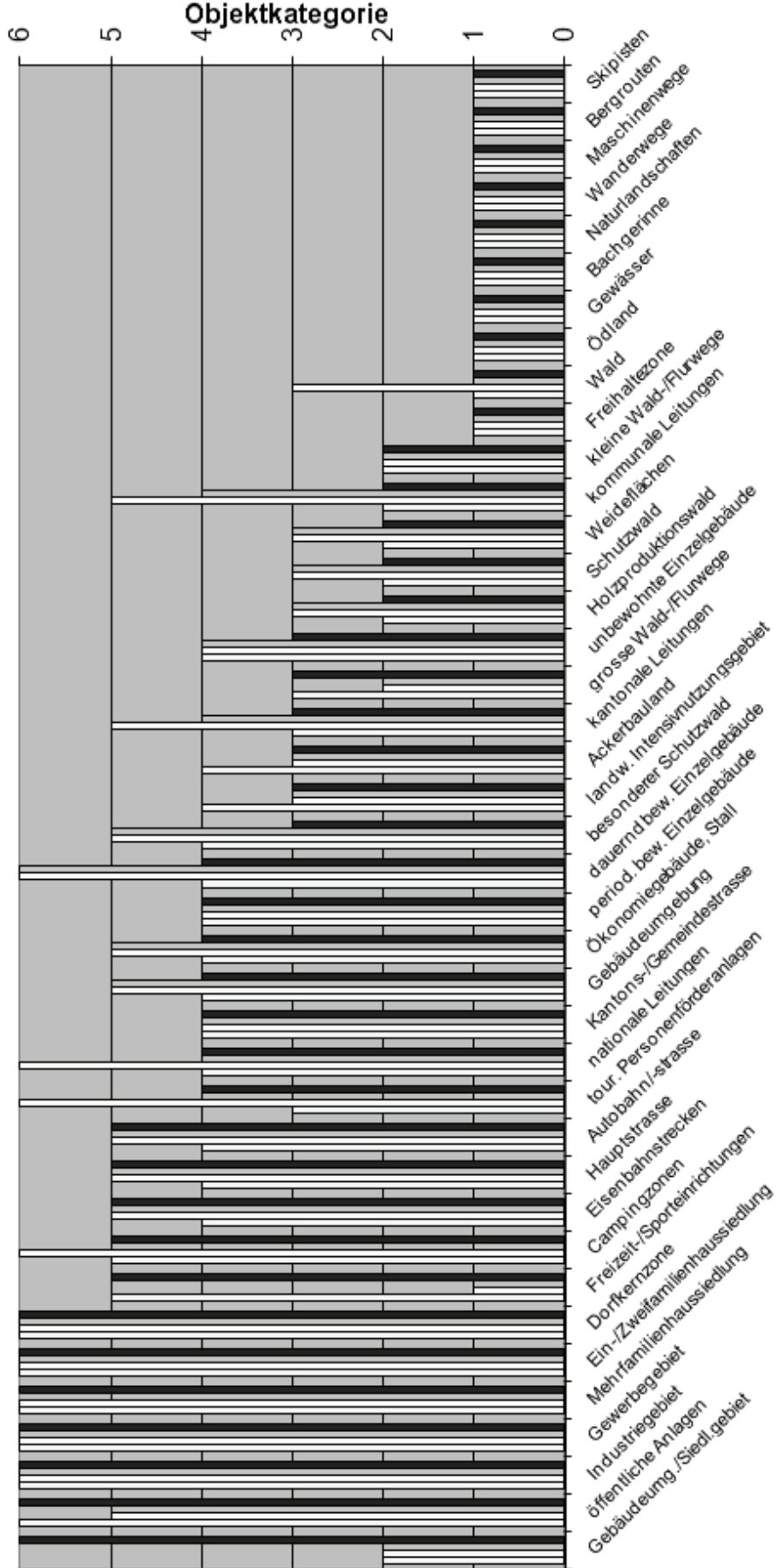


\section{Fallbeispiel Engelberg}

\section{XV.I Projektpriorisierung Managementkonzept nach Gefahrenprozessen}

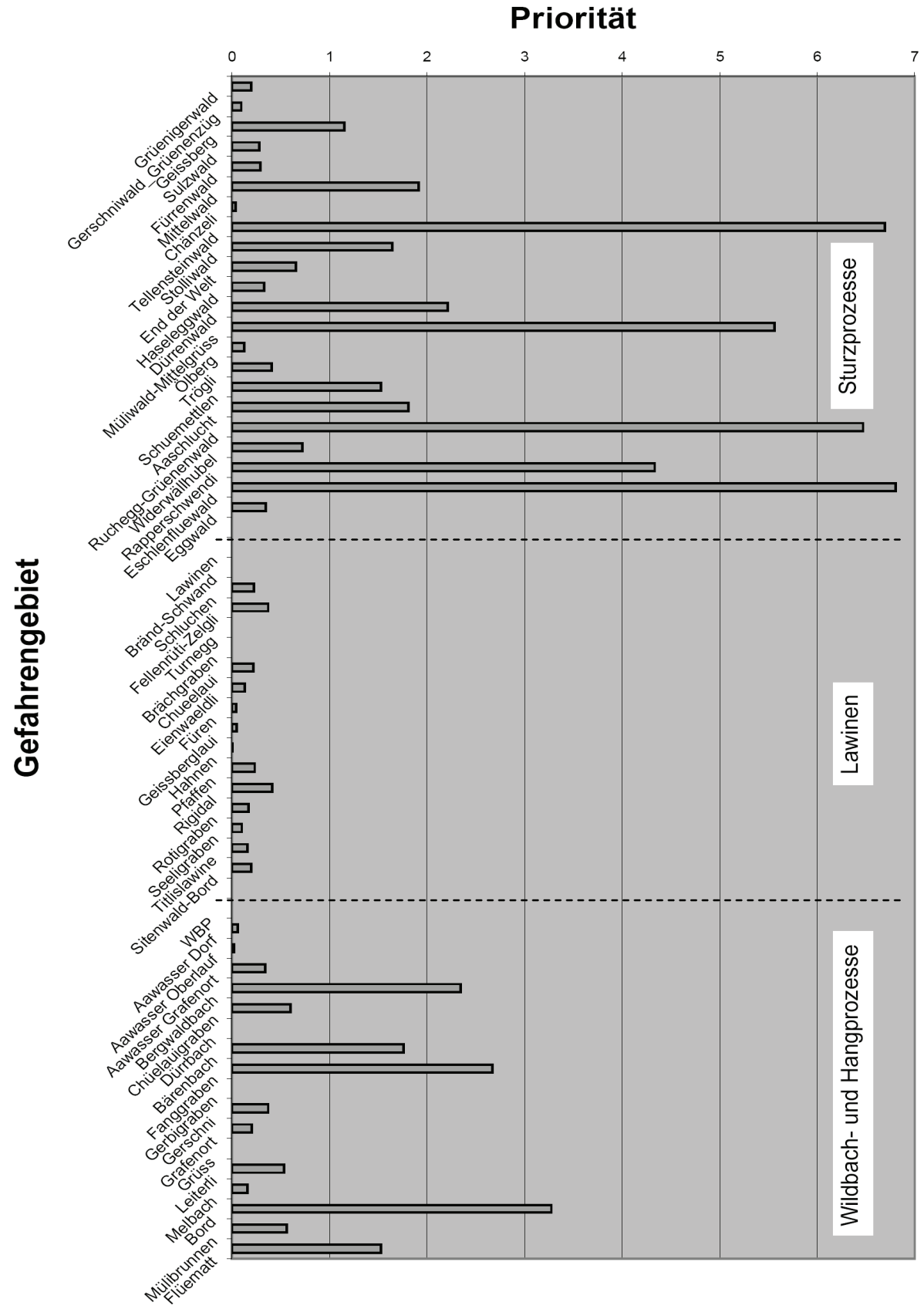




\section{XV.II Projektpriorisierung Managementkonzept rangiert}

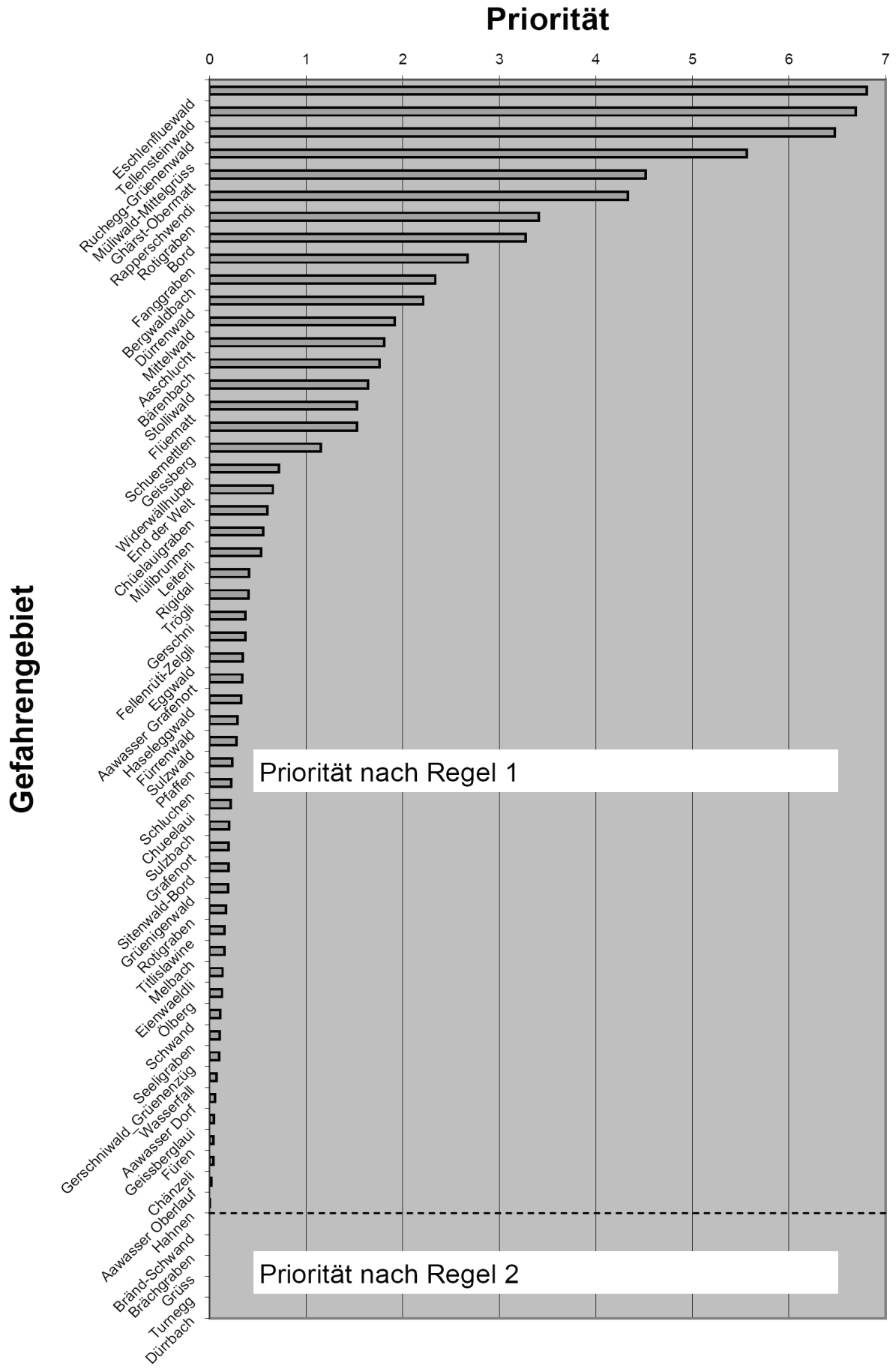




\section{Fallbeispiel Sarnenraa, Bewertung Projektvarianten}

\section{XVI.I Bewertung und Gewichtung durch Experten}

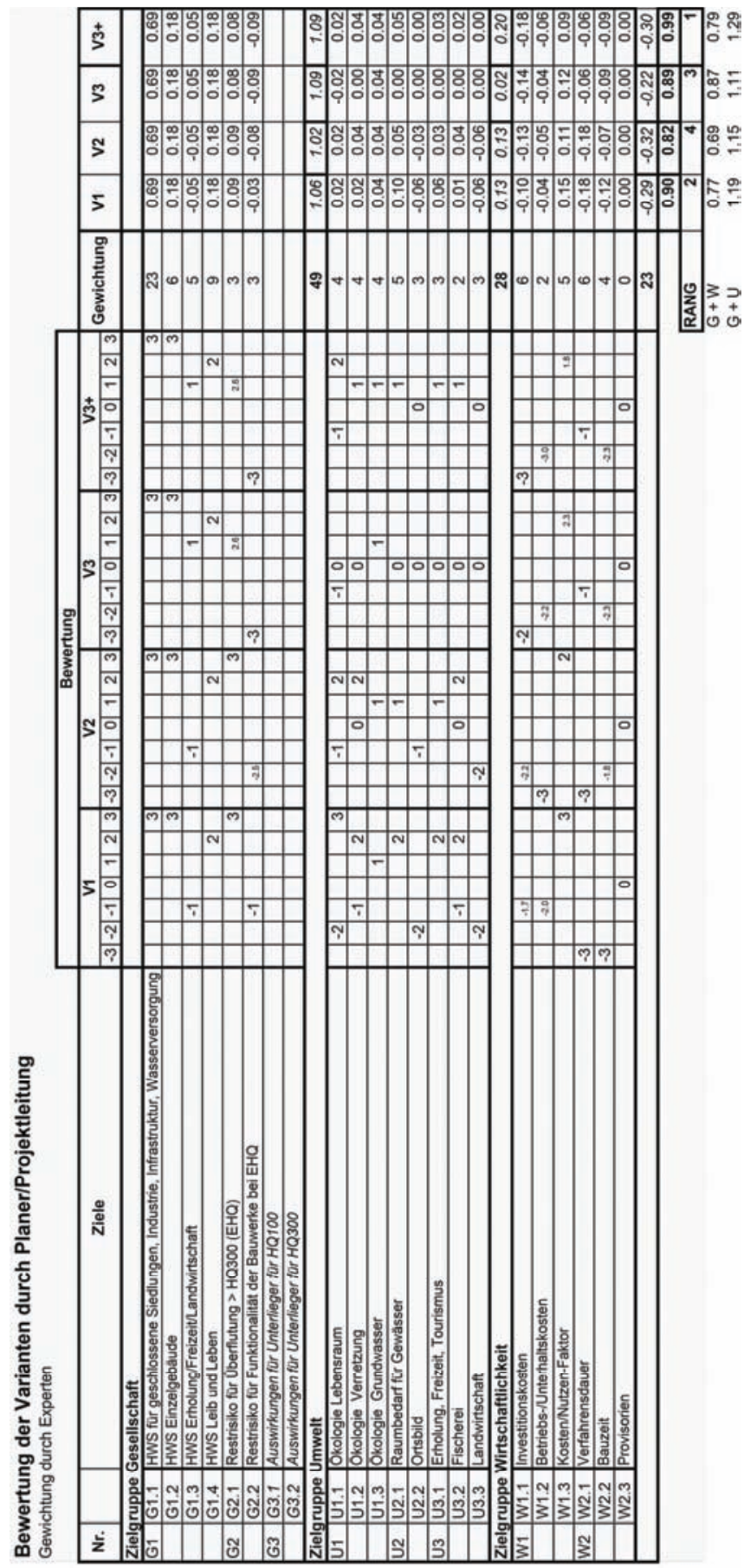


ISBN 978-3-7281-3282-6 (Print) ISBN 978-3-7281-3390-8

DOI Nr.: $10.3218 / 3390-8$ 